LBL-36079

\title{
Study of Radicals, Clusters and Transition State Species by Anion Photoelectron Spectroscopy
}

Don Wesley Arnold

Ph.D. Thesis

Department of Chemistry

University of California

and

Chemical Sciences Division

Lawrence Berkeley Laboratory

University of California

Berkeley, California 94720

August 1994

This work was supported in part by the Director, Office of Energy Research, Office of Basic Energy Sciences,

Chemical Sciences Division of the U.S. Department of Energy under Contract No. DE-AC03-76SF00098, and in part by the National Science Foundation. 


\title{
Study of Radicals, Clusters and Transition State Species by Anion Photoecelecron Spectroscopy
}

\author{
Copyright (C) 1994
}

by Don Wesley Arnold

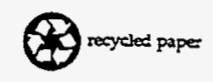

The U.S. Department of Energy has to right to use this thesis for any purpose whatsoever including the right to reproduce all or any part thereof 


\section{DISCLAIMER}

This report was prepared as an account of work sponsored by an agency of the United States Government. Neither the United States Government nor any agency thereof, nor any of their employees, make any warranty, express or implied, or assumes any legal liability or responsibility for the accuracy, completeness, or usefulness of any information, apparatus, product, or process disclosed, or represents that its use would not infringe privately owned rights. Reference herein to any specific commercial product, process, or service by trade name, trademark, manufacturer, or otherwise does not necessarily constitute or imply its endorsement, recommendation, or favoring by the United States Government or any agency thereof. The views and opinions of authors expressed herein do not necessarily state or reflect those of the United States Government or any agency thereof. 


\section{DISCLAIMER}

Portions of this document may be illegible in electronic image products. Images are produced from the best available original document. 


\begin{abstract} by

Don Wesley Arnold

\author{
Doctor of Philosophy in Chemistry \\ University of California at Berkeley
}

Professor Daniel M. Neumark, Chair
\end{abstract}

Study of Radicals, Clusters and Transition State Species by Anion Photoelectron Spectroscopy

Free radicals, elemental and van der Waals clusters and transition state species for bimolecular chemical reactions are investigated using anion photoelectron spectroscopy. Several low-lying electronic states of ozone have been identified via photoelectron spectroscopy of $\mathrm{O}_{3}^{-}$. A characterization of these states is important to models for atmospheric ozone reaction kinetics. The fluoroformyloxyl radical, $\mathrm{FCO}_{2}$, has been investigated, providing vibrational frequencies and energies for two electronic states. The technique has also been employed to make the first direct observation and characterization of the $\mathrm{NNO}_{2}$ molecule. Several electronic states are observed for this species which is believed to play a role as a reactive intermediate in the $\mathrm{N}+$ $\mathrm{NO}_{2}$ reaction. The experimental results for all three of these radicals are supplemented by $a b$ initio investigations of their molecular properties.

The clusters investigations include studies of elemental carbon clusters $\left(C_{2}^{-}-C_{11}^{-}\right)$, and van der Waals clusters $\left(\mathrm{X}^{-}\left(\mathrm{CO}_{2}\right)_{\mathrm{n}}, \mathrm{X}=\mathrm{I}, \mathrm{Br}, \mathrm{Cl} ; \mathrm{n} \leq 13\right.$ and $\left.\mathrm{I}^{-}\left(\mathrm{N}_{2} \mathrm{O}\right)_{\mathrm{n}=1-11}\right)$. Primarily linear clusters are observed for the smaller carbon clusters, while the spectra of the larger clusters contain contribution. from cyclic anion photodetachment. Very interesting ion-solvent interactions are observed in the $\mathrm{X}^{-}\left(\mathrm{CO}_{2}\right)_{\mathrm{n}}$ clusters.

The transition state regions for several bimolecular chemical reactions have also been investigated by photodetachment of a negative ion precursor possessing a geometry similar to 
that of the transition state species. These spectra show features which are assigned to motions of the unstable neutral complex existing between reactants and products. Photodetachment of $\mathrm{FH}_{2}^{-}$ provides a very sensitive probe of the accuracy of proposed $\mathrm{F}+\mathrm{H}_{2} \rightarrow \mathrm{FH}+\mathrm{H}$ potential surfaces in the transition state region. Comparison with three-dimensional quantum simulations indicate that the new ab initio SW potential energy surface is very accurate. The transition state regions of the $\mathrm{O}+\mathrm{H}_{2} \mathrm{O}$ and $\mathrm{OH}+\mathrm{H}_{2} \mathrm{O}$ reactions are investigated by photodetachment of the $\mathrm{O}^{-}\left(\mathrm{H}_{2} \mathrm{O}\right)$ and $\mathrm{H}_{3} \mathrm{O}_{2}^{-}$anions. Ab initio calculated $v_{3}$ potential curves are used to model the data in a FranckCondon analysis. Photoelectron spectra of the $\mathrm{IH}^{-}(\mathrm{M})_{\mathrm{n}}$ anions $\left(\mathrm{M}=\mathrm{CO}_{2}, \mathrm{~N}_{2} \mathrm{O} ; \mathrm{n} \leq 5\right)$ provide information about reaction dynamics in clusters and how they differ from gas phase dynamics. 
Table of Contents

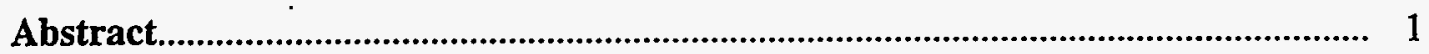

Dedication ............................................................................................................. iii

Table of Contents........................................................................................................ iv

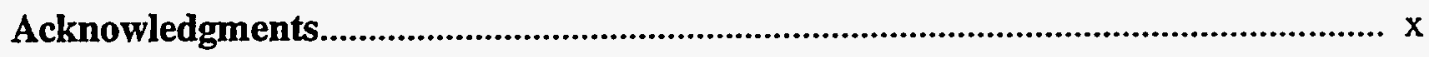

Chapter 1. Introduction

1. Applications of Anion Photoelectron Spectroscopy............................................... 1

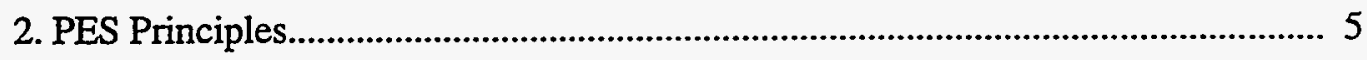

2.1 Bound Molecules............................................................................................... 5

2.2 Transition States, Dissociative States and Reactive Intermediates.................... 8

3. Experimental Technique......................................................................................... 12

Chapter 2. Study of the Low-Lying Electronic States of Ozone by Photodetachment of $\mathrm{O}_{3}^{-}$

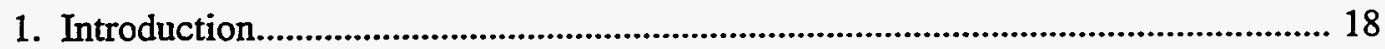

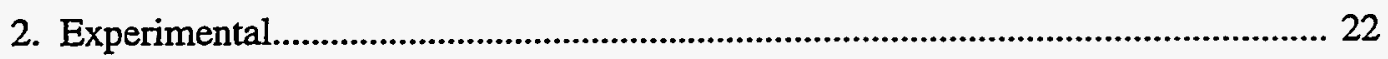

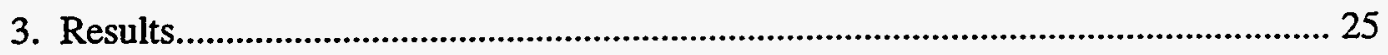

4. Analysis and Discussion..................................................................................... 32

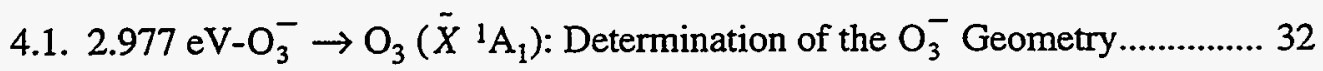

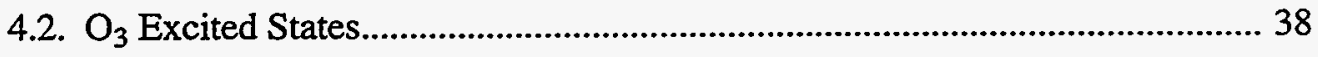

4.2.1. Assignment of the $\mathrm{O}_{3}$ Excited Electronic States................................... 38

4.2.2. $\mathrm{O}_{3}$ Excited State Dissociation Dynamics............................................. 43

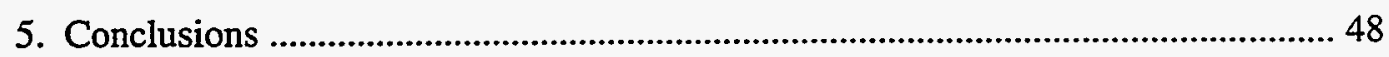

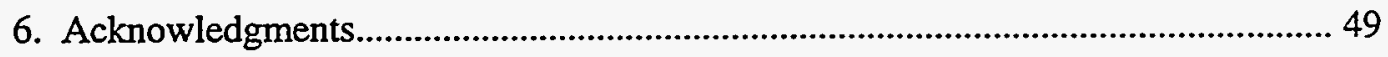


Chapter 3. Study of $\mathrm{N}_{2} \mathrm{O}_{2}$ by Photodetachment of $\mathrm{N}_{2} \mathrm{O}_{2}^{-}$

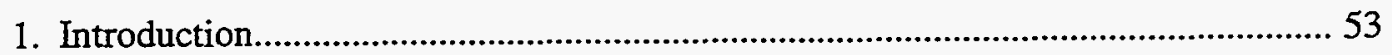

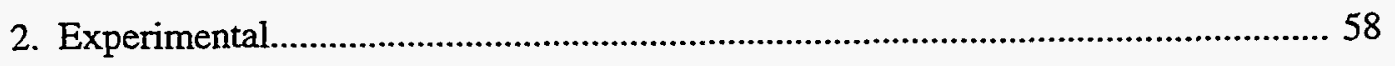

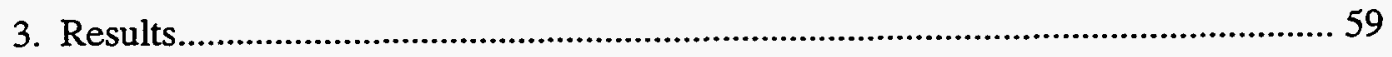

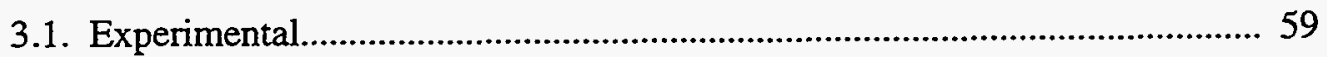

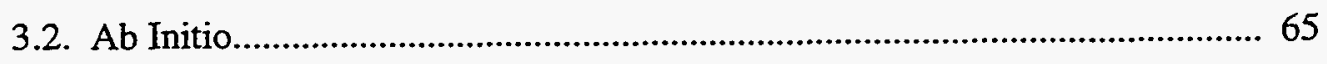

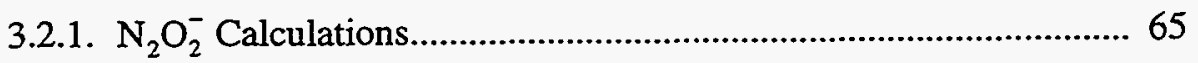

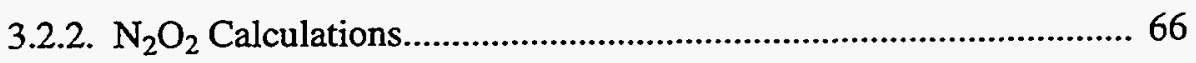

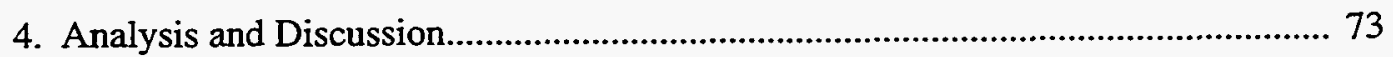

4.1. Franck-Condon Analysis ......................................................................... 74

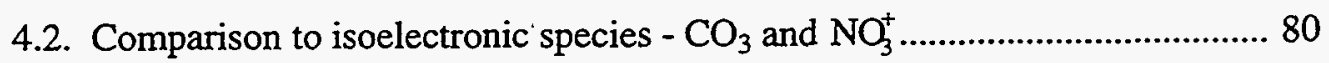

4.3. Roles as reactive intermediates.......................................................... 82

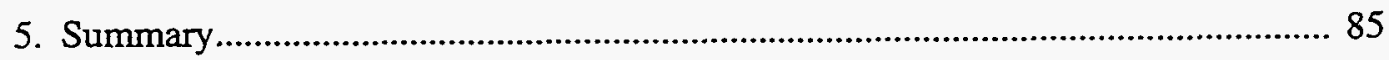

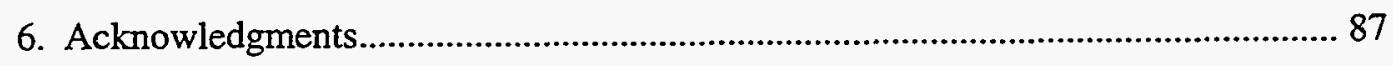

\section{Chapter 4. Vibrationally Resolved Spectra of $C_{2}-C_{11}$ by Anion}

\section{Photoelectron Spectroscopy}

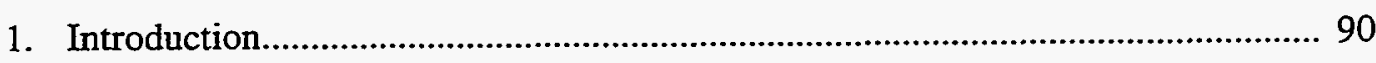

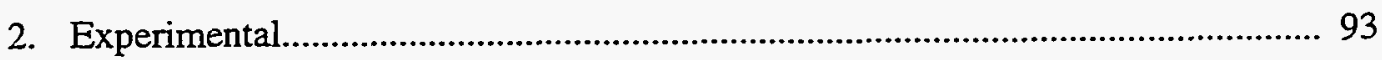

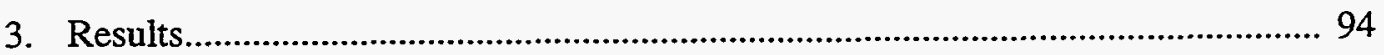

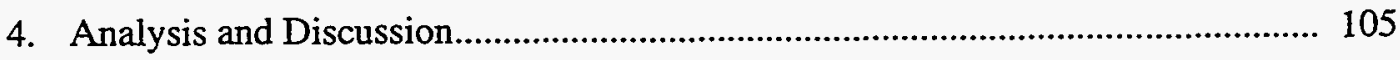

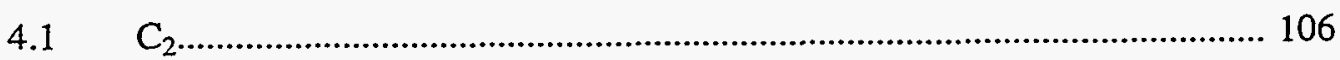

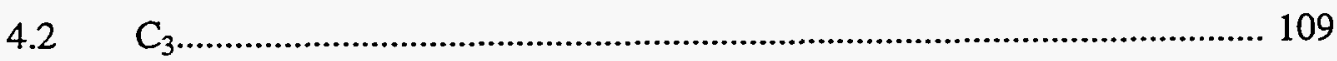

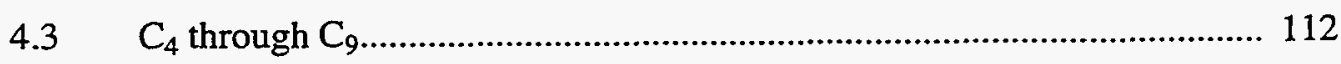

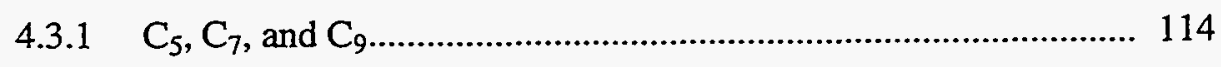

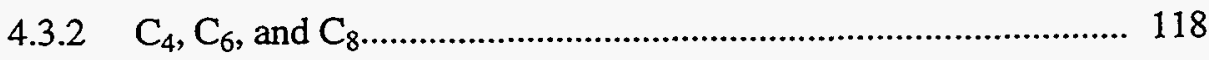

$4.4 \quad \mathrm{C}_{10}, \mathrm{C}_{11}$, and Nonlinear Anion Photodetachment............................... 121 


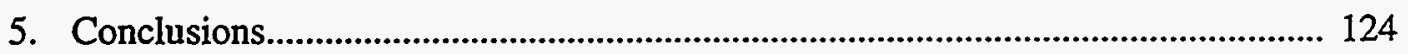

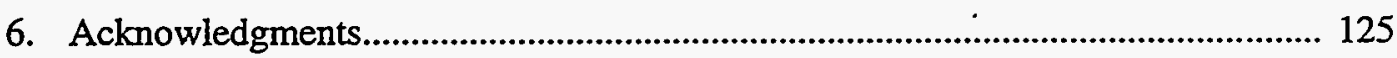

Chapter 5. Study of the Fluoroformyloxyl Radical and Halogen-Carbon

Dioxide Clusters by Photodetachment of $\mathrm{FCO}_{2}^{-}$and

$\mathrm{X}^{-}\left(\mathrm{CO}_{2}\right)[\mathrm{X}=\mathrm{I}, \mathrm{Br}$, and $\mathrm{Cl}]$

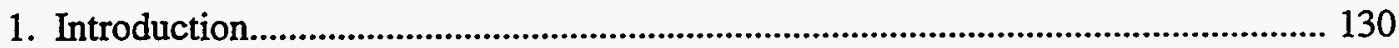

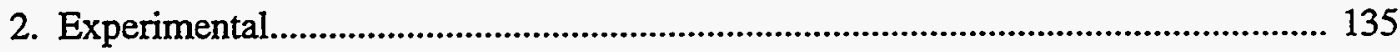

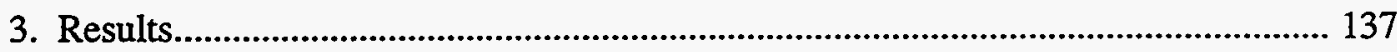

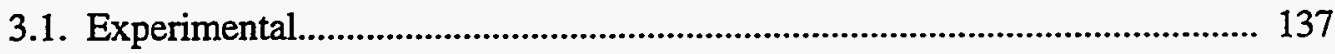

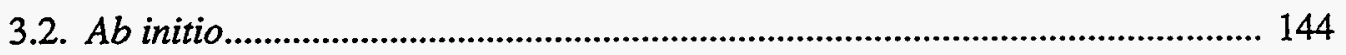

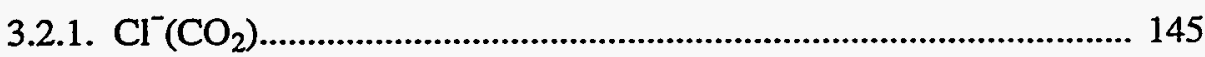

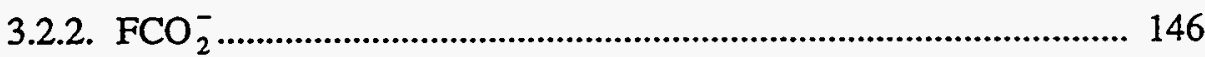

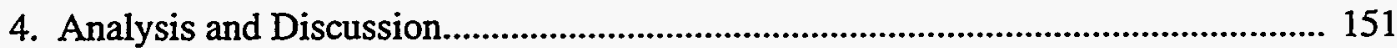

4.1. $\mathrm{X}=\mathrm{I}, \mathrm{Br}$, and $\mathrm{Cl}$

4.1.1. Electron Affinities and Thermochemistry................................ 151

4.1.2. Underlying Structure.................................................................. 154

4.1.3. Geometry and Bonding................................................................ 156

4.1.4. Electrostatic Models................................................................ 160

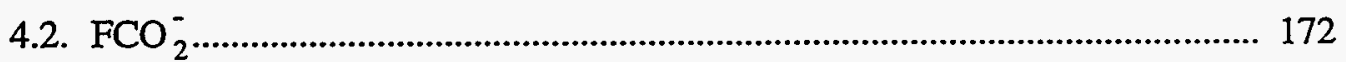

4.2.1. Spectroscopy and Franck-Condon Analysis............................. 172

4.2.2. Electron Affinity and Thermochemistry..................................... 181

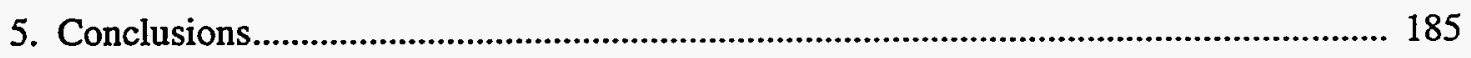

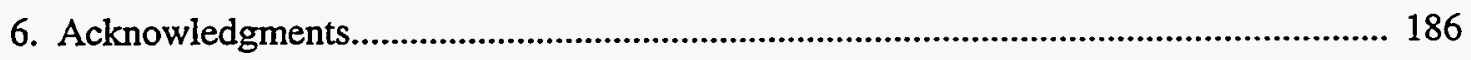


Chapter 6. Study of $\mathrm{I}^{-}\left(\mathrm{CO}_{2}\right)_{\mathrm{n}}, \mathrm{Br}^{-}\left(\mathrm{CO}_{2}\right)_{\mathrm{n}}$, and $\mathrm{I}^{-}\left(\mathrm{N}_{2} \mathrm{O}\right)_{\mathrm{n}}$ Clusters by Anion

\section{Photoelectron Spectroscopy}

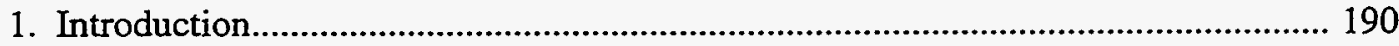

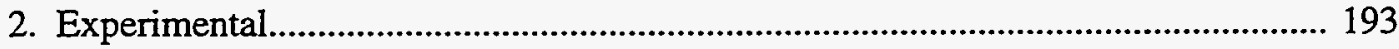

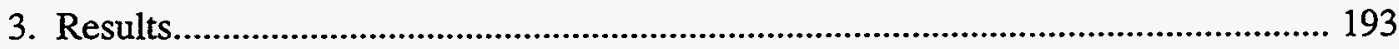

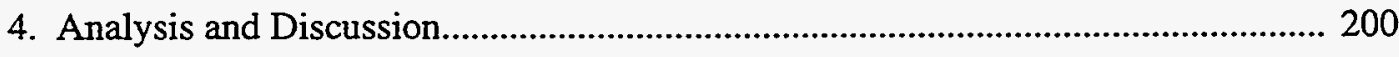

4.1. Thermodynamics and Geometries........................................................... 200

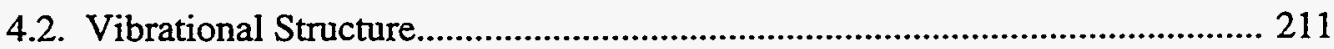

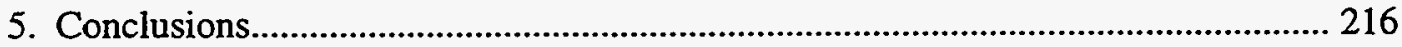

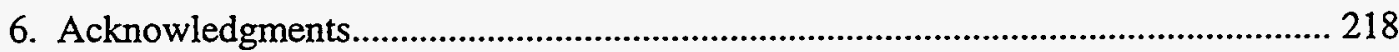

\section{Chapter 7. Study of the $\mathrm{F}+\mathrm{H}_{2}$ Transition State by Photodetachment of $\mathrm{FH}_{2}^{-}$}

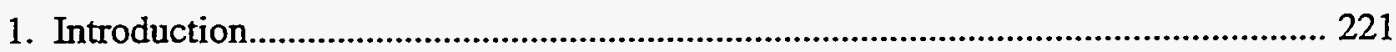

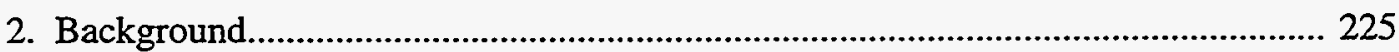

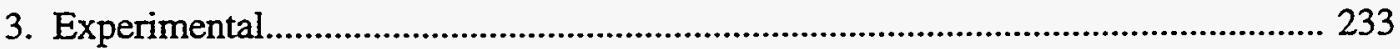

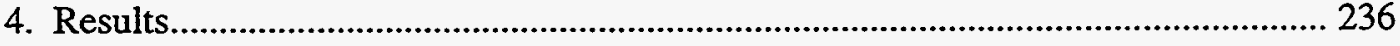

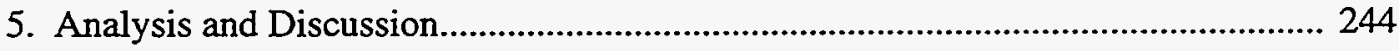

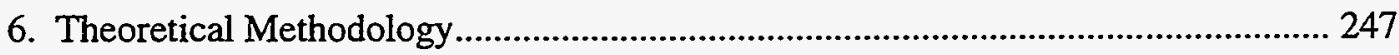

6.1. Calculation of $A b$ initio Potential Surface................................................. 247

6.2. Calculation of Franck-Condon Simulations.............................................. 248

7. Comparison between Theory and Experiment...................................................... 248

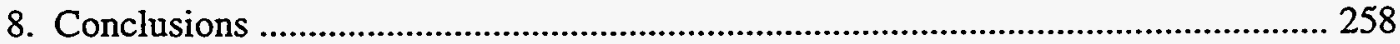

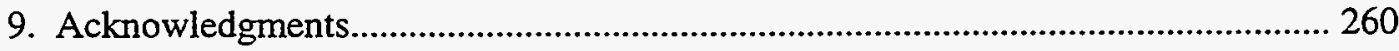


Chapter 8. Study of the Transition State Region of the $\mathrm{O}+\mathrm{H}_{2} \mathrm{O}$ and $\mathrm{OH}+\mathrm{H}_{2} \mathrm{O}$

Reactions by Photodetachment of $\mathrm{O}^{-}\left(\mathrm{H}_{2} \mathrm{O}\right)$ and $\mathrm{OH}^{-}\left(\mathrm{H}_{2} \mathrm{O}\right)$

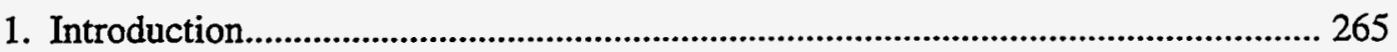

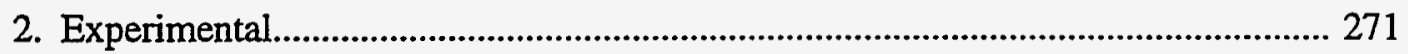

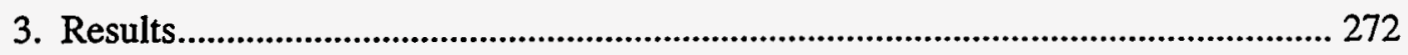

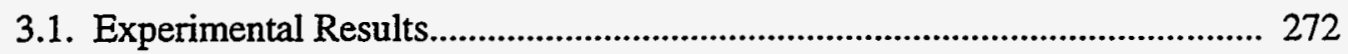

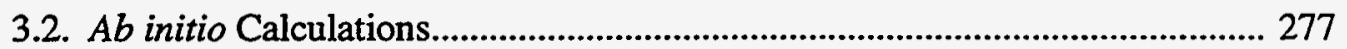

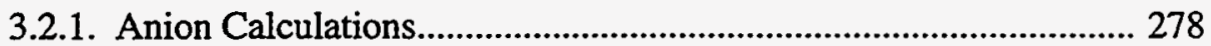

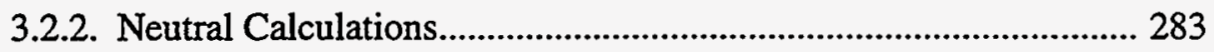

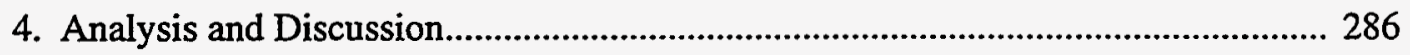

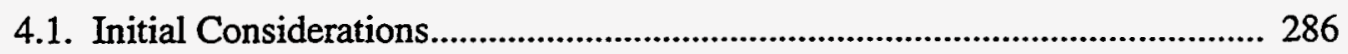

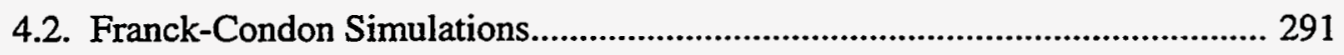

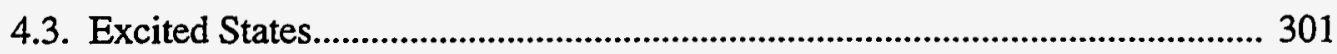

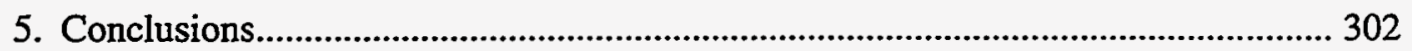

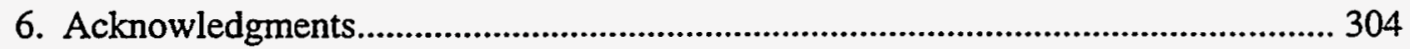

\section{Chapter 9. The Study of the I + HI Chemical Reaction Dynamics in Clusters by} Photodetachment of $\mathrm{IHI}^{-}(\mathrm{M})_{\mathrm{n}}\left\{\mathrm{M}=\mathrm{Xe}, \mathrm{N}_{2} \mathrm{O}\right.$, and $\left.\mathrm{CO}_{2}\right\}$

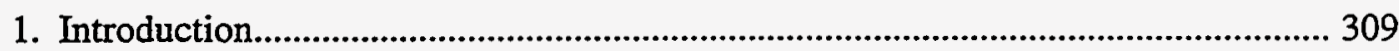

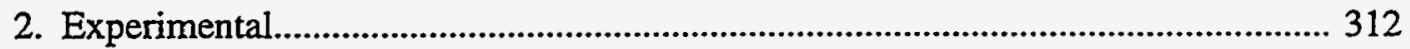

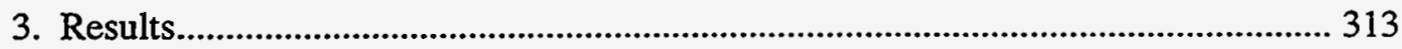

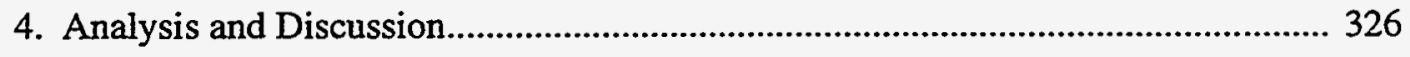

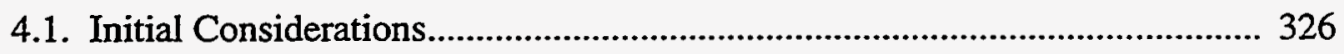

4.2. Change in Peak Spacings and Franck-Condon Simulations......................... 330

4.2.1. One-dimensional Simulations................................................... 331

4.2.2. Two-Dimensional Simulations........................................................ 335

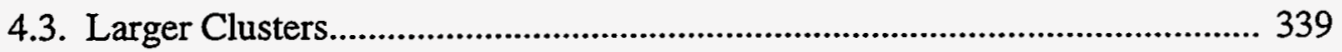


4.4. Feature "A" and Possible Anion Geometries.................................................. 342

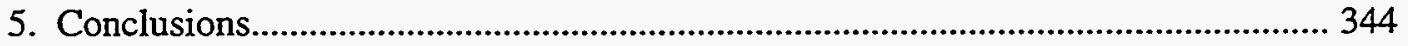

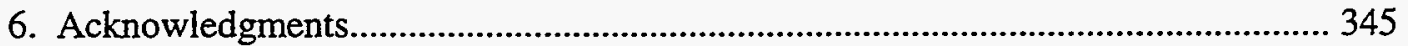

Appendix A. PES: The Franck-Condon Analysis Program for Anion

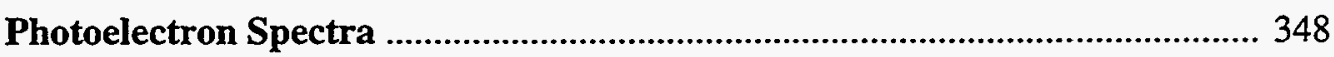

Appendix B. Publications from Graduate Work ................................................. 466 


\section{Acknowledgments}

Working in Professor Daniel Neumark's research group for the past several years has been a great experience. I offer my thanks to Dan for allowing me to work on such a flexible and productive experimental apparatus during my time at Berkeley. His enthusiasm and scientific drive have provided as an exemplary model for his graduate students. It is a really great feeling to have a supportive advisor who gets excited when your long hours turn into interesting results. Dan's advice, wisdom and direction have been an essential part of the projects to be presented in this dissertation.

Of course, there have been many co-workers that I must thank for providing an environment in which I have been able to learn so much and actually enjoy coming to D-level of Latimer Hall when the California sun was offering a variety of other activities. A great deal of credit goes to the former students in the group that built the photoelectron spectrometer: Dr. Ricardo Metz, Dr. Theofanis Kitsopoulos, and Dr. Alexandra Weaver. Thanks go to Ricky for development of the DVR codes (part of which is incorporated into the FCF code in Appendix A) and to Theo for his lively conversations. I hope to deliver my thanks to Theo in person some day. While Ricky and Theo went on to develop new experiments in Dan's group, Alex remained a Stonehenger and carried out part of the task of turning me into a 'trained photoelectron spectroscopist'. I hope I have achieved that title. I must also thank her for an invitation to the Telluride Workshop where I learned a great deal about atmospheric chemistry. The remainder of the training chore was relegated to Dr. Stephen E. Bradforth. I will recall the years of working with Steve as my most rewarding and enjoyable times at Berkeley. I hope to find a co-worker that is as enjoyable to work with as Steve again. An additional thanks goes to Steve for his superior performance as a best man and champion Zachary's eater.

Dr. Douglas R. Cyr joined the Dan's group the same time that I did and undertook the job of building Dan's third machine. It has been a pleasure to work with Doug and watch the FRBM grow to his size after many late nights with R.E.M. (the music not the sleep). I also would like to 
thank him, and Lejla, for letting us watch their two little girls (Ana and Jelena) grow up (at least for a couple of years). Dr. Robert Continetti came into the group to work on the same project but bought an expertise and gusto that profoundly influenced the entire group. Since his departure to San Diego and the land of the triple-coincidence experiments, Dr. David Leahy has brought a fresh approach to many problems, great advice and a passion for mexican food. DaveO has done a great job keeping the computers going and allocating space for my giant G92 CPU hogs.

The PES machine is abound with operators as I write. Eun Ha Kim has taken the helm to explore beyond $\mathrm{HCOO}$. Cangshan Xu will get that reflectron on the machine that I never managed to get built. Dr. Esther de Beer and Dr. Gordon Burton (our requisite tall Canadian) bring their fresh sciencific approaches to the project as postdocs. I wish them well and I am happy that Dan found someone who can replace (dare I say, improve) the bad jokes and puns that leave with me.

There are several other group members that deserve mention. Dr. Irene Waller served as my best double-play partner at Berkeley. Dr. Georg Reiser gave me wonderful advice about visiting the Grand Canyon. Good luck to Yuexhing and Ivan as they takeover the ZEKE apparatus and to Jeff who will someday run Dan's first ultrafast system. Of course, unlimited thanks go to Cheryn Gliebe for providing her experience, friendship and dosages of reality.

A particularly special thanks goes to the remaining member of Neumark Group (but not for much longer) - Caroline Chick Arnold (a.k.a. the ZEKE queen). Amazingly enough, after learning of my sports mania and often strange behavior, she still agreed to marry me. I am very grateful for all the love and support she has given, particularly during the hectic time of thesis preparation.

Additional thanks go to my preliminary exam committee: Prof. R. J. Saykally, Prof. H. S. Johnston, Prof. L. Moretto, and Prof. S. P. Davis. Special thanks go to Professor Johnston for an inspirational discussion before my second examination and to Professor Saykally for advice on a somewhat diverse set of topics. 
Tim Robinson has provided an invaluable amount of time helping to get some great color graphics generated and getting the $a b$ initio programs organized on the graphics facility computers. Harry Chiladakis provided very useful information about para- $\mathrm{H}_{\mathbf{2}}$ preparation and how to keep explosive gas mixtures under control.

Outside of the Berkeley environment, I would like to thank several people who have provided advice for the various projects described in this dissertation: Dr. David Manolopoulos, Professor Stuart Anderson, Dr. Krishnan Raghavachari, Dr. Matt Braunstein, and Dr. Russell Pack. I am grateful to the National Science Foundation for three years of financial support. I have to thank several people from my undergraduate years for allowing me participate in their research programs: Prof. Laura Philips and Prof. Paul Houston at Comell University and Dr. William Marinelli and Dr. Steve Davis at PSI, Inc (Andover, MA). The experience I gained in their programs provided much of my motivation to pursue physical chemistry at Berkeley. I was also very lucky to have very supportive high school teachers: Mr. and Mrs. Dalton, Ms.

Hutchisson, Mr. Rose, Ms. McClean, Mr. Jacobs, Mr. Keys all inspired me in their own ways to begin this journey and achieve as much as possible.

Many friends have played a key role in forming my experience at Berkeley. The UPTOP hoops crowd provided weekly (or more) physical punishment, particularly the salad days crowd, Andy, Tyler, Bas, Dave and DK. Karl Hanold has always provided an optimistic viewpoint - at least I only had to deal with Luciano for 4 or 5 hours (not 6 years). Rick and Avery were good friends and housemates. Thanks to the Disclexics for a champion's wardrobe.

Finally, I would like to thank my family. The support of my parents John and Audrey, grandparents Mary and Lawrence and brother Jon throughout the past 27 years has been a formative part of my direction and success. I would also like to thank my new in-laws Mock-nJock, Steve and Anne for welcoming me into their family.

This research was supported by the Air Force Office of Scientific of Scientific Research under Contract No. AFOSR-91-0084 and Contract No. F49620-94-1-0115.

This work was supported under the Director, Office of Energy Research, Office of Basic Energy Sciences, Chemical Sciences Division of the U. S. Department of Energy under Contract No. DE-AC03-76SF00098. 


\section{Chapter 1. Introduction}

\subsection{Applications of Anion Photoelectron Spectroscopy}

The following chapters describe experiments using anion photoelectron spectroscopy to study several different chemical systems. They illustrate the capability of the method to provide new and important results in several sub-fields of chemistry. As will be seen, the experiments provide new information about systems that have been very difficult to study by conventional techniques. The studies involve a variety of chemical systems including free radicals (Chapters 2, 3, and 5), elemental clusters (Chapter 4), van der Waals clusters (Chapters 5,6 , and 9) and transition state species of bimolecular chemical reactions (Chapters 7 - 9).

The relatively turbulent conditions of most radical (discharge, photolysis, etc.) and cluster (laser ablation, supersonic molecular beam, etc.) sources produce a wide range of species, charged and uncharged. Thus, in addition to the target species many other chromophores can contribute to the data obtained by conventional spectroscopic methods. Often, emission from (or absorption by) several species in such a mixture can complicate a spectrum beyond interpretation.

For many species, anion photoelectron spectroscopy can circumvent these problems. Free radicals, by their open-shell nature, typically have electron affinities that are on the order of a few electron-volts (eV). Thus, the stable negative ion corresponding to the radical of interest can be easily generated (usually), mass-selected and photodetached by a laser in the visible or near-UV wavelength range. By this method, one is able to isolate the molecule of interest and investigate its electronic and vibrational properties.

Another advantage offered by anion photoelectron spectroscopy, in addition to the combined mass selectivity and moderate spectral resolution $\left(\sim 65 \mathrm{~cm}^{-1}\right)$, is the capability to observe transitions to electronic states that are forbidden in direct absorption or emission experiments (a. k. a. dark states). This capability results from the one-electron detachment 
propensity rule for photoelectron spectroscopy. ${ }^{1}$ As an example, consider the case of $\mathrm{O}_{3}^{-}$ photodetachment to be discussed in Chapter 2. Equation (1) shows the first several photodetachment transitions from the ozonide anion ground electronic state,

$$
\mathrm{O}_{3}^{-} \tilde{\mathrm{X}}^{2} \mathrm{~B}_{1}\left(\ldots 1 b_{2}^{2} 6 a_{2}^{2} 4 b_{2}^{2} 1 a_{2}^{2} 2 b_{1}^{1}\right) \stackrel{h v}{\longrightarrow} e^{-}+\mathrm{O}_{3} \begin{aligned}
& \left(\ldots 1 b_{1}^{2} 6 a_{1}^{2} 4 b_{2}^{2} 1 a_{2}^{2} 2 b_{1}^{0}\right)-{ }^{1} \mathrm{~A}_{1} \\
& \left(\ldots 1 b_{1}^{2} 6 a_{1}^{2} 4 b_{2}^{2} 1 a_{2}^{1} 2 b_{1}^{1}\right)-{ }^{3} \mathrm{~B}_{2} l^{1} \mathrm{~B}_{2} \\
& \left(\ldots 16 a_{1}^{2} 4 b_{2}^{1} 1 a_{2}^{2} 2 b_{1}^{1}\right)-{ }^{3} \mathrm{~A}_{2} l^{1} \mathrm{~A}_{2} \\
& \left(\ldots 1 b_{1}^{2} 6 a_{1}^{1} 4 b_{2}^{2} 1 a_{2}^{2} 2 b_{1}^{1}\right)-{ }^{3} \mathrm{~B}_{1}{ }^{1} \mathrm{~B}_{1}
\end{aligned}
$$

Of these $\mathrm{O}_{3}$ electronic states that are accessible by $\mathrm{O}_{3}^{-}$photodetachment, all but the ${ }^{1} \mathrm{~B}_{2}$ and ${ }^{1} \mathrm{~B}_{1}$ states are 'dark states'. While conventional techniques had been used to investigate the electronic structure of the ozone molecule, much confusion remained about the several lowlying dark electronic states. The energetics of these states relative to the dissociation asymptote of the $\mathrm{O}_{3}$ molecule are quite important to atmospheric chemists since the existence of a bound excited electronic state could play a major role in ozone photodissociation/recombination kinetics. Several of these states have been identified and characterized by photoelectron spectroscopy of $\mathrm{O}_{3}^{-}$(Chapter 2).

Similarly, dark (and bright) states are observed for the $\mathrm{N}_{2} \mathrm{O}_{2}$ and $\mathrm{FCO}_{2}$ radicals in Chapters 3 and 5. In fact, the results in Chapter 3 mark the first experimental characterization of the $\mathrm{N}-\mathrm{NO}_{2}$ form of the $\mathrm{N}_{2} \mathrm{O}_{2}$ molecule.

Chapters 4 - 6 describe studies of elemental carbon clusters and van der Waals clusters. Although the cluster sources used in these experiments generate a broad distribution of clusters, mass-selection prior to spectroscopic interrogation removes any uncertainty about the species being investigated. In general, cluster studies aim to determine the evolution of chemical properties from the atomic to the bulk scale. ${ }^{2}$ Such studies can be made of both elemental clusters, where the atom-by-atom addition should eventually lead to the observation of the bulk 
elemental properties, and mixed clusters where observed solute-solvent interactions provide information about solvation processes.

Anion photoelectron spectroscopy has also been applied to a completely different field of chemistry - reaction dynamics. In the study of chemical reactions, one would ideally determine a fully characterized potential energy surface that governs the course of the reaction. ${ }^{3}$ With such a potential energy surface in hand it is possible to calculate rates of reaction, product state distributions, and a host of other observables. However, the accurate determination of the potential energy surface for a general reaction remains a formidable problem.

Perhaps the most important region of a reaction surface (and the most difficult to characterize) is the transition state region. This region governs the simultaneous bond rupture and formation which occurs as the reactants are converted into products. In fact, some reaction rate theories (i.e., transition state theory and its variants) ${ }^{4}$ are based upon the assumption that the saddle point of a potential surface represents the "point of no return" in a chemical reaction.

Until recently, the direct study of reactive 'transition state' species has been hampered by the very short lifetime of these complexes, which typically exist for only for a vibrational period or less. Results of asymptotic experiments have been extrapolated back to the transition state in attempts to characterize the transition state region of reactions. However, they do not provide a direct characterization of the transition state region. Accurate calculations are quite difficult for transition state regions since they require the inclusion of a large amount of electron correlation or configuration interaction using very large basis sets.

Techniques have been developed within the last decade to examine the transition state region directly. These methods study the transition state region by preparing the transition state species from a well-defined precursor molecule and observing the dissociation dynamics of the transient complex by various spectroscopic methods. With the development of 'ultrafast' lasers, one approach has been to study reaction dynamics in 'real time' using pump-probe techniques. 
The first pulse 'synthesizes' the transient species from the precursor molecule and the dissociation dynamics of the unstable complex are probed by a second, time-delayed laser pulse. Recent reviews exist for this method which has proven quite useful. 5

The approach used to obtain the results presented in Chapters $7-9$ is anion photoelectron spectroscopy of stable negative ions. In this technique the precursor, a negative ion, is photodetached to generate the transient species and the photoelectron spectrum provides information about the shape of the potential energy surface in the transition state region. Considering a simple hydrogen exchange between two species $A$ and $B$,

$$
\mathrm{AH}+\mathrm{B} \rightarrow \mathrm{A}+\mathrm{HB} \quad(2)
$$

one can imagine the reaction going through the $[\mathrm{A} \cdots \mathrm{H} \cdots \mathrm{B}]^{\ddagger}$ activated complex. As we have shown previously for several reactions of this type, the anion, $\mathrm{AHB}^{-}$, can exist in a geometry that is very similar to that which is expected for the transition state complex. As for bound molecules, the equilibrium geometry of the anion determines what region of the neutral reaction surface is accessible by photodetachment. Thus, if the $\mathrm{AHB}^{-}$species resembles the $[\mathrm{A} \cdots \mathrm{H} \cdots \mathrm{B}]^{\frac{1}{*}}$ transition state species, measurements of the photoelectron kinetic energy (eKE) distribution following photodetachment of $\mathrm{AHB}^{-}$provides information about the transition state region of the reaction shown in Equation (2).

The transition state regions of many bimolecular chemical reactions have been investigated using this technique. These include heavy-light-heavy hydrogen transfer reactions $(\mathrm{X}+\mathrm{HY} \rightarrow \mathrm{XH}+\mathrm{Y} ; \mathrm{X}, \mathrm{Y}=\mathrm{I}, \mathrm{Br}, \mathrm{Cl} \text {, and } \mathrm{F})^{6}$ and hydrogen abstraction reactions by fluorine $\left(\mathrm{OH}+\mathrm{F} \rightarrow \mathrm{O}+\mathrm{HF} ; \mathrm{RH}+\mathrm{F} \rightarrow \mathrm{R}+\mathrm{HF} ; \mathrm{R}=\mathrm{CH}_{3} \mathrm{O}, \mathrm{C}_{2} \mathrm{H}_{5} \mathrm{O}\right) .{ }^{7}$ Chapter 7 describes results for the prototypical $\mathrm{F}+\mathrm{H}_{2}$ reaction in which significantly improved data sets extend the results previously obtained by A. Weaver and S. E. Bradforth using the same apparatus. ${ }^{8,9,10}$ Chapter 8 discusses results that describe the transition state region of two fundamental hydroxyl radical 
reactions. Chapter 9 describes another extension of this technique where reaction dynamics in clusters are studied by photodetachment of solvated precursor anions, $\mathrm{IHI}^{-}(\mathrm{M})_{\mathrm{n}}$.

\subsection{PES Principles}

\subsubsection{Bound Molecules}

In the studies of molecules and clusters to be presented in Chapters 2 through 6 , the experiments involve the photodetachment of a stable negative ion, $\mathrm{X}^{-}$, using a photon of a known energy, hv, as illustrated by Equation (3) and Figure 1.1,

$$
\mathrm{X}^{-} \stackrel{\mathrm{hv}}{\longrightarrow} \mathrm{X}+\mathrm{e}^{-}
$$

to produce a neutral molecule and an electron.1 The energy of the photon is partitioned between the detached electron ( $\mathrm{KKE} \equiv$ electron Kinetic Energy) and the remaining molecule according to Equation (4)

$$
\mathrm{eKE}=\mathrm{hv}-\mathrm{EA}-\mathrm{E}_{\mathrm{int}}^{\mathrm{o}}+\mathrm{E}_{\mathrm{int}}^{-}
$$

In this expression, $E A$ is the adiabatic electron affinity of $X$, and $E_{\text {int }}^{\circ}$ and $E_{i n t}^{-}$represent the internal energy (i.e., electronic, vibrational, rotational, etc.) of the neutral and anionic species. Since the anion internal energies are reasonably well controlled in the experiments, a measurement of the eKE's will yield the internal energy states of the neutral molecule in a straightforward manner.

Figure 1.1. Schematic diagram illustrating the anion photoelectron spectroscopy technique. The figure shows the photodetachment of a species, $\mathrm{X}^{-}$, to three different electronic states of the neutral, $X$ by a fixed frequency photon, hv. See text for details. 


\section{Anion Photoelectron Spectroscopy}

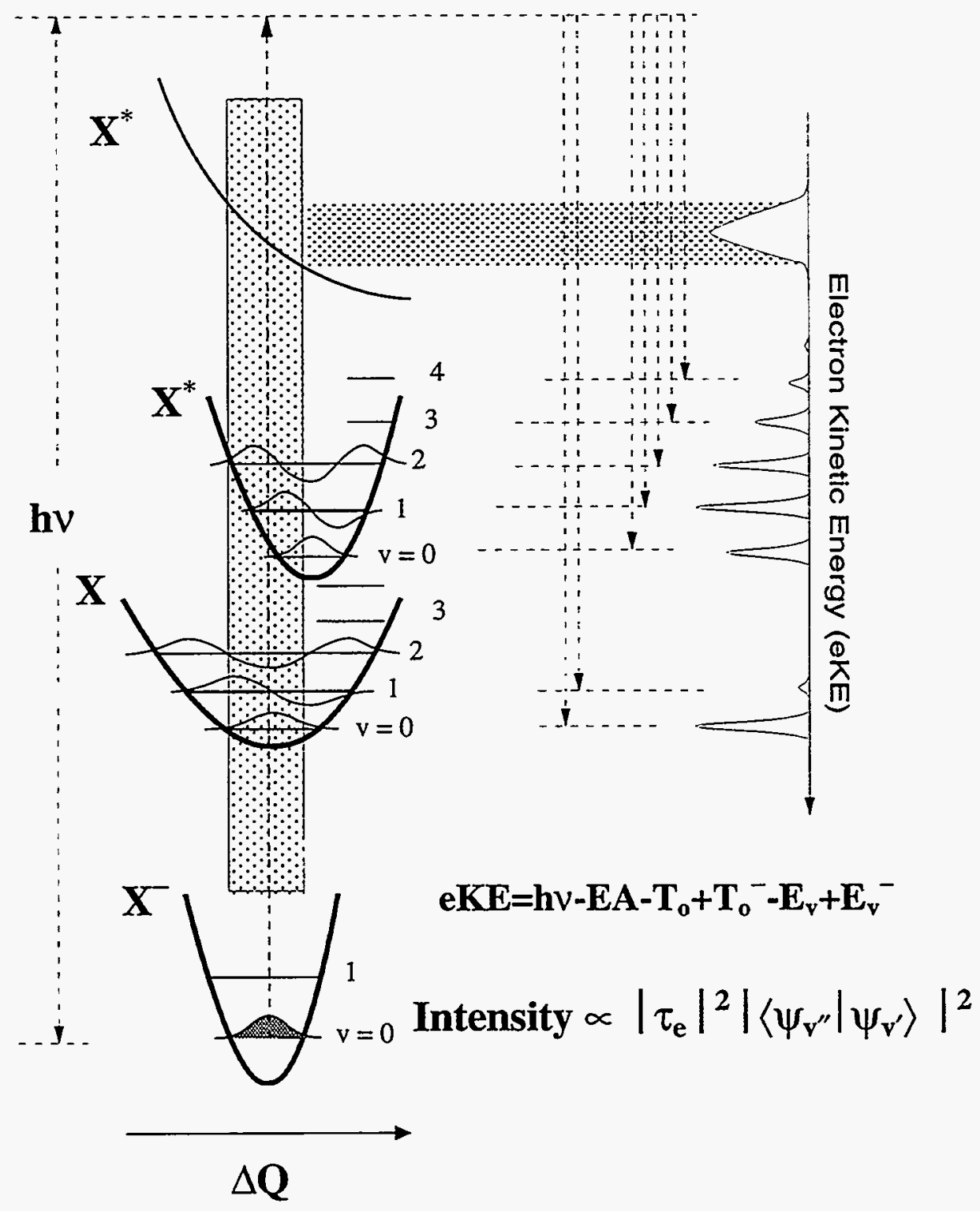

Figure 1.1 
In addition to the energetic information provided by the measured eKEs, the relative yields of electrons as a function of eKE can provide information about the relative geometries of the anion and the neutral. This is most easily illustrated by discussing the photodetachment of a diatomic anion. Due to the different time scales for nuclear and electronic motion, electron photodetachment is usually well described within the Franck-Condon approximation. In this case, the relative yield of photoelectrons as a function of energy is determined by the overlap of the vibrational wave functions for different vibrational levels of the anion, $\psi_{v^{\prime \prime}}$, and the neutral, $\Psi_{v^{\prime}}$, as expressed in Equation (5),

$$
\mathrm{I} \propto \mathrm{v}_{\mathrm{e}} \cdot\left|\tau_{\mathrm{e}}\right|^{2} \cdot\left|\left\langle\Psi_{\mathrm{v}^{\prime \prime}}(R) \mid \Psi_{\mathrm{v}^{\prime}}(R)\right\rangle\right|^{2}
$$

The intensity also has a straightforward dependence on the asymptotic velocity of the electron and on the electronic transition dipole moment which is not expected to change significantly over the energy range of a vibrational progression.

The relationship between Equation (5) and the peak intensities is illustrated schematically in Figure 1.1. In this case, $\mathrm{X}$ is a diatomic molecule and $\mathrm{Q}$ is the bond length. Anion photodetachment transitions to two different bound electronic states of the neutral are shown, where the two neutral electronic states have different bond lengths. In both cases, since photodetachment from only the ground anion vibrational state is considered, the peak spacing is indicative of the neutral vibrational level spacing. The figure illustrates how the small difference in geometry results in a very short vibrational progression and a large displacement results in a long vibrational progression. It is this bond length dependence of the vibrational wave functions that allows geometrical information to be extracted from the peak intensities of the photoelectron spectra. Polyatomic geometrical information can also be obtained quite easily within the separable parallel normal coordinate approximation using a slight modification of the ideas described above. Demonstrations of such determinations are given in 
Chapters 1,2,5 and 6. A brief description of the methodology and the FORTRAN code developed for doing these calculations is given in Appendix A.

\subsubsection{Transition States, Dissociative States and Reactive Intermediates}

The application of anion photoelectron spectroscopy to the study of transition state species for bimolecular reactions has been one of the most exciting recent developments in chemical reaction dynamics. ${ }^{11}$ The technique is illustrated in Figure 1.2 using an example which will be discussed in detail in Chapter 7. In the top portion, the coordinate is simply an idealized reaction coordinate for the reaction,

$$
\mathrm{F}+\mathrm{H}_{2} \rightarrow \mathrm{H}+\mathrm{HF} \quad(6)
$$

The bottom portion of the figure is a two-dimensional contour plot of a semi-empirical potential energy surface for the same reaction. The shaded regions indicate the Franck-Condon region for photodetachment, which is determined by projection of the ground state vibrational wave function of the $\mathrm{FH}_{2}^{-}$anion onto the neutral reaction surface.

Since the neutral potential surface does not support a set of fully-bound eigenstates, the relationship between the $\mathrm{eKE}$ of photodetached electrons and the energy of the neutral

Figure 1.2. Schematic diagram illustrating the use of anion photoelectron spectroscopy to study the transition state species of a bimolecular reaction, in particular the $\mathrm{F}$ $+\mathrm{H}_{2}$ reaction. The top portion of the figure shows the photodetachment of a bound negative ion to the transition state along a generic neutral reaction coordinate. The bottom figure shows a two dimensional $\mathrm{F}+\mathrm{H}_{2}$ reaction surface. The saddle point is marked with an ' $\mathrm{X}$ ' and the shaded region designates the Franck-Condon region for photodetachment, determined by projection of the anion vibrational wave function onto the reaction surface. 


\section{Anion Photoelectron Spectroscopy of Transition State Species}

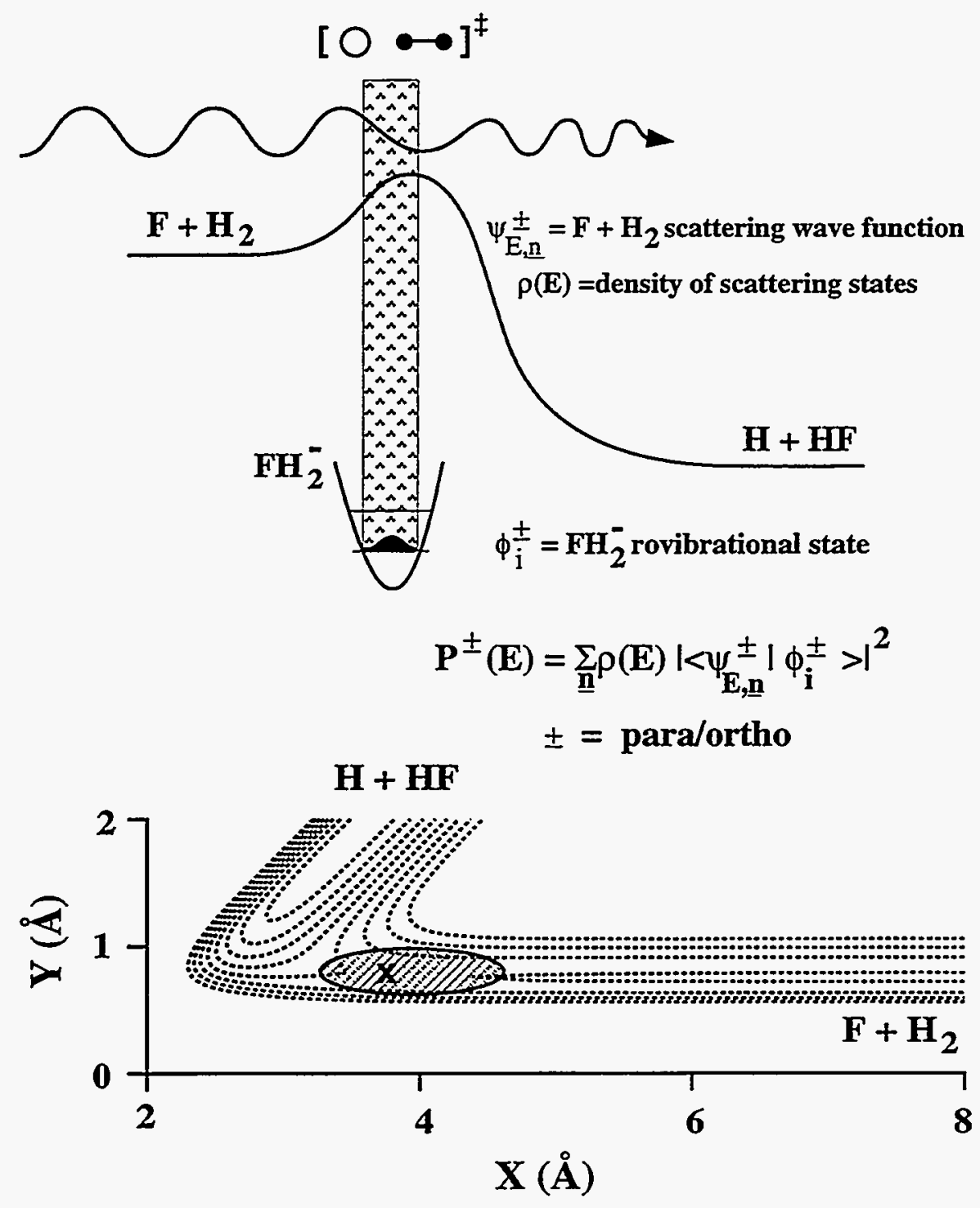

Figure 1.2 
complex, $E_{\text {int }}$, is different than that given for bound molecules in Equation (4). For these experiments, the relationship is given by Equation (7)

$$
\mathrm{eKE}=\mathrm{hv}-\mathrm{D}_{\mathrm{o}}-\mathrm{EA}+\mathrm{ZPE} \mathrm{E}^{(-)}-\mathrm{E}_{\mathrm{int}}
$$

In Equation (7), $\mathrm{D}_{\mathrm{o}}$ represents the dissociation energy of the $\mathrm{AHB}^{-}$anion into $\mathrm{A}^{-}+\mathrm{HB}$ (or $\mathrm{AH}$ $\left.+\mathrm{B}^{-}\right), \mathrm{EA}$ is the electron affinity of the $\mathrm{A}\left(\right.$ or $\mathrm{B}$ ) fragment and $\mathrm{ZPE}\left(^{-}\right)$is the vibrational zero point energy of the anion. In this case, $E_{\text {int }}$ is the energy if the unstable neutral complex with respect to the dissociation asymptote. ${ }^{12}$

Due to the unbound nature of the transition state complex, calculation of the spectral intensities is also more complicated than for a bound molecule. As a simple example, the top curve in Figure 1.1 schematically illustrates the photodetachment of a diatomic anion to a dissociative electronic state of the neutral diatom. The figure illustrates, in the limit of the classical reflection principle, how the shape and steepness of the potential energy surface determines the distribution of eKEs observed in the photoelectron spectrum.

For the diatom with this type of potential energy curve one would observe only a broad unstructured feature in the spectrum. However, polyatomic systems, including transition state species, have pseudo-bound vibrational coordinates which are perpendicular to (or not wellcoupled to) the dissociative coordinate of the complex. If the time-scale for motion along a pseudo-bound coordinate is comparable to, or shorter than, the time required for dissociation, vibrational structure may appear in the spectrum. ${ }^{13}$ These effects are discussed in greater detail, not only in the transition state results of Chapters 7,8 , and 9 , but also in the analysis of the ozone spectrum (Chapter 2) where much of the structure in the spectrum lies above the dissociation asymptote.

To predict the eKE distributions for photodetachment to the transition state region, one must calculate the Franck-Condon overlap between the anion an neutral, as in the case of bound 
molecules. However, for the transition state spectra, scattering wave functions are calculated for the neutral reaction surface which vary as a function of energy above the asymptotic energy of separated products. These wave functions can be calculated by several methods ${ }^{14}$ and used in the simulation of the photoelectron spectra of the $\mathrm{AHB}^{-}$species according to Eq. (8),

$$
I \propto \sum_{n}\left|\left\langle\Psi_{n}(E) \mid \Psi_{i}\right\rangle\right|^{2}
$$

Here, $\psi_{\mathrm{i}}$ is the anion ground state wave function and $\psi_{\mathrm{n}}(\mathrm{E})$ is a properly normalized scattering wave function for the asymptotic state, $n$, at an energy $E$ (which is related to eKE according to Eq. (7)). Simulations of the $\mathrm{FH}_{2}^{-}$photoelectron spectra generated by this method are shown in Chapter 7.

Alternatively, photoelectron spectra can be simulated within the time-dependent quantum mechanical framework. ${ }^{15,16}$ In this case, the anion wave function is propagated on the neutral reaction surface using the time-dependent Schrodinger equation, the solution of which is

$$
|\phi(t)\rangle=e^{i \hat{H} t / \hbar}|\phi(0)\rangle \quad(9)
$$

In Eq. (9), $e^{-i \hat{H} t / \hbar}$ is the time evolution operator where $\hat{H}$ is the Hamiltonian for the neutral reaction surface. The overlap of $\phi(t)$ with $\phi(0)$, the autocorrelation function,

$$
\mathrm{C}(\mathrm{t})=\langle\phi(0) \mid \phi(t)\rangle
$$

yields the photoelectron spectrum through a Fourier transform,

$$
\sigma(E)=\int_{-\infty}^{\infty} e^{i E t / \hbar} C(t) d t
$$


The simulations of the $\mathbb{I H}^{-}(\mathrm{M})$ data presented in Chapter 9 as calculated using a twodimensional wave packet program developed by S. E. Bradforth and based upon the methods of Kosloff and Kosloff. 15

\subsection{Experimental Technique}

Since full descriptions of the experimental apparatus can be found in the dissertations of Alexandra Weaver ${ }^{9}$ and Stephen Bradforth, ${ }^{10}$ previous students on this project, only a brief description of the apparatus and data collection will be given for introductory purposes. In the following chapters, details specific to each experiment will accompany the data presentation and discussion.

A schematic diagram of the experimental apparatus is shown in Figure 1.3. The experiment begins with the formation of a negative ion that upon photodetachment will provide information about the neutral species that we want to study. In the experiments described in this dissertation, ions are formed using two different techniques: electron bombardment and laser vaporization. The first of these, developed in the Lineberger laboratory, ${ }^{17}$ generates ions at the intersection of a pulsed molecular beam and a $1 \mathrm{keV}$ electron beam near the orifice of the pulsed valve through a variety of dissociative attachment and clustering processes. The second ion source is a Smalley-type source ${ }^{18}$ where a rotating translating rod is vaporized with a laser pulse and the resultant plasma, containing the negative ions, is entrained in the carrier gas pulse from the molecular beam valve. In both sources, the anions relax rotationally and vibrationally after collisions with carrier gas atoms in the continuing molecular beam expansion resulting in rotationally and vibrationally cold anions which may be studied further.

The next stage of the experiment separates the ion of interest from the other ions

Figure 1.3. Schematic diagram of the dual time-of-flight anion photoelectron spectrometer. 


\section{Anion Photoelectron Spectrometer}

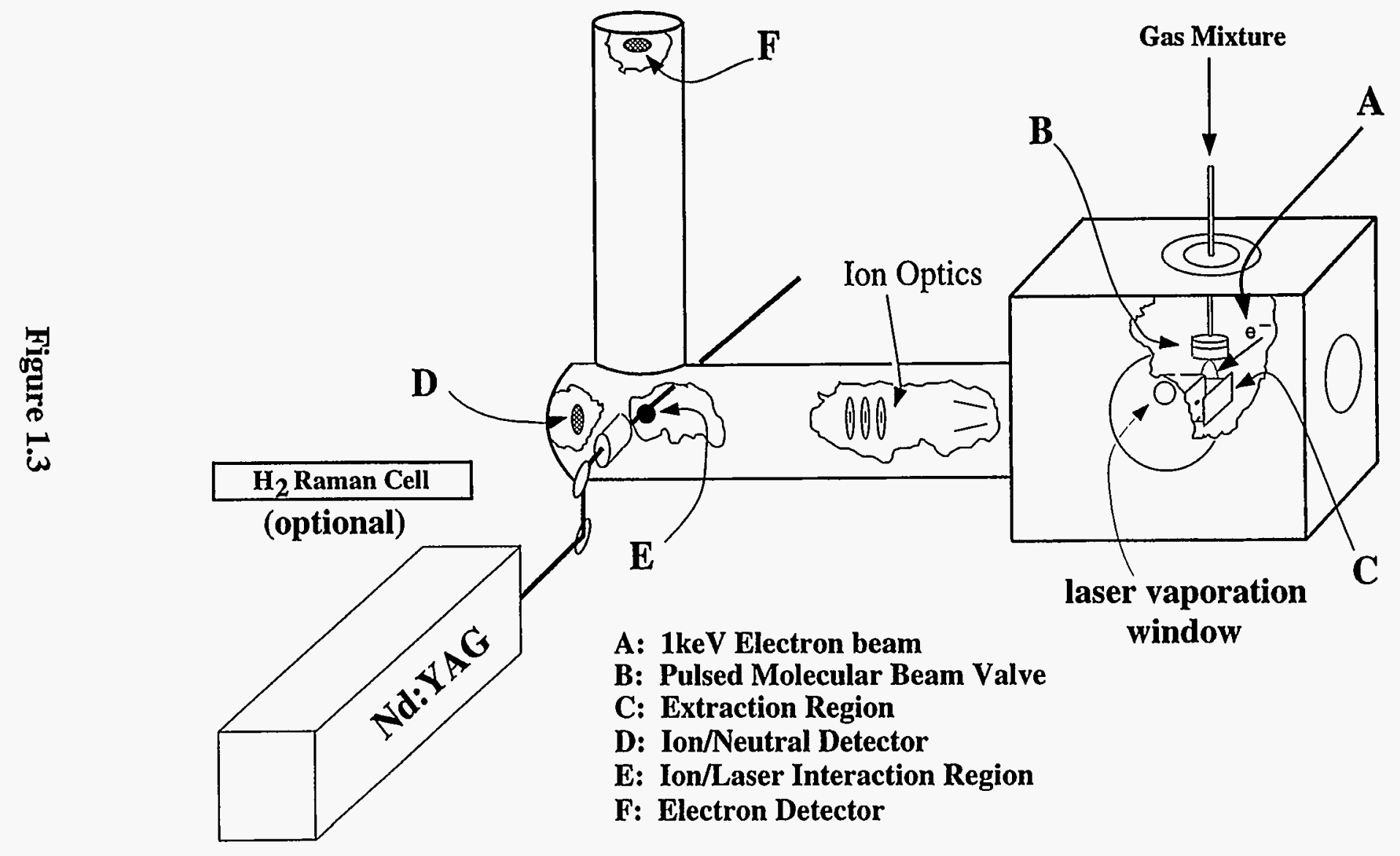


formed in the source region by time-of-flight mass spectrometry. A Wiley-McLaren-type mass spectrometer ${ }^{19}$ provides a mass resolution of $M / \Delta M \sim 250$. The mass spectrometer is now being converted to a linear mass reflectron similar to the type developed by Mamyrin and Shmikk ${ }^{20}$ and employed by Cheshnovsky. ${ }^{21}$ This modification should enhance the mass resolution significantly for the study of ions with much larger masses.

After the ions have separated spatially according to their mass, they enter the detector region where they are selectively photodetached by a properly timed pulse from a fixed frequency Nd:YAG laser (QuantaRay DCR3). Time-of-flight measurements are made on those electrons which reach the dual microchannel plate detector located at the end of a 1 meter fieldfree flight tube. By calibration to the spectra of ions with well-characterized neutral electron affinities and internal energy states, the time-of-flight measurements are converted to eKEs. In the experiments described in the following chapters, the electron arrival times were measured with a 5 ns accuracy using an transient recorder (LeCroy TR8828C). Recently, by replacing the transient recorder with a digital oscilloscope (Tektronix Model TDS544A), 1 ns accuracies can be achieved for the time-of-flight measurements. At present the eKE resolution is $\sim 8 \mathrm{meV}$ at an $\mathrm{eKE}$ of $0.65 \mathrm{eV}$ and degrades as $\mathrm{E}^{3 / 2}$.

In the following chapters, different resolution values appear as a result of alterations made to the detector region since the apparatus was first constructed. For example, an increase in the diameter of the microchannel plates improved the electron collection efficiency at a slight cost to the spectral resolution (from 8 to $11 \mathrm{meV}$ ). This resolution was recently regained, however, by adjustment of the detector electronics to decrease the effects of stray electric fields which were present near the detector face.

This apparatus can also be used to study the photoelectron angular distributions. For photodetachment to a particular electronic state, the partial photodetachment cross-section is given by: ${ }^{22}$ 


$$
\frac{d \sigma}{d \Omega}=\frac{\sigma_{t o t}(e K E)}{4 \pi} \cdot\left(1+\frac{\beta(e K E)}{2} \cdot\left(3 \cos ^{2} \theta-1\right)\right)
$$

In Eq. (12), $\sigma_{\text {tot }}(\mathrm{eKE})$ is the total cross-section for photodetachment to the electronic state, $\beta(\mathrm{eKE})$ is the asymmetry parameter $(-1 \leq \beta \leq 2)$ and $\theta$ is the angle between the electric vector of the laser and the direction of electron collection. The asymmetry parameter is not expected to change rapidly for transitions within an electronic state but can be very different for transitions to different electronic states. Thus, according to Eq. (12), two electronic states which have different asymmetry parameters will have different photoelectron distributions. These can be detected by monitoring the photoelectron spectrum as the laser polarization is rotated. This type of study has proven extremely valuable in several of the experiments to be discussed in the following chapters. 


\section{References}

1J. W. Rabalais, Principles of Ultraviolet Photoelectron Spectroscopy, (Wiley, New York, 1977); J. Berkowitz, Photoabsorption, Photoionization and Photoelectron Spectroscopy, (Academic Press, New York, 1979).

${ }^{2}$ G. Benedek, T. P. Martin and G. Pacchioni, Eds., Elemental and Molecular Clusters, (Springer, Berlin, 1988); P. J. Reynolds, Ed., On Clusters and Clustering: From Atoms to Fractals, (Elsevier Science, Amsterdam, 1993); R. S. Berry, Chem. Rev. 93, 2379 (1993).

${ }^{3}$ R. D. Levine and R. B. Bernstein, Molecular Reaction Dynamics and Chemical Reactivity, (Oxford University Press, New York, 1987) and references therein.

${ }^{4}$ K. J. Laidler, Chemical Kinetics, (Harper and Row, New York, 1987); J. I. Steinfeld, J. S. Francisco, and W. L. Hase, Chemical Kinetics and Dynamics, (Prentice-Hall, Englewood Cliffs, 1989).

${ }^{5}$ A. H. Zewail, J. Phys. Chem. 97, 12427 (1993); L. R. Khundkar and A. H. Zewail, Ann. Rev. Phys. Chem. 41, 15 (1990).

${ }^{6}$ A. Weaver, R. B. Metz, S. E. Bradforth, and D. M. Neumark, J. Phys. Chem. 92, 5558 (1988); S. E. Bradforth, A. Weaver, D. W. Arnold. R. B. Metz, and D. M. Neumark, J. Chem. Phys. 92, 7205 (1990); R. B. Metz, A. Weaver, S. E. Bradforth, T. N. Kitsopoulos, and D. M. Neumark, J. Phys. Chem. 94, 1377 (1990).

${ }^{7}$ S. E. Bradforth, D. W. Arnold, R. B. Metz, A. Weaver, and D. M. Neumark, J. Phys. Chem. 95, 8066 (1991).

${ }^{8}$ A. Weaver, R. B. Metz, S. E. Bradforth, and D. M. Neumark, J. Chem. Phys. 93, 5352 (1990); A. Weaver and D. M. Neumark, Faraday Discuss. Chem. Soc. 91, 5 (1991); S. E. Bradforth, D. W. Arnold, D. M. Neumark, and D. E. Manolopoulos, J. Chem. Phys. 99, 6345 (1993).

${ }^{9}$ A. Weaver, Ph. D. Thesis, University of California, Berkeley (1991).

${ }^{10}$ S. E. Bradforth, Ph. D. Thesis, University of California, Berkeley (1992).

${ }_{11}$ R. B. Metz, S. E. Bradforth, and D. M. Neumark, Adv. Chem. Phys. 91, 1 (1992); D. M. Neumark, Ann. Rev. Phys. Chem. 43, 153 (1992); D. M. Neumark, Acc. Chem. Res. 26, 33 (1993).

${ }^{12}$ Any dissociation asymptote can be chosen as long as the other values in Eq. (6) are consistent with the choice.

${ }^{13}$ A very good discussion with many references can be found in: R. Schinke, Photodissociation Dynamics, (Cambridge University Press, New York, 1993), Chapters 7-9.

${ }^{14}$ See W. H. Miller, Acc. Chem. Res. 26, 174 (1993) and references therein.

${ }^{15}$ D. Kosloff and R. Kosloff, J. Comput. Chem. 52, 35 (1983); R. Kosloff, J. Phys. Chem. 92, 2087 (1988).

${ }^{16}$ E. J. Heller, Acc. Chem. Res. 14, 368 (1981). 
${ }^{17}$ M. A. Johnson, M. L. Alexander, and W. C. Lineberger, Chem. Phys. Lett. 122, 285 (1984).

${ }^{18}$ T. G. Dietz, M. A. Duncan, D. E. Powers, and R. E. Smalley, J. Chem. Phys. 74, 6511 (1981).

${ }^{19}$ W. C. Wiley and I. H. McLaren, Rev. Sci. Instrum. 26, 1150 (1955).

20B. A. Mamyrin and D. V. Shmikk, Sov. Phys. JETP 49, 762 (1979).

${ }^{21}$ G. Markovich, R. Giniger, M. Levin, and O. Cheshnovsky, Z. Phys. D 20, 69 (1991).

22J. Cooper and R. N. Zare, J. Chem. Phys. 48, 942 (1968). 


\title{
Chapter 2: Study of Low-Lying Electronic States of Ozone by Anion Photoelectron Spectroscopy of $\mathrm{O}_{3}^{-\dagger}$
}

\begin{abstract}
The low-lying electronic states of ozone are studied using anion photoelectron spectroscopy of $\mathrm{O}_{3}^{-}$. The spectra show photodetachment transitions from $\mathrm{O}_{3}^{-}$to the $\tilde{\mathrm{X}}^{1} \mathrm{~A}_{1}$ ground state and to the five lowest lying electronic states of the ozone molecule, namely the ${ }^{3} \mathrm{~B}_{2},{ }^{3} \mathrm{~A}_{2},{ }^{1} \mathrm{~A}_{2},{ }^{3} \mathrm{~B}_{1}$, and ${ }^{1} \mathrm{~B}_{1}$ states. The geometry of the ozonide anion determined from a Franck-Condon analysis of the $\mathrm{O}_{3} \overline{\mathrm{X}}{ }^{1} \mathrm{~A}_{1}$ ground state spectrum agrees reasonably well with previous work. The excited state spectra are dominated by bending vibrational progressions which, for some states, extend well above the dissociation asymptote without noticeable lifetime broadening effects. Preliminary assignments are based upon photoelectron angular distributions and comparison with $a b$ initio calculations. None of the excited states observed lies below the ground state dissociation limit of $\mathrm{O}_{3}$ as suggested by previous experimental and theoretical results.
\end{abstract}

\subsection{Introduction}

As the stratospheric $\mathrm{O}_{3}$ concentration decreases, so does the protection it offers from harmful ultraviolet radiation. Annual polar ozone depletion has been well documented, particularly in the southern hemisphere. ${ }^{1}$ As the polar depletion worsens annually, regions of lower latitude are increasingly affected, in both the southern and northern hemispheres, resulting in a growing public awareness and concern. ${ }^{2,3}$ The grave impact which an unchecked ozone depletion will have on the Earth's biological systems has provided the impetus for the present global research effort directed towards determining the causes and effects of ozone

\footnotetext{
${ }^{\dagger}$ Published in the Journal of Chemical Physics, Vol. 101, No. 1, July 15, 1994.
} 
depletion. Of the many chemical reactions involved in ozone depletion, the most attention has been given to $\mathrm{O}_{3}$ destruction cycles catalyzed by the chemical by-products which result from photolysis of man-made chlorofluorocarbons (CFCs) and nitrogen oxides. ${ }^{4}$

In order to understand the underlying chemistry and physics of ozone depletion, a wide range of laboratory experimental studies and high level theoretical calculations have been directed toward the characterization of the $\mathrm{O}_{3}$ dissociation dynamics and electronic structure. While the electronic spectrum of ozone has been studied extensively, a full characterization is far from complete. The diradical character of ozone leads to the existence of several low-lying excited states, the orbital occupations of which are given in Table $I$. The ${ }^{1} B_{2} \leftarrow \tilde{X}{ }^{1} A_{1}$ transition is responsible for the intense Hartley band centered at $39,000 \mathrm{~cm}^{-1}(-5 \mathrm{eV})$. Ab initio theory predicts that the other five excited states of ozone listed in Table I lie less than $3 \mathrm{eV}$ $\left(\sim 24000 \mathrm{~cm}^{-1}\right)$ above the $\mathrm{O}_{3}\left(\tilde{\mathrm{X}}^{1} \mathrm{~A}_{1}\right)$ state and that the lowest of these lie near the dissociation threshold of the ground state $\left(\mathrm{D}_{0}=1.05 \mathrm{eV} ; \mathrm{D}_{\mathrm{e}}=1.13 \mathrm{eV}\right) .5,6,7,8$ Transitions from the ground state to most of these excited states are nominally dipole-forbidden, complicating their study by absorption spectroscopy. While these electronic states are not directly involved in absorption of ultraviolet photons, they could play a significant role in ozone recombination kinetics. As a result, atmospheric ozone models and detection methods may be significantly influenced by the properties of these states. This paper describes experiments performed in our laboratory in which we employ anion photoelectron spectroscopy of $\mathrm{O}_{3}^{-}$to detect and better characterize the low-lying electronic states of ozone.

The complexity of the $\mathrm{O}_{3}$ electronic structure below $3 \mathrm{eV}$ is readily apparent from the ozone absorption spectrum. Chappuis ${ }^{9}$ and Wulf ${ }^{10}$ observed diffuse absorption bands in the visible and near-infrared, respectively, several decades ago, but made no electronic state assignments to the data. The weak, diffuse bands which were observed lie between 9000 and $22000 \mathrm{~cm}^{-1}$, with a spectral maximum at $16600 \mathrm{~cm}^{-1}$. Considering the low-lying $\mathrm{O}_{3}$ electronic states (Table 1 ), only the ${ }^{1} B_{1} \leftarrow \tilde{X}{ }^{1} A_{1}$ transition is symmetry allowed from the ground state, 
while the ${ }^{1} A_{2} \leftarrow \tilde{X}{ }^{1} A_{1}$ transition is vibronically allowed via the $v_{3}$ antisymmetric stretch. The remaining three electronic states are triplet states whose absorption transitions from the ground state are spin-forbidden. The observation of two absorption bands in this region implies some form of coupling to enable absorption to at least one 'dark' electronic state.

The congested electronic structure of $\mathrm{O}_{3}$ has lead to several contradictory interpretations of the absorption data. Based upon early $a b$ initio calculations, the Chappuis and Wulf bands were assigned to the ${ }^{1} \mathrm{~B}_{1} \leftarrow \tilde{\mathrm{X}}{ }^{1} \mathrm{~A}_{1}$ and the ${ }^{1} \mathrm{~A}_{2} \leftarrow \tilde{\mathrm{X}}{ }^{1} \mathrm{~A}_{1}$ transitions, respectively. 11,12 These assignments, however, were insufficient to explain fully the diffuse nature of the Chappuis band or the dissociation dynamics observed by Valentini and coworkers. ${ }^{13}$ Vaida et al. ${ }^{14}$ assigned the Chappuis band to overlapping transitions to the ${ }^{1} \mathrm{~A}_{2}$ state and an unidentified triplet state. This assignment, based on the weakness of the transition $(f \sim$ $3.2 \times 10^{-5}$ ) and the enhancement of the Chappuis band relative to the Hartley band in condensed phase absorption, ${ }^{15}$ is also questionable as it left no apparent absorption feature to account for the allowed ${ }^{1} B_{1} \leftarrow \tilde{X}{ }^{1} A_{1}$ transition.

A recent set of experimental and theoretical studies has provided further insight into the assignment of $\mathrm{O}_{3}$ absorption spectrum. Anderson and co-workers ${ }^{16}$ recently utilized isotopic substitution to determine the origins of both the Chappuis and Wulf bands, and have observed rotational structure in the first vibronic transitions of the Wulf band. Theoretical efforts by Braunstein et al. ${ }^{6}$ and Banichevich et al. ${ }^{8}$ indicate that the Chappuis band results from the significant interaction between the ${ }^{1} B_{1}$ and ${ }^{1} A_{2}$ states. This analysis implies that the lower-lying Wulf band is due to transitions to one or more triplet states. Of these, the most likely candidate is considered to be the ${ }^{3} A_{2} \leftarrow \tilde{X}{ }^{1} A_{1}$ transition which becomes allowed through a spin-orbit coupling to the ${ }^{1} \mathrm{~B}_{2}$ state $\left(\mathrm{T}_{\mathrm{e}}=30,000 \mathrm{~cm}^{-1}\right) \cdot 6,16$

Several techniques other than photoabsorption have been used to study $\mathrm{O}_{3}$ in the visible and near IR regions, some of which have lead researchers to conclude that bound excited states exist below the ground state dissociation limit. ${ }^{17}$ Kinetics measurements find a discrepancy for 
the $\mathrm{O}\left({ }^{3} \mathrm{P}\right)+\mathrm{O}_{2}\left(\tilde{\mathrm{X}}^{3} \Sigma_{\mathrm{g}}^{-}\right) \stackrel{k_{\text {recomb }}}{\longrightarrow} \mathrm{O}_{3}\left(\tilde{\mathrm{X}}^{1} A_{1}\right)$ recombination rate depending upon whether the rate is determined by measuring the disappearance of $O\left({ }^{3} \mathrm{P}\right)\left(\mathrm{k}_{\mathrm{recomb}}=6 \times 10^{-34} \mathrm{~cm}^{6} \mathrm{~s}^{-1}\right)^{18}$ or the appearance of $\mathrm{O}_{3}\left(\mathrm{X}^{1} \mathrm{~A}_{1}\right)\left(\mathrm{k}_{\text {recomb }}=3 \times 10^{-34} \mathrm{~cm}^{6} \mathrm{~s}^{-1}\right) .{ }^{19}$ Bair and co-workers ${ }^{20}$ attribute this discrepancy to the efficient formation $(-60 \%)$ of a bound excited electronic state. Two sets of emission measurements have been attributed to low-lying electronic states. Von Rosenberg and Trainor ${ }^{21}$ discuss the possible assignment of an observed emission peak at $6.6 \mu \mathrm{m}$ to the ${ }^{3} \mathrm{~B}_{2} \rightarrow{ }^{1} \mathrm{~A}_{1}$ transition, corresponding to an excitation energy of $-1450 \mathrm{~cm}^{-1}$. Shi and Barker ${ }^{22}$ postulate that a $1.9 \mu \mathrm{m}$ emission $\left(0.652 \mathrm{eV}, 5,263 \mathrm{~cm}^{-1}\right)$ originates from an unidentified excited triplet electronic state of $\mathrm{O}_{3}$. McGrath et al. ${ }^{23}$ observe a transient absorption feature with a maximum at $320 \mathrm{~nm}$ after primary excitation of $\mathrm{O}_{3}$ in the 540-650 $\mathrm{nm}$ region (Chappuis band). They assign the intermediate as the $\mathrm{O}_{3}\left({ }^{1} \mathrm{~A}_{2}\right)$ state. Swanson and Celotta ${ }^{24}$ studied $\mathrm{O}_{3}$ using electron energy loss spectroscopy (EELS) and observed signal in their data which they attribute to low-lying triplet states. From the analysis of their data, they extrapolated the existence of energy levels for the ${ }^{3} \mathrm{~B}_{2}$ state which are bound with respect to dissociation. Their data are particularly relevant to the present results because the same $\mathrm{O}_{3}$ electronic states can be observed using EELS and anion photoelectron spectroscopy. However, anion photoelectron spectroscopy has the distinct advantages of mass selectivity and higher spectral resolution. Although this abundance of research has been performed on the visible / near IR region of the $\mathrm{O}_{3}$ spectrum, no complete state assignment exists and some uncertainty remains concerning whether there are bound excited electronic states which should be considered in atmospheric ozone models.

In this paper, photoelectron spectroscopy of $\mathrm{O}_{3}^{-}$is used to provide a more complete picture of the $\mathrm{O}_{3}$ electronic and vibrational structure. Previously, Novick et al. ${ }^{25}$ measured the photoelectron spectrum of $\mathrm{O}_{3}^{-}$at photodetachment photon energies of 2.540 and $3.407 \mathrm{eV}$; at these energies, only the $\mathrm{O}_{3}$ ground state is accessible. The present work explores the properties of several $\mathrm{O}_{3}$ excited electronic states by using higher photon energies ( 4.657 and $5.822 \mathrm{eV}$ ) 
Table I: Single Reference Configuration of $\mathrm{O}_{3}$ and $\mathrm{O}_{3}^{-}$Electronic States

\begin{tabular}{||l|l|}
\hline Electronic State & Configuration \\
\hline $\mathrm{O}_{3} \tilde{\mathrm{X}}{ }^{1} \mathrm{~A}_{1}$ & $\ldots 1 \mathrm{~b}_{1}^{2} 6 \mathrm{a}_{1}^{2} 4 \mathrm{~b}_{2}^{2} 1 \mathrm{a}_{2}^{2} 2 \mathrm{~b}_{1}^{0}$ \\
\hline${ }^{3} \mathrm{~B}_{2} /{ }^{1} \mathrm{~B}_{2}$ & $\ldots 1 \mathrm{~b}_{1}^{2} 6 \mathrm{a}_{1}^{2} 4 \mathrm{~b}_{2}^{2} 1 \mathrm{a}_{2}^{1} 2 \mathrm{~b}_{1}^{1}$ \\
\hline${ }^{3} \mathrm{~A}_{2} /{ }^{1} \mathrm{~A}_{2}$ & $\ldots 1 \mathrm{~b}_{1}^{2} 6 \mathrm{a}_{1}^{2} 4 \mathrm{~b}_{2}^{1} 1 \mathrm{a}_{2}^{2} 2 \mathrm{~b}_{1}^{1}$ \\
\hline${ }^{3} \mathrm{~B}_{1} /{ }^{1} \mathrm{~B}_{1}$ & $\ldots 1 \mathrm{~b}_{1}^{2} 6 \mathrm{a}_{1}^{1} 4 \mathrm{~b}_{2}^{2} 1 \mathrm{a}_{2}^{2} 2 \mathrm{~b}_{1}^{1}$ \\
\hline \hline $\mathrm{O}_{3}^{-} \tilde{\mathrm{X}}^{2} \mathrm{~B}_{1}$ & $\ldots 1 \mathrm{~b}_{1}^{2} 6 \mathrm{a}_{1}^{2} 4 \mathrm{~b}_{2}^{2} 1 \mathrm{a}_{2}^{2} 2 \mathrm{~b}_{1}^{1}$ \\
\hline
\end{tabular}

and higher resolution electron detection. As demonstrated previously, ${ }^{26,27,28}$ anion photoelectron spectroscopy is complementary to absorption techniques because one is often able to observe optically 'dark' states. Considering the single determinant orbital occupation of $\mathrm{O}_{3}^{-}$in its $\tilde{\mathrm{X}}\left({ }^{2} \mathrm{~B}_{1}\right)$ ground state, a one-electron photodetachment of the ozonide anion can yield the $\mathrm{O}_{3}$ ground state and the six excited states in Table I. Based upon excitation energies predicted by $a b$ initio calculations $5,6,7,8$ and the electron affinity of $\mathrm{O}_{3}$ determined by Novick et al., ${ }^{25}$ all but one of these states, the ${ }^{1} \mathrm{~B}_{2}$ state, should be energetically accessible at $4.657 \mathrm{eV}$. Photodetachment transitions from $\mathrm{O}_{3}^{-}$to all the other five excited states of $\mathrm{O}_{3}$ do appear in our anion photoelectron spectra. We also obtain the geometry of the $\mathrm{O}_{3}^{-}$anion by analyzing the transition to the $\mathrm{O}_{3}$ ground state, and this is compared to previous results.

\subsection{Experimental}

The apparatus employed in these experiments is a dual time-of-flight anion photoelectron spectrometer. While details of the apparatus have been given elsewhere, ${ }^{29} \mathrm{a}$ general overview with specifics relevant to the present results will be provided here. In the experiment, anions are generated at the intersection of a pulsed molecular beam and a $1 \mathrm{keV}$ 
electron beam focused near the orifice of the piezoelectric molecular beam valve ${ }^{30}$ operated at $20 \mathrm{~Hz}$. As the molecular beam expansion proceeds, the anions relax vibrationally and rotationally by collisions with the carrier gas. The cooled anions are extracted perpendicularly and injected into a Wiley-McLaren type time-of-flight mass spectrometer. ${ }^{31}$ The mass-selected $\mathrm{O}_{3}^{-}$ions are selectively photodetached by a properly timed $8 \mathrm{~ns}$ light pulse from a Nd:YAG laser. Electron kinetic energies (eKEs) are determined from field-free time-of-flight measurements made on photodetached electrons reaching microchannel plates located at the end of a one meter flight tube. The linearly polarized laser beam can be rotated with respect to the direction of electron collection to study photoelectron angular distributions. This is a very important feature of the experiment which aids the data analysis. When studying the photoelectron angular distributions, the laser polarization is alternated between the desired polarizations in data collection increments of $\sim 40000$ laser shots. The final data result from a summation of those spectra obtained using the same polarization. This procedure ensures that differences in the spectra do not result from a long-term drift of experimental conditions. The resolution of the photoelectron spectrometer is $7 \mathrm{meV}$ at $0.65 \mathrm{eV}$ and degrades as (eKE) ${ }^{3 / 2}$ at higher energies. The spectra presented are averaged for approximately 400,000 laser shots each.

Generation of ozonide anions was accomplished by two 'synthetic' routes. Initially, $\mathrm{O}_{2}$ was expanded through the molecular beam valve, at a backing pressure of 4 bar. $\mathrm{O}^{-}$anions, formed at the molecular beam/electron beam intersection, react with $\mathrm{O}_{2}$ to form $\mathrm{O}_{3}^{-}$,

$$
\mathrm{O}^{-}+\mathrm{O}_{2} \stackrel{\mathrm{M}}{\longrightarrow} \mathrm{O}_{3}^{-}
$$

The third body, $\mathrm{M}=\mathrm{O}_{2}$, carries away excess energy deposited in the $\mathrm{O}_{3}^{-}$internal degrees of freedom upon anion formation $\left(\mathrm{D}_{0}\left(\mathrm{O}_{2}-\mathrm{O}^{-}\right) \sim 1.7 \mathrm{eV}\right)$. The resulting photoelectron spectra, not shown, indicate that this ion formation mechanism leads to significant excitation of the $\mathrm{O}_{3}^{-}$ 
$v_{3}$ antisymmetric stretch. "Hot bands" resulting from the detachment of vibrationally excited $\mathrm{O}_{3}^{-}$anions led to spectral congestion, necessitating the use of second method of ozonide synthesis which produced colder anions. An ozone gas mixture $\left(\sim 0.2 \% \mathrm{O}_{3} / 10 \% \mathrm{He} / \sim 90 \%\right.$ $\mathrm{Ne}$ ) is made by passing the $\mathrm{He} / \mathrm{Ne}$ mixture over $\mathrm{O}_{3}$ adsorbed onto silica gel at $-78^{\circ} \mathrm{C}$. Ozonide anions are generated by expanding this gas mixture, at a pressure of $1.5 \mathrm{bar}$, through molecular beam valve, as above. In this case, the $\mathrm{O}_{3}^{-}$is most likely produced by electron attachment processes. Ions generated in this way are more efficiently cooled (see Sec. IV A) leading to a less congested spectrum. The data presented here were obtained using the second method of $\mathrm{O}_{3}^{-}$preparation.

At the ion densities necessary to obtain sufficient photoelectron signal for $\mathrm{O}_{3}^{-}$, the energies of the photodetached electrons are affected by a Coulombic repulsion between the detached electron and the remaining packet of $\mathrm{O}_{3}^{-}$ions which are not photodetached. Compensation for this 'space-charge' effect is made by first measuring the magnitude of the 'space-charge shift' for calibration ions (i.e., $\mathrm{O}_{2}^{-}, \mathrm{Cl}^{-}, \mathrm{Br}^{-}$and $\mathrm{I}$ ) at the same ion densities that were used for $\mathrm{O}_{3}^{-}$data collection. The $\mathrm{O}_{3}^{-}$data is then corrected by this amount $(\sim 5 \mathrm{meV})$. In addition to the overall shift of the spectrum to higher eKE, there is also a slight broadening of the spectral features $(\sim 3 \mathrm{meV})$. The photoelectron spectra obtained using different photon energies provide information about different regions of the ozone electronic structure as a result of the $(\mathrm{eKE})^{3 / 2}$ dependence of the experimental resolution. The data sets described below were collected using three different photodetachment energies, two of which are the fourth $(266 \mathrm{~nm}$, $4.657 \mathrm{eV} ; 16 \mathrm{~mJ} /$ pulse) and fifth $(213 \mathrm{~nm} ; 5.822 \mathrm{eV} ; 5 \mathrm{~mJ} / \mathrm{pulse})$ harmonics of the Nd:YAG laser. The other wavelength ( $416 \mathrm{~nm} ; 2.977 \mathrm{eV} ; 5 \mathrm{~mJ} / \mathrm{pulse})$ is the first Stokes Raman line generated by focusing the third harmonic of the Nd:YAG laser $(355 \mathrm{~nm} ; 3.549 \mathrm{eV} ; 50$ $\mathrm{mJ} /$ pulse $)$ into a high pressure $(-20 \mathrm{bar}) \mathrm{H}_{2}$ cell. 


\subsection{Results}

The photoelectron spectrum of $\mathrm{O}_{3}^{-}$measured with a photodetachment energy (hv) of $2.977 \mathrm{eV}$ is shown in Figure 2.1a. Only transitions to the $\mathrm{O}_{3}\left(\overrightarrow{\mathrm{X}}{ }^{1} \mathrm{~A}_{1}\right)$ ground state are energetically accessible at this photon energy. In general, for a transition to an $\mathrm{O}_{3}$ electronic state with term value $T_{0}$, the eKE is related to the internal energy of the neutral by

$$
\mathrm{eKE}=\mathrm{hv}-\mathrm{EA}-\mathrm{T}_{\mathrm{o}}-\mathrm{E}_{\mathrm{v}}^{\mathrm{o}}+\mathrm{E}_{\mathrm{v}}^{-}
$$

Here, EA is the electron affinity of $\mathrm{O}_{3}$, and $\mathrm{E}_{\mathrm{v}}^{\circ}$ and $\mathrm{E}_{\mathrm{v}}^{-}$are the vibrational energies above the zero point of the neutral and anion, respectively. According to Eq. (2), peaks at higher eKE correspond to lower internal energy states of the neutral molecule. The spectrum shown in Fig. 2.1a consists of vibrational progressions in the totally symmetric $v_{1}$ and $v_{2}$ modes of the $\mathrm{O}_{3}(\tilde{\mathrm{X}}$ ${ }^{1} \mathrm{~A}_{1}$ ) ground state, the origin of which is labeled 'A' at $\mathrm{eKE}=0.874 \mathrm{eV}$. Peak ' $\mathrm{a}$ ' is a "hot band" resulting from detachment of vibrationally excited $\mathrm{O}_{3}^{-}$anions with $v_{1}=1$. Peak positions and assignments are summarized in Table II. Since the geometric parameters and vibrational frequencies of the $\mathrm{O}_{3}$ ground state have been accurately determined, this spectrum can be used in conjunction with a Franck-Condon analysis to determine the geometry of the ozonide anion (see Sec. 2.4.1).

Figures $2.2 \mathrm{a}$ and $2.2 \mathrm{~b}$ show $\mathrm{O}_{3}^{-}$spectra collected at a photon energy of $4.657 \mathrm{eV}$, and laser polarization angles of $\theta=90^{\circ}$ and $0^{\circ}$, respectively. The transitions to the $\mathrm{O}_{3}\left(\overline{\mathrm{X}}{ }^{1} \mathrm{~A}_{1}\right)$ ground state occur at $\mathrm{eKE}>2.0 \mathrm{eV}$; the peaks are broadened, relative to the corresponding features in Fig. 2.1a, due to the higher energy of the detected electrons. In Fig 2.2a, a long progression begins at $1.378 \mathrm{eV}$, peak ' $a$ ', with an average peak spacing of $555 \pm 50 \mathrm{~cm}^{-1}$

Figure 2.1: (a) Photoelectron spectrum of $\mathrm{O}_{3}^{-}$measured using a photodetachment energy of $2.977 \mathrm{eV}$ and (b) Franck-Condon simulation of data. 


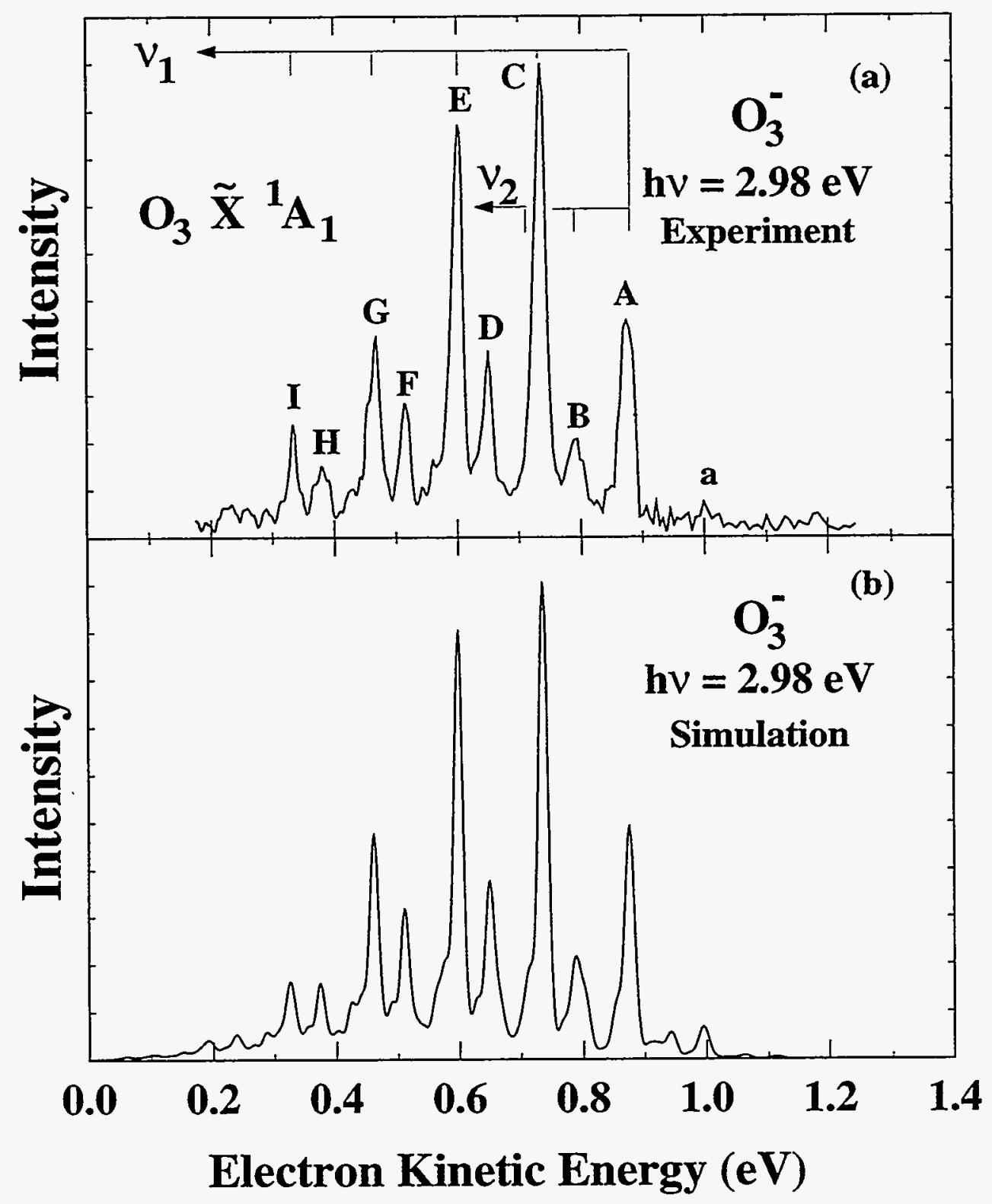

Figure 2.1 
Table II: Peak Positions and Assignments ${ }^{\mathrm{a}}$ for the $2.977 \mathrm{eV} \mathrm{O}_{3}^{-}$Spectrum

\begin{tabular}{|c|c|c|}
\hline Peak & eKE (eV) & Assignment \\
\hline $\mathrm{a}$ & 1.000 & $1_{1}^{0} 2_{0}^{0}$ \\
\hline $\mathrm{A}$ & 0.874 & $0-0$ \\
\hline $\mathrm{B}$ & 0.789 & $1_{0}^{0} 2_{0}^{1}$ \\
\hline $\mathrm{C}$ & 0.737 & $1_{0}^{1} 2_{0}^{0}$ \\
\hline $\mathrm{D}$ & 0.649 & $1_{0}^{1} 2_{0}^{1}$ \\
\hline $\mathrm{E}$ & 0.598 & $1_{0}^{2} 2_{0}^{0}$ \\
\hline $\mathrm{F}$ & 0.511 & $1_{0}^{2} 2_{0}^{1}$ \\
\hline $\mathrm{G}$ & 0.462 & $1_{0}^{3} 2_{0}^{0}$ \\
\hline $\mathrm{H}$ & 0.374 & $1_{0}^{3} 2_{0}^{1}$ \\
\hline $\mathrm{I}$ & 0.326 & $1_{0}^{4} 2_{0}^{0}$ \\
\hline
\end{tabular}

a) Assignment notation: $\left(v_{1}\right)_{v^{\prime \prime}}^{v^{\prime \prime}}\left(v_{2}\right)_{v^{\prime \prime}}^{v^{\prime \prime}}$

evolving into a very congested feature beginning at $\sim 0.9 \mathrm{eV}$. Peak positions are given in Table III. Based upon agreement with the maximum in the Chappuis band absorption, ${ }^{16 \mathrm{~b}}$ the peak at $\sim 0.5 \mathrm{eV}$ is assigned as the transition to the ${ }^{1} \mathrm{~B}_{1}$ state $\left(\mathrm{T}_{\mathrm{e}}=2.046 \mathrm{eV}^{16}\right)$. By default, the long progression must represent transitions to one or more of the lower lying "optically forbidden" states of $\mathrm{O}_{3}$. In contrast to $\mathrm{O}_{3}$ absorption experiments, $\mathrm{O}_{3}^{-}$photodetachment transitions to these

Figure 2.2: Photoelectron spectra of $\mathrm{O}_{3}^{-}$measured using a photodetachment energy of $4.657 \mathrm{eV}$ at laser polarization angles $\theta=90^{\circ}$ (a) and $0^{\circ}$ (b). The laser polarization angle, $\theta$, is the angle between the laser $\vec{E}$ vector and the direction of electron detection. State assignments in Figure $2 \mathrm{a}$ are discussed in Section 2.4.2.1. In Figure 2.2b, labels " $\mathrm{A}$ " and " $\mathrm{B}$ " indicate the dissociation asymptotes for the $\mathrm{O}\left({ }^{3} \mathrm{P}\right)+\mathrm{O}_{2}\left(\mathrm{X}^{3} \Sigma_{\mathrm{g}}^{-}\right)$and the $\mathrm{O}\left({ }^{3} \mathrm{P}\right)+\mathrm{O}_{2}\left(\mathrm{a}^{1} \Delta_{\mathrm{g}}\right)$ dissociation channels, respectively. 


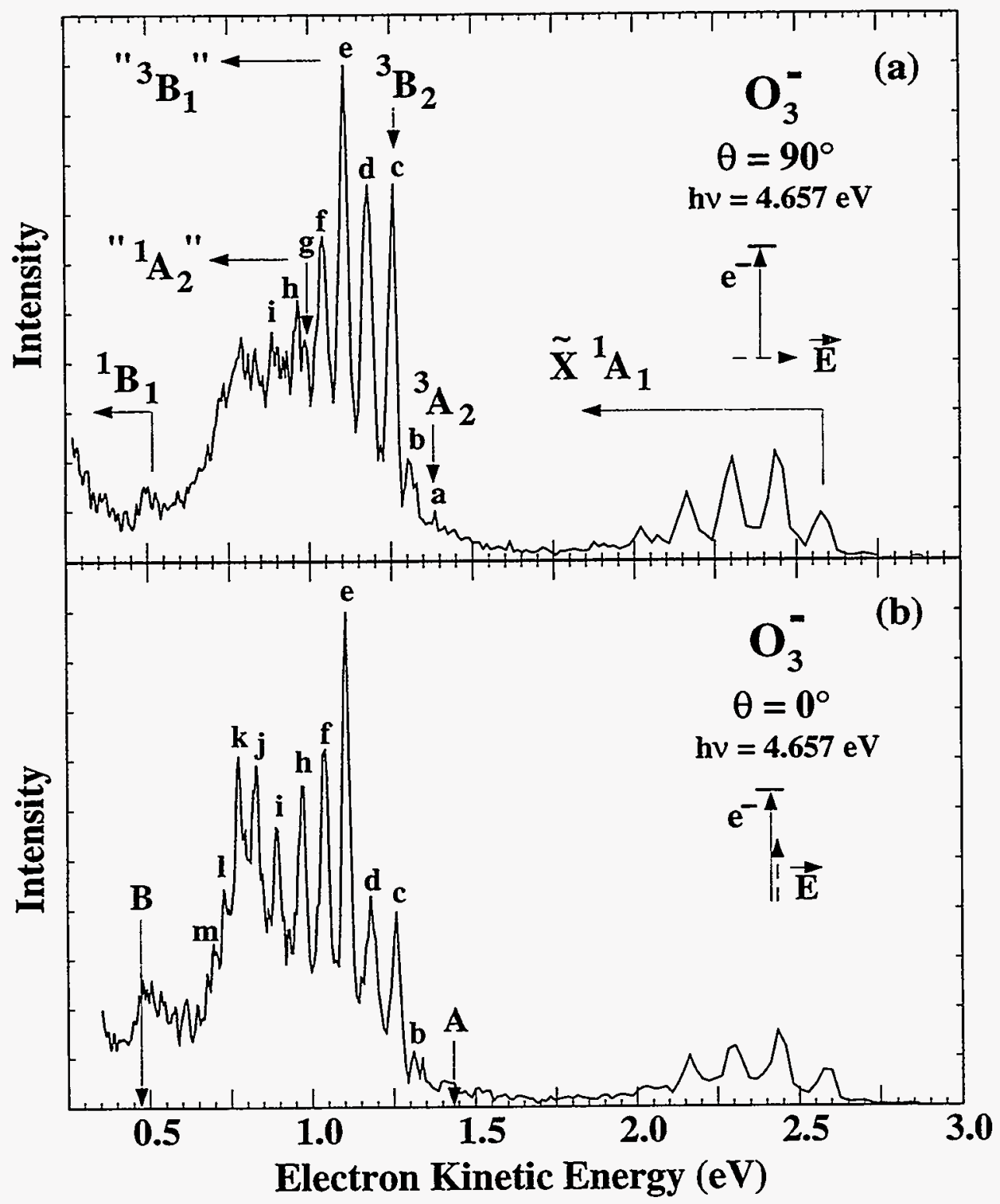

Figure 2.2 
Table III: Peak Positions for the $4.657 \mathrm{eV} \mathrm{O}_{3}^{-}$Spectrum

\begin{tabular}{|c|c|c|}
\hline Peak & eKE $(\mathrm{eV})$ & Width ${ }^{\mathrm{a}}$ (FWHM; eV) \\
\hline \hline $\mathrm{a}$ & 1.378 & - \\
\hline $\mathrm{b}$ & 1.304 & 0.037 \\
\hline $\mathrm{c}$ & 1.257 & 0.032 \\
\hline $\mathrm{d}$ & 1.177 & 0.047 \\
\hline $\mathrm{e}$ & 1.105 & 0.032 \\
\hline $\mathrm{f}$ & 1.039 & 0.040 \\
\hline $\mathrm{g}$ & 0.984 & $\sim 0.025^{\mathrm{b}}$ \\
\hline $\mathrm{h}$ & 0.967 & $\sim 0.025^{\mathrm{b}}$ \\
\hline $\mathrm{i}$ & 0.890 & $\mathrm{c}$ \\
\hline $\mathrm{j}$ & 0.827 & $\mathrm{c}$ \\
\hline $\mathrm{k}$ & 0.772 & $\mathrm{c}$ \\
\hline $\mathrm{l}$ & 0.726 & $\mathrm{c}$ \\
\hline $\mathrm{m}$ & 0.694 & $\mathrm{c}$ \\
\hline
\end{tabular}

a Peak widths determined from $\theta=90^{\circ}$ data.

b Estimate based upon deconvolution into two Gaussian peaks with FWHM $=0.025 \mathrm{eV}$.

c Estimated FWHM $<0.025 \mathrm{eV}$ on top of background structure.

states are vibrationally resolved and are more intense than the transition to the ${ }^{1} \mathrm{~B}_{1}$ state.

While the peaks in the long progression of Figure 2.2a are approximately evenly spaced, there are several indications that this is not simply a progression in a single vibrational mode of an $\mathrm{O}_{3}$ excited state. For example, Table III shows that there are significant variations in the peak spacings and widths. The intensity distribution is quite irregular, and does not resemble the typical Franck-Condon profile for a single vibrational mode. Rather, it appears that this progression is actually composed of a series of overlapping transitions. To 
characterize it further, it is useful to examine the dependence of the features in the photoelectron spectrum on the laser polarization direction.

The photoelectron angular distribution resulting from $\mathrm{O}_{3}^{-}$photodetachment to a particular $\mathrm{O}_{3}$ electronic state is given by Equation (3), ${ }^{32}$ where $\sigma_{\text {tot }}(\mathrm{eKE})$ is the total crosssection for photodetachment, $\beta$ (eKE) is the asymmetry parameter $(-1 \leq \beta \leq 2)$, and $\theta$ is the angle between the laser polarization and the direction of electron collection:

$$
\frac{\mathrm{d} \sigma}{\mathrm{d} \Omega}=\frac{\sigma_{t o t}(\mathrm{eKE})}{4 \pi} \cdot\left(1+\beta(\mathrm{eKE}) \cdot\left(3 \cos ^{2} \theta-1\right)\right)
$$

The asymmetry parameter, $\beta$, is not expected to change rapidly for transitions to different vibrational levels of the same neutral electronic state, but it can be very different for transitions to different electronic states. Hence, a marked variation of peak intensities with laser polarization provides a means of determining the presence of overlapping transitions to multiple electronic states.

There are significant differences between the spectra in Figures $2.2 \mathrm{a}$ and $2.2 \mathrm{~b}$. Peaks ' $\mathrm{c}$ ' and ' $\mathrm{d}$ ' are considerably less intense in the $\theta=0^{\circ}$ spectrum, and the intensity discontinuity between peaks ' $d$ ' and 'e' is much more noticeable in Figure 2.2b. At lower eKE, peaks ' $h$ ' and ' $i$ ' are more intense in the $\theta=0^{\circ}$ spectrum, and several new peaks ('j'-'m') are apparent on top of what was only a broad unstructured feature in the $\theta=90^{\circ}$ spectrum. Additional polarization studies are shown in Figs. 2.3a and 2.3b. These are $\mathrm{O}_{3}^{-}$photoelectron spectra collected at $\theta=$ $90^{\circ}$ and $\theta=0^{\circ}$, respectively, using a $5.822 \mathrm{eV}$ photodetachment photon energy. The spectral features, broadened due to their positions at higher eKEs, exhibit a polarization dependence similar to that observed in the $4.657 \mathrm{eV}$ data. This confirms that the polarization effects seen in

Figure 2.3: Photoelectron spectra of $\mathrm{O}_{3}^{-}$measured using a photodetachment energy of $5.822 \mathrm{eV}$ at laser polarization angle $\theta=90^{\circ}$ (a) and $0^{\circ}$ (b). Peak labels are consistent with those used in Figure 2.2. 


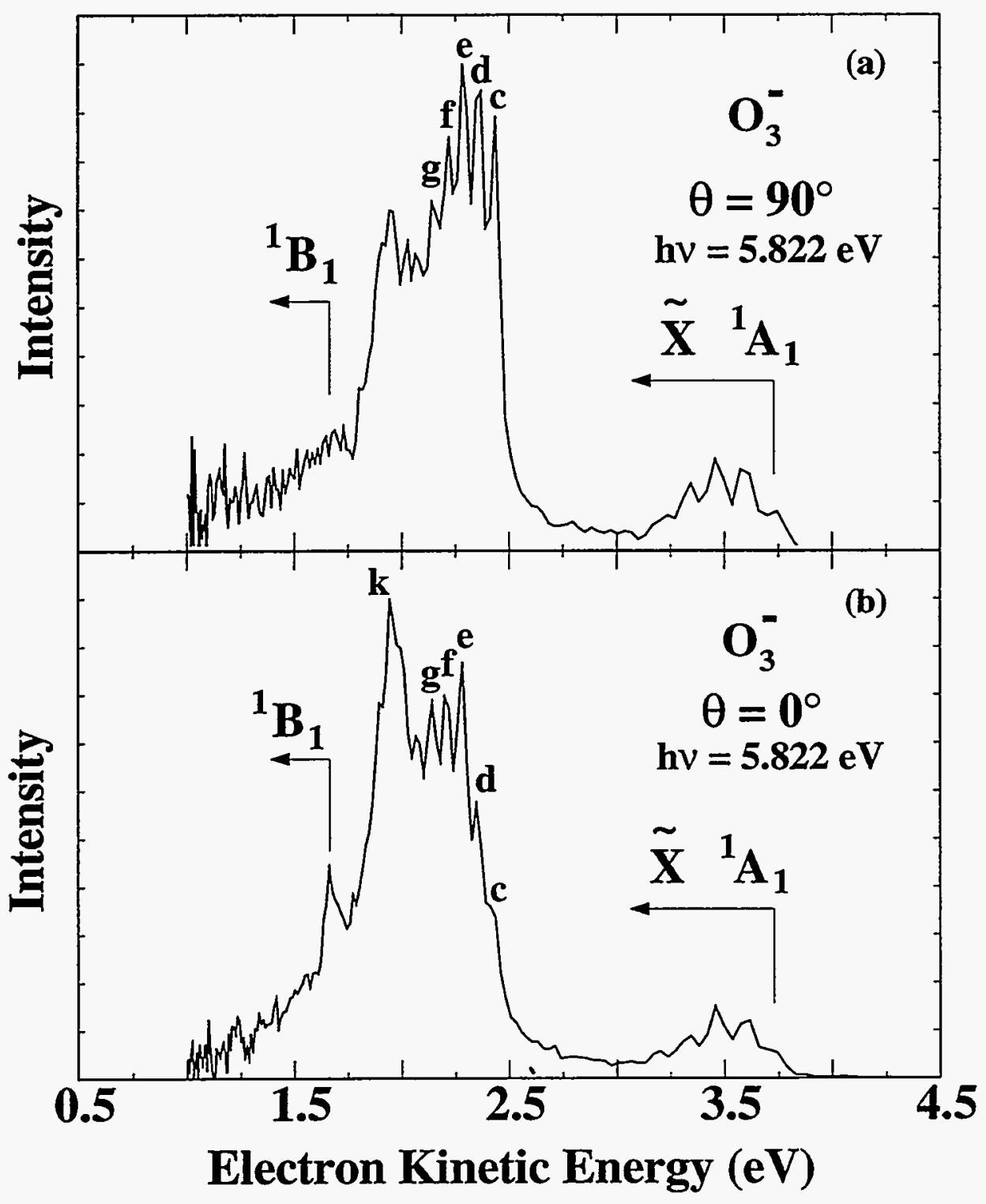

Figure 2.3 
Figure 2.2 are not due to the energy dependence of the asymmetry parameter, but rather to the existence of overlapping transitions to multiple electronic states. Detachment transitions to the ${ }^{1} \mathrm{~B}_{1}$ state become more prominent in the $5.822 \mathrm{eV}$ spectrum and exhibit a strong polarization dependence as well. The spectra show no evidence of higher lying electronic states which would appear at lower eKE.

The intensities and polarization dependence of the peaks in Figures 2.2a and $2.2 \mathrm{~b}$ suggest that the long progression beginning at $\mathrm{eKE}=1.378 \mathrm{eV}$ consists of transitions to four electronic states of $\mathrm{O}_{3}$, with origins at peaks 'a', 'c', 'e', and ' $h$ '. These states lie between the $\mathrm{O}_{3}$ ground state and the ${ }^{1} \mathrm{~B}_{1}$ excited state. The remaining low-lying excited states are the ${ }^{3} \mathrm{~A}_{2},{ }^{3} \mathrm{~B}_{2}$, ${ }^{3} B_{1}$, and ${ }^{1} A_{2}$ states, and it appears we are observing transitions to all of these. The specific assignment of the features in the photoelectron spectra to these states will be discussed further in Sec. 2.4.2.

\subsection{Analysis and Discussion}

The data presented in Sec. 2.3 provide information about the ground state and the five lowest-lying electronic states of $\mathrm{O}_{3}$. In Sec. 2.4.1, the analysis of the $2.977 \mathrm{eV} \mathrm{O}-$ photoelectron spectrum is discussed. A Franck-Condon analysis of the $\mathrm{O}_{3}\left(\tilde{\mathrm{X}}{ }^{1} \mathrm{~A}_{1}\right)$ ground state spectrum provides the ozonide geometry. In Sec. 2.4 .2 , the $4.657 \mathrm{eV}$ spectrum of the ozone excited states is considered and compared with previous experimental and theoretical results (Table IV).

\subsection{1 $2.977 \mathrm{eV}-\mathrm{O}_{3}^{-} \rightarrow \mathrm{O}_{3}\left(\overline{\mathrm{X}}^{1} \mathrm{~A}_{1}\right)$ : Determination of the $\mathrm{O}_{3}^{-}$geometry}

While Novick et al. ${ }^{25}$ previously obtained photoelectron spectra of $\mathrm{O}_{3}^{-}$, the present results are of sufficiently higher resolution to resolve the $\mathrm{O}_{3}\left(\tilde{\bar{X}}{ }^{1} \mathrm{~A}_{1}\right) v_{2}$ bending progression which was not observed in their data. The electron affinity of ozone, determined from the position of the 0-0 peak in the spectrum and Eq. (2), $\mathrm{EA}\left(\mathrm{O}_{3}\right)=2.103 \pm 0.004 \mathrm{eV}$, agrees well 
with the value previously determined by Novick et al. ${ }^{25}$ using threshold photodetachment $\left(\mathrm{EA}\left(\mathrm{O}_{3}\right)=2.1028 \pm 0.0025 \mathrm{eV}\right)$

Table IV: Calculated and Experimental Properties of Electronic States of Ozone

\begin{tabular}{|c|c|c|c|c|c|c|c|}
\hline State & Theory & $\mathbf{r}_{\mathbf{e}}(\AA)$ & $\theta_{\mathrm{e}}(\mathrm{deg})$ & $\omega_{1}\left(\mathbf{c m}^{-1}\right)$ & $\omega_{2}\left(\mathbf{c m}^{-1}\right)^{*}$ & $\mathbf{T}_{\mathrm{e}}(\mathrm{eV})$ & Ref \\
\hline $\mathrm{O}_{3}{ }^{1} \mathrm{~A}_{1}$ & $\begin{array}{c}\text { POL-CI } \\
\text { MCSCF + CI } \\
\text { MRD-CI } \\
-\end{array}$ & $\begin{array}{c}1.299 \\
1.277 \\
1.29 \\
1.2717\end{array}$ & $\begin{array}{c}116 \\
116.1 \\
116.0 \\
116.7\end{array}$ & $\begin{array}{c}1235.0 \\
1173.0 \\
1105 \\
1135 \\
\end{array}$ & $\begin{array}{c}707.0 \\
737.1 \\
704 \\
716 \\
\end{array}$ & $\begin{array}{l}0.0 \\
0.0 \\
0.0 \\
0.0\end{array}$ & $\begin{array}{l}a \\
b \\
d \\
f\end{array}$ \\
\hline${ }^{3} B_{2}$ & $\begin{array}{c}\text { POL-CI } \\
\text { MCSCF + CI } \\
\text { MRD-CI } \\
-\end{array}$ & $\begin{array}{c}1.382 \\
1.360 \\
1.34 \\
-\end{array}$ & $\begin{array}{c}107.9 \\
108.3 \\
108.5 \\
- \\
\end{array}$ & $\begin{array}{c}1112.0 \\
1176.4 \\
1276 \\
- \\
\end{array}$ & $\begin{array}{c}645.0 \\
624.6 \\
600 \\
580(50)^{\mathrm{h}} \\
\end{array}$ & $\begin{array}{c}0.92 \\
1.09 \\
1.10 \\
\mathrm{~T}_{0}=1.30\end{array}$ & $\begin{array}{l}\text { a } \\
\mathrm{c} \\
\mathrm{d} \\
\mathrm{g}\end{array}$ \\
\hline${ }^{3} \mathrm{~A}_{2}$ & $\begin{array}{c}\text { POL-CI } \\
\text { MCSCF + CI } \\
\text { MRD-CI } \\
- \\
-\end{array}$ & $\begin{array}{c}1.366 \\
1.348 \\
1.36 \\
- \\
- \\
\end{array}$ & $\begin{array}{c}99.7 \\
101.5 \\
103.6 \\
- \\
- \\
\end{array}$ & $\begin{array}{c}1167.0 \\
1224.0 \\
1288 \\
- \\
-\end{array}$ & $\begin{array}{c}535.0 \\
552.8 \\
552 \\
528(15) \\
530(50)^{\mathrm{h}}\end{array}$ & $\begin{array}{c}1.35 \\
1.34 \\
0.86 \\
\mathrm{~T}_{\mathrm{o}}=1.18 \\
\mathrm{~T}_{0}=1.18\end{array}$ & $\begin{array}{l}\text { a } \\
c \\
d \\
\text { e } \\
\text { g }\end{array}$ \\
\hline${ }^{3} B_{1}$ & $\begin{array}{c}\text { POL-CI } \\
\text { MCSCF + CI } \\
\text { MRD-CI } \\
\text { Expt. }\end{array}$ & $\begin{array}{c}1.347 \\
1.343 \\
1.36 \\
-\end{array}$ & $\begin{array}{c}123.8 \\
121.3 \\
123.5 \\
-\end{array}$ & $\begin{array}{c}915.0 \\
932.1 \\
1083 \\
- \\
\end{array}$ & $\begin{array}{c}518.0 \\
520.4 \\
662 \\
560(50)^{\mathrm{h}} \\
\end{array}$ & $\begin{array}{c}1.74 \\
1.78 \\
1.27 \\
\mathrm{~T}_{0}=1.45\end{array}$ & $\begin{array}{l}\text { a } \\
\text { c } \\
\text { d } \\
\text { g }\end{array}$ \\
\hline${ }^{1} A_{2}$ & $\begin{array}{c}\text { POL-CI } \\
\text { MCSCF + CI } \\
\text { MRD-CI } \\
\text { Expt. }\end{array}$ & $\begin{array}{c}1.374 \\
1.351 \\
1.34 \\
- \\
\end{array}$ & $\begin{array}{c}100.7 \\
101.5 \\
100.0 \\
- \\
\end{array}$ & $\begin{array}{c}1160.0 \\
1182.7 \\
1093 \\
- \\
\end{array}$ & $\begin{array}{c}537.0 \\
598.2 \\
675 \\
690(100)^{\mathrm{i}} \\
\end{array}$ & $\begin{array}{c}1.66 \\
1.57 \\
1.44 \\
\mathrm{~T}_{0} \sim 1.6 \\
\end{array}$ & $\begin{array}{l}a \\
b \\
d \\
g\end{array}$ \\
\hline${ }^{1} B_{1}$ & $\begin{array}{c}\text { POL-CI } \\
\text { MCSCF + CI } \\
\text { MRD-CI }\end{array}$ & $\begin{array}{c}1.370 \\
1.362 \\
1.35\end{array}$ & $\begin{array}{l}117.7 \\
116.2 \\
117.2\end{array}$ & $\begin{array}{c}965.0 \\
1004.6 \\
1091\end{array}$ & $\begin{array}{c}489.0 \\
509.4 \\
476\end{array}$ & $\begin{array}{l}2.06 \\
2.01 \\
1.82\end{array}$ & d \\
\hline${ }^{1} B_{2}$ & $\begin{array}{c}\text { POL-CI } \\
\text { MRD-CI }\end{array}$ & $\begin{array}{c}1.405 \\
1.38\end{array}$ & $\begin{array}{l}108.4 \\
110.1\end{array}$ & $\overline{1235}$ & $\overline{574}$ & $\begin{array}{l}5.54 \\
4.34\end{array}$ & $\begin{array}{l}\mathrm{a} \\
\mathrm{d}\end{array}$ \\
\hline
\end{tabular}

* Values in parentheses are estimated experimental uncertainties.
a) Polarization Configuration Interaction - Reference 5a.
b) Multi-Reference Self-Consistent Field + Configuration Interaction - Reference 6 a.
c) Multi-Reference Self-Consistent Field + Configuration Interaction - Reference $6 \mathrm{~b}$.
d) Multi-Reference with Single and Double Excitations + Configuration Interaction - Reference 7.
e) Reference 16.
f) Reference 35 .
g) Present work.
h) Values are based upon Franck-Condon simulations (not shown) which enable deconvolution of varied peak widths and spacings.
i) Based upon least-squares analysis of peaks ' $h$ ' through ' $m$ ' giving $\omega_{e}=694 \mathrm{~cm}^{-1}$ and $x_{e} \omega_{e}=42 \mathrm{~cm}^{-1}$. 
The length of the observed vibrational progressions provides information about the changes in equilibrium geometry between $\mathrm{O}_{3}^{-}$and $\mathrm{O}_{3}$. The long symmetric stretch progression indicates that there is a significant difference between the bond lengths of the anion and neutral. The shorter progression in the bending mode shows that the bond angles also differ but not as substantially. To determine the direction of these geometry changes, it is useful to consider the molecular orbital from which the electron is detached to form the $\mathrm{O}_{3} \tilde{\mathrm{X}}{ }^{1} \mathrm{~A}_{1}$ ground state. As indicated in Table $I$, the HOMO of the ozonide anion is a $b_{1}$ antibonding $\pi$ orbital. Removal of this electron should lead to a bond length decrease and a bond angle increase, making $\mathrm{r}_{\mathrm{O}-\mathrm{O}}\left(\mathrm{O}_{3}\right)<\mathrm{r}_{\mathrm{O}-\mathrm{O}}\left(\mathrm{O}_{3}^{-}\right)$and $\theta\left(\mathrm{O}_{3}\right)>\theta\left(\mathrm{O}_{3}^{-}\right)$.

Through the use of a Franck-Condon analysis and normal mode calculations, a quantitative determination of the ozonide anion geometry is possible following, for example, the treatment of the $\mathrm{NO}_{2}^{-}$photoelectron spectrum of Ervin et al. ${ }^{33}$ The normal modes, $Q_{i}$, are assumed to be separable and parallel (i.e., the form of the normal coordinates in the anion and neutral are the same), in which case the transition intensity, I, for the process,

$$
\mathrm{O}_{3}^{-}\left(\mathrm{v}_{1}^{\prime \prime}, \mathrm{v}_{2}^{\prime \prime}, \mathrm{v}_{3}^{\prime \prime}\right) \stackrel{\mathrm{hv}}{\longrightarrow} \mathrm{O}_{3}\left(\mathrm{v}_{1}^{\prime}, \mathrm{v}_{2}^{\prime}, \mathrm{v}_{3}^{\prime}\right)+\mathrm{e}^{-}
$$

is given by, ${ }^{34}$

$$
\mathrm{I} \propto \mathrm{v} \cdot\left|\tau_{\mathrm{e}}\right|^{2} \cdot \prod_{\mathrm{i}=1}^{3}\left|\left\langle\psi_{\mathrm{v}_{\mathrm{i}}^{\prime}}\left(Q_{i}\right) \mid \Psi_{\mathrm{v}_{\mathrm{i}}^{\prime \prime}}\left(Q_{i}\right)\right\rangle\right|^{2}
$$

The Franck-Condon factor, $\left|\left\langle\psi_{v_{i}^{\prime}} \mid \psi_{v_{i}^{\prime \prime}}\right\rangle\right|^{2}$, is the spatial overlap of the vibrational wave functions for the $i^{\text {th }}$ vibrational mode of the anion, $\Psi_{v_{i}^{\prime \prime}}$, and neutral, $\Psi_{v_{i}^{\prime}}$. The electronic transition dipole moment, $\tau_{e}$, is assumed to be constant over the energy range spanned by a 
vibrational progression for a particular electronic state, and $v$ is the asymptotic velocity of the detached electron.

The Franck-Condon factors (FCFs) are calculated using known structural parameters $\left(\mathrm{r}_{\mathrm{e}}=1.2717 \AA, \theta_{\mathrm{e}}=116.7^{\circ}\right)$, vibrational frequencies and primary anharmonicities $\left(\omega_{1}=1135\right.$ $\left.\mathrm{cm}^{-1}, \omega_{2}=716 \mathrm{~cm}^{-1}, \omega_{3}=1089 \mathrm{~cm}^{-1}, \mathrm{x}_{11}=4.9 \mathrm{~cm}^{-1}, \mathrm{x}_{22}=1.0 \mathrm{~cm}^{-1}, \mathrm{x}_{33}=10.6 \mathrm{~cm}^{-1}\right)$ of the ozone ground state..$^{35}$ The $v_{1}$ and $v_{2}$ modes of $\mathrm{O}_{3}$ are modeled with Morse potentials derived from these vibrational parameters. Anharmonic effects are most important for the $v_{1}$ symmetric stretch mode, as this is the longest progression in the spectrum. The $v_{3}$ normal coordinate change, $\Delta \mathrm{Q}_{3}$, is constrained to be zero by symmetry, and anharmonicity of this mode is neglected in the simulation shown in Fig. 2.1. As the gas phase vibrational frequencies and anharmonicities for the anion vibrational modes are not as accurately established, the frequencies are adjusted in the simulation to obtain the best agreement with the data (i.e., peak ' $a$ ' in Figure 2.1) and previous observations (see Table V). The $\mathrm{O}_{3}^{-}$harmonic frequencies employed in the simulation are $\omega_{1}=975 \mathrm{~cm}^{-1}, \omega_{2}=550 \mathrm{~cm}^{-1}$, and $\omega_{3}=880 \mathrm{~cm}^{-1}$. A vibrational temperature of $550 \mathrm{~K}$ was assumed in the simulations. At this temperature, most of the anions $(\sim 65 \%)$ are in their ground vibrational state. As a result, anion anharmonicities have minor effects on the simulations which contribute to the stated uncertainties below. The calculated FCFs are convoluted with the experimental resolution and an additional $4 \mathrm{meV}$ Gaussian peak to account for unresolved rotational contours and 'space-charge' broadening effects (see Sec. II).

The simulation in Fig. $1 \mathrm{~b}$ is obtained by varying the $v_{1}$ and $v_{2}$ normal coordinate displacements between the anion and neutral to obtain the best agreement with the experimental spectrum. It is possible to determine the $\mathrm{O}_{3}^{-}$geometry, provided we can determine the sign of the normal coordinate displacements. Based on the molecular orbital considerations discussed above, one expects a longer bond length and more acute bond angle in the anion. These expectations are supported by considering effects due to anharmonicity, which break the symmetry of the harmonic oscillator potential. Specifically, the $v_{1}$ 
anharmonicity leads to a more attractive potential at longer bond lengths. As a result, no acceptable simulation for the spectrum could be calculated if the bond length displacement was in the opposite direction (i.e., when $\mathrm{r}_{\mathrm{O}-\mathrm{o}}\left(\mathrm{O}_{3}\right)>\mathrm{r}_{\mathrm{O}-\mathrm{o}}\left(\mathrm{O}_{3}^{-}\right)$). For the $\mathrm{v}_{2}$ bending mode, the anion is assumed to have a more acute bond angle than the neutral and a more repulsive wall at smaller bond angles.

Once the normal mode displacements are determined, the geometry changes and hence the $\mathrm{O}_{3}^{-}$geometry are determined using an FG-matrix vibrational analysis. ${ }^{36}$ In the parallel mode approximation, the same force constants are used in the $\mathrm{F}$ matrix for both the anion and neutral; in this case, the $\mathrm{O}_{3}$ force constants are those determined by Hennig et al. ${ }^{37}$ The overall analysis yields $\mathrm{r}_{\mathrm{e}}\left(\mathrm{O}_{3}^{-}\right)=1.36 \pm 0.02 \AA$ and $\theta_{\mathrm{e}}\left(\mathrm{O}_{3}^{-}\right)=111.8 \pm 2.0^{\circ} .38$

Comparison of the ozonide geometry obtained from this analysis with other experiments and theoretical studies finds reasonable agreement in many cases and a slight disagreement in others. Excellent agreement is found with the results of a vibrationally resolved $\mathrm{O}_{3}^{-}$photodetachment total cross-section measurement $\left[\mathrm{EA}\left(\mathrm{O}_{3}\right)=2.082 \pm 0.040 \mathrm{eV}\right.$; $\mathrm{r}\left(\mathrm{O}_{3}^{-}\right)=1.3414 \pm 0.0300 \AA ; \theta\left(\mathrm{O}_{3}^{-}\right)=112.6 \pm 2.0^{\circ} \mathrm{J}$ by Wang et al. ${ }^{39}$ Matrix isolation spectroscopy results ${ }^{40}$ determine $\theta_{\mathrm{e}}\left(\mathrm{O}_{3}^{-}\right)$bond angles between $105^{\circ}$ and $119^{\circ}$. Since alkali counterions may distort the geometry of the anion, $\mathrm{O}_{3}^{-}$has been studied in an Ar matrix without counterions ${ }^{41}$ to yield $\theta_{\mathrm{e}}\left(\mathrm{O}_{3}^{-}\right)=110 \pm 5^{\circ}$. The isoelectronic species, $\mathrm{SO}_{2}^{-}$, undergoes a similar geometric rearrangement upon photodetachment $\left(\Delta r_{0}=0.09 \AA, \Delta \theta_{0}=3.9^{\circ}\right) .42$ Our ozonide geometry does not lie on the seam of geometries derived from a Franck-Condon analysis of the photoelectron spectrum obtained by Novick et al. ${ }^{25}$ This disagreement stems from the effect which the newly resolved bending progression produces in the normal coordinate analysis. Recent $a b$ initio calculations predict $\mathrm{O}_{3}^{-}$bond lengths in agreement with the present results but predicts a larger bond angle than that determined from the photoelectron spectrum (see Table V). Our geometry changes are close to those obtained by Cederbaum et al. ${ }^{43}$ in their calculation of the $\mathrm{O}_{3}$ radiative attachment spectrum. 
Table V: Calculated and Experimental Properties of the Ozonide Anion Ground State

\begin{tabular}{|c|c|c|c|c|c|c|}
\hline $\mathrm{O}_{3}^{-}-\tilde{\mathrm{X}}^{2} \mathrm{~B}_{1}$ & $\mathbf{r}_{e}(\AA)$ & $\theta_{e}(\mathrm{deg})$ & $\omega_{1}\left(\mathrm{~cm}^{-1}\right)$ & $\omega_{2}\left(\mathrm{~cm}^{-1}\right)$ & $\omega_{3}\left(\mathbf{c m}^{-1}\right)$ & Ref. \\
\hline \multirow[t]{3}{*}{ Theory } & 1.385 & 115.4 & 976 & 552 & - & $\mathbf{a}$ \\
\hline & 1.35 & 114.5 & - & - & - & $\mathrm{b}$ \\
\hline & 1.361 & 115.4 & 992 & 572 & 879 & c \\
\hline \multirow[t]{6}{*}{ Expt. } & - & $110 \pm 5$ & - & - & 800 & $d$ \\
\hline & $1.3414(0.03)$ & $112.6 \pm 2.0$ & - & - & - & $\mathrm{e}$ \\
\hline & - & - & $\begin{array}{l}\text { a) } 790 \pm 50 \\
\text { b) } 928 \pm 50\end{array}$ & $\begin{array}{l}\text { a) } 419 \pm 20 \\
\text { b) } 403 \pm 20\end{array}$ & - & $\mathrm{f}$ \\
\hline & - & - & $975 \pm 10$ & $590 \pm 10$ & - & $\mathrm{g}$ \\
\hline & \multicolumn{2}{|c|}{ see text and Ref 25} & $982 \pm 50$ & $550 \pm 50$ & - & $\mathrm{h}$ \\
\hline & $1.36 \pm 0.02$ & $111.7 \pm 2.0$ & $975 \pm 50$ & $550 \pm 50$ & $880 \pm 50^{*}$ & $\mathrm{i}$ \\
\hline
\end{tabular}

* Determined from sequence bands of unpublished spectrum of vibrationally excited $\mathrm{O}_{3}^{-}$.

a) K. A. Peterson, R. C. Mayrhofer and R. C. Woods, J. Chem. Phys. 93, 5020 (1990).

b) W. Koch, G. Frenking, G. Steffen, D. Reinen, M. Jansen, and W. Assenmacher, J. Chem. Phys. 99, 1271 (1993).

c) R. González-Luque, M. Merchán, P. Borowski and B. O. Roos, Theor. Chim. Acta 86, 467 (1993).

d) Reference 41.

e) Reference 39

f) J. F. Hiller and M. L Vestal, J. Chem. Phys 74, 6096 (1981).

g) P. C. Cosby, J. T. Moseley, J. R. Peterson, and J. H. Ling, J. Chem. Phys. 69, 2771 (1978)

h) Reference 25 .

i) Present work. 


\subsection{2. $\mathrm{O}_{3}$ Excited States}

As discussed in Sec. 2.3, the $4.657 \mathrm{eV} \mathrm{O}_{3}^{-}$spectrum appears to represent photodetachment transitions to the ground state and the five lowest-lying excited states of the ozone molecule. In this section, these excited state bands will be discussed in more detail. Comparisons will be made with other experimental results and with the predictions of $a b$ initio calculations in an effort to assign features of the spectrum to specific $\mathrm{O}_{3}$ electronic states. In addition, we will consider the exceptional amount of vibrational structure seen in the $\mathrm{O}_{3}^{-}$ photoelectron spectrum, much of which lies well above the dissociation asymptotes of the electronic states observed.

\subsubsection{Assignment of the $\mathrm{O}_{3}$ excited electronic states}

The intensity distribution and polarization dependence of the spectral features in Fig. 2.2 imply that transitions to five excited $\mathrm{O}_{3}$ states occur in the $\mathrm{hv}=4.657 \mathrm{eV}$ photoelectron spectrum. In addition to the ${ }^{1} \mathrm{~B}_{1}$ state at $\mathrm{eKE}=0.5 \mathrm{eV}$, excited state origins occur at eKEs of $1.378,1.257,1.105$ and $0.97 \mathrm{eV}$ (peaks 'a', 'c', 'e', and ' $h$ ', respectively). These correspond to excitation energies $\left(\mathrm{T}_{\mathrm{o}}\right)$ of $1.18,1.30,1.45$, and $-1.6 \mathrm{eV}$, respectively. As indicated in the Fig. $2.2 \mathrm{a}$, the origin of the Wulf band, as determined by Anderson and co-workers $\left(\mathrm{T}_{0}=1.18 \pm 0.01\right.$ $\mathrm{eV}),{ }^{16}$ is expected to lie at $\mathrm{eKE}=1.371 \mathrm{eV}$ in reasonable agreement with the position of peak ' $a$ '. Anderson and co-workers have recently obtained independent evidence that excited states may exist at excitation energies of 1.29 and $1.45 \mathrm{eV}$ using absorption and isotopic substitution techniques. ${ }^{44}$ These energies are in excellent agreement with the positions of peaks 'c' and ' $e$ ', respectively. No evidence for an excited state with $T_{0} \sim 1.6 \mathrm{eV}$ has been found in any absorption spectra as of yet.

The assignment of these features to specific electronic states is complicated by the considerable spectral overlap among the bands. As a first step, it is useful to address only geometric considerations. As discussed in Sec. 2.4.1, the geometric differences between the anion and neutral govern the Franck-Condon factors and thus the vibrational profile for each 
electronic state. Based on the $a b$ initio geometries in Tables $I V$ and $V$, one expects significant bending mode excitation upon photodetachment of the ozonide anion for the ${ }^{3} \mathrm{~A}_{2},{ }^{3} \mathrm{~B}_{1}$, and ${ }^{1} \mathrm{~A}_{2}$ states and very little vibrational excitation for the ${ }^{3} B_{2}$ and ${ }^{1} B_{1}$ states. Since the bond lengths of the excited states are approximately equal to that determined for the anion, very little excitation of the symmetric stretch is expected for any of the excited states observed. As a general rule, excitation of non-totally symmetric vibrational modes $\left(v_{3}\right)$ is not expected unless a large frequency difference exists between the anion and the neutral in that mode.

$A b$ initio predictions of the excited state energies (see Table IV) are also helpful in assigning the spectra. High level $a b$ initio calculations have predicted various orderings of the electronic states, depending upon the level of theory employed. ${ }^{5-8}$ While all of the states are fairly low-lying, the ${ }^{3} \mathrm{~A}_{2}$ and ${ }^{3} \mathrm{~B}_{2}$ states are usually found to be the lowest excited states and fluctuate above or below the dissociation asymptote of $\mathrm{O}_{3}$ depending upon the calculation performed.

Summarizing our expectations based on ab initio calculations, the two lowest excited states are the ${ }^{3} \mathrm{~A}_{2}$ state with a long vibrational progression and the ${ }^{3} \mathrm{~B}_{2}$ state with a short progression. However, the energetic ordering of the two states is undetermined. These two states are expected to lie below the ${ }^{1} \mathrm{~A}_{2}$ and the ${ }^{3} \mathrm{~B}_{1}$ states, both of which should have long bending progressions. To agree with the Chappuis band assignment, the ${ }^{1} \mathrm{~A}_{2}$ should be energetically proximal to the ${ }^{1} \mathrm{~B}_{1}$ state.

Turning to the photoelectron spectra in Fig. 2.2, it appears that peaks 'a' and ' $b$ ' are the beginning of a long vibrational progression, whereas peaks ' $c$ ' and ' $d$ ' are part of a considerably shorter progression. From the above discussion, we would assign peaks 'a' and 'c' to the origins of the ${ }^{3} \mathrm{~A}_{2}$ and ${ }^{3} \mathrm{~B}_{1}$ states, respectively. As a more quantitative comparison between theory and experiment, one can easily generate a simulated photoelectron based upon the output of $a b$ initio calculations within the separable normal mode approximation. These simulations require the calculation of geometries and force constant matrices so that the normal coordinate 
displacements can be calculated. Since configuration interaction $(\mathrm{CI})$ is known to be significant for most of the $\mathrm{O}_{3}$ electronic states considered, the calculations must include these effects. We have carried out such calculations with single and double excitations (SCF-CISD) within a standard 6-31G basis set using the Gaussian 92 package. ${ }^{45}$ As we are only interested in the totally symmetric modes, the calculation is restricted to geometries with $C_{2 v}$ symmetry. While not as extensive as previously published calculations, the geometries and frequencies are in reasonable agreement ${ }^{46}$ with the higher level results in Table IV, so the force constants should be sufficiently accurate for our purposes.

For each $\mathrm{O}_{3}$ excited state, the normal coordinates for the $v_{1}$ and $v_{2}$ modes are obtained by diagonalization of the $a b$ initio Cartesian force constant matrix, and the normal coordinate displacements between the anion (using our experimentally determined geometry) and each neutral state are determined within the parallel mode approximation. We then calculate Franck-Condon factors for the two anion $\rightarrow$ neutral transitions as in Sec. 2.4.1, again assuming an anion vibrational temperature of $550 \mathrm{~K}$.

The resulting simulations for the ${ }^{3} \mathrm{~A}_{2}$ and ${ }^{3} \mathrm{~B}_{2}$ states are shown in Fig. $2.4 \mathrm{a}$ and $2.4 \mathrm{~b}$, respectively. As expected, the bending progression for the transition to the ${ }^{3} \mathrm{~B}_{2}$ state is considerably shorter than that of the ${ }^{3} \mathrm{~A}_{2}$ state. In Fig. $2.4 \mathrm{c}$ and $2.4 \mathrm{~d}$, these simulations are superimposed on the experimental spectra at laser polarization angles $\theta=0^{\circ}$ and $90^{\circ}$, respectively. Here the ${ }^{3} \mathrm{~A}_{2}$ state is assigned to the Wulf band beginning at $\mathrm{eKE}=1.378 \mathrm{eV}$,

Figure 2.4: [(a) and (b)] Franck-Condon calculations for photodetachment to the $\mathrm{O}_{3}{ }^{3} \mathrm{~A}_{2}$ and ${ }^{3} \mathrm{~B}_{2}$ electronic states using $a b$ initio calculated geometries and vibrational frequencies and the experimentally determined $\mathrm{O}_{3}^{-}$geometry (See Sec 2.4 .2 for details). [(c) and (d)] Simulations (solid lines) are scaled and summed to fit first several peaks in the $4.657 \mathrm{eV}$ experimental spectra (dashed lines) collected at laser polarizations of $\theta=0^{\circ}$ and $90^{\circ}$. Origins of ${ }^{3} \mathrm{~A}_{2}$ and ${ }^{3} \mathrm{~B}_{2}$ states are assumed to be peaks ' $a$ ' and ' $c$ ', respectively. Scaling factors are $\left(0.42 \cdot{ }^{3} \mathrm{~A}_{2}+0.3 \cdot{ }^{3} \mathrm{~B}_{2}\right)$ in Figure $2.4 \mathrm{c}$ and $\left(0.53 \cdot{ }^{3} \mathrm{~A}_{2}+0.53 \cdot{ }^{3} \mathrm{~B}_{2}\right)$ in Figure $2.4 \mathrm{~d}$. 


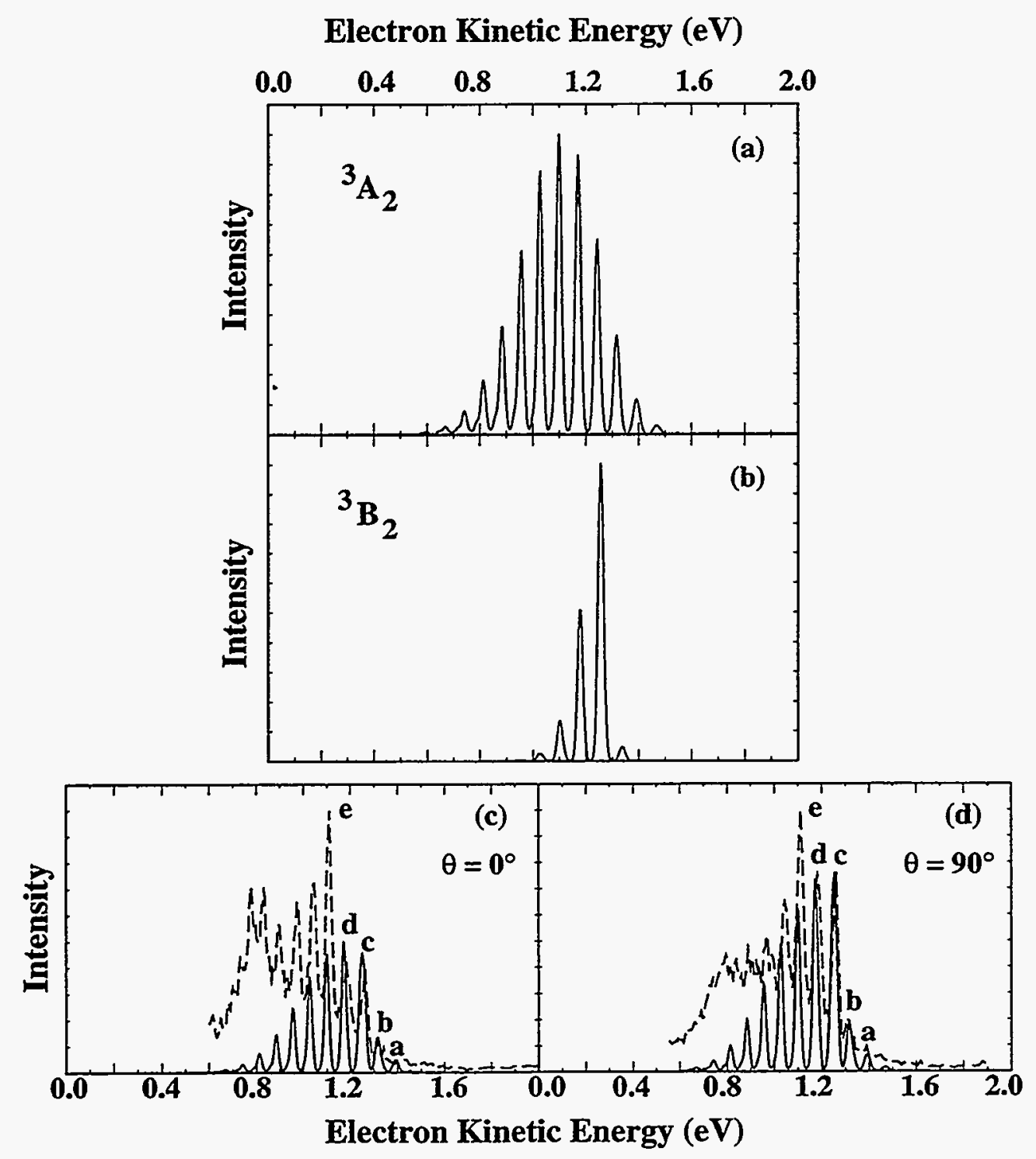

Figure 2.4 
peak ' $a$ ', and the ${ }^{3} \mathrm{~B}_{2}$ state to the state beginning at $\mathrm{eKE}=1.257 \mathrm{eV}$, peak 'c'. By combining scaled ${ }^{3} \mathrm{~A}_{2}$ and ${ }^{3} \mathrm{~B}_{2}$ simulations, as indicated in the Figure caption, one can approximately simulate the peak intensities at both laser polarizations for peaks ' $a$ '-' $d$ '. This cannot be done if the assignment of the origins is reversed. Beginning at peak 'e', another state begins to contribute to the spectrum, hence its greater intensity and different polarization dependence. Overall, the simulations support our assignment of the ${ }^{3} \mathrm{~A}_{2}$ and ${ }^{3} \mathrm{~B}_{2}$ states. Our assignment of the ${ }^{3} \mathrm{~A}_{2}$ state is consistent with that of Braunstein et al. ${ }^{6 \mathrm{~b}}$

The two electronic states which remain unassigned are the ${ }^{3} B_{1}$ and the ${ }^{1} A_{2}$ states. As mentioned above, long progressions are expected for both the ${ }^{1} \mathrm{~A}_{2}$ and the ${ }^{3} \mathrm{~B}_{1}$ states. However, one of the two remaining states in the spectra, beginning at peak 'e', has only has medium $v_{2}$ length progression '. Thus the simple considerations of geometry and energy are insufficient to determine a specific assignment of these states. No additional assistance is gained from calculated vibrational frequencies for these states since at each level of theory investigated, both states have similar $v_{2}$ frequencies (Table IV). However, the recent $a b$ initio calculation by Banichevich and Peyerimhoff ${ }^{7}$ predicts the ${ }^{1} A_{2}$ state to lie $0.17 \mathrm{eV}$ above the ${ }^{3} B_{1}$ state, suggesting that the origins of the two states should be assigned to peaks ' $h$ ' and 'e', respectively, with $\mathrm{T}_{0}$ values of -1.6 and $1.45 \mathrm{eV}$. This assignment, particularly that of the ${ }^{1} \mathrm{~A}_{2}$ origin, must be considered as tentative, however. While the most consistent assignment of the ${ }^{1} \mathrm{~A}_{2}$ origin is to peak ' $h$, it is not definitive due to the spectral congestion in this region.

As mentioned above, the variation of peak widths in the spectrum most likely results from the convolution of nearly overlapping transitions to different electronic states. Deconvolution of the irregular peak widths and spacings through a Franck-Condon analysis (not shown) provides the $v_{2}$ vibrational frequencies for the ${ }^{3} \mathrm{~A}_{2},{ }^{3} \mathrm{~B}_{2}$, and ${ }^{3} \mathrm{~B}_{1}$ electronic states. The values, which differ from the raw spacings of the peak centers, are included in Table IV along with the excitation energies of these states determined from the data. 
The one aspect of the spectra not discussed so far is the broad feature around eKE $=$ $0.75 \mathrm{eV}$ in Figure 2.2a. This band could result from very rapid dissociation of the higher lying vibrational levels of one the $\mathrm{O}_{3}$ excited states, most likely the one with its origin at peak ' $e$ '. Alternatively, the width of this feature could be entirely heterogeneous, and due instead to overlapping vibrational transitions from several electronic states. While one might hope to distinguish between these possibilities based via laser polarization effects, the polarization dependence of this feature is difficult to determine as it is obscured by peaks ' $\mathrm{i}-\mathrm{-} l \mathrm{l}$ ' in Figure $2.2 \mathrm{~b}$.

\subsubsection{2 $\mathrm{O}_{3}$ excited state dissociation dynamics}

We next consider in greater detail what the photoelectron spectrum of the $\mathrm{O}_{3}$ excited states reveals about their energetics and dissociation dynamics. All of the $\mathrm{O}_{3}$ excited states accessed in the photoelectron spectrum lie above the dissociation threshold to ground state $O$ $\left({ }^{3 P}\right)+\mathrm{O}_{2}\left(\mathrm{X}^{3} \Sigma_{\mathrm{g}}^{-}\right)$products. Since all of the low-lying excited states of $\mathrm{O}_{3}$ have been accounted for, this means there are no bound excited electronic states of $\mathrm{O}_{3}$. This result is relevant to atmospheric chemistry since it rules out the possibility of a bound excited state affecting the $\mathrm{O}+\mathrm{O}_{2}$ recombination rate; the discrepancy in this rate measurement discussed in the Introduction must come from another source.

Since all of the excited states have sufficient energy to dissociate, the presence of so much well-resolved vibrational structure in the excited state region of the photoelectron spectrum is of considerable interest. The observation of vibrational structure in transitions to

Figure 2.5: Correlation diagram for $\mathrm{O}_{3}$ in $\mathrm{C}_{2 \mathrm{v}}$ symmetry adapted from Hay and Dunning. $5 c$ The relative energies of the electronic states have been modified to reflect the preliminary assignments made from the spectra presented. The ${ }^{1} \mathrm{~A}_{2}$ and ${ }^{3} \mathrm{~B}_{1}$ ordering is tentative. The arrows marked ' $a$ ', ' $b$ ', and ' $c$ ' indicate the highest energies accessible using the 2.977, 4.657, and $5.822 \mathrm{eV}$ photodetachment energies, respectively. 


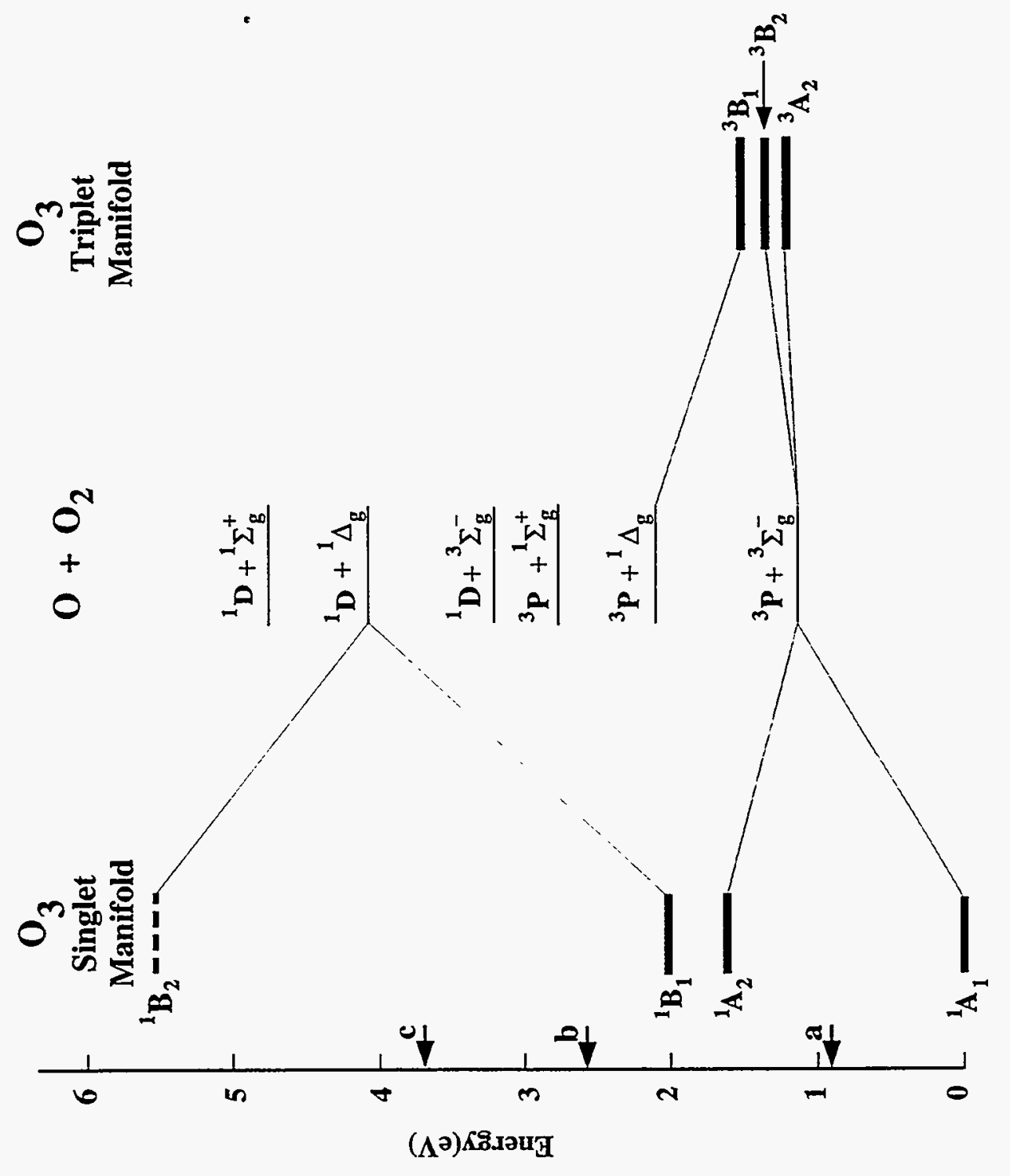

Figure 2.5 
dissociative electronic states in both absorption and photoelectron spectra is well known, and can occur through a variety of mechanisms. The simplest explanation for the current case is suggested by the correlation diagram in Figure 5, adapted from the work of Hay and Dunning. $5 \mathrm{c}$ This shows that the ${ }^{3} \mathrm{~A}_{2},{ }^{3} \mathrm{~B}_{2}$, and ${ }^{1} \mathrm{~A}_{2}$ states correlate to ground state $\mathrm{O}\left({ }^{3} \mathrm{P}\right)+\mathrm{O}_{2}\left(\mathrm{X}^{3} \Sigma_{\mathrm{g}}^{-}\right)$ products, whereas the ${ }^{3} B_{1}$ and ${ }^{1} B_{1}$ states correlate to excited $O\left({ }^{3} \mathrm{P}\right)+\mathrm{O}_{2}\left(\mathrm{a}^{1} \Delta_{\mathrm{g}}\right)$ and $\mathrm{O}\left({ }^{1} \mathrm{D}\right)+$ $\mathrm{O}_{2}$ (a ${ }^{1} \Delta_{\mathrm{g}}$ ) products, respectively. Thus, both of the latter states lie below their respective asymptotic channels. One can therefore imagine that the structure in the transitions to the ${ }^{3} \mathrm{~B}_{1}$ and ${ }^{1} B_{1}$ states arises because they are long-lived states that undergo predissociation. On the other hand, the lower three states lie above the asymptotic channel to which they correlate. Even if these states are purely repulsive along the dissociation coordinate (the $Q_{3}$ antisymmetic stretch coordinate in the Franck-Condon region), structure can arise in the spectrum if the dissociating molecule undergoes vibrational motion along the bound symmetric stretch and bend coordinates on the same time scale as dissociation. Such a mechanism has been proposed for structured absorption bands in several small molecules. ${ }^{47,48}$

As an exemplary application of this latter mechanism to the electronic spectroscopy of $\mathrm{O}_{3}$, Braunstein and $\mathrm{Pack}^{6 \mathrm{~b}}$ have simulated the $\mathrm{O}_{3}{ }^{3} \mathrm{~A}_{2} \leftarrow{ }^{1} \mathrm{~A}_{1}$ band, which is allowed via spinorbit coupling between the ${ }^{3} \mathrm{~A}_{2}$ and ${ }^{1} \mathrm{~B}_{2}$ states (the latter is responsible for the very strong Hartley band around $250 \mathrm{~nm}$ ). Even though the ${ }^{3} \mathrm{~A}_{2}$ potential energy surface was assumed to be repulsive along the $\mathrm{Q}_{3}$ coordinate, the simulated spectrum shows a resolved bend progression that approximately matches the structure seen in the Wulf band of $\mathrm{O}_{3}$. However, a very recent reinvestigation of the Wulf band by Anderson and co-workers uncovered resolved rotational structure in the first few vibrational bands. ${ }^{16}$ While the above mechanism enables vibrational structure to survive for dissociative states, the presence of this rotational structure suggests the existence of a barrier to dissociation along the $\mathrm{Q}_{3}$ coordinate. Anderson et al. estimate the height of this barrier to be on the order of $\sim 0.1 \mathrm{eV}$. 
A more complex mechanism has been proposed by Braunstein et al. ${ }^{6}$ and Banichevich et al. ${ }^{8}$ to explain the diffuse structure observed in the $\mathrm{O}_{3}$ Chappuis band. Specifically, when $\mathrm{O}_{3}$ is restricted to $C_{2 v}$ symmetry, the ${ }^{1} B_{1}$ and ${ }^{1} A_{2}$ states intersect near the Franck-Condon region for absorption from the $\mathrm{O}_{3}$ ground state. However, both states have ${ }^{1} \mathrm{~A}^{\prime \prime}$ symmetry in the $\mathrm{C}_{\mathrm{s}}$ point group, so the two states repel each other if there is any displacement along the $\mathrm{O}_{3}$ antisymmetric stretch coordinate. This results in a conical intersection when the full three dimensional potential energy surfaces for the two states are considered. While the lower $1^{1} \mathrm{~A}$ " state is repulsive along the $\mathrm{Q}_{3}$ coordinate, the upper $2{ }^{1} \mathrm{~A}$ " state (the "cone" state) can support vibrational levels which are bound along all three coordinates, although they couple to the lower repulsive state via non-adiabatic interactions. Both sets of calculations indicate that the 2 ${ }^{1} \mathrm{~A}$ " state is responsible for the partially resolved vibrational structure in the $\mathrm{O}_{3}$ Chappuis band. A conical intersection between the ${ }^{3} \mathrm{~A}_{2}$ and ${ }^{3} \mathrm{~B}_{1}$ states is also predicted to occur near the Franck-Condon region, but the effect this has on the absorption spectrum has not been considered in detail.

The same mechanisms responsible for structure in the absorption spectrum can be applied to excited state region of the $\mathrm{O}_{3}^{-}$photoelectron spectrum. However, it is noteworthy that the photoelectron spectrum is more structured than the absorption spectrum; one might have instead expected the photoelectron spectrum to be more congested because transitions to more electronic states are allowed. Several possible causes for these differences could arise from the different Franck-Condon regions for photodetachment vs. absorption. For example, while photodetachment of $\mathrm{O}_{3}^{-}$to the $\mathrm{O}_{3}$ excited states primarily excites the $v_{2}$ bending mode, the $\mathrm{O}_{3}{ }^{1} \mathrm{~A}_{2} \leftarrow{ }^{1} \mathrm{~A}_{1}$ transition will excite both the bend and the $v_{1}$ symmetric stretch, further congesting the absorption spectrum. One should also consider the proximity of the FranckCondon region to the conical intersection between the ${ }^{1} B_{1}$ and ${ }^{1} A_{2}$ states, which occurs near $\theta$ $=120^{\circ}$ over a wide range of $\mathrm{O}_{3}$ bond lengths. Since the $\mathrm{O}_{3}^{-}$bond angle is smaller than that of the neutral (see Sec IV A), the photodetachment Franck-Condon region is farther from the 
conical intersection than that for absorption. For $\mathrm{r}_{0-0}=1.35 \AA$, approximately the bond length of the anion, Braunstein and $\mathrm{Pack}^{6}$ clearly illustrate that as the bond angle is changed from $\theta$ $120^{\circ}$ the potential energy surface along the $Q_{3}$ coordinate of the $1^{1} \mathrm{~A}^{\prime \prime}$ state becomes less repulsive. The $1^{1} \mathrm{~A}^{\prime \prime}$ state flattens along $\mathrm{Q}_{3}$ due to weaker interaction with the upper $2{ }^{1} \mathrm{~A}^{\prime \prime}$ state at the anion geometry. The flatter surface may lead to slower dissociation and a more structured spectrum. Similar effects may also occur in the triplet manifold where the conical intersection occurs at approximately the same bond angle as for the singlet states. The lower energy triplet surfaces are already closer to the dissociation limit so that they must be less repulsive along the dissociation coordinate anyway.

Photodetachment and absorption transitions to the $\mathrm{O}_{3}\left({ }^{1} \mathrm{~A}_{2}\right)$ state differ in another significant respect. In absorption, the ${ }^{1} \mathrm{~A}_{2} \leftarrow{ }^{1} \mathrm{~A}_{1}$ transition is only vibronically allowed via the antisymmetric stretch; thus, this absorption process necessarily includes $v_{3}$ excitation. Along the $Q_{3}$ coordinate, the ${ }^{1} A_{2}$ potential energy surface has a maximum at $Q_{3}=0$ and slopes downward to $\mathrm{O}+\mathrm{O}_{2}$ dissociation products at $\left|\mathrm{Q}_{3}\right|>0$. The effect of this on the dissociation dynamics can best be pictured in the language of wave packets. ${ }^{47 c}$ As Braunstein and Pack 6 point out, the initial $t=0$ wave packet placed on the ${ }^{1} A_{2}$ surface by absorption has a node at the relatively flat $Q_{3}=0$ barrier and the greatest amplitude at a non zero value of $Q_{3}$ on the repulsive part of the potential. As a consequence, simulated spectra of the ${ }^{1} A_{2} \leftarrow{ }^{1} A_{1}$ transition show no resolved vibrational structure since the wave packet rapidly moves out of the Franck-Condon region to dissociation products. In contrast, the photodetachment transition from $\mathrm{O}_{3}^{-}$to the $\mathrm{O}_{3}{ }^{1} \mathrm{~A}_{2}$ state is fully allowed. In this case, the $t=0$ wave packet will be the Franck-Condon projection of the anion ground state vibrational wave function onto the ${ }^{1} \mathrm{~A}_{2}$ surface. By symmetry, the maximum amplitude of this wave packet will occur at $\mathrm{Q}_{3}=0$ where the first derivative of the surface along the $Q_{3}$ coordinate is zero by definition. Recurrences along the bound normal coordinates, which are necessary for observing vibrational structure, are more likely to occur under these conditions. These considerations indicate that 
photodetachment should produce a more structured ${ }^{1} \mathrm{~A}_{2}$ spectrum than absorption; the extent of the difference depends upon the actual steepness of the dissociation pathway in the vicinity of the barrier.

Given the complicated interactions between the $\mathrm{O}_{3}$ excited states discussed above, it is perhaps surprising that the excited state region of the $\mathrm{O}_{3}^{-}$photoelectron spectrum is as regular as it is. Clearly, this spectrum warrants a more detailed treatment than given here. For example, realistic simulations of the photoelectron spectrum should consider the effects of the multiple conical intersections in the $\mathrm{O}_{3}$ excited state manifold. Strong vibronic coupling in the vicinity of these intersections ${ }^{49}$ requires considerably more sophisticated simulation methods than the separable Franck-Condon model used in this paper. We hope that the data presented here stimulate further theoretical studies of this fundamentally important molecule.

\subsection{Conclusions}

We have presented photoelectron spectra of $\mathrm{O}_{3}^{-}$obtained using photodetachment energies of $2.977,4.657$ and $5.822 \mathrm{eV}$. From the $2.977 \mathrm{eV}$ spectrum, we determine a geometry for the ozonide anion $\left(\mathrm{r}_{\mathrm{e}}\left(\mathrm{O}_{3}^{-}\right)=1.36 \pm 0.02 \AA\right.$ and $\left.\theta_{e}\left(\mathrm{O}_{3}^{-}\right)=111.8 \pm 2^{\circ}\right)$. The 4.657 and 5.822 eV spectra show evidence of transitions to multiple electronic states. Laser polarization studies indicate that we are observing transitions to five excited states of $\mathrm{O}_{3}$ below $3 \mathrm{eV}$ in energy: the ${ }^{3} \mathrm{~A}_{2},{ }^{3} \mathrm{~B}_{2},{ }^{3} \mathrm{~B}_{1},{ }^{1} \mathrm{~A}_{2}$, and ${ }^{1} \mathrm{~B}_{1}$ states. Of importance to atmospheric chemists is the fact that we do not observe any evidence of electronic states lying below the ground state dissociation asymptote. Simulations of the data and comparison with published $a b$ initio results indicate that the three lowest-lying electronic states have a ${ }^{1} \mathrm{~A}_{1},{ }^{3} \mathrm{~A}_{2},{ }^{3} \mathrm{~B}_{2}$, energetic ordering, with the states lying at $T_{0}$ values of $0.0,1.18,1.30 \mathrm{eV}$, respectively. $T_{0}$ values of 1.45 and $-1.6 \mathrm{eV}$ are proposed for the ${ }^{3} \mathrm{~B}_{1}$ and ${ }^{1} \mathrm{~A}_{2}$ states, respectively; this assignment is more tentative, however. The large amount of vibrational structure above the dissociation limits of the excited states suggests that interesting dissociation dynamics occurs on these surfaces. The vibrational 
structure and polarization dependence of the data presented here serves as an excellent guide to determining the form and interaction of the low-lying electronic state potential energy surfaces, and it is hoped that these results will stimulate theoretical efforts along these lines.

\subsection{Acknowledgments}

We would like to thank Professor S. M. Anderson for stimulating discussion and communication of unpublished results. We thank Dr. M. Braunstein for communication of unpublished results and $\mathrm{P}$. Ludowise for providing $\mathrm{O}_{3}$. This work has been sponsored by the United States Air Force Office of Scientific Research under contract number AFOSR-91-0084. 


\section{References}

${ }^{1}$ S. Solomon, Rev. Geophys. 26, 131 (1988).

${ }^{2}$ R. Stolarski, R. Bojkov, L. Bishop, C. Zerefos, J. Staehelin, and J. Zawodny, Science 256, 342 (1992).

3 J. B. Kerr and C. T. McElroy, Science 262, 1032 (1993).

${ }^{4}$ S. Solomon, Nature 347, 347 (1990); D. J. Hofmann, S. J. Oltmans, J. M. Harris, S. Solomon, T. Deshler, and B. J. Johnson, Nature 359, 283 (1992).

5 a) P. J. Hay, T. H. Dunning, and W. A. Goddard II, Chem. Phys. Lett. 23, 457 (1973); b) D. Grimbert and A. Devaquet, Mol. Phys. 27, 831 (1974); c) P. J. Hay and T. H. Dunning, Jr., J. Chem. Phys. 67, 2290 (1977); d) K. H. Thunemann, S. D. Peyerimhoff, and R. J. Buenker, J. Mol. Spectrosc. 70, 432 (1978); e) R. O. Jones, J. Chem. Phys. 82, 325 (1985); f) A. Banichevich, S. D. Peyerimhoff, and F. Grein, Chem. Phys. Lett. 173, 1 (1990); g) M. Barysz, M. Rittby, and R. J. Bartlett, Chem. Phys. Lett. 193, 373 (1992).

6 a) M. Braunstein, P. J. Hay, R. L. Martin, and R. T. Pack, J. Chem. Phys. 95, 8239 (1991); b) M. Braunstein and R. T. Pack, J. Chem. Phys. 96, 6378 (1992).

${ }^{7}$ A. Banichevich and S. D. Peyerimhoff, Chem. Phys. 174, 93 (1993).

${ }^{8}$ A. Banichevich, S. D. Peyerimhoff, J. A. Beswick, and O. Atabek, J. Chem. Phys. 96, 6580 (1992).

9 M. J. Chappuis, C. R. Acad. Sci. (Paris) 91, 1985 (1880).

10 O. R. Wulf, Proc. Natl. Acad. Sci. 16, 507 (1930).

11 P. J. Hay and W. A. Goddard III, Chem. Phys. Lett. 14, 46 (1972).

12 R. S. Mulliken, Can. J. Chem. 36, 10 (1958).

13 H. B. Levene, J.-C. Nieh, and J. J. Valentini, J. Chem. Phys. 87, 2583 (1987).

14 V. Vaida, D. J. Donaldson, S. J. Strickler, S. L. Stephens, and J. W. Birks, J. Phys. Chem. 93, 506 (1989).

15 A. D. Kirshenbaum and A. G. Streng, J. Chem. Phys. 35, 1440 (1961).

16 a) S. M. Anderson, P. Hupalo, and K. Mauersberger, J. Chem. Phys. 99, 737 (1993); b) S. M. Anderson, J. Maeder, and K. Mauersberger, J. Chem. Phys. 94, 6351 (1991); c) S. M. Anderson, J. Morton, and K. Mauersberger, J. Chem. Phys. 93, 3826 (1990).

17P. G. Burton and M. D. Harvey, Nature 266, 826 (1977).

${ }^{18}$ C. L. Lin and M. T. Leu, Int. J. Chem. Kin. 14, 417 (1982) and references therein. 
${ }^{19}$ C. W. von Rosenberg Jr., and D. W. Trainor, J. Chem. Phys. 61, 2442 (1974).

20 J. R. Locker, J. A. Joens, and E. J. Bair, J. Photochem. 36, 235 (1987); T. Kleindienst, J. R. Locker, and E. J. Bair, J. Photochem. 12, 67 (1980).

${ }^{21}$ C. W. von Rosenberg, Jr. and D. W. Trainor, J. Chem. Phys. 63, 5348 (1975).

22 J. Shi and J. R. Barker, J. Phys. Chem. 94, 8390 (1990).

${ }^{23}$ W. D. McGrath , J. M. Maguire, A. Thompson, and J. Trocha-Grimshaw, Chem. Phys. Lett. 102, 59 (1983).

${ }^{24}$ N. Swanson and R. J. Celotta, Phys. Rev. Lett. 35, 783 (1975).

${ }^{25}$ S. E. Novick, P. C. Engelking, P. L. Jones, J. H. Futrell, and W. C. Lineberger, J. Chem. Phys. 70, 2652 (1979).

26 "Photoelectron Spectroscopy of Negative Ions", in Advances in Gas Phase Chemistry, Vol. 1 (N. G. Adams and L. M. Babcock, Eds.) JAP Press, Greenwich, CT, 1992, pp.121-166.

${ }^{27}$ K. M. Ervin, J. Ho, and W. C. Lineberger, J. Chem. Phys. 91, 5974 (1989); D. G. Leopold, K. K. Murray, A. E. Stevens Miller, and W. C. Lineberger, J. Chem. Phys. 83, 4849 (1985); H. B. Ellis, Jr. and G. B. Ellison, J. Chem. Phys. 78, 6541 (1983).

28 A. Weaver, D. W. Arnold, S. E. Bradforth, and D. M. Neumark, J. Chem. Phys. 94, 1740 (1991); A. Weaver, R. B. Metz, S. E. Bradforth, and D. M. Neumark, J. Chem. Phys. 90, 2070 (1989).

${ }^{29}$ R. B. Metz, A. Weaver, S. E. Bradforth, T. N. Kitsopoulos, and D. M. Neumark, J. Phys. Chem. 94, 1377 (1990).

${ }^{30}$ D. Proch and T. Trickl, Rev. Sci. Instrum. 60, 713 (1989).

31 W. C. Wiley and I. H. McLaren, Rev. Sci. Instrum. 26, 1150 (1955).

32 J. Cooper and R. N. Zare, J. Chem. Phys. 48, 942 (1968).

${ }^{33}$ K. M. Ervin, J. Ho, and W. C. Lineberger, J. Phys. Chem. 92, 5405 (1988).

${ }^{34}$ H. S. W. Massey, Negative Ions (Cambridge University Press, Cambridge, 1976); K. Ervin, J. Ho, and W. C. Lineberger, J. Chem. Phys 91, 5974 (1991).

${ }^{35}$ T. Tanaka and Y. Morino, J. Mol. Spectrosc. 33, 538 (1970); R. H. Hughes, J. Chem. Phys. 24, 131 (1956); R. Trambarulo, S. N. Ghosh, C. A. Burrus, Jr., and W. Gordy, J. Chem. Phys. 21, 538 (1953);

${ }^{36}$ E. B. Wilson, Jr., J. C. Decius, P. C. Cross, Molecular Vibrations (Dover, New York, 1980).

37 P. Hennig and G. Strey, Z. Naturforsch. A 31, 244 (1976). 
${ }^{38}$ This value of $\mathrm{r}_{\mathrm{e}}\left(\mathrm{O}_{3}^{-}\right)$does not account for the difference between $\mathrm{r}_{\mathrm{e}}\left(\mathrm{O}_{3}^{-}\right)$and $\mathrm{r}_{\mathrm{o}}\left(\mathrm{O}_{3}^{-}\right)$resulting from anion vibrational anharmonicities which are presently undetermined. Likewise for the value of $\theta_{e}\left(\mathrm{O}_{3}^{-}\right)$.

${ }^{39}$ L. J. Wang, S. B. Woo, and E. M. Helmy, Phys. Rev. A 35, 759 (1987).

${ }^{40}$ R. C. Spiker, Jr. and L. Andrews, J. Chem. Phys. 59, 1851 (1973); G. Steffen, W. Hesse, M. Jansen, and D. Reinen, Inorg. Chem. 30, 1923 (1991).

${ }^{41}$ M. E. Jacox and D. E. Milligan, J. Mol. Spectrosc. 43, 148 (1972); M. E. Jacox and D. E. Milligan, Chem. Phys. Lett. 14, 518 (1972).

${ }^{42}$ M. R. Nimlos and G. B. Ellison, J. Phys. Chem. 90, 2574 (1986).

${ }^{43}$ L. S. Cederbaum, W. Domcke, and W. von Niessen, Mol. Phys. 33, 1399 (1977).

${ }^{44}$ S. M. Anderson and K. Mauersberger, J. Geophys. Res., (submitted for publication).

45 Gaussian 92, Revision C, M. J. Frisch, G. W. Trucks, M. Head-Gordon, P. M. W. Gill, M. W. Wong, J. B. Foresman, B. G. Johnson, H. B. Schlegel, M. A. Robb, E. S. Replogle, R. Gomperts, J. L. Andres, K. Raghavachari, J. S. Binkley, C. Gonzalez, R. L. Martin, D. J. Fox, D. J. Defrees, J. Baker, J. J. P. Stewart, and J. A. Pople, Gaussian, Inc., Pittsburgh PA, 1992.

46 At the SCF-CISD/6-31G level of theory we obtain: ${ }^{3} \mathrm{~A}_{2}-\mathrm{r}_{0-0}=1.365 \AA, \theta=98.12^{\circ}, \omega_{1}=1349 \mathrm{~cm}^{-1}$, $\omega_{2}=580 \mathrm{~cm}^{-1} ;{ }^{3} \mathrm{~B}_{2} \mathrm{r}_{0-0}=1.402 \AA, \theta=108.56^{\circ}, \omega_{1}=1380 \mathrm{~cm}^{-1}, \omega_{2}=579 \mathrm{~cm}^{-1}$.

47 a) R. Schinke and V. Engel, J. Chem. Phys. 93, 3252 (1990); b) K. C. Kulander and J. C. Light, J. Chem. Phys. 73, 4337 (1980); c) E. J. Heller, J. Chem. Phys. 68, 3891 (1978); d) R. T. Pack, J. Chem. Phys. 65, 4765 (1976).

${ }^{48}$ R. Schinke, Photodissociation Dynamics, (Cambridge University Press, Cambridge, 1993), Chapter 8.

${ }^{49}$ H. Koppel, W. Domcke, and L. S, Cederbaum, Adv. Chem. Phys. 57, 59 (1984). 


\title{
Chapter 3. Study of $\mathrm{N}_{2} \mathrm{O}_{2}$ by Photoelectron Spectroscopy of $\mathrm{N}_{2} \mathrm{O}_{2}^{-}$
}

\begin{abstract}
Photoelectron spectra of the $\mathrm{NNO}_{2}^{-}$anion, collected at $\mathrm{hv}=4.657$ and $5.822 \mathrm{eV}$, are presented. Both the data and calculations support a $C_{2 v}$ geometry for the anion and the observed neutral electronic states. Transitions to several electronic states of the previously unobserved $\mathrm{N}_{2} \mathrm{O}_{2}$ molecule contain significant amounts of resolved vibrational structure. All of the observed transitions lie above the dissociation asymptotes for $\mathrm{N}_{2}+\mathrm{O}_{2}, \mathrm{NO}+\mathrm{NO}$, and $\mathrm{O}$ $+\mathrm{N}_{2} \mathrm{O}$. Several of the features lie above the $\mathrm{N}+\mathrm{NO}_{2}$ and $\mathrm{N}_{2}+\mathrm{O}+\mathrm{O}$ asymptotes. Assignments are proposed based upon Franck-Condon simulations based upon the results of $a b$ initio calculations for several electronic states of the $\mathrm{N}_{2} \mathrm{O}_{2}$ molecule. Both the $\mathrm{NNO}_{2}^{-}$and the $\mathrm{N}_{2} \mathrm{O}_{2}$ species are considered in terms of their roles as reactive intermediates in the $\mathrm{O}^{-}+\mathrm{N}_{2} \mathrm{O}$ and $\mathrm{N}+\mathrm{NO}_{2}$ chemical reactions.
\end{abstract}

\subsection{Introduction}

The widespread interest in nitrogen oxide chemistry results from the important roles which $\mathrm{NO}_{\mathrm{x}}$ species play in combustion, atmospheric, and pollution chemistry, ${ }^{1,2,3,4}$ The rather complex and interdependent nitrogen oxide cycles include both neutral and ion-molecule reactions. In many cases, the complexity of these reactions results from the availability of multiple product channels. Often several studies are necessary to determine which reactions actually occur under a specified set of experimental conditions.

As examples, consider the two reactions which are most closely related to the present study. Fig. 3.1 is a schematic energetics diagram in which the primary channels are shown for the $\mathrm{O}^{-}+\mathrm{N}_{2} \mathrm{O}$ and $\mathrm{N}+\mathrm{NO}_{2}$ reactions. The reaction of the $\mathrm{O}^{-}$anion with $\mathrm{N}_{2} \mathrm{O}$ has been studied for many years. While there are several energetically accessible pathways for this reaction, 


$$
\begin{array}{ll}
\mathrm{O}^{-}+\mathrm{N}_{2} \mathrm{O} \rightarrow \mathrm{NO}^{-}+\mathrm{NO} & \Delta \mathrm{H}=-2.8 \mathrm{kcal} / \mathrm{mole} \\
\mathrm{O}^{-}+\mathrm{N}_{2} \mathrm{O} \rightarrow \mathrm{NO}+\mathrm{NO}+e^{-} & \Delta \mathrm{H}=-2.3 \mathrm{kcal} / \mathrm{mole} \\
\mathrm{O}^{-}+\mathrm{N}_{2} \mathrm{O} \rightarrow \mathrm{O}_{2}^{-}+\mathrm{N}_{2} & \Delta \mathrm{H}=-55.6 \mathrm{kcal} / \mathrm{mole} \\
\mathrm{O}^{-}+\mathrm{N}_{2} \mathrm{O} \rightarrow \mathrm{O}_{2}+\mathrm{N}_{2}+e^{-} & \Delta \mathrm{H}=-45.4 \mathrm{kcal} / \mathrm{mole}
\end{array}
$$

only the first channel has been observed experimentally. This reaction was proposed ${ }^{5,6}$ to proceed through a long-lived intermediate formed by the attack of an $\mathrm{O}^{-}$anion onto the central nitrogen atom of the $\mathrm{N}_{2} \mathrm{O}$. Recent studies of product distributions using isotopically labeled reactants support this proposed mechanism.7,8

The second reaction, $\mathrm{N}+\mathrm{NO}_{2}$, has been studied using various kinetics techniques in order to determine the important channels and the product branching ratios. For this reaction, several channels are exothermic:

$$
\begin{array}{ll}
N+\mathrm{NO}_{2} \rightarrow \mathrm{N}_{2} \mathrm{O}+\mathrm{O} & \Delta \mathrm{H}=-42 \mathrm{kcal} / \mathrm{mole} \\
N+\mathrm{NO}_{2} \rightarrow \mathrm{NO}+\mathrm{NO} & \Delta \mathrm{H}=-78 \mathrm{kcal} / \mathrm{mole} \\
N+\mathrm{NO}_{2} \rightarrow N_{2}+\mathrm{O}_{2} & \Delta \mathrm{H}=-121 \mathrm{kcal} / \mathrm{mole} \\
N+\mathrm{NO}_{2} \rightarrow \mathrm{N}_{2}+2 \mathrm{O} & \Delta \mathrm{H}=-2 \mathrm{kcal} / \mathrm{mole}
\end{array}
$$

Unlike the anion reaction considered above, kinetics measurements indicate that all four of the channels occur to some extent. The overall rate of reaction for ground state nitrogen atoms with nitrogen dioxide $\left(\mathrm{k}_{\mathrm{tot}}=1 \times 10^{-12}-2 \times 10^{-11} \mathrm{~cm}^{3} / \mathrm{molecule} \cdot \mathrm{sec}\right)$ has been measured by various techniques. $9,10,11,12$ The branching ratios for reactions (5) - (8) were determined to be $0.43 \pm$ $0.04,0.33 \pm 0.07,0.10 \pm 0.12$, and $0.13 \pm 0.11$, respectively, by Phillips and Schiff. ${ }^{13}$ For the reaction of $\mathrm{NO}_{2}$ with a combination excited state $\mathrm{N}\left({ }^{2} \mathrm{D}\right)$ and $\mathrm{N}\left({ }^{2} \mathrm{P}\right)$ atoms, the branching ratio has been determined $\{1.00(5) ; 0.65(6) 0.27[(7)+(8)]\}$ but the overall reaction rate was determined to be much slower $\left(k_{t o t}=3.3 \times 10^{-13} \mathrm{~cm}^{3}\right.$ molecule-1 $\left.\mathrm{s}^{-1}\right)$ than the ground state

Figure 3.1: Schematic energetics diagram for the $\mathrm{O}^{-}+\mathrm{N}_{2} \mathrm{O}$ and $\mathrm{N}+\mathrm{NO}_{2}$ reactions. The energies (not to scale) are shown as a function of generic reaction coordinates which are not the same for the two reactions. 


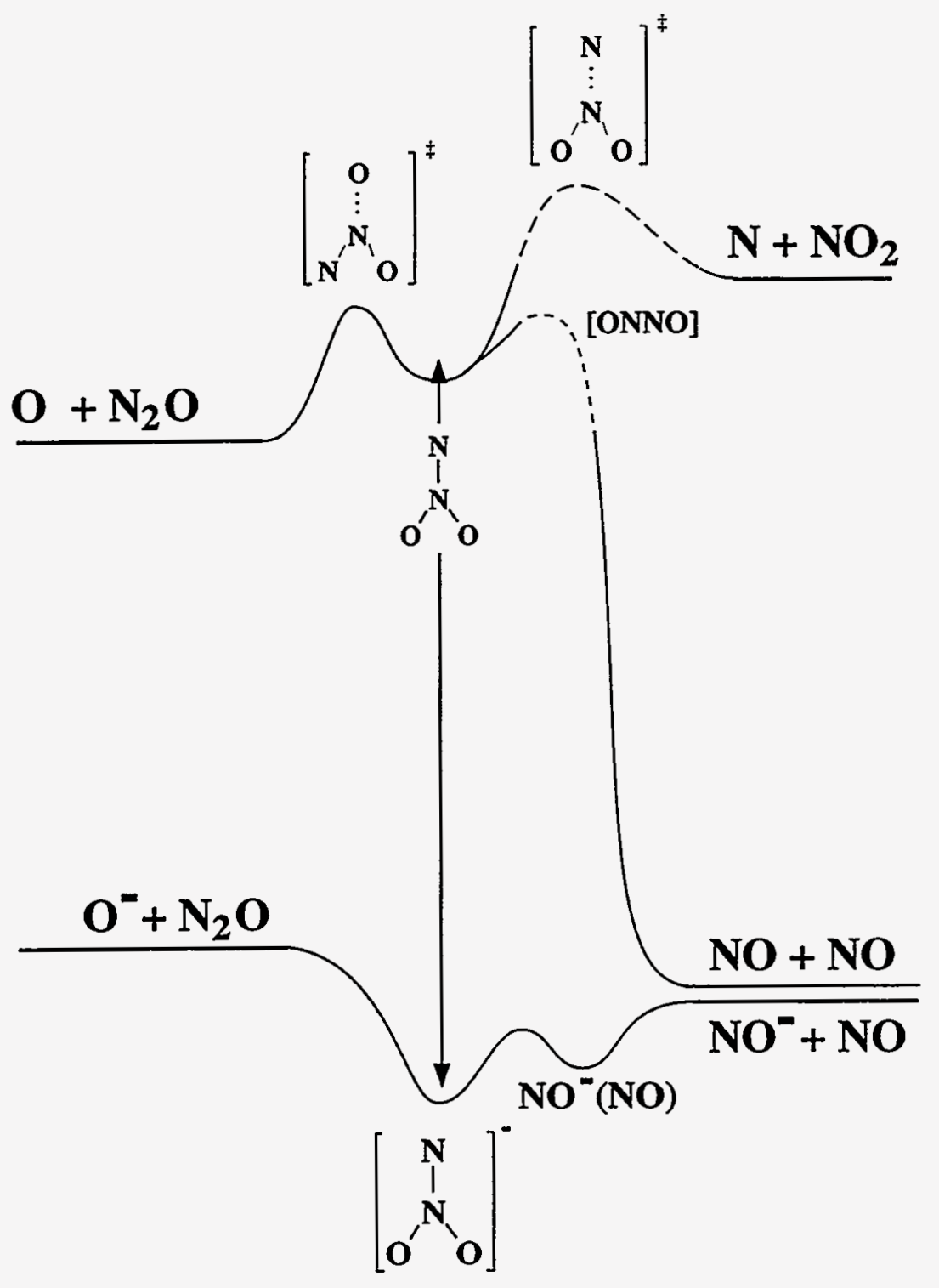

Figure 3.1 
reaction..$^{14}$ Reactions (5) - (8) are also proposed to proceed via a mechanism whereby an attack onto the central nitrogen atom leads to the formation of an intermediate which decays to the various channels. ${ }^{11}$ No experiments have been performed to confirm this mechanism for the $\mathrm{N}$ $+\mathrm{NO}_{2}$ reaction.

Common to both the $\mathrm{O}^{-}+\mathrm{N}_{2} \mathrm{O}$ and $\mathrm{N}+\mathrm{NO}_{2}$ reactions is the proposed geometry of the intermediate species. As shown schematically in Fig. 3.1 the intermediate for both reactions consists of a central nitrogen atom bound to two oxygen atoms and another nitrogen atom. These two molecules are the subject of the present investigation. We isolate the $\mathrm{N}_{2} \mathrm{O}_{2}^{-}$using molecular beam and mass spectrometric techniques. The anion is then photodetached to form the $\mathrm{N}_{2} \mathrm{O}_{2}$ neutral species. Interrogation of the photoelectron kinetic energy provides information about both the anion and the neutral species.

While no observation of the $\mathrm{N}_{2} \mathrm{O}_{2}^{-}$anion was reported in several early studies of the $\mathrm{O}^{-}$ $+\mathrm{N}_{2} \mathrm{O}$ reaction, Moruzzi and Dakin ${ }^{5}$ found that the ion could be collisionally stabilized by increasing reaction chamber pressures ( $>0.3$ Torr). The reaction mechanism proposed to model their data included the $\mathrm{N}_{2} \mathrm{O}_{2}^{-}$anion as a long-lived unstable intermediate $\left(\tau \sim 10^{-7} \mathrm{sec}\right)$ that was stabilized by $\mathrm{N}_{2} \mathrm{O}$ collisions with a rate of $\mathrm{k}=1 \times 10^{-11} \mathrm{~cm}^{3} / \mathrm{molecule} \cdot \mathrm{sec}$. The $\mathrm{C}_{2 \mathrm{v}}$ $\mathrm{NNO}_{2}^{-}$was subsequently isolated and identified in a matrix by Milligan and Jacox. ${ }^{15}$ Further evidence for this structure was found in later matrix studies by Jacox ${ }^{16}$ and by Hacaloglu et $a l .{ }^{17}$ A force constant analysis of the absorption features for isotopically substituted $\mathrm{NNO}_{2}^{-}$ showed that the $\mathrm{N}-\mathrm{N}$ bond is slightly stronger than a single bond while the $\mathrm{N}-\mathrm{O}$ bonds are very weak. 16

In a photoelectron study of $\left[\mathrm{N}_{2} \mathrm{O}_{2}^{-}\right]$, Posey and Johnson ${ }^{18}$ were able to collect three very dissimilar and exclusive spectra for the $\left[\mathrm{N}_{2} \mathrm{O}_{2}^{-}\right]$anion by varying the starting materials used for ion formation. Their ion source, like to one employed here, consists of a crossed molecular beam and a $1 \mathrm{keV}$ electron beam. Beginning with either a $5 \% \mathrm{O}_{2} / \mathrm{N}_{2}$ gas mixture or a $10 \% \mathrm{NO} / \mathrm{Ar}$ mixture, they characterized two different forms ${ }^{19}$ of the $\left[\mathrm{N}_{2} \mathrm{O}_{2}^{-}\right]$anion $[(\mathrm{I})$ 
$\mathrm{O}_{2}^{-}\left(\mathrm{N}_{2}\right)$ and (III) $\left.\mathrm{NO}^{-}(\mathrm{NO})\right]$ according to the photoelectron spectra which were obtained. However, using a $3.495 \mathrm{eV}$ photodetachment energy, no direct photoelectron signal was observed for species (II) which was formed using pure $\mathrm{N}_{2} \mathrm{O}$ in the molecular beam expansion. They observed photodissociation of (II) into $\mathrm{O}^{-}+\mathrm{N}_{2} \mathrm{O}$ by detection of a sequential two-photon process in which the $\mathrm{O}^{-}$photodetachment signal was detected after the anion photodissociation (i.e., $\mathrm{NNO}_{2}^{-}+\mathrm{hv} \rightarrow \mathrm{O}^{-}+\mathrm{N}_{2} \mathrm{O}$ ).

As illustrated in Fig. 3.1, both (I) and (II) are proposed ${ }^{18}$ as intermediates in reaction (1). The full isotopic scrambling observed for this reaction 7,8 suggests that the reaction proceeds through both (II) and (I) by a sequential process beginning with the $\mathrm{O}^{-}$attack on the central $\mathrm{N}$ atom of the $\mathrm{N}_{2} \mathrm{O}$ to form $\left[\mathrm{N}_{2} \mathrm{O}_{2}^{-}\right]$in which the two oxygen atoms are equivalent. A rearrangement to form $(\mathrm{I})$ is followed by dissociation into $\mathrm{NO}^{-}$and $\mathrm{NO}$ where the $\mathrm{O}$ atoms have lost knowledge of their origin.

In contrast to the moderate number of anion investigations, little consideration has been given to the $\mathrm{C}_{2 \mathrm{v}}$ form of $\mathrm{N}_{2} \mathrm{O}_{2}$. No experimental studies of the $\mathrm{NNO}_{2}$ species have been completed but Michels et al., ${ }^{20}$ have investigated the molecule with theoretical methods. In their $a b$ initio investigation, they find that the ${ }^{1} \mathrm{~A}_{1}$ electronic state of the $\mathrm{C}_{2 \mathrm{v}} \mathrm{NNO}_{2}$ structure is located at a potential energy minimum at the HF/6-31G* level of theory. However, there is only a very small barrier ( $2 \mathrm{~kJ} / \mathrm{mole}$ ) for rearrangement to a lower energy $\mathrm{C}_{\mathrm{s}}$ structure of the form O-O-N-N. Similarly to $\mathrm{N}_{2} \mathrm{O}_{2}^{-}$, the neutral $\mathrm{N}_{2} \mathrm{O}_{2}$ is proposed to have multiple stable isomers. In addition to the $\mathrm{C}_{2 \mathrm{v}}$ structure studied here, and the metastable $\mathrm{O}-\mathrm{O}-\mathrm{N}-\mathrm{N}$ structure studied by Michels et al. ${ }^{20}$ there is also the NO dimer which has been the subject of several investigations and exists in both the cis- and trans-forms with a very weak $\mathrm{N}-\mathrm{N}$ bond. $21,22,23,24$

In this paper, we make a further investigation of the (II) species $\left(\mathrm{NNO}_{2}^{-}\right)$and the corresponding neutral $\left(\mathrm{N}-\mathrm{NO}_{2}\right)$ by a combination of anion photoelectron spectroscopy and $a b$ initio calculations. By collecting photoelectron spectra of the $\left[\mathrm{N}_{2} \mathrm{O}_{2}^{-}\right]$species (II) at higher photon energies than used by Posey and Johnson, ${ }^{18}$ we observe photodetachment transitions 
from a $\mathrm{C}_{2 \mathrm{v}} \mathrm{NNO}_{2}^{-}$anion to several electronic states of the neutral species, $\mathrm{NNO}_{2}$. This represents the first observation of the $\mathrm{C}_{2 v} \mathrm{~N}_{2} \mathrm{O}_{2}$ molecule. The spectra contain much wellresolved vibrational structure, even though the observed spectral features lie well above the energetic asymptotes for dissociation of the $\mathrm{N}_{2} \mathrm{O}_{2}$ species. With the assistance of $a b$ initio calculations for the anion and neutral, assignments are proposed for these states.

\subsection{Experimental}

A dual time-of-flight anion photoelectron spectrometer which has been previously described in detail ${ }^{25}$ is employed for these experiments. For the present experiments, $\mathrm{N}_{2} \mathrm{O}_{2}^{-}$is generated at the intersection of a pulsed molecular beam and a $1 \mathrm{keV}$ electron beam. ${ }^{26}$ The molecular beam was operated by expanding neat $\mathrm{N}_{2} \mathrm{O}$ at a backing pressure of $\sim 1.5$ bar through a $0.020^{\prime \prime}$ orifice at $20 \mathrm{~Hz}$ with a piezoelectric valve. The $\mathrm{N}_{2} \mathrm{O}_{2}^{-}$anions are believed to form through the mechanism outlined in Eq. (9):

$$
\begin{gathered}
\mathrm{N}_{2} \mathrm{O}+\mathrm{e}^{-} \rightarrow \mathrm{O}^{-}+\mathrm{N}_{2} \\
\mathrm{O}^{-}+\mathrm{N}_{2} \mathrm{O}+\mathrm{M} \rightarrow \mathrm{N}_{2} \mathrm{O}_{2}^{-}+\mathrm{M}
\end{gathered}
$$

After formation, the anions relax vibrationally and rotationally by collisions with the carrier gas atoms in the continuing molecular beam expansion.

The cooled ions are extracted into a Wiley-McLaren-type time-of-flight mass spectrometer ${ }^{27}$ where they are separated by mass from other anions formed in the source region. The $\mathrm{N}_{2} \mathrm{O}_{2}^{-}$anions are selectively photodetached by a properly timed $8 \mathrm{~ns}$ laser pulse. Photoelectron kinetic energies are determined by time-of-flight measurements through a 1 meter field-free tube perpendicular to the anion velocity vector. The resolution of the apparatus is $11 \mathrm{meV}$ for electrons with $0.65 \mathrm{eV}$ of electron kinetic energy (eKE) and degrades as $\mathrm{eKE}^{3 / 2}$. 
For these experiments the 4th (266 nm; $4.657 \mathrm{eV} ; 15 \mathrm{~mJ} / \mathrm{pulse})$ and 5th harmonics (213 nm; $5.822 \mathrm{eV} ; 6 \mathrm{~mJ} /$ pulse) of a pulsed Nd:YAG laser are employed for photodetachment.

For experiments performed at $213 \mathrm{~nm}$, scattered photons generate background signal through interactions with the surfaces in the detector region. A background spectrum, collected using the same laser power used during data collection, is fitted to a smooth function which is scaled and subtracted from the data to correct for the moderate level background.

\subsection{Results}

\subsubsection{Experimental}

Figures 3.2 and 3.3 show the photoelectron spectra of $\mathrm{N}_{2} \mathrm{O}_{2}^{-}$collected with photodetachment energies of 4.657 and $5.822 \mathrm{eV}$, respectively. The spectra represent the intensity of electron signal as a function of electron kinetic energy (eKE) where

$$
e K E=h v-E A-T_{0}-E_{v}^{o}+E_{v}^{-} \quad(10)
$$

In Eq. (10), hv is the photon energy, EA is the electron affinity of the $\mathrm{N}_{2} \mathrm{O}_{2}$ species and $\mathrm{T}_{0}$ is the term value for the various electronic states. $E_{v}^{o}$ and $E_{v}^{-}$represent in the vibrational energy (above the zero point) of $\mathrm{N}_{2} \mathrm{O}_{2}$ and $\mathrm{N}_{2} \mathrm{O}_{2}^{-}$, respectively.

The $4.657 \mathrm{eV}$ spectrum, Fig. 3.2, contains a single band consisting long series of

Figure 3.2: Photoelectron spectrum of $\mathrm{N}_{2} \mathrm{O}_{2}^{-}$collected at $\mathrm{hv}=4.657 \mathrm{eV}$. The inset points out the signal from $\mathrm{O}^{-}$photodetachment that occurs after dissociation of $\mathrm{N}_{2} \mathrm{O}_{2}^{-}$. Also in the inset are arrows ' 1 ' and ' 2 ' indicating the $\mathrm{NO}+\mathrm{NO}$ and $\mathrm{O}+\mathrm{N}_{2} \mathrm{O}$ dissociation asymptotes.

Figure 3.3: Photoelectron spectrum of $\mathrm{N}_{2} \mathrm{O}_{2}^{-}$collected at hv $=5.822 \mathrm{eV}$. Arrows '2', '3', and ' 4 ' indicate the $\mathrm{O}+\mathrm{N}_{2} \mathrm{O}, \mathrm{N}+\mathrm{N}_{2} \mathrm{O}$, and $\mathrm{N}_{2}+\mathrm{O}+\mathrm{O}$ dissociation asymptotes. 


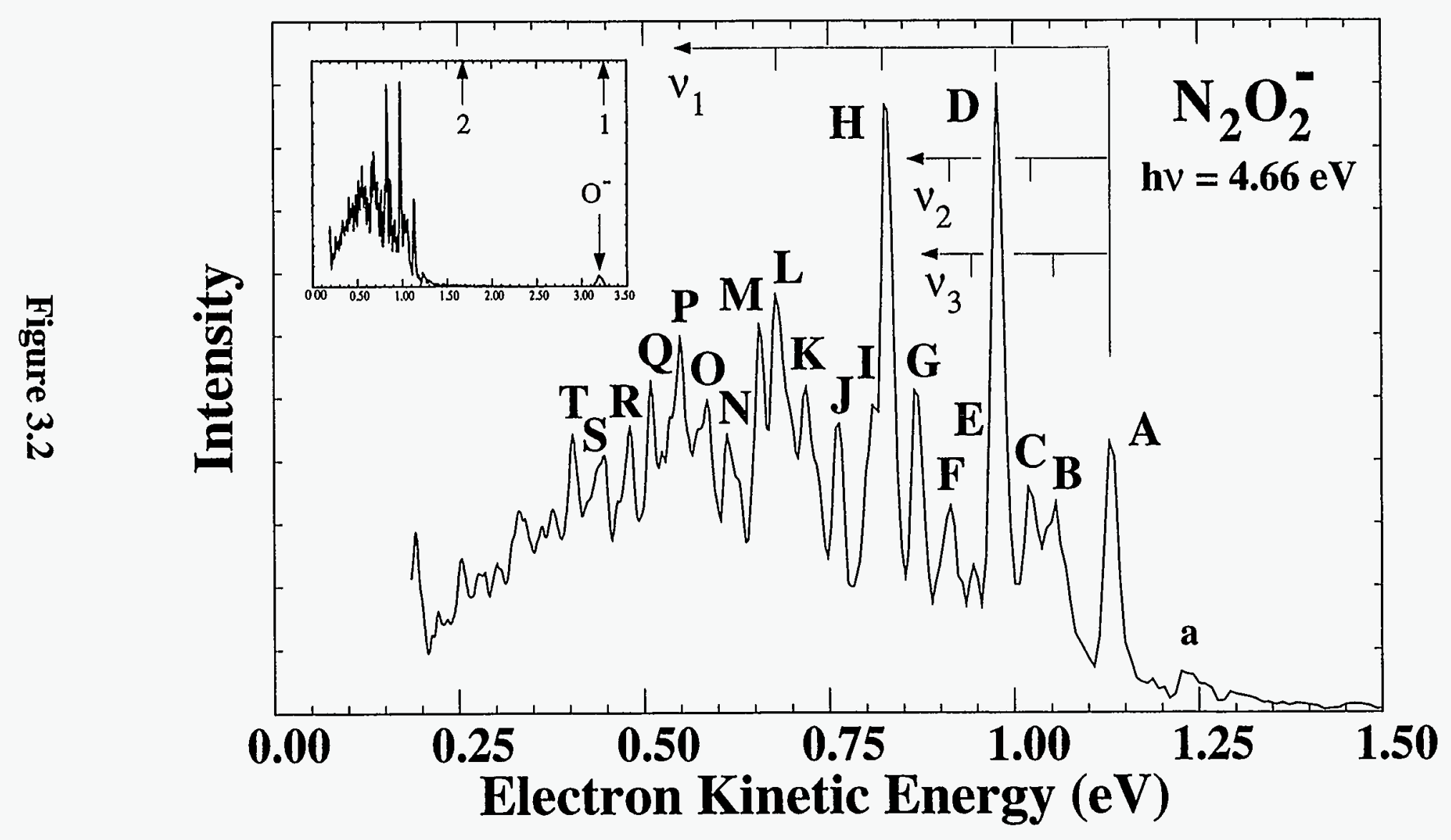




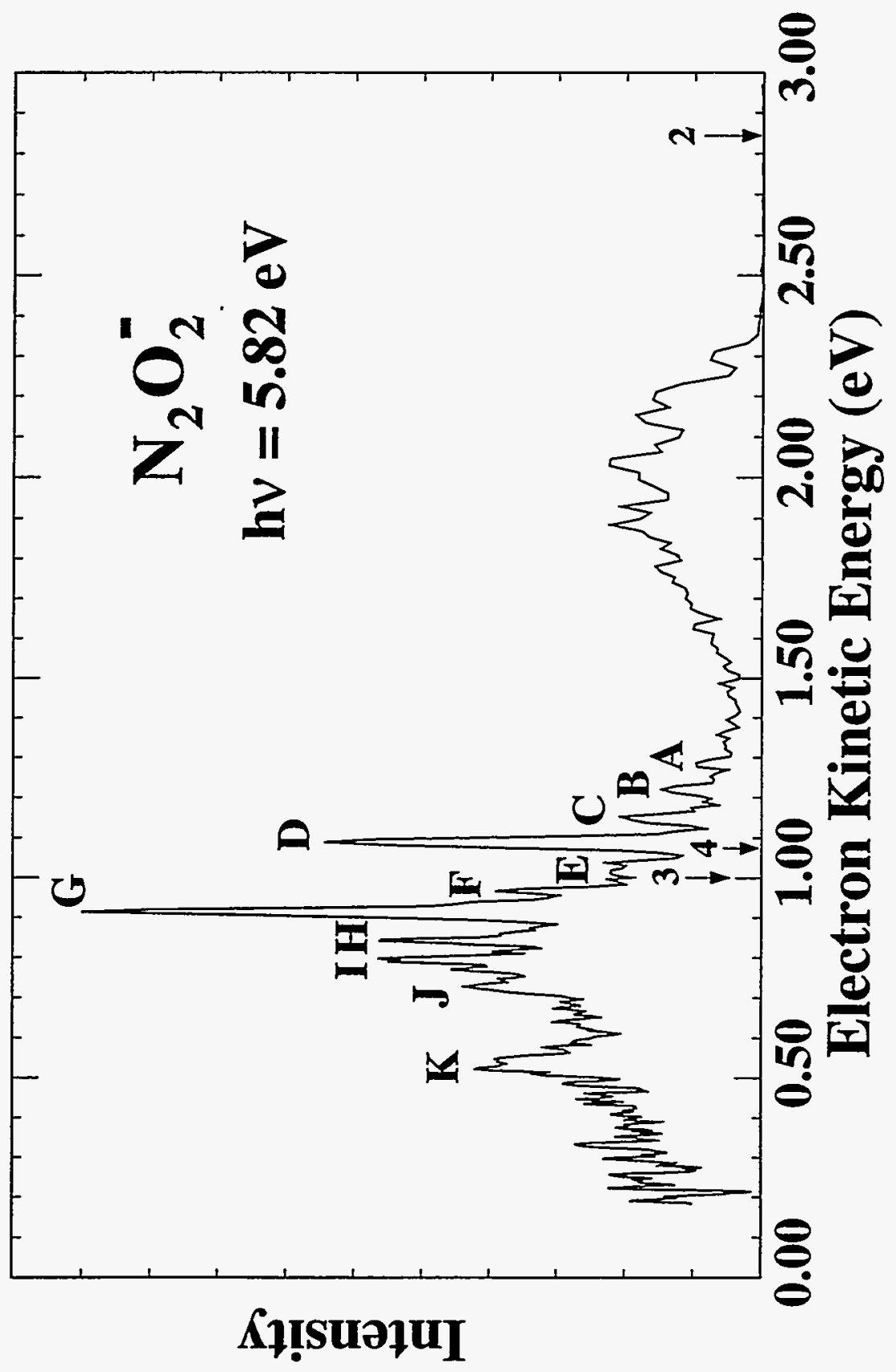

Figure 3.3 
resolved peaks beginning at $1.126 \mathrm{eV}$ and continuing to lower eKE. The peak positions are summarized in Table I. As indicated in the figure, progressions in three different vibrational modes can be discerned in the data. The A, D, H, L progression is described by $\omega_{e}=1240 \mathrm{~cm}^{-1}$ and $x_{e} \omega_{e}=7 \mathrm{~cm}^{-1}$. The other two modes have frequencies of 880 and $680 \mathrm{~cm}^{-1}$ and also appear to be quite anharmonic. Although the peaks are reasonably well resolved at the beginning of the band, the large number of resultant combination bands leads to congestion at lower eKE. The peak labeled ' $a$ ' corresponds to photodetachment transitions originating from vibrationally excited anions. This assignment to a 'hot band' is based upon the dependence of this feature's intensity upon the ion source conditions. The A-a separation provides an anion vibrational frequency of $\sim 950 \mathrm{~cm}^{-1}$.

Table I: Peak Positions for $4.657 \mathrm{eV}$ data

\begin{tabular}{|c|c|}
\hline Peak & Position (eKE) \\
\hline A & 1.126 \\
\hline B & 1.055 \\
\hline C & 1.016 \\
\hline D & 0.969 \\
\hline E & 0.942 \\
\hline F & 0.905 \\
\hline G & 0.861 \\
\hline H & 0.820 \\
\hline I & 0.795 \\
\hline J & 0.755 \\
\hline K & 0.711 \\
\hline L & 0.671 \\
\hline M & 0.649 \\
\hline N & 0.613 \\
\hline O & 0.586 \\
\hline P & 0.549 \\
\hline Q & 0.509 \\
\hline R & 0.481 \\
\hline S & 0.445 \\
\hline T & 0.404 \\
\hline
\end{tabular}


As labeled by arrow marked ' $\mathrm{O}^{-1}$ in the Fig. 3.2 inset, there is also a single peak in the $\mathrm{N}_{2} \mathrm{O}_{2}^{-}$spectrum which is well separated from the other spectral features. This feature, located at $3.19 \mathrm{eV}$, corresponds to the $\mathrm{O}^{-}$photodetachment transitions as observed by Posey and Johnson. ${ }^{18}$ The intensity of this transition exhibits a higher order laser power dependence than the remainder of the spectral features in agreement with their assignment as a sequential twophoton process in which the $\mathrm{N}_{2} \mathrm{O}_{2}^{-}$anion is photodissociated by the first photon to yield $\mathrm{O}^{-}$and $\mathrm{N}_{2} \mathrm{O}$ followed by photodetachment of the $\mathrm{O}^{-}$by a second photon. No peaks are observed at the eKEs where $\mathrm{NO}^{-}$or $\mathrm{O}_{2}^{-}$photodetachment signal is expected indicating that dissociation of the $\mathrm{N}_{2} \mathrm{O}_{2}^{-}$to yield $\mathrm{NO}^{-}$or $\mathrm{O}_{2}^{-}$does not occur at detectable levels at $\mathrm{hv}=4.66 \mathrm{eV}$. In agreement with the work of Posey and Johnson, ${ }^{18}$ we do not observe any signal corresponding to the $\mathrm{NO}^{-}(\mathrm{NO})$ or the $\mathrm{O}_{2}^{-}\left(\mathrm{N}_{2}\right)$ species when $\mathrm{N}_{2} \mathrm{O}$ is used as the starting material for $\mathrm{N}_{2} \mathrm{O}_{2}^{-}$formation.

According to Fig. 3.1 and Eq. (11), the measured eKE of a photoelectron can be related to the asymptotic energy of the $\mathrm{N}_{2} \mathrm{O}_{2}$ dissociation products,

$$
e K E=h v-D_{o}^{-}-E A_{\mathrm{prod}}-E_{\mathrm{int}}^{o}+E_{\mathrm{int}}^{-}
$$

Here, $D_{o}^{-}$is the dissociation energy of $\mathrm{N}_{2} \mathrm{O}_{2}^{-}$into neutral and anion fragments, the latter of which has an electron affinity of $\mathrm{EA}_{\text {prod. }}$. The other terms are the same as for Eq. (10). Thus, the eKE distribution provides information about the energetic stability of $\mathrm{N}_{2} \mathrm{O}_{2}$ with respect to various dissociation pathways.

The energetic asymptotes for dissociation of the $\mathrm{N}_{2} \mathrm{O}_{2}$ molecule calculated using Eq. (11) are shown along the top axis of the Fig. 3.2 inset. Arrow ' 1 ' at $\mathrm{eKE}=3.25 \mathrm{eV}$ indicates the $\mathrm{NO}+\mathrm{NO}$ limit and arrow ' 2 ', represents the $\mathrm{O}+\mathrm{N}_{2} \mathrm{O}$ asymptote. These values are determined using Eq. (11), known reaction exothermicities, and an upper limit for $\mathrm{N}_{2} \mathrm{O}_{2}^{-}$dissociation of 1.5 eV. This limit for $D_{0}\left(\mathrm{O}^{-} \cdots \mathrm{N}_{2} \mathrm{O}\right)$ has been recently determined in a separate experiment performed in this laboratory which will be described briefly in Section 3.4.3. Thus, all of the 
observed vibrational features lie above both of these asymptotes, suggesting that significant barriers exist along both dissociation coordinates for the $\mathrm{N}_{2} \mathrm{O}_{2}$ molecule.

In the $5.822 \mathrm{eV}$ photoelectron spectrum of $\mathrm{N}_{2} \mathrm{O}_{2}^{-}$, Fig. 3.3, the features of the band observed in Fig. 3.2 are significantly broadened due to their higher eKE. The arrows marked '2', '3', and '4' indicate the $\mathrm{O}+\mathrm{N}_{2} \mathrm{O}, \mathrm{N}+\mathrm{NO}_{2}$, and $\mathrm{N}_{2}+\mathrm{O}+\mathrm{O}$ dissociation asymptotes, respectively. Beginning at $1.286 \mathrm{eV}$, a second set of spectral features appears in the spectrum. In general, the peaks are unevenly spaced and appear to result from overlapping transitions to multiple electronic states of $\mathrm{N}_{2} \mathrm{O}_{2}$. The peak positions are summarized in Table $\mathrm{II}$. In addition to the $540 \mathrm{~cm}^{-1}$ progression (A-C) beginning at $1.286 \mathrm{eV}$, there are two dominant narrow features at 1.089 and $0.914 \mathrm{eV}$ which do not appear to belong to belong to a single vibrational progression. There are several smaller features that seem to belong to vibrational progressions beginning at $A, D$ and perhaps $G$. There is also a significantly broader feature, ' $K$ ', which occurs at $\sim 0.525 \mathrm{eV}$ with a width of $\sim 60 \mathrm{meV}$ (i.e., much broader than the experimental resolution of $\sim 10 \mathrm{meV}$ in this energy region).

Table II: Peak Positions for $5.821 \mathrm{eV}$ data

\begin{tabular}{|c|c|}
\hline Peak & $\begin{array}{c}\text { Position } \\
\text { (eKE) }\end{array}$ \\
\hline \hline A & 1.286 \\
\hline B & 1.220 \\
\hline C & 1.152 \\
\hline D & 1.089 \\
\hline E & 1.035 \\
\hline F & 0.967 \\
\hline G & 0.914 \\
\hline H & 0.842 \\
\hline I & 0.794 \\
\hline$J$ & 0.728 \\
\hline K & 0.525 \\
\hline
\end{tabular}


Due to the relatively low cross-section for photodetachment and the competition with the photodissociation process described above, it was necessary to use unusually high ion densities in order to obtain a reasonable signal-to-noise ratio. At these higher ion levels the energy of the photodetached electron is affected by Coulombic interactions with the remaining undetached ions in the laser interaction region. This interaction shifts the spectrum to higher eKE and broadens the observed spectral features. The magnitude of the 'space-charge shift' is determined by measurement the shift experienced by calibration ions at the same ion densities used for the $\mathrm{N}_{2} \mathrm{O}_{2}^{-}$data collection. Through this procedure, we find the data to be shifted by $8 \mathrm{meV}$ and peaks to be broadened by $\sim 2-3 \mathrm{meV}$. The energy axis of the spectra in Fig. 3.2 and 3.3 has been corrected accordingly.

\subsection{2. $A b$ initio Calculations}

For assistance in the assignment of the photoelectron spectra, $a b$ initio calculations for $\mathrm{N}_{2} \mathrm{O}_{2}^{-}$and $\mathrm{N}_{2} \mathrm{O}_{2}$ have been performed using the Gaussian92 package. ${ }^{28}$ The standard $6-31+\mathrm{G}^{*}$ basis is employed in HF and MP2 calculations of the optimized geometries and vibrational frequencies. The anion results, discussed first in Sec. 3.3.2.1, will be followed by a discussion of calculations for the lowest $\mathrm{N}_{2} \mathrm{O}_{2}$ singlet state and four $\mathrm{N}_{2} \mathrm{O}_{2}$ triplet states.

\subsubsection{1. $\mathrm{N}_{2} \mathrm{O}_{2}^{-}$Calculations}

The $\mathrm{N}_{2} \mathrm{O}_{2}^{-}$ground state is predicted to be the ${ }^{2} \mathrm{~B}_{2}$ state with the orbital occupation $\left(\ldots 4 b_{2}^{2} 2 b_{1}^{2} 8 a_{1}^{2} 1 a_{2}^{2} 5 b_{2}^{1}\right)$. Investigations of other possible anion electronic states (i.e., ${ }^{2} \mathrm{~A}_{2},{ }^{2} \mathrm{~B}_{1}$, and ${ }^{2} A_{1}$ in $C_{2 v}$ symmetry and the ${ }^{2} A^{\prime \prime}$ state in $C_{s}$ symmetry) at the HF/6-31+G* level of theory show that they lie higher in energy. Support for this assignment is found by comparison with the isoelectronic $\mathrm{NO}_{3}$ and $\mathrm{FCO}_{2}$ molecules. Weaver et al. ${ }^{29}$ assign the ground state of the $\mathrm{D}_{3 \mathrm{~h}}$ symmetric $\mathrm{NO}_{3}$ molecule as the $\tilde{\mathrm{X}}^{2} \mathrm{~A}_{2}^{\prime}$ state which, as Walsh shows, ${ }^{30}$ correlates to the ${ }^{2} \mathrm{~B}_{2}$ state in the $\mathrm{C}_{2 \mathrm{v}}$ point group. The ground state of the isoelectronic $\mathrm{FCO}_{2}$ radical has also been assigned as the $\tilde{\mathrm{X}}{ }^{2} \mathrm{~B}_{2}$ electronic state. ${ }^{31,32}$ 
As shown in Table III, the geometry optimizations at both the HF/6-31+G* and the MP2/6-31+G* levels of theory predict a $C_{2 v}$ symmetric species with three nearly equal bond angles and bond lengths. The geometrical parameters are illustrated in Figure 3.4a. The $\mathrm{N}_{2} \mathrm{O}_{2}^{-}$ vibrational frequencies have also been calculated at both levels of theory. The results agree reasonably well with the experimental $\mathrm{N}_{2} \mathrm{O}_{2}^{-}$vibrational frequencies measured in the matrix isolation experiments. Through a the force constant analysis of these vibrational frequencies, Hacaloglu et al. ${ }^{17}$ estimate the $\angle \mathrm{ONO}$ angle of $\mathrm{N}_{2} \mathrm{O}_{2}^{-}$to be between $105^{\circ}$ and $120^{\circ}$. The angle is calculated to be $119.2^{\circ}$ and $120.3^{\circ}$ at the HF and MP2 levels of theory, respectively.

\subsubsection{2. $\mathrm{N}_{2} \mathrm{O}_{2}$ Calculations}

In the case of the neutral $\mathrm{N}_{2} \mathrm{O}_{2}$ molecule, we have investigated the properties of several electronic states which can be produced by one-electron photodetachment of the $\mathrm{N}_{2} \mathrm{O}_{2}^{-}$anion. For example, removal of an electron from the $5 b_{2}$ orbital of $\mathrm{N}_{2} \mathrm{O}_{2}^{-}$anion forms the ${ }^{1} \mathrm{~A}_{1}$ state of $\mathrm{N}_{2} \mathrm{O}_{2}$ in $\mathrm{C}_{2 v}$ symmetry. The geometry optimization of the $\mathrm{N}_{2} \mathrm{O}_{2}$ species with this orbital occupation provided rather interesting results which are summarized in Table $\mathrm{V}$. When the $\mathrm{N}_{2} \mathrm{O}_{2}$ molecule was constrained to have $\mathrm{C}_{2 v}$ symmetry, two stationary points were located on the potential energy surface. Force constant analyses revealed that one of these geometries was located at a potential energy minimum while the other was a first order transition state with an imaginary frequency for the $\mathrm{NO}_{2}$ antisymmetric stretch.

The ${ }^{1} \mathrm{~A}_{1}$ state located at a minimum is similar to that determined by Michels et al. ${ }^{20}$ at the HF/6-31G* level of theory. The calculation shows that a significant interaction exists between the two oxygen atoms where the ONO angle is only $66^{\circ}$ and the O-O separation is only $1.59 \AA$ at the $M P 2 / 6-31+G^{*}$ level of theory (c.f. $\mathrm{R}_{\mathrm{N}-\mathrm{O}}\left(\mathrm{N}_{2} \mathrm{O}_{2}\right)=1.49 \AA ; \mathrm{R}_{\mathrm{N}-\mathrm{O}}\left(\mathrm{N}_{2} \mathrm{O}_{2}^{-}\right)=1.25 \AA$;

Figure 3.4: Definition of parameters used in calculation of $a b$ initio geometries for the $C_{2 v}$ (top) and $\mathrm{C}_{\mathrm{s}}$ (bottom) species. 


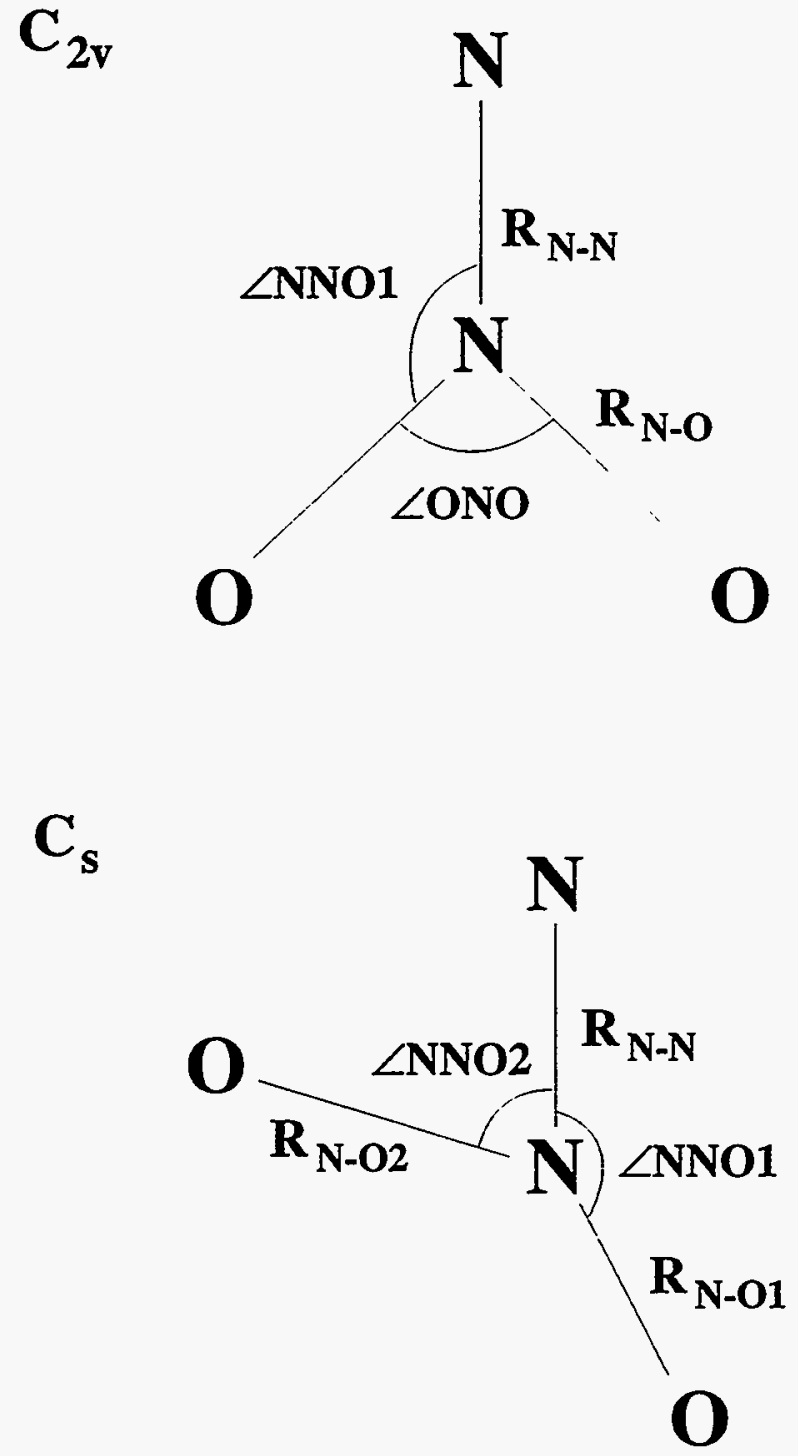

Figure 3.4 
Table III: $\boldsymbol{A} b$ initio Results for Anion ${ }^{a}$

\begin{tabular}{|c|c|c|c|c|c|c|c|c|c|c|c|}
\hline Theory & Energy & $\mathrm{R}_{\mathrm{N}-\mathrm{N}}$ & $\mathrm{R}_{\mathrm{N}-\mathrm{O}}$ & $\mathrm{R}_{0.0}$ & $\angle \mathrm{ONO}$ & $\omega_{1}$ & $\omega_{2}$ & $\omega_{3}$ & $\omega_{4}$ & $\omega_{5}$ & $\omega_{6}$ \\
\hline UHF/6-31+G* & -258.494495 & 1.253 & 1.254 & 2.189 & $121.6^{\circ}$ & 1582 & 1086 & 692 & 836 & 1434 & 654 \\
\hline $\mathrm{MP} 2 / 6-31+\mathrm{G}^{*}$ & -259.178980 & 1.270 & 1.253 & 2.164 & $119.4^{\circ}$ & 1411 & 1241 & 679 & 998 & 1538 & 609 \\
\hline Expt & 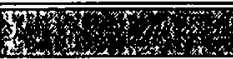 & W & W6 & $\sqrt{431}$ & 48 & (3) & (1) & 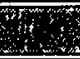 & (r) & W. & 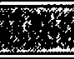 \\
\hline Ar matrix & $\ldots$ & $\ldots$ & -- & -.. & $\cdots$ & 1356 & 1004 & $\ldots$ & $\ldots$ & 1199 & $\overline{-1--}$ \\
\hline Ne matrix ${ }^{c}$ & --. & --- & --. & --- & --- & 1359 & 1008 & -- & -- & 1205.5 & $-\cdots$ \\
\hline
\end{tabular}

${ }^{a}$ Bond lengths in $\AA$, energy in au (hartrees) and vibrational frequencies in $\mathrm{cm}^{-1}$. See Fig. 3.4 for parameter definitions.

$b$ References 16 and 17.

$c$ Reference 16. 
Table IV: $A b$ initio Results for $\mathrm{N}_{2} \mathrm{O}_{2}$ Singlet Calculations ${ }^{a}$

\begin{tabular}{|c|c|c|c|c|c|c|}
\hline \multicolumn{7}{|c|}{$\mathrm{C}_{2 \mathrm{v}}$ Symmetry } \\
\hline State & & $\mathrm{HF} / 6-31+\mathrm{G}^{*}$ & $\mathrm{MP} 2 / 6-31+\mathrm{G}^{*}$ & Mode & $\mathrm{HF} / 6-31+\mathrm{G}^{*}$ & $\mathrm{MP} 2 / 6-31+\mathrm{G}^{*}$ \\
\hline${ }^{1} \mathrm{~A}_{1}$ & $\mathrm{R}_{\mathrm{N}-\mathrm{N}}(\AA)$ & 1.302 & 1.253 & $\omega_{1}$ & 1535 & 1415 \\
\hline \multirow[t]{5}{*}{ (A) } & $\mathrm{R}_{\mathrm{N}-\mathrm{O}}(\AA)$ & 1.190 & 1.243 & $\omega_{2}$ & 1178 & 1121 \\
\hline & $\mathrm{R}_{\mathrm{O}-\mathrm{O}}(\AA)$ & 2.169 & 2.276 & $\omega_{3}$ & 692 & 575 \\
\hline & $\angle O N O$ & $131.4^{\circ}$ & $132.6^{\circ}$ & $\omega_{4}$ & 876 & 728 \\
\hline & Energy & -258.332951 & -259.014352 & $\omega_{5}$ & 1761 & 1696 \\
\hline & & & & $\omega_{6}$ & $176 \mathbf{i}$ & $824 \mathbf{i}$ \\
\hline${ }^{1} \mathrm{~A}_{1}$ & $\mathrm{R}_{\mathrm{N}-\mathrm{N}}(\AA)$ & 1.127 & 1.138 & $\omega_{1}$ & 2197 & 2358 \\
\hline \multirow[t]{5}{*}{ (B) } & $\mathrm{R}_{\mathrm{N}-\mathrm{O}}(\AA)$ & 1.350 & 1.494 & $\omega_{2}$ & 1179 & 1155 \\
\hline & $\mathrm{R}_{\mathrm{O}-\mathrm{O}}(\AA)$ & 1.478 & 1.592 & $\omega_{3}$ & 895 & 628 \\
\hline & $\angle O N O$ & $66.4^{\circ}$ & $64.4^{\circ}$ & $\omega_{4}$ & 510 & 520 \\
\hline & Energy & -258.293541 & -259.030722 & $\omega_{5}$ & 668 & 1089 \\
\hline & & & & $\omega_{6}$ & 459 & 165 \\
\hline \multicolumn{7}{|c|}{$\mathrm{C}_{\mathrm{s}}$ Symmetry } \\
\hline \multirow[t]{6}{*}{${ }^{1} \mathrm{~A}^{\prime}$} & $\mathrm{R}_{\mathrm{N} \cdot \mathrm{N}}(\AA)$ & 1.202 & 1.242 & $\omega_{1}$ & 2029 & 2190 \\
\hline & $\mathrm{R}_{\mathrm{N}-\mathrm{OI}}(\AA)$ & 1.168 & 1.231 & $\omega_{2}$ & 1390 & 1237 \\
\hline & $\mathrm{R}_{\mathrm{N}-\mathrm{O} 2}(\AA)$ & 1.313 & 1.460 & $\omega_{3}$ & 1098 & 1005 \\
\hline & $\angle \mathrm{NNO} 1$ & $146.6^{\circ}$ & $152.7^{\circ}$ & $\omega_{4}$ & 657 & 631 \\
\hline & $\angle \mathrm{NNO} 2$ & $80.0^{\circ}$ & $73.2^{\circ}$ & $\omega_{5}$ & 468 & 304 \\
\hline & Energy & -258.341391 & -259.070351 & $\omega_{6}$ & 733 & 534 \\
\hline
\end{tabular}

a Bond lengths given in $\AA$, energy in au (hartree) and vibrational frequencies in $\mathrm{cm}^{-1}$.

See Fig. 3.4 for parameter definitions. 
$\left.\mathrm{R}_{\mathrm{O}-\mathrm{O}}\left(\mathrm{N}_{2} \mathrm{O}_{2}^{-}\right)=2.16 \AA\right)$. The $\mathrm{O}-\mathrm{O}$ antibonding character of the $5 \mathrm{~b}_{2}$ orbital hinders such bonding in the anion and results in the larger $\mathrm{N}_{2} \mathrm{O}_{2}^{-} \angle \mathrm{ONO}$ angle. The extreme difference in the geometries of this neutral state and the anion ground state will be very important in the data analysis.

At the other stationary point, the saddle point species is calculated to lie $0.44 \mathrm{eV}$ above the $C_{2 v}$ minimum at the MP2/6-31+G* level of theory. Upon removal of the $C_{2 v}$ symmetry constraint, the saddle point species relaxes significantly to a $C_{s}\left({ }^{1} A^{\prime}\right)$ structure with one acute NNO angle and one obtuse NNO angle. One of the NO bond lengths is significantly longer (1.49 $\AA$ ) than the other $(1.14 \AA)$. This $C_{s}$ minimum energy structure lies $1.52 \mathrm{eV}$ below the saddle point geometry and $1.08 \mathrm{eV}$ below the $C_{2 v}$ minimum at the MP2/6-31+G* level of theory. Force constant analyses at each stationary point yield the frequencies shown in Table IV.

Removal of electrons from the $1 a_{2}, 8 a_{1}, 4 b_{2}$, and $2 b_{1}$ orbitals can generate either singlet or triplet states of the $B_{1}, B_{2}, A_{1}$ or $A_{2}$ state symmetries, respectively. For the present analysis, only the triplet states will be considered. Preliminary investigations of the open-shell singlet states by UHF methodology were found to suffer from significant spin-contamination. While further calculations are not pursued here, additional discussion of the singlet states is given in $\operatorname{Sec}$ 3.4.2. We have investigated the triplet states that can result from $\mathrm{N}_{2} \mathrm{O}_{2}^{-}$photodetachment at the HF and MP2 levels as for the closed-shell singlet states discussed above. A summary of the geometry optimizations and vibrational frequency calculations for the triplet states is given in Table V. Minima were located in the potential energy surfaces for each of the states in $C_{2 v}$ symmetry at the HF/6-31+G* level of theory. However, the MP2/6-31+G* force constant analysis indicates that the ${ }^{3} \mathrm{~B}_{1}$ state is located at a saddle point. Since this saddle point lies $\sim 3$ $\mathrm{eV}$ above the ${ }^{3} \mathrm{~A}_{2}$ state and the other three states are located at potential energy minima (at both levels of theory), no investigations were made of $\mathrm{C}_{\mathrm{s}}$ geometries for the triplet states. 
Table V: $A b$ initio Results for $\mathrm{N}_{2} \mathrm{O}_{2}$ Triplet Calculations ${ }^{a}$

\begin{tabular}{|c|c|c|c|c|c|c|c|c|c|c|c|}
\hline${ }^{3} \mathrm{~A}_{2}$ & Energy & $R_{n-n}$ & $R_{n-0}$ & $R_{n-0}$ & $\angle \mathrm{ONO}$ & $\omega_{1}$ & $\omega_{2}$ & $\omega_{3}$ & $\omega_{4}$ & $\omega_{5}$ & $\omega_{6}$ \\
\hline UHF/6-31+G* & -258.41 & 1.405 & 2.143 & 1.18 & 129.4 & 1576 & 1050 & 716 & 777 & 1868 & 565 \\
\hline $\mathrm{MP} 2 / 6-31+\mathrm{G}^{*}$ & -259.059695 & 1.441 & 2.228 & 1.230 & 129.8 & 1431 & 902 & 625 & 638 & 1815 & 483 \\
\hline${ }^{3} \mathrm{~A}_{1}$ & 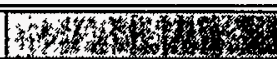 & (3) & 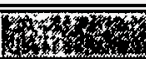 & 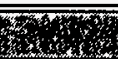 & 8 & 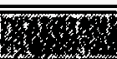 & 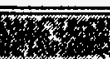 & Fen & 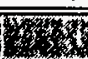 & (1) & 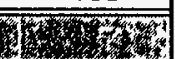 \\
\hline$\overline{\mathrm{UHF}} / 6-31+\mathrm{G}^{*}$ & -258.425991 & 1.225 & 2.015 & 1.270 & 105.0 & 1543 & 1038 & 608 & 608 & 1288 & 576 \\
\hline $\mathrm{MP} 2 / 6-31+\mathrm{G}^{*}$ & -258.997166 & 1.182 & 1.994 & 1.294 & 100.8 & 1972 & 1142 & 649 & 819 & 1776 & 585 \\
\hline${ }^{3} \mathrm{~B}_{2}$ & W & 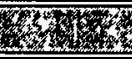 & ${ }^{2}$ & 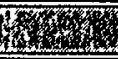 & Wh & & 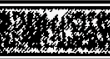 & (y) & 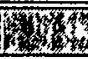 & 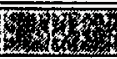 & 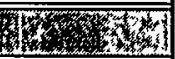 \\
\hline $\mathrm{UHF} / 6-31+\mathrm{G}^{*}$ & -258.3418507 & 1.144 & 2.217 & 1.388 & 106.0 & 2037 & 869 & 495 & 618 & 977 & 561 \\
\hline $\mathrm{MP} 2 / 6-31+\mathrm{G}^{*}$ & -258.9539203 & 1.188 & 2.344 & 1.311 & 126.8 & 1646 & 959 & 611 & 541 & 1164 & 312 \\
\hline${ }^{3} \mathrm{~B}_{1}$ & Tho & 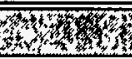 & 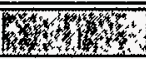 & 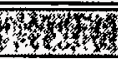 & Ther & 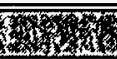 & 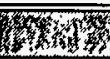 & & 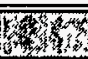 & 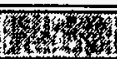 & 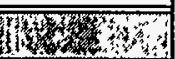 \\
\hline & -258.375947 & 1.282 & 2.166 & & & 1634 & & & & & \\
\hline $\mathrm{MP} 2 / 6-31+\mathrm{G}^{*}$ & -258.951237 & 1.248 & 2.167 & 1.301 & 112.8 & 1467 & 1022 & 618 & 627 & 571 & $1180 \mathrm{i}$ \\
\hline
\end{tabular}

a Bond lengths given in $\AA$, energy in au (hartree) and vibrational frequencies in $\mathrm{cm}^{-1}$. See Fig. 3.4 for parameter definitions. For the ${ }^{3} \mathrm{~A}_{2}$ electronic state, $v_{1}$ is the NO symmetric stretch (s. s.), $v_{2}$ is the NN s. s. and $v_{3}$ is the $\mathrm{NO}_{2}$ bend. For the other three states, $v_{1}$ is the NN s. s., $v_{2}$ is the NO s. s. and $v_{3}$ is the $\mathrm{NO}_{2}$ bend. 
Table VI: Calculated Electron Detachment Energies ${ }^{a}$

\begin{tabular}{|cccc|}
\hline State & $\mathrm{ADE}^{b}$ & $\mathrm{ADE}+\mathrm{ZPE}^{c}$ & $\mathrm{VDE}^{d}$ \\
\hline${ }^{1} \mathrm{~A}^{\prime}$ & 2.907 & 2.871 & $4.024^{e}$ \\
${ }^{3} \mathrm{~A}_{2}$ & 2.916 & 2.952 & 3.409 \\
${ }^{1} \mathrm{~A}_{1}$ & 4.033 & 4.068 & 4.024 \\
${ }^{3} \mathrm{~A}_{1}$ & 4.278 & 4.250 & 4.783 \\
${ }^{3} \mathrm{~B}_{1}$ & 5.172 & $5.109 f$ & 5.414 \\
${ }^{3} \mathrm{~B}_{2}$ & 5.252 & 5.329 & 5.394 \\
\hline
\end{tabular}

${ }^{a} \mathrm{MP} 4 / 6-311+\mathrm{G}^{*}$ energy calculated at the MP2/6-31+G* optimized geometry. All energies in $\mathrm{eV}$.

${ }^{b}$ Adiabatic Detachment Energy.

c $\mathrm{ADE}$ plus zero point energy (ZPE) correction using MP2/6-31+G* vibrational frequencies.

$d$ Vertical Detachment Energy.

$e$ Note that this is the same energy as for ${ }^{1} \mathrm{~A}_{1}$ state because both states coincide at $\mathrm{C}_{2 \mathrm{v}}$ symmetry.

$f \mathrm{ZPE}$ correction made using the $\mathrm{HF} / 6-31+\mathrm{G}^{*}$ frequencies because an imaginary frequency results at the MP2/6-31+G* level.

At the HF level, the calculations result in the ${ }^{3} \mathrm{~A}_{1}<{ }^{3} \mathrm{~A}_{2}<{ }^{3} \mathrm{~B}_{1}<{ }^{3} \mathrm{~B}_{2}$ energetic ordering of the electronic states. Inclusion of electron correlation at the MP2 level changes the ordering to ${ }^{3} \mathrm{~A}_{2}<{ }^{3} \mathrm{~A}_{1}<{ }^{3} \mathrm{~B}_{2}<{ }^{3} \mathrm{~B}_{1}$. Due to this reordering as a result of electron correlation, further investigation of the energies of the triplet states relative to each other, to the singlet states and to the anion were made by calculating the MP4/6-311+G* energy for each state at their respective MP2/6-31+G* optimized geometries. This results in a ${ }^{3} A_{2}<{ }^{3} A_{1}<{ }^{3} B_{1}<{ }^{3} B_{2}$ ordering of the states. From these results the adiabatic electron detachment energies (ADEs) have been calculated for each state. ${ }^{33}$ A listing of these energies are given in Table VI. A zero-point corrected energy is also given for each $\mathrm{ADE}$ where the zero-point energy is determined from the calculated vibrational frequencies. At this level of theory, we see that the ${ }^{1} \mathrm{~A}^{\prime}$ and ${ }^{3} \mathrm{~A}_{2}$ states are separated (including the ZPE correction) by only $81 \mathrm{meV}$. Thus, it is unclear from these calculations which state is the $\mathrm{N}_{2} \mathrm{O}_{2}$ ground electronic state. The table also 
includes vertical detachment energies (VDEs) determined by calculation the MP4/6-311+G* energy of each electronic state at the MP2/6-31+G* optimized geometry of the $\mathrm{N}_{2} \mathrm{O}_{2}^{-}$anion.

\subsection{Analysis and Discussion}

As described in the Results section, the spectra represent photodetachment transitions from the $\mathrm{C}_{2 \mathrm{v}} \mathrm{N}_{2} \mathrm{O}_{2}^{-}$anion to more than one electronic state of the $\mathrm{N}_{2} \mathrm{O}_{2}$ molecule. The next step in the data analysis is the identification of the final states involved in the photodetachment transitions. As discussed in Section 3.3.2.2, photodetachment of an electron from one the four highest-lying orbitals of the $\mathrm{N}_{2} \mathrm{O}_{2}^{-}$anion can produce $\mathrm{N}_{2} \mathrm{O}_{2}$ electronic states of all four symmetries associated with the $C_{2 v}$ point group. The data provide two types of information which can be used to make the assignments: 1) the energetic separation of the electronic states and 2) the vibrational profile for each electronic band. The state energetics can be compared directly to the $a b$ initio calculations or to the spectra of isoelectronic species. However, the vibrational profile for polyatomic species is determined by the Franck-Condon factors for photodetachment. These depend upon a combination of the geometry change upon photodetachment and the vibrational force constants of both the anion and neutral.

In the following sections, the photoelectron spectra will be considered in more detail. With the assistance of $a b$ initio calculations and Franck-Condon analyses, assignments of the spectral features to vibronic states of the $\mathrm{N}_{2} \mathrm{O}_{2}$ molecule will be proposed in Sec. 3.4.1. Since so little previous information is available about $\mathrm{N}_{2} \mathrm{O}_{2}$, comparisons will be drawn with the results for the isoelectronic $\mathrm{CO}_{3}$ and $\mathrm{NO}_{3}^{+}$molecules, which are also rather limited, in $\mathrm{Sec}$ 3.4.2. The results of the thermodynamic calculations will then be considered in Sec. 3.4 .3 with reference to the role of $\mathrm{N}_{2} \mathrm{O}_{2}$ and $\mathrm{N}_{2} \mathrm{O}_{2}^{-}$as metastable reactive intermediates in the $\mathrm{N}+\mathrm{NO}_{2} \rightarrow$ $\mathrm{O}+\mathrm{N}_{2} \mathrm{O}$ and $\mathrm{O}^{-}+\mathrm{N}_{2} \mathrm{O} \rightarrow \mathrm{NO}^{-}+\mathrm{NO}$ reactions, respectively. 


\subsubsection{Franck-Condon Analysis}

The calculated geometries and force constants for $\mathrm{N}_{2} \mathrm{O}_{2}^{-}$and $\mathrm{N}_{2} \mathrm{O}_{2}$ will now be used to predict the $a b$ initio vibrational profiles which are expected for photodetachment to several of the $\mathrm{N}_{2} \mathrm{O}_{2}$ electronic states. By comparison to the experimental data, we can gain further insight into the photodetachment transitions which are responsible for the data. For the simulations, Franck-Condon factors for the photodetachment process will be calculated within the separable parallel normal mode approximation. For each electronic state, the normal coordinate displacements are obtained by standard matrix methods using the ab initio Cartesian force constant matrix and the optimized geometries for the anion and neutral. These normal coordinate displacements are used to determine the Franck-Condon overlap between $\mathrm{N}_{2} \mathrm{O}_{2}^{-}$and $\mathrm{N}_{2} \mathrm{O}_{2}$. The photodetachment transition intensity, $\mathrm{I}$, for the process

$$
\mathrm{N}_{2} \mathrm{O}_{2}^{-}\left(\mathrm{v}_{1}^{\prime \prime}, \mathrm{v}_{2}^{\prime \prime}, \mathrm{v}_{3}^{\prime \prime}\right) \stackrel{\mathrm{hv}}{\longrightarrow} \mathrm{N}_{2} \mathrm{O}_{2}\left(\mathrm{v}_{1}^{\prime}, \mathrm{v}_{2}^{\prime}, \mathrm{v}_{3}^{\prime}\right)+e^{-}
$$

is calculated according to Eq. (11)

$$
I \propto v_{e} \cdot\left|\tau_{e}\right|^{2} \cdot \prod_{i=1}^{6}\left|\left\langle\Psi_{v_{i}^{\prime}}\left(Q_{i}\right) \mid \Psi_{v_{i}^{\prime}}\left(Q_{i}\right)\right\rangle\right|^{2}
$$

where $v_{e}$ is the asymptotic velocity of the photodetached electron and $\tau_{e}$ is the electronic transition dipole which is considered to be a constant for a each electronic state. In the separable parallel normal mode approximation, the Franck-Condon factors (FCFs) for each

vibrational mode, $\left|\left\langle\psi_{v_{i}^{\prime}}\left(Q_{i}\right) \mid \psi_{v_{i}^{\prime \prime}}\left(Q_{i}\right)\right\rangle\right|^{2}$, are calculated as the spatial overlap of the vibrational wave functions of the anion, $\Psi_{v_{i}^{*}}$, and the neutral, $\psi_{v_{i}^{\prime}}$, for that mode. Combination bands are calculated as products of single-mode FCFs. The resultant stick spectrum is convoluted with the experimental resolution function for comparison to the experimental data.

In general, a photodetachment transition between an anion and a neutral possessing the same symmetry (i.e., $\mathrm{C}_{2 \mathrm{v}}$ ) will only excite totally symmetric vibrational modes of the neutral. 
In the case of the $C_{2 v}$ geometry, there are three symmetric modes, best labeled as the NN stretch, the NO stretch and the $\mathrm{NO}_{2}$ bend. For the other three non-symmetric normal modes, only transitions to even vibrational levels of the neutral from the anion ground state have non-zero Franck-Condon overlap. Significant excitation of these modes occurs only if there is a large difference in the anion and neutral vibrational frequencies for that mode. For the photodetachment of the $C_{2 v}$ anion to generate the $C_{s}$ neutral, transitions are allowed to the symmetric modes of the $C_{s}$ state (i.e., $v_{1}-v_{5} ; v_{6}$ is the non-totally-symmetric out-of-plane motion).

According to Eq. (11), the length of a vibrational progression observed in the anion PES depends, in general, upon the difference in geometry between the anion and the neutral. A significant geometrical rearrangement upon photodetachment results in a long vibrational progression, whereas if the anion and neutral have very similar geometries, the spectrum is dominated by the $0-0$ transition. For example, based upon comparison of the anion and neutral HF $a b$ initio calculations, one would expect a significant amount of vibrational excitation for photodetachment to the ${ }^{3} \mathrm{~B}_{2}$ state but less excitation for transitions to the ${ }^{3} \mathrm{~B}_{1}$ state.

In the case of photodetachment transitions to the ${ }^{1} \mathrm{~A}_{1}$ state, the geometry change is so dramatic (i.e., the $\angle \mathrm{ONO}$ angle changes by $56^{\circ}$ at the MP2 level), that the Franck-Condon intensity is extremely spread out over many vibrational levels for this state. A similar situation occurs for the ${ }^{1} \mathrm{~A}$ ' state in which one of the NNO angles changes by $47^{\circ}$. This suggests that we do not observe any transitions to the ${ }^{1} \mathrm{~A}^{\prime}$ or ${ }^{1} \mathrm{~A} 1$ states of $\mathrm{N}_{2} \mathrm{O}_{2}$ in our $\mathrm{N}_{2} \mathrm{O}_{2}^{-}$photoelectron spectra. Thus, if the ' $\mathrm{A}$ ' state is the ground electronic state, we are not able to determine experimentally the adiabatic electron affinity of $\mathrm{N}_{2} \mathrm{O}_{2}$ or derive thermochemical information such as the heat of formation of the species.

The geometries of the triplet states, however, are much closer to that of the anion.

Figure 3.5: Ab initio simulations at $\mathrm{HF} / 6-31+\mathrm{G}^{*}$ for the $\mathrm{N}_{2} \mathrm{O}_{2}^{-}$photoelectron transitions to the triplet electronic states of $\mathrm{N}_{2} \mathrm{O}_{2}$ molecule. 


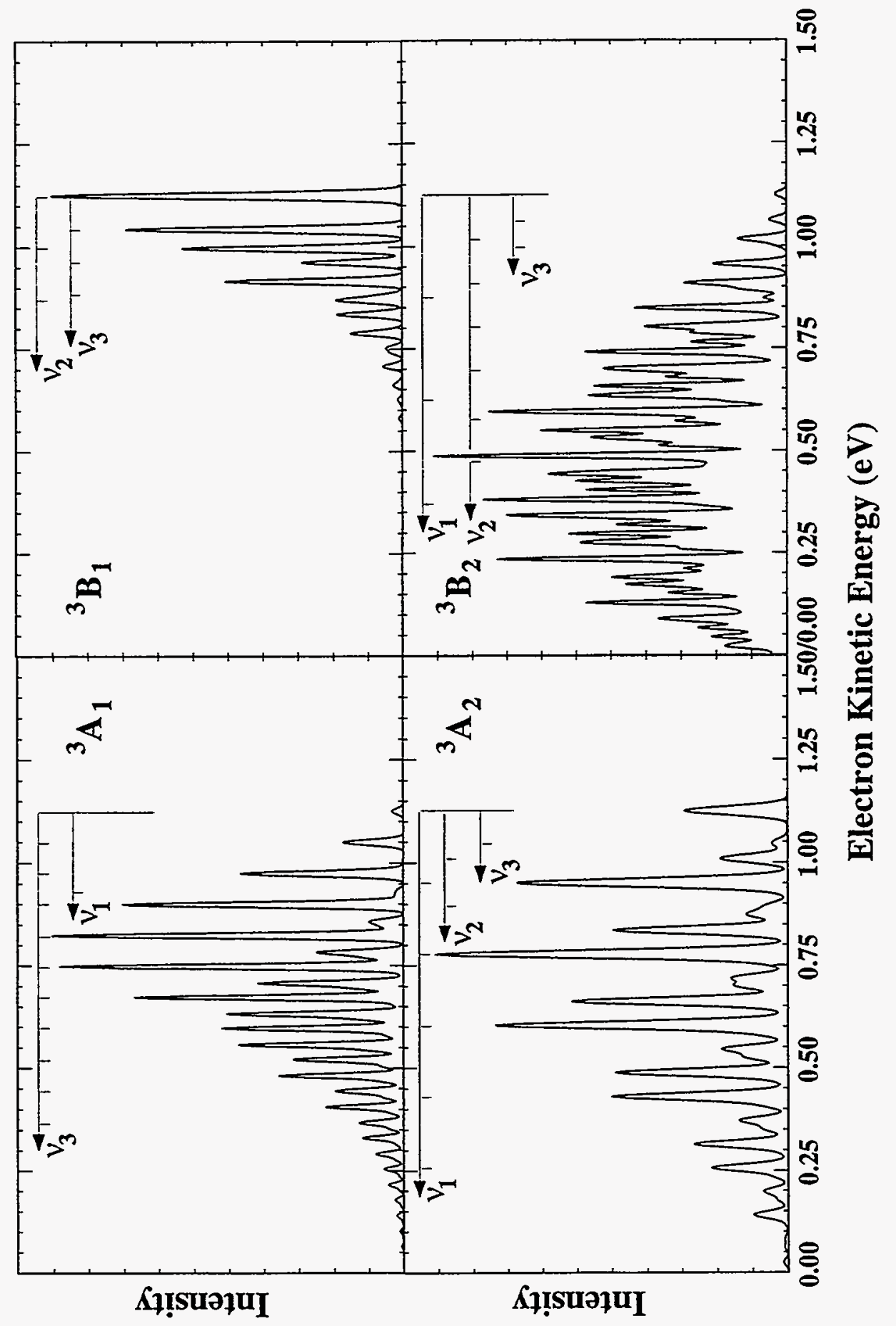

Figure 3.5 
Figure 3.5 shows the Franck-Condon simulations for the ${ }^{3} \mathrm{~A}_{1},{ }^{3} \mathrm{~A}_{2},{ }^{3} \mathrm{~B}_{1}$, and ${ }^{3} \mathrm{~B}_{2}$ electronic states using the results of the HF $a b$ initio calculated geometries and neutral force constants. In the simulations, the 0-0 transition is placed at $1.126 \mathrm{eV}$ for purposes of comparison with the 4.657 $\mathrm{eV}$ photoelectron spectrum. Each state has its own characteristic profile. While the ${ }^{3} \mathrm{~B}_{1}$ state is dominated by the $0-0$ transition, the other three states have reasonably long progressions in at least one vibrational mode. The ${ }^{3} \mathrm{~A}_{1}$ state primarily has a long progression in the $\mathrm{NO}_{2}$ bending mode with a slight excitation of the NN stretch $\left(v_{1}\right)$, while the ${ }^{3} \mathrm{~A}_{2}$ state is dominated by the NO stretch $\left(v_{1}\right)$ with shorter progression in the $\mathrm{NO}_{2}$ bend $\left(v_{3}\right)$ and the NN stretch $\left(v_{2}\right)$. Significant excitation of two modes [NN $\left(v_{1}\right)$ and NO $\left(v_{2}\right)$ stretches] in the ${ }^{3} B_{2}$ spectrum leads to congestion of the spectrum by combination bands.

Figure 3.6 shows the simulations of the photoelectron spectrum using the MP2 calculated geometries and force constants. The different equilibrium geometries and force constants force constants which result from the MP2 calculations can result in very different simulated spectra relative to the HF simulations. The greatest change occurs in the ${ }^{3} \mathrm{~B}_{2}$ simulation where a larger than average electron correlation effect is observed in the geometry calculation. In this case, activity in the $v_{2}$ mode is greatly decreased relative to the HF simulation. Recall that the force constant analysis of the ${ }^{3} \mathrm{~B}_{1}$ state at the MP2 level produced an imaginary frequency for the $\mathrm{NO}_{2}$ antisymmetric stretch (a.s.). This mode is not included in the simulation for the ${ }^{3} \mathrm{~B}_{1}$ state. This does not affect the simulation significantly because the displacement along that coordinate in the $C_{2 v}$ symmetric species is zero by symmetry. If, in fact, there is a lower energy structure in $\mathrm{C}_{\mathrm{s}}$ symmetry, which has not been investigated here, the $\mathrm{NO}_{2}$ a.s. would be excited upon photodetachment in the simulated spectrum.

In comparison to the experimental data, it is clear that the observed progression in the $4.657 \mathrm{eV}$ data does not represent transitions to the singlet states because there is negligible

Figure 3.6: Ab initio simulations at MP2/6-31+G* for the $\mathrm{N}_{2} \mathrm{O}_{2}^{-}$photoelectron transitions to the triplet electronic states of $\mathrm{N}_{2} \mathrm{O}_{2}$ molecule. 


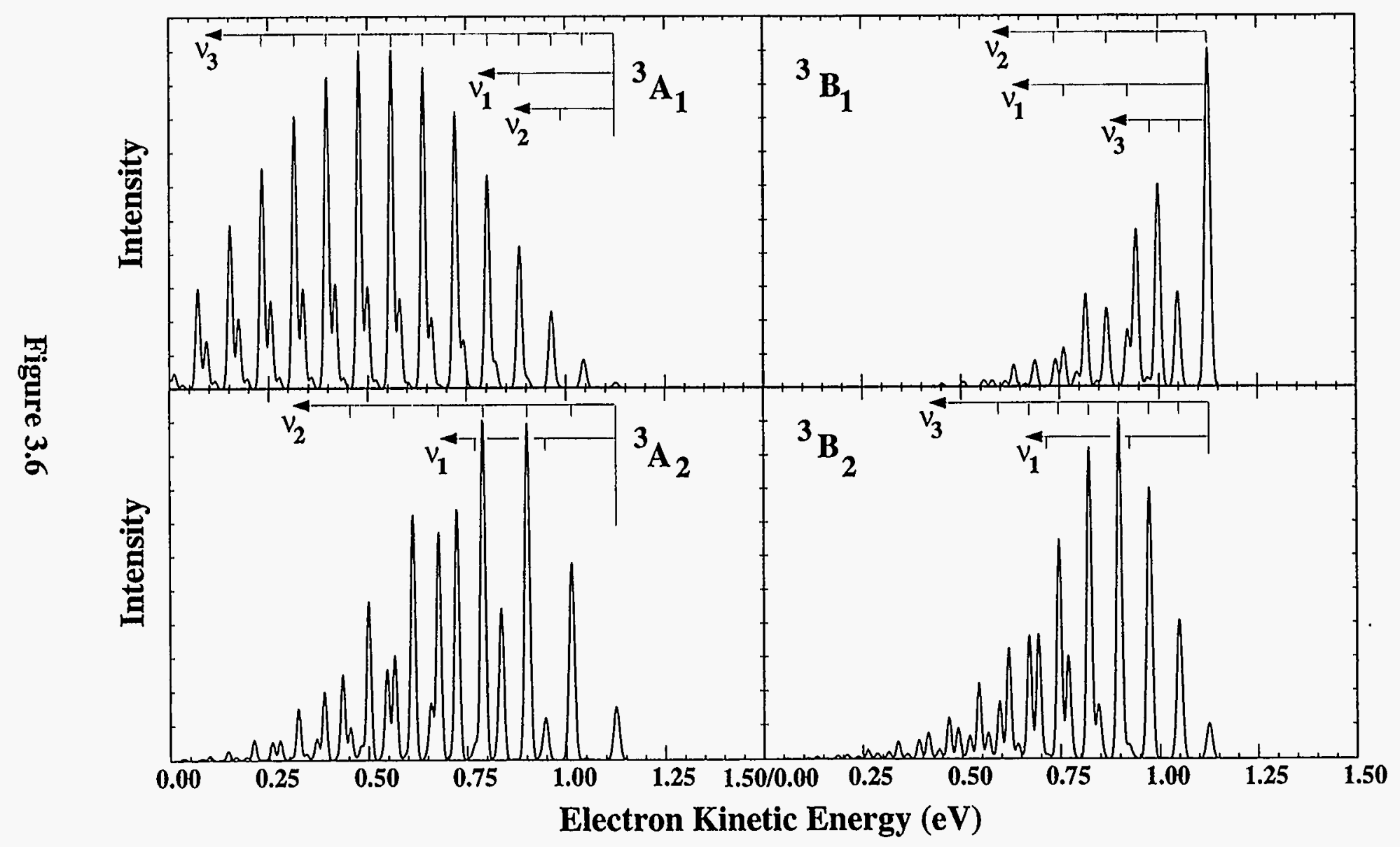


Franck-Condon overlap to these states. Instead, the most likely candidates are the ${ }^{3} \mathrm{~A}_{2}$ and the ${ }^{3} \mathrm{~A}_{1}$ states since they are calculated to be the lowest triplet states at the MP4 level of theory. Comparison of the data to the simulations suggests that the best assignment would be the ${ }^{3} \mathrm{~A}_{2}$ state because the $a b$ initio simulation models the observed vibrational profile is reasonably well, particularly the HF simulation. Energetically, the position of peak 'A' at $1.126 \mathrm{eV}$ corresponds to a $\mathrm{ADE}$ of $3.531 \mathrm{eV}$. This is significantly larger than the calculated value of $2.907 \mathrm{eV}$ for the ${ }^{3} \mathrm{~A}_{2}$ state but much less than the calculated $\mathrm{ADE}=4.278 \mathrm{eV}$ of the ${ }^{3} \mathrm{~A}_{1}$ state. The observed vibrational frequencies, which are quite anharmonic for all three modes, are in reasonable agreement with calculated vibrational frequencies.

In the $5.822 \mathrm{eV}$ data, the progression beginning at peak $\mathrm{A}(\mathrm{eKE}=1.286 \mathrm{eV})$ is in very good agreement with the calculated result for the ${ }^{3} \mathrm{~A}_{1}$ state not only energetically but also with respect to the appearance of the vibrational profile. Assuming peak A to be the 0-0 transition to the ${ }^{3} \mathrm{~A}_{1}$ state, the experimental $\mathrm{ADE}$ is $4.536 \mathrm{eV}$ which is reasonably close the calculated ${ }^{3} \mathrm{~A}_{1}$ value of $4.278 \mathrm{eV}$. The simulations predict $\mathrm{NO}_{2}$ bend excitation at both the HF and MP2 levels at a frequency $\left(v_{3} \sim 600-650 \mathrm{~cm}^{-1}\right)$ close to the observed value of $540 \pm 40 \mathrm{~cm}^{-1}$.

This progression continues under the two most intense features which appear in the spectrum. The positions of peaks $\mathrm{D}$ and $\mathrm{G}$ at 1.089 and $0.914 \mathrm{eV}$ correspond to ADEs of 4.733 and $4.908 \mathrm{eV}$, which both agree reasonably well with the MP4 calculated value for the ${ }^{3} \mathrm{~B}_{1}$ state $(5.172 \mathrm{eV})$. Since no higher lying features are observed to indicate that the peaks belong to a single vibrational progression, peaks $D$ and $G$ may represent $0-0$ transitions to two different electronic states which have geometries near that of the anion. Fig. 3.5 and 3.6 show that only the ${ }^{3} B_{1}$ simulation is dominated by the $0-0$ transition while all of the other states considered here have long progressions and can not explain the second intense feature. In this case, the second peak may result from transitions to an additional electronic state, either singlet or triplet, which has not been considered here. 
However, peaks $D$ and $G\left(\Delta=1411 \mathrm{~cm}^{-1}\right)$ could also be members of a single $\mathrm{N}-\mathrm{N}$ stretch vibrational progression in an electronic state which dissociates at higher energies. This is consistent with the sharp drop-off of resolved vibrational structure after peak $J$ and the broad appearance of peak $\mathrm{K}$ suggesting that the neutral vibrational excitation induced by photodetachment leads to dissociation of the neutral complex. This is reasonable in light of the fact that all of the structure observed in the spectrum lies well above the asymptotic energies for dissociation to $\mathrm{O}+\mathrm{N}_{2} \mathrm{O}$ and $\mathrm{NO}+\mathrm{NO}$ and peaks $\mathrm{F}-\mathrm{K}$ lie above not only the $\mathrm{N}+\mathrm{NO}_{2}$ but also the $\mathrm{N}_{2}+\mathrm{O}+\mathrm{O}$ dissociation asymptote. In this case, peak $\mathrm{D}$ represents the origin of a neutral electronic state with a geometry which is different from that of the anion, perhaps the ${ }^{3} \mathrm{~B}_{2}$ state.

\subsubsection{Comparison to isoelectronic species $-\mathrm{CO}_{3}$ and $\mathrm{NO}_{3}^{+}$}

Comparison to isoelectronic species often provides insight to the properties of a new, as yet uncharacterized system. While the $\mathrm{CO}_{3}$ molecule has been observed experimentally in matrix isolation experiments, ${ }^{34,35,36}$ no observations have been reported for the $\mathrm{NO}_{3}^{+}$cation. Both the $\mathrm{CO}_{3}$ and $\mathrm{NO}_{3}^{+}$molecules have been the subject of theoretical investigations in which several structures and excited electronic states have been investigated. Most of the debate for these isoelectronic molecules concerns the relative stability of the $C_{2 v}$ and $D_{3 h}$ geometries, an issue which is not of concern here. Unlike $\mathrm{CO}_{3}$ and $\mathrm{NO}_{3}^{+}$, a distortion to $\mathrm{C}_{\mathrm{s}}$ symmetry lowers the energy of the singlet $\mathrm{N}_{2} \mathrm{O}_{2}$ in the $\mathrm{HF}$ and MP2 calculations.

Experimental observations suggest that the $\mathrm{CO}_{3}$ molecule possesses $\mathrm{C}_{2 \mathrm{v}}$ symmetry and a unique acute angle $\left(\theta-70^{\circ}\right)$ in its ${ }^{1} A_{1}$ closed-shell ground state. Early theoretical treatments supported these findings. ${ }^{37,38} \mathrm{~A} \mathrm{D}_{3 \mathrm{~h}}$ structure was briefly proposed ${ }^{39}$ as the actual geometry but further investigations $s^{40,41}$ have concluded that the $C_{2 v}$ geometry is, indeed, energetically favored over the $D_{3 h}$ species. The acute angle of the $C_{2 v} C O_{3}$ species is very similar to that found for the $\mathrm{C}_{2 \mathrm{v}}$ (B) structure of the $\mathrm{N}_{2} \mathrm{O}_{2}^{1} \mathrm{~A}_{1}$ electronic state $\left[\theta(\mathrm{ONO})=64^{\circ}\right]$. Froese et al..$^{41}$ 
calculated the MP2/6-31G* geometry of the ${ }^{3} \mathrm{~A}_{1}$ state of $\mathrm{CO}_{3}(\mathrm{CO} 1=1.313 \AA ; \mathrm{CO} 2=\mathrm{CO} 3=$ $1.259 \AA ; \theta(\mathrm{O} 2 \mathrm{CO} 3)=113.4^{\circ}$ ), which can be compared to the geometry for the $\mathrm{N}_{2} \mathrm{O}_{2}{ }^{3} \mathrm{~A}_{1}$ state presented above $\left[N N=1.182 \AA ; N O=1.294 \AA ; \theta(O N O)=101^{\circ}\right]$. While the bond relative lengths of the axial and symmetric oxygen atoms is reversed in $\mathrm{CO}_{3}$ as compared to $\mathrm{N}_{2} \mathrm{O}_{2}$, the acute angles are similar. They find that this $\mathrm{CO}_{3}{ }^{3} \mathrm{~A}_{1}$ state lies $(0.99 \mathrm{eV})$ above the $\mathrm{C}_{2 \mathrm{v}}$ structure at the MP4/6-31G*//MP2/6-31G* level of theory. This is much larger than the $\mathrm{N}_{2} \mathrm{O}_{2}$ ${ }^{1} \mathrm{~A}_{1}-{ }^{3} \mathrm{~A}_{1}$ splitting of $0.24 \mathrm{eV}$. No other states were available for direct comparison.

Castro et al..$^{40}$ present MBPT calculations of the four lowest-lying triplet states of $\mathrm{CO}_{3}$. The geometries obtained for each state can be readily compared to the corresponding states of $\mathrm{N}_{2} \mathrm{O}_{2}$. At the MBPT(4) level of theory using a split valence plus polarization basis, they find that the $\mathrm{CO}_{3}$ triplet states are ordered as ${ }^{3} \mathrm{~A}_{1}<{ }^{3} \mathrm{~A}_{2}<{ }^{3} \mathrm{~B}_{2}<{ }^{3} \mathrm{~B}_{1}$. The states are found to lie $0.89,1.06,1.07$ and $1.16 \mathrm{eV}$ above the singlet $C_{2 v}$ minimum energy species, respectively. The relative ordering of the states differs from those calculated for $\mathrm{N}_{2} \mathrm{O}_{2}$. More importantly, the singlet state of the $\mathrm{CO}_{3}$ appears to be much more stable, with respect to the triplet states, than that of the $\mathrm{N}_{2} \mathrm{O}_{2}$ molecule.

Canuto et al., ${ }^{38}$ used a first order polarization propagation method to calculate the vertical excitation energies of the open-shell singlet states of the $\mathrm{CO}_{3}$ molecule. The results of these calculations showed that the singlet states lie at energies well above the ground state. The lowest excited singlet state was found to be the ${ }^{1} \mathrm{~B}_{1}$ state at $2.47 \mathrm{eV}$ and the rest of the states were about $5 \mathrm{eV}$ or more above the $\mathrm{C}_{2 \mathrm{v}}$ ground state. It is likely that a similar situation will occur in the case of $\mathrm{N}_{2} \mathrm{O}_{2}$, justifying the neglect of these states in our analysis.

Boehm and Lohr ${ }^{42}$ have calculated HF optimized geometries for several electronic states of the nitroxylium cation $\left(\mathrm{NO}_{3}^{+}\right)$. In a similar analysis to that presented here, they calculated the contribution of electron correlation effects to the energy of each species using MP methods through fourth-order for a selected set of these states. They find a $D_{3 h}{ }^{1} A_{1}$ ' ground state for the $\mathrm{NO}_{3}^{+}$molecule lying $0.94 \mathrm{eV}$ below the $\mathrm{C}_{2 \mathrm{v}}{ }^{1} \mathrm{~A}_{1}$ state. Among the several states 
considered, they also calculate MP4SDTQ/DZP energies for the ${ }^{3} \mathrm{~B}_{2}$ and ${ }^{3} \mathrm{~B}_{1}$ electronic states which are predicted to lie 1.96 and $2.17 \mathrm{eV}$ above the ${ }^{1} \mathrm{~A}_{1}{ }^{\prime}$ state and 1.02 and $1.27 \mathrm{eV}$ above the ${ }^{1} \mathrm{~A}_{1}$ state. Due to the high energies of the ${ }^{3} \mathrm{~A}_{2}$ and ${ }^{3} \mathrm{~A}_{1}$ states of $\mathrm{NO}_{3}^{+}$at the $\mathrm{HF}$ level, higher level calculations were not pursued. Again, the calculations indicate that the splitting between the $\mathrm{C}_{2 \mathrm{v}}$ singlet and triplet states of $\mathrm{NO}_{3}^{+}$is considerably larger that observed for $\mathrm{N}_{2} \mathrm{O}_{2}$.

Thus, the comparison of $\mathrm{N}_{2} \mathrm{O}_{2}$ with $\mathrm{CO}_{3}$ and $\mathrm{NO}_{3}^{+}$species shows interesting similarities and differences. All three of the species have fairly low-lying triplet states. While the geometries of the triplet states are comparable between the molecules, there are reasonable differences in bond lengths and bond angles. In the singlet manifold, the $\mathrm{C}_{s}$ minimum energy structure of the $\mathrm{N}_{2} \mathrm{O}_{2}$ molecule has no counterpart in the $\mathrm{CO}_{3}$ or $\mathrm{NO}_{3}^{+}$electronic spectra. However, the energetic separation between the $\mathrm{N}_{2} \mathrm{O}_{2}$ singlet and triplet states appears to be smaller than those of the $\mathrm{CO}_{2}$ and $\mathrm{NO}_{3}^{+}$molecules.

\subsubsection{Roles as Reactive Intermediates}

Shown in Fig. 3.7 is a schematic energy diagram which summarizes the findings presented here and compares these results to the asymptotic energies of the separated reactants and products for both the anion and the neutral. All energies are with respect to the anion ground state, in $\mathrm{eV}$. The left column indicates the experimentally observed transitions which have been assigned to $0-0$ transitions in the 4.657 and $5.822 \mathrm{eV} \mathrm{N} \mathrm{O}_{2}^{-}$photoelectron spectra. Arrows indicating the photon energies used in the experiments are shown against the left axis. The central column contains the energetic ordering of the electronic states according to the $a b$ initio calculations at the MP4/6-311+G*//MP2/6-31+G* level of theory (including the ZPE correction). On the right are the anion and neutral dissociation asymptotes.

Figure 3.7: Schematic energetics diagram for the $\mathrm{N}_{2} \mathrm{O}_{2}^{-} / \mathrm{N}_{2} \mathrm{O}_{2}$ system. Included are the $a b$ initio calculated energies of the electronic states, the observed state origins and the energetic asymptotes for dissociation of both the anion and the neutral. 


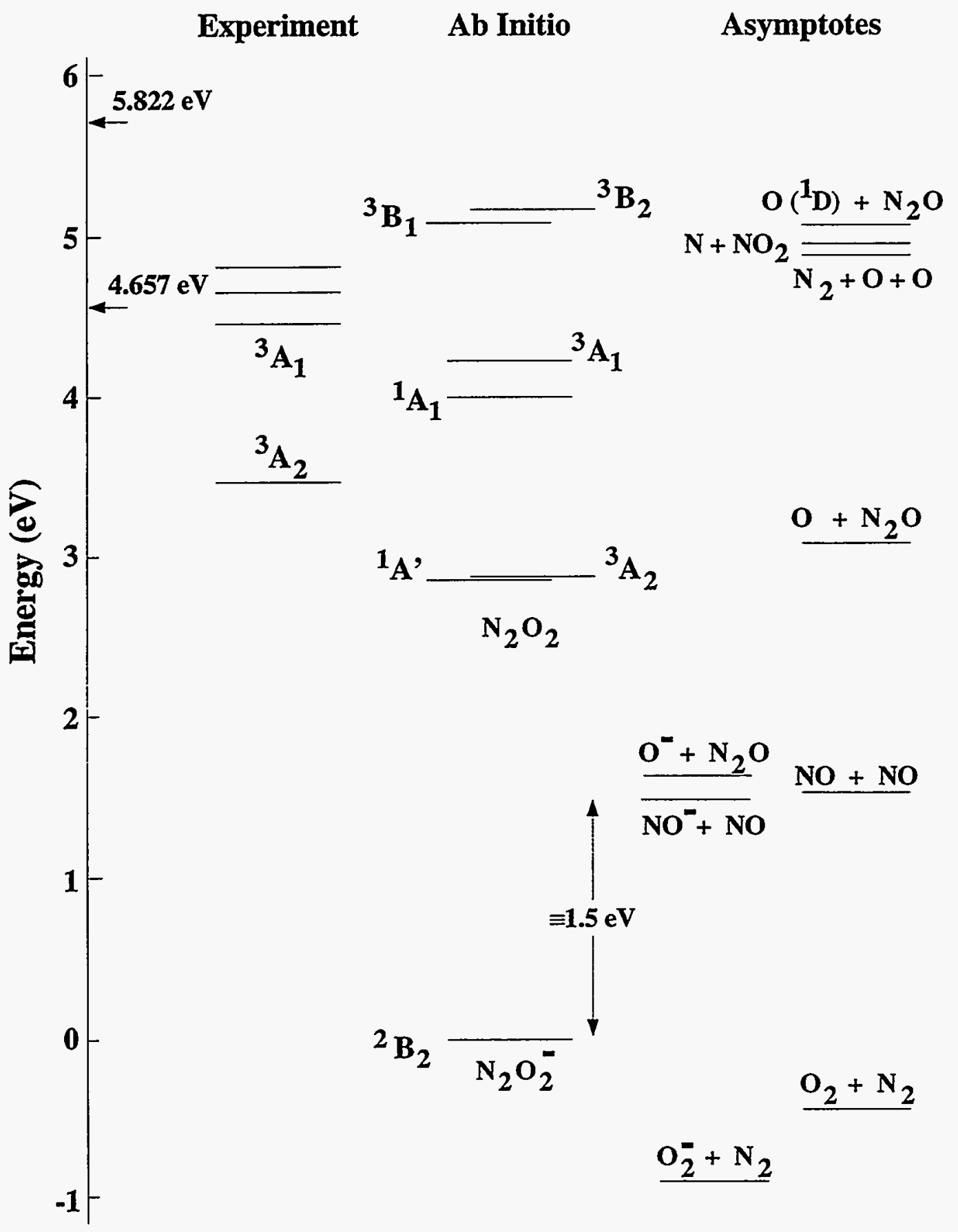

Figure 3.7 
As illustrated in Figure 3.7, the anion, in its ground state, is bound with respect to dissociation by $\leq 1.5 \mathrm{eV}$. This value has been determined recently in this laboratory in a study of the $\mathrm{N}_{2} \mathrm{O}_{2}^{-}$photodissociation dynamics. ${ }^{43}$ The energy release resulting from $\mathrm{N}_{2} \mathrm{O}_{2}^{-}$ dissociation $(\lambda=500 \mathrm{~nm})$ in a fast $(8 \mathrm{keV})$ ion beam were measured using a time-and-position sensitive coincidence detection scheme. The limit for the dissociation is determined from the maximum observed kinetic energy release to both the $\mathrm{O}^{-}+\mathrm{N}_{2} \mathrm{O}$ and $\mathrm{NO}^{-}+\mathrm{NO}$ dissociation channels. The $\mathrm{D}_{\mathrm{o}}\left(\mathrm{O}^{-} \cdots \mathrm{N}_{2} \mathrm{O}\right)$ values determined from both channels, combined with the known reaction exothermicity, agree to within $10 \%$ ( $3 \mathrm{kcal} / \mathrm{mole}$ ). A more complete description of these experiments will be provided in a future publication.

On the basis of several kinetics measurements, it is proposed that the mildly exothermic reaction (1) proceeds through a long-lived $\mathrm{N}_{2} \mathrm{O}_{2}^{-}$intermediate. Since the formation of $\mathrm{N}_{2} \mathrm{O}_{2}^{-}$ most likely involves the $\mathrm{O}^{-}$attack onto the central $\mathrm{N}$-atom, the long lifetime of the intermediate reaction complex is not surprising because the coordinates for ion formation from $\mathrm{O}^{-}+\mathrm{N}_{2} \mathrm{O}$ are quite different from the dissociation coordinate into $\mathrm{NO}^{-}+\mathrm{NO}$. From these results, the $\mathrm{NNO}_{2}^{-}$ well depth has been better established. However, the rest of the reaction surface (See Fig. 3.1) remains uncharacterized. Thus, questions remain concerning the nature of the second minimum (i.e., for the $\mathrm{NO}^{-}(\mathrm{NO})$ species) and the height of the barrier between the two minima and their role in the $\mathrm{O}^{-}+\mathrm{N}_{2} \mathrm{O}$ reaction.

In the early studies of the $\mathrm{N}+\mathrm{NO}_{2}$ reaction by Clyne and Thrush, ${ }^{11}$ they proposed a $\mathrm{C}_{2 \mathrm{v}}$ structure as a transition state for the reaction to form $\mathrm{N}_{2} \mathrm{O}+\mathrm{O}$. While different transition state species might be expected for the other channels available, they proposed that the $C_{2 v}$ species was involved for all pathways based upon the constant ratio of reaction rates as a function of temperature. This suggests that the intermediate must exist long enough to undergo a significant rearrangement before dissociation to $\mathrm{NO}+\mathrm{NO}$.

In the reaction of ground state $\mathrm{N}\left({ }^{4} S\right)$ atoms with ground state $\mathrm{NO}_{2}\left(\tilde{X}^{2} \mathrm{~A}_{1}\right)$ the reaction proceeds on a triplet reaction surface and can proceed adiabatically to all of the observed 
products through $\mathrm{C}_{\mathrm{s}}$ intermediates which must be formed in the reactions generating $\mathrm{O}+\mathrm{N}_{2} \mathrm{O}$ and $\mathrm{NO}+\mathrm{NO}$. In $\mathrm{C}_{2 \mathrm{v}}$ symmetry, the singly occupied orbitals of the $\mathrm{N}\left({ }^{4} \mathrm{~S}\right)$ atoms have $\mathrm{A}_{2}, \mathrm{~B}_{1}$, and $\mathrm{B}_{2}$ irreducible representations while the $\mathrm{HOMO}$ of the $\mathrm{NO}_{2}$ molecule is a half-filled $\mathrm{a}_{1}$ orbital primarily localized on the $\mathrm{N}$-atom. Thus the reaction of $\mathrm{N}+\mathrm{NO}_{2}$ along a $\mathrm{C}_{2 \mathrm{v}}$ constrained pathway can generate the ${ }^{3} \mathrm{~A}_{1},{ }^{3} \mathrm{~B}_{2}$ and the ${ }^{3} \mathrm{~B}_{1}$ electronic states of the $\mathrm{NNO}_{2}$ species. These can, in turn, generate both $\mathrm{A}^{\prime}$ and $\mathrm{A}^{\prime \prime}$ states by distortion to $\mathrm{C}_{\mathrm{s}}$ symmetry. As discussed above, we believe the $\mathrm{N}_{2} \mathrm{O}_{2}^{-}$photoelectron spectrum at hv $=5.822 \mathrm{eV}$ contains transitions to the ${ }^{3} \mathrm{~A}_{1}$ state and perhaps the ${ }^{3} \mathrm{~B}_{1}$ and/or the ${ }^{3} \mathrm{~B}_{2}$ states. Thus, the photoelectron spectrum of $\mathrm{N}_{2} \mathrm{O}_{2}^{-}$provides information about the properties of the intermediate for the $\mathrm{N}+$ $\mathrm{NO}_{2}$ reactions.

As mentioned in Sec. 3.3 and 3.4.1 and shown in Fig. 3.7, all of the vibrational structure observed in the photoelectron spectrum of $\mathrm{N}_{2} \mathrm{O}_{2}^{-}$lies above the energetic asymptotes for dissociation of the $\mathrm{N}_{2} \mathrm{O}_{2}$ complex into $\mathrm{O}+\mathrm{N}_{2} \mathrm{O}$ and $\mathrm{N}_{2}+\mathrm{O}_{2}$. The amount of vibrational structure observed in the spectrum implies the existence of a significant barrier to dissociation to these products indicating that the reaction surfaces have fairly deep minima along the reaction coordinate. The sharp drop-off in signal and the increased width of feature $\mathrm{K}$ may indicate the onset of significant dissociation and thus provide an indication of the barrier height. Features A, D and $\mathrm{H}$ (the possible $0-0$ transitions) all lie very close to the energy of the $\mathrm{N}+\mathrm{NO}_{2}$ reactants. Thus, the barrier to dissociation of the $\mathrm{N}_{2} \mathrm{O}_{2}$ complex will most likely play an important role in the $\mathrm{N}+\mathrm{NO}_{2}$ reaction dynamics. Further kinetics studies may reveal whether these minima lead to the formation of long-lived reaction intermediates as in the case of the $\mathrm{O}^{-}+\mathrm{N}_{2} \mathrm{O}$ reaction.

!.

\subsection{Summary}

We have presented the photoelectron spectra of $\mathrm{N}_{2} \mathrm{O}_{2}^{-}$collected at $\mathrm{hv}=4.657$ and $5.822 \mathrm{eV}$. By using the $\mathrm{O}^{-}+\mathrm{N}_{2} \mathrm{O}+\mathrm{M}$ ion formation mechanism we are able to from 
exclusively the $\mathrm{C}_{2 \mathrm{v}} \mathrm{NNO}_{2}^{-}$species, as observed by Posey and Johnson. ${ }^{18}$ Photodetachment of this anion produces the previously unobserved $\mathrm{NNO}_{2}$ molecule. The spectra contain resolved vibrational progressions for several electronic states of this species. All of the observed features correspond to transitions to states of the $\mathrm{N}_{2} \mathrm{O}_{2}$ molecule which are energetically unstable with respect to dissociation to at least three, and as many as six dissociation asymptotes.

$A b$ initio calculations are presented for the geometries and vibrational frequencies of both the anion and neutral species. The $\mathrm{N}_{2} \mathrm{O}_{2}^{-}$anion $\tilde{X}{ }^{2} \mathrm{~B}_{2}$ ground state is found to be planar with nearly equal bond lengths and bond angles. For $\mathrm{N}_{2} \mathrm{O}_{2}$, several electronic states which are accessible by one-electron photodetachment of the anion were investigated. Properties of the ${ }^{1} \mathrm{~A}_{1},{ }^{3} \mathrm{~A}_{2},{ }^{3} \mathrm{~A}_{1},{ }^{3} \mathrm{~B}_{2}$ and ${ }^{3} \mathrm{~B}_{1}$ (in $\mathrm{C}_{2 v}$ symmetry) and ${ }^{1} \mathrm{~A}^{\prime}$ (in $\mathrm{C}_{\mathrm{s}}$ symmetry) electronic states were calculated. At the highest level of theory considered, MP4/6-311+G*//MP2/6-31+G*, the lowest states, ${ }^{3} \mathrm{~A}_{2}$ and ${ }^{1} \mathrm{~A}$, were found to be nearly isoenergetic. The remaining states were ordered energetically as ${ }^{1} \mathrm{~A}_{1}<{ }^{3} \mathrm{~A}_{1}<{ }^{3} \mathrm{~B}_{1}<{ }^{3} \mathrm{~B}_{2}$ at their respective equilibrium geometries. The calculations indicate that the triplet states are most likely to be observed in the photoelectron spectra based upon the geometry of each state with respect to the anion geometry. Simulated spectra are presented using the results of $a b$ initio calculations for the lowest singlet and the four lowest triplet electronic states. Several tentative assignments are made are made for the observed transitions based upon comparison with the $a b$ initio results and simulations.

Due to the significant geometrical differences between the anion and the singlet electronic states of the $\mathrm{N}_{2} \mathrm{O}_{2}$ molecule, transitions to these states are not observed in the photoelectron spectra. Thus, while the $a b$ initio calculations predict the ${ }^{1} \mathrm{~A}^{\prime}$ and the ${ }^{3} \mathrm{~A}_{2}$ electronic states to be nearly isoenergetic, we are unable to determined the actual ordering of these states and accordingly we are unable to determine the ground state of $\mathrm{N}_{2} \mathrm{O}_{2}$ or the electron affinity of the species. If the ${ }^{3} A_{2}$ state is the ground state, the EA of $\mathrm{N}_{2} \mathrm{O}_{2}$ is $3.531 \pm$ $0.014 \mathrm{eV}$. 
Both the anion and the neutral species studied here, $\mathrm{N}_{2} \mathrm{O}_{2}^{-}$and $\mathrm{N}_{2} \mathrm{O}_{2}$, appear to be reactive intermediates in the $\mathrm{O}^{-}+\mathrm{N}_{2} \mathrm{O}$ and $\mathrm{N}+\mathrm{NO}_{2}$ reactions, respectively. The latter reaction can occur on triplet reactions surfaces containing the $C_{2 v}$ species which are observed in the present study. The observed vibrational structure indicates the existence of significant barriers to dissociation of the $\mathrm{N}_{2} \mathrm{O}_{2}$ complex which will play a role in the $\mathrm{N}+\mathrm{NO}_{2}$ reaction dynamics. Further kinetics measurements are necessary to determine the extent of these effects.

\subsection{Acknowledgments}

Special thanks to D. J. Leahy, D. L. Osborn and E. M. Ross for determining $\mathrm{D}_{0}\left(\mathrm{~N}_{2} \mathrm{O}_{2}{ }^{-}\right)$ upon request. This work has been supported by the United States Air Force Office of Scientific Research under Contract No. F49620-94-1-0115. 


\section{References}

${ }^{1}$ A. G. Gaydon and H. G. Wolfhard, Flames, (John Wiley and Sons, New York, 1979).

${ }^{2}$ M. J. McEwan and L. F. Phillips, Chemistry of the Atmosphere, (John Wiley and Sons, New York, 1975).

${ }^{3}$ R. P. Wayne, Chemistry of Atmospheres, (Oxford University Press, New York, 1985).

${ }^{4}$ R. F. Sawyer, Eighteenth Symposium (International) on Combustion, p.1 (The Combustion Institute, Pittsburgh, 1980).

5J. F. Paulson, Adv. Chem. Ser. 58, 28 (1966); J. F. Paulson, J. Chem. Phys. 52, 959 (1970).

6J. L. Moruzzi and J. T. Dakin, J. Chem. Phys. 49, 5000 (1968).

${ }^{7}$ R. A. Morris, A. A. Viggianio, and J. F. Paulson, J. Chem. Phys. 92, 3448 (1990).

${ }^{8}$ S. E. Barlow and V. M. Bierbaum, J. Chem. Phys. 92, 3442 (1990).

${ }^{9}$ G. B. Kistiakowsky and G. G. Volpi, J. Chem. Phys. 27, 1141 (1957).

${ }^{10}$ G. J. Verberke and C. A. Winkler, J. Phys. Chem. 64, 319 (1960).

${ }^{11}$ M. A. A. Clyne and B. A. Thrush, Trans. Faraday. Soc. 57, 69 (1961); M. A. A. Clyne and I. S. McDermid, J. Chem. Soc. Faraday Trans. 1 71, 2189 (1975); M. A. A. Clyne and Y. Ono, Chem. Phys. 69, 381 (1982).

${ }^{12}$ D. Husain and N. K. H. Slater, J. Chem. Soc. Faraday Trans. 76, 606 (1980).

${ }^{13}$ L. F. Phillips and H. I. Schiff, J. Chem. Phys. 42, 3171 (1965).

${ }^{14}$ R. Iwata, R. A. Ferrieri, and A. P. Wolf, J. Phys. Chem. 90, 6722 (1986).

${ }^{15}$ D. E. Milligan and M. E. Jacox, J. Chem. Phys. 55, 3404 (1971).

${ }^{16}$ M. E. Jacox, J. Chem. Phys. 93, 7622 (1990).

${ }^{17}$ J. Hacaloglu, S. Suzer, and L. Andrews, J. Chem. Phys. 94, 1759 (1990).

${ }^{18}$ L. A. Posey and M. A. Johnson, J. Chem. Phys. 88, 5383 (1988).

${ }^{19}$ The numbering sheme is consistent with that of Ref. 18.

${ }^{20} \mathrm{H}$. H. Michaels and J. A. Montgomery, Jr., J. Chem. Phys. 88, 7248 (1988).

${ }^{21}$ W. A. Guillory and C. E. Hunter, J. Chem. Phys. 50, 3516 (1969).

${ }^{22}$ S. G. Kukolich, J. Amer. Chem. Soc. 104, 4715 (1982); J. Mol. Spectr. 98, 80 (1983). 
${ }^{23}$ T.-K. Ha, Theoret. Chim. Acta 58, 125 (1981).

${ }^{24}$ R. D. Harcourt, J. Mol. Struct. 206, 253 (1990).

${ }^{25}$ R. B. Metz, A. Weaver, S. E. Bradforth, T. N. Kitsopoulos, and D. M. Neumark, J. Phys. Chem. 94, 1377 (1990).

${ }^{26}$ M. A. Johnson, M. L. Alexander, and W. C. Lineberger, Chem. Phys. Lett. 112, 285 (1984).

27W. C. Wiley and I. H. McLaren, Rev. Sci. Instrum. 26, 1150 (1955).

${ }^{28}$ Gaussian 92, Revision C, M. J. Frisch, G. W. Trucks, M. Head-Gordon, P. M. W. Gill, M. W. Wong, J. B. Foresman, B. G. Johnson, H. B. Schlegel, M. A. Robb, E. S. Replogle, R. Gomperts, J. L. Andres, K. Raghavachari, J. S. Binkley, C. Gonzalez, R. L. Martin, D. J. Fox, D. J. Defrees, J. Baker, J. J. P. Stewart, and J. A. Pople, Gaussian, Inc., Pittsburgh PA, 1992.

${ }^{29}$ A. Weaver, D. W. Arnold, S. E. Bradforth and D. M. Neumark, J. Chem. Phys. 94, 1740 (1991).

${ }^{30}$ A. D. Walsh, J. Chem. Soc. 2301 (1953).

${ }^{31}$ M. M. Maricq, J. J. Szente, Z. Li, and J. S. Francisco, J. Chem Phys. 98, 784 (1993).

32D. W. Arnold, S. E. Bradforth, E. H. Kim, and D. M. Neumark, J. Chem. Phys. (submitted for publication).

${ }^{33} \mathrm{For}$ the ${ }^{3} \mathrm{~B}_{1}$ state, this value is reported even though the force constant analysis at the MP2 level indicates that it is a saddle point species.

${ }^{34}$ N. G. Moll, D. R. Clutter, and W. E. Thompson, J. Chem. Phys. 45, 4469 (1966).

${ }^{35}$ E. Weissberger, W. H. Breckenridge, and H. Taube, J. Chem. Phys. 47, 1764 (1967).

36M. E. Jacox and D. E. Milligan, J. Chem. Phys. 54, 919 (1971).

37J. A. Pople, U. Seeger, R. Seeger, and P. von R. Schleyer, J. Comput. Chem. 1, 199 (1980).

${ }^{38}$ S. Canuto and G. H. F. Diercksen, Chem. Phys. 120, 173 (1988).

${ }^{39}$ W. J. van DeGuchte, J. P. Zwart and J. J. C. Mulder, J. Mol. Struct. THEOCHEM 152, 213 (1987).

${ }^{40}$ M. A. Castro, S. Canuto, and A. M. Simas, Chem. Phys. Lett. 177, 98 (1991).

${ }^{41}$ R. D. J. Froese and J. D. Goddard, J. Phys. Chem. 97, 7484 (1993).

${ }^{42}$ R. C. Boehm and L. L. Lohr, J. Comput. Chem. 12, 119 (1991).

${ }^{43}$ D. J. Leahy, D. L. Osborn, E. M. Ross, and D. M. Neumark, private communication. 


\title{
Chapter 4: Vibrationally Resolved Spectra Of $C_{2}-C_{11}$ by Anion Photoelectron Spectroscopy ${ }^{\dagger}$
}

\begin{abstract}
Anion photoelectron spectroscopy has been employed to obtain vibrationally resolved spectra of the carbon molecules $C_{2}$ through $C_{11}$. The spectra of $C_{2}^{-}$through $C_{9}^{-}$are dominated by linear anion to linear neutral photodetachment transitions. Linear to linear transitions contribute to the $\mathrm{C}_{11}^{-}$spectrum, as well. From these spectra, vibrational frequencies and electron affinities are determined for the linear isomers of $C_{2}-C_{9}$ and $C_{11}$. The term value is also obtained for the first excited electronic states of linear $C_{4}$. The spectra of $C_{10}^{-}$and $C_{11}^{-}$ show evidence for transitions involving cyclic anions and/or neutrals. Similar types of transitions are identified in the spectra of other smaller molecules, specifically $\mathrm{C}_{6}^{-}, \mathrm{C}_{8}^{-}$, and to a lesser extent $\mathrm{C}_{5}^{-}$.
\end{abstract}

\subsection{Introduction}

For several decades researchers have studied pure carbon molecules, attempting to elucidate their physical properties and the processes governing their formation. Carbon molecules have been identified as intermediates in soot formation, ${ }^{1}$ they exist in the vapor above heated graphite, ${ }^{2}$ and they have been detected in interstellar space, ${ }^{3}$ being produced in giant carbon stars. Recently, the verification of the icosahedral structure of the $\mathrm{C}_{60}$ molecule $\mathrm{e}^{4,5}$ has launched a new investigation of the formation processes of such novel cage structures. An excellent review of the vast amount of research performed on carbonaceous species through April 1989 has been given by Weltner and van Zee. ${ }^{6}$ Given the abundance of research on these molecules, surprisingly little is definitely known about the physical properties of pure carbon

†Published in The Journal of Chemical Physics 95, 8753 (1991) 
molecules containing more than three atoms. Before a model for the formation of the large complexes such as the fullerenes and soot can be fully developed, fundamental information about the building block carbon molecules must be compiled. Determination of the molecular properties of the smaller carbon molecules and how these properties change with molecular size should provide a more complete understanding of these processes.

One of the more controversial issues encountered in carbon molecule research is the determination of the lowest energy molecular geometries. Until recently, this question was addressed mainly by $a b$ initio calculations. Early molecular orbital (MO) calculations by Pitzer and Clementi $^{7}$ and Hoffman ${ }^{8}$ predicted that the carbon molecules would have cumulenic linear structures until reaching the size of $\mathrm{C}_{10}$, at which time the energy stabilization gained by the formation of an additional bond would be larger than the destabilization created by ring strain and it would form a monocyclic ring. Recent experiments by Saykally, Bernath, Amano, and their co-workers ${ }^{3,9,10,11}$ as well as higher levels of theory 12,13 have confirmed this hypothesis for the odd-numbered carbon molecules (up to $\mathrm{C}_{9}$ ), finding linear $\mathrm{D}_{\text {oh }}$ geometries for the ${ }^{1} \Sigma_{g}^{+}$ ground state of each of these species. ${ }^{13,14}$ However, calculations predict that planar monocyclic ${ }^{1} \mathrm{~A}_{\mathrm{g}}$ isomers exist for even-numbered carbon molecules as small as $\mathrm{C}_{4}$, with energies near those of the linear ${ }^{3} \Sigma_{g}^{-}$species. The relative energies of the two forms vary depending upon the level of the calculation, and the energy separations are often less than the error limits of the calculations. Even very high levels of $a b$ initio theory predict that the cyclic forms of these molecules may be energetically more stable than their linear counterparts. ${ }^{15}$ However, calculations considering entropic effects have shown that the high temperatures of natural formation conditions thermodynamically favor linear carbon molecules over their nonlinear counterparts. ${ }^{16}$

Most quantitative experimental information about carbon molecules containing more than three atoms has been obtained during the last five years. Researchers have employed ESR techniques to detect the linear forms of several even-numbered species in low-temperature 
matrices. ${ }^{17}$ Yang et al. ${ }^{18,19}$ used anion photoelectron spectroscopy to obtain electron affinities and electronic structure for carbon molecules with up to 84 carbon atoms. They deduced that they were observing the linear forms of $C_{n}(n=2-9)$ and monocyclic ring forms of $C_{n}(n=10-29)$. Absorption experiments have been performed, both in matrices and in the gas phase. However, conclusive assignment of spectral peaks is often difficult due to the presence of multiple species with varying numbers of carbon atoms. As a result, several bands originally assigned in matrix isolation spectroscopy ${ }^{20}$ have necessarily been reassigned by techniques which are more molecule-specific. Isotope studies in matrices have clearly assigned vibrational frequencies for the linear forms of $\mathrm{C}_{4},{ }^{21} \mathrm{C}_{5},{ }^{22}$ and $\mathrm{C}_{6}{ }^{23}$ High resolution gas-phase spectra have been obtained for linear forms of $\mathrm{C}_{4},{ }^{24} \mathrm{C}_{5},{ }^{10} \mathrm{C}_{7},{ }^{11}$ and $\mathrm{C}_{9},{ }^{14}$ yielding rotational constants and at least one vibrational frequency for each. ${ }^{25}$ While most experimentalists studying carbon molecules with less than ten atoms have detected only the linear forms of the molecules, evidence for nonlinear isomers has been obtained by researchers using the Coulomb Explosion Imaging (CEN) technique, who reported the photodetachment of cyclic forms of $\mathrm{C}_{4}^{-}, \mathrm{C}_{5}^{-}$, and $\mathrm{C}_{6}^{-} \cdot{ }^{26}$

Using an anion photoelectron spectroscopy technique similar to that of Yang et al., ${ }^{18}$ but at considerably higher resolution, we have obtained vibrationally resolved photoelectron spectra of the anions $\mathrm{C}_{2}^{-}$through $\mathrm{C}_{11}^{-}$. The use of an anion precursor allows study of the single mass-selected neutral molecule of interest, circumventing chromophore uncertainties encountered in absorption experiments. The photoelectron experiment also complements infrared absorption experiments by providing vibrational frequencies which are infrared inactive. In addition, since anion photodetachment is a vertical process, the length of the observed vibrational progression provides information about the difference in geometry between the anion and the neutral. The spectra presented here, with the exception of $\mathrm{C}_{10}^{-}$, show evidence for transitions between linear carbon anions and linear carbon neutrals. Electron affinities (EA's) are measured for all the linear carbon molecules. Vibrational frequencies are determined for many of the linear neutral carbon species and an excited electronic state is 
assigned for $\mathrm{C}_{4}$. In addition, the photoelectron spectra of $\mathrm{C}_{10}^{-}$and $\mathrm{C}_{11}^{-}$, and to a lesser extent those of $\mathrm{C}_{5}^{-}, \mathrm{C}_{6}^{-}$and $\mathrm{C}_{8}^{-}$, show contributions from what are believed to be non-linear isomers of the carbon anions. The structures of the non-linear anions cannot be determined from these spectra, but it is apparent that photodetachment of these anions results in a significant geometrical reorganization of the neutral.

\subsection{Experimental}

The apparatus used in these experiments is a modified version of our previously described anion time-of-flight photoelectron spectrometer. ${ }^{27}$ Carbon anions are generated in a Smalley-type laser vaporization/pulsed molecular beam source. ${ }^{28} \mathrm{~A} \mathrm{XeCl}$ excimer laser is focused onto a rotating and translating graphite rod $\left(0.25^{\prime \prime}\right.$ diameter). The resulting plasma is swept through a $1 \mathrm{~cm}$ long, $0.25 \mathrm{~cm}$ diameter channel by helium carrier gas pulsed from a molecular beam valve (General Valve Series 9), operated at a backing pressure of about 5 atmospheres. The gas mixture expands, allowing relaxation of molecular vibrations and rotations by collisions with the carrier gas atoms. The anions generated in the plasma are injected into a Wiley-McLaren-type time-of-flight mass spectrometer ${ }^{29}$ with a pulsed electric field. After acceleration to an energy of $1 \mathrm{keV}$, the ions separate out by mass and are detected by a microchannel plate detector. The mass resolution of the instrument, $M / \Delta M$, is approximately 150 . The ion of interest is selectively detached by a properly timed pulse of light from a pulsed Nd:YAG photodetachment laser. After photodetachment, a dual microchannel plate detector at the end of a one meter field-free flight tube detects a small fraction $(\approx 0.01 \%)$ of the detached electrons. Time-of-flight analysis yields electron kinetic

energies (eKE); the instrumental resolution is $8 \mathrm{meV}$ at $0.65 \mathrm{eV}$ and degrades as $(\mathrm{eKE})^{3 / 2}$ at higher electron kinetic energies.

The experiments described below were performed with the third and fourth harmonic frequencies (355 nm, $3.49 \mathrm{eV}$ and $266 \mathrm{~nm}, 4.66 \mathrm{eV}$, respectively) of a Nd:YAG laser. The 
plane-polarized laser beam can be rotated using a half-wave plate. In the spectra shown, unless otherwise specified, the laser beam is polarized at $\theta=54.7^{\circ}$ (magic angle) ${ }^{30}$ with respect to the direction of electron collection. The spectra presented here are averaged for $100,000-500,000$ laser shots at $20 \mathrm{~Hz}$ repetition rate, and smoothed by convolution with a $5 \mathrm{meV}$ FWHM Gaussian. In order to account for the small background electron signal which results from scattered light interacting with the photodetachment chamber surfaces in the $4.66 \mathrm{eV}$ spectra, a background spectrum is collected, smoothed and subtracted from the data.

\subsection{Results}

The photoelectron spectra of the odd-numbered carbon anions, $C_{2 n+1}^{-}(n=1-4)$, obtained using a photon energy of $4.66 \mathrm{eV}$, are presented in Fig. 4.1. In these and all other photoelectron spectra, the electron kinetic energy (eKE) is related to the internal energy of the neutral molecule by the expression:

$$
\mathrm{eKE}=h v-E A-\mathrm{T}_{\mathrm{o}}+\mathrm{T}_{\mathrm{o}}^{-}-\mathrm{E}_{\mathrm{v}}^{\circ}+\mathrm{E}_{\mathrm{v}}^{-}
$$

Here, $h v$ is the laser photon energy, EA is the electron affinity of the neutral species, $\mathrm{T}_{0}^{0}$ and $\mathrm{T}_{0}^{-}$are the term values of the specific neutral and anion electronic states, respectively. $\mathrm{E}_{\mathrm{v}}^{0}$ and $E_{v}^{-}$are the vibrational energies (above the zero point energy) of the neutral and anion, respectively. Rotational contributions to molecular internal energy are neglected. As indicated by Equation (1), the peaks occurring at lowest eKE in the photoelectron spectrum correspond to the highest internal energy states of the neutral.

As $\mathrm{n}$ increases for the $\mathrm{C}_{2 \mathrm{n}+1}$ molecules, the spectral features shift to lower eKE, indicating an increase in electron affinity. In the $\mathrm{C}_{3}^{-}$spectrum, there are poorly resolved

Figure 4.1: Photoelectron spectra of $\mathrm{C}_{3}^{-}, \mathrm{C}_{5}^{-}, \mathrm{C}_{7}^{-}$and $\mathrm{C}_{9}^{-}$at $266 \mathrm{~nm}$. Arrows indicate electron affinity of linear carbon chain. 


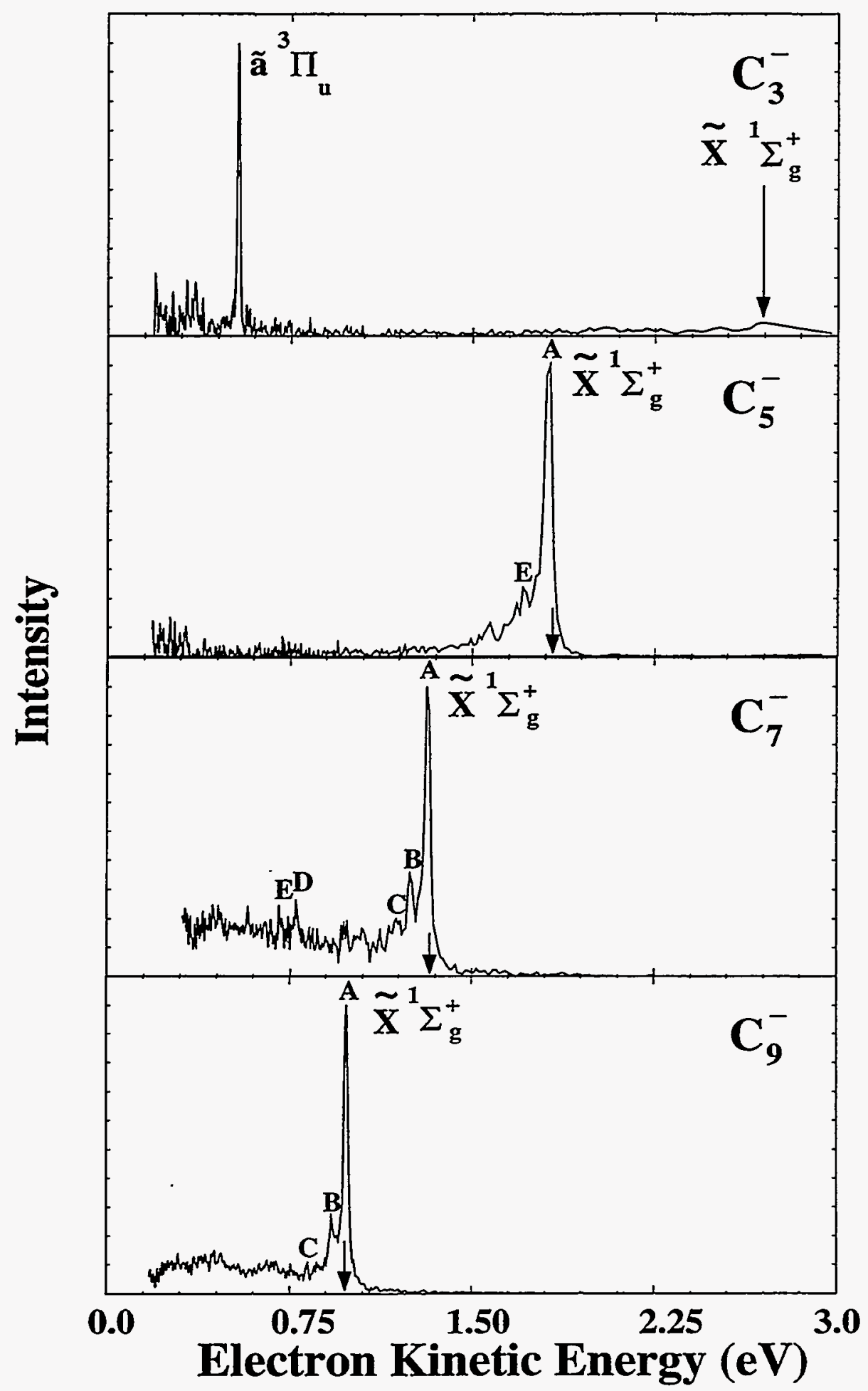

Figure 4.1 
features at high eKE which correspond to the ground state of $\mathrm{C}_{3}$. However, there is a single well-resolved peak at low $\mathrm{eKE}$ which corresponds to an excited electronic state of $\mathrm{C}_{3}$. In each of the spectra of $\mathrm{C}_{5}^{-}, \mathrm{C}_{7}^{-}$, and $\mathrm{C}_{9}^{-}$, there is a short, congested progression extending to lower eKE.

Since our experimental resolution degrades at higher eKE, experiments using a lower photon energy $(3.49 \mathrm{eV})$ yield better resolved spectra of the ground state progressions of $\mathrm{C}_{3}$ and $\mathrm{C}_{5}$, (Figs. 4.2 and 4.3). The $\mathrm{C}_{3}^{-}$spectrum contains two peaks which have multiple shoulders. The peaks and shoulders are indicated with letters and arrows, respectively. The higher resolution spectrum of $\mathrm{C}_{5}^{-}$contains many small peaks in addition to the large peak $\mathrm{A}$, indicating that vibrational excitation of $C_{5}$ occurs upon photodetachment of the $C_{5}^{-}$anion. Peak positions and assignments for the $\mathrm{C}_{5}^{-}, \mathrm{C}_{7}^{-}$, and $\mathrm{C}_{9}^{-}$spectra, discussed in more detail below, are summarized in Table I.

The photoelectron spectra obtained at $4.66 \mathrm{eV}$ for the even carbon anions, $C_{2 n}^{-}(n=1-4)$, are shown in Fig. 4.4. As was the case for the $C_{2 n+1}^{-}$spectra, the $C_{2 n}^{-}$spectra strongly resemble each other, but the electron affinities of the even numbered species are higher than their neighboring $\mathrm{C}_{2 \mathrm{n}+1}$ counterparts. In the $\mathrm{C}_{2}^{-}$and $\mathrm{C}_{4}^{-}$spectra, there are several peaks extending over a larger energy range than the progressions of the $C_{2 n+1}^{-}$spectra, with a somewhat irregular intensity pattern. Only limited portions of the $\mathrm{C}_{6}^{-}$and $\mathrm{C}_{8}^{-}$spectra are obtainable with the 4.66

Figure 4.2: Photoelectron spectrum of $\mathrm{C}_{3}^{-}$at $355 \mathrm{~nm}$ (top); Simulations of $\mathrm{C}_{3}^{-}$photoelectron spectrum at $355 \mathrm{~nm}$ using a separable harmonic oscillator approximation and $a\left(v_{2} \times v\right)$ cross-section of MORBID potential energy surface. Both simulations are performed at $0.002 \mathrm{eV}$ resolution to illustrate effect of anharmonicity upon $v_{2}$ bend mode progression intensities.

Figure 4.3: Photoelectron spectrum of $\mathrm{C}_{5}^{-}$at $355 \mathrm{~nm}$. The inset shows an expanded spectrum including labels discussed in text.

Figure 4.4: Photoelectron spectra of $\mathrm{C}_{2}^{-}, \mathrm{C}_{4}^{-}, \mathrm{C}_{6}^{-}$and $\mathrm{C}_{8}^{-}$at $266 \mathrm{~nm}$. Arrows indicate electron affinity of linear carbon chain. 


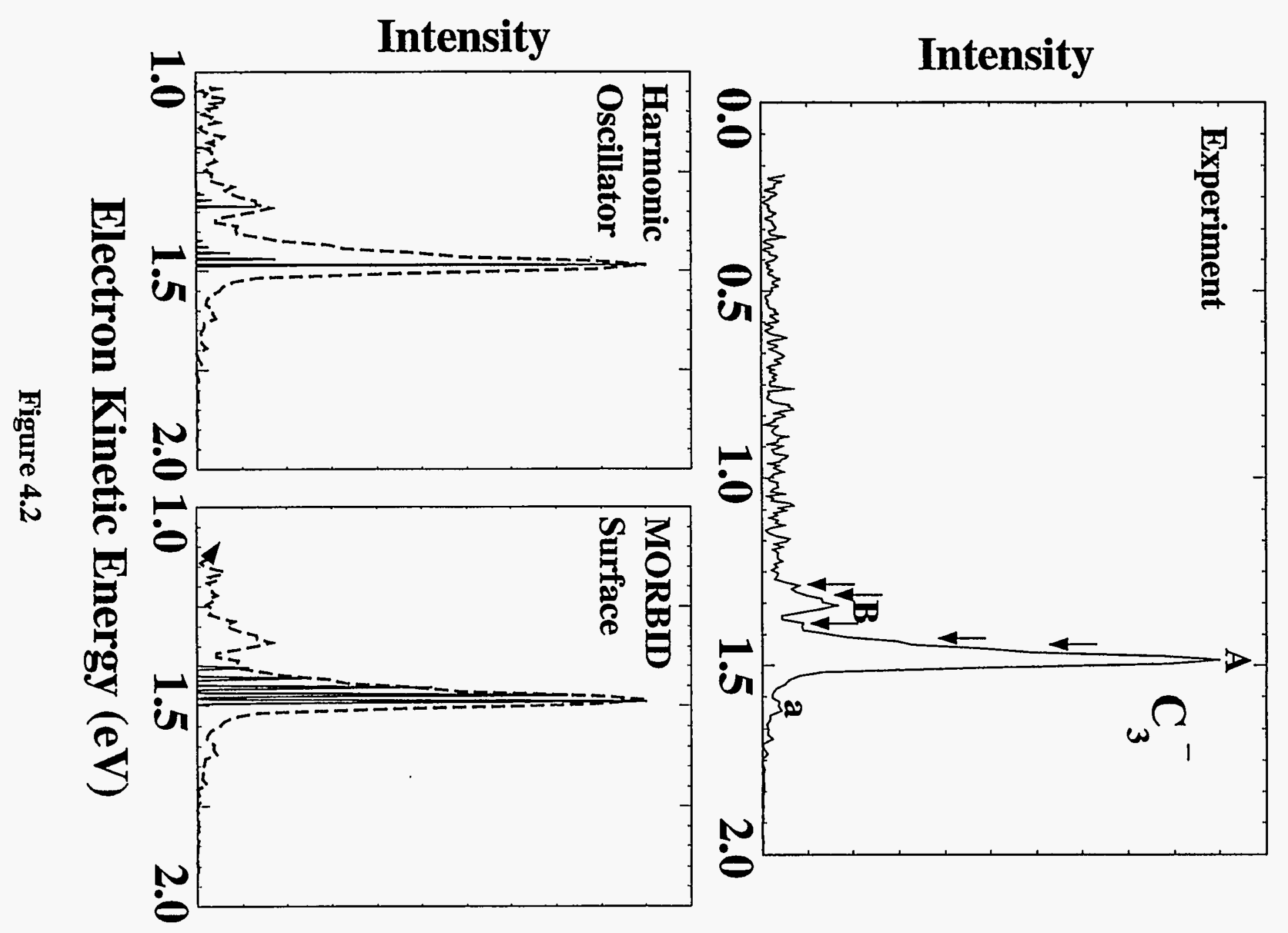




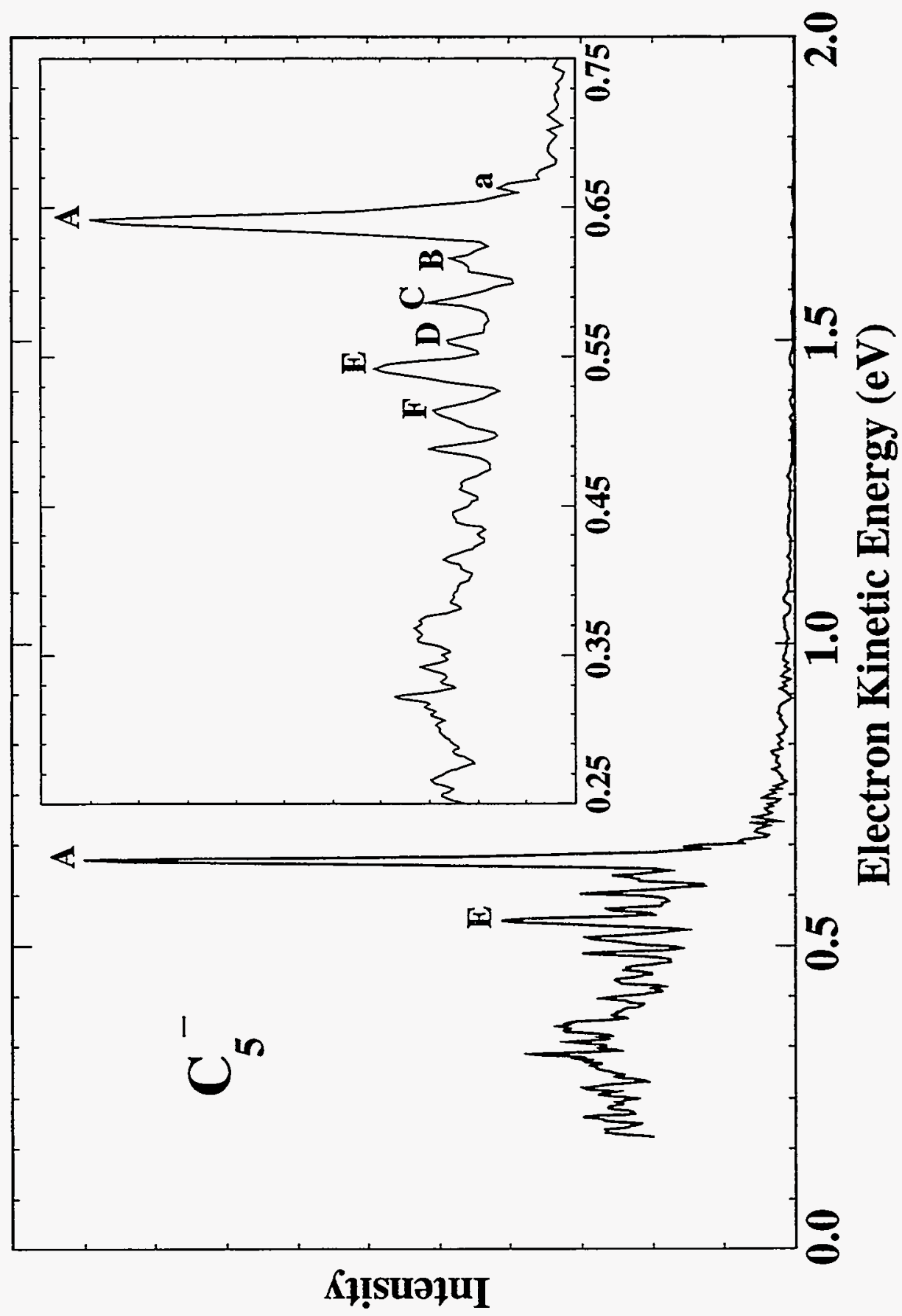

Figure 4.3 


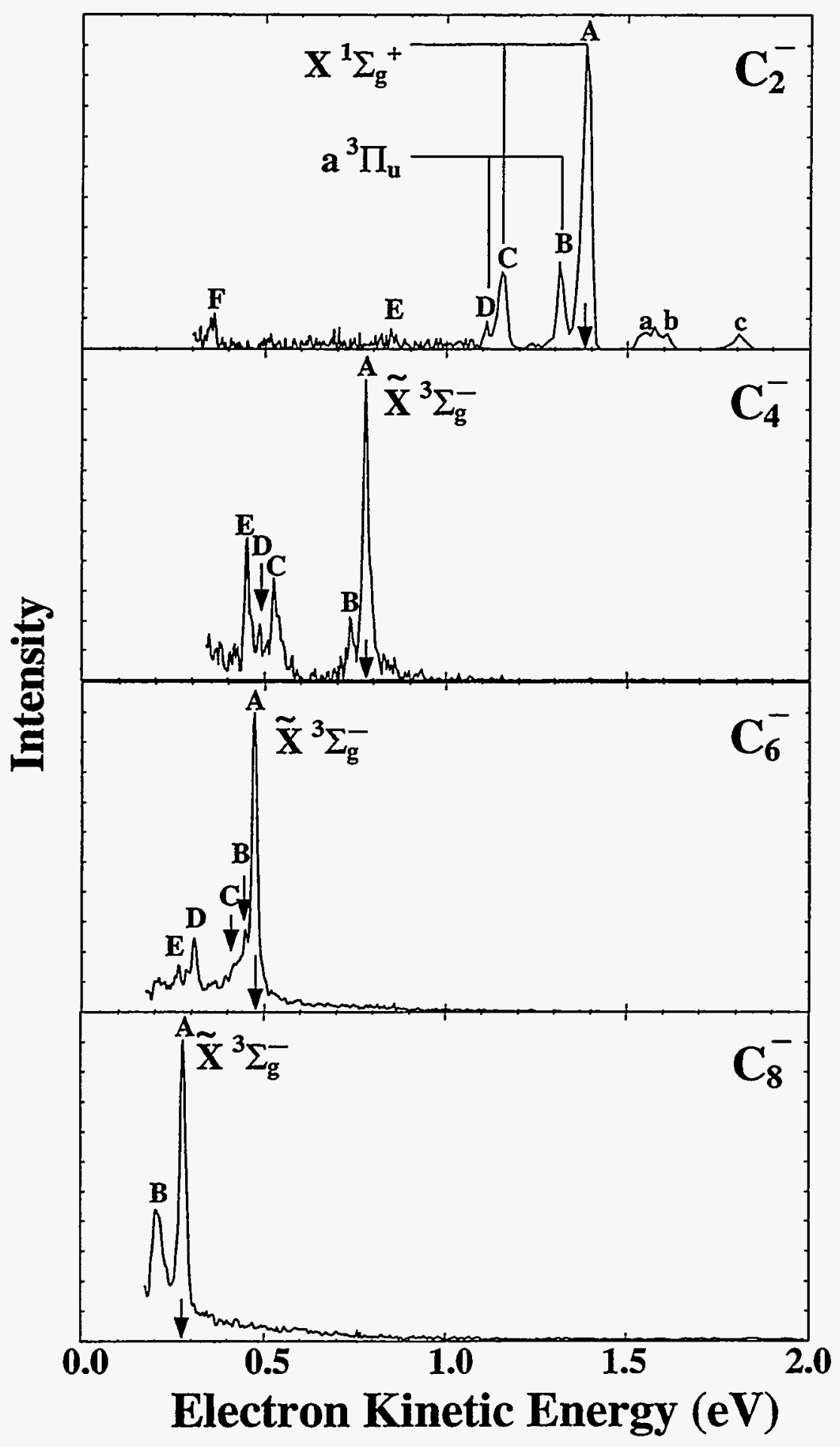

Figure 4.4 
Table I: Peak positions and assignments for the $\mathrm{C}_{5}^{-}, \mathrm{C}_{7}^{-}$, and $\mathrm{C}_{9}^{-}$photoelectron spectra.

\begin{tabular}{c|c|c|c|c}
\hline \hline Molecule & Peak & Position & $\begin{array}{c}\text { Splitting from } \\
\text { Origin }\left(\mathrm{cm}^{-1}\right)\end{array}$ & Assignment \\
\hline \hline \multirow{6}{*}{$\mathrm{C}_{5}{ }^{\mathrm{a}}$} & $\mathrm{A}$ & 0.641 & 0 & Origin \\
\cline { 2 - 5 } & $\mathrm{B}$ & 0.616 & 202 & $7_{0}^{2}$ \\
\cline { 2 - 5 } & $\mathrm{C}$ & 0.586 & 444 & $5_{0}^{2}$ \\
\cline { 2 - 5 } & $\mathrm{D}$ & 0.560 & 653 & $5_{0}^{2} 7_{0}^{2}$ \\
\cline { 2 - 5 } & $\mathrm{E}$ & 0.542 & 798 & $2_{0}^{1}$ \\
\cline { 2 - 5 } & $\mathrm{F}$ & 0.514 & 1024 & $6_{0}^{2}$ \\
\hline
\end{tabular}

a) The $v_{2}$ mode is a symmetric stretch and the $v_{5}, v_{6}$ and $v_{7}$ modes are bending modes, see Fig $8 b$.

\begin{tabular}{c|c|c|c|c}
\hline $\mathrm{C}^{\mathrm{b}}$ & $\mathrm{A}$ & 1.302 & 0 & Origin \\
\cline { 2 - 5 } & $\mathrm{B}$ & 1.234 & 548 & $3_{0}^{1}$ \\
\hline $\mathrm{C}$ & 1.179 & 992 & $7_{0}^{2}$ \\
\hline & $\mathrm{D}$ & 0.773 & 4267 & $4_{0}^{2}$ \\
\hline
\end{tabular}

b) The $v_{3}$ mode is a symmetric stretch, $v_{4}$ is an antisymmetric stretch and $v_{7}$ is a bending mode.

\begin{tabular}{l|c|c|c|c}
\hline $\mathrm{C}^{\mathrm{c}}$ & $\mathrm{A}$ & 0.978 & 0 & Origin \\
\cline { 2 - 5 } & $\mathrm{B}$ & 0.918 & 484 & $4_{0}^{1}$ \\
\cline { 2 - 5 } & $\mathrm{C}$ & 0.822 & 1258 & $3_{0}^{\mathrm{l}}$ \\
\hline
\end{tabular}

c) The $v_{3}$ and $v_{4}$ modes are symmetric stretches. 
$\mathrm{eV}$ photon energy due to the high EA's of $\mathrm{C}_{6}$ and $\mathrm{C}_{8}$. The peak positions and assignments for the $\mathrm{C}_{2}^{-}$spectrum are in Table II, and those for the $\mathrm{C}_{4}^{-}$and $\mathrm{C}_{6}^{-}$spectra are listed Table III.

Table II: Peak positions and assignments for the $\mathrm{C}_{2}^{-}$photoelectron spectrum.

\begin{tabular}{c|c|c}
\hline \hline Peak & Position & Assignment ${ }^{\mathrm{a}}\left(\mathrm{C}_{2} \leftarrow \mathrm{C}_{2}^{-}\right)$ \\
\hline \hline $\mathrm{A}$ & 1.384 & $\mathrm{X}^{1} \Sigma_{g}^{+}\left(\mathrm{v}^{\prime}=0\right) \leftarrow \mathrm{X}^{2} \Sigma_{g}^{+}\left(\mathrm{v}^{\prime \prime}=0\right)$ \\
\hline $\mathrm{B}$ & 1.310 & $\mathrm{a}^{3} \Pi_{\mathrm{u}}\left(\mathrm{v}^{\prime}=0\right) \leftarrow \mathrm{X}^{2} \Sigma_{g}^{+}\left(\mathrm{v}^{\prime \prime}=0\right)$ \\
\hline $\mathrm{C}$ & 1.153 & $\mathrm{X}^{1} \Sigma_{g}^{+}\left(\mathrm{v}^{\prime}=1\right) \leftarrow \mathrm{X}^{2} \Sigma_{g}^{+}\left(\mathrm{v}^{\prime \prime}=0\right)$ \\
\hline $\mathrm{D}$ & 1.111 & $\mathrm{a}^{3} \Pi_{\mathrm{u}}\left(\mathrm{v}^{\prime}=1\right) \leftarrow \mathrm{X}^{2} \Sigma_{g}^{+}\left(\mathrm{v}^{\prime \prime}=0\right)$ \\
\hline $\mathrm{E}$ & 0.849 & $\mathrm{~A}{ }^{1} \Pi_{\mathrm{u}}\left(\mathrm{v}^{\prime}=0\right) \leftarrow \mathrm{A}^{2} \Pi_{\mathrm{u}}\left(\mathrm{v}^{\prime \prime}=0\right)$ \\
\hline $\mathrm{F}$ & 0.362 & $\mathrm{~A}{ }^{1} \Pi_{\mathrm{u}}\left(\mathrm{v}^{\prime}=0\right) \leftarrow \mathrm{X}^{2} \Sigma_{g}^{+}\left(\mathrm{v}^{\prime \prime}=0\right)$ \\
\hline $\mathrm{a}$ & 1.542 & $\mathrm{a}^{3} \Pi_{\mathrm{u}}\left(\mathrm{v}^{\prime}=0\right) \leftarrow \mathrm{X}^{2} \Sigma_{g}^{+}\left(\mathrm{v}^{\prime \prime}=1\right)$ \\
\hline $\mathrm{b}$ & 1.609 & $\mathrm{X}^{1} \Sigma_{g}^{+}\left(\mathrm{v}^{\prime}=0\right) \leftarrow \mathrm{X}^{2} \Sigma_{g}^{+}\left(\mathrm{v}^{\prime \prime}=1\right)$ \\
\hline $\mathrm{c}$ & 1.804 & $\mathrm{a}^{3} \Pi_{\mathrm{u}}\left(\mathrm{v}^{\prime}=0\right) \leftarrow \mathrm{A}^{2} \Pi_{\mathrm{u}}\left(\mathrm{v}^{\prime \prime}=0\right)$ \\
\hline \hline
\end{tabular}

a) For each peak assigned, there are also underlying sequence bands which are unresolved in the spectrum.

Photoelectron spectra of $C_{10}^{-}$and $C_{11}^{-}$, taken with a $4.66 \mathrm{eV}$ photodetachment energy, are displayed in Fig. 4.5. These two spectra have a significantly different appearance than the spectra of $C_{2}^{-}$through $C_{9}^{-}$. The $C_{10}^{-}$spectrum, Fig. 4.5, contains broad unresolved band structure possibly indicative of transitions involving multiple electronic states. However, in the $\mathrm{C}_{11}^{-}$spectrum, there are resolved peaks superimposed upon a broad spectrum which resembles the $\mathrm{C}_{10}^{-}$spectrum. A long low-intensity tail extends to the high eKE regions of both spectra. These long tails also appear in the $\mathrm{C}_{6}^{-}$and $\mathrm{C}_{8}^{-}$spectra and with significantly less intensity in the $C_{5}^{-}$spectrum. This is discussed further in Section 4.4.4.

Data collected for $\mathrm{C}_{4}^{-}$at two different laser polarizations are shown in Fig. 4.6. The important difference between the two spectra is the variation of the relative intensities of peaks 
Table III: Peak positions and assignments for the $\mathrm{C}_{4}^{-}$and $\mathrm{C}_{6}^{-}$photoelectron spectra.

\begin{tabular}{|c|c|c|c|c|}
\hline Molecule & Peak & Position & $\begin{array}{l}\text { Splitting from } \\
\text { Origin }\left(\mathrm{cm}^{-1}\right)\end{array}$ & Assignment \\
\hline \multirow[t]{5}{*}{$\mathrm{C}_{4}^{\mathrm{a}}$} & A & 0.778 & 0 & Origin \\
\hline & B & 0.736 & 339 & $4_{0}^{1}$ \\
\hline & $\mathrm{C}$ & 0.526 & 2032 & $1_{0}^{1}$ \\
\hline & $\mathrm{D}$ & 0.487 & 2347 & $1_{0}^{1} 4_{0}^{1}$ \\
\hline & $E$ & 0.451 & 2637 & ${ }^{1} \Delta_{g}^{b}$ \\
\hline
\end{tabular}

a) The $v_{1}$ mode is a symmetric stretch and $v_{4}$ is a bend mode, see Figure $4.8 \mathrm{a}$.

b) Peak $E$ is assigned to an excited electronic state, see text.

\begin{tabular}{c|c|c|c|c}
\hline $\mathrm{C}_{6}{ }^{\mathrm{c}}$ & $\mathrm{A}$ & 0.475 & 0 & Origin \\
\cline { 2 - 5 } & $\mathrm{B}$ & 0.451 & 194 & Sequenceband \\
\hline $\mathrm{C}$ & 0.369 & 855 & $3_{0}^{1}$ or $7_{0}^{2}$ \\
\hline $\mathrm{D}$ & 0.312 & 1315 & ${ }^{1} \Delta_{\mathrm{g}}{ }^{\mathrm{d}}$ \\
\hline $\mathrm{E}$ & 0.269 & 1662 & Sequence band \\
\hline
\end{tabular}

c) Both $v_{8}$ and $v_{9}$ are bend modes while $v_{2}$ is a symmetric stretch.

Figure 4.5: Photoelectron spectra of $\mathrm{C}_{10}^{-}$and $\mathrm{C}_{11}^{-}$at $266 \mathrm{~nm}$. Arrows indicate estimated electron affinity for monocyclic isomer.

Figure 4.6: Photoelectron spectra of $\mathrm{C}_{4}^{-}$at $266 \mathrm{~nm}$ showing laser polarization dependence of peaks $B$ and $D$. Laser polarization angles are $\theta=55^{\circ}$ and $\theta=90^{\circ}$ with respect to direction of electron collection. 


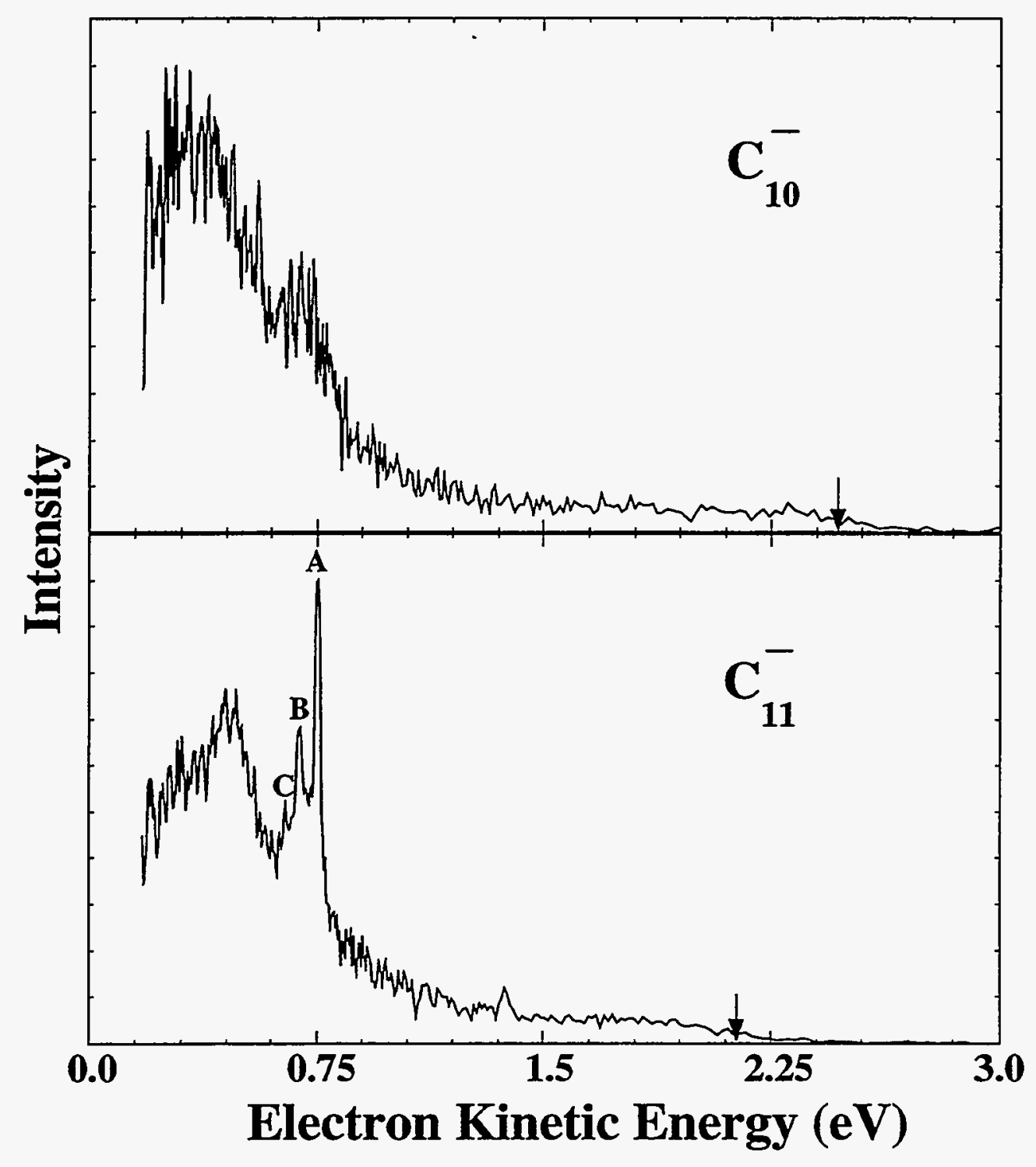

Figure 4.5 


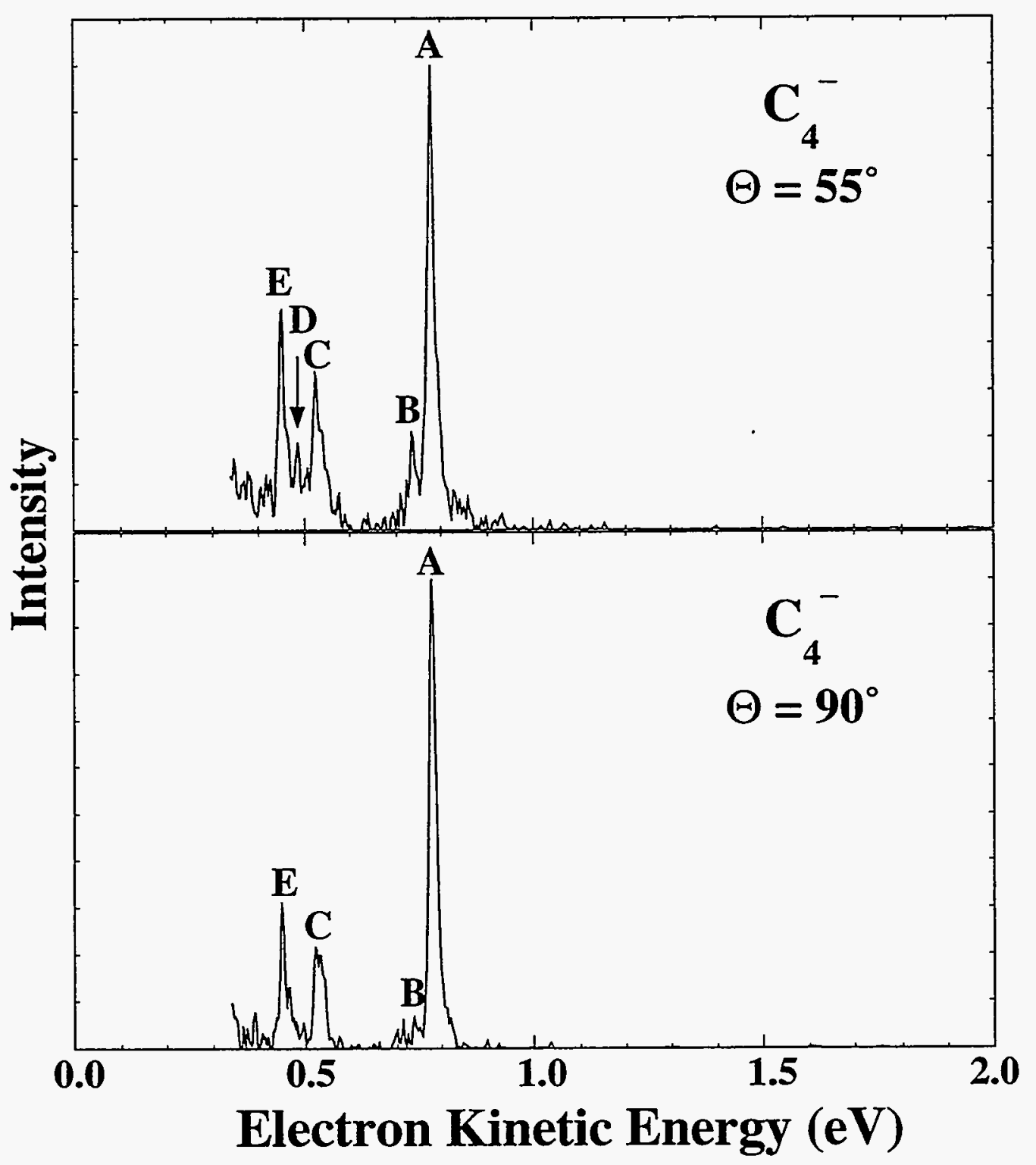

Figure 4.6 
$\mathrm{B}$ and $\mathrm{D}$ as a function of laser polarization angle; these peaks are essentially absent in the $\theta=$ $90^{\circ}$ spectrum. This behavior will be addressed in greater detail below.

\subsection{Analysis and Discussion}

In this section, the $C_{2}^{-}$and $C_{3}^{-}$photoelectron spectra are analyzed in considerable detail and used to lay the framework for the discussion of the larger carbon molecules. The spectroscopy of $C_{2}$ (Ref. 31 and 32) and $C_{3}$ (Ref. 9 and 33) has been studied intensely for several years using a variety of techniques, including anion photoelectron spectroscopy. ${ }^{18,34,35}$ Therefore, the discussion of these systems will be limited to the new information provided by the photoelectron spectra presented here.

The spectra of the larger molecules are treated more qualitatively. The analyses for all the spectra presented here are done within the Franck-Condon approximation. The transition intensity, $I$, for the process,

$$
\mathrm{C}_{n}^{-}\left(\mathrm{v}^{\prime \prime}\right) \stackrel{\mathrm{w} y}{\longrightarrow} \mathrm{C}_{\mathrm{n}}\left(\mathrm{v}^{\prime}\right)+\mathrm{e}^{-}
$$

is governed by the expression,

$$
I \propto\left|\tau_{e}\right|^{2}\left|\left\langle\Psi_{v^{\prime \prime}} \mid \Psi_{v^{\prime}}\right\rangle\right|^{2}
$$

Here $\tau_{\mathrm{e}}$ is the electronic transition dipole moment and the Franck-Condon factor, $\left|\left\langle\Psi_{\mathrm{v}^{\prime}} \mid \Psi_{\mathrm{v}^{\prime}}\right\rangle\right|^{2}$, depends upon the spatial overlap of the vibrational wavefunctions of the anion and the neutral. ${ }^{36}$ In this approximation, it is assumed that $\tau_{\mathrm{e}}$ does not change significantly over the spatial range covered by the nuclear wavefunction and is treated as a constant in the spectral simulations. 


\subsection{1 $\mathrm{C}_{2}$}

Diatomic carbon, $\mathrm{C}_{2}$, has been thoroughly investigated using both absorption and emission spectroscopy. ${ }^{37} \mathrm{C}_{2}^{-}$, one of the few anions known to possess bound excited electronic states, ${ }^{38}$ has also been well characterized. ${ }^{39}$ Recently, Ervin and Lineberger have obtained a vibrationally resolved photoelectron spectrum of the $C_{2}^{-}$anion. 35 The use of a higher photodetachment energy $(4.66 \mathrm{eV})$ in the experiments described here reveals transitions to excited vibrational levels and an excited electronic state of $\mathrm{C}_{2}$ which could not be seen by Ervin and Lineberger.

Shown in Fig. 4.7 is the $\mathrm{C}_{2}^{-}$photoelectron spectrum obtained at $4.66 \mathrm{eV}$ photodetachment energy. The spectrum has been simulated (Fig. 4.7, bottom) using molecular constants obtained from high resolution data ${ }^{32}$ by varying the vibrational temperature, electron affinity and relative intensities of different electronic transitions. Both $C_{2}$ and $C_{2}^{-}$have lowlying excited electronic states. As a result, several photodetachment transitions are energetically accessible using the $4.66 \mathrm{eV}$ detachment photon energy. Some peak assignments are indicated in Fig. 7 and details are given in Table II. One-electron photodetachment of $\mathrm{C}_{2}^{-} \mathrm{X}$ ${ }^{2} \Sigma_{g}^{+}\left(\ldots 2 \sigma_{u}^{2} 1 \pi_{u}^{4} 3 \sigma_{g}^{1}\right)$ can produce the $C_{2} X^{1} \Sigma_{g}^{+}\left(\ldots 2 \sigma_{u}^{2} 1 \pi_{u}^{4}\right), a^{3} \Pi_{u}$ and $A^{1} \Pi_{u}$ states $\left(\ldots 2 \sigma_{u}^{2} 1 \pi_{u}^{3} 3 \sigma_{g}^{1}\right)$ for the latter excited states, $T_{e}=0.089 \mathrm{eV}$ and $1.040 \mathrm{eV}$, respectively). ${ }^{32}$ Peaks A and B in Fig. 4.7 are the origins of the $C_{2} X{ }^{1} \Sigma_{g}^{+} \leftarrow C_{2}^{-} X^{2} \Sigma_{g}^{+}$and $C_{2} a^{3} \Pi_{u} \leftarrow C_{2}^{-} X^{2} \Sigma_{g}^{+}$transitions, respectively. The $C_{2} X^{1} \Sigma_{g}^{+}\left(v^{\prime}=1\right) \leftarrow C_{2}^{-} X^{2} \Sigma_{g}^{+}\left(v^{\prime \prime}=0\right)$ and $C_{2}$ a ${ }^{3} \Pi_{u}\left(v^{\prime}=1\right) \leftarrow C_{2}^{-} X^{2} \Sigma_{g}^{+}$ $\left(v^{\prime \prime}=0\right)$ transitions, which were near the cutoff region for the energy analyzer of the Lineberger experiment, ${ }^{35}$ are clearly defined in Fig. 4.7 (peaks $C$ and D, respectively). At even lower eKE, peak $F$ represents the origin of the $C_{2} A^{1} \Pi_{u} \leftarrow C_{2}^{-} X^{2} \Sigma_{g}^{+}$transition. From peak $A$, we obtain a

Figure 4.7: Photoelectron spectrum of $\mathrm{C}_{2}^{-}$at $266 \mathrm{~nm}$ and best-fit Franck-Condon simulation using EA, temperature and relative electronic dipole transition moments as variables. 


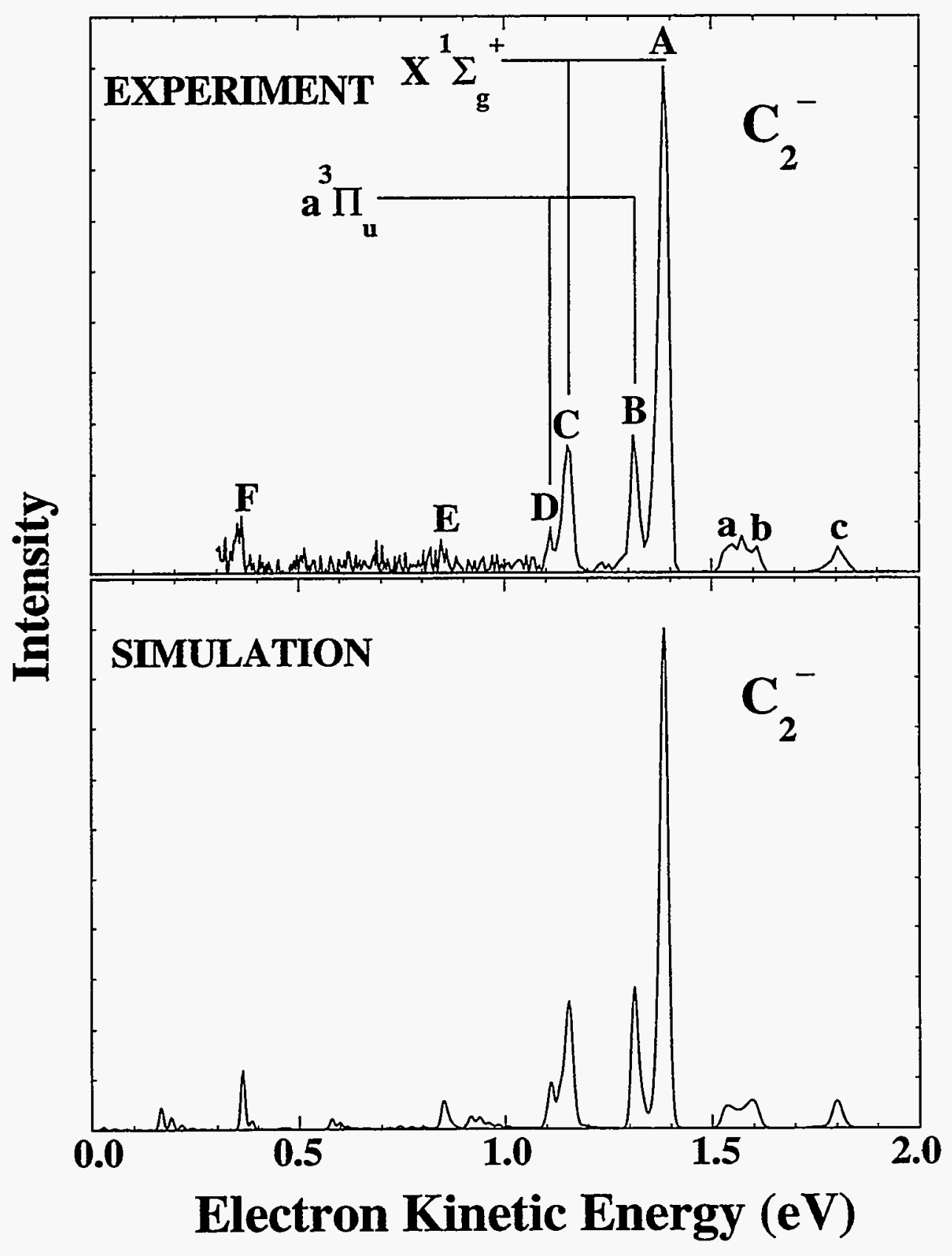

Figure 4.7 
value of $3.273 \pm 0.008 \mathrm{eV}$ for $C_{2}$. This agrees well with the value of $3.269 \pm 0.006 \mathrm{eV}$ recently measured by Ervin and Lineberger. 35,40

It is apparent in the $\mathrm{C}_{2}^{-}$spectrum that peaks corresponding to $\pi$-electron photodetachment from the $\mathrm{C}_{2}^{-} \mathrm{X}^{2} \Sigma_{\mathrm{g}}^{+}$state (peaks $\mathrm{B}, \mathrm{D}$ and $\mathrm{F}$ ) are consistently less intense than the those representing $\sigma$-electron photodetachment from the same electronic state of $\mathrm{C}_{2}^{-}$(peaks $\mathrm{A}$ and $\mathrm{C})$. This pattern suggests that the photodetachment cross-section for removal of a $3 \sigma$ electron from this state is higher than that for removal of a $1 \pi$ electron from the $\mathrm{C}_{2}^{-} \mathrm{X}^{2} \Sigma_{\mathrm{g}}^{+}$ state.

As in Lineberger's spectrum, the $C_{2} X{ }^{1} \Sigma_{g}^{+}\left(v^{\prime}=0\right) \leftarrow C_{2}^{-} X^{2} \Sigma_{g}^{+}\left(v^{\prime \prime}=1\right)$ and $C_{2}$ a ${ }^{3} \Pi_{u}$ $\left(v^{\prime}=0\right) \leftarrow C_{2}^{-} X^{2} \Sigma_{g}^{+}\left(v^{\prime \prime}=1\right)$ 'hot' band transitions occur (labelled as a and b, respectively). While the vibrational distribution of the ions produced in our laser vaporization source could be controlled to a considerable extent for the other carbon anions, $\mathrm{C}_{2}^{-}$could only be generated under conditions that produced anions with considerable vibrational excitation. The best fit to the spectrum was obtained assuming a vibrational temperature of $3000 \mathrm{~K}$. In addition to the 'hot bands', the anion vibrational excitation gives rise to many sequence bands under the $v^{\prime}=0$ and the $v^{\prime}=1$ peaks of the progressions in the $X^{1} \Sigma_{g}^{+}$and the a ${ }^{3} \Pi_{u}$ states of $C_{2}$.

The spectrum also shows photodetachment transitions from the low-lying $C_{2}^{-} A^{2} \Pi_{u}$ first excited state. Signal resulting from the photodetachment of this anion state is observed because its lifetime $\left(\tau_{\mathrm{rad}} \approx 50 \mu \mathrm{s}\right)^{41}$ is comparable to the amount of time between anion formation and photodetachment. One-electron transitions can occur from $\mathrm{C}_{2}^{-} \mathrm{A}$ ${ }^{2} \Pi_{u}\left(\ldots 2 \sigma_{u}^{2} 1 \pi_{u}^{3} 3 \sigma_{u}^{2}, \mathrm{~T}_{\mathrm{e}}=0.494 \mathrm{eV}\right)^{39}$ to $\mathrm{C}_{2}$ in the ${ }^{3} \Pi_{\mathrm{u}}, \mathrm{b}{ }^{3} \Sigma_{\mathrm{g}}^{-}\left(\ldots 2 \sigma_{u}^{2} 1 \pi_{u}^{2} 3 \sigma_{u}^{2}, \mathrm{~T}_{\mathrm{e}}=0.798 \mathrm{eV}\right)^{31}$ and $A^{1} \Pi_{u}$ excited states. Peak $c$ in the spectrum is assigned to the origin of the $C_{2}$ a ${ }^{3} \Pi_{u} \leftarrow$ $\mathrm{C}_{2}^{-} \mathrm{A}^{2} \Pi_{\mathrm{u}}$ transition. Label $\mathrm{E}$ in the spectrum represents the energy at which signal is expected for the $C_{2} \quad A{ }^{1} \Pi_{u} \leftarrow C_{2}^{-}$A ${ }^{2} \Pi_{u}$ transition; a very small peak may be present there in the experimental spectrum. Electrons resulting from the $\mathrm{C}_{2} \mathrm{~b}^{3} \Sigma_{\mathrm{g}}^{-} \leftarrow \mathrm{C}_{2}^{-} \mathrm{A}{ }^{2} \Pi_{\mathrm{u}}$ transition are 
expected to appear at $\mathrm{eKE} \approx 1.1 \mathrm{eV}$, close to peak $\mathrm{D}$. However, there does not appear to be a significant contribution to the spectrum from this transition.

\subsection{2 $\mathrm{C}_{3}$}

$\mathrm{C}_{3}$ has been shown to be a linear but floppy molecule. The $\mathrm{C}_{3}^{-}$anion is predicted to be linear ${ }^{42}$ with a ${ }^{2} \Pi_{\mathrm{g}}\left(\ldots 4 \sigma_{g}^{2} 3 \sigma_{u}^{2} 1 \pi_{u}^{4} 1 \pi_{g}^{1}\right)$ ground state which is considerably more rigid than $\mathrm{C}_{3}$ due to the partly filled $\pi_{\mathrm{g}}$ orbital. The $4.66 \mathrm{eV}$ photoelectron spectrum of $\mathrm{C}_{3}^{-}$, Fig. 4.1 , shows transitions to the two electronic states of $C_{3}$ which are energetically accessible via one-electron photodetachment of $\mathrm{C}_{3}^{-}$. While photodetachment of the $1 \pi$-electron leaves $\mathrm{C}_{3}$ in its $\bar{X}^{1} \Sigma_{g}^{+}$ ground state $\left(. . .4 \sigma_{g}^{2} 3 \sigma_{u}^{2} 1 \pi_{u}^{4}\right)$, removal of the $3 \sigma_{u}$-electron produces the $C_{3} \tilde{a}^{3} \Pi_{u}$ first excited state $\left(. .4 \sigma_{g}^{2} 3 \sigma_{u}^{1} 1 \pi_{u}^{4} 1 \pi_{g}^{1}\right)$. The spectrum of $C_{3}^{-}$obtained with a photodetachment energy of 3.49 $\mathrm{eV}$ (Fig. 4.2) reveals vibrational details of the $\mathrm{C}_{3} \tilde{X}^{1} \Sigma_{g}^{+}$ground state.

Understanding the features of this spectrum requires consideration of the FranckCondon principle for molecules with more than one vibrational mode. For an anion and neutral belonging to the same symmetry point group, transitions can occur from the anion ground state to any quantum state of a totally symmetric vibrational mode (e.g., $v_{1}$ for $C_{3}$ ). Excitation of these modes occurs primarily when there is a difference in bond lengths between the anion and neutral. Typically, excitation occurs in those vibrational modes which most strongly resemble the change in geometry upon anion photodetachment. For non-totally symmetric vibrational modes (e.g., $v_{2}$ and $v_{3}$ for $C_{3}$ ), symmetry forbids transitions from the anion ground state to odd quanta of excitation in the neutral. From the anion ground state, only transitions to even quanta of these neutral vibrational modes will be observed, and transitions to states with $v>0$ only occur when there is a large difference in vibrational frequency between the anion and the neutral. For example, little excitation is expected in the antisymmetric stretch of $C_{3}$ since the $v_{3}$ frequencies are predicted ${ }^{42}$ to be comparable for $\mathrm{C}_{3}$ and $\mathrm{C}_{3}^{-}$

In the $3.49 \mathrm{eV}$ photoelectron spectrum of $\mathrm{C}_{3}^{-}$(Fig. 4.2), peaks $\mathrm{A}$ and $\mathrm{B}$ are assigned to the $0-0$ and the $1_{0}^{1}$ members of the $C_{3} \tilde{X}^{1} \Sigma_{g}^{+} \leftarrow C_{3}^{-} \tilde{X}^{2} \Pi_{g}$ transition, respectively. Our peak 
spacing of $1200 \pm 100 \mathrm{~cm}^{-1}$ for the neutral symmetric stretch agrees well with the value of $1224.5 \mathrm{~cm}^{-1}$ from higher resolution studies. ${ }^{43,44,45}$ The dominance of peak A indicates a fairly small bond length difference between $\mathrm{C}_{3}^{-}$and $\mathrm{C}_{3}$, in agreement with the ab initio results. ${ }^{42}$ Peak a is a hot band assigned to the $1_{1}^{0}$ transition, providing a frequency for the $C_{3}^{-} v_{1}$ symmetric stretch of $1075 \pm 100 \mathrm{~cm}^{-1}$, in good agreement with ab initio results $\left(1175 \mathrm{~cm}^{-1}\right) .42$ The intensity of peak a indicates that the anion vibrational temperature is less than $450 \mathrm{~K}$.

Peaks $\mathrm{A}$ and $\mathrm{B}$ are considerably broader $(\approx 0.15 \mathrm{eV})$ than the experimental resolution (approx. $0.025 \mathrm{eV}$ ). This breadth is due to underlying vibrational structure which appears as a series of poorly resolved shoulders (indicated by arrows). Lineberger and co-workers partially resolve these transitions in their higher resolution $C_{3}^{-}$photoelectron spectrum. ${ }^{46}$ These shoulders result from two types of transitions. The first type, which provides most of the intensity for the shoulders, is the $2_{0}^{2 n}$ progression in the $C_{3}$ bend. The second type is the $1_{n}^{n}$ sequence band progression resulting from the $149 \mathrm{~cm}^{-1}$ frequency difference for $v_{1}$ between $C_{3}$ and $\mathrm{C}_{3}^{-}$.

The $C_{3}$ bending mode has a fundamental frequency 9,47 of $63 \mathrm{~cm}^{-1}$, which is significantly different from the calculated anion bending frequency $\left(v_{2} \approx 300 \mathrm{~cm}^{-1}\right) .42$ In addition, the neutral bending mode is very anharmonic and couples to both of the other $\mathrm{C}_{3}$ vibrational modes. ${ }^{45}$ All of these effects can produce excitation of the neutral upon anion photodetachment, and the latter two effects create a vibrational pattern for $\mathrm{C}_{3}$ which is poorly described by a separable normal mode approximation. $9,45,48$

The inadequacy of the normal mode approximation for describing the $\mathrm{C}_{3}$ bend can been seen in Fig. 4.2. This shows the results of a Franck-Condon calculation assuming separable harmonic oscillators for the $v_{1}$ symmetric stretch and the $v_{2}$ degenerate bend. The anion wavefunction is generated assuming an anion bending frequency of $300 \mathrm{~cm}^{-1}$ based upon $a b$ initio predictions, ${ }^{42,49}$ and a symmetric stretch frequency of $1075 \mathrm{~cm}^{-1}$ (discussed below). The simulation shows some excitation of the neutral bend due to the large difference between the $C_{3}$ 
and $\mathrm{C}_{3}^{-}$bend frequencies. However, the frequency difference alone does yield sufficiently bend excitation in $\mathrm{C}_{3}$.

In order to account for this discrepancy, an exact quantum mechanical calculation ${ }^{50}$ of eigenvalues and Franck-Condon intensities, Fig. 4.2, was performed on a two dimensional cross-section $\left(v_{2} \times v_{2}\right)$ of the semi-empirical MORBID ${ }^{51}$ potential energy surface. ${ }^{52}$ This potential energy surface, generated by fitting Rohlfing's laser induced fluorescence data, ${ }^{45}$ includes the anharmonicity and vibrational coupling present between all three vibrational modes. The anion wavefunction is the same as that used in the harmonic oscillator simulation. Since the calculation considers only the bend mode, the changes in the simulation from the harmonic oscillator results are due to anharmonicity along the $C_{3} v_{2}$ coordinate. It is clear from this simulation that the extreme anharmonicity of the bend mode drastically changes the intensities of the $2_{0}^{2 n}$ transitions. Although the experimental spectra do not resolve all this structure, the simulations demonstrate qualitatively that the excitation of the bending mode is due to two effects: the change in frequency of the bending mode upon photodetachment and, more importantly, the floppiness, or anharmonicity, of the $C_{3}$ bend mode.

Removal of the $\sigma_{u}$-electron from the $C_{3}^{-}$anion leaves the neutral with the $\ldots 4 \sigma_{g}^{2} 3 \sigma_{u}^{1} 1 \pi_{u}^{4} 1 \pi_{g}^{1}$ electronic configuration corresponding to either the $C_{3} \tilde{a}^{3} \Pi_{u}$ or $\tilde{A}^{1} \Pi_{u}$ states. Peak $B$ in the $4.66 \mathrm{eV}$ photoelectron spectrum of $C_{3}^{-}$, Fig. 4.1, is assigned to the $0-0$ transition to the $\tilde{a}^{3} \Pi_{u}$ first excited state placing it $2.118 \pm 0.026 \mathrm{eV}$ above the ground state. While the $\tilde{a}^{3} \Pi_{u}$ state has been observed in matrix emission experiments due to an intersystem crossing from the $\tilde{A}^{1} \Pi_{u}$ state, ${ }^{44,53}$ the term value for $C_{3} \tilde{a}^{3} \Pi_{u}$ had not previously been directly measured in the gas phase because the $\tilde{a}^{3} \Pi_{u} \leftarrow \tilde{X}^{1} \Sigma_{g}^{+}$transition is optically spin-forbidden. Our $\mathrm{T}_{\mathrm{o}}$ for the $\tilde{a}^{3} \Pi_{u}$ state agrees well with matrix values obtained by Weltner and McLeod ${ }^{44}$ (2.117 eV in $\mathrm{Ne}, 2.100 \mathrm{eV}$ in $\mathrm{Ar}$ ) and Bondybey and English ${ }^{53}(2.117 \mathrm{eV}$ in $\mathrm{Ne}$ ) and the calculations of Peric-Radic et al. $(2.04 \mathrm{eV}) .{ }^{54}$ The dominance of the $0-0$ transition for the $\tilde{a}^{3} \Pi_{u}$ state indicates a very small difference in geometry between $C_{3}^{-}$and the first excited state 
of $C_{3}$. This agrees with geometry calculations for $C_{3}$ and $C_{3}^{-}, 42,54$ as well as intuition, because the detached electron originates from the effectively non-bonding $C_{3}^{-} \quad \sigma_{u}$-orbital. The $r_{0}$ bond length for the $\tilde{a}^{3} \Pi_{u}$ state is $1.298 \AA .9 \mathrm{~b}$

\subsection{3 $\mathrm{C}_{4}$ through $\mathrm{C}_{9}$}

The photoelectron spectra of $\mathrm{C}_{4}^{-}$through $\mathrm{C}_{9}^{-}$show many similarities. Each is dominated by a sharp peak (labelled $A$ in each spectrum) and contains several smaller peaks at lower eKE. Peak $A$ is assigned to the $C_{n}\left(v^{\prime}=0\right) \leftarrow C_{n}^{-}\left(v^{\prime \prime}=0\right)$ transition in each spectrum. As in $\mathrm{C}_{3}$, the dominance of this transition indicates only a small change in geometry upon photodetachment. As discussed earlier, there is experimental and theoretical evidence for the existence of linear neutral carbon molecules with up to 9 atoms. In addition, $a b$ initio calculations predict linear ground state structures for all $\mathrm{C}_{\mathrm{n}}^{-}(\mathrm{n} \leq 6)$ anions. 55 Based upon the appearance of our spectra and these other results, the peaks in the $C_{4}^{-}$through $C_{9}^{-}$spectra are assigned to transitions between the linear forms of the anion and the neutral. The electron affinities for the linear carbon molecules are then determined from the position of peak $A$ in each spectrum. The observed EA's for linear $C_{2}-C_{9}$ (and $C_{11}$ ) are compiled in Table IV. Contributions from sequence bands, uneven rotational contours, or spin-orbit splittings have not been assessed and so the EA may deviate slightly from the value presented. These effects are the basis for the error bars reported. The uncertainties vary as a function of the eKE at the origin of the spectrum. Also listed for comparison are the EA's obtained from other experiments and from $a b$ initio calculations. The EA's of the even molecules are consistently higher than their neighboring odd-numbered counterparts. In general, the EA's agree with the values obtained by Yang et al. ${ }^{18}$ at significantly lower resolution and confirm the even-odd alternation of ground state symmetries for small carbon molecules. The EA's calculated by Adamowicz ${ }^{56}$ appear to be consistently low by $\approx 0.5 \mathrm{eV}$ for the $\mathrm{C}_{2 \mathrm{n}}$ molecules and by $\approx 0.4 \mathrm{eV}$ for the $\mathrm{C}_{2 \mathrm{n}+1}$ molecules. 
Table IV: Electron affinities for linear carbon molecules.

\begin{tabular}{|c|c|c|c|}
\hline \multirow{2}{*}{ Molecule } & \multicolumn{2}{|c|}{ Electron Affinity $(\mathrm{eV})^{\mathrm{a}}$} & \multirow{2}{*}{$\begin{array}{c}A b \text { initio } \\
\text { Results } \\
(\mathrm{eV})\end{array}$} \\
\hline & Present Work & Other & \\
\hline $\mathrm{C}_{2}$ & $3.273(.008)$ & $\begin{array}{c}3.269(.006)^{b} \\
3.30(0.1)^{c}\end{array}$ & $\begin{array}{c}3.112^{\mathrm{e}} \\
3.43^{\mathrm{f}} \\
\end{array}$ \\
\hline $\mathrm{C}_{3}$ & $1.995(.025)$ & $\begin{array}{c}1.981(.020)^{\mathrm{d}} \\
1.95(0.1)^{\mathrm{c}}\end{array}$ & $\begin{array}{l}2.0 \mathrm{~g}, \mathrm{~h} \\
1.58^{\mathrm{i}} \\
\end{array}$ \\
\hline $\mathrm{C}_{4}$ & $3.882(.010)$ & $3.7(0.1)^{c}$ & $\begin{array}{l}3.39 \mathrm{j} \\
3.41^{\mathrm{i}}\end{array}$ \\
\hline $\mathrm{C}_{5}$ & $2.839(.008)$ & $2.8(0.1)^{c}$ & $2.43^{i}$ \\
\hline $\mathrm{C}_{6}$ & $4.185(.006)$ & $4.1(0.1)^{c}$ & $3.69^{i}$ \\
\hline $\mathrm{C}_{7}$ & $3.358(.014)$ & $3.1(0.1)^{c}$ & - \\
\hline $\mathrm{C}_{8}$ & $4.379(.006)$ & $4.42(0.1)^{\mathrm{c}}$ & - \\
\hline $\mathrm{C}_{9}$ & $3.684(.010)$ & $3.70(0.1)^{c}$ & - \\
\hline $\mathrm{C}_{10}$ & - & - & - \\
\hline $\mathrm{C}_{11}$ & $3.913(.008)$ & $4.0(0.1)^{\mathrm{c}}$ & - \\
\hline
\end{tabular}

a) Uncertainties given in parentheses.

b) Reference 35 .

c) Reference 18 .

d) Reference 34 .

e) J. A. Nichols and J. Simons, J. Chem. Phys. 86, 6972 (1987).

f) M. Zeitz, S. D. Peyerimhoff, and R. J. Buenker, Chem. Phys. Lett. 64, 243 (1979).

g) Reference 42.

h) K. K. Sunil, A. Orendt, and K. D. Jordan, Chem. Phys. 89, 245 (1984).

i) Reference 56.

j) Reference 64 . 
Most of the smaller peaks in the spectra presented here can be assigned to either vibrational progressions of the neutral or 'hot bands'. As discussed above, in photoelectron spectroscopy, excitation is seen principally in totally symmetric vibrational modes upon photodetachment. The combination of this principle and $a b$ initio calculations of vibrational frequencies provides much of the basis for the assignments presented.

In addition to the vibrational progression assignments, some of the smaller peaks are assigned to excited electronic states of the linear neutral molecules (see discussion of $\mathrm{C}_{4}, \mathrm{C}_{6}$, and $\mathrm{C}_{8}$ ). In the spectra of $\mathrm{C}_{6}^{-}, \mathrm{C}_{8}^{-}$and $\mathrm{C}_{5}^{-}(3.49 \mathrm{eV} \text { spectrum only })^{57}$ there are long tails extending to high eKE, which are assigned to transitions involving non-linear carbon neutrals and/or anions. The analysis of the linear $C_{n}^{-}(n \leq 9)$ is divided into two sections: one for molecules with an odd number of carbon atoms $\left(\mathrm{C}_{2 n+1}\right)$ and the other for those with an even number of carbon atoms $\left(\mathrm{C}_{2 \mathrm{n}}\right)$. The linear $\mathrm{C}_{2 \mathrm{n}+1}$ molecules are all closed-shell, ${ }^{1} \Sigma_{g}^{+}$, species while the linear $\mathrm{C}_{2 \mathrm{n}}$ molecules all have open-shell ${ }^{3} \Sigma_{g}^{-}$ground states.

\subsubsection{1 $\mathrm{C}_{5}, \mathrm{C}_{7}$ and $\mathrm{C}_{9}$}

The photoelectron spectrum of $C_{5}^{-}$recorded with a photon energy of $4.66 \mathrm{eV}$, Fig. 4.1, shows a short vibrational progression of the neutral. At higher resolution (3.49 eV, Fig. 4.3), it is evident that several modes, including symmetric stretch and non-totally symmetric bend modes, are excited upon photodetachment of the anion. The forms of these vibrational modes are shown in Fig. 4.8b. The assignments of the peaks in the $\mathrm{C}_{5}$ spectra (Table $\mathrm{D}$ ) are based upon $a b$ initio results. ${ }^{13,58}$ Peaks $\mathrm{B}, \mathrm{C}$ and $\mathrm{F}$ are assigned to the $7_{0}^{2}, 5_{0}^{2}$, and $6_{0}^{2}$ transitions, respectively. The bend frequencies obtained from these assignments are $2 v_{5} / 2=222 \mathrm{~cm}^{-1}$ and $2 v_{7} / 2=101 \mathrm{~cm}^{-1}$, in agreement with the tentative assignments proposed by Moazzen-Ahmadi et al., ${ }^{10}$ and $2 \mathrm{v}_{6} / 2=512 \mathrm{~cm}^{-1}$. The uncertainty in these values is approximately $45 \mathrm{~cm}^{-1}$. Peak

Figure 4.8: $\quad$ Forms of normal modes for (a) $\mathrm{C}_{4}$ and (b) $\mathrm{C}_{5}$. 
a)

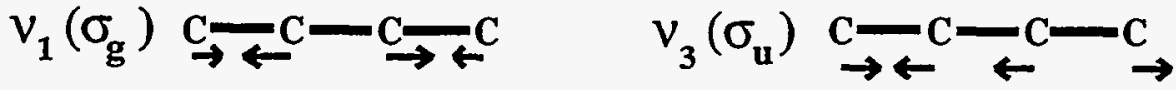

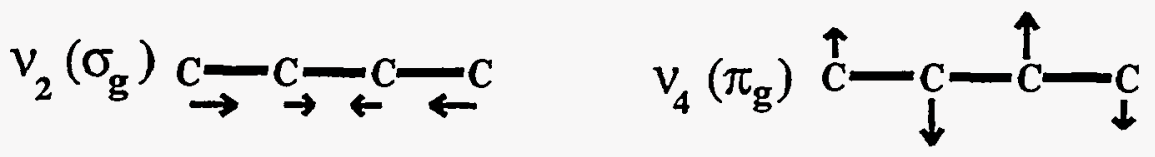

$$
\begin{aligned}
& v_{5}\left(\pi_{u}\right) \stackrel{\uparrow}{\mathrm{C}}-\underset{\downarrow}{\mathrm{C}}-\underset{\downarrow}{\mathrm{C}}=\stackrel{\uparrow}{\mathrm{C}}
\end{aligned}
$$

b)

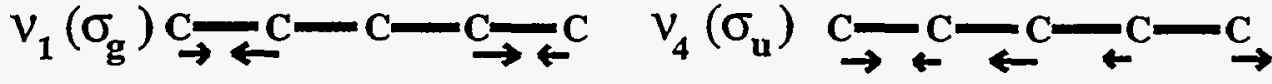

$$
\begin{aligned}
& v_{2}\left(\sigma_{g}\right) \underset{\rightarrow}{\mathrm{C}} \underset{\rightarrow}{\rightarrow} \mathrm{C} \underset{\leftarrow}{\mathrm{C}} \underset{\leftarrow}{\leftarrow} \quad \mathrm{v}_{5}\left(\pi_{\mathrm{g}}\right) \stackrel{\uparrow}{\mathrm{C}}-\underset{\downarrow}{\mathrm{C}}-\mathrm{C}-\mathrm{T}-\mathrm{C}-\mathrm{C} \\
& v_{3}\left(\sigma_{u}\right) \underset{\leftarrow}{C}-\mathrm{C} \underset{\rightarrow}{\rightarrow}-\mathrm{C}-\mathrm{C} \quad v_{6}\left(\pi_{\mathrm{u}}\right) \underset{\downarrow}{\mathrm{C}-\mathrm{T}}-\mathrm{C}-\stackrel{\mathrm{T}}{\downarrow}-\mathrm{C}-\mathrm{C} \\
& v_{7}\left(\pi_{\mathrm{u}}\right) \stackrel{\uparrow}{\mathrm{C}}-\mathrm{C}-\mathrm{C}-\underset{\downarrow}{\downarrow} \mathrm{C}-\stackrel{\uparrow}{\mathrm{C}}
\end{aligned}
$$

Figure 4.8 
Table V: Calculated and experimental frequencies for $\mathrm{C}_{5}\left(\mathrm{~cm}^{-1}\right)$

\begin{tabular}{|c|c|c|c|c|c|c|c|c|}
\hline Reference & Calculation & $\begin{array}{c}v_{1} \\
\left(\sigma_{g}\right) \\
\end{array}$ & $\begin{array}{c}v_{2} \\
\left(\sigma_{g}\right) \\
\end{array}$ & $\begin{array}{c}v_{3} \\
\left(\sigma_{u}\right) \\
\end{array}$ & $\begin{array}{c}v_{4} \\
\left(\sigma_{u}\right) \\
\end{array}$ & $\begin{array}{c}v_{5} \\
\left(\pi_{g}\right) \\
\end{array}$ & $\begin{array}{c}v_{6} \\
\left(\pi_{u}\right)\end{array}$ & $\begin{array}{c}v_{7} \\
\left(\pi_{u}\right) \\
\end{array}$ \\
\hline Botschwina $^{a}$ & CEPA-1 & 2008 & 792 & 2169 & 1478 & 209 & 570 & 119 \\
\hline Kurtz $^{b}$ & MBPT(2)/6-31G* & 2018 & 786 & 2358 & 1471 & 281 & 480 & 131 \\
\hline Gijbels $^{c}$ & MP2/6-31G* & 1877 & 731 & 2193 & 1368 & 261 & 453 & 121 \\
\hline \multirow[t]{2}{*}{ Raghavacharid $^{d}$} & $\mathrm{HF} / 6-31 \mathrm{G}^{*}$ & 1998 & 768 & 2110 & 1469 & 200 & 583 & 101 \\
\hline & Experiment & & & & & & & \\
\hline Weltner ${ }^{e}$ & IR matrix & $(1904)^{f}$ & $(785)^{\mathrm{f}}$ & 2164 & - & -- & -- & -- \\
\hline Amanog & IR gas-phase & -- & -- & 2169 & -- & 218 & -- & 118 \\
\hline Saykally & IR gas-phase & -- & -- & 2169 & $\cdots$ & -- & -- & -- \\
\hline Present Work & UV-PES & -- & 798 & -- & -- & 222 & 512 & 101 \\
\hline
\end{tabular}

a) P. Botschwina and P. Sebald, Chem. Phys. Lett. 160, 485 (1989).

b) Reference 58 (a).

c) Reference 58 (c); Scaled by 0.93 .

d) Reference 13; Scaled by 0.89 .

e) Reference 22 .

f) Predicted by force constant analysis.

g) Reference 10 (b and c).

h) Reference 10 (a). 
$\mathrm{D}$ corresponds to the $5_{0}^{2} 7_{0}^{2}$ combination band. Peak $\mathrm{E}$ is assigned to the $2_{0}^{1}$ transition providing a symmetric stretch frequency of $v_{2}=798 \pm 45 \mathrm{~cm}^{-1}$. Vala et al., ${ }^{22}$ using force constants obtained from their $v_{3}$ measurement in the matrix environment, predict frequencies of 1904 $\mathrm{cm}^{-1}$ and $785 \mathrm{~cm}^{-1}$ for the $v_{1}$ and $v_{2}$ symmetric stretch modes. Our value for $v_{2}$ agrees well with their prediction. The $a b$ initio and experimental frequencies for $\mathrm{C}_{5}$ are compiled in Table V.

In addition to these assigned peaks, there are several other peaks to low eKE which do not appear in the $4.66 \mathrm{eV}$ spectrum due to the lower resolution there. Although some of these peaks could potentially be assigned to $\mathrm{C}_{5}$ vibrations based upon agreement with $a b$ initio results, higher resolution $\left(10 \mathrm{~cm}^{-1}\right)$ threshold photodetachment results obtained in this laboratory indicate that these peaks may actually be due to transitions involving another electronic state of $\mathrm{C}_{5} .59$ This assignment is supported by a comparison of the two $\mathrm{C}_{5}^{-}$spectra presented here. The peaks to low eKE are relatively more intense in the $3.49 \mathrm{eV}$ spectrum than in the $4.66 \mathrm{eV}$ spectrum. It is well-documented that the partial cross-sections for different photoionization transitions have different energy dependences. ${ }^{60}$ It appears that the partial photodetachment cross-section for the transition to the excited state of $\mathrm{C}_{5}$ decreases relative to the $\tilde{X}^{1} \Sigma_{g}^{+} \leftarrow \tilde{X}^{2} \Sigma_{g}^{+}$transition with increasing photodetachment energy. In agreement with this observation, there was little or no signal observed for this transition in the $\mathrm{C}_{5}^{-}$ photoelectron spectrum obtained by Yang et al. ${ }^{18}$ using a photodetachment energy of $7.9 \mathrm{eV}$.

The photoelectron spectrum of $C_{7}^{-}$obtained with a photon energy of $4.66 \mathrm{eV}$, Fig. 4.1, is similar to that of $\mathrm{C}_{5}^{-}$. Resolution limitations at higher $\mathrm{eKE}$ combined with additional low frequency bending vibrations of $\mathrm{C}_{7}$ result in fewer fully resolved vibrational features, but some of the peaks can be assigned (Table $\mathrm{n}$ ) using $a b$ initio results for the vibrational frequencies. ${ }^{58}$ Peak $B$ is assigned to the $3_{0}^{1}$ transition, providing a symmetric stretch vibrational frequency of $v_{3}=548 \pm 90 \mathrm{~cm}^{-1}$. As in $\mathrm{C}_{5}^{-}$, bending modes are excited upon photodetachment of $\mathrm{C}_{7}^{-}$. Peak C, tentatively assigned to the $7_{0}^{2}$ transition, gives the bend frequency of $2 v_{7} / 2=496 \pm 110 \mathrm{~cm}^{-1}$. 
The spacing of peak $D$ from the origin agrees well with the value obtained for the $v_{4}$ antisymmetric stretch mode by Heath and Saykally. ${ }^{11}$ However, as in $\mathrm{C}_{5}$, peaks D and $\mathrm{E}$ (and structure to lower eKE) may result from transitions involving an excited electronic state of the neutral.

While the $\mathrm{C}_{9}^{-}$spectrum, Fig. 4.1 , resembles the spectra of $\mathrm{C}_{5}^{-}$and $\mathrm{C}_{7}^{-}$, it primarily shows two symmetric stretch vibrational progressions. Peaks $B$ and $C$ are assigned to the $4_{0}^{1}$ and $3_{0}^{1}$ transitions providing symmetric stretch frequencies of $v_{3}=1258 \pm 50 \mathrm{~cm}^{-1}$ and $v_{4}=484$ $\pm 48 \mathrm{~cm}^{-1}$.

All of the odd-numbered molecules discussed thus far show excitation in the breathingtype symmetric stretch (e.g., $v_{2}$ for $C_{5}$, Fig. $8 b$ ), which is the lowest frequency symmetric stretch in all cases. This suggests that all the $\mathrm{C}-\mathrm{C}$ bonds for these systems change in the same manner upon photodetachment, whether it be to lengthen or shorten. The ab initio results for the $C_{3} / C_{3}^{-}$(Ref. 42) and $C_{5} / C_{5}^{-}$(Ref. 61) systems indicate that all the neutral bond lengths are shorter than those of the ions, in agreement with these results. In addition, as the chain grows in length, the frequency of this mode decreases: $v_{1}\left(C_{3}\right)=1200 \mathrm{~cm}^{-1} ; v_{2}\left(C_{5}\right)=798 \mathrm{~cm}^{-1}, v_{3}\left(C_{7}\right)$ $=548 \mathrm{~cm}^{-1}$; and $v_{4}\left(\mathrm{C}_{9}\right)=484 \mathrm{~cm}^{-1}$. Since the electronic structure is expected to be very similar for these molecules, one might expect comparable force constants for similar types of vibrational modes. As a result, the decrease in frequency as a function of chain length results mainly from the increase in reduced mass of the longer chains.

\subsubsection{2 $\mathrm{C}_{4}, \mathrm{C}_{6}$ and $\mathrm{C}_{8}$}

The $4.66 \mathrm{eV}$ photoelectron spectrum of $\mathrm{C}_{4}^{-}$shown in Fig. 4.4 has four resolved peaks to the low eKE side of the origin. Theoretical calculations for $\mathrm{C}_{4}$ predict two nearly isoenergetic isomers which have been considered for the ground state: a ${ }^{3} \Sigma_{g}^{-} D_{\infty h}$ linear structure and a ${ }^{1} A_{g}$ $\mathrm{D}_{2 \mathrm{~h}}$ rhombic structure. At the highest levels of theory, the two are separated by as little as 1 $\mathrm{kcal} / \mathrm{mole} .^{62.63}$ The electron affinity of $\mathrm{C}_{4}$ determined from the $\mathrm{C}_{4}^{-}$spectra, $3.882 \pm 0.010 \mathrm{eV}$, 
agrees reasonably well with Yang et al.'s value of $3.7 \pm 0.1 \mathrm{eV} .{ }^{18}$ From their CEI results, Algranati et al. ${ }^{26}$ report an electron affinity of the rhombic isomer of $\mathrm{C}_{4}$ as $2.1 \pm 0.1 \mathrm{eV}$. Comparison with Watts et al.'s calculated EA for $\mathrm{C}_{4}$ of $3.39 \mathrm{eV}^{64}$ and Adamowicz's ${ }^{56} \mathrm{EA}^{\prime} \mathrm{s}$ for the linear and rhombic isomers of $\mathrm{C}_{4}(3.45 \mathrm{eV}$ and $2.03 \mathrm{eV}$, respectively) further supports the assignment of the $\mathrm{C}_{4}^{-}$photoelectron spectral features to a linear anion $\rightarrow$ linear neutral photodetachment process.

Peak $\mathrm{C}$ is assigned to the $1_{0}^{1}$ transition, providing a symmetric stretch vibrational frequency of $v_{1}=2032 \pm 50 \mathrm{~cm}^{-1}\left(a b\right.$ initio value ${ }^{58}$ for $\left.v_{1}=2150 \mathrm{~cm}^{-1}\right)$. According to recent geometry calculations for linear $\mathrm{C}_{4}$ and $\mathrm{C}_{4}^{-}$at the SDQ-MBPT(4) level of theory, ${ }^{64}$ upon photodetachment of $\mathrm{C}_{4}^{-}$the outer bonds will shorten while the inner bond will stretch. The strong resemblance of this geometry change to the $v_{1}$ symmetric stretch normal coordinate, shown in Fig 4.8a, suggests that excitation of this mode will occur upon photodetachment, in agreement with the present results.

Peaks $\mathrm{B}$ and $\mathrm{D}$ in the $\mathrm{C}_{4}^{-}$spectrum, are located at $0.736 \mathrm{eV}$ and $0.487 \mathrm{eV} \mathrm{eKE}$, respectively. On the basis of ab initio frequency calculations alone, ${ }^{58,62}$ peak $\mathrm{B}$, located $339 \pm$ $55 \mathrm{~cm}^{-1}$ from the origin, could be assigned to either the $5_{0}^{2}$ or the $4_{0}^{1}$ transition. The recent measurement $t^{21 b}$ of the $v_{5}$ vibrational frequency $\left(v_{5}=172.4 \mathrm{~cm}^{-1}\right)$ agrees well with the $A-B$ peak spacing. The $4_{0}^{1}$ transition is symmetry forbidden within the Franck-Condon approximation, but can occur in the presence of vibronic coupling to a nearby electronic state. While this transition might normally be excluded from consideration, the strong polarization dependence of peaks $B$ and $D$ relative to the other peaks suggests that vibronic coupling may indeed be occurring. As observed by Ervin and Lineberger ${ }^{35}$ in their $\mathrm{C}_{2} \mathrm{H}^{-}$photoelectron spectrum, a signature of non-Franck-Condon allowed transitions is that the polarization dependence of these peaks differs from that of the Franck-Condon allowed transitions. The $4_{0}^{1}$ transition can occur if the $v_{4}=1$ level is coupled to the $v_{4}=0$ or 2 levels of a nearby $\Pi$ electronic state. $A b$ initio calculations predict that a $\mathrm{C}_{4}$ excited state of the appropriate 
symmetry $\left({ }^{3} \Pi_{\mathrm{g}}\right)$ lies just $1.00 \mathrm{eV}$ above the ${ }^{3} \Sigma_{g}^{-}$ground state. ${ }^{65}$ While the assignment of peak B to the $5_{0}^{2}$ transition is certainly possible, the polarization dependence of the intensity of such a transition is difficult to explain. The assignment of peak $B$ to the $4_{0}^{1}$ transition provides a bending vibrational frequency of $v_{4}=339 \pm 55 \mathrm{~cm}^{-1}$. Due to its similar polarization dependence and appropriate spacing, peak $\mathrm{D}$ can be assigned as the $1_{0}^{1} 4_{0}^{1}$ combination band.

Although the relative intensity of peak $E$ does not vary significantly as a function of laser polarization, it is assigned as a transition to an excited state of linear $C_{4}\left(T_{0}=0.327 \pm\right.$ $0.006 \mathrm{eV}$ ). This assignment is based upon two major factors: 1) no reasonable vibrational state assignment can be made which agrees the frequencies available from theoretical and experimental results and 2) two excited electronic states are predicted to lie in the vicinity of peak $E$. From the electronic configuration of linear $C_{4}, \ldots 1 \pi_{u}^{4} 4 \sigma_{u}^{2} 5 \sigma_{g}^{2} 1 \pi_{g}^{2}$, three electronic states can be formed: ${ }^{3} \Sigma_{g}^{-},{ }^{1} \Sigma_{g}^{+}$, and ${ }^{1} \Delta_{\mathrm{g}}$. Ab initio calculations and high resolution gas phase absorption experiments agree that the ground state of linear $\mathrm{C}_{4}$ is ${ }^{3} \Sigma_{g}^{-} \cdot 24,62$ Therefore, peak $\mathrm{E}$ can be assigned to either the ${ }^{1} \Delta_{g}$ or the ${ }^{1} \Sigma_{g}^{+}$electronic states which have been calculated as nearly isoenergetic states lying between $0.25 \mathrm{eV}$ and $0.75 \mathrm{eV} .65,66$ Based upon Hund's rules and Liang et al.'s ab initio results, ${ }^{67}$ discussed below, peak $E$ is assigned to the ${ }^{1} \Delta_{\mathrm{g}}$ excited state.

The $4.66 \mathrm{eV} \mathrm{C}_{6}^{-}$photoelectron spectrum, Fig. 4.4, contains several partially resolved peaks to the low eKE side of the origin, peak $A$. A higher resolution $\left(\approx 10 \mathrm{~cm}^{-1}\right)$ threshold photodetachment spectrum, recently obtained in our laboratory 68 better resolves peak $\mathrm{C}$, which lies $480 \mathrm{~cm}^{-1}$ from the origin. Based on the photoelectron spectra of the odd carbon cluster anions, it is tempting to assign peak $\mathrm{C}$ to excitation of the lowest frequency symmetric stretch in $\mathrm{C}_{6}$ (the $3_{0}^{1}$ transition). Ab initio calculations, ${ }^{13,58}$ however, predict that the frequency of this mode is $\sim 150-200 \mathrm{~cm}^{-1}$ larger than the A-C spacing. Alternatively, agreement is found between the A-C spacing and twice the predicted $v_{7}$ frequency, indicating the possible assignment of peak $C$ to the $7_{0}^{2}$ transition, (a $\pi_{\mathrm{g}}$ bending mode). While peak $\mathrm{B}$, which lies 194 
$\mathrm{cm}^{-1}$ from the origin, could be assigned to the $9_{0}^{2}$ transition (a $\pi_{\mathrm{u}}$ bending mode) on the basis of comparison with $a b$ initio values, ${ }^{13,58}$ this peak does not appear in the threshold photodetachment spectrum. It may instead be a sequence band transition from vibrationally excited $\mathrm{C}_{6}^{-}$which does not appear in the threshold spectrum because of differing ion source conditions in the two experiments; our experience with $\mathrm{Si}_{2}^{-}$(Ref. 69) indicates that the source configuration in the threshold experiment produces somewhat colder ions.

Peak D, located at $0.312 \mathrm{eV}$, is $1315 \mathrm{~cm}^{-1}$ above the origin. This peak spacing does not agree with any of the calculated vibrational frequencies for linear $\mathrm{C}_{6}$. The nearest agreement is with the $v_{2}$ symmetric stretch (ab initio value ${ }^{58 c}$ for $v_{2}=1759 \mathrm{~cm}^{-1}$ ). In accord with this assignment, peak $\mathrm{E}$ is most likely a sequence band in combination with the $2_{0}^{1}$ transition. It is also possible that peak $\mathrm{D}$ represents the transition to the ${ }^{1} \Delta_{\mathrm{g}}$ excited electronic state of linear $\mathrm{C}_{6}$, predicted to lie $\sim 1200 \mathrm{~cm}^{-1}$ above the ground state. 67,70

Due to the high electron affinity of $C_{8}$ relative to $C_{4}$ and $C_{6}$, the amount of information obtained from the $\mathrm{C}_{8}^{-}$spectrum is not as abundant. The spectrum, Fig. 4.4, contains two peaks ( $A$ and $B$ ) at very low eKE separated by $\approx 565 \mathrm{~cm}^{-1}$. This lies close to the predicted value of the $v_{4}$ symmetric stretch vibration in $\mathrm{C}_{8},{ }^{58}$ so peak $\mathrm{B}$ is tentatively assigned to the $4_{0}^{1}$ transition. It is also possible that peak $B$ represents the low-lying ${ }^{1} \Delta_{\mathrm{g}}$ excited electronic state of $C_{8}$ (predicted $\mathrm{T}_{\mathrm{e}}=1130 \mathrm{~cm}^{-1}$ ) 67 based upon calculations by Liang et al. which predict a decreasing ${ }^{3} \Sigma_{g}^{-}-{ }^{1} \Delta_{g}$ splitting as a function of increasing chain length in the linear even-numbered carbon molecules. ${ }^{67}$

\subsection{4 $\mathrm{C}_{10}, \mathrm{C}_{11}$ and Non-linear Anion Photodetachment}

The photoelectron spectra of $\mathrm{C}_{10}^{-}$and $\mathrm{C}_{11}^{-}$, Fig. 4.5, have a different appearance than the other spectra presented. The $\mathrm{C}_{10}^{-}$spectrum consists of several broad unresolved features. The $\mathrm{C}_{11}^{-}$spectrum has similar broad features, but also exhibits three sharp peaks (labelled as $\mathrm{A}, \mathrm{B}$ and C). These three peaks taken alone strongly resemble the linear anion $\rightarrow$ linear neutral 
transitions seen in the $\mathrm{C}_{2 \mathrm{n}+1}^{-}$spectra. We therefore assign peak $\mathrm{A}$ to the linear $\rightarrow$ linear origin and peaks $B$ and $C$, spaced $440 \mathrm{~cm}^{-1}$ and $830 \mathrm{~cm}^{-1}$, respectively, from peak $A$ to transitions to vibrationally excited levels of linear $C_{11}$. The approximately equal spacing of the three peaks suggests that they may belong to a single vibrational progression, most likely in the breathing mode analogous to the $v_{4}$ symmetric stretch in $C_{9}$. The electron affinity determined for the linear $\mathrm{C}_{11}$ molecule, $3.913 \pm 0.010 \mathrm{eV}$, compares well with Yang et al.'s ${ }^{18}$ assignment of the linear $C_{11}$ electron affinity as $4.00 \pm 0.1 \mathrm{eV}$. The $C_{10}^{-}$spectrum, in contrast, shows no evidence for linear $\rightarrow$ linear transitions.

We next consider the broad structure in the $\mathrm{C}_{10}^{-}$and $\mathrm{C}_{11}^{-}$spectra. Every ab initio calculation for $\mathrm{C}_{10}$ has predicted a monocyclic ground state with the lowest linear isomer considerably higher in energy; Schaeffer predicts an energy difference of $2.9 \mathrm{eV}$, for example. ${ }^{71}$ It is therefore reasonable to assign the $C_{10}^{-}$spectrum to transitions to one or more electronic states of the cyclic $\mathrm{C}_{10}$ isomer. Based upon its similarity to the $\mathrm{C}_{10}^{-}$spectrum, the broad structure in the $C_{11}^{-}$spectrum is also assigned to a transition to cyclic $C_{11}$. Thus, the $C_{11}^{-}$ spectrum exhibits transitions to both the linear and cyclic forms of $\mathrm{C}_{11}$.

A more difficult question pertains to the structures of the $C_{10}^{-}$and $C_{11}^{-}$anions which yield the broad features in the two spectra. Specifically, are these features due to linear anion $\rightarrow$ cyclic neutral transitions or cyclic anion $\rightarrow$ cyclic neutral transitions? The $\mathrm{C}_{11}^{-}$spectrum suggests the latter to be the case. If only the linear $C_{11}^{-}$anions were responsible, then it is difficult to understand why the integrated intensity of the three sharp peaks assigned to the linear $\rightarrow$ linear transition is so much smaller than that of the broad features. A more reasonable explanation is that both the cyclic and linear isomers of $\mathrm{C}_{11}^{-}$are present in the ion beam, and that these are responsible for the broad features and the narrow peaks, respectively, in the spectrum. Yang et al. ${ }^{18}$ used similar reasoning to explain how their $\mathrm{C}_{11}^{-}$photoelectron spectrum changed as a function of ion source conditions. 
For $\mathrm{C}_{10}$ and $\mathrm{C}_{11}$, the assignment of the long tails in the spectra to the cyclic anion $\rightarrow$ cyclic neutral transitions allows us to estimate the electron affinities of the cyclic molecules. Based upon the eKE at which these long tails approach baseline, we can approximate the EA's of cyclic $C_{10}$ and $C_{11}$ to be $2.2 \pm 0.1 \mathrm{eV}$ and $1.5 \pm 0.1 \mathrm{eV}$, respectively. These values are indicated by arrows in the $C_{10}^{-}$and $C_{11}^{-}$photoelectron spectra, Fig. 4.5.

One problem with the assignment of the broad features to the cyclic $\rightarrow$ cyclic transitions is that these features extend over at least $2 \mathrm{eV}$ of electron kinetic energy, implying a substantial difference in geometry between the anion and the neutral and/or the presence of overlapping electronic transitions. Both of these possibilities appear reasonable in light of the $a b$ initio study by Liang and Schaeffer, ${ }^{71}$ which predicts three close-lying cyclic isomers of $\mathrm{C}_{10}$ : two cumulenic forms (one with $D_{5 h}$ symmetry and one with $D_{10 h}$ symmetry) and one acetylenic form $\left(D_{5 h}\right)$. The bond lengths and angles are quite different among these three isomers. One can therefore envision transitions between cyclic forms of the anion and neutral involving a considerable change in geometry. Whether such a change is sufficient to explain the broad features in the $C_{10}^{-}$and $C_{11}^{-}$spectra will require calculations of the anion geometries and a multidimensional Franck-Condon simulation of the spectrum.

As noted previously, the $\mathrm{C}_{6}^{-}, \mathrm{C}_{8}^{-}$, and $\mathrm{C}_{5}^{-}$photoelectron spectra show low-intensity 'tails' on the high eKE side of the sharp structure of the spectra (for $\mathrm{C}_{5}^{-}$, the tail is only visible in the $3.49 \mathrm{eV}$ spectrum). These tails extend for nearly $1 \mathrm{eV}$, suggesting that they are not simply 'hot bands'. Based on our interpretation of the $C_{10}^{-}$and $C_{11}^{-}$spectra, we believe that the tails are due to transitions involving cyclic forms of the anion and/or neutral molecules. $A b$ initio calculations indicate that while the cyclic and linear forms of neutral $\mathrm{C}_{6}$ are nearly degenerate, the cyclic form of the anion lies $1.4 \mathrm{eV}$ above the linear ground state. 55 This suggests that the tail in the $\mathrm{C}_{6}^{-}$spectrum results from the presence of cyclic $\mathrm{C}_{6}^{-}$in the ion beam, and that either cyclic $\rightarrow$ cyclic or cyclic $\rightarrow$ linear transitions are occurring. Similar explanations account for the tails in the $\mathrm{C}_{5}^{-}$and $\mathrm{C}_{8}^{-}$spectra. 
Note that Feldman et al. ${ }^{26}$ claim to observe the cyclic forms of $\mathrm{C}_{5}^{-}$and $\mathrm{C}_{6}^{-}$with relatively low electron binding energies in their Coulomb Explosion Imaging experiments. Their $\mathrm{Cs}^{+}$bombardment ion source lacked the cooling provided by a supersonic jet, so one might expect considerably higher percentage of vibrationally excited cyclic anions in their experiment as compared to ours. In any case, our explanation of the tails in our photoelectron spectra is consistent with their earlier work.

The most controversial 'cyclic vs. linear' debate concerns the structure of $\mathrm{C}_{4}$, as evidenced by the number of recent theoretical results cited in the discussion of the $\mathrm{C}_{4}^{-}$ photoelectron spectrum. Unlike the spectra of the other even-numbered carbon molecules, there is no evidence in the $\mathrm{C}_{4}^{-}$spectra that a detectable number of cyclic anions are photodetached in our experiment. Thus, because we see no evidence for transitions to cyclic $\mathrm{C}_{4}$, we cannot say anything about its stability relative to linear $\mathrm{C}_{4}$.

\subsection{Conclusions}

Vibrationally resolved spectra of the carbon molecules $C_{2}$ through $C_{11}$ have been obtained using anion photoelectron spectroscopy. The spectra of $\mathrm{C}_{2}^{-}$through $\mathrm{C}_{9}^{-}$are dominated by transitions between the linear forms of the anions and neutrals. Electron affinities are determined for the linear isomers of $\mathrm{C}_{2}-\mathrm{C}_{9}$ and $\mathrm{C}_{11}$ with a typical uncertainty of approximately $0.010 \mathrm{eV}$. The spectra confirm the even-odd alternation of electronic structure seen by Yang et al. ${ }^{18}$ In addition, several vibrational frequencies (including symmetric stretch, antisymmetric stretch and bending modes) are determined for these linear species. The spectra of the odd clusters show excitation of the breathing mode symmetric stretch upon photodetachment and there is a decrease in the frequency of this mode as the carbon chain length increases. The 3.49 $\mathrm{eV}$ photoelectron spectrum of $\mathrm{C}_{5}^{-}$shows evidence for a low-lying excited electronic state. A possible transition to an excited state of $\mathrm{C}_{4}$ is observed as well. 
Several of the spectra show evidence for photodetachment transitions involving nonlinear isomers of the anion and/or neutral. The spectra of $\mathrm{C}_{10}^{-}$and $\mathrm{C}_{11}^{-}$show broad features which appear to result from transitions between cyclic anions and cyclic neutral clusters. For $\mathrm{C}_{5}^{-}, \mathrm{C}_{6}^{-}$and $\mathrm{C}_{8}^{-}$, the spectra suggest that a small number of cyclic anions in our ion beam are detached to form either cyclic or linear neutrals.

It is clear from these results that anion photoelectron spectroscopy can provide a wealth of information about these intriguing molecules. Planned experiments at higher photodetachment energy will yield a more complete picture of the low-lying excited electronic states of the carbon clusters. In addition, higher resolution $\left(6-10 \mathrm{~cm}^{-1}\right)$ investigations employing threshold photodetachment spectroscopy $59,68,69,72$ are currently in progress.

\subsection{Acknowledgements}

Support from the Office of Naval Research under contract No. N0014-87-0495 is gratefully acknowledged. We would like to thank Dr. J. M. L. Martin and Dr. K. Raghavachari for communication of unpublished results. 


\section{References}

IP. Gerhardt, S. Loffler, and K. H. Homann, Chem. Phys. Lett. 137, 306 (1987).

2R. E. Honig, J. Chem. Phys. 22, 126 (1954).

${ }^{3}$ P. F. Bernath, K. H. Hinkle, and J. J. Keady, Science 244, 562 (1989).

${ }^{4}$ W. Krätschmer, K. Fostiropoulos, and D. R. Huffman, Chem. Phys. Lett. 170, 167 (1990).

5J. M. Hawkins, A. Meyer, T. A. Lewis, S. Loren, and F. J. Hollander, Science 252, 312 (1991).

${ }^{6}$ W. Weltner, Jr. and R. J. van Zee, Chem. Rev. 89, 1713 (1989).

${ }^{7}$ K. S. Pitzer and E. Clementi, J. Am. Chem. Soc. 81, 4477 (1959).

${ }^{8} \mathrm{R}$. Hoffman, Tetrahedron 22, 521 (1966).

${ }^{9}$ (a) C. A. Schmuttenmaer, R. C. Cohen, N. Pugliano, J. R. Heath, A. L. Cooksy, K. L. Busarow, and R. J. Saykally, Science 249, 897 (1990); (b) H. Sasada, T. Amano, C. Jarman, and P. F. Bernath, J. Chem. Phys 94, 2401 (1991).

${ }^{10}$ (a) J. R. Heath, A. L. Cooksy, M. H. W. Gruebele, C. A. Schmuttenmaer, and R. J. Saykally, Science 244, 564 (1989); (b) N. Moazzen-Ahmadi, A. R. W. McKellar, and T. Amano, J. Chem. Phys. 91, 2140 (1989); (c) N. Moazzen-Ahmadi, A. R. W. McKellar, and T. Amano, Chem. Phys. Lett. 157, 1 (1989).

11(a) J. R. Heath, R. A. Sheeks, A. L. Cooksy, and R. J. Saykally, Science 249, 895 (1990); (b) J. R. Heath and R. J. Saykally, J. Chem. Phys. 94, 1724 (1991).

${ }^{12}$ R. A. Whiteside, R. Krishnan, D. J. Defrees, J. A. Pople, and P. von R. Schleyer, Chem. Phys. Lett. 78, 538 (1981).

${ }^{13} \mathrm{~K}$. Raghavachari and J. S. Binkley, J. Chem. Phys. 87, 2191 (1987).

14J. R. Heath and R. J. Saykally, J. Chem. Phys. 93, 8392 (1990).

${ }^{15}$ K. Raghavachari, R. A. Whiteside, and J. A. Pople, J. Chem. Phys. 85, 6623 (1986).

${ }^{16}$ (a) Z. Slanina, Chem. Phys. Lett. 142, 512 (1987); (b) Z. Slanina, Chem. Phys. Lett. 173, 164 (1990).

${ }^{17}$ (a) R. J. van Zee, R. F. Ferrante, K. J. Zeringue, W. Weltner, Jr., and D. W. Ewing, J. Chem. Phys. 88, 3465 (1988); (b) R. J. van Zee, R. F. Ferrante, K. J. Zeringue, and W. Weltner Jr., J. Chem. Phys. 86, 5212 (1987); (c) H. M. Cheung and W. R. M. Graham, J. Chem. Phys. 91, 6664 (1989).

${ }^{18}$ S. Yang, K. J. Taylor, M. J. Craycraft, J. Conceicao, C. L. Pettiette, O. Cheshnovsky, and R. E. Smalley, Chem. Phys. Lett. 144, 431 (1988).

${ }^{19}$ S. H. Yang, C. L. Pettiette, J. Conceicao, O. Cheshnovsky, and R. E. Smalley, Chem. Phys. Lett. 139, 233 (1987).

${ }^{20}$ K. R. Thompson, R. L. DeKock, and W. Weltner, Jr., J. Am. Chem. Soc. 93, 4688 (1971). 
21(a) L. N. Shen and W. R. M. Graham, J. Chem. Phys. 91, 5115 (1989); (b) P. A. Withey, L. N. Shen, and W. R. M. Graham, J. Chem. Phys. 95, 820 (1991); (c) L. N. Shen, P. A. Withey, and W. R. M. Graham, J. Chem. Phys. 94, 2395 (1991).

${ }^{22}$ M. Vala, T. M. Chandrasekhar, J. Szczepanski, R. van Zee, and W. Weltner Jr., J. Chem. Phys. 90, 595 (1989).

${ }^{23}$ M. Vala, T. M. Chandrasekhar, J. Szczepanski, and R. Pellow, High Temp. Sci. 27, 19 (1990).

24J. R. Heath and R. J. Saykally, J. Chem. Phys. 94, 3271 (1991).

${ }^{25}$ One vibrational frequency is obtained for $\mathrm{C}_{4}, \mathrm{C}_{7}$, and $\mathrm{C}_{9}$, however, Moazzen-Ahmadi et al. (Reference 21) determine three frequencies for $C_{5}$, by assigning hot band transitions.

${ }^{26}$ (a) H. Feldman, D. Kella, E. Malkin, E. Miklazky, Z. Vager, J. Zajfman, and R. Naaman, J. Chem. Soc. Far. Trans. 86, 2469 (1990); (b) M. Algranati, H. Feldman, D. Kella, E. Malkin, E. Miklazky, R. Naaman, Z. Vager, and J. Zajfman, Isr. J. Chem. 30, 79 (1990); (c) M. Algranati, H. Feldman, D. Kella, E. Malkin, E. Miklazky, R. Naaman, Z. Vager, and J. Zajfman, J. Chem. Phys. 90,4617 (1989).

${ }^{27}$ R. B. Metz, A. Weaver, S. E. Bradforth, T. N. Kitsopoulos, and D. M. Neumark, J. Chem. Phys. 94, 1377 (1990).

${ }^{28}$ O. Cheshnovsky, S. H. Yang, C. L. Pettiette, M. J. Craycraft, and R. E. Smalley, Rev. Sci. Instr. 58, 2131 (1987).

${ }^{29}$ W. C. Wiley and I. H. McLaren, Rev. Sci. Instrum. 26, 1150 (1955).

${ }^{30}$ A. Weaver, D. W. Arnold, S. E. Bradforth, and D. M. Neumark, J. Chem. Phys. 94, 1740 (1991).

31(a) E. A. Ballik and D. A. Ramsay, Astrophys. J. 137, 84 (1963); (b) E. A. Ballik and D. A. Ramsay, Astrophys. J. 137, 61 (1963).

${ }^{32} \mathrm{~K}$. P. Huber and G. Herzberg, Molecular Spectra and Molecular Structure IV: Constants of Diatomic Molecules (Van Nostrand Reinhold, New York, 1977).

33(a) K. Kawaguchi, K. Matsumura, H. Kanamori, and E. Hirota, J. Chem. Phys. 91, 1953 (1989); (b) G. W. Lemire, Z. Fu, Y. M. Hamrick, S. Taylor, and M. D. Morse, J. Phys. Chem. 93, 2313 (1989); (c) L. Gausset, G Herzberg, A Lagerqvist, and B. Rosen, Disc. Far. Soc. 35, 113 (1963).

34J. M. Oakes and G. B. Ellison, Tetrahedron 42, 6263 (1986).

${ }^{35}$ K. M. Ervin and W. C. Lineberger, J. Phys. Chem. 95, 1167 (1991).

36J. W. Rabalais, Principles of Ultraviolet Photoelectron Spectroscopy (Wiley, New York, 1977).

${ }^{37}$ For a full list of references see Weltner and van Zee review, Reference 6.

${ }^{38}$ (a) W. C. Lineberger and T. A. Patterson, Chem. Phys. Lett. 13, 40 (1972); (b) G. Herzberg and A. Lagerqvist, Can. J. Phys. 46, 2363 (1968). 
39(a) R. D. Mead, U. Hefter, P. A. Schultz, and W. C. Lineberger, J. Chem. Phys. 82, 1723 (1985); (b) B. D. Rehfuss, D. J. Liu, B. M. Dinelli, M. F. Jagod, W. C. Ho, M. W. Crofton, and T. Oka, J. Chem. Phys. 89, 129 (1988).

${ }^{40}$ This corrected the previous value measured from autodetachment experiments; P. L. Jones, R. D. Mead, B. E. Kohler, S. D. Rosner, and W. C. Lineberger, J. Chem. Phys. 73, 4419 (1980).

${ }^{41}$ P. Rosmus and H. Werner, J. Chem. Phys. 80, 5085 (1984).

${ }^{42}$ K. Raghavachari, Chem. Phys. Lett. 171, 249 (1990).

${ }^{43}$ A. J. Merer, Can. J. Phys. 45, 4103 (1967).

${ }^{44}$ W. Weltner Jr. and D. McLeod Jr., J. Chem. Phys. 40, 1305 (1964).

${ }^{45}$ E. A. Rohlfing, J. Chem. Phys. 91, 4531 (1989).

${ }^{46} \mathrm{M}$. Polak, M. Gilles, and C. Lineberger, private communication.

${ }^{47}$ L. Gausset, G. Herzberg, A. Lagerqvist, and B. Rosen, Astrophys. J. 142, 45 (1965).

48(a) F. J. Northrup and T. J. Sears, J. Opt. Soc. Am. B 7, 1924 (1990); (b) E. A. Rohlfing and J. E. M. Goldsmith, J. Opt. Soc. Am. B 7, 1915 (1990).

${ }^{49}$ While the bending mode of $\mathrm{C}$ is Renner-Teller active (as are the bending modes for all the carbon anions), no consideration is made for this effect in the simulations. Rather, the $v_{2}$ mode is considered as a doubly degenerate harmonic oscillator with a frequency set at approximately the average of the frequencies calculated in Ref. 42.

${ }^{50}$ Time-dependent wave packet propagation technique is employed using the surface described. For further details see: S. E. Bradforth, A. Weaver, D. W. Arnold, R. B. Metz, and D. M. Neumark, J. Chem. Phys. 92, 7205 (1990).

${ }^{51}$ Morse Oscillator Rigid Bender Internal Dynamics.

${ }^{52}$ (a) P. Jensen, Collect. Czech. Chem. Commun. 54, 1209 (1989); (b) P. Jensen, J. Mol. Spect. 128, 478 (1988).

${ }^{53}$ V. E. Bondybey and J. H. English, J. Chem. Phys. 68, 4641 (1978).

54J. Peri_-Radi_, J. Römelt, S. D. Peyerimhoff, and R. J. Buenker, Chem. Phys. Lett. 50, 344 (1977).

55K. Raghavachari, Z. Phys. D. 12, 61 (1989).

${ }^{56}$ L. Adamowicz, J. Chem. Phys. 93, 6685 (1990).

${ }^{57}$ The tail appears in the $3.49 \mathrm{eV}$ spectrum as a result of better sensitivity and improved resolution at lower eKE.

58 (a) J. Kurtz and L. Adamowicz, Astrophys. J. 370, 784 (1991); (b) J. M. L. Martin, J. P. François, and R. Gijbels, J. Comp. Chem. 12, 52 (1991); (c) J. M. L. Martin, J. P. François, and R. Gijbels, J. Chem. Phys. 93, 8850 (1990). 
${ }^{59}$ T. N. Kitsopoulos, C. J. Chick, Y. Zhao, and D. M. Neumark, J. Chem. Phys. 95,5479 (1991).

$60 \mathrm{~J}$. W. Berkowitz, Photoabsorption, Photoionization and Photoelectron Spectroscopy, (Academic Press, New York, 1979) pp. 155-357.

61(a) J. M. L. Martin, private communication; (b) K. Raghavachari, private communication.

62J. M. L. Martin, J. P. François, and R. Gijbels, J. Chem. Phys. 94, 3753 (1991).

${ }^{63}$ V. Parasuk and J. Almlöf, J. Chem. Phys. 94, 8172 (1991).

64J. D. Watts, I. Cernusak, and R. J. Bartlett, Chem. Phys. Lett. 178, 259 (1991).

${ }^{65}$ G. Pacchioni and J. Koutecký, J. Chem. Phys. 88, 1066 (1988).

66(a) D. H. Magers, R. J. Harrison, and R. J. Bartlett, J. Chem. Phys. 84, 3284 (1986); (b) A. V.

Nemukhin, N. F. Stepanov, and A. A. Safonov, Teor. Eksp. Khim. 18, 608 (1982).

${ }^{67}$ C. Liang and H. F. Schaefer III, Chem. Phys. Lett. 169, 150 (1990).

${ }^{68}$ C. J. Chick, Y. Zhao, T. N. Kitsopoulos, and D. M. Neumark, J. Chem. Phys. 97, 6121 (1992).

${ }^{69}$ T. N. Kitsopoulos, C. J. Chick, Y. Zhao, and D. M. Neumark, J. Chem. Phys. 95, 1441 (1991).

70V. Parasuk and J. Almlöf, J. Chem. Phys. 91,1137 (1989).

${ }^{71}$ C. Liang and H. F. Schaefer III, J. Chem. Phys. 93, 8844 (1990).

${ }^{72}$ (a) T. N. Kitsopoulos, I. M. Waller, J. G. Loeser, and D. M. Neumark, Chem. Phys. Lett. 159, 300

(1989); (b) T. N. Kitsopoulos, C. J. Chick, Y. Zhao, and D. M. Neumark, J. Chem. Phys. 95, 1441 (1991). 


\title{
Chapter 5. Study of Halogen-Carbon Dioxide Clusters and the Fluoroformyloxyl Radical by Photodetachment of $\mathrm{X}^{-}\left(\mathrm{CO}_{2}\right)[\mathrm{X}=\mathrm{I}, \mathrm{Cl}, \mathrm{Br}]$ and $\mathrm{FCO}_{2}^{-}$
}

\begin{abstract}
Anion photoelectron spectroscopy has been employed to study clusters of the form $\mathrm{X}^{-}\left(\mathrm{CO}_{2}\right)(\mathrm{X}=\mathrm{I}, \mathrm{Br}, \mathrm{Cl}$ and $\mathrm{F})$. The results show that the charge-quadrupole interaction within the primarily electrostatically bound clusters, $\mathrm{I}\left(\mathrm{CO}_{2}\right), \mathrm{Br}^{-}\left(\mathrm{CO}_{2}\right)$, and $\mathrm{Cl}^{-}\left(\mathrm{CO}_{2}\right)$, is strong enough to distort the $\mathrm{CO}_{2}$ molecule in the nearly T-shaped clusters by as much as $10^{\circ}$ $\left(\mathrm{Cl}^{-}\left(\mathrm{CO}_{2}\right)\right) . A b$ initio calculations and electrostatic models are used to describe the geometry and bonding of these clusters. The photoelectron spectrum of $\mathrm{FCO}_{2}^{-}$shows transitions to both the $\overline{\mathrm{X}}{ }^{2} \mathrm{~B}_{2}$ ground and the $\overline{\mathrm{A}}{ }^{2} \mathrm{~A}_{2}$ first excited electronic states of the covalently bound $\mathrm{FCO}_{2}$ molecule. The previously unobserved $\overline{\mathrm{A}}{ }^{2} \mathrm{~A}_{2}$ state is measured to lie $0.579 \mathrm{eV}$ above the ground state. Vibrational frequencies are assigned with the assistance of $a b$ initio calculations. The $\mathrm{FCO}_{2}$ heat of formation is determined to be $\Delta_{f} \mathrm{H}_{298}^{\circ}\left(\mathrm{FCO}_{2}\right)=-85.2 \pm 2.8 \mathrm{kcal} / \mathrm{mole}$. While both the $\mathrm{FCO}_{2}^{-}$and $\mathrm{FCO}_{2}$ are more strongly bound than the other halide- $\mathrm{CO}_{2}$ clusters, the $\mathrm{C}-\mathrm{F}$ bonds are very weak relative to $\mathrm{C}-\mathrm{F}$ bonds found in other halocarbon compounds.
\end{abstract}

\subsection{Introduction}

The central role which solvation plays in the chemical sciences is well recognized. As a result, the fundamental basis of solvation remains an active topic of chemical physics. The major goal of these studies is to generate a model which can explain and predict solvation dynamics based upon the chemical properties of each component of a solute/solvent pair. As a first step, it is necessary to understand the interaction of simple binary complexes. Then, changes which occur in the interactions as additional solvent molecules are added can be studied with respect to higher order, longer range interactions which become important in bulk solvation. In this paper, the first of a two part series, we present photoelectron spectra of $\mathrm{X}^{-}\left(\mathrm{CO}_{2}\right)$ complexes $[\mathrm{X}=\mathrm{I}, \mathrm{Br}, \mathrm{Cl}, \mathrm{F}]$ which demonstrate how the $\mathrm{X}-\mathrm{CO}_{2}$ bonding character 
changes from a very weak interaction to a covalent bond, in both the anion and the neutral, as $\mathrm{X}$ varies from I to $\mathrm{F}$. The second paper, which follows, describes how the cluster properties change as additional $\mathrm{CO}_{2}$ molecules are added to the anion cluster.

The nature of the interaction between halide anions and the $\mathrm{CO}_{2}$ molecule has been studied for several years. Spears and co-workers ${ }^{1}$ studied the $\mathrm{F}^{-}-\mathrm{CO}_{2}$ interaction both experimentally and theoretically. From their studies, they found that electrostatic calculations for the complex were insufficient to describe their measured $C-F$ bond strength $(\Delta H \sim 18$ $\mathrm{kcal} / \mathrm{mole}^{\mathrm{lb}}$ ). McMahon and co-workers ${ }^{2}$ used ion cyclotron resonance (ICR) techniques to measure the gas phase Lewis acidities of many systems and bracketed the value of the $\mathrm{CO}_{2}$ fluoride affinity as $\mathrm{D}_{0}\left(\mathrm{~F}^{-}-\mathrm{CO}_{2}\right)=31.7+/-2 \mathrm{kcal} / \mathrm{mole}$. Kinetic and thermodynamic determinations have been made for the formation of the other $\mathrm{X}^{-}\left(\mathrm{CO}_{2}\right)$ clusters as well. High pressure mass spectrometry data 3,4 indicate that $\mathrm{Cl}^{-}, \mathrm{Br}^{-}$, and $\mathrm{I}^{-}$are bound much more weakly to $\mathrm{CO}_{2}$, ( $\mathrm{D}_{\mathrm{o}} \sim 5-8 \mathrm{kcal} / \mathrm{mole}$ in order of decreasing $\mathrm{X}-\mathrm{C}$ bond strength). This significant difference in bond energies implies that a qualitatively different type of bonding is present for $\mathrm{FCO}_{2}^{-}$, relative to the other $\mathrm{X}^{-}\left(\mathrm{CO}_{2}\right)$ complexes.

Two of the anions have been studied previously by spectroscopic methods. Ault ${ }^{5}$ investigated the matrix isolated $\mathrm{FCO}_{2}^{-}$anion complexed with a $\mathrm{Cs}^{+}$counterion. Three vibrational frequencies were measured and assigned to the CF symmetric stretch $\left(883 \mathrm{~cm}^{-1}\right)$, the CO symmetric stretch $\left(1316 \mathrm{~cm}^{-1}\right)$ and the $\mathrm{CO}$ antisymmetric stretch $\left(1749 \mathrm{~cm}^{-1}\right)$. Photoelectron spectra have been previously reported for the $\mathrm{I}^{-}\left(\mathrm{CO}_{2}\right)_{\mathrm{n}}$ clusters. ${ }^{6,7}$ In addition, the $\Gamma\left(\mathrm{CO}_{2}\right)$ cluster has been investigated using negative ion zero electron kinetic energy (ZEKE) spectroscopy by our research group. ${ }^{8}$

Information on the neutral $\mathrm{XCO}_{2}$ complexes is limited to the above photodetachment studies of the $\mathrm{I}\left(\mathrm{CO}_{2}\right)$ cluster and a few studies of the $\mathrm{FCO}_{2}$ radical. The $\mathrm{FCO}_{2}$ radical has been proposed as a reactive intermediate the $\mathrm{O}+\mathrm{FCO} \rightarrow \mathrm{F}+\mathrm{CO}_{2}$ reaction. ${ }^{9}$ This reaction has been determined to be an important contributor to the $\mathrm{F}$ atom production in $\mathrm{O}_{2} / \mathrm{CF}_{4}$ plasmas 
employed to etch $\mathrm{Si}$ or $\mathrm{SiO}_{2}$ surfaces commonly used in the fabrication of microelectronics devices. ${ }^{10}$ In addition to several theoretical investigations ${ }^{9,11}$ of the $\mathrm{FCO}_{2}$ radical and the $\mathrm{O}+$ FCO reaction pathways, Francisco and co-workers ${ }^{12}$ have recently observed an absorption band of the $\mathrm{FCO}_{2}$ radical which they assign as the $\overline{\mathrm{B}}^{2} \mathrm{~A}_{1} \leftarrow \overline{\mathrm{X}}{ }^{2} \mathrm{~B}_{2}$ transition. ${ }^{13}$ From their data they determine the dissociation half-life of $\mathrm{FCO}_{2}$ be at least 3 seconds at 300 Torr and $295 \mathrm{~K}$. Wallington et al. ${ }^{14}$ studied the kinetics of the $\mathrm{FCO}_{2}$ radical reactions with $\mathrm{NO}$ and $\mathrm{O}_{3}$. Although their measured reaction rates suggest that the $\mathrm{FCO}_{2}$ radical will not play a significant role in stratospheric chemistry, the $\mathrm{FCO}_{2}$ radical remains a participant in the hydrofluorocarbon (HFC) degradation process, which is of growing importance as these chlorofluorocarbon (CFC) substitutes find widespread application. Although kinetic measurements have been made, little thermodynamic information is available for the $\mathrm{FCO}_{2}$ radical. The $\mathrm{FCO}_{2}^{-}$photoelectron spectra presented here provide the F-C bond strength as well as spectroscopic information about the ground and first excited states of the fluoroformyloxyl radical. These results are complementary to those of the previous absorption study.

Another issue addressed in this paper, related to Spears early electrostatic treatment of the $\mathrm{F}^{-}-\mathrm{CO}_{2}$ complex, ${ }^{1}$ concerns the nature of the chemical bonding involved in the $\mathrm{X}^{-}\left(\mathrm{CO}_{2}\right)$ anions. Electrostatic calculations of cluster properties using rigid molecular subunits do not allow for quantum mechanical electron exchange or deformation of the component molecules. For the 16 valence electron $\mathrm{CO}_{2}$, the $\mathrm{AB}_{2}$ Walsh diagram (Fig. 5.1) shows that the $\mathrm{CO}_{2}$ molecule is stabilized by distortion from linearity when an electron is placed in the LUMO $\left(2 \pi_{u}^{*}\right)$ orbital. This is verified experimentally by the OCO angles, $\alpha$, of metastable $\mathrm{CO}_{2}^{-}(\alpha=$ $\left.134^{\circ}\right)^{15}$ and the $\mathrm{CO}_{2}\left(\tilde{\mathrm{A}}^{1} \mathrm{~B}_{2}\left(\Delta_{\mathrm{u}}\right)\right)$ excited state $\left(\alpha=122 \pm 2^{\circ}\right),{ }^{16}$ which is formed by promotion of an electron from the $\left(1 \pi_{g}\right)$ HOMO orbital to the $\left(4 a_{1}\right)$ component of the $\left(\pi_{u}^{*}\right)$ LUMO. Population of the $\mathrm{CO}_{2}$ LUMO also increases the bending mode vibrational frequency from 667

Figure 5.1. Molecular orbital correlation (Walsh) diagram for an $\mathrm{AB}_{2}$ molecule. Adapted from Ref. 15. 


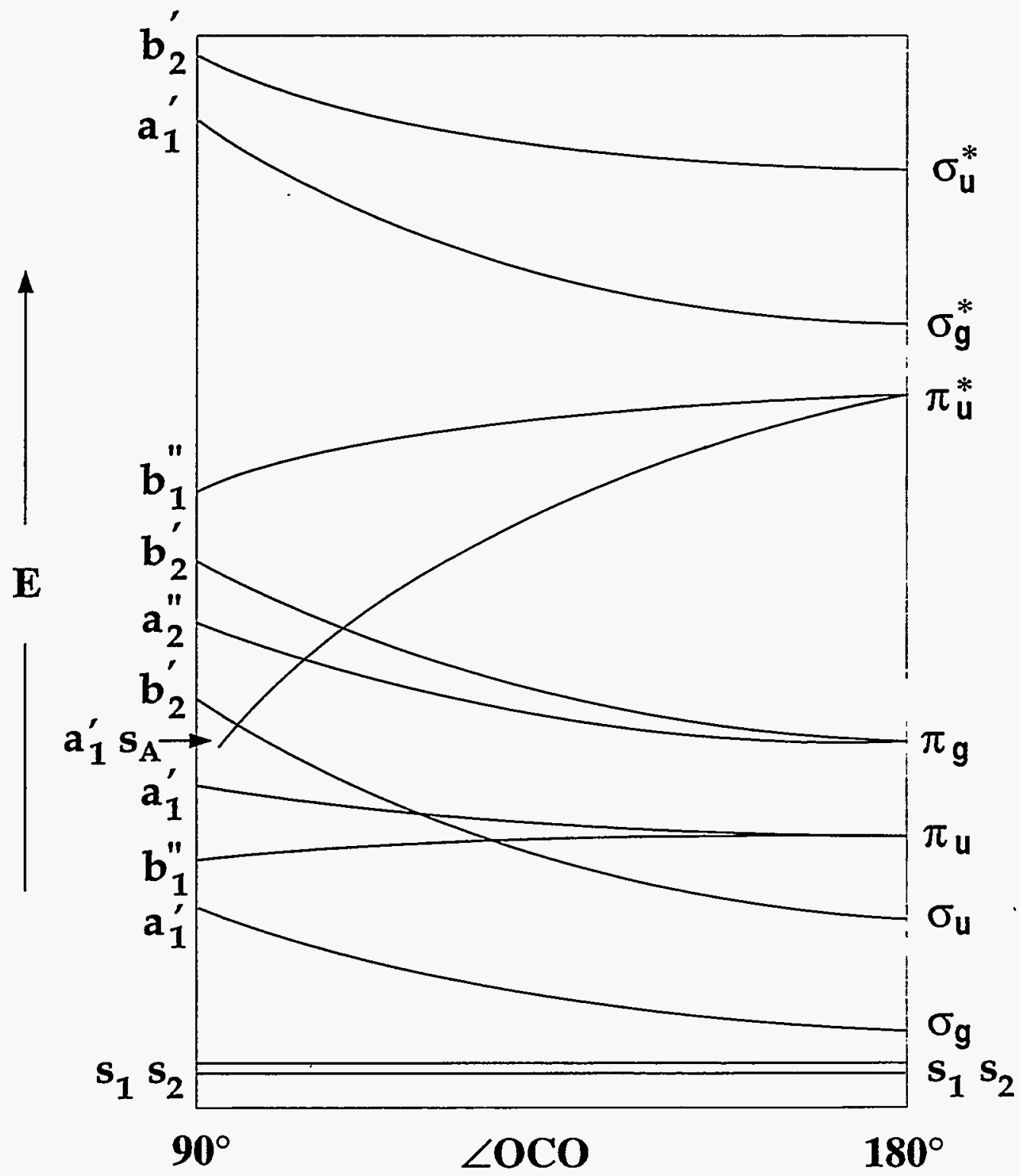

Figure 5.1 
$\mathrm{cm}^{-1}$ for $\mathrm{CO}_{2}$ to $849 \mathrm{~cm}^{-1}$ for $\mathrm{CO}_{2}^{-} \cdot{ }^{17}$

In anion-molecule complexes, it is possible for charge transfer (CT) effects to occur which lead to significant changes in bonding character and/or geometrical arrangement. As will be seen below, the photoelectron spectra of the $\mathrm{X}^{-}\left(\mathrm{CO}_{2}\right)$ complexes show that clusters of $\mathrm{CO}_{2}$ with the various halide anions contain nonlinear $\mathrm{CO}_{2}$ molecules. The extent of the distortion increases as the halide changes from $\mathrm{I}^{-}$to $\mathrm{F}^{-}$. If $\mathrm{CT}$ occurs in these complexes, the $\mathrm{CO}_{2}$ should distort from linearity. The distortion may be accompanied by an increased bending frequency. However, in addition to the charge transfer mechanism, the $\mathrm{CO}_{2}$ distortion may result from electrostatic interactions. Although briefly addressed by other investigators ${ }^{4}$ it is interesting to consider the role which each of these mechanisms plays in the $\mathrm{CO}_{2}$ distortion for the different halide anions in light of the photoelectron spectroscopy results presented here. In a related study, Cyr et al. have recently found that $\mathrm{CT}$ occurs in $\mathrm{I}^{-}\left(\mathrm{CH}_{3} \mathrm{I}\right)$ leading to a significant elongation of the $\mathrm{C}-\mathrm{I}$ bonds of the $\mathrm{CH}_{3} \mathrm{I}$ molecule. ${ }^{18}$

From the photoelectron spectra, the actual degree of $\mathrm{CO}_{2}$ distortion in each complex can be determined. In addition, we infer the halide- $\mathrm{CO}_{2}$ interaction energy through the electron affinity of the neutral complex. The spectra also provide information about the open-shell halide / closed-shell $\mathrm{CO}_{2}$ interaction of the van der Waals complex. In the case of $\mathrm{Cl}\left(\mathrm{CO}_{2}\right)$, $\mathrm{Br}\left(\mathrm{CO}_{2}\right)$, and $\mathrm{I}\left(\mathrm{CO}_{2}\right)$, a perturbation of the halogen electronic structure by the weak van der Waals interaction is large enough to be observed in the $\mathrm{X}^{-}\left(\mathrm{CO}_{2}\right)$ photoelectron spectra. By comparison of the $\mathrm{X}^{-}\left(\mathrm{CO}_{2}\right)$ spectra to bare halide spectra, the nature of $\mathrm{X}-\mathrm{C}$ bond (covalent vs. electrostatic) in the complex is obtained. The results indicates that the $\mathrm{X}^{-}\left(\mathrm{CO}_{2}\right)$ complexes, $\mathrm{X}$ $=\mathrm{I}, \mathrm{Br}$, and $\mathrm{Cl}$, are primarily electrostatically bound while the $\mathrm{FCO}_{2}^{-}$has a covalent, but weak, C-F bond.

As a part of the data analysis, ab initio calculations for $\mathrm{FCO}_{2}^{-}, \mathrm{FCO}_{2}$ and $\mathrm{Cl}^{-}\left(\mathrm{CO}_{2}\right)$ have been performed. The $\mathrm{Cl}^{-}\left(\mathrm{CO}_{2}\right)$ results are compared to the results of the electrostatic calculations used to investigate the nature of the $\mathrm{X}-\mathrm{C}$ bonding. The $\mathrm{FCO}_{2}^{-}$and $\mathrm{FCO}_{2}$ results 
are instrumental in the assignment of the observed spectral features for the ground and first excited electronic states of $\mathrm{FCO}_{2}$.

\subsection{Experimental}

The apparatus employed in these experiments is a dual time-of-flight anion photoelectron spectrometer. A schematic diagram of the apparatus is shown in Fig. 5.2. Since a full description of the spectrometer has been given previously, ${ }^{19}$ only a general overview with details relevant to these experiments will be given. By intersecting a pulsed molecular beam(1) with a $1 \mathrm{keV}$ electron beam(2), ${ }^{20}$ the anions of interest are formed as in Eq. (1).

$$
\begin{aligned}
& \mathrm{R}-\mathrm{X}+\mathrm{e}^{-} \rightarrow \mathrm{R}+\mathrm{X}^{-} \\
& \mathrm{X}^{-}+\mathrm{CO}_{2} \stackrel{\mathrm{M}}{\longrightarrow} \mathrm{X}^{-}\left(\mathrm{CO}_{2}\right)
\end{aligned}
$$

For these experiments, halide ions, $\mathrm{X}^{-}=\mathrm{F}^{-}, \mathrm{Cl}^{-}, \mathrm{Br}^{-}$, and $\mathrm{I}^{-}$, are generated by dissociative attachment of low-energy $(\sim 1 \mathrm{eV})$ secondary electrons to $\mathrm{NF}_{3}, \mathrm{CF}_{2} \mathrm{Cl}_{2}, \mathrm{HBr}$ and $\mathrm{HI}$, respectively. The molecular beam expansion typically consists of a 5\% mixture of the halogen source in $\mathrm{CO}_{2}$ at a backing pressure of $\sim 4$ atmospheres. As the expansion proceeds, the halide ions cluster, or react, with the carrier gas, $\mathrm{M}=\mathrm{CO}_{2}$, and the adduct relaxes rotationally and vibrationally by further collisions with the carrier gas during the remainder of the expansion.

The cooled ions are injected by a pulsed negative potential at (3) into a WileyMcLaren-type time-of-flight mass spectrometer ${ }^{21}$ where they separate according to mass. Ion levels are optimized with ion optics (4) to obtain maximum signal at the microchannel plate detector (5). The ion of interest is photodetached (6) by a properly timed $8 \mathrm{~ns}$ laser pulse propagating perpendicular to the anion velocity vector. Energies of the photoelectrons are

Figure 5.2. Schematic diagram of the dual time-of-flight anion photoelectron spectrometer. 


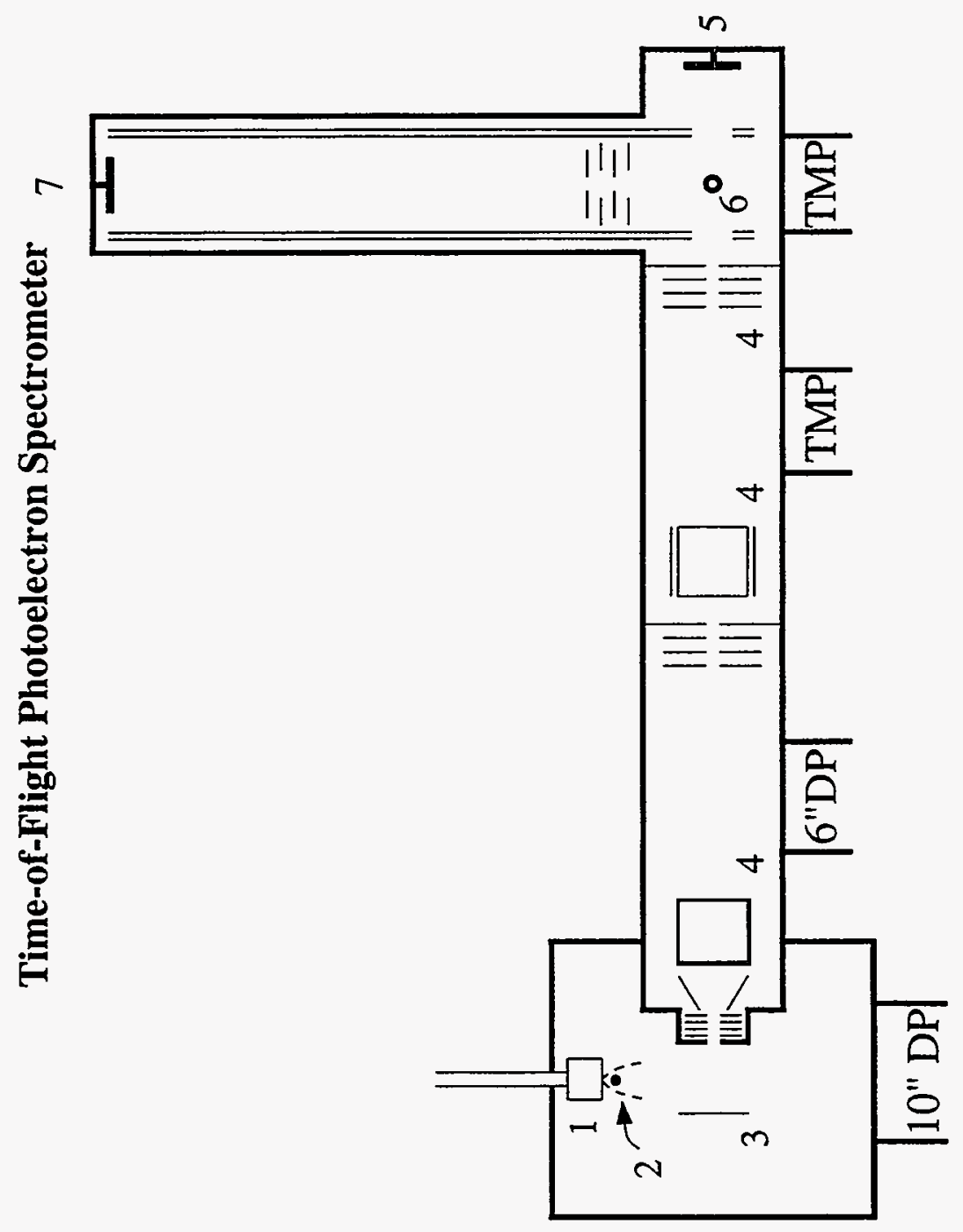

Figure 5.2 
determined by measurement of their time-of-flight through a 1 meter field-free flight tube to a second microchannel plate detector (7). The resolution of the apparatus is $0.011 \mathrm{eV}$ for electrons with $0.65 \mathrm{eV}$ of electron kinetic energy (eKE) and degrades as $\mathrm{eKE}^{3 / 2}$. In the $\mathrm{FCO}_{2}^{-}$ spectra, the resolution is slightly decreased by 'space-charge' effects, to $0.015 \mathrm{eV}$ at $0.65 \mathrm{eV}$ (see $\operatorname{Sec} 5.4 .2 .1)$. For these experiments, the $4^{\text {th }}(266 \mathrm{~nm} ; 4.657 \mathrm{eV} ; 15 \mathrm{~mJ} / \mathrm{pulse})$ and $5^{\text {th }}$ harmonics (213 nm; $5.822 \mathrm{eV} ; 6 \mathrm{~mJ} /$ pulse) of a Nd:YAG pulsed laser (QuantaRay DCR3), are employed for photodetachment. The plane polarized laser beam can be rotated with a halfwave plate in order to study photoelectron angular distributions.

Background electrons are generated by $213 \mathrm{~nm}$ photons which scatter and interact with the surfaces inside the detector region of the apparatus. Although laser and electron baffles are employed to discriminate against these background electrons, the $213 \mathrm{~nm}$ photons generate background levels which require a background subtraction procedure. A background spectrum, collected with the same laser power used during data collection, is fitted to a smooth function. This function is scaled and subtracted from the data to correct for the moderate level background signal.

\subsection{RESULTS}

\subsubsection{Experimental}

Shown in Figure 5.3 are the photoelectron spectra obtained for $\mathrm{I}^{-}\left(\mathrm{CO}_{2}\right), \mathrm{Br}^{-}\left(\mathrm{CO}_{2}\right)$, and $\mathrm{Cl}^{-}\left(\mathrm{CO}_{2}\right)$ at $\mathrm{hv}=4.657 \mathrm{eV}$. Spectra of the bare halide atoms are shown in the corresponding insets. The spectra represent intensity of electron signal as a function of electron kinetic energy (eKE) where

Figure 5.3.Photoelectron spectra of $\mathrm{I}^{-}\left(\mathrm{CO}_{2}\right), \mathrm{Br}^{-}\left(\mathrm{CO}_{2}\right)$ and $\mathrm{Cl}^{-}\left(\mathrm{CO}_{2}\right)$ using a $4.657 \mathrm{eV}$ photodetachment energy. Spectra of $\mathrm{I}^{-}, \mathrm{Br}^{-}$and $\mathrm{Cl}^{-}$are shown, for comparison, in the insets. 


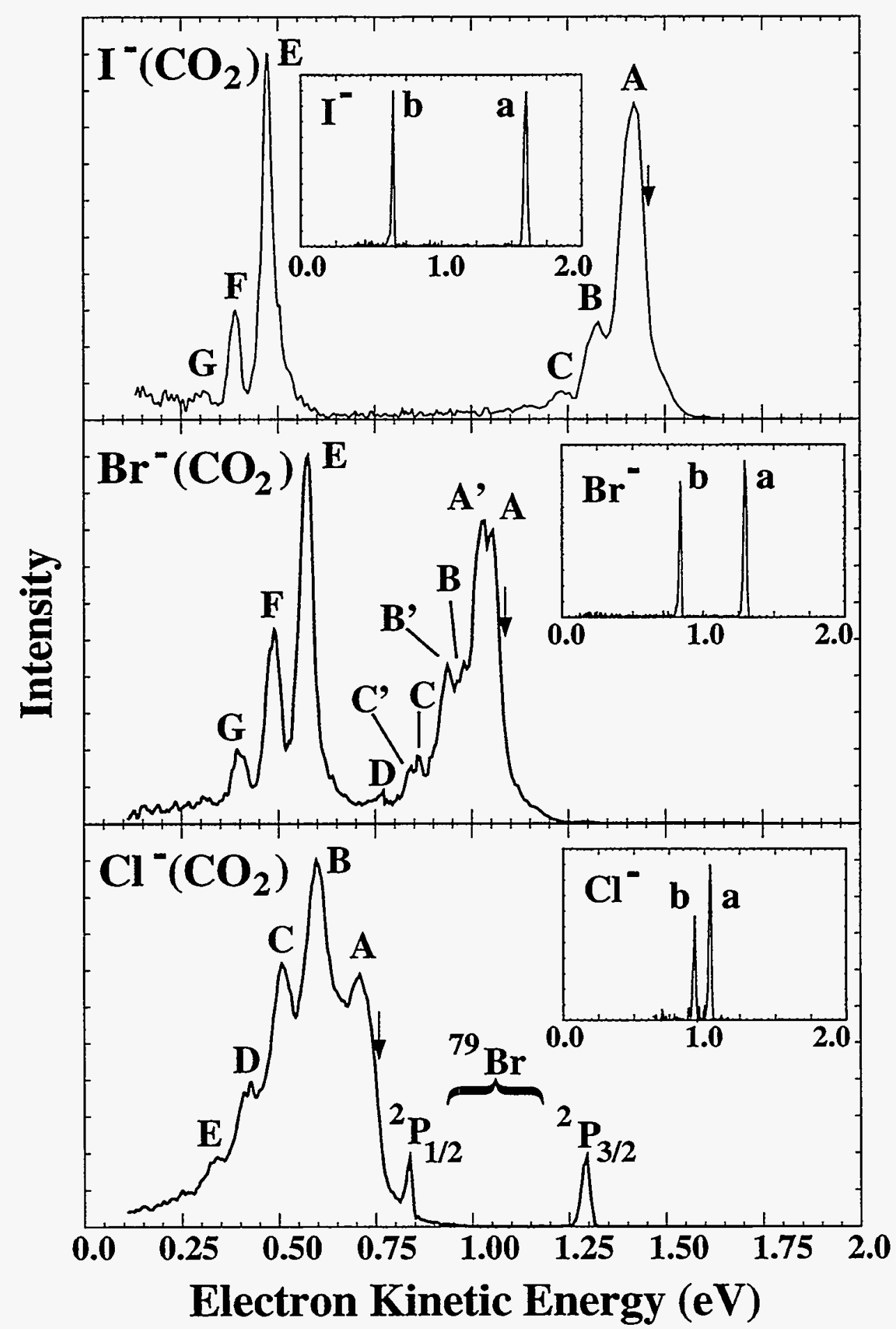

Figure 5.3 


$$
\mathrm{eKE}=\mathrm{hv}-\mathrm{EA}-\mathrm{T}_{0}-\mathrm{E}_{\mathrm{v}}^{0}+\mathrm{E}_{\mathrm{v}}^{-}
$$

In Eq. (2), $h v$ is the laser photon energy, EA is the electron affinity of the neutral species, $\mathrm{T}_{0}$ is the term value of the neutral electronic state. $\mathrm{E}_{\mathrm{v}}^{0}$ and $\mathrm{E}_{\mathrm{v}}^{-}$are the vibrational energies (above the zero point energy) of the neutral and anion, respectively. Rotational contributions to the molecular internal energy are neglected.

In the insets in Fig. 5.3, the two peaks in each atomic halide spectrum represent photodetachment transitions from the closed-shell anion to the two spin-orbit states of the neutral halogen atom. At higher eKE, peak ' $a$ ' is the ${ }^{2} \mathrm{P}_{3 / 2}$ ground state while peak ' $b$ ', at lower eKE, represents the ${ }^{2} \mathrm{P}_{1 / 2}$ excited state. The EA and the spin-orbit splitting energies are well known for the halogens and are given in Table I.

Table I: Halogen Electron Affinities and Spin-Orbit Splittings

\begin{tabular}{|c|c|c|}
\hline Halogen & Electron Affinity & Spin-Orbit Splitting \\
\hline \hline $\mathrm{F}$ & $3.401190 \mathrm{eV}$ & $0.0510 \mathrm{eV}^{\mathrm{b}}$ \\
\hline $\mathrm{Cl}$ & $3.61269 \mathrm{eV}$ & $0.10940 \mathrm{eV}^{\mathrm{b}}$ \\
\hline $\mathrm{Br}$ & $3.36590 \mathrm{eV}$ & $0.4569 \mathrm{eV}^{\mathrm{c}}$ \\
\hline $\mathrm{I}$ & $3.0591 \mathrm{eV}$ & $0.94268 \mathrm{eV}^{\mathrm{c}}$ \\
\hline
\end{tabular}

a T. R. Miller in CRC Handbook of Chemistry and Physics, 72nd Edition, ed. D.

R. Lide, CRC Press (1991), p.10-180.

b S. Baskin and J. O. Stoner, Jr., Atomic Energy Level and Grotian

Diagrams, Vol 2, North Holland Publishing Company, New York (1978).

c C. E. Moore, Atomic Energy Levels, Vol I, NSRDS-NBS 35 (1971).

Some qualitative differences between the $\mathrm{X}^{-}$and the $\mathrm{X}^{-}\left(\mathrm{CO}_{2}\right)$ data are apparent upon first observation. The $\mathrm{I}^{-}\left(\mathrm{CO}_{2}\right)$ and $\mathrm{Br}^{-}\left(\mathrm{CO}_{2}\right)$ photoelectron spectra consist of two vibrational progressions separated by the respective spin-orbit splittings of the bare halide atoms. The 
$\mathrm{Cl}^{-}\left(\mathrm{CO}_{2}\right)$ spectrum contains only one progression because the vibrational spacings are near the spin-orbit splitting of $\mathrm{Cl}$. A shift of the spectral features to lower eKE, with respect to the bare halide spectra, is observed for all of the $\mathrm{X}^{-}\left(\mathrm{CO}_{2}\right)$ spectra. The peaks for each of the spectra are significantly broader than the experimental resolution. A summary of peak positions and widths are summarized in Table II.

Table II: Peak Positions and Widths for $\mathrm{X}^{-}\left(\mathrm{CO}_{2}\right)$ Photoelectron Spectra [X $=\mathrm{Cl}, \mathrm{Br}, \mathrm{\Gamma}]$

\begin{tabular}{|c|c|c|c|c|c|c|c|c|}
\hline \multirow[t]{2}{*}{ Peak } & \multicolumn{2}{|c|}{$\mathrm{Cl}^{-}\left(\mathrm{CO}_{2}\right)$} & \multicolumn{4}{|c|}{$\mathrm{Br}^{-}\left(\mathrm{CO}_{2}\right)$} & \multicolumn{2}{|c|}{$\mathrm{I}^{-}\left(\mathrm{CO}_{2}\right)$} \\
\hline & $\begin{array}{l}\mathrm{eKE} \\
(\mathrm{eV})\end{array}$ & FWHM & $\begin{array}{l}\text { eKE } \\
(\mathrm{eV})\end{array}$ & FWHM & $\begin{array}{l}\mathrm{eKE} \\
(\mathrm{eV})\end{array}$ & FWHM & $\begin{array}{l}\mathrm{eKE} \\
(\mathrm{eV})\end{array}$ & FWHM \\
\hline A & 0.709 & 0.09 & 1.058 & 0.036 & $\mathrm{~A}^{\prime} 1.033$ & -- & 1.418 & 0.068 \\
\hline $\mathrm{B}$ & 0.598 & 0.08 & 0.984 & 0.036 & $B^{\prime}-0.941$ & $\ldots$ & 1.326 & 0.059 \\
\hline $\mathrm{C}$ & 0.510 & 0.07 & 0.868 & 0.036 & $C^{\prime}-0.847$ & $-\cdots$ & 1.235 & 0.060 \\
\hline $\mathrm{D}$ & 0.425 & 0.07 & 0.775 & 0.036 & - & -- & - & - \\
\hline$E$ & 0.338 & 0.06 & 0.580 & 0.045 & - & -- & 0.472 & 0.034 \\
\hline$F$ & - & - & 0.492 & 0.052 & - & $\cdots$ & 0.389 & 0.034 \\
\hline$G$ & - & -- & 0.400 & 0.42 & - & -- & 0.307 & 0.033 \\
\hline
\end{tabular}

The $\mathrm{I}^{-}\left(\mathrm{CO}_{2}\right)$ spectrum contains two bands, each consisting of several peaks, separated by $0.948 \pm 0.010 \mathrm{eV}$, which is equal to the iodine spin-orbit splitting within experimental uncertainty. Thus, to first order, these bands can be labeled as $I\left({ }^{2} \mathrm{P}_{3 / 2}\right) \cdot \mathrm{CO}_{2}$ and $\mathrm{I}\left({ }^{2} \mathrm{P}_{1 / 2}\right) \cdot \mathrm{CO}_{2}$. Peaks $\mathrm{A}$ and $\mathrm{E}$ are shifted to lower eKE by $0.181 \mathrm{eV}$ relative to peaks $\mathrm{a}$ and $\mathrm{b}$ in the $\mathrm{I}^{-}$ spectrum. Within each set of peaks, a progression extends to lower eKE. The average spacing for the progression beginning at $0.472 \mathrm{eV}$ is $\sim 665 \mathrm{~cm}^{-1}$. All of the peaks in the spectrum are significantly broader than the experimental resolution as a result of underlying structure which is not resolved. ${ }^{8}$ Additionally, the peaks at high eKE are significantly broader (68 meV FWHM) than those at low eKE (33 meV FWHM).

The two bands in the $\mathrm{Br}^{-}\left(\mathrm{CO}_{2}\right)$ spectrum, separated by $0.460 \pm 0.008 \mathrm{eV}$, are shifted by $0.233 \mathrm{eV}$ relative to the $\mathrm{Br}^{-}$spectrum. However, the peaks in the $\mathrm{Br}\left({ }^{2} \mathrm{P}_{3 / 2}\right) \cdot \mathrm{CO}_{2}$ progression, at high eKE, are split into doublet pairs. Deconvolution of the feature into two Gaussian shaped 
peaks reveals that the two peaks of the doublet are separated by about $280 \mathrm{~cm}^{-1}$. The raw spacing of the progression is $-750 \mathrm{~cm}^{-1}$.

The $\mathrm{Cl}^{-}\left(\mathrm{CO}_{2}\right)$ spectrum appears as one long progression of broad peaks. However, the A-B separation $\left(-900 \mathrm{~cm}^{-1}\right)$ is greater than the remaining peak spacings which are about 700 $\mathrm{cm}^{-1}$. The comparable energies of the vibrational spacings and the $\mathrm{Cl}$ spin-orbit splitting (880 $\mathrm{cm}^{-1}$ ) suggests that the extra width of the peaks in the spectrum results from nearly overlapping transitions to the two spin-orbit bands of the neutral. Peak $\mathrm{A}$ is displaced by $0.355 \mathrm{eV}$ with respect to the $\mathrm{Cl}^{2} \mathrm{P}_{3 / 2} \leftarrow \mathrm{Cl}^{-}$peak in the inset. As indicated in the figure, the ${ }^{35} \mathrm{Cl}^{-}\left(\mathrm{CO}_{2}\right)$ spectrum contains contributions from ${ }^{79} \mathrm{Br}^{-}$detachment at higher eKE $(0.834$ and $1.300 \mathrm{eV})$ as a result of their identical masses. These 'contaminant' peaks serves as a check of calibration accuracy.

Figure 5.4 contains the photoelectron spectra of $\mathrm{FCO}_{2}^{-}$obtained using a $5.822 \mathrm{eV}$ photodetachment energy. A spectrum of $\mathrm{F}^{-}$, also collected at the $5.822 \mathrm{eV}$ photon energy, is shown in the inset. The spin-orbit splitting of fluorine $(0.0501 \mathrm{eV})$ is too small to be resolved with this photodetachment energy because the electrons have $>2.3 \mathrm{eV}$ of kinetic energy where the spectral resolution is approximately $60 \mathrm{meV}$. Peak A in the $\mathrm{FCO}_{2}^{-}$spectrum is located at a much lower eKE than the $\mathrm{F}^{-}$peak. This shift $(0.824 \mathrm{eV})$ is much larger than those observed in the other $\mathrm{X}^{-}\left(\mathrm{CO}_{2}\right)$ spectra. Additionally, the $\mathrm{FCO}_{2}^{-}$spectrum is very different from those of the other $\mathrm{X}^{-}\left(\mathrm{CO}_{2}\right)$ complexes. The observed features are better characterized as transitions between two molecular species than as photodetachment transitions of a solvated fluoride anion. The wealth of vibrational structure present in the $\mathrm{FCO}_{2}^{-}$spectrum covers the energy range from 1.597 to $<0.5 \mathrm{eV}$. The peak positions are summarized in Table III. A least-squares

Figure 5.4. Photoelectron spectra of $\mathrm{FCO}_{2}^{-}$using a $5.822 \mathrm{eV}$ photodetachment energy. The data are collected at two laser polarizations, as indicated. A spectrum of $\mathrm{F}^{-}$is shown in the inset for comparison. The arrow labeled ' $a$ ' indicates the dissociation $\mathrm{F}+\mathrm{CO}_{2}$ dissociation asymptote for the fluoroformyloxyl radical. 


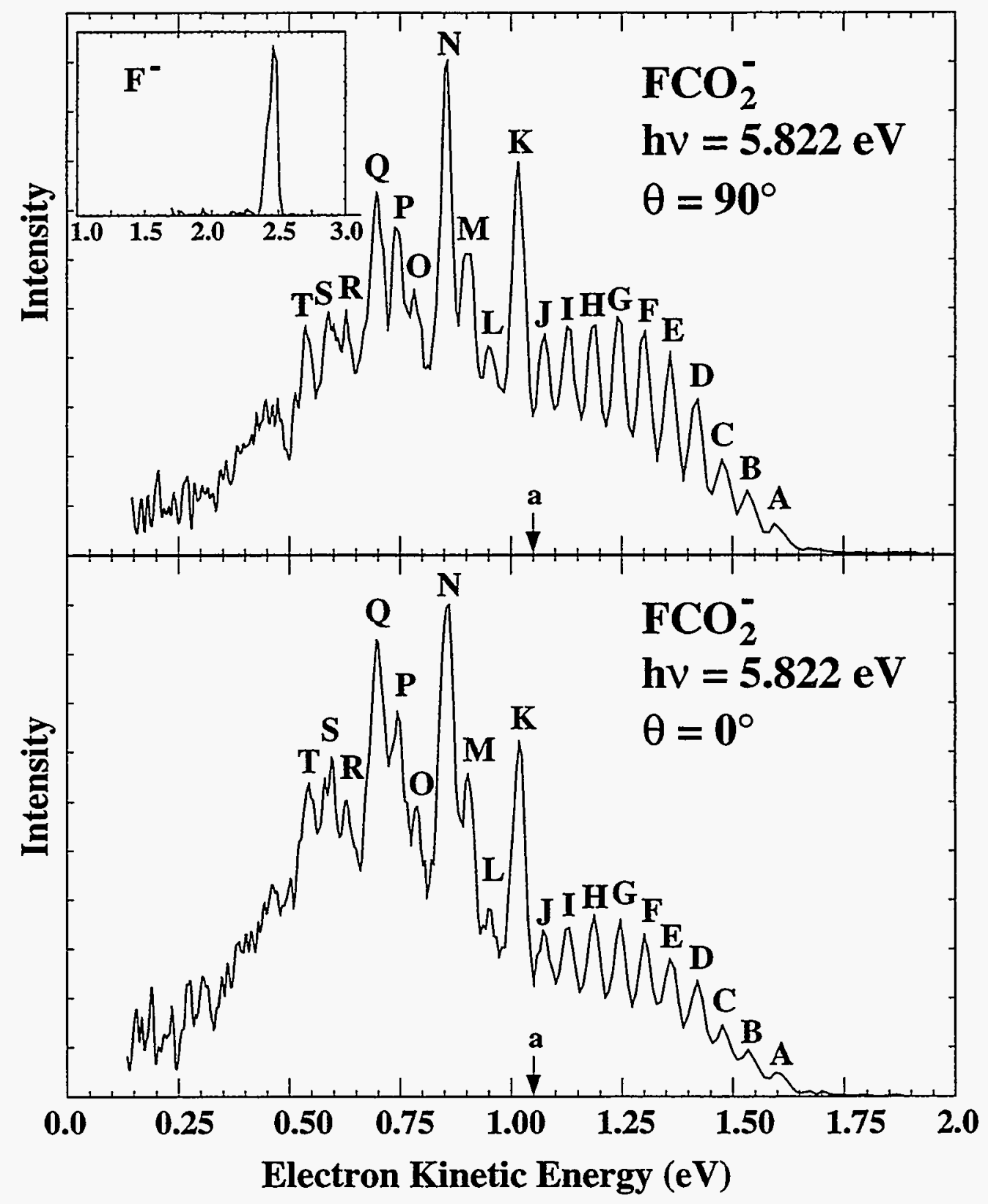

Figure 5.4 
Table III: Peaks Positions for $\mathrm{FCO}_{2}^{-}$Photoelectron Spectra

\begin{tabular}{|c|c|c|}
\hline Peak & eKE $(\mathrm{eV})$ & Energy $\left(\mathrm{cm}^{-1}\right)$ \\
\hline \hline A & 1.595 & 0 \\
\hline B & 1.534 & 492 \\
\hline C & 1.476 & 960 \\
\hline D & 1.419 & 1420 \\
\hline E & 1.360 & 1895 \\
\hline F & 1.300 & 2379 \\
\hline G & 1.246 & 2815 \\
\hline H & 1.186 & 3299 \\
\hline I & 1.129 & 3758 \\
\hline J & 1.075 & 4194 \\
\hline K & 1.016 & 4670 \\
\hline L & 0.950 & 5202 \\
\hline M & 0.904 & 5573 \\
\hline N & 0.858 & 5944 \\
\hline O & 0.785 & 6863 \\
\hline P & 0.744 & 6863 \\
\hline Q & 0.698 & 7234 \\
\hline R & 0.628 & 7799 \\
\hline S & 0.592 & 8090 \\
\hline T & 0.540 & 8509 \\
\hline
\end{tabular}

analysis of the peak spacings in progression A-J provides values of $\omega_{e}=485 \mathrm{~cm}^{-1}$ and $\mathrm{x}_{e} \omega_{e}=2$ $\mathrm{cm}^{-1}$. Beginning at peak $\mathrm{K}$, three progressions and their combination bands can be discerned in the irregularly spaced peaks. The average spacings of these three progressions are $540 \mathrm{~cm}^{-1}$, $905 \mathrm{~cm}^{-1}$, and $1280 \mathrm{~cm}^{-1}$.

As mentioned in Sec. 5.2, the photoelectron angular distributions can be studied by varying the direction of the laser polarization. Equation (3) gives the expression for the differential photodetachment cross-section, ${ }^{22}$ where $\sigma_{\text {tot }}(\mathrm{eKE})$ is the total cross-section for photodetachment, $\beta(\mathrm{eKE})$ is the asymmetry parameter $(-1 \leq \beta \leq 2)$ and $\theta$ is the angle between the electric vector of the laser beam and the direction of electron collection:

$$
\frac{\mathrm{d} \sigma}{\mathrm{d} \Omega}=\frac{\sigma_{\mathrm{tot}}(\mathrm{E})}{4 \pi}\left(1+\beta(\mathrm{E})\left(\frac{3}{2} \cos ^{2} \theta-\frac{1}{2}\right)\right)
$$


While the asymmetry parameter is not expected to change significantly over the energy range spanned by the vibrational progression, it is possible that $\beta$ will differ for transitions to different electronic states. For electronic states with different $\beta(\mathrm{eKE})$ functions, Eq. (3) shows that a change in laser polarization angle, $\theta$, results in a variation of relative peak intensities for the states. Figure 5.4 shows the $\mathrm{FCO}_{2}^{-}$data collected at two different laser polarizations, as indicated. The intensity of progression A-J decreases by approximately $20 \%$ upon rotation from $\theta=90^{\circ}$ to $\theta=0^{\circ}$, relative to peaks K-T. According to Eq. (3), this polarization dependence suggests that photodetachment transitions to two different electronic states are contributing to the spectrum; one state begins at peak $\mathrm{A}$ while the second begins at peak $\mathrm{K}$. An analysis the $\mathrm{FCO}_{2}^{-}$spectrum, including peak assignments, is given in Sec. 5.4.2.3.

As found previously for the isoelectronic $\mathrm{NO}_{3}^{-}$anion, ${ }^{44} \mathrm{FCO}_{2}^{-}$has an extremely low cross-section for photodetachment. As a result, unusually high ion levels were required to obtain a satisfactory signal-to-noise ratio. At such high ion levels, the energy of the departing electron is significantly affected by Coulombic interactions with remaining undetached $\mathrm{FCO}_{2}^{-}$ ions in the laser interaction region. This interaction shifts the spectrum to higher eKE and broadens observed spectral features. The magnitude of the 'space-charge' shift is determined by measurement of the shift for calibration ions (i.e., $\mathrm{I}^{-}, \mathrm{Br}^{-}, \mathrm{Cl}^{-}$, and $\mathrm{F}^{-}$) at the same ion densities used for the $\mathrm{FCO}_{2}^{-}$data collection. For the $\mathrm{FCO}_{2}^{-}$data, the shift is determined to be $\sim 0.035$ $\mathrm{eV}$. Through the same comparison, the broadening of the peaks is estimated to be $\sim 0.005 \mathrm{eV}$. The data in Fig. 5.4 and Fig. 5.15 have been corrected by the appropriate amount.

\subsection{2. $A b$ initio Calculations}

The following two sections describe $a b$ initio calculations performed to aid the interpretation of the data presented above. These calculations are aimed at determining the 
geometries of $\mathrm{Cl}^{-}\left(\mathrm{CO}_{2}\right), \mathrm{FCO}_{2}^{-}$, and $\mathrm{FCO}_{2}$. For $\mathrm{FCO}_{2}$, several electronic states are investigated and the results are used in the assignment of the observed spectral features.

\subsubsection{1. $\mathrm{Cl}^{-}\left(\mathrm{CO}_{2}\right)$}

Previous calculations ${ }^{4}$ at the $S C F / 4-31 G+p$ level of theory for this cluster show that the $\mathrm{CO}_{2}$ is slightly bent in the $\mathrm{Cl}^{-}\left(\mathrm{CO}_{2}\right)$ cluster. The accurate calculation of the properties of weakly bound complexes requires the inclusion of electron correlation using very large basis sets due to the large spatial extent and the weak long-range nature of the dominant forces involved (i.e., dispersion and induction forces). In the calculation of anion complexes, like $\mathrm{Cl}^{-}\left(\mathrm{CO}_{2}\right)$, the basis set must also include diffuse functions which can describe the negative charge on the complex. ${ }^{23}$

Geometry optimizations have been carried out for the $\mathrm{Cl}^{-}\left(\mathrm{CO}_{2}\right)$ complex at several levels of theory. At the SCF and Moller-Plesset (MP2 and MP4) levels of theory, calculations are carried out using $6-31+G^{*}$ and $6-311+G^{*}$ basis sets to determine the equilibrium geometry. The calculations were carried out using the Gaussian92 ab initio package. ${ }^{24}$ The results of the calculations are summarized in Table IV. There is surprising agreement between the HF/431G*, MP2/6-31+G* and MP2/6-311+G* results for this weakly bound complex. In addition to the geometry optimizations, scans along the $\mathrm{OCO}$ angle are calculated with $\mathrm{R}_{\mathrm{C}-\mathrm{Cl}}$ and $\mathrm{R}_{\mathrm{C}-\mathrm{O}}$ fixed at $3.0 \AA$ and $1.143 \AA$, respectively. These geometric parameters are chosen for comparison to electrostatic calculations to be described below. ${ }^{25}$ The results of these calculations will be discussed in Sec. 5.4.1.3.

With respect to the $\angle \mathrm{OCO}$ angle in the $\mathrm{Cl}^{-}\left(\mathrm{CO}_{2}\right)$ complex, two trends are apparent in the calculated results. As the size of basis set is increased for the same level of theory, the equilibrium OCO angle consistently becomes more linear. As more electron correlation is included, the $\mathrm{CO}_{2}$ distorts from linearity. The $\mathrm{R}_{\mathrm{C}-\mathrm{Cl}}$, bond length decreases as electron correlation is added, but increases as the basis set is enlarged. 
Table IV: $A b$ initio Calculations for $\mathrm{Cl}^{-}\left(\mathrm{CO}_{2}\right)$

\begin{tabular}{|l|c|c|c|c|}
\hline Level of Theory & Energy $(\mathrm{au})$ & $\mathrm{R}_{\mathrm{Cl}-\mathrm{C}}(\AA)$ & $\mathrm{R}_{\mathrm{C}-\mathrm{O}}(\AA)$ & $\angle \mathrm{OCO}(\mathrm{deg})$ \\
\hline \hline $\mathrm{RHF} / 4-31 \mathrm{G}^{*}$ & -646.520925 & 2.879 & 1.146 & 168.2 \\
\hline $\mathrm{RHF} / 6-31+\mathrm{G}^{*}$ & -647.186564 & 3.241 & 1.145 & 173.2 \\
\hline $\mathrm{RHF} / 6-311+\mathrm{G}^{*}$ & -647.265090 & 3.256 & 1.137 & 173.6 \\
\hline $\mathrm{MP} 2 / 6-31+\mathrm{G}^{*}$ & -647.799755 & 2.974 & 1.183 & 170.9 \\
\hline $\mathrm{MP} 2 / 6-311+\mathrm{G}^{*}$ & -647.920816 & 2.992 & 1.172 & 171.6 \\
\hline MP4/6-31+G* & -647.839376 & 2.943 & 1.190 & 169.9 \\
\hline MP4/6-311+G* & -647.962047 & 2.972 & 1.180 & 170.9 \\
\hline Expt. & - & - & - & $169.2 \pm 2.5$ \\
\hline
\end{tabular}

The lower-level HF/4-31G* calculation shows the greatest $\mathrm{CO}_{2}$ distortion of all of the calculations performed. While moderate levels of theory predict less distortion, the predicted $\angle \mathrm{OCO}$ decreases as the level of theory is improved. The MP4/6-311+G* population analysis for the $\mathrm{Cl}^{-}\left(\mathrm{CO}_{2}\right)$ cluster indicates that $\sim 6 \%$ of the total negative charge on the complex has migrated from the $\mathrm{Cl}^{-}$anion to the $\mathrm{CO}_{2}$ molecule. The $\mathrm{CO}_{2}$ distortion is correlated with the degree of charge transfer which ranges from $\sim 3.5 \%$ to $\sim 7 \%$ depending upon the level of theory. Whether the $\mathrm{CO}_{2}$ distortion can be entirely attributed to a charge transfer process is further addressed in $\operatorname{Sec} 5.4$.

\subsubsection{2. $\mathrm{FCO}_{2}^{-}$}

Both $\mathrm{FCO}_{2}$ and $\mathrm{FCO}_{2}^{-}$have been investigated theoretically at various levels of ab initio theory. Previous geometry optimizations ${ }^{26}$ of $\mathrm{FCO}_{2}^{-}$have been limited to SCF calculations which do not account for electron correlation. In order to obtain more quantitative results which may be compared to the data, we have extended these results to the MP2, MP4 and QCISD $^{27}$ levels of theory with, in some cases, larger basis sets than previously employed. The calculations were performed using the Gaussian92 ab initio calculation package ${ }^{24}$ and the results are summarized in Table $\mathrm{V}$.

The results show that the $\mathrm{F}-\mathrm{C}$ bond is quite long relative to other $\mathrm{C}-\mathrm{F}$ bonds (i.e., $\mathrm{R}_{\mathrm{C}-\mathrm{F}}\left(\mathrm{CH}_{3} \mathrm{~F}=1.39 \AA\right)$. The OCO bond angle varies by as much as $6^{\circ}$ depending upon the level 
Table V: $A b$ initio Calculations for $\mathrm{FCO}_{2}^{-} \overline{\mathrm{X}}{ }^{1} \mathrm{~A}_{1}$ Ground State Properties

\begin{tabular}{|c|c|c|c|c|c|c|c|c|c|c|}
\hline Level of Theory & Energy (au) & $\mathrm{R}_{\mathrm{FC}}(\AA)$ & $\mathrm{R}_{\mathrm{CO}}(\AA)$ & $\angle O C O$ & $\begin{array}{c}\omega_{1} \\
\text { (a1) } \\
\end{array}$ & $\begin{array}{c}\omega_{2} \\
\text { (a1) } \\
\end{array}$ & $\begin{array}{c}\omega_{3} \\
\text { (a1) } \\
\end{array}$ & $\begin{array}{c}\omega_{4} \\
\text { (b1) } \\
\end{array}$ & $\begin{array}{c}\omega_{5} \\
(\mathrm{~b} 2) \\
\end{array}$ & $\begin{array}{c}\omega_{6} \\
\text { (b2) } \\
\end{array}$ \\
\hline $\mathrm{HF} / 6-31 \mathrm{G}$ & -286.970713 & 1.454 & 1.232 & $135.4^{\circ}$ & 1371 & 894 & 655 & 841 & 1906 & 602 \\
\hline $\mathrm{HF} / 6-31+\mathrm{G}^{\mathrm{a}}$ & -286.994634 & 1.475 & 1.229 & $136.2^{\circ}$ & 1327 & 815 & 622 & 834 & 1856 & 585 \\
\hline $\mathrm{HF} / 6-31+\mathrm{G}^{\mathrm{b}}$ & -286.994634 & 1.475 & 1.229 & $136.2^{\circ}$ & 1339 & 816 & 625 & 838 & 1860 & 587 \\
\hline $\mathrm{HF} / 6-31+\mathrm{G}^{*}$ & -287.112049 & 1.410 & 1.212 & $135.2^{\circ}$ & 1440 & 893 & 672 & 920 & 1974 & 638 \\
\hline $\mathrm{MP} 2 / 6-31 \mathrm{G}$ & -287.437503 & 1.563 & 1.261 & $137.1^{\circ}$ & 1226 & 762 & 526 & 717 & 1827 & 512 \\
\hline $\mathrm{MP} 2 / 6-31+\mathrm{G}$ & -287.489520 & 1.664 & 1.253 & $140.7^{\circ}$ & 1181 & 674 & 390 & 684 & 1816 & 454 \\
\hline MP2/6-31G* & -287.736034 & 1.478 & 1.236 & $136.4^{\circ}$ & 1338 & 815 & 585 & 804 & 1940 & 565 \\
\hline $\mathrm{MP} 2 / 6-31+\mathrm{G}^{*}$ & -287.799822 & 1.517 & 1.234 & $138.0^{\circ}$ & 1290 & 724 & 507 & 783 & 1894 & 535 \\
\hline $\mathrm{MP} 2 / 6-311 \mathrm{G}$ & -287.568112 & 1.595 & 1.253 & $138.2^{\circ}$ & 1202 & 729 & 485 & 706 & 1817 & 493 \\
\hline $\mathrm{MP} 2 / 6-311+\mathrm{G}$ & -287.603201 & 1.677 & 1.247 & $141.3^{\circ}$ & 1170 & 667 & 275 & 682 & 1810 & 449 \\
\hline MP2/6-311G* & -287.892138 & 1.486 & 1.225 & $137.2^{\circ}$ & 1326 & 792 & 564 & 820 & 1949 & 570 \\
\hline $\mathrm{MP} 2 / 6-311+\mathrm{G}^{*}$ & -287.927429 & 1.505 & 1.225 & $137.9^{\circ}$ & 1294 & 738 & 510 & 801 & 1900 & 550 \\
\hline MP4/6-31+G & -287.510246 & 1.715 & 1.259 & $141.9^{\circ}$ & -- & -- & -- & -- & -- & -- \\
\hline $\mathrm{MP} 4 / 6-31+\mathrm{G}^{*}$ & -287.816161 & 1.539 & 1.238 & $138.5^{\circ}$ & - & -- & - & - & -- & - \\
\hline MP4/6-311+G* & -287.959000 & 1.523 & 1.230 & $138.4^{\circ}$ & & & & & & \\
\hline QCISD/6-31G* & -287.740084 & 1.459 & 1.235 & $135.9^{\circ}$ & 1352 & 845 & 609 & 819 & 1914 & 578 \\
\hline Experiment ${ }^{\mathfrak{c}}$ & -- & -- & -- & -- & 1316 & 883 & $\cdots$ & -- & 1749 & -- \\
\hline
\end{tabular}


of theory employed. In all cases, the OCO bond angle is very near that determined for the $\mathrm{CO}_{2}^{-}$ anion $\left(134^{\circ}\right) .^{15}$ The results also show that the $\mathrm{CO}_{2}$ bending frequency $\left(\omega_{2}\right)$ is $720-900 \mathrm{~cm}^{-1}$, near the $883 \mathrm{~cm}^{-1}$ frequency observed for matrix isolated $\mathrm{FCO}_{2}^{-}$by Ault. 5 This suggests that perhaps the matrix absorption feature would be better assigned as the $\mathrm{CO}_{2}$ bend rather than the C-F stretch $\left(\omega_{3}\right)$ which is predicted to have a significantly lower frequency. The reassignment to the $\mathrm{CO}_{2}$ bending vibration is consistent with 1) the observed isotopic shift in ${ }^{18} \mathrm{O}$ substitution studies, 2) the expected increase in this $\mathrm{CO}_{2}$ bending force constant when the electron is transferred from the $\mathrm{F}^{-}$to the $\mathrm{CO}_{2} \mathrm{LUMO}$, and 3) the expected low frequency $\mathrm{C}-\mathrm{F}$ stretch for such a long, and relatively weak, C-F bond.

Based upon the results in Table $\mathrm{V}$, it appears that the $\mathrm{FCO}_{2}^{-}$geometrical parameters are reasonably well converged at the MP4/6-311+G* level of theory. It is interesting to note that these results agree quite well with the results of the lower level MP2/6-31+G* calculations. Increasing the basis set from $6-31+\mathrm{G}^{*}$ to the $6-311+\mathrm{G}^{*}$ has little effect on the calculated vibrational frequencies at the MP2 level of theory, but there is a slight dependence on the inclusion of diffuse and polarization functions.

Geometry optimizations and vibrational force constant analyses for four electronic states of $\mathrm{FCO}_{2}\left({ }^{2} \mathrm{~B}_{2},{ }^{2} \mathrm{~A}_{2},{ }^{2} \mathrm{~A}_{1}\right.$, and $\left.{ }^{2} \mathrm{~B}_{1}\right)$ have also been performed. The results of these calculations are summarized in Table VI. Also included in the table are the results of previous calculations for the ${ }^{2} \mathrm{~B}_{2}$ and ${ }^{2} \mathrm{~A}_{1}$ electronic states of $\mathrm{FCO}_{2}$ by Francisco and co-workers. ${ }^{12}$ Due to the high excitation energies found for the ${ }^{2} A_{1}$ and ${ }^{2} B_{1}$ electronic states at the $M P 2 / 6-31+G^{*}$ level of theory $\left(\mathrm{T}_{\mathrm{e}}=1.5 \mathrm{eV}\right.$ and $5.9 \mathrm{eV},{ }^{28}$ respectively), no calculations beyond this level were performed for these states because they most likely will not contribute to the data presented here. Experimentally, Maricq et al. ${ }^{12}$ have observed a set of absorption features between 13,000 and $17,000 \mathrm{~cm}^{-1}$ which they assign as the $\tilde{\mathrm{B}}{ }^{2} \mathrm{~A}_{1} \leftarrow \tilde{\mathrm{X}}{ }^{2} \mathrm{~B}_{2}$ transition. The term value which they measure $\left(T_{0}\left({ }^{2} A_{1}\right)=1.630 \mathrm{eV}\right)$ agrees well with their calculated value $\left(T_{0}\left({ }^{2} A_{1}\right)=\right.$ $1.606 \mathrm{eV}$ ). The two remaining electronic states, ${ }^{2} \mathrm{~B}_{2}$ and ${ }^{2} \mathrm{~A}_{2}$, are significantly affected by 
Table VI: $A b$ initio Calculations for $\mathrm{FCO}_{2}$ ground and excited electronic states

\begin{tabular}{|c|c|c|c|c|c|c|c|c|c|c|c|}
\hline$\tilde{\mathbf{x}}^{2} \mathrm{~B}_{2}$ & Energy $(\mathrm{au})^{\mathrm{c}}$ & $\mathrm{T}_{\mathrm{e}}(\mathrm{eV})$ & $\mathrm{R}_{\mathrm{FC}}(\AA)$ & $\mathrm{R}_{\mathrm{CO}}(\AA)$ & $\angle O C O$ & $\begin{array}{c}\omega_{1} \\
(\mathrm{a} 1)\end{array}$ & $\begin{array}{c}\omega_{2} \\
(\mathrm{al})\end{array}$ & $\begin{array}{c}\omega_{3} \\
\text { (a1) }\end{array}$ & $\begin{array}{c}\omega_{4} \\
\text { (b1) }\end{array}$ & $\begin{array}{c}\omega_{5} \\
\text { (b2) }\end{array}$ & $\begin{array}{c}\omega_{6} \\
\text { (b2) }\end{array}$ \\
\hline UHF/6-31+G* & -286.978331 & 0.41 & 1.291 & 1.218 & $117.8^{\circ}$ & 1722 & 1101 & 601 & 908 & 2703 & 633 \\
\hline $\mathrm{MP} 2 / 6-31 \mathrm{G}^{* a}$ & -287.6193 & 0.0 & 1.324 & 1.244 & $117.4^{\circ}$ & 1626 & 1016 & 557 & 880 & 3401 & 574 \\
\hline $\mathrm{MP} 2 / 6-31+\mathrm{G}^{*}$ & -287.632652 & 0.0 & 1.331 & 1.245 & $117.8^{\circ}$ & 1585 & 991 & 546 & 870 & 3290 & 566 \\
\hline $\mathrm{MP} 2 / 6-311+\mathrm{G}^{*}$ & -287.770014 & 0.0 & 1.318 & 1.237 & $118.2^{\circ}$ & -- & -- & -- & -- & --. & -- \\
\hline $\mathrm{MP} 4 / 6-31+\mathrm{G}$ & -287.341920 & 0.0 & 1.409 & 1.284 & $120.8^{\circ}$ & 1350 & 845 & 521 & 681 & 2125 & 503 \\
\hline QCISD/6-31G*b,c & -287.618133 & 0.0 & 1.326 & 1.245 & $118.8^{\circ}$ & 1580 & 1007 & 537 & -- & -- & -- \\
\hline Experiment $^{\mathrm{a}}$ & - & 0.0 & -- & -- & - & - & -- & 520 & -- & -- & -. \\
\hline Present Results & -- & 0.0 & -- & - & - & $1465(70)$ & $950(70)$ & $500(70)$ & - & - & -- \\
\hline \multicolumn{12}{|l|}{$\tilde{\mathbf{A}}^{2} \mathrm{~A}_{2}$} \\
\hline $\mathrm{UHF} / 6-31+\mathrm{G}^{*}$ & -286.993254 & 0.0 & 1.311 & 1.244 & $124.6^{\circ}$ & 1565 & 1067 & 659 & 660 & 705 & 544 \\
\hline $\mathrm{MP} 2 / 6-31 \mathrm{G}^{*}$ & -287.584751 & $d$ & 1.337 & 1.248 & $124.8^{\circ}$ & 1531 & 1031 & 637 & 584 & 614 & $1397 \mathbf{i}$ \\
\hline $\mathrm{MP} 2 / 6-31+\mathrm{G}^{*}$ & -287.602790 & d & 1.346 & 1.248 & $125.3^{\circ}$ & 1499 & 998 & 630 & 564 & 604 & $1400 \mathbf{i}$ \\
\hline $\mathrm{MP} 2 / 6-311 \mathrm{G}$ & -287.400777 & d & 1.399 & 1.267 & $126.1^{\circ}$ & 1360 & 901 & 608 & 725 & 565 & $889 \mathbf{i}$ \\
\hline MP4/6-31+G & -287.309680 & d & 1.418 & 1.279 & $126.8^{\circ}$ & 1309 & 868 & 595 & 714 & 551 & $646 i$ \\
\hline QCISD/6-31G* & -287.602032 & 0.44 & 1.339 & 1.262 & $125.1^{\circ}$ & 1480 & 1003 & 616 & 746 & 658 & 384 \\
\hline Present Results & -- & $\mathrm{T}_{0}=0.579$ & -- & - & -- & $1274(40)$ & $903(40)$ & $532(40)$ & -- & -- & $\cdots$ \\
\hline$\tilde{\mathbf{B}}^{2} \mathrm{~A}_{1}$ & & & & & & & (4) & & & & \\
\hline UHF/6-31+G* & -286.892389 & 2.74 & 1.350 & 1.222 & $137.0^{\circ}$ & 1272 & 798 & 679 & 849 & 1735 & 581 \\
\hline $\mathrm{MP} 2 / 6-31 \mathrm{G}^{* a}$ & -287.5671 & 1.42 & 1.360 & 1.260 & $137.8^{\circ}$ & 1283 & 1048 & 520 & 742 & 2413 & 566 \\
\hline $\mathrm{MP} 2 / 6-31+\mathrm{G}^{*}$ & -287.578778 & 1.47 & 1.367 & 1.263 & $137.8^{\circ}$ & 1262 & 1033 & 611 & 732 & 2356 & 558 \\
\hline QCISD/6-31G*a & 287.5559 & 1.69 & 1.370 & 1.258 & $137.0^{\circ}$ & -- & -- & -- & -- & -- & -- \\
\hline Experiment $^{\mathrm{a}}$ & & $T_{0}=1.63$ & -- & -- & -- & -- & 1110 & 610 & 840 & 2820 & - \\
\hline${ }^{2} \mathrm{~B}_{1}$ & (4) & & 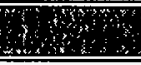 & $\therefore \therefore 8$ & & $3 \%$ & 13 & 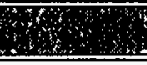 & nanks & sin & Sest \\
\hline UHF/6-31+G* & -286.755039 & $\overline{6.08^{c}}$ & 1.287 & 1.322 & $70.0^{\circ}$ & 1893 & 1058 & 725 & $1616 i$ & 1231 & 442 \\
\hline $\mathrm{MP} 2 / 6-31+\mathrm{G}^{*}$ & -287.416961 & 5.89 & 1.315 & 1.358 & $75.4^{\circ}$ & & & & & & \\
\hline
\end{tabular}

a) Reference 12 ; b) Geomtries from Ref. 12, vibrational frequencies from present work; c) MP2 energies are energies obtained after annihilation of spin contamination; MP4 are the MP4SDTQ energies; d) Force constant analysis indicates that geometry is not located at a potential energy minimum, but rather at a saddle point; e) See Ref. 46. 
changes in basis set and the degree of electron correlation considered in the calculation. At the $\mathrm{HF} / 6-31+\mathrm{G}^{*}$ level of theory, the ${ }^{2} \mathrm{~A}_{2}$ state is predicted to be the ground state of the fluoroformyloxyl radical with the ${ }^{2} \mathrm{~B}_{2}$ and ${ }^{2} \mathrm{~A}_{1}$ states lying $0.406 \mathrm{eV}$ and $2.74 \mathrm{eV}$ above the ground state respectively. Inclusion of electron correlation effects by either perturbative Moller-Plesset (MP2 or MP4) or CI (QCISD) methods changes the energetic ordering of the lower two states, consistently predicting the ${ }^{2} \mathrm{~B}_{2}$ state as the ground state.

For the ${ }^{2} \mathrm{~A}_{2}$ state, calculations using the MP methods leads to a more significant problem. Force constant analyses at both the MP2 and MP4 levels of theory suggest that this $\mathrm{C}_{2 \mathrm{v}}$ excited state species, which results from detachment of an electron from the $\left(1 \mathrm{a}_{2}\right)$ orbital of $\mathrm{FCO}_{2}^{-}$, is not located at a potential minimum. Rather, the imaginary frequency calculated for the $v_{6}$ vibrational mode $\left(\mathrm{CO}_{2}\right.$ asymmetric stretch) suggests that the $\mathrm{C}_{2 \mathrm{v}}$ species is a transition state structure between two lower-symmetry ${ }^{2} \mathrm{~A} "$ structures. Lowering the symmetry of the molecule, the MP2/6-31+G* calculations predict a $C_{s}$ structure which lies $0.1 \mathrm{eV}$ below the $C_{2 v}$ 'saddle point' structure. 29

By analogy with ab initio calculations for the $\mathrm{HCO}_{2}$ and $\mathrm{NO}_{2}$ molecules, 30,31 it is likely that calculations for the fluoroformyloxyl radical will be affected by the symmetry-breaking phenomenon which results from the inability of some levels of theory to describe the resonance of the electronic structure which leads to equal bond lengths ( $\mathrm{CO}$ or $\mathrm{NO}$ ) in the $\mathrm{HCO}_{2}$ and $\mathrm{NO}_{2}$ radicals. For these molecules, single reference wave functions tend to localize the unpaired electron, resulting one $\mathrm{CO}$ (or NO) double bond and one $\mathrm{CO}$ (or $\mathrm{NO}$ ) single bond rather than a symmetric $C_{2 v}$ structure. Configuration interaction calculations (QCISD) are performed to allow a more flexible description of the possible resonance structures. As shown in Table VI, the QCISD/6-31+G* calculation predicts a $\mathrm{C}_{2 \mathrm{v}}$ structure which exists at a potential minimum with the vibrational frequencies given. This result is very important for the analysis of the $\mathrm{FCO}_{2}^{-}$photoelectron spectrum. For comparison to the data, the ${ }^{2} \mathrm{~B}_{2}$ state properties are also calculated at the QCISD/6-31G* level of theory. 


\subsection{Analysis and Discussion}

In the following sections, we will treat the data presented above in more detail. Due to the first order distinction in the appearance of the data, the $\mathrm{X}^{-}\left(\mathrm{CO}_{2}\right)$ clusters $(\mathrm{X}=\mathrm{I}, \mathrm{Br}, \mathrm{Cl})$ will be treated separately from the $\mathrm{FCO}_{2}^{-}$anion. For the $\mathrm{X}^{-}\left(\mathrm{CO}_{2}\right)$ data, we will discuss the most likely geometry for the anions and discuss the difference in the electronic structure for the neutral complexes as one goes from $\mathrm{I}$ to $\mathrm{Br}$ to $\mathrm{Cl}$. In the process, we will apply the Distributed Multipole Analysis (DMA) model of Stone and co-workers ${ }^{32}$ in order to better understand the nature of the forces between the halide anion and the $\mathrm{CO}_{2}$ molecule for the $\mathrm{X}^{-}\left(\mathrm{CO}_{2}\right)$ clusters. In the case of $\mathrm{Cl}^{-}\left(\mathrm{CO}_{2}\right), a b$ initio calculations are compared with the experimental results. For the $\mathrm{FCO}_{2}^{-}$spectrum, the electronic and vibrational structure of the fluoroformyloxyl radical is studied from a more spectroscopic viewpoint with the aid of the ab initio results.

\subsection{1. $\mathrm{X}=\mathrm{I}, \mathrm{Br}, \mathrm{Cl}$}

\subsubsection{Electron Affinities and Thermodynamics}

From the data in Fig. 5.3, the most readily derived information is the electron affinity (EA) for each of the $\mathrm{X}\left(\mathrm{CO}_{2}\right)$ complexes. For $\mathrm{Br}^{-}\left(\mathrm{CO}_{2}\right)$ and $\mathrm{Cl}^{-}\left(\mathrm{CO}_{2}\right)$, the EA is determined using Eq. (2) for the 0-0 transition in the spectrum. $\mathrm{EA}\left[\mathrm{I}^{-}\left(\mathrm{CO}_{2}\right)\right]=3.225 \pm 0.001 \mathrm{eV}$ has previously been determined using higher resolution ZEKE spectroscopy. ${ }^{8}$ Based upon comparison of photoelectron spectrum with higher resolution ZEKE spectra collected for I$\left(\mathrm{CO}_{2}\right)$, the 0-0 transition for each $\mathrm{X}^{-}\left(\mathrm{CO}_{2}\right)$ spectrum is assumed to lie at the position indicated by an arrow in Fig. 5.3. Using the values for the ground state origins of the spectra, the EA's of the $\mathrm{Br}\left(\mathrm{CO}_{2}\right)$ and $\mathrm{Cl}\left(\mathrm{CO}_{2}\right)$ complexes are determined to be $3.582 \pm 0.017$, and $3.907 \pm 0.010 \mathrm{eV}$, respectively. The different error bars result from the eKE dependence of the spectrometer resolution function.

The EA's of $\mathrm{X}\left(\mathrm{CO}_{2}\right)$ clusters can be combined with the measured values for the $\mathrm{X}^{-}-\left(\mathrm{CO}_{2}\right)$ interaction energies to determine the $\mathrm{X}-\mathrm{CO}_{2}$ interaction energy. The diagram in Fig. 
5.5 shows that the observed spectral shift results from the difference in the $D_{0}\left(X-C O_{2}\right)$ and $D_{0}\left(X^{-}-C_{2}\right)$ energies. The shift of the $X^{-}\left(C_{2}\right)$ spectrum to lower eKE relative to the $X^{-}$ spectrum is attributed to the stronger bond of the charged cluster. From this diagram, the $\mathrm{D}_{\mathrm{o}}\left(\mathrm{X}-\mathrm{CO}_{2}\right)$ value is derived as

$$
\mathrm{D}_{\mathrm{o}}\left(\mathrm{X}-\mathrm{CO}_{2}\right)=\mathrm{EA}(\mathrm{X})+\mathrm{D}_{\mathrm{o}}\left(\mathrm{X}^{-}-\mathrm{CO}_{2}\right)-\mathrm{EA}\left(\mathrm{X}\left(\mathrm{CO}_{2}\right)\right)
$$

High pressure mass spectrometry (HPMS) clustering studies provide $\Delta H^{\circ}$ values which are readily converted to $D_{0}$ using the vibrational frequencies of the clusters. If the $C-X$ frequencies for all the anion complexes are on the order of that measured for $\mathrm{I}^{-}\left(\mathrm{CO}_{2}\right), 64 \mathrm{~cm}^{-1}$, then the $\mathrm{D}_{0}\left(\mathrm{X}^{-}-\mathrm{CO}_{2}\right)$ values will be approximately $0.25 \mathrm{kcal} / \mathrm{mole}$ larger than the measured $\Delta \mathrm{H}_{0,1}^{0}\left(\mathrm{X}^{-}-\mathrm{CO}_{2}\right)$ values. Keesee et al. ${ }^{3}$ measure $\Delta \mathrm{H}_{0,1}^{0}\left(\mathrm{Cl}^{-}-\mathrm{CO}_{2}\right)=8.0 \pm 0.1 \mathrm{kcal} / \mathrm{mole}(0.347 \pm$ $0.004 \mathrm{eV})$ and $\Delta \mathrm{H}_{0,1}^{0}\left(\mathrm{~T}^{-}-\mathrm{CO}_{2}\right)=5.6 \pm 0.1 \mathrm{kcal} / \mathrm{mole}(0.243 \pm 0.004 \mathrm{eV})$. Hiraoka et al. ${ }^{4}$ measure $\Delta \mathrm{H}_{0,1}^{0}\left(\mathrm{Cl}^{-}-\mathrm{CO}_{2}\right)=7.6 \mathrm{kcal} /$ mole $(0.330 \mathrm{eV}), \Delta \mathrm{H}_{0,1}^{0}\left(\mathrm{Br}^{-}-\mathrm{CO}_{2}\right)=6.7 \mathrm{kcal} / \mathrm{mole}(0.290$ $\mathrm{eV})$, and $\Delta \mathrm{H}_{0,1}^{0}\left(\mathrm{I}^{-}-\mathrm{CO}_{2}\right)=4.7 \mathrm{kcal} / \mathrm{mole}(0.204 \mathrm{eV})$. Approximate $\mathrm{D}_{\mathrm{o}}\left(\mathrm{X}^{-}-\left(\mathrm{CO}_{2}\right)\right.$ values can be calculated and substituted along with the EA's of $\mathrm{X}$ and $\mathrm{X}\left(\mathrm{CO}_{2}\right)$ into Eq. (13) providing $D_{0}\left(X-C_{2}\right)$ values which are quite small $(0.5-1 \mathrm{kcal} / \mathrm{mole})$ with error bars on the same order of magnitude. Thus, as expected, the neutral complexes are very weakly bound van der Waals clusters and an accurate determination of their binding energies will require a higher resolution ZEKE investigation. Such a study of the $\mathrm{I}\left(\mathrm{CO}_{2}\right)$ cluster finds $\mathrm{D}_{\mathrm{o}}\left(\mathrm{I}-\mathrm{CO}_{2}\right)=0.0445 \pm 0.0036$ eV.8

Figure 5.5. Schematic diagram illustrating the thermodynamic relationships between the anionic and neutral clusters. These relationships are used to derive Eq. (4) in the text. 


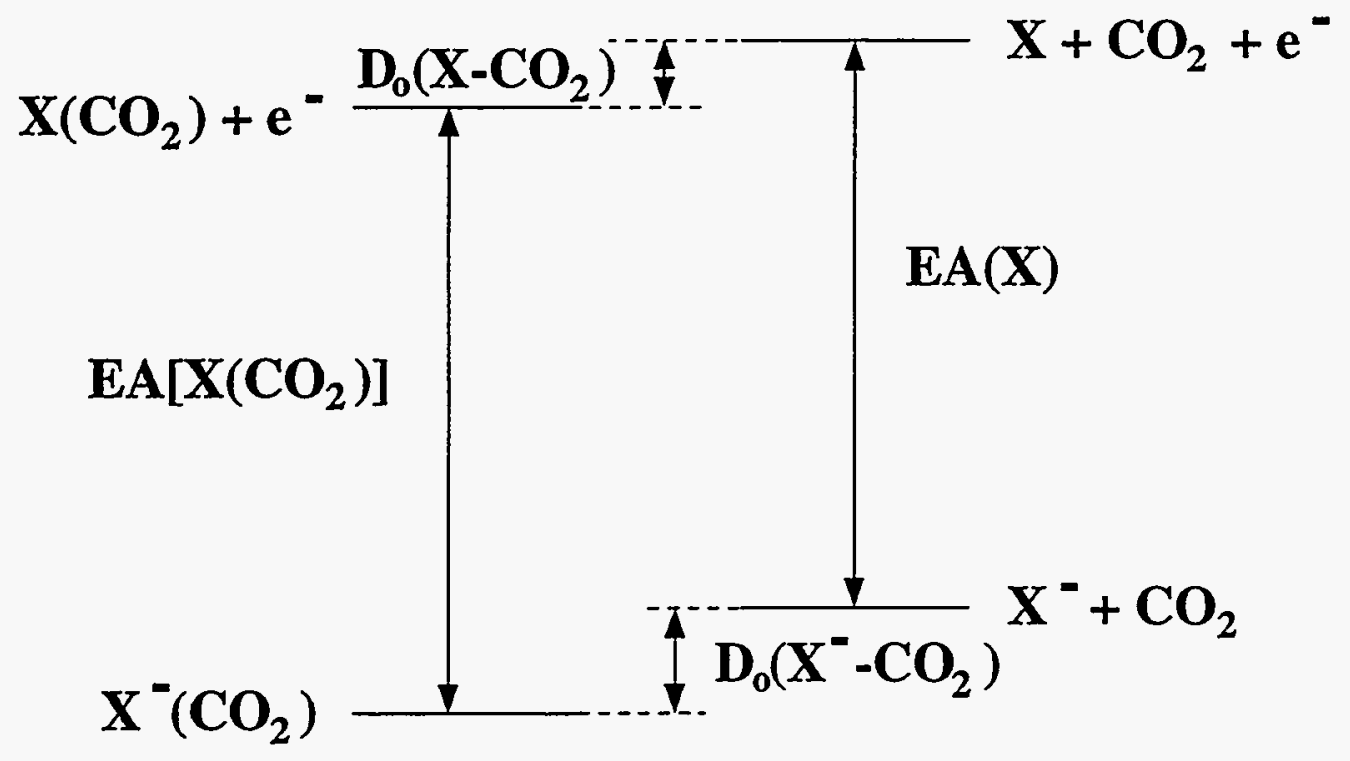

Figure 5.5 


\subsubsection{Underlying Structure}

As mentioned in Sec. 5.2, the peak widths in the $\mathrm{X}^{-}\left(\mathrm{CO}_{2}\right)$ spectra are significantly broader than the experimental resolution. In addition, the widths of the $I^{-}\left(\mathrm{CO}_{2}\right)$ and $\mathrm{Br}^{-}\left(\mathrm{CO}_{2}\right)$ ground state peaks are broader than the excited state features. Since hot band and rotational contributions should not differ significantly between the ground and excited states other effects must account for the differing peak widths. Two effects will be addressed here which contribute to the peak widths. The first contribution, which affects the ground and excited states approximately equally, results from excitation of the $\mathrm{X}-\mathrm{CO}_{2}$ van der Waals stretch by photodetachment of the $\mathrm{X}^{-}\left(\mathrm{CO}_{2}\right)$ anions. As a result of the significant difference in the interaction energies of the anion and neutral, the $\mathrm{X}-\mathrm{CO}_{2}$ distance increases upon photodetachment. While the van der Waals progression is not resolved in the data presented here due to our limited resolution, the extent of the van der Waals progression for one of the clusters, $\mathrm{I}^{-}\left(\mathrm{CO}_{2}\right)$, has been studied in greater detail in this laboratory using ZEKE spectroscopy 8

In addition to the van der Waals progressions, there is an electronic effect which contributes only to the ground state peaks in the data. The correlation diagram for a closedshell atom $(\mathrm{Rg}) /$ open-shell atom $(\mathrm{X})$ interaction, which is analogous to that for $\mathrm{X}\left(\mathrm{CO}_{2}\right)$, is shown in Fig. 5.6. The degeneracy of the ${ }^{2} \mathrm{P}_{3 / 2}$ state of the halogen atom is lifted by the approach of the $\mathrm{CO}_{2}$ molecule which breaks the isotropic spatial symmetry of the bare halogen atom. As seen in the figure, this interaction leads to three electronic states, identified as $\mathrm{X} 1 / 2$, I $3 / 2$ and II $1 / 2$ according to the projection of the total electronic angular momentum onto the

symmetry axis of the cluster. The extra width of the 'ground state' peak in the $\mathrm{I}^{-}\left(\mathrm{CO}_{2}\right)$

Figure 5.6. Correlation diagram for an open-shell atom/closed-shell atom interaction showing the three electronic states (X 1/2, I $3 / 2$ and II 1/2) which arise from this interaction. 


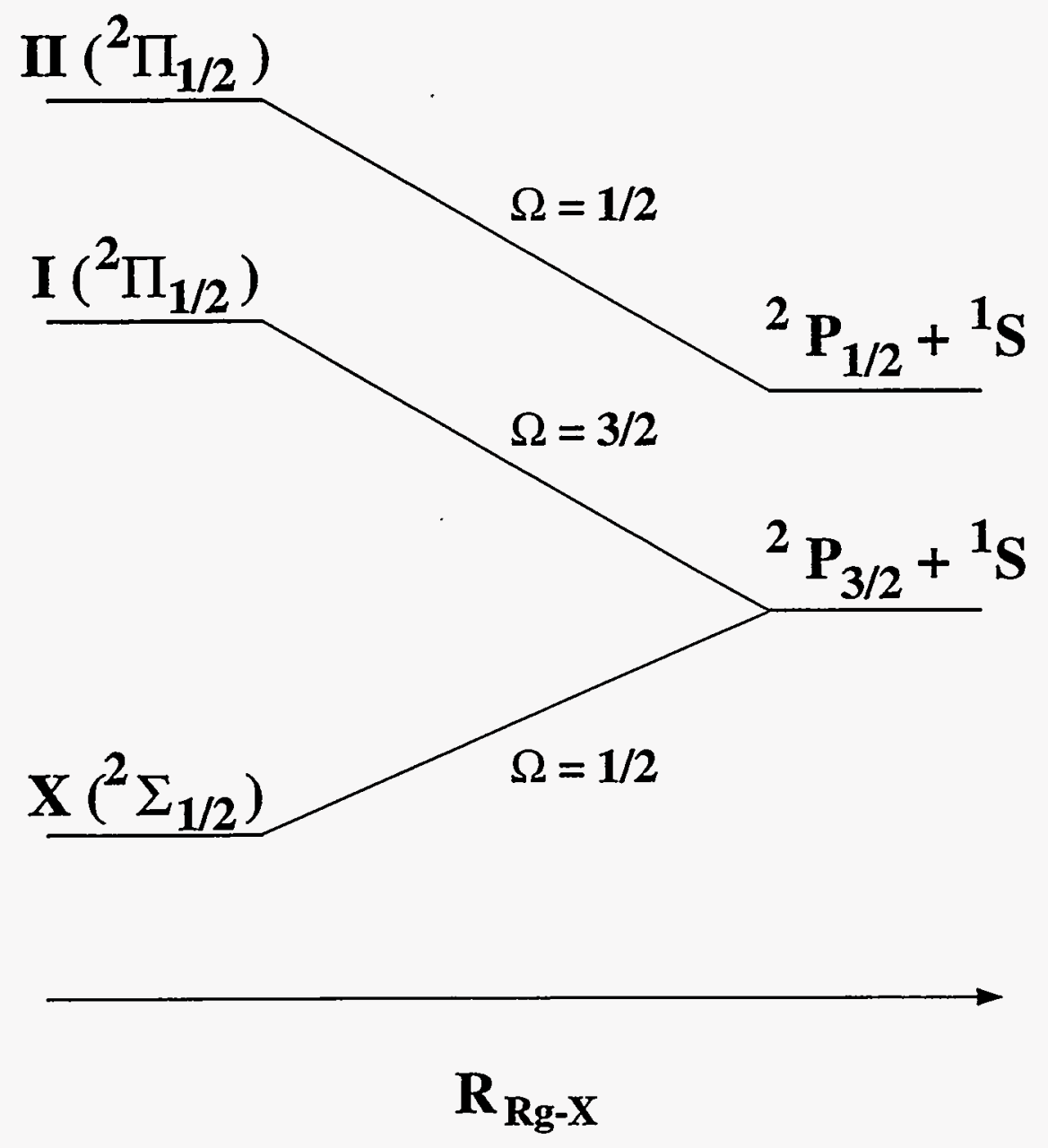

Figure 5.6 
spectrum results from this splitting $\left(225 \mathrm{~cm}^{-1}\right)^{8}$ which is too small to be cleanly resolved in the photoelectron spectrum. However, the larger $280 \mathrm{~cm}^{-1}$ splitting can be observed in the $\mathrm{Br}^{-}$ $\left(\mathrm{CO}_{2}\right)$ spectrum, indicating that the $\mathrm{Br}\left(\mathrm{CO}_{2}\right)$ interaction is stronger than that of $\mathrm{I}\left(\mathrm{CO}_{2}\right)$. As mentioned above, the small spin-orbit splitting of the $\mathrm{Cl}$ atom leads to significant congestion of the $\mathrm{Cl}^{-}\left(\mathrm{CO}_{2}\right)$ spectrum. Simulations in the following section provide further insight into the nature of the underlying electronic and vibrational structure for this spectrum.

\subsubsection{Geometry and Bonding}

In the analysis of the vibrational structure present in the $\mathrm{X}^{-}\left(\mathrm{CO}_{2}\right)$ data presented above, the geometry of the anion which is photodetached in the experiment must initially be determined. The simplest approach is to consider the electrostatics involved. As a $\mathrm{CO}_{2}$ molecule approaches the halide anion, the electrostatic interaction between the two is dominated by the charge-quadrupole interaction,

$$
\mathrm{U}_{\mathrm{q}-\theta}=\frac{\mathrm{q} \theta}{2 \mathrm{R}^{3}}\left(3 \cos ^{2} \xi-1\right)
$$

In Eq. (5), $\mathrm{q}=-1$ is the halide charge and $\theta=-4.3 \times 10^{-26}$ esu $\cdot \mathrm{cm}^{2}$ is the $\mathrm{CO}_{2}$ quadrupole moment. ${ }^{33}$ The negative sign of $\theta$ indicates that the carbon atom is positively charged, relative to the two oxygen atoms. According to Eq. (5), the minimum energy configuration for the halide anion separated from a rigid $\mathrm{CO}_{2}$ molecule by a distance, $\mathrm{R}$, occurs when $\xi=90^{\circ}$. The variable, $\xi$, is the angle between the $\mathrm{CO}_{2}$ symmetry axis and the $\mathrm{X}-\mathrm{C}$ bond. In a related study, Duncan and co-workers ${ }^{34}$ study a complex in which a $\mathrm{CO}_{2}$ molecule is clustered to a cation, $\mathrm{Mg}^{+}\left(\mathrm{CO}_{2}\right)$. In accord with the prediction of Eq. (5), they determined that the complex possesses a linear geometry.

The vibrational structure in the $\mathrm{X}^{-}\left(\mathrm{CO}_{2}\right)$ spectra indicates that this very simple model does not fully describe the geometry of the anion clusters. Of the three $\mathrm{CO}_{2}$ vibrational 
frequencies $\left(v_{1}=1388 \mathrm{~cm}^{-1} ; v_{2}=667 \mathrm{~cm}^{-1} ; 35\right.$ and $\left.v_{3}=2349.2 \mathrm{~cm}^{-1}\right),{ }^{36}$ the spacings of the peaks in the $\mathrm{X}^{-}\left(\mathrm{CO}_{2}\right)$ spectra $\left(\sim 60 \mathrm{~cm}^{-1}\right.$ for $\Gamma^{-}\left(\mathrm{CO}_{2}\right)$ and $\sim 750 \mathrm{~cm}^{-1}$ for $\mathrm{Br}^{-}\left(\mathrm{CO}_{2}\right)$ and $\left.\mathrm{Cl}^{-}\left(\mathrm{CO}_{2}\right)\right)$ most closely match the bending frequency. This indicates that this progression is best attributed to the excitation of the $\mathrm{CO}_{2}$ bending mode of the neutral $\mathrm{X}\left(\mathrm{CO}_{2}\right)$ complex by photodetachment of the $\mathrm{X}^{-}\left(\mathrm{CO}_{2}\right)$ cluster. The excitation observed in the spectrum shows that the anion must be bent in either the anion or neutral clusters. As mentioned above, the neutral interaction is an order of magnitude weaker than that of the anion. In a charge-quadrupole interaction, like that of the $\mathrm{X}^{-}\left(\mathrm{CO}_{2}\right)$ cluster, the charge feels an attraction for the center of the quadrupole and a repulsion for ends of the quadrupole. A strong interaction of this type will distort the $\mathrm{CO}_{2}$ molecule; thus, the $\mathrm{CO}_{2}$ distortion most likely occurs in the anion cluster.

In these complexes, the $\mathrm{CO}_{2}$ bending vibration involves primarily motion of the carbon and oxygen atoms while the halogen/halide atom remains relatively stationary. Thus it is reasonable to approximate the $\mathrm{CO}_{2}$ bending mode of the $\mathrm{X}\left(\mathrm{CO}_{2}\right)$ complex by the analytic normal modes of the free $\mathrm{CO}_{2}$ molecule, $\mathrm{QCO}_{2}$. Within this approximation, quantitative information about the $\mathrm{CO}_{2}$ distortion in the anion cluster can be determined through a FranckCondon analysis of the data. In photoelectron spectroscopy, the intensity of the vibrational peaks, I, are determined by the Franck-Condon factors (FCFs), as in Eq. (6)

$$
\mathrm{I} \propto \mathrm{v}_{e} \cdot\left|\tau_{e}\right| 2 \cdot\left|\left\langle\Psi_{v^{\prime}}\left(\mathrm{Q}_{\mathrm{CO}_{2}}\right) \mid \Psi_{v^{\prime \prime}}\left(\mathrm{Q}_{\mathrm{CO}_{2}}\right)\right\rangle\right|^{2}
$$

Here, $\tau_{\mathrm{e}}$ is the electronic transition dipole, which is assumed to be constant for a particular electronic state and $v_{e}$ is the asymptotic velocity of the detached electron. ${ }^{37}$ The FCF, $\left|\left\langle\Psi_{v_{i}^{\prime}} \mid \Psi_{v_{i}^{\prime}}\right\rangle\right|^{2}$, is the spatial overlap between anion and neutral wave functions for the $\mathrm{CO}_{2}$ bending vibration. Thus, in this approximation, the length of the progression is determined by the change in the OCO bond angle upon photodetachment of the $\mathrm{X}^{-}\left(\mathrm{CO}_{2}\right)$ complex. 
Calculation of the FCF's results in a stick spectrum which, when convoluted with the experimental resolution function, can be compared with the experimental data.

For each spectrum, the change in OCO angle was varied to obtain the best agreement of the convoluted FCF's with the experimental peak intensities. The $\mathrm{CO}_{2}$ bending vibrational frequency is assumed to be the same in the anion and the neutral and is set to the observed experimental spacing. In Fig. 5.7, the Franck-Condon simulations are shown with the experimental data (circles). In the $\mathrm{I}^{-}\left(\mathrm{CO}_{2}\right)$ and $\mathrm{Br}^{-}\left(\mathrm{CO}_{2}\right)$ data, only the $\mathrm{X}\left({ }^{2} \mathrm{P}_{1 / 2}\right) \cdot \mathrm{CO}_{2}$ state is fit for simplicity. A normal mode analysis of the optimized parameters in the simulations gives the $\mathrm{OCO}$ angles for the $\mathrm{I}^{-}\left(\mathrm{CO}_{2}\right)$ and $\mathrm{Br}^{-}\left(\mathrm{CO}_{2}\right)$ clusters $\left(174.5 \pm 1.5^{\circ}\right.$ and $172.2 \pm 1.5^{\circ}$, respectively).

The congestion of the $\mathrm{Cl}^{-}\left(\mathrm{CO}_{2}\right)$ spectrum prevents a similar simple treatment of the $\mathrm{Cl}$ $\left({ }^{2} \mathrm{P}_{1 / 2}\right) \cdot \mathrm{CO}_{2}$ state. Therefore, all three electronic states of the neutral cluster (X 1/2, I $3 / 2$ and II 1/2; see Sec. 5.4.1.2) are included in the simulation and convoluted together for comparison with the data. The same normal coordinate displacement is used for all three states. The total intensity of the II $1 / 2$ state is varied manually with respect to the other two states which are given equal relative intensities. In the simulations, the vibrational frequencies, assumed to be equal for all three states, are also varied as are the splittings between the $\mathrm{X}, \mathrm{I}$, and $\mathrm{II}$ states. The peaks are convoluted with the experimental resolution plus an additional $30 \mathrm{meV}$ Gaussian, to account for unresolved van der Waals progressions, by comparison with the other $\mathrm{X}^{-}\left(\mathrm{CO}_{2}\right)$ spectra. In Fig. 5.7, the $\mathrm{Cl}^{-}\left(\mathrm{CO}_{2}\right)$ experimental spectrum is superimposed upon two FranckCondon simulations. One has been convoluted to the experimental resolution function and the other is a stick spectrum so that individual transitions can be seen more clearly. The

Figure 5.7. Photoelectron spectra (circles) of $\mathrm{I}^{-}\left(\mathrm{CO}_{2}\right), \mathrm{Br}^{-}\left(\mathrm{CO}_{2}\right)$ and $\mathrm{Cl}^{-}\left(\mathrm{CO}_{2}\right)$ and FranckCondon simulations convoluted with the experimental resolution (solid line) and with a stick spectrum for $\mathrm{Cl}^{-}\left(\mathrm{CO}_{2}\right)$. 


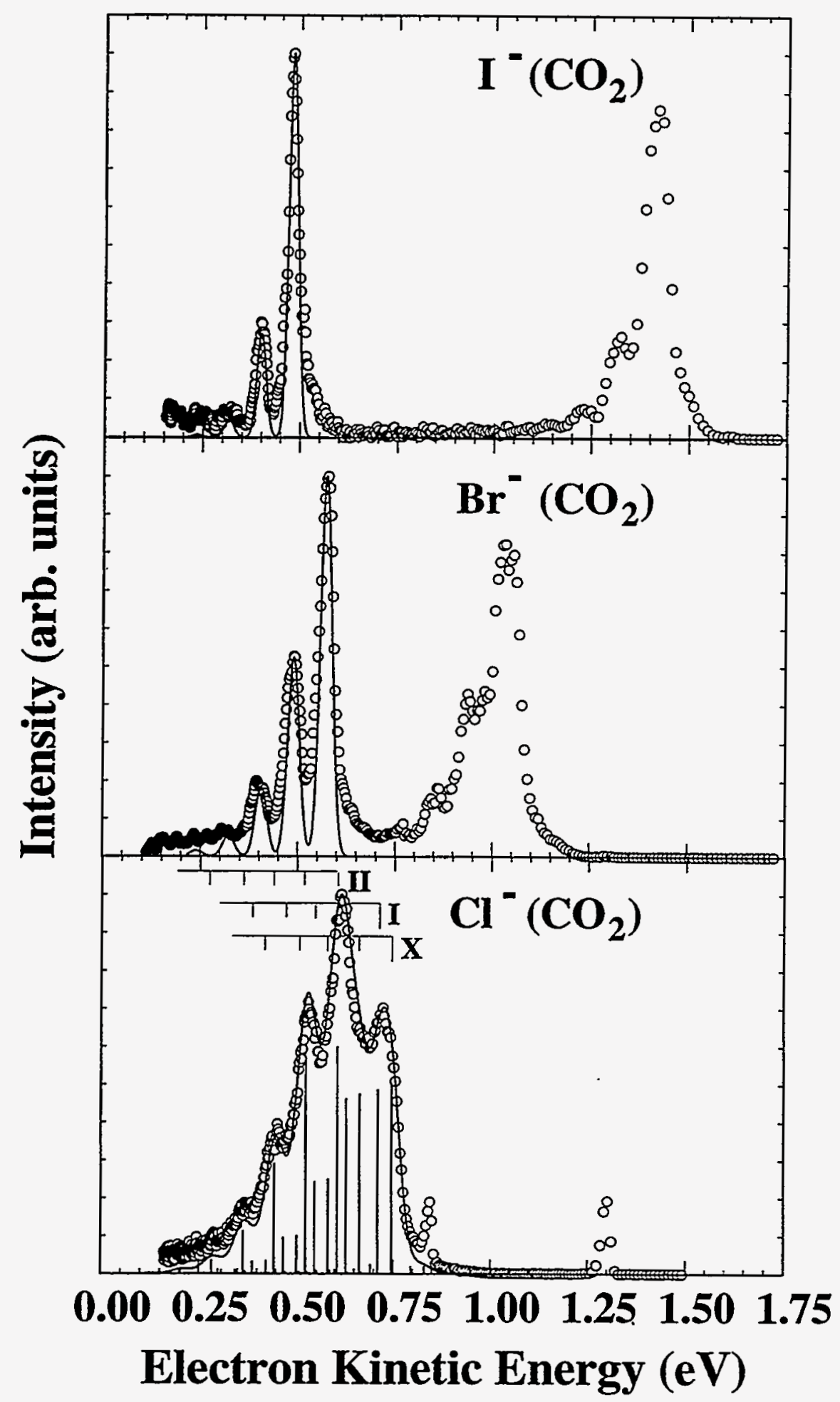

Figure 5.7 
parameters determined from the fit indicate that the vibrational frequency is $\sim 700 \pm 50 \mathrm{~cm}^{-1}$. The splitting between the $X 1 / 2$ and $I 3 / 2$ states is $35 \mathrm{meV}$ and the $I 3 / 2-\Pi 1 / 2$ splitting is 104 $\mathrm{meV}$. The larger uncertainties for these values, $10 \mathrm{meV}$, are due to the congestion of the transitions. As a comparison, Aquilanti and co-workers determine the X-I and I- $I I$ splittings for $\mathrm{Cl}-\mathrm{Ar}$ (4 and $108 \mathrm{meV}), \mathrm{Cl}-\mathrm{Kr}$ (8 and $107 \mathrm{meV}), \mathrm{Cl}-\mathrm{Xe}(18$ and $107 \mathrm{meV})$ and $\mathrm{Cl}_{-} \mathrm{CH}_{4}(16$ and $106 \mathrm{meV}$ ) using beam scattering methods. ${ }^{38}$ The larger splittings for the $\mathrm{Cl}-\mathrm{CO}_{2}$ are reasonable since the $\mathrm{CO}_{2}$ quadrupole leads to a stronger intermolecular interaction than is present in the systems studied by Aquilanti. From the optimized change in normal coordinate, the OCO bond angle in $\mathrm{Cl}^{-}\left(\mathrm{CO}_{2}\right)$ is determined to be $169.2 \pm 2.5^{\circ}$.

The simulations show that the $\mathrm{CO}_{2}$ molecule is distorted in the cluster anions and that the distortion increases as the halide decreases in size. With this observed trend, it is interesting to consider the physical basis of the distortion which occurs in the anion clusters. There are two possible mechanisms by which the $\mathrm{CO}_{2}$ can be distorted in the $\mathrm{X}^{-}\left(\mathrm{CO}_{2}\right)$ complex: (1) electrostatic interaction between the $\mathrm{X}^{-}$charge and the $\mathrm{CO}_{2}$ molecule where the negative charge is completely localized on the halide anion, and (2) charge transfer from the halide to the $\mathrm{CO}_{2}$ molecule which can decrease the $\mathrm{OCO}$ bond angle as discussed in the Introduction. Thus the questions arise: Is the distortion of the $\mathrm{CO}_{2}$ molecule attributable purely to the electrostatic interaction or is there a significant amount of charge transfer from the halide to the $\mathrm{CO}_{2}$ ? Does the nature of this interaction change significantly as the halide is changed from $\mathrm{I}^{-}$to $\mathrm{Cl}^{-}$?

\subsubsection{Electrostatic Models}

$A b$ initio calculations for the $\mathrm{Cl}^{-}\left(\mathrm{CO}_{2}\right)$ complex, discussed in Sec. 5.3.2.1, predict a bent $\mathrm{CO}_{2}$ subunit but provided little insight into the mechanism for this distortion. Therefore, two electrostatic calculations, which neglect quantum mechanical charge exchange between $\mathrm{X}^{-}$ and $\mathrm{CO}_{2}$, are presented. These calculations attempt to address the bonding in the $\mathrm{I}^{-}, \mathrm{Br}^{-}$and 
$\mathrm{Cl}^{-}$clusters and to determine how the energy of the complex changes as a function of the OCO angle. By using various approximations for the electrostatic interactions and the degree of charge transfer from the halide to the $\mathrm{CO}_{2}$, we hope to model the bonding for these complexes.

The electrostatic calculations differ in their treatment of the $\mathrm{CO}_{2}$ molecule. The simplest electrostatic model (EM) assumes the quadrupole of the $\mathrm{CO}_{2}$ can be modeled as three point charges, one on each of the three atoms. In the other model, the $\mathrm{CO}_{2}$ electrostatic properties are determined using the Distributed Multipole Analysis (DMA) of Stone and coworkers. ${ }^{39}$ The electrostatic properties of the $\mathrm{CO}_{2}$ molecule, in both models, are determined by $a b$ initio calculations using the CADPAC $a b$ initio package. ${ }^{39}$ The energy and electrostatic properties of the $\mathrm{CO}_{2}$ molecule are determined at the $\mathrm{HF} / 6-31+\mathrm{G}^{*}$ level of theory at several $\theta_{\text {oco }}$ angles between $165^{\circ}$ and $179^{\circ}$. The $\mathrm{CO}$ bond length is held at $1.1433 \AA$, the value determined in a geometry optimization of $\mathrm{CO}_{2}$ at the $\mathrm{HF} / 6-31+\mathrm{G}^{*}$ level of theory. Experiment determines the $\mathrm{CO}_{2}$ bond length to be $1.158 \AA .40$

Once the electrostatic properties of the $\mathrm{CO}_{2}$ are calculated, the $\mathrm{X}^{-}-\mathrm{CO}_{2}$ interaction energy is calculated using classical electrostatic interaction formulae. ${ }^{41}$ The halide is treated as a polarizable negative charge located at the distance from the halide to the $\mathrm{CO}_{2}$ center of mass, $\mathrm{R}_{\mathrm{X}-\mathrm{CO}_{2}}$, along the $\mathrm{C}_{2 \mathrm{v}}$ symmetry axis of the complex. The polarizability of the halides, taken from Sangachin et al., 42 are 3.82, 5.16, and 7.53 $\AA^{3}$ for $\mathrm{Cl}^{-}, \mathrm{Br}^{-}$, and $\mathrm{I}^{-}$, respectively. Although the polarizability of the halides is included in the calculation to account for induction forces, the effect of the induction forces (quadrupole-induced dipole, etc.) on the energies calculated at different geometries is found to be relatively insignificant $(<1 \%)$. Another induction term, the $\mathrm{X}^{-}-\mathrm{CO}_{2}$ charge-induced dipole term, will simply add a constant factor as a function of the $\mathrm{CO}_{2}$ angle because the $\mathrm{CO}_{2}$ polarizability will not vary significantly over the geometry changes considered. For this reason it is not included in the model. 
The EM model uses the partial charges of the $\mathrm{CO}_{2}$ atoms output by CADPAC at each $\theta_{\text {oco }}$ calculated. These charges are substituted into Eq. (7) to determine the electrostatic interaction energy for each value of $\theta_{\text {oco }}$.

$$
\mathrm{U}_{\text {elec }}=\sum_{a} \frac{q_{x} q_{a}}{r_{x a}}+\text { induction terms }
$$

In Eq. (7), $\mathrm{q}_{\mathrm{x}}=-1$ is the halide charge and $\mathrm{q}_{\mathrm{a}}$ is the partial charge of atom ' $\mathrm{a}$ ' in the $\mathrm{CO}_{2}$ molecule. As shown in Fig. 5.8, $\mathrm{r}_{\mathrm{xa}}$ is the distance between the halide and atom ' $\mathrm{a}$ ' at a particular bond angle. In all of the electrostatic calculations, the $\mathrm{CO}_{2}$ center-of-mass is held constant with respect to the $\mathrm{X}^{-}$position as the $\mathrm{OCO}$ angle is changed. One specific value of $\mathrm{R}_{\mathrm{X}-\mathrm{CO}_{2}}$ is chosen based upon our previous $\mathrm{ZEKE}$ results for $\mathrm{I}^{-}\left(\mathrm{CO}_{2}\right)$. The other $\mathrm{R}_{\mathrm{X}-\mathrm{CO}_{2}}$ values are chosen to cover the range which might be expected to occur in the $\mathrm{X}^{-}\left(\mathrm{CO}_{2}\right)$ complexes (i.e., $\left.2.5 \AA<\mathrm{R}_{\mathrm{X}-\mathrm{CO}_{2}}<4.0 \AA\right)$.

In addition to the electrostatic interactions, the $\mathrm{CO}_{2}$ distortion energy must be considered. This distortion energy is included in the $a b$ initio calculation of the total energy of the $\mathrm{CO}_{2}$ molecule. At each angle the calculated energy of the $\mathrm{CO}_{2}$ molecule, $\mathrm{U}_{\mathrm{HF}}^{\mathrm{CO}_{2}}(\varphi)$, is added to the calculated electrostatic potential energy to obtain the total energy:

$$
\mathrm{U}_{\text {tot }}(\varphi)=\mathrm{U}_{\mathrm{HF}}^{\mathrm{CO}_{2}}(\varphi)+\mathrm{U}_{\text {elec }}(\varphi)
$$

Equation (8) represents the sum of two competing terms. The first term, describing the energy of the free $\mathrm{CO}_{2}$ molecule, favors $\theta_{\text {oco }}=180^{\circ}$ while the second term, describing the interaction between the $\mathrm{X}^{-}$and $\mathrm{CO}_{2}$, drives a distortion to $\theta_{\text {oco }}<180^{\circ}$. The magnitude of this distorting

Figure 5.8. Schematic diagram of $\mathrm{X}^{-}\left(\mathrm{CO}_{2}\right)$ complex with coordinates used in the electrostatic calculations. 


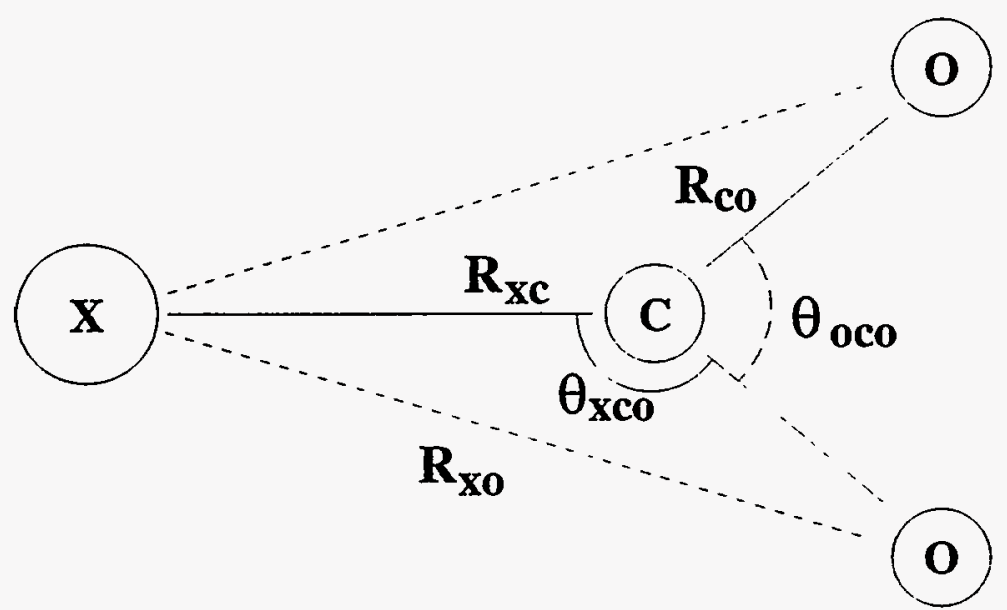

Figure 5.8 
force is dependent upon $\mathrm{R}_{\mathrm{X}-\mathrm{CO}_{2}}$. In the following discussion, the minima of all of the $U_{\text {tot }}(\varphi)$ functions calculated using Eq. (8) are offset to zero (or another appropriate value) for ease of comparison.

A more sophisticated treatment of the electrostatic forces is based upon a DMA analysis of the $\mathrm{CO}_{2}$ molecule. Stone, ${ }^{39}$ Buckingham, ${ }^{43}$ and their co-workers have successfully used the Distributed Multipole Analysis to calculate the equilibrium geometries of several van der Waals complexes. They find that the structures of the van der Waals complexes can usually be calculated by accurately determining the electrostatic properties of the molecules which comprise the clusters. The DMA approach differs from the simpler electrostatic model (EM) in that it considers the electrostatic fields of the electron charge distribution within each monomer. This results in a more detailed electrostatic field for each monomer than considered in the EM calculations. These calculations have previously been used for neutral clusters composed of rigid molecular subunits. Our application represents an extension of their method because we allow the $\mathrm{CO}_{2}$ molecules to distort as part of the energy optimization of the charged cluster. In the limit that the $\mathrm{X}^{-}\left(\mathrm{CO}_{2}\right)$ complexes are governed by purely electrostatic interactions, the DMA approach should predict geometries near that which we observe experimentally.

We now summarize the procedure followed during the DMA calculations. At each $\theta_{\text {oco, }}$, an $a b$ initio wave function for $\mathrm{CO}_{2}$, calculated using the Hartree-Fock Self Consistent Field method (SCF), is analyzed to produce a charge density map. This map includes effects from both electronic and nuclear charges. Then, a set of points is defined within the $\mathrm{CO}_{2}$ molecular framework which are used as centers for electrostatic multipole expansions. Five expansion centers are used in the calculations: one on each atom and one at each $\mathrm{CO}$ bond midpoint. The expansion coefficients are adjusted to give a best fit to the quantum mechanically determined charge distribution. At the HF/6-31+G* level of theory, the $\mathrm{CO}_{2}$ 
quadrupole is calculated to be $-4.05 \times 10^{-26} \mathrm{esu} \cdot \mathrm{cm}^{2}$ which is in reasonable agreement with the experimentally determined value of $-4.3 \times 10^{-26} \mathrm{esu} \cdot \mathrm{cm}^{2} .33$

In these calculations, the multipole expansion includes terms through hexadecapole at each expansion center. Once the multipoles are calculated, the energy of interaction between the halide and the $\mathrm{CO}_{2}$ is calculated from

$$
U_{\text {elec }}(\varphi)=\sum_{a A} \sum_{b B} U_{a b}(\varphi)
$$

where

$$
\begin{aligned}
& \left(4 \pi \varepsilon_{0}\right) U_{a b}(\varphi)=\frac{q^{a} q^{b}}{R}-R_{\alpha}\left(\mu_{\alpha}^{b} q^{a}-\mu_{\alpha}^{a} q^{b}\right) / R^{3}+ \\
& \left(3 R_{\alpha} R_{\beta}-R^{2} \delta_{\alpha \beta}\right)\left(q^{a} \theta_{\alpha \beta}^{b}+q^{b} \theta_{\alpha \beta}^{a}-3 \mu_{\alpha}^{a} \mu_{\beta}^{b}\right) / 3 R^{5}+\ldots
\end{aligned}
$$

$\mathrm{R}$ is the vector between site $\mathrm{a}$ in the $\mathrm{A}$ monomer and site $\mathrm{b}$ in the $\mathrm{B}$ monomer. In our case, $\mathrm{A}$ is the halide anion, the only electrostatic component of which is the charge, $\mathrm{q}^{\mathrm{a}}$, and $\mathrm{B}$ is the $\mathrm{CO}_{2}$ molecule. In this expression, $q, \mu$, and $\theta$ represent the charge, dipole and quadrupole moments at the sites a or $\mathrm{b}$. The angle $\varphi$ is the XCO angle of the complex. Since A only has a charge, Eq. (10) simplifies significantly to give:

$$
\left(4 \pi \varepsilon_{0}\right) U_{a b}=\frac{q^{a} q^{b}}{R}-R_{\alpha}\left(\mu_{\alpha}^{b} q^{a}\right) / R^{3}+\ldots
$$

Additionally, at each $\theta_{\text {oco, }}$, terms for the induction forces, discussed above, are included (but not shown in Eq. (10) or Eq. (11)). These include terms which account for the induction effects of the $\mathrm{CO}_{2}$ multipoles, through the quadrupole, at each geometry considered. As the $\mathrm{CO}_{2}$ bends, the dipole which forms becomes increasingly attracted to the negative charge of the halide atom, but the induction effects remain quite small. As in the EM calculations, Eq. (8) is used to calculate the total energy, $\mathrm{U}_{\text {tot }}(\varphi)$, by including the calculated Hartree-Fock energy of the $\mathrm{CO}_{2}$ molecule. 
In earlier DMA calculations for van der Waals clusters, ${ }^{43}$ a hard-sphere potential was used as a model for the repulsive potential between monomers. Since no attempt is made in this study to determine the $\mathrm{R}_{\mathrm{X}-\mathrm{CO}_{2}}$ distance in the $\mathrm{X}^{-}\left(\mathrm{CO}_{2}\right)$ cluster, this is not included. Rather, the effective potential energy curves for the $\mathrm{X}^{-}\left(\mathrm{CO}_{2}\right)$ curves are calculated at the same $\mathrm{R}_{\mathrm{X}}-\mathrm{CO}_{2}$ distances chosen for the EM calculations.

A comparison of the electrostatic calculations is shown in Fig. 5.9 where $\mathrm{R}_{\mathrm{X}-\mathrm{CO}_{2}}=3.8$ $\AA$, the bond length determined for the $\mathrm{I}^{-}\left(\mathrm{CO}_{2}\right)$ complex from the analysis of the ZEKE spectrum $^{8}$ for this complex. The plot shows the total energy, $U_{\text {tot }}(\varphi)$ as a function of the XCO bond angle, $\varphi$, over the range $90^{\circ}$ to $98^{\circ}$. For all of the calculations, a minimum in the energy of the complex occurs at a geometry away from the linear $\mathrm{CO}_{2}$ configuration. At this $\mathrm{R}_{\mathrm{X}-\mathrm{CO}_{2}}$ distance, the calculations predict approximately the same distortion of the $\mathrm{CO}_{2}, \angle \mathrm{OCO} \sim 175-$ $176^{\circ}$. These agree well with the experimentally determined $\angle \mathrm{OCO}=174.5 \pm 1.5^{\circ}$ for $\mathrm{I}^{-}\left(\mathrm{CO}_{2}\right)$. If $\mathrm{R}_{\mathrm{X}-\mathrm{CO}_{2}}$ is decreased, greater $\theta_{\mathrm{oco}}$ distortion occurs. Calculations at four different $\mathrm{R}_{\mathrm{X}-\mathrm{CO}_{2}}$ distances are shown in Fig. 5.10. The calculated minimum energy OCO angles, $\theta_{\min }$, for the calculations (Table VIII) are determined from the derivatives of a polynomial fit to the calculated data points. At each $\mathrm{R}_{\mathrm{X}-\mathrm{CO}_{2}}$ distance, $\mathrm{DMA}$ predicts less $\mathrm{CO}_{2}$ distortion than $\mathrm{EM}$. In principle, the DMA method should provide a more accurate description than EM because the increased flexibility of the multipole expansion allows for a better description of the potential field of the $\mathrm{CO}_{2}$ molecule. The increasing deviation between the EM and DMA models as $\mathrm{R}_{\mathrm{X}-\mathrm{CO}_{2}}$ decreases may result because the local potential becomes more important at the smaller $\mathrm{X}^{-}-\mathrm{CO}_{2}$ distances, where the DMA calculation more accurately accounts for the local potential

Figure 5.9. Plot of normalized $\mathrm{X}^{-}\left(\mathrm{CO}_{2}\right)$ energy as function of the $\mathrm{XCO}$ angle for the electrostatic calculations described in the text. The energies of the minima in the curves are separated by $10 \mathrm{meV}$ for clarity.

Figure 5.10. Results of Distributed Multipole Analysis (DMA) and simple electrostatic (EM) calculations of energy of the $\mathrm{X}^{-}\left(\mathrm{CO}_{2}\right)$ clusters as a function of the $\mathrm{XCO}$ bond angle. See text for calculation details. 


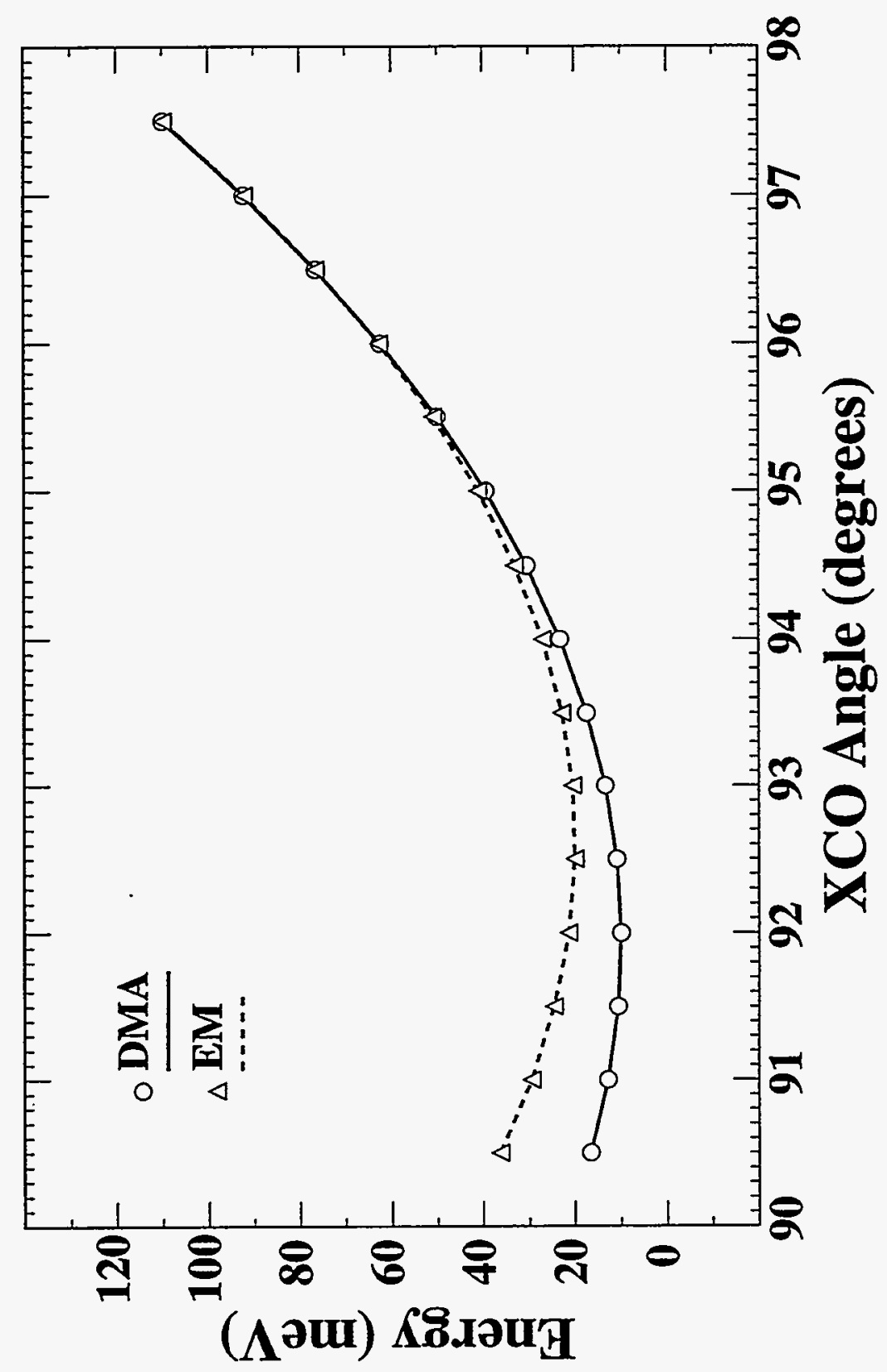

Figure 5.9 


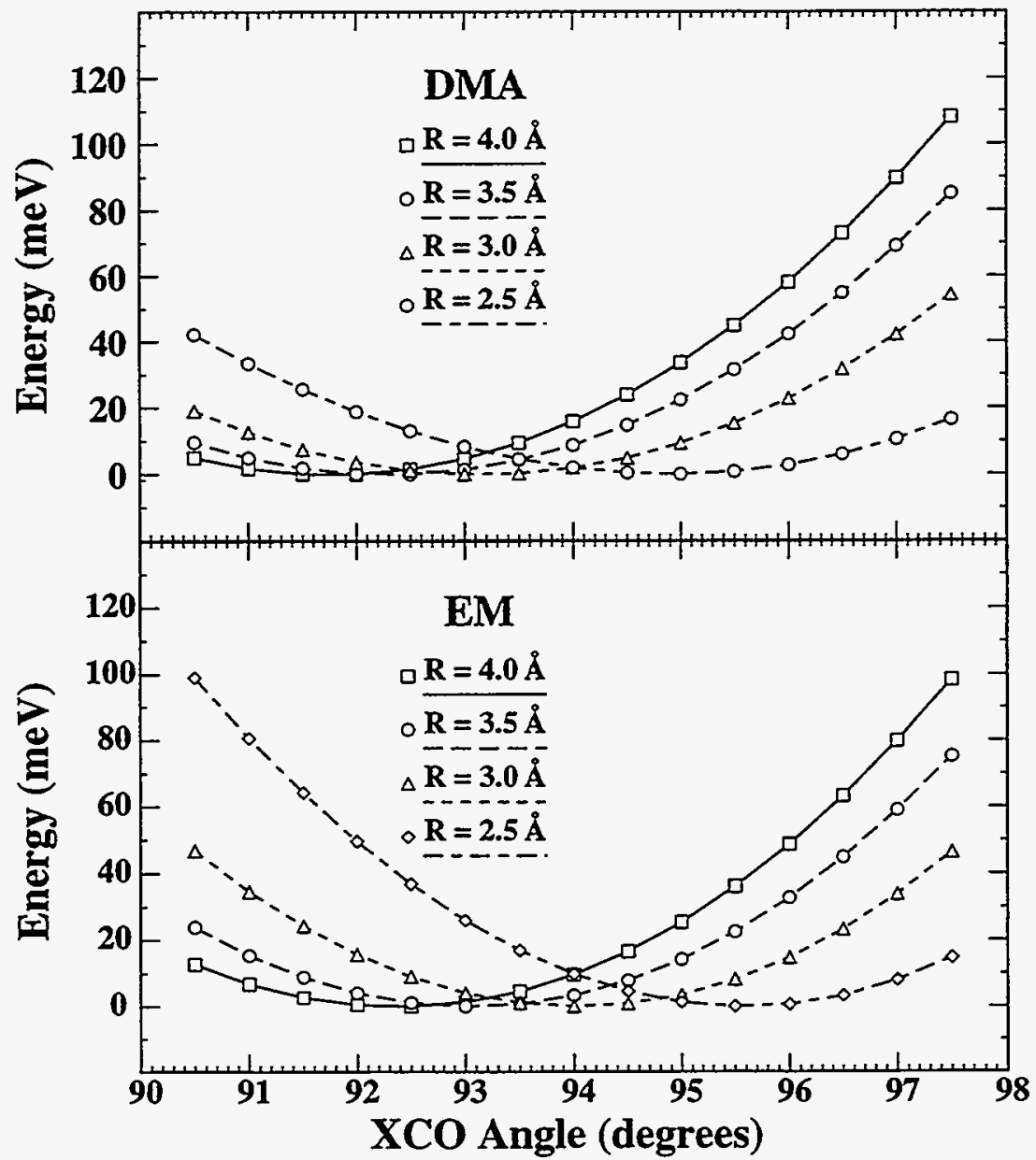

Figure 5.10 
field.

Table VII: DMA Analysis Results from Optimum OCO Angle in $\mathrm{X}^{-}\left(\mathrm{CO}_{2}\right)$

\begin{tabular}{|c|c|c|}
\hline $\mathrm{R}_{\mathrm{X}-\mathrm{C}}(\AA) \backslash$ Calculation & $\mathrm{EM}$ & $\mathrm{DMA}$ \\
\hline 2.5 & 168.8 & 170.3 \\
3.0 & 172.0 & 173.7 \\
3.5 & 174.0 & 175.7 \\
4.0 & 175.4 & 176.4 \\
\hline
\end{tabular}

While the $\mathrm{R}_{\mathrm{X}-\mathrm{CO}_{2}}$ distances for the $\mathrm{Cl}^{-}\left(\mathrm{CO}_{2}\right)$ and $\mathrm{Br}^{-}\left(\mathrm{CO}_{2}\right)$ clusters have not been experimentally determined, a comparison can be made between the electrostatic calculations, the $a b$ initio results for $\mathrm{Cl}^{-}\left(\mathrm{CO}_{2}\right)$ and the Franck-Condon analysis of the $\mathrm{Cl}^{-}\left(\mathrm{CO}_{2}\right)$ photoelectron spectrum. The MP2 and MP4 geometry optimizations for $\mathrm{Cl}^{-}\left(\mathrm{CO}_{2}\right)$ suggest that the $R_{C-C l}$ distance is $\sim 3.0 \AA$. Comparison of the MP2 potential scan along $\theta_{\text {oco }}$ with the electrostatic calculations for $\mathrm{R}_{\mathrm{X}-\mathrm{C}}=3.0 \AA$ is shown in Fig. 5.11. In these electrostatic calculations, no center of mass correction is made so that the same $R_{\mathrm{X}-\mathrm{C}}$ distances are used at each $\theta_{\text {oco }}$ in the electrostatic calculations and the MP2 potential surface scan. Excellent agreement is found with the EM calculation. As above, the DMA expansion predicts less $\mathrm{CO}_{2}$ distortion which does not agree with the $a b$ initio results. The $\theta_{\min }=171^{\circ}$ resulting from the highest level calculation performed (MP4/6-311+G* in Table IV) is just within range of the experimental result of $169.2 \pm 2.5^{\circ}$ The DMA result, $\theta_{\min }=172^{\circ}$, lies outside of the experimental uncertainty.

The small discrepancy between the electrostatic and ab initio results for the $\mathrm{Cl}^{-}\left(\mathrm{CO}_{2}\right)$ cluster may have many sources. Assuming the DMA expansion provides an accurate accounting of the electrostatic interactions in the $\mathrm{X}^{-}\left(\mathrm{CO}_{2}\right)$ clusters and that the ab initio results

Figure 5.11. Comparison of electrostatic calculations using $\mathrm{R}_{\mathrm{X}-\mathrm{C}}=3.0 \AA$ with results of $a b$ initio calculations of the energy of the $\mathrm{Cl}^{-}\left(\mathrm{CO}_{2}\right)$ complex as a function of the $\mathrm{XCO}$ bond angle. 


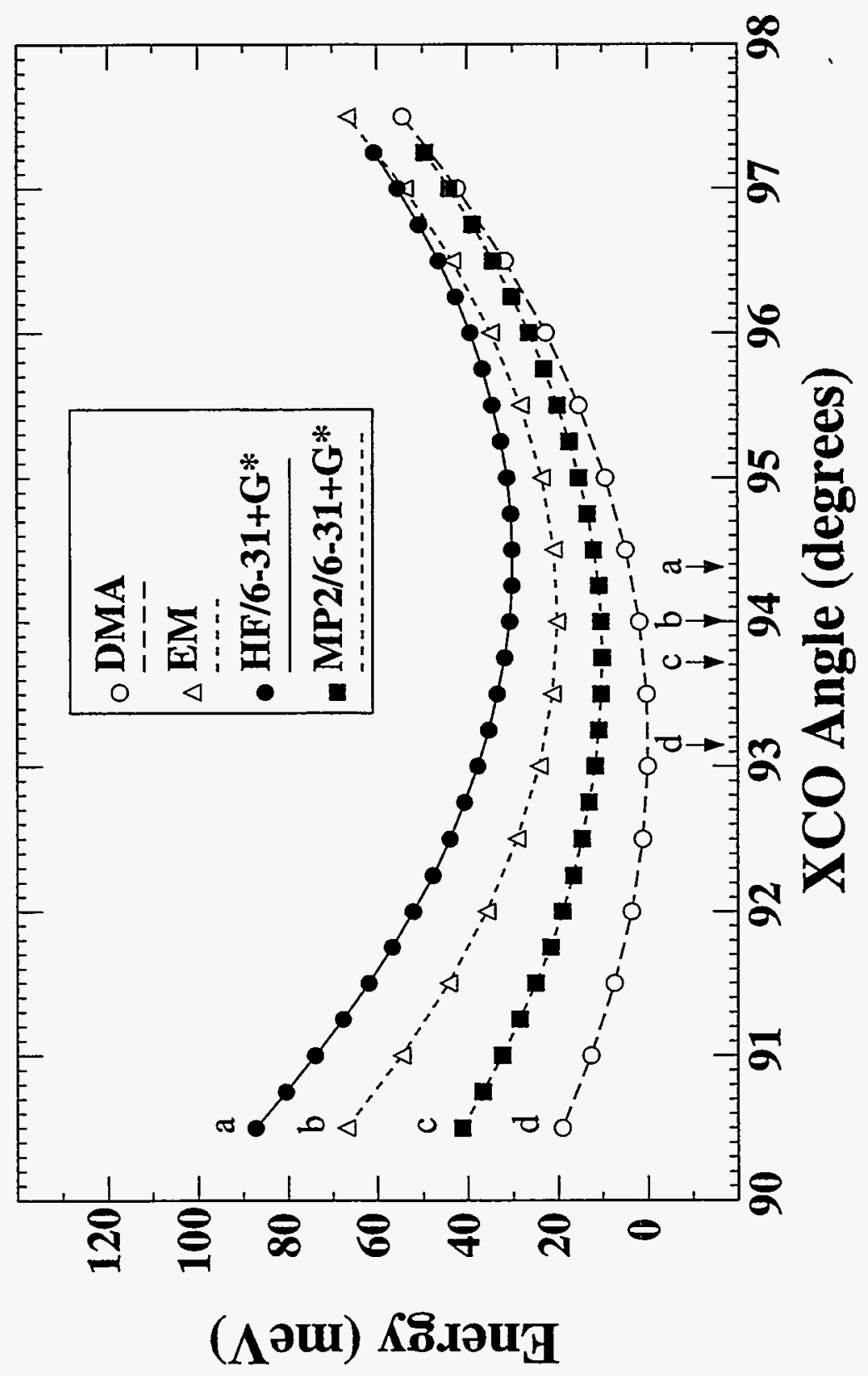

Figure 5.11 
are accurate, the results in Fig. 5.11 show that the electrostatic interactions do not fully account for the observed $\mathrm{CO}_{2}$ distortion by $\mathrm{Cl}^{-}$. As mentioned in Sec. 5.3.2.2, the ab initio calculations indicate that $\sim 6 \%$ of the negative charge is transferred from the chloride to the $\mathrm{CO}_{2}$ molecule at the MP4/6-311+G* level of theory, the effect of which would be to bend the $\mathrm{CO}_{2}$ molecule. Thus, provided the $a b$ initio calculations are correct and DMA is the best of the electrostatic calculations, the results suggest that a small amount of charge migration may occur from the $\mathrm{Cl}^{-}$to the $\mathrm{CO}_{2}$.

Another possible explanation for the difference between DMA and the $a b$ initio results may be the inaccuracy of the HF/6-31+G* wave function on which the DMA is based. The HF underestimation of the $\mathrm{CO}_{2}$ quadrupole necessarily leads to an underestimation of the $\mathrm{CO}_{2}$ distortion. Electrostatic calculations using more accurate $\mathrm{CO}_{2}$ electrostatic properties should lead to a greater $\mathrm{CO}_{2}$ distortion.

As shown in Fig. 5.11, the $R_{X-C}$ dependence of $\theta_{\min }$ is significant. Assuming the DMA model to be correct, the $\mathrm{R}_{\mathrm{X}-\mathrm{CO}_{2}}$ distances can be approximated by finding the $\mathrm{R}_{\mathrm{X}-\mathrm{CO}_{2}}$ values which provide the observed $\mathrm{CO}_{2}$ distortions. This approach suggests that the $\mathrm{R}_{\mathrm{X}-\mathrm{CO}_{2}}$ values are $\sim 3.4, \sim 2.8$ and $\sim 2.6 \AA$ for the $\mathrm{I}^{-}\left(\mathrm{CO}_{2}\right), \mathrm{Br}^{-}\left(\mathrm{CO}_{2}\right)$ and $\mathrm{Cl}^{-}\left(\mathrm{CO}_{2}\right)$ clusters, respectively. Since no repulsive terms are included in the calculations, these values are smaller than other observed or calculated values. For example, Zhao et al. find $\mathrm{R}_{\mathrm{I}-\mathrm{C}}=3.8 \AA$ and for $\mathrm{Cl}^{-}\left(\mathrm{CO}_{2}\right)^{-}$the $\mathrm{Cl}-\mathrm{C}$ separation is calculated to be $3.0 \AA$ at the MP4/6-311+G* level of theory (Table IV). These distances can also be compared with the ionic radii or van der Waals radii of the components of the $\mathrm{X}^{-}\left(\mathrm{CO}_{2}\right)$ complexes. As a simple approximation, we choose to sum the ionic radius of the halide $\left(\mathrm{I}^{-}=2.20 \AA ; \mathrm{Br}^{-}=1.96 \AA ; \mathrm{Cl}^{-}=1.81 \AA\right)$ with the van der Waals radius of a carbon atom $(\sim 1.45 \AA)$ to approximate a 'hard sphere' $\mathrm{R}_{\mathrm{X}-\mathrm{C}}$ distance. The ionic and van der Waals radii of the halides are very similar. Doing so, we find $R_{X-C}$ distances of $3.65 \AA, 3.41 \AA$, and $3.26 \AA$ for $\mathrm{X}=\mathrm{I}, \mathrm{Br}$ and $\mathrm{Cl}$, respectively. These values are larger than those 'predicted' by the DMA analysis and the $a b$ initio calculations (in the case of $\mathrm{Cl}^{-}\left(\mathrm{CO}_{2}\right)$ ). However, the hard sphere 
distances for $\mathrm{I}^{-}\left(\mathrm{CO}_{2}\right)$ is smaller than the observed distance (i.e., $3.65 \AA$ vs. $3.8 \AA$ ) while the reverse is true for $\mathrm{Cl}^{-}\left(\mathrm{CO}_{2}\right)(3.26 \AA$ vs. $3.0 \AA)$. This trend suggests that the nature of the binding evolves as the halide is changed from $\mathrm{I}^{-}$to $\mathrm{Cl}^{-}$, perhaps as a result of increasing charge transfer from the $\mathrm{X}^{-}$to the $\mathrm{CO}_{2}$ molecule.

The major point which results from the EM, DMA, and ab initio calculations is that electrostatic interactions provide the primary mechanism for the distortion of the $\mathrm{CO}_{2}$ molecule by the $\mathrm{I}^{-}, \mathrm{Br}^{-}$and $\mathrm{Cl}^{-}$halide anions, but that charge transfer from $\mathrm{X}^{-}$to $\mathrm{CO}_{2}$ may increase as $\mathrm{X}$ becomes smaller. The dominant role of electrostatic interactions in the $\mathrm{X}^{-}\left(\mathrm{CO}_{2}\right)$ clusters is consistent with the results of high pressure mass spectrometry results ${ }^{3,4}$ where the sequential binding energies for $\mathrm{X}^{-}\left(\mathrm{CO}_{2}\right)_{\mathrm{n}}$ clusters are relatively constant for the first few $\mathrm{CO}_{2}$ molecules which attach to the halide.

\subsection{2. $\mathrm{FCO}_{2}^{-}$}

\subsubsection{Spectroscopy and Franck-Condon Analysis}

As discussed in Sec. 5.3.1.1, the $\mathrm{FCO}_{2}^{-}$photoelectron spectrum (Fig. 5.4) contains contributions from photodetachment transitions to two different $\mathrm{FCO}_{2}$ electronic states. The states of $\mathrm{FCO}_{2}$ which may be observed by this technique are those which can be formed by oneelectron photodetachment of $\mathrm{FCO}_{2}^{-}$. Removal of an electron from any of the four highest-lying occupied molecular orbitals of the $\mathrm{FCO}_{2}^{-} \tilde{\mathrm{X}}{ }^{1} \mathrm{~A}_{1}$ ground state $\left(. .\left(2 \mathrm{~b}_{1}\right)^{2}\left(8 \mathrm{a}_{1}\right)^{2}\left(5 \mathrm{~b}_{2}\right)^{2}\left(1 \mathrm{a}_{2}\right)^{2}\right)$ will lead to the formation of the ${ }^{2} \mathrm{~A}_{2},{ }^{2} \mathrm{~B}_{2},{ }^{2} \mathrm{~A}_{1}$, and ${ }^{2} \mathrm{~B}_{1}$ electronic states of the $\mathrm{FCO}_{2}$ radical, in $\mathrm{C}_{2 \mathrm{v}}$ symmetry. The three orbitals are shown in Fig. 5.12. The assignment of the electronic states observed in the spectrum is assisted by ab initio calculations and previous observations for $\mathrm{FCO}_{2}$ and other isoelectronic molecules.

The QCISD/6-31G* $a b$ initio calculations predict the ${ }^{2} \mathrm{~B}_{2}$ state to be the ground state lying 0.437 and $1.692 \mathrm{eV}$ below the ${ }^{2} \mathrm{~A}_{2}$ and ${ }^{2} \mathrm{~A}_{1}$ electronic states, respectively. As mentioned earlier, the ${ }^{2} \mathrm{~A}_{1}$ and ${ }^{2} \mathrm{~B}_{1}$ states lie above the energy which is accessible by photodetachment 
with a $5.822 \mathrm{eV}$ photon energy. In accord with these results, the progression beginning at peak $A$ is assigned to transitions to the $\bar{X}{ }^{2} B_{2}$ ground state. This is consistent with the previous assignment of the $\tilde{\mathrm{B}}{ }^{2} \mathrm{~A}_{1} \leftarrow \tilde{\mathrm{X}}{ }^{2} \mathrm{~B}_{2}$ absorption by Maricq et al. ${ }^{12}$ The irregular set of peaks beginning at peak $\mathrm{K}$ and extending to lower $\mathrm{KEE}$ is assigned as photodetachment transitions to the $\tilde{\mathrm{A}}{ }^{2} \mathrm{~A}_{2}$ state. Weaver et al. ${ }^{44}$ find that the lowest-lying excited state of the isoelectronic $D_{3 \mathrm{~h}}$ $\mathrm{NO}_{3}$ molecule is the doubly degenerate ${ }^{2} \mathrm{E}$ " state, which lies $0.868 \pm 0.014 \mathrm{eV}$ above the ${ }^{2} \mathrm{~A}_{2}{ }^{\prime}$ ground state. This is consistent with the state ordering for $\mathrm{FCO}_{2}$ since the $\mathrm{D}_{3 \mathrm{~h}}{ }^{2} \mathrm{E}$ " state correlates to the $C_{2 v}{ }^{2} B_{1}$ and ${ }^{2} A_{2}$ states and the ${ }^{2} A_{2}$ s state correlates to the ${ }^{2} B_{2}$ state.

Further support for these assignments is obtained through a Franck-Condon analysis of the vibrational features observed in the spectra of both electronic states. For the $\overline{\mathrm{X}}{ }^{2} \mathrm{~B}_{2}$ ground state, the $\omega_{e}=485 \mathrm{~cm}^{-1}$ and $x_{e} \omega_{e}=2 \mathrm{~cm}^{-1}$ values determined from a least squares analysis of the peak positions A-J agree marginally with the $v_{3}{ }^{\prime \prime}=520 \mathrm{~cm}^{-1}$ value determined from hot bands in the absorption data. ${ }^{9}$ Similar agreement is found with the calculated $v_{3}$ frequency (Table VI). However, simulations of the spectrum, discussed below, reveal that the peaks in this progressions are not solely attributable to a single progression in the $v_{3}$ vibrational mode.

Due to symmetry, photodetachment of a negative ion primarily results in the excitation of totally symmetric vibrational modes. Shown in Fig. $5.12 \mathrm{~b}$ are the three symmetric modes of $\mathrm{FCO}_{2}$ : the $\mathrm{C}-\mathrm{F}$ stretch, $\mathrm{C}-\mathrm{O}$ stretch and $\mathrm{CO}_{2}$ bend. The degree to which each of these modes are excited upon photodetachment, or the length of the observed vibrational progression for each mode, is determined by the difference in geometries and force constants of the $\mathrm{FCO}_{2}^{-}$anion and the $\mathrm{FCO}_{2}$ radical. In terms of molecular orbitals (MOs), Fig. 5.12 shows that the removal of an electron from either the $\left(5 b_{2}\right)$ or the $\left(1 a_{2}\right)$ orbital of $\mathrm{FCO}_{2}^{-}$will lead to a relatively smaller OCO

Figure 5.12. Relevant molecular orbitals (a, top) and vibrational normal coordinates (b, bottom) for $\mathrm{FCO}_{2}^{-}$. MOs are based upon the results of a QCISD/6-31G* ab initio calculation. 
a)
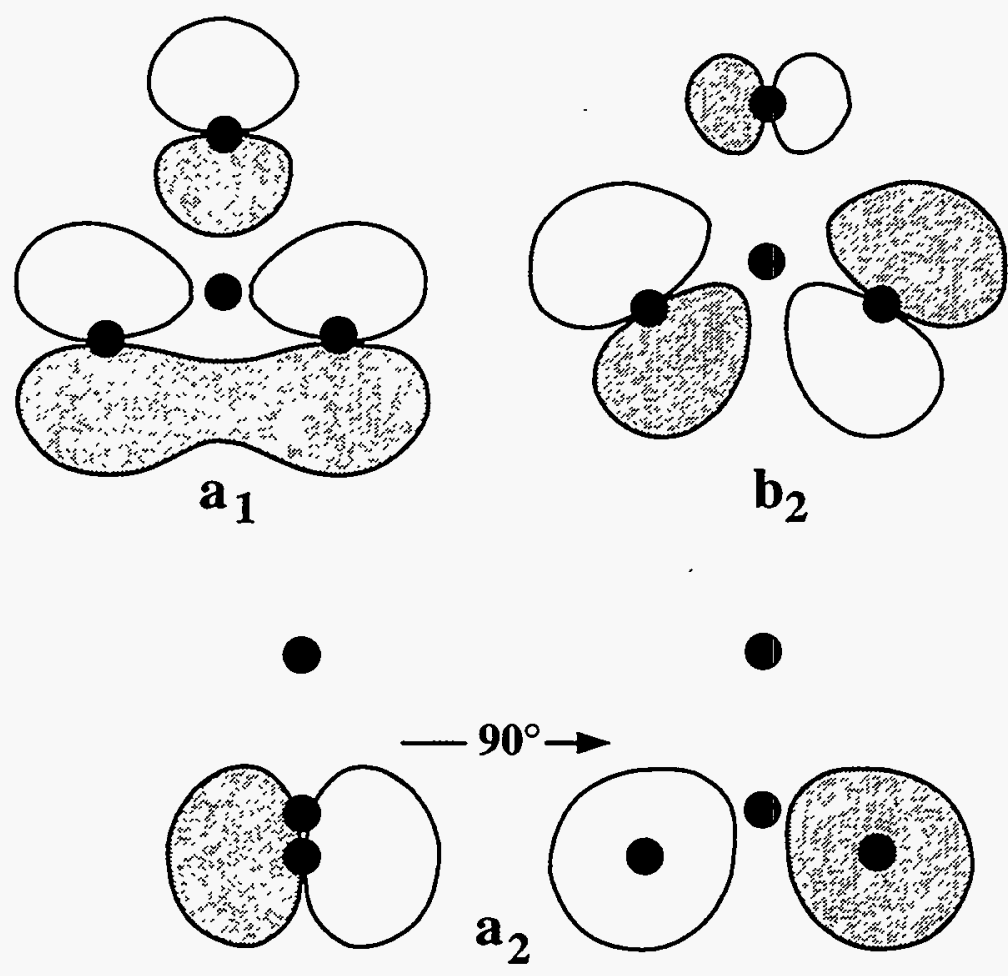

b)

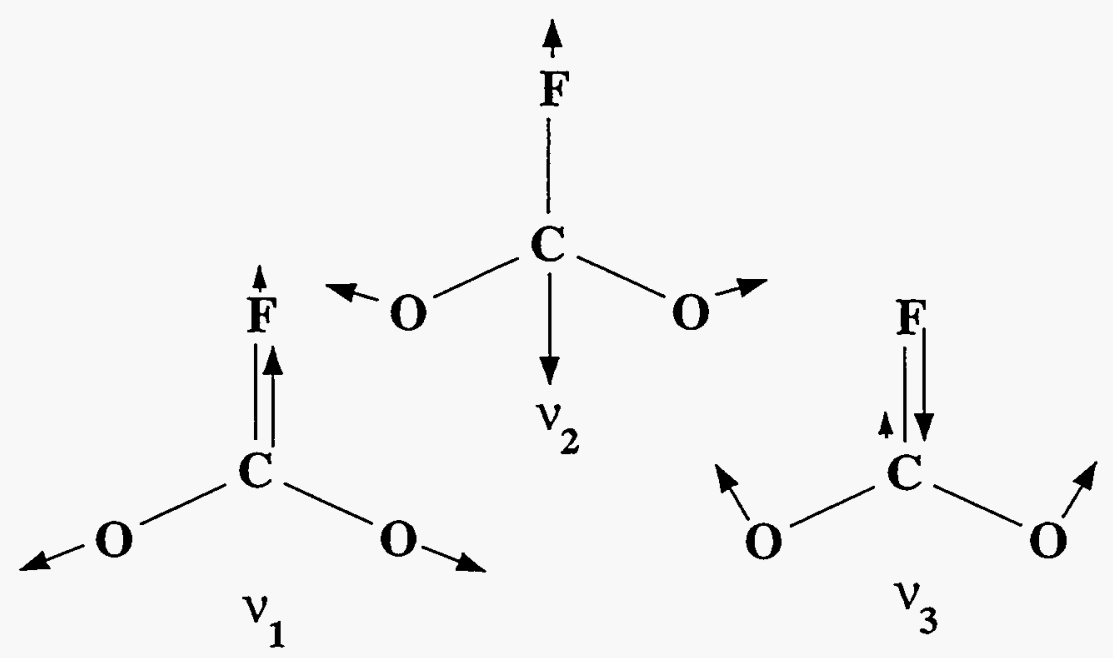

Figure 5.12 
angle for the neutral $\mathrm{FCO}_{2}$ radical. Qualitatively, the $a b$ initio calculations (Tables $\mathrm{V}$ and VI) indicate that all three modes will be excited for both the ${ }^{2} \mathrm{~B}_{2}$ and ${ }^{2} \mathrm{~A}_{2}$ states because the $\mathrm{FCO}_{2}^{-}$ and $\mathrm{FCO}_{2}$ geometries are quite different.

In Sec. 5.4.1.3, the $\mathrm{CO}_{2}$ distortion in the $\mathrm{X}^{-}\left(\mathrm{CO}_{2}\right)$ clusters was determined using a Franck-Condon analysis. By assuming a reasonable geometry for the $\mathrm{X}\left(\mathrm{CO}_{2}\right)$ neutral, the anion geometry was readily determined. In the case of $\mathrm{FCO}_{2}^{-}$, no reasonable assumption can be made for geometry of the covalent neutral species. However, it is possible to compare, quantitatively, the $\mathrm{FCO}_{2}^{-}$photoelectron spectrum with simulations based upon the ab initio results presented in Sec. 5.3.2.2.

The calculated geometries and force constant matrices (Sec. 5.3.2.2) can easily be used to predict a photoelectron spectrum of $\mathrm{FCO}_{2}^{-}$. By standard matrix methods, ${ }^{45}$ the $a b$ initio force constants are used to convert the calculated geometry difference between the anion and neutral into displacements, $\Delta \mathrm{Q}$, along each neutral normal mode. The resultant $\Delta \mathrm{Q}$ values are used as input for the calculation of Franck-Condon overlap between $\mathrm{FCO}_{2}^{-}$and $\mathrm{FCO}_{2}$. The FranckCondon principle determines the photodetachment transition intensities, I, according to Eq. (12):

$$
\mathbf{I}=\mathrm{v}_{\mathrm{e}} \cdot\left|\tau_{\mathrm{e}}\right|^{2} \cdot \prod_{i=1}^{6}\left|\left\langle\psi_{\mathrm{v}_{\mathrm{i}}^{\prime}}\left(Q_{i}\right) \mid \psi_{\mathrm{v}_{\mathrm{i}}^{\prime}}\left(Q_{i}\right)\right\rangle\right|^{2}
$$

As in Eq. (3), $v_{e}$ is the asymptotic velocity of the detached electron and $\tau_{e}$ is the electronic transition dipole. The major approximation of this analysis is that the vibrational normal modes of the anion and neutral are separable and parallel (i.e., no Duschinsky rotation). Unlike Eq. (3), the 'multidimensionality' of the problem leads to combination bands which are calculated as a product of Franck-Condon factors for each mode. The Franck-Condon factor, $\left|\left\langle\Psi_{v_{i}^{\prime}} \mid \Psi_{v_{i}^{\prime \prime}}\right\rangle\right|^{2}$, as before, is related to the spatial overlap of the vibrational wave functions for the $\mathrm{i}^{\text {th }}$ mode of the anion,$\Psi_{v_{i}^{\prime \prime}}$, and neutral, $\Psi_{v_{i}^{\prime}}$. The resultant stick spectrum is convoluted with the experimental 
resolution function plus an additional $10 \mathrm{meV}$ Gaussian to account for space-charge effects and rotational contours for comparison with the experimental data.

For the $\overline{\mathrm{X}}{ }^{2} \mathrm{~B}_{2}$ ground state, a Franck-Condon analysis using the QCISD/6-31G* geometries and force constants ${ }^{46}$ for $\mathrm{FCO}_{2}^{-}$and $\mathrm{FCO}_{2}$ leads to the simulations shown in Fig. 5.13. The stick spectrum shows that all three symmetric vibrational modes are moderately excited upon photodetachment of $\mathrm{FCO}_{2}^{-}$. However, when the FCF's are convoluted with the experimental resolution, Fig. 5.13, a long series of peaks results which resemble the ground state spectrum of $\mathrm{FCO}_{2}$. Since the three symmetric vibrational frequencies are nearly multiples of $\sim 500 \mathrm{~cm}^{-1}$, the individual peaks for each progression are not resolved, but rather convoluted into a progression which might easily be interpreted as a progression in a single vibrational mode peaking at $v^{\prime}=7$. In fact, the Franck-Condon analysis indicates that for the $v_{1}, v_{2}$, and $v_{3}$ modes, the most intense transitions occur at $v_{1}^{\prime}=0, v_{2}^{\prime}=1$, and $v_{3}^{\prime}=2$, respectively.

The significant amount of vibrational structure in the spectrum of the $\overline{\mathrm{A}}{ }^{2} \mathrm{~A}_{2}$ state, beginning at peak $K$, suggests that more than one vibrational mode is excited upon photodetachment of $\mathrm{FCO}_{2}^{-}$to the first excited state of $\mathrm{FCO}_{2}$. However, better separation of the vibrational frequencies results in a less convoluted spectrum as compared to the ground state. Using the QCISD/6-31G* optimized parameters for $\mathrm{FCO}_{2}^{-}$and the $\mathrm{FCO}_{2}{ }^{2} \mathrm{~A}_{2}$ state, the FranckCondon analysis produces a simulation (Fig. 5.14) which agrees remarkably well with observed spectral profile and spacings. As indicated in the Figure, all three totally symmetric vibrational modes are excited upon photodetachment. The excellent agreement between the experimental

Figure 5.13. Franck-Condon simulations for the $\mathrm{FCO}_{2} \overline{\mathrm{X}}{ }^{2} \mathrm{~B}_{2} \leftarrow \mathrm{FCO}_{2}^{-} \overline{\mathrm{X}}{ }^{1} \mathrm{~A}_{1}$ photodetachment process predicted by QCISD/6-31G* calculated geometries and force constants for anion and neutral. The Franck-Condon factors are convoluted with the experimental resolution in the top frame while the stick spectrum is shown at bottom. The simulations show that the experimentally observed progression represents moderate excitation of all three totally symmetric vibrational modes upon photodetachment of $\mathrm{FCO}_{2}^{-}$. 


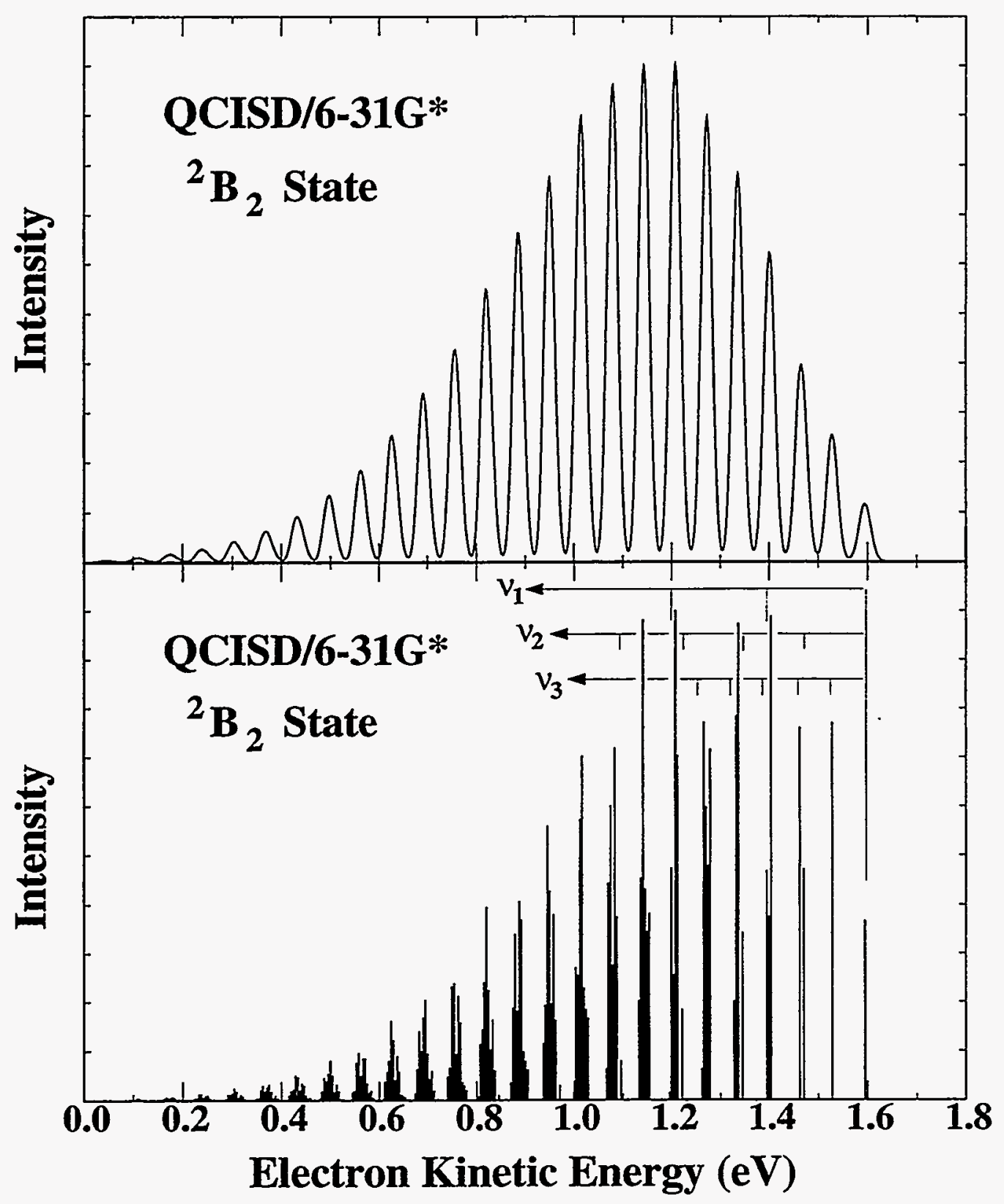

Figure 5.13 
photoelectron spectrum and the $a b$ initio simulations indicates that the QCISD/6-31G* calculated results are reasonably accurate.

The $a b$ initio simulations serve as a starting point to fit the spectrum within the FranckCondon approximation. Shown in Fig. 5.15 is a best fit simulation of the data assuming that peaks $A$ and $K$ represent the origins of the $\bar{X}{ }^{2} B_{2}$ and $\tilde{A}{ }^{2} A_{2}$ states of the $F_{C O}$ radical, respectively. The simulation was generated beginning with those shown in Fig. 5.13a and Fig. 5.14a and adjusting the changes in normal coordinates and the neutral vibrational frequencies to obtain the best agreement with the experimental peak intensities and shapes. The $\mathrm{FCO}_{2}^{-}$ frequencies employed are also adjusted in the simulation at a vibrational temperature of $300 \mathrm{~K}$. The optimized values (Table VII) do not differ significantly from the matrix values determined by Ault. ${ }^{5}$ In the case of the $\mathrm{CO}$ symmetric stretch of the $\mathrm{FCO}_{2}{ }^{2} \mathrm{~A}_{2}$ excited state, a Morse potential with $\omega_{\mathrm{e}}=1320 \mathrm{~cm}^{-1}$ and $\mathrm{x}_{\mathrm{e}} \omega_{\mathrm{e}}=8 \mathrm{~cm}^{-1}$ was used to match the anharmonicity observed for that mode in the spectrum.

Given the normal coordinate displacements, we can determine the geometries of the neutral states if we assume the $a b$ initio $\mathrm{FCO}_{2}^{-}$geometry is correct. In this analysis, the signs of the normal coordinate displacements are chosen to be consistent with those predicted by the $a b$ initio force constants and geometries. Projection of the optimized normal coordinate displacements onto the raw $a b$ initio transformation matrices provides the geometries of the $\bar{X}$ ${ }^{2} \mathrm{~B}_{2}$ and $\tilde{\mathrm{A}}{ }^{2} \mathrm{~A}_{2}$ states of the $\mathrm{FCO}_{2}$ radical. The geometries which result from this analysis are given in Table IX. The agreement with the $a b$ initio values is excellent for the ${ }^{2} \mathrm{~A}_{2}$ state and

Figure 5.14. Franck-Condon simulation for the $\mathrm{FCO}_{2} \tilde{\mathrm{A}}{ }^{2} \mathrm{~A}_{2} \leftarrow \mathrm{FCO}_{2}^{-} \tilde{\mathrm{X}}{ }^{1} \mathrm{~A}_{1}$ photodetachment process predicted by QCISD/6-31G* calculated geometries and force constants for anion and neutral.

Figure 5.15. Franck-Condon simulation (top) for both the $\mathrm{FCO}_{2} \tilde{\mathrm{X}}^{2} \mathrm{~B}_{2} \leftarrow \mathrm{FCO}_{2}^{-} \tilde{\mathrm{X}}{ }^{1} \mathrm{~A}_{1}$ and the $\mathrm{FCO}_{2} \tilde{\mathrm{A}}{ }^{2} \mathrm{~A}_{2} \leftarrow \mathrm{FCO}_{2}^{-} \tilde{\mathrm{X}}^{1} \mathrm{~A}_{1}$ photodetachment transitions in which the normal coordinate displacements and vibrational frequencies have been adjusted to obtain the best agreement with the experimental data.(bottom). 


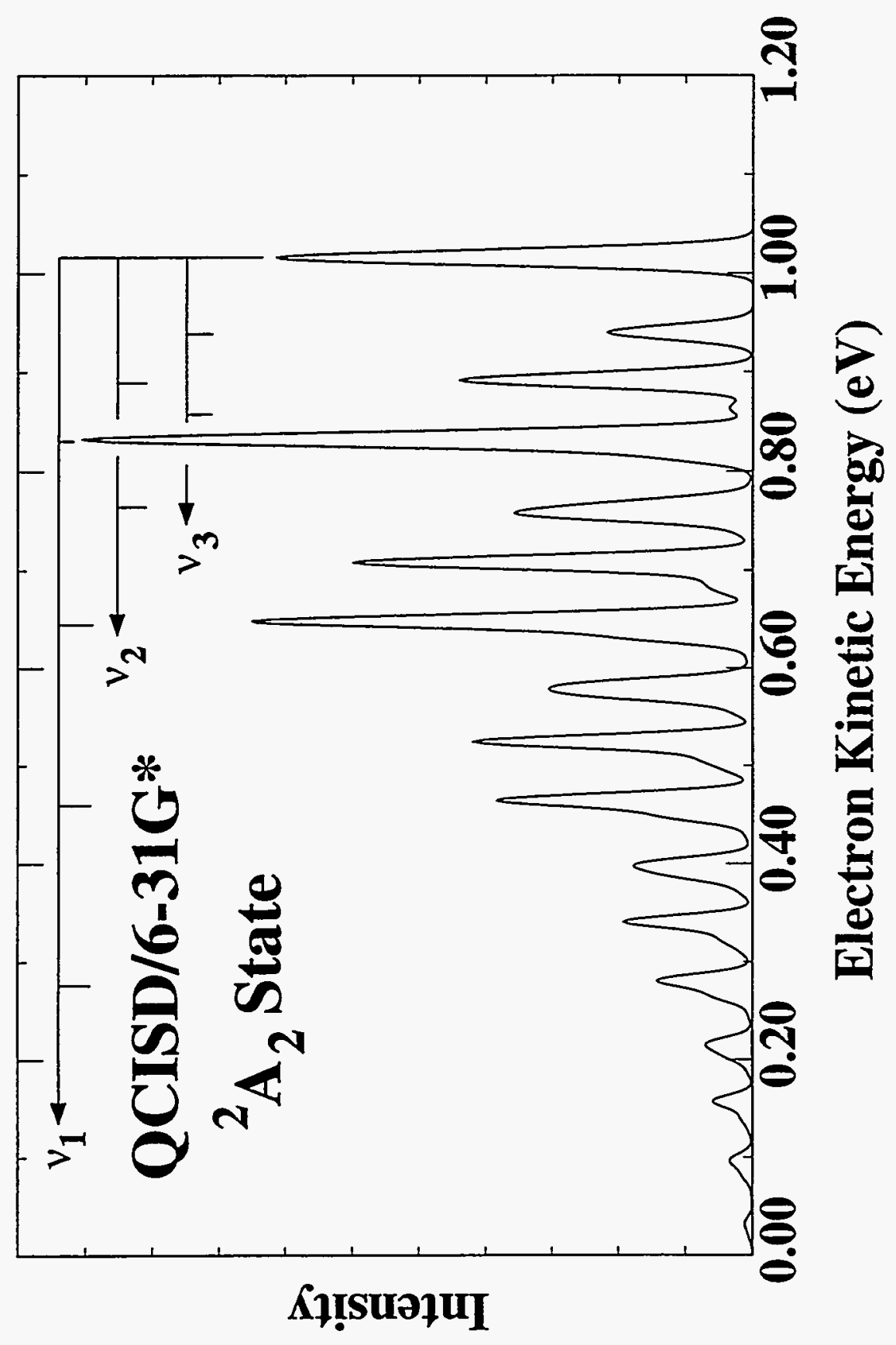

Figure 5.14 


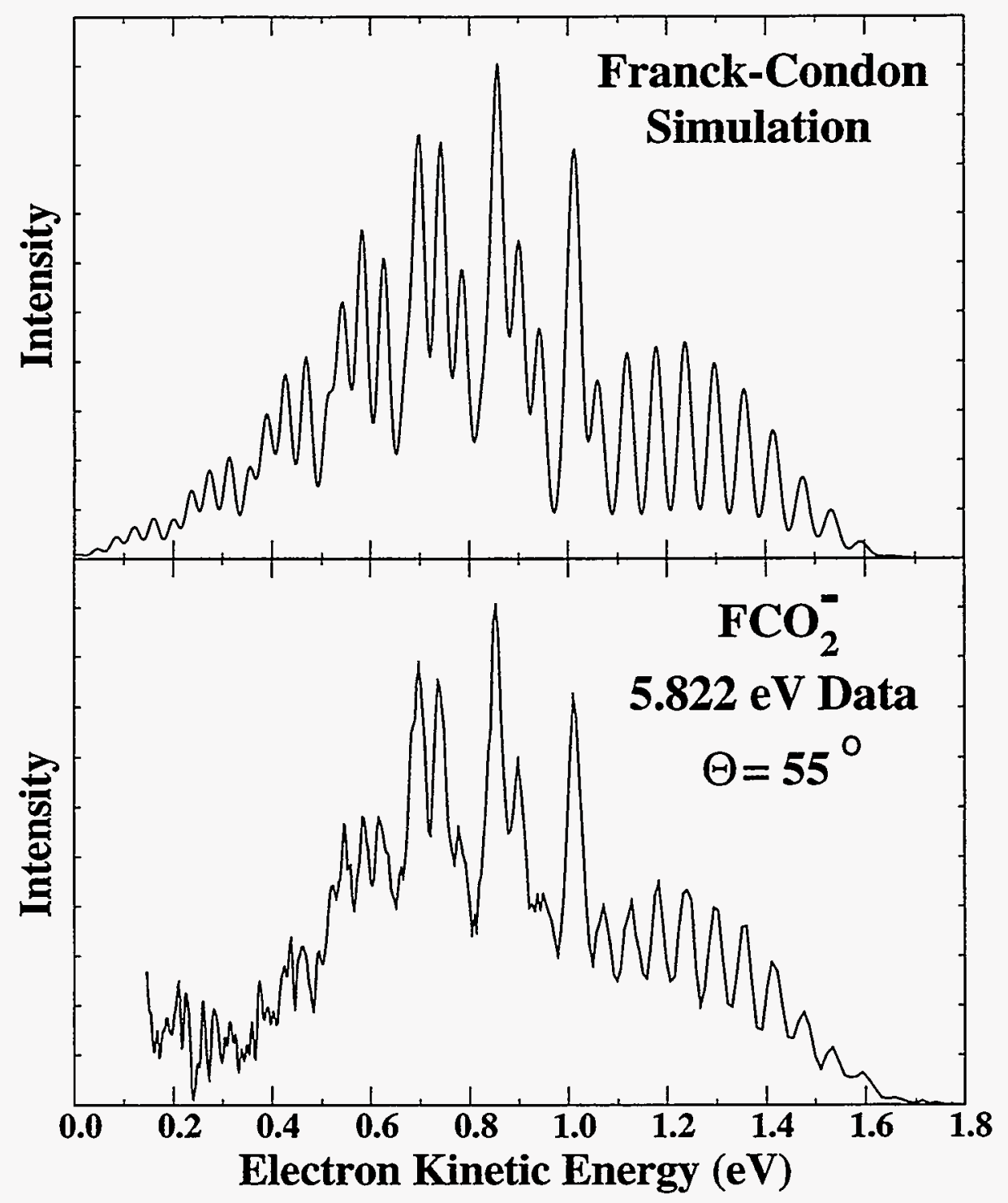

Figure 5.15 
reasonably good for the ground state. Of course, the accuracy of the neutral geometries determined from this FC analysis is dependent upon the accuracy of the anion geometry and the neutral force constant matrices, as well as the simplifications made in the analysis (i.e., separable and parallel modes). However, the overall agreement of the calculated and fit neutral geometries further supports the assignments.

Table IX: Optimized Parameters for Franck-Condon Simulation of the $\mathrm{FCO}_{2}^{-}$Photoelectron Spectrum

\begin{tabular}{|c|c|c|c||}
\hline \hline State & $\mathrm{FCO}_{2}{ }^{2} \mathrm{~B}_{2}$ & $\mathrm{FCO}_{2}{ }^{2} \mathrm{~A}_{2}$ & $\mathrm{FCO}_{2}^{-1} \mathrm{~A}_{1}$ \\
\hline \hline $\mathrm{T}_{0} \mathrm{eV}$ & 0.0 & 0.576 & 0.0 \\
$\omega_{1}\left(\mathrm{~cm}^{-1}\right)$ & $1465(70)$ & $1310(40)$ & 1399 \\
$\mathrm{x}_{11} \omega_{1}\left(\mathrm{~cm}^{-1}\right)$ & 0.0 & $8.0(4)$ & 0.0 \\
$\omega_{2}\left(\mathrm{~cm}^{-1}\right)$ & $950(70)$ & $920(40)$ & 899 \\
$\omega_{3}\left(\mathrm{~cm}^{-1}\right)$ & $500(70)$ & $577(40)$ & 596 \\
$\mathrm{R}_{\mathrm{FC}}$ & $1.38 \AA^{\mathrm{a}}$ & $1.33 \AA^{\mathrm{b}}$ & $1.459 \AA^{\mathrm{c}}$ \\
$\mathrm{R}_{\mathrm{CO}}$ & $1.23 \AA^{\mathrm{a}}$ & $1.26 \AA^{\mathrm{b}}$ & $1.235 \AA^{\mathrm{c}}$ \\
$\theta_{\mathrm{OCO}}$ & $119^{\circ \mathrm{a}}$ & $125^{\circ \mathrm{b}}$ & $135.9^{\circ \mathrm{c}}$ \\
\hline
\end{tabular}

aMP2/6-31+G* ${ }^{2} \mathrm{~B}_{2}$ force constants used. ${ }^{b} \mathrm{QCISD} / 6-31+\mathrm{G}^{*} \mathrm{~A}_{2}$ force constants used.

cValues fixed to those calculated at the QCISD/6-31G* level of theory.

\subsubsection{Electron Affinity and Thermochemistry}

As in Sec. 5.4.1.1, Eq. (2) shows that the EA of $\mathrm{FCO}_{2}$ is readily calculated as the difference between the photon energy and the eKE of the 0-0 transition. Although the intensity of peak ' $\mathrm{A}$ ' is quite low, the Franck-Condon simulations in the previous section support the assignment of this feature as the 0-0 transition. With this assignment, according to Eq. (2), we find $\mathrm{EA}\left(\mathrm{FCO}_{2}\right)=4.277 \pm 0.030 \mathrm{eV}$. The sizable uncertainty for the experimental electron affinity results from the high $\mathrm{eKE}$ of peak ' $\mathrm{A}$ ' combined with the uncertainty associated with the necessary 'space-charge' correction. The MP2/6-31+G* and MP2/6-311+G* calculations predict $\mathrm{EA}\left(\mathrm{FCO}_{2}\right)$ values (4.549 and $4.295 \mathrm{eV}$, respectively) near the experimentally determined value. Calculations using basis sets without diffuse functions underestimate 
$\mathrm{EA}\left(\mathrm{FCO}_{2}\right)$ significantly (i.e., $\mathrm{EA}\left(\mathrm{FCO}_{2}\right)=3.176$ and $3.319 \mathrm{eV}$ at the $\mathrm{MP} 2 / 6-31 \mathrm{G}^{*}$ and QCISD/6-31G* levels, respectively), illustrating the importance of diffuse functions for the accurate calculations of anion energies.

With our value for $\mathrm{EA}\left(\mathrm{FCO}_{2}\right)$, it is possible to determine the value of $\mathrm{D}_{\mathrm{o}}\left(\mathrm{F}-\mathrm{CO}_{2}\right)$ using Eq. (4) as in Sec. 5.4.1.1. Using ion cyclotron resonance, McMahon and co-workers ${ }^{2}$ bracket the fluoride affinity of carbon dioxide as $\mathrm{D}_{0}\left(\mathrm{~F}^{-}-\mathrm{CO}_{2}\right)=31.7 \pm 2 \mathrm{kcal} / \mathrm{mole}$. Hiraoka et al. ${ }^{4}$ find a slightly higher value of $32.3 \mathrm{kcal} /$ mole but report no estimated uncertainty for their value. Substitution of McMahon's dissociation energy into Eq. (4) yields $\mathrm{D}_{0}\left(\mathrm{~F}-\mathrm{CO}_{2}\right)=11.5 \pm 3$ $\mathrm{kcal} / \mathrm{mole}(0.5 \mathrm{eV})$, substantially larger than the value of $\mathrm{D}_{0}\left(\mathrm{~F}-\mathrm{CO}_{2}\right)=3.6 \mathrm{kcal} / \mathrm{mole}$ calculated by Francisco and co-workers. ${ }^{47}$ The $\mathrm{D}_{0}\left(\mathrm{FCO}_{2}\right)$ value will be larger than $11.5 \mathrm{kcal} / \mathrm{mole}$ if peak A represents photodetachment to a vibrationally excited level of the $\mathrm{FCO}_{2} \tilde{\mathrm{X}}{ }^{2} \mathrm{~B}_{2}$ ground state rather than a transition to the origin of this state.

Carbon-fluorine bond strengths for other halocarbon compounds are measured to be $\sim 100 \mathrm{kcal} / \mathrm{mole}$. Some example values ${ }^{48}$ are $\mathrm{D}_{\mathrm{o}}\left(\mathrm{H}_{3} \mathrm{C}-\mathrm{F}\right)=109.9 \pm 1 \mathrm{kcal} / \mathrm{mole}, \mathrm{D}_{0}\left(\mathrm{~F}_{3} \mathrm{C}-\mathrm{F}\right)=$ $130.5 \pm 1 \mathrm{kcal} / \mathrm{mole}, \mathrm{D}_{\mathrm{o}}\left(\mathrm{Cl}_{3} \mathrm{C}-\mathrm{F}\right)=101.9 \pm 1$ and $\mathrm{D}_{\mathrm{o}}\left(\mathrm{F}_{2} \mathrm{HC}-\mathrm{F}\right)=85.3 \pm 1 \mathrm{kcal} / \mathrm{mole}$. Compared to other $\mathrm{C}-\mathrm{F}$ bond strengths, both the $\mathrm{FCO}_{2}^{-}$and $\mathrm{FCO}_{2}$ molecules have quite weak $\mathrm{C}-\mathrm{F}$ bonds. As McMahon and Northcott ${ }^{2 \mathrm{~b}}$ point out for $\mathrm{FCO}_{2}^{-}$(and for $\mathrm{FCO}_{2}$ ), the C-F bond is weak because its formation requires a perturbation of the strong $\pi$-bonding of the $\mathrm{CO}_{2}$ molecule.

It is also interesting to compare the C-F bond lengths and bond dissociation energies for $\mathrm{FCO}_{2}$ and $\mathrm{FCO}_{2}^{-}$. Although the $\mathrm{C}-\mathrm{F}$ bond enthalpy of $\mathrm{FCO}_{2}^{-}$is measured to be approximately triple that of $\mathrm{FCO}_{2}$, the $\mathrm{C}-\mathrm{F}$ bond length is predicted to be $\sim 0.2 \AA$ longer in the anion. All of the neutral $\mathrm{FCO}_{2}$ electronic states studied are predicted to have $\mathrm{C}-\mathrm{F}$ bond lengths on the order of $1.35 \AA$, even though the unpaired electron occupies a different orbitals for each state. Thus, it is likely that the $\mathrm{C}-\mathrm{F}$ bond extension in $\mathrm{FCO}_{2}^{-}$results from the diffuse nature of the additional charge rather than from the bonding character of the orbital in which the extra electron resides. 
Another system for comparison is the isovalent $\mathrm{HCO}_{2} / \mathrm{HCO}_{2}^{-}$pair. Comparison of these values to those for $\mathrm{HCO}_{2}\left(\mathrm{D}_{\mathrm{o}}\left(\mathrm{H}-\mathrm{CO}_{2}\right)=11 \pm 3 \mathrm{kcal} / \mathrm{mole}\right)$ and $\mathrm{HCO}_{2}^{-}\left(\mathrm{D}_{\mathrm{o}}\left(\mathrm{H}^{-}-\mathrm{CO}_{2}\right)=74\right.$ $\pm 3 \mathrm{kcal} / \mathrm{mole}$ ) is quite interesting. 49 While the $D_{0}$ 's for the neutrals are nearly identical, the anions differ by more than a factor of two. The main factor which contributes to this difference is the higher electron affinity of fluorine (Table D) relative to that of hydrogen $(0.754209 \pm$ $0.0000033 \mathrm{eV}) .50$

The $\mathrm{FCO}_{2}$ heat of formation, $\Delta_{f} \mathrm{H}_{298}^{o}\left(\mathrm{FCO}_{2}\right)$ can be obtained from the measured $\mathrm{EA}\left(\mathrm{FCO}_{2}\right)=4.277 \pm 0.030 \mathrm{eV}$, the $\mathrm{FCO}_{2}^{-}$heat of formation, $\Delta_{f} H_{298}^{o}\left(\mathrm{FCO}_{2}^{-}\right)=-185 \pm 2.6$ $\mathrm{kcal} / \mathrm{mole}(8.02 \mathrm{eV} \pm 0.11 \mathrm{eV})$, and a combination of measured and calculated vibrational frequencies. In Eq. (13),

$$
\begin{aligned}
\Delta_{f} H_{298}^{o}\left(F C O_{2}\right)= & \Delta_{f} H_{298}^{o}\left(F C O_{2}^{-}\right)+E A\left(F C O_{2}\right)+\frac{5}{2} R T \\
& +\int_{0 K}^{298 K}\left[C_{p}\left(F C O_{2}\right)-C_{p}\left(F C O_{2}^{-}\right)\right] d T
\end{aligned}
$$

the $5 / 2 \mathrm{RT}$ term $(1.481 \mathrm{kcal} / \mathrm{mole} ; 0.064 \mathrm{eV})$ accounts for the heat capacity of the electron. ${ }^{51}$ The three symmetric stretch vibrational frequencies used in the FC simulations (Table IX) are combined with the MP4/6-31+G vibrational frequencies for the other three modes of the neutral and the QCISD/6-31G* frequencies of the anion to calculate the heat capacities of $\mathrm{FCO}_{2}$ and $\mathrm{FCO}_{2}^{-}$. Substitution into Eq. (13) yields $\Delta_{f} H_{298}^{o}\left(\mathrm{FCO}_{2}\right)=-85.2 \pm 2.8 \mathrm{kcal} / \mathrm{mole}(-3.69 \pm 0.12$ $\mathrm{eV})$.

In light of the weak $\mathrm{C}-\mathrm{F}$ bond found in the $\mathrm{FCO}_{2}$ radical, the amount of vibrational structure which is observed in the $\mathrm{FCO}_{2}^{-}$photoelectron spectrum is quite surprising. Both the ${ }^{2} \mathrm{~B}_{2}$ and ${ }^{2} \mathrm{~A}_{2}$ electronic states of $\mathrm{FCO}_{2}$ correlate adiabatically to the $\mathrm{F}\left({ }^{2} \mathrm{P}\right)+\mathrm{CO}_{2}\left({ }^{1} \Sigma_{\mathrm{g}}^{+}\right)$ dissociation products. ${ }^{9}$ According to Eq. (14), the measured eKE of a photoelectron can be related to the asymptotic energy of the $\mathrm{F}+\mathrm{CO}_{2}$ ground state dissociation products, 


$$
e K E=h v-D_{o}\left(F C O_{2}^{-}\right)-E A(F)-E_{\text {int }}^{o}+E_{\text {int }}^{-}
$$

establishing the energetic stability of the $\mathrm{FCO}_{2}$ radical with respect to dissociation. The asymptotic dissociation energy, indicated in the $\mathrm{FCO}_{2}^{-}$photoelectron spectra by the arrow labeled ' $a$ ', is calculated using the $\mathrm{D}_{0}\left(\mathrm{~F}^{-}-\mathrm{CO}_{2}\right)$ and $\mathrm{EA}(\mathrm{F})$ values given above and is accurate to $\sim 0.1 \mathrm{eV}$. While all of the ${ }^{2} \mathrm{~B}_{2}$ vibrational peaks lie below the dissociation asymptote, those for the ${ }^{2} \mathrm{~A}_{2}$ state extend well above the energy required for dissociation into ground state $\mathrm{F}+\mathrm{CO} 2$ products. Thus, the peaks represent photodetachment transitions to metastable vibrational levels of $\mathrm{FCO}_{2}$ which are energetically unstable with respect to dissociation into $\mathrm{F}+\mathrm{CO}_{2}$ products. The peaks, known to be broadened due to space-charge effects and combination band congestion, remain reasonably well resolved at energies more than $0.5 \mathrm{eV}$ above the dissociation limit for the ${ }^{2} \mathrm{~A}_{2}$ state, the origin of which actually lies just above the dissociation asymptote. The retention of reasonably narrow peak widths indicates that molecules excited to these vibrational levels must survive for at least a vibrational period, and perhaps longer, before dissociation.

This behavior suggests that either a potential energy barrier prevents prompt dissociation. Francisco et al. ${ }^{9}$ did not find a barrier saddle point along the $\mathrm{FCO}_{2}{ }^{2} \mathrm{~B}_{2} \rightarrow \mathrm{F}\left({ }^{2} \mathrm{P}\right)$ $+\mathrm{CO}_{2}\left({ }^{1} \Sigma_{g}^{+}\right)$dissociation coordinate at the MP2/3-21G level of theory. A similar search was also made along the dissociation coordinate of the lower symmetry ${ }^{2} \mathrm{~A}$ ' structure predicted by MP2 theory, which correlates to the ${ }^{2} \mathrm{~A}_{2}$ state in $\mathrm{C}_{2 v}$ symmetry. Here they found a barrier of $0.72 \mathrm{eV}$ with respect to the bound species $(0.66 \mathrm{eV}$ with respect to the separated products). The existence of such a barrier for the ${ }^{2} \mathrm{~A}_{2}$ state is sufficient to explain the observed vibrational structure for that state. 


\subsection{Conclusions}

Negative ion photoelectron spectroscopy has been used to investigate the $\mathrm{X}^{-}\left(\mathrm{CO}_{2}\right)$ clusters $(\mathrm{X}=\mathrm{I}, \mathrm{Br}, \mathrm{Cl}$ and $\mathrm{F})$. The spectra indicate that the bonding in $\Gamma^{-}\left(\mathrm{CO}_{2}\right), \mathrm{Br}^{-}\left(\mathrm{CO}_{2}\right)$ and $\mathrm{Cl}^{-}\left(\mathrm{CO}_{2}\right)$ clusters is primarily electrostatic, being dominated by a charge-quadrupole interaction. This interaction leads to a distortion of the $\mathrm{CO}_{2}$ molecule producing Y-shaped clusters. Franck-Condon analyses of the data show that the $\mathrm{OCO}$ angle in the $\mathrm{I}^{-}\left(\mathrm{CO}_{2}\right)$, $\mathrm{Br}^{-}\left(\mathrm{CO}_{2}\right)$ and $\mathrm{Cl}^{-}\left(\mathrm{CO}_{2}\right)$ clusters is $174.5 \pm 1.5^{\circ}, 172.2 \pm 1.5^{\circ}$ and $169.2 \pm 2.5^{\circ}$, respectively. Electrostatic calculations are performed which reproduce the observed distortion for the $I^{-}\left(\mathrm{CO}_{2}\right)$ cluster to within experimental uncertainty. Reasonable agreement is found between experimental and $a b$ initio results for the $\mathrm{Cl}^{-}\left(\mathrm{CO}_{2}\right)$ cluster. The $a b$ initio results and their comparison to the electrostatic calculations indicate that a small but increasing amount $(\sim 5 \%)$ of charge migration may occur from $\mathrm{X}^{-}$to $\mathrm{CO}_{2}$ as $\mathrm{X}$ becomes smaller.

The $\mathrm{FCO}_{2}^{-}$photoelectron spectrum shows that both the $\mathrm{FCO}_{2}^{-}$and $\mathrm{FCO}_{2}$ molecules have more covalent bonding character than the other systems studied. However, these bonds are also found to be fairly weak $\left(\mathrm{D}_{0}\left(\mathrm{~F}^{-}-\mathrm{CO}_{2}\right)=31.7 \pm 2 \mathrm{kcal} / \mathrm{mole} \mathrm{e}^{2,4}\right.$ and $\mathrm{D}_{\mathrm{o}}\left(\mathrm{F}-\mathrm{CO}_{2}\right)=11.5$ $\mathrm{kcal} / \mathrm{mole}$ ) when compared to other known C-F chemical bonds ( $100 \mathrm{kcal} / \mathrm{mole})$. The weakness of the C-F bond is attributed to the stability of the C-O bonds which must be lost in the formation of the $\mathrm{C}-\mathrm{F}$ bond. The $\mathrm{FCO}_{2}$ heat of formation is determined to be $\Delta_{f} H_{298}^{o}\left(\mathrm{FCO}_{2}\right)$ $=-85.2 \pm 2.8 \mathrm{kcal} / \mathrm{mole}$. Two electronic states of $\mathrm{FCO}_{2}$ are observed. These are assigned as

the $\tilde{\mathrm{X}}{ }^{2} \mathrm{~B}_{2}$ ground state and the previously unobserved $\tilde{\mathrm{A}}{ }^{2} \mathrm{~A}_{2}$ first excited state. The spectra exhibit vibrational progressions which extend well above the dissociation asymptote for both of these states indicating that a substantial barrier to dissociation exists for the ${ }^{2} \mathrm{~A}_{2}$ state. FranckCondon simulations of the data using $a b$ initio geometries and force constants calculated for the $\mathrm{FCO}_{2}^{-}$anion and the $\mathrm{FCO}_{2}{ }^{2} \mathrm{~B}_{2}$ and ${ }^{2} \mathrm{~A}_{2}$ electronic states at the QCISD/6-31G* level of theory reproduce the major features of the experimental data remarkably well. 


\subsection{Acknowledgments}

This work has been supported by the United States Air Force Office of Scientific Research under Contract No. F49620-94-1-0115. We thank Professor Martin Head-Gordon for helpful suggestions with the $a b$ initio calculations. 


\section{References}

${ }^{1}$ (a) K. G. Spears, J. Chem. Phys. 57, 1850 (1972); (b) K. G. Spears and E. E. Ferguson, J. Chem. Phys. 59, 4174 (1973).

2 (a) J. W. Larson and T. B. McMahon, J. Am. Chem. Soc. 107, 766 (1985); (b) T. B. McMahon and C. J. Northcott, Can. J. Chem. 56, 1069 (1978).

${ }^{3}$ R. G. Keesee, N. Lee, and A. W. Castleman, Jr., J. Chem. Phys. 73, 2195 (1980).

${ }^{4}$ K. Hiraoka, S. Mizuse, and S. Yamabe, J. Chem. Phys. 87, 3647 (1987); K. Hiraoka, T. Shoda, K. Morise, S. Yamabe, E. Kawai, and K. Hirao, J. Chem. Phys. 84, 2091 (1986).

5B. S. Ault, Inorg. Chem. 21, 756 (1982).

${ }^{6}$ G. Markovich, R. Giniger, M. Levin, and O. Cheshnovsky, Z. Phys. D 20, 69 (1991).

7D. W. Arnold, S. E. Bradforth, E. H. Kim, and D. M. Neumark, J. Chem. Phys. 97,9468 (1992).

8Y. Zhao, C. C. Arnold, and D. M. Neumark, J. Chem. Soc. Faraday Trans. 2 89, 1449 (1993).

9J. S. Francisco and A. Ostafin, J. Phys. Chem. 94, 6337 (1990).

${ }^{10} \mathrm{G}$. Hancock and D. E. Heard, J. Chem. Soc. Faraday Trans. 2 87, 1039 (1991); ibid., 1045 (1991).

${ }^{11}$ J. S. Francisco, A. N. Goldstein, Z. Li, Y. Zhao, and I. H. Williams, J. Phys. Chem. 94, 4791 (1990); J. S. Francisco, Chem. Phys. Lett. 163, 375 (1989); J. S. Francisco and A. N. Goldstein, Chem. Phys. 127, 73 (1988).

12M. M. Maricq, J. J. Szente, Z. Li, and J. S. Francisco, J. Chem. Phys. 98, 784 (1993).

${ }^{13}$ M. M. Maricq, J. J. Szente, G. A Khitrov, and J. S. Francisco, J. Chem. Phys. 98, 9522 (1993).

${ }^{14}$ T. J. Wallington, T. Ellermann, O. J. Nielsen, and J. Sehested, J. Phys. Chem. 98, 2346 (1994).

15D. Ovenall and D. H. Whiffen, Mol. Phys. 4, 135 (1961); J. E. Bennett, B. Mile, and A. Thomas, Trans. Far. Soc. 61, 2357 (1965).

16R. N. Dixon, Proc. Roy. Soc. 275A, 431 (1963).

${ }^{17} \mathrm{~K}$. O. Hartman and I. C. Hisatsune, J. Chem. Phys. 44, 1913 (1966).

${ }^{18}$ D. M. Cyr, M. G. Scarton, and M. A. Johnson, J. Chem. Phys. 99, 4869 (1993).

${ }^{19}$ R. B. Metz, A. Weaver, S. E. Bradforth, T. N. Kitsopoulos, and D. M. Neumark, J. Phys. Chem. 94, 1377 (1990).

${ }^{20}$ M. A. Johnson, M. L. Alexander and W. C. Lineberger, Chem. Phys. Lett. 112, 285 (1984).

${ }^{21}$ W. C. Wiley and I. H. McLaren, Rev. Sci. Instrum. 26, 1150 (1955). 
22 J. Cooper and R. N. Zare, J. Chem. Phys. 48, 942 (1968).

23J. Simons, J. Phys. Chem. 95, 1017 (1991); J. Simons and K. D. Jordan, Chem. Rev. 87, 535 (1987).

${ }^{24}$ Gaussian 92, Revision C, M. J. Frisch, G. W. Trucks, M. Head-Gordon, P. M. W. Gill, M. W. Wong, J. B. Foresman, B. G. Johnson, H. B. Schlegel, M. A. Robb, E. S. Replogle, R. Gomperts, J. L. Andres, K. Raghavachari, J. S. Binkley, C. Gonzalez, R. L. Martin, D. J. Fox, D. J. Defrees, J. Baker, J. J. P. Stewart, and J. A. Pople, Gaussian, Inc., Pittsburgh PA, 1992.

${ }^{25}$ The $\mathrm{C}-\mathrm{O}$ bondlength is the same bond length used in electrostatic calculations described in Sec IV A 2 . The value results from an optimization of the $\mathrm{CO}_{2}$ geometry at the HF/6-31+G* level of theory.

${ }^{26}$ M. T. Nguyen, J. Mol. Struct. 133, 269 (1985).

${ }^{27}$ Quadratic Configuration Interaction with Single and Double excitations.

${ }^{28}$ Note that a force constant analysis indicates that the geometry calculated for the ${ }^{2} \mathrm{~B}_{1}$ state is not at a potential energy minimum but at a saddle point.

${ }^{29}$ The calculated geometry for the $C_{s}$ species at MP2/6-31+G*: $R_{C F}=1.339 \AA ; R_{C O}=1.170 \AA ; R_{C O}=$ $1.392 \AA ; \angle \mathrm{OCO}^{\prime}=126.6^{\circ} ; \angle \mathrm{FCO}=124.7^{\circ}$.

${ }^{30}$ A. D. McLean, B. H. Lengsfield III, J. Pacansky, and Y. Ellinger, J. Chem. Phys. 83, 3567 (1985).

${ }^{31}$ N. A. Burton, Y. Yamaguchi, I. L. Alberts, and H. F. Schaefer III, J. Chem. Phys. 95, 7466 (1991); C. P. Blahous III, B. F. Yates, Y. Xie, and H. F. Schaefer III, J. Chem. Phys. 93, 8105 (1990).

${ }^{32}$ A. J. Stone, Chem. Phys. Lett. 83, 233 (1981); S. L. Price, A. J. Stone, and M. Alderton, Mol. Phys. 32, 987 (1984); A. J. Stone and M. Alderton, Mol. Phys. 56, 1047 (1985).

${ }^{33}$ M. R. Battaglia, A. D. Buckingham, D. Neumark, R. K. Pierens, and J. H. Williams, Mol. Phys. 43, 1015 (1981).

${ }^{34}$ C. S. Yeh, K. F. Willey, D. F. Robbins, J. S.Pilgrim, and M. A. Duncan, J. Chem. Phys. 98, 1867

(1993); C. S. Yeh, K. F. Willey, D. L. Robbins, and M. A. Duncan, J.Chem. Phys. 96, 7833 (1992).

${ }^{35}$ M. A. Pariseau, I. Suzuki, and J. Overend, J. Chem. Phys. 42, 2335 (1965).

${ }^{36} \mathrm{G}$. Herzberg, Molecular Spectra and Molecular Structure III. Electronic Spectra and Electronic Structure of Polyatomic Molecules (Krieger, Malabar, 1991).

${ }^{37}$ H. S. W. Massey, Negative Ions (Cambridge University Press, Cambridge, 1976); K. Ervin, J. Ho, and W.C. Lineberger, J. Chem. Phys. 91, 5974 (1991).

38V. Aquilanti, D. Cappelletti, and F. Pirani, J. Chem. Soc. Faraday Trans. 89, 1467 (1993).

${ }^{39}$ R. D. Amos and J. E. Rice, 'CADPAC: The Cambridge Analytical Derivatives Package', issue 4.0, Cambridge, 1987.

${ }^{40}$ I. Suzuki, J. Mol. Spectrosc. 25, 479 (1968).

${ }^{41}$ A. D. Buckingham, Quart. Rev. Chem. Soc. London. 13, 183 (1959). 
${ }^{42}$ A. A. S. Sangachin and J. Shanker, J. Chem. Phys. 90, 1061 (1989).

${ }^{43}$ A. D. Buckingham and P. W. Fowler, Can J. Chem. 63, 2018 (1985); A. D. Buckingham, P. W. Fowler, and A. J. Stone, Int. Rev. Phys. Chem. 5, 107 (1986).

${ }^{44}$ A. Weaver, D. W. Arnold, S. E. Bradforth, and D. M. Neumark, J. Chem. Phys. 94, 1740 (1991).

${ }^{45}$ E. B. Wilson, Jr., J. C. Decius, P. C. Cross, Molecular Vibrations (Dover, New York, 1980).

${ }^{46}$ Although the antisymmetric stretch force constants (fc's) suffer from numerical errors, the normal coordinates appear to be less affected and are used for comparison to the ${ }^{2} \mathrm{~A}_{2}$ results. Simulations using HF and MP2 fc's produce very similar results.

47From Ref. 9, using their PMP4SDTQ/6-31G*//UMP2/6-31G* + $\triangle$ ZPE/UMP2/6-31+G* values.

${ }^{48}$ D. F. McMillen and D. M. Golden, Ann. Rev. Phys. Chem. 33, 493 (1982).

${ }^{49}$ E. H. Kim, S. E. Bradforth, D. W. Arnold, and D. M. Neumark, J. Chem. Phys. (in preparation).

50R. D. Mead, A. E. Stevens, and W. C. Lineberger in Gas Phase Ion Chemistry 3, ed. M. T. Bowers, (1983).

${ }^{51}$ S. G. Lias, J. E. Bartmess, J. F. Liebman, J. L. Holmes, R. D. Levin, and W. G. Mallard, J. Phys. Chem. Ref. Data 17, Supplement 1, pp. 5-30 (1988). 


\title{
Chapter 6: Study of $\mathrm{I}^{-}\left(\mathrm{CO}_{2}\right)_{\mathrm{n}}, \mathrm{Br}^{-}\left(\mathrm{CO}_{2}\right)_{\mathrm{n}}$ and $\mathrm{I}^{-}\left(\mathrm{N}_{2} \mathrm{O}\right)_{\mathrm{n}}$ clusters by Anion Photoelectron Spectroscopy
}

\begin{abstract}
Photoelectron spectra of the $\mathrm{I}^{-}\left(\mathrm{CO}_{2}\right)_{\mathrm{n}=1-13}, \mathrm{I}^{-}\left(\mathrm{N}_{2} \mathrm{O}\right)_{\mathrm{n}=1-12}$, and $\mathrm{Br}^{-}\left(\mathrm{CO}_{2}\right)_{\mathrm{n}=1-11}$ clusters are presented. The spectra provide information about the stepwise solvation of the bromide and iodide anions and about the size of the first solvation shells in these clusters. The data suggest that significantly different solute-solvent interactions exist in the three sets of clusters studied here. The $\mathrm{X}^{-}\left(\mathrm{CO}_{2}\right)_{\mathfrak{n}}$ spectra exhibit resolved progressions which are assigned to in-phase $\mathrm{CO}_{2}$ solvent bending vibrations in the neutral clusters. These vibrations are excited by photodetachment of anion clusters in which the $\mathrm{CO}_{2}$ molecules are distorted from linearity by a charge-quadrupole interaction. The lengths of vibrational progressions observed in the $\mathrm{X}^{-}\left(\mathrm{CO}_{2}\right)_{\mathrm{n}=1-4}$ spectra suggest that the $\mathrm{CO}_{2}$ molecules occupy equivalent sites when $\mathrm{X}=\mathrm{I}$ but not when $X=B r$. An observed change in peak widths and reappearance of vibrational structure for the larger $\mathrm{I}^{-}\left(\mathrm{CO}_{2}\right)_{\mathrm{n}}$ clusters, through $\mathrm{I}^{-}\left(\mathrm{CO}_{2}\right)_{13}$ (40 atoms; mass $=699$ amu), provides information about the perturbation of the halogen electronic structure by the solvent molecules.
\end{abstract}

\subsection{Introduction}

The study of molecular clusters has developed into a diverse field directed towards understanding the evolution of molecular interactions from simple gas-phase dimers to bulk chemical material. One would like to determine how the properties of the cluster change as additional units are added and at what point the cluster properties become indistinguishable from those of the bulk. The study of clusters, in general, involves the determination of how the properties of clusters change according to their size and/or composition and many techniques have been developed for this purpose. ${ }^{1}$ 
Several studies of clusters have now been completed using anion photodetachment techniques. The moderate spectral resolution and mass selectivity of anion photoelectron spectroscopy is well-suited for these studies because it allows the study the electronic and vibrational properties of an isolated, identified cluster. Anion photodetachment studies of clusters can, for the most part, be divided into two groups which depend upon the degree to which the negative charge in the cluster is delocalized. Several studies have been completed on elemental and molecular clusters, $A_{n}^{-}$, which are typically characterized as covalently bound clusters in which the negative charge is fairly delocalized. 2,3,4,5,6,7 This paper deals with clusters of a second type, $\mathrm{X}^{-}(\mathrm{M})_{\mathrm{n}}$, in which the charge remains localized on a central chromophore, $X^{-}$, which interacts with solvent molecules, $M$. The spectra of the $X^{-}(M)_{n}$ clusters typically retain the primary spectral features of the $\mathrm{X}^{-}$species because of the relatively weak X-M interactions. $8,9,10,11$ Deviations between the $\mathrm{X}^{-}$and the $\mathrm{X}^{-}(\mathrm{M})_{\mathrm{n}}$ spectra offer insight into the $\mathrm{X}^{-}+\mathrm{n} \cdot \mathrm{M}$ solvation energetics and dynamics.

A solute molecule, or ion, typically interacts most strongly with the nearest neighbor solvent molecules. Molecules outside of this 'solvent shell' are somewhat shielded from the solute molecule and the secondary interactions are relatively weak. The interactions of solvated ions with outer solvent molecules are quite dependent on the ability of the solvent to effectively mask a charge. In bulk terms, this ability is measured by the dielectric constant which, on the molecular scale, is dependent on the electrostatic properties of the individual solvent molecules.

Anion PES offers a means of studying several properties of solvation shells which form around negative ions. Cheshnovsky and co-workers ${ }^{9}$ have used this technique to study hydrated iodide clusters, $\mathrm{I}^{-}\left(\mathrm{H}_{2} \mathrm{O}\right)_{\mathrm{n} \leq 60}$. From their results, they suggest the formation of a solvent shell around $\mathrm{I}^{-}$composed of $6 \mathrm{H}_{2} \mathrm{O}$ molecules. They also assign features in some of their spectra to another isomer of the cluster in which the $\Gamma$ resides on the surface of a water cluster rather than in the center. In some cases, such as those to be presented below, the 
photoelectron spectra also offer insight into the structure and dynamical behavior of the solvent shell.

In this paper, photoelectron spectra are presented for $I^{-}\left(N_{2} \mathrm{O}\right)_{n=1-12}, I^{-}\left(\mathrm{CO}_{2}\right)_{n=1-13}$, and $\mathrm{Br}^{-}\left(\mathrm{CO}_{2}\right)_{\mathrm{n}=1-11}$. Markovich et al. ${ }^{10}$ previously obtained $\mathrm{I}^{-}\left(\mathrm{CO}_{2}\right)_{\mathrm{n}=1-7}$ photoelectron spectra in a study of the solvation energetics of these clusters. In other related studies, we have compared the interaction of a single $\mathrm{CO}_{2}$ molecule with different halide ions and halogens ${ }^{12}$ and studied the $\mathrm{I}^{-}$interaction with several $\mathrm{CO}_{2}$ molecules. ${ }^{13}$ The $\mathrm{I}^{-}\left(\mathrm{CO}_{2}\right)$ complex has also been studied at higher resolution in this laboratory using threshold photodetachment (ZEKE) spectroscopy. ${ }^{14}$

In our previous Communication, we presented the $\mathrm{I}^{-}\left(\mathrm{CO}_{2}\right)_{\mathrm{n}=1-13}$ photoelectron spectra. As a result of our higher spectral resolution, these photoelectron spectra showed pronounced vibrational progressions which were not observed by Markovich et al. ${ }^{10}$ The spectra provide information about the interaction between the $I^{-} /$chromophore and the $\mathrm{CO}_{2}$ solvent molecules as a function of cluster size. In particular, the spectra clearly illustrate a significant $\mathrm{CO}_{2}$ solvent perturbation by the core ion.

In this study, we include spectra of $\mathrm{Br}^{-}\left(\mathrm{CO}_{2}\right)_{n}$ and $\mathrm{I}^{-}\left(\mathrm{N}_{2} \mathrm{O}\right)_{n}$ in order to study the evolution of the solute-solvent interactions as a function of solute, solvent and size. The two solvent species, $\mathrm{N}_{2} \mathrm{O}$ and $\mathrm{CO}_{2}$, are isoelectronic and the two ions are both halides, $\mathrm{I}^{-}$and $\mathrm{Br}^{-}$. Nonetheless, as will be seen, the spectra clearly show that the ion-solvent interactions for all three systems studied are different. While both the $\mathrm{I}^{-}\left(\mathrm{CO}_{2}\right)_{\mathrm{n}}$ and the $\operatorname{Br}^{-}\left(\mathrm{CO}_{2}\right)_{\mathrm{n}}$ spectra suggest that the similar ion- $\mathrm{CO}_{2}$ interactions exist in two types of clusters, the similarity of spectra diverges rapidly as a function of cluster size and suggest that the two halides have different size solvent shells. The $\mathrm{I}^{-}\left(\mathrm{N}_{2} \mathrm{O}\right)_{\mathrm{n}}$ spectra, which differ significantly from those of the $\mathrm{CO}_{2}$ clusters, show that a more weakly interacting $\mathrm{N}_{2} \mathrm{O}$ is not distorted by the ion core and forms a larger solvent shell around $\mathrm{I}^{-}$than $\mathrm{CO}_{2}$. 


\subsection{Experimental}

The apparatus employed in these experiments, a dual time-of-flight anion photoelectron spectrometer, has been described in detail previously. ${ }^{15}$ An overview of the apparatus, with details relevant to the present results, will be summarized here. Anion clusters are generated at the intersection of a pulsed molecular beam and a $1 \mathrm{keV}$ electron beam. ${ }^{16}$ In the ion source, $\Gamma$ and $\mathrm{Br}^{-}$ions are formed by dissociative attachment of low-energy $(\sim 1 \mathrm{eV})$ secondary electrons to $\mathrm{HI}$ and $\mathrm{HBr}$. The $\mathrm{HI}$ (or $\mathrm{HBr}$ ) is seeded $(\sim 1 \%)$ in a carrier gas $\left(\mathrm{N}_{2} \mathrm{O}\right.$ or $\left.\mathrm{CO}_{2}\right)$ and expanded through a piezoelectric valve operated at $20 \mathrm{~Hz}$ with a stagnation pressure of $\sim 3-4 \mathrm{~atm}$. As the free jet expansion evolves, the ions cluster with the carrier gas and relax rotationally and vibrationally.

The cooled ions are extracted into a Wiley-McLaren-type time-of-flight mass spectrometer ${ }^{17}$ where they separate according to mass. The ion of interest is selectively photodetached by a properly timed 8 ns laser pulse. Photoelectron energies are determined by time-of-flight measurements in a $1 \mathrm{~m}$ field-free flight tube. The resolution of the apparatus is $0.011 \mathrm{eV}$ for electrons with $0.65 \mathrm{eV}$ of electron kinetic energy (eKE) and degrades as (eKE) $)^{3 / 2}$. For these experiments, the 4th $(266 \mathrm{~nm} ; 4.657 \mathrm{eV} ; 15 \mathrm{~mJ} / \mathrm{pulse})$ and 5 th $(213 \mathrm{~nm} ; 5.822 \mathrm{eV} ; 6$ $\mathrm{mJ} / \mathrm{pulse}$ ) harmonics of a Nd:YAG pulsed laser are employed for photodetachment. The 213 $\mathrm{nm}$ photons generate background signal through the interactions of scattered light with the surfaces of the detector chamber. A background spectrum, collected using the same laser power used during data collection, is fitted to a smooth function which is scaled and subtracted from the data to correct for the moderate level background signal.

\subsection{Results}

For comparison to the cluster data, photoelectron spectra of $\mathrm{I}^{-}$and $\mathrm{Br}^{-}$using a $4.657 \mathrm{eV}^{-}$ photodetachment energy are shown in Fig. 6.1. The spectra represent electron signal as a function of electron kinetic energy (eKE) where 


$$
\mathrm{eKE}=\mathrm{hv}-\mathrm{EA}-\mathrm{T}_{\mathrm{o}}-\mathrm{E}_{\mathrm{v}}^{\circ}+\mathrm{E}_{\mathrm{v}}^{-}
$$

In Eq. (1), hv is the laser photon energy, EA is the electron affinity of the neutral cluster and $\mathrm{T}_{\mathrm{o}}$ is the term value of the neutral electronic state. In the case of the polyatomic spectra presented below, $E_{v}^{\circ}$ and $E_{v}^{-}$are the vibrational energies (above the zero point) of the neutral and anion, respectively. The two peaks in each halide spectrum (Fig. 6.1) represent photodetachment transitions from the closed-shell halide anions to the two spin-orbit states of the halogen atoms. The peaks at high and low eKE in each spectrum represent photodetachment transitions to the halogen ground $\left({ }^{2} \mathrm{P}_{3 / 2}\right)$ and excited $\left({ }^{2} \mathrm{P}_{1 / 2}\right)$ spin-orbit states, respectively. The ${ }^{2} \mathrm{P}_{3 / 2}$ peaks are slightly broader because they occur at higher eKE. The electron affinities and spin-orbit splitting for each of these atoms are well-known and given in Table I.

Table I: Halogen Electron Affinities and Spin-Orbit Splittings

\begin{tabular}{|c|c|c|}
\hline Halogen & Electron Affinity $^{\mathrm{a}}$ & Spin-Orbit Splitting $^{\mathrm{b}}$ \\
\hline \hline $\mathrm{Br}$ & $3.36590 \mathrm{eV}$ & $0.4569 \mathrm{eV}$ \\
\hline $\mathrm{I}$ & $3.0591 \mathrm{eV}$ & $0.94268 \mathrm{eV}$ \\
\hline
\end{tabular}

a T. R. Miller in CRC Handbook of Chemistry and Physics, 72nd Edition, ed. D. R. Lide, CRC Press (1991), p. 10-180.

b C. E. Moore, Atomic Energy Levels, Vol. I, NSRDS-NBS 35 (1971).

Shown in Fig. 6.2 are the photoelectron spectra collected for the $I^{-}\left(\mathrm{N}_{2} \mathrm{O}\right)_{n}$ clusters, $n=$

Figure 6.1. Photoelectron spectra of $\mathrm{I}^{-}$and $\mathrm{Br}^{-}$collected using a photodetachment energy of $4.657 \mathrm{eV}$.

Figure 6.2. Photoelectron spectra of $\mathrm{I}^{-}\left(\mathrm{N}_{2} \mathrm{O}\right)_{n}, \mathrm{n}=1-12$, collected using a photodetachment energy of $4.657 \mathrm{eV}$. 


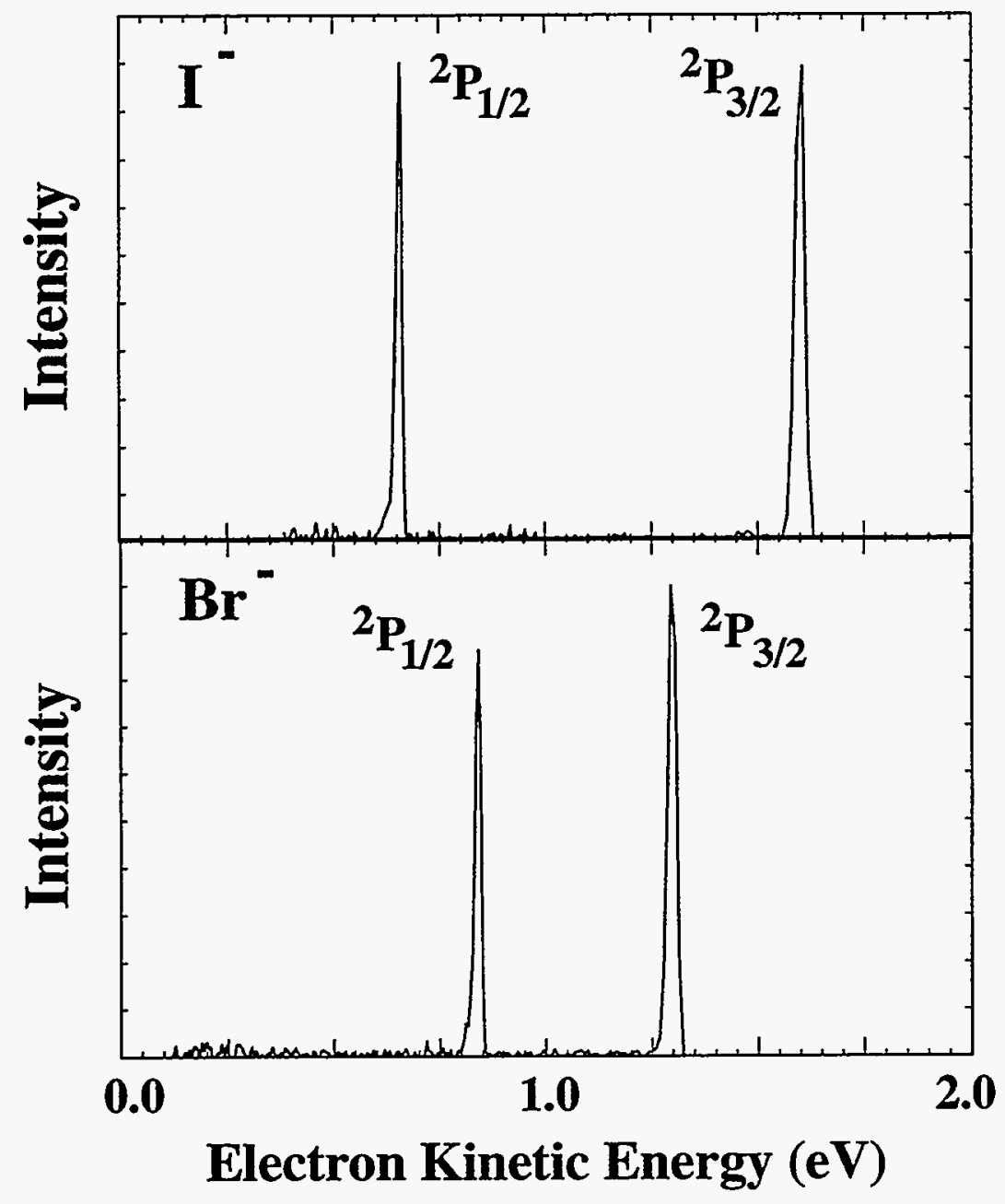

Figure 6.1 


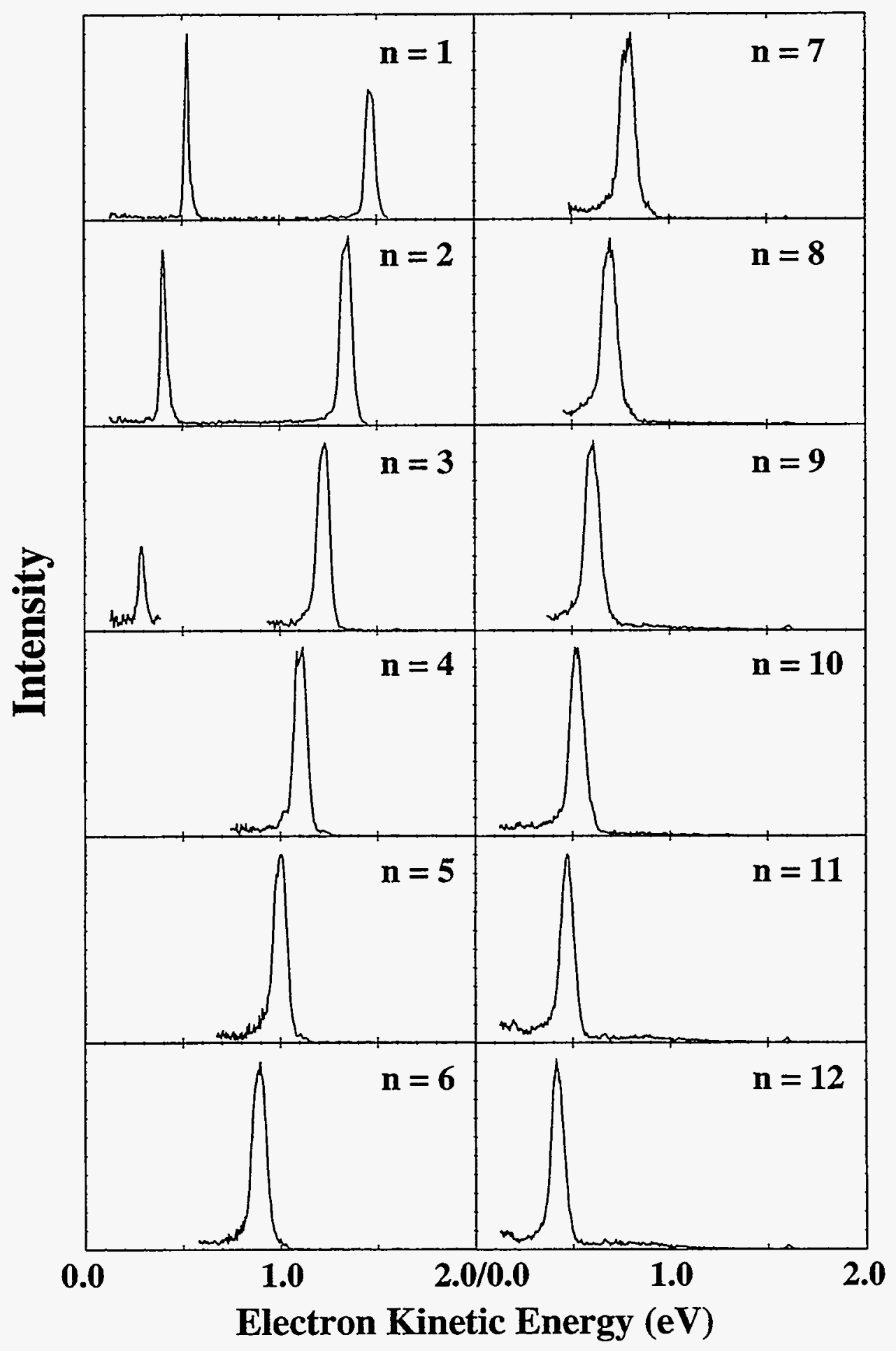

Figure 6.2 
$1-12$, using a photodetachment energy of $4.657 \mathrm{eV}$. For $\mathbf{n}=1-3$, the spectra contain two peaks separated by an energy equal to that of the iodine spin-orbit splitting. To first order, these peaks can be labeled as $I\left({ }^{2} \mathrm{P}_{3 / 2}\right) \cdot\left(\mathrm{N}_{2} \mathrm{O}\right)_{n}$ and $\mathrm{I}\left({ }^{2} \mathrm{P}_{1 / 2}\right) \cdot\left(\mathrm{N}_{2} \mathrm{O}\right)_{n}$ and likewise for the $\mathrm{I}\left(\mathrm{CO}_{2}\right)_{n}$ and $\mathrm{Br}\left(\mathrm{CO}_{2}\right)_{\mathrm{n}}$ clusters. The most obvious change which occurs as a function of cluster size is the consistent shift of the spectra to lower eKE. For the $\mathrm{I}^{-}\left(\mathrm{N}_{2} \mathrm{O}\right)_{3}$ cluster, the excited state peak intensity is affected by the cutoff of the experimental detection efficiency. For the larger clusters, the excited state is energetically out of range for the photodetachment energy used. The peaks at high eKE are significantly broader than the peaks at low eKE. The peak widths are larger than would be expected by the experimental resolution function for individual transitions, suggesting the presence of underlying structure.

Figure 6.3 displays the photoelectron spectra of $\mathrm{I}^{-}\left(\mathrm{CO}_{2}\right)_{\mathrm{n}}$ and $\mathrm{Br}^{-}\left(\mathrm{CO}_{2}\right)_{\mathrm{n}}, \mathrm{n}=1,2$ collected using a $4.657 \mathrm{eV}$ photodetachment energy. As in the $\mathrm{I}^{-}\left(\mathrm{N}_{2} \mathrm{O}\right)_{\mathrm{n}}$ spectra (Figure 2), the peaks shift to lower energy as the cluster size is increased and the general spin-orbit splitting of the halogen atom is preserved. However, the $\mathrm{X}^{-}\left(\mathrm{CO}_{2}\right)_{\mathrm{n}}$ spectra show additional structure for each electronic state which extends to low eKE. As discussed previously, ${ }^{12,13}$ the additional peaks in the spectrum result from excitation of the $\mathrm{CO}_{2}$ bending vibration upon photodetachment. The length of the vibrational progression increases as a function of cluster size. The peaks at high eKE are significantly broader than the peaks at low eKE. In the case of $\mathrm{Br}^{-}\left(\mathrm{CO}_{2}\right)$, an additional splitting of the peaks in the $\mathrm{Br}\left({ }^{2} \mathrm{P}_{3 / 2}\right) \cdot \mathrm{CO}_{2}$ band is discernible.

Figures 6.4 and 6.5 contain the photoelectron spectra of $\mathrm{I}^{-}\left(\mathrm{CO}_{2}\right)_{n}$, for $\mathrm{n}=0-13$, and $\mathrm{Br}^{-}\left(\mathrm{CO}_{2}\right)_{\mathrm{n}}$, for $\mathrm{n}=0$ - 11 collected using a $5.822 \mathrm{eV}$ photodetachment energy, respectively. In

Figure 6.3. Photoelectron spectra of $\Gamma^{-}\left(\mathrm{CO}_{2}\right)_{n}$ and $\mathrm{Br}^{-}\left(\mathrm{CO}_{2}\right)_{n}, \mathrm{n}=1-2$, using a photodetachment energy of $4.657 \mathrm{eV}$.

Figure 6.4. Photoelectron spectra of $\mathrm{I}^{-}\left(\mathrm{CO}_{2}\right)_{\mathrm{n}}, \mathrm{n}=0-13$, collected using a photodetachment energy of $5.822 \mathrm{eV}$. 


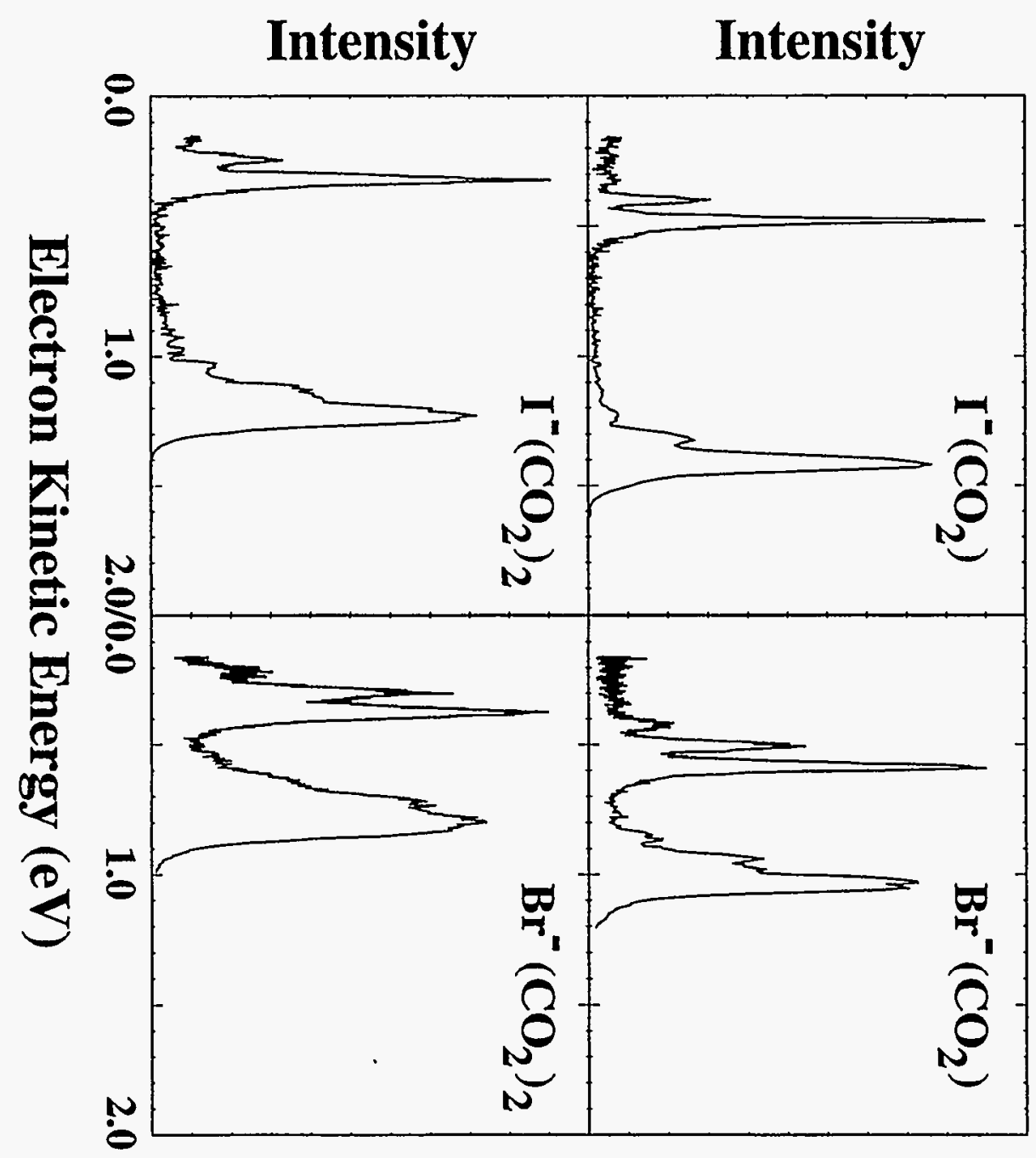




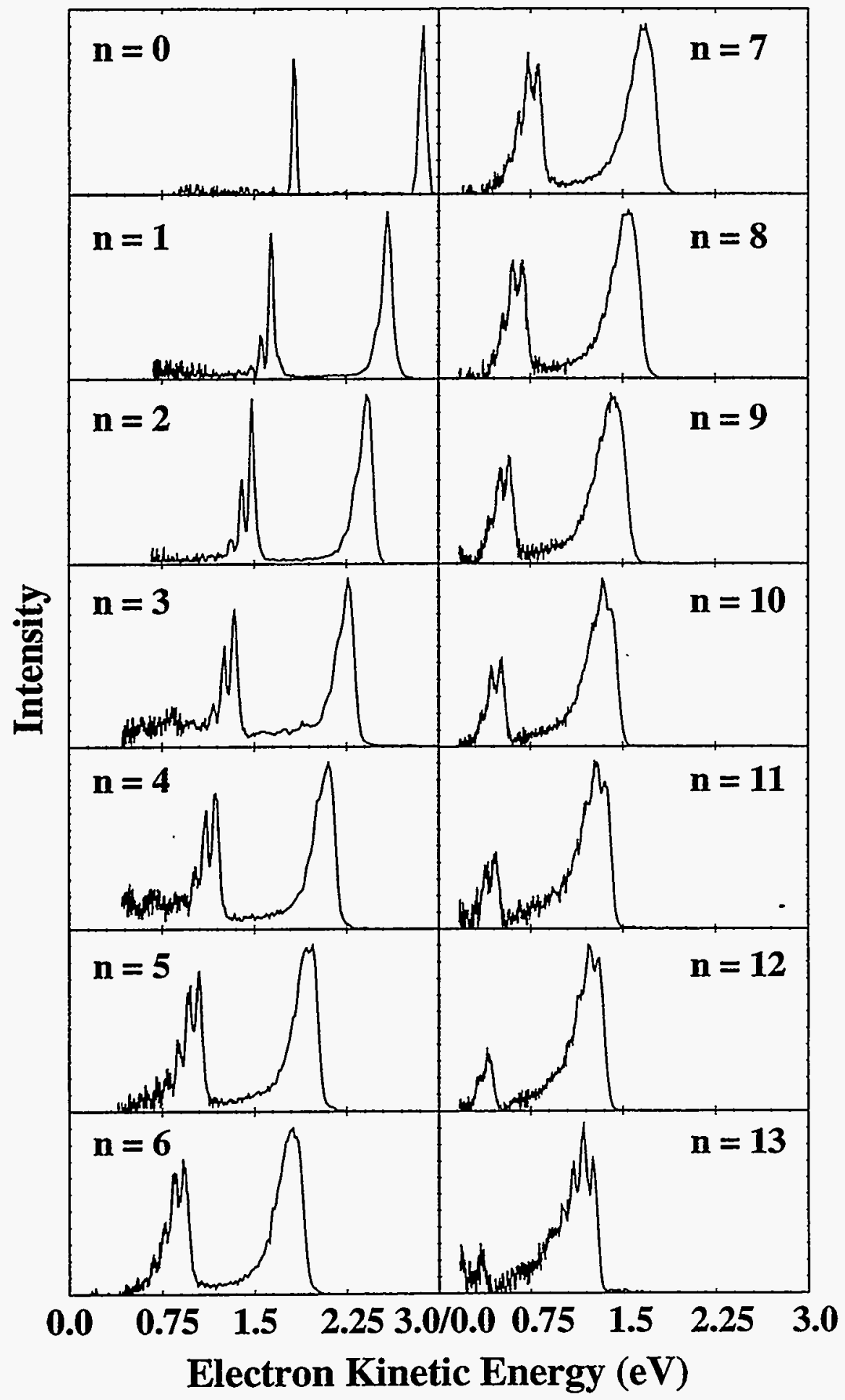

Figure 6.4 
both sets of data, the vibrational progression in the ${ }^{2} P_{1 / 2}$ band becomes more extended as $n$ is increased through $n=5$. Beyond this point the progression is no longer clearly resolved in the $\mathrm{Br}^{-}\left(\mathrm{CO}_{2}\right)_{\mathrm{n}}$ data. However, the $\mathrm{I}^{-}\left(\mathrm{CO}_{2}\right)_{\mathrm{n}}$ spectra exhibit excited state vibrational progressions until the intensity of the ${ }^{2} \mathrm{P}_{1 / 2}$ state is degraded by the detector cutoff function and becomes inaccessible using the $5.822 \mathrm{eV}$ photon energy (i.e., $\mathrm{n}=11$ ). An notable feature of this data set is the reappearance of vibrational structure in the ${ }^{2} \mathrm{P}_{3 / 2}$ band as the size increases past $\mathrm{n}=9$, culminating in a distinct progression for the largest cluster studied, $\Gamma^{-}\left(\mathrm{CO}_{2}\right)_{13}$. This is truly remarkable because the progression represents the concerted motion of 40 atoms within a cluster that has a mass of $699 \mathrm{amu}$. No such reappearance of vibrational structure occurs in the $\mathrm{Br}^{-}\left(\mathrm{CO}_{2}\right)_{\mathrm{n}}$ data set.

\subsection{Analysis and Discussion}

In the following sections, we will treat the data in more detail. The solvation thermodynamics are analyzed in Sec. 6.4.1, comparing the $\mathrm{CO}_{2}$ and $\mathrm{N}_{2} \mathrm{O}$ clusters as a function of size. As part of the interpretation of the thermodynamic information, the dominant bonding interactions and the geometries of the complexes are considered. In Sec. 6.4.2, the vibrational structure observed in the $\mathrm{X}^{-}\left(\mathrm{CO}_{2}\right)_{\mathrm{n}}$ photoelectron spectra are discussed in terms of the cluster size and symmetry.

\subsubsection{Thermodynamics and Geometries}

As illustrated in Figure 6.6, the shift of the photoelectron spectra to lower eKE as a function of cluster size results from the different interactions of the solvent molecule with the anion and neutral. Assuming the 0-0 transition can be identified in the photoelectron spectrum,

Figure 6.5. Photoelectron spectra of $\mathrm{Br}^{-}\left(\mathrm{CO}_{2}\right)_{\mathrm{n}}, \mathrm{n}=0-11$, collected using a photodetachment energy of $5.822 \mathrm{eV}$. 


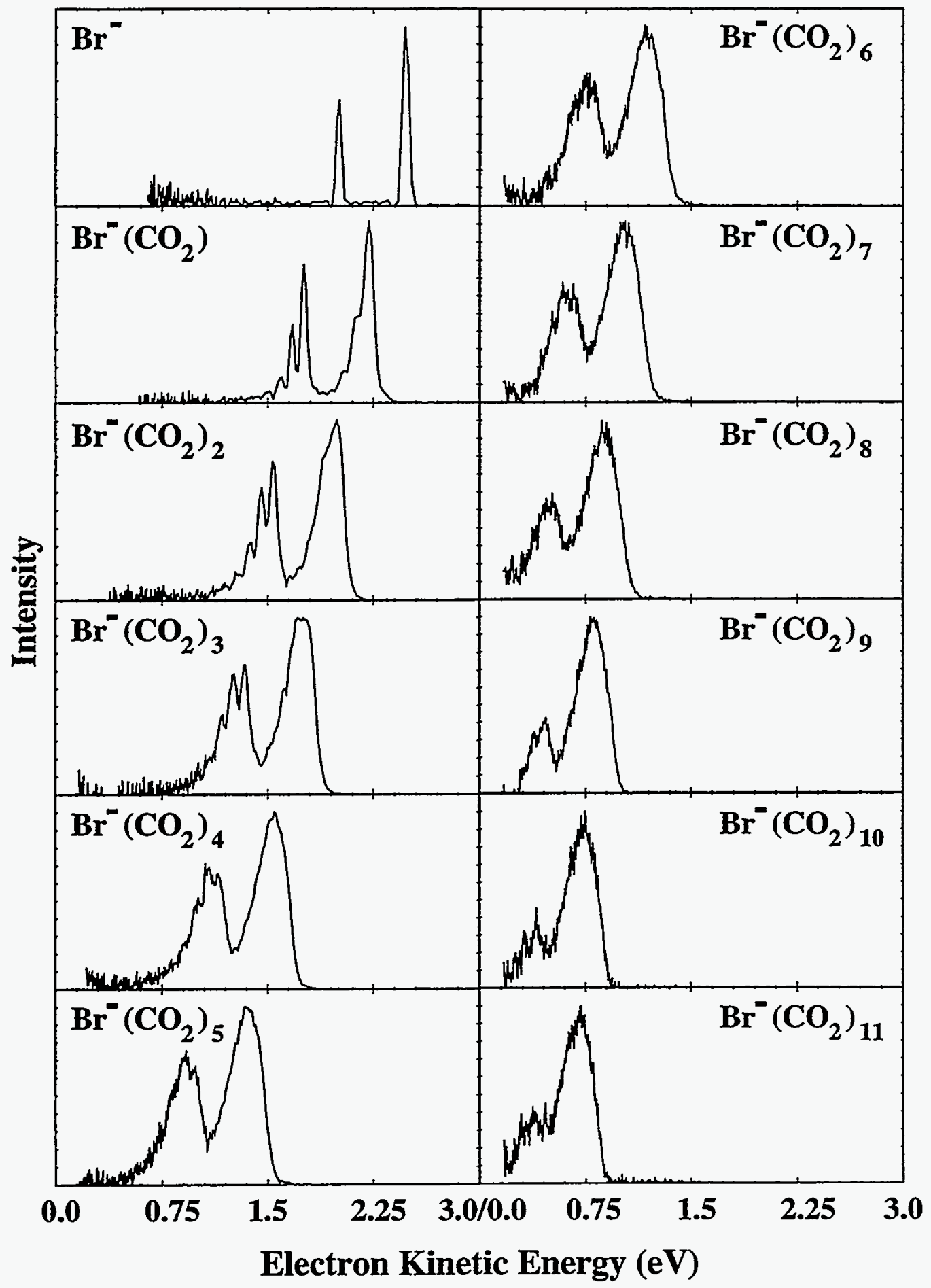

Figure 6.5 
Eq. (2) shows the relationship between the EAs of the two clusters and the charge stabilization provided by a solvent molecule, $\Delta \mathrm{E}_{\mathrm{n}, \mathrm{n}-\mathrm{l}}$,

$$
E A_{n}=E A_{n-1}+\Delta E_{n, n-1}^{a}-\Delta E_{n, n-1}^{n}
$$

The charge-dipole (or charge-quadrupole) interaction which accounts for most of the $\mathrm{X}^{-}(\mathrm{M})$ anion cluster binding energy $\left(\Delta \mathrm{E}_{\mathrm{n}, \mathrm{n}-\mathrm{l}}^{\mathrm{a}}\right)$ is an order of magnitude larger ${ }^{14}$ than the force holding the neutral $X(M)$ cluster together $\left(\Delta E_{n, n-1}^{n}\right)$. Thus, according the Eq. (2), a larger EA results and causes the observed spectral shift. These spectral shifts, which are strongly correlated with the anion stepwise solvation energies ( $\mathrm{SSE}=\Delta \mathrm{E}_{\mathrm{n}, \mathrm{n}-1}^{\mathrm{a}}$ ), provide an excellent means of studying the change in solvation dynamics as the cluster size increases.

For a particular cluster, an additional solvent molecule can stabilize the halide charge by an energy near that of the previous solvent molecules if it occupies an equivalent site in the cluster. If the molecule adds to a different interaction site, or if a significant rearrangement occurs, the stabilization energy will be different, most likely smaller. A special case of this behavior occurs when a solvent shell forms around the anion. After the formation of such a shell, the next solvent molecule which adds to the cluster encounters a well-shielded halide charge resulting in a weaker interaction. As a result, the incremental stabilization of the anion is less and a significantly smaller spectral shift occurs. ${ }^{8-10}$ As further solvent shells form, the stabilization of the charge continually decreases as a result of further charge shielding. Since the interactions among the solvent molecules will be identical for the anion and neutral, the

Figure 6.6. Schematic diagram of the thermodynamics of solvation for a single solvent molecule. The difference between $\Delta \mathrm{H}_{\text {solv }}^{\mathrm{a}}$ and $\Delta \mathrm{H}_{\text {solv }}^{\mathrm{n}}$ leads to the observed spectral shift upon solvation. 


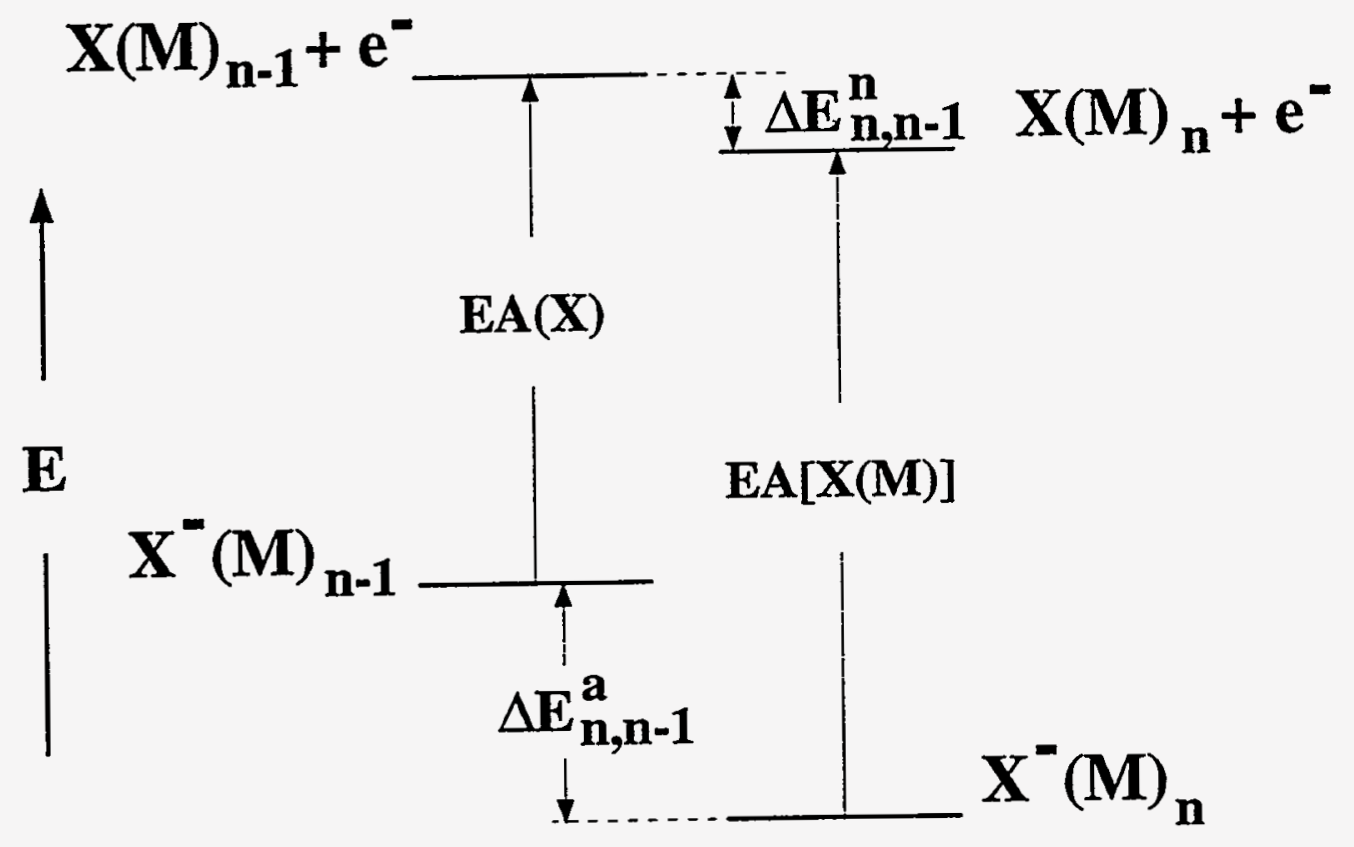

Figure 6.6 
spectral shift will approach zero as the cluster size approaches the bulk limit.

To determined the size of the solvation shells which form in the $X^{-}(M)_{n}$ clusters, we can plot the SSEs as a function of cluster size. Two sources of peak broadening contribute the uncertainty in the position of the ground state 0-0 transition. The peaks are broadened due to unresolved underlying structure which results primarily from excitation of van der Waals progressions upon photodetachment. The second source of broadening which affects only the ground state features results from a splitting of the halogen ${ }^{2} \mathrm{P}_{3 / 2}$ ground state degeneracy by the solvent molecules. ${ }^{14}$ For consistency, the measurement of the EAs (used to determine the SSEs) are made at $25 \%$ of the full height of the peak at highest eKE for each cluster. The peak position and SSE's are summarized in Table II.

Table II: Spectral Shifts as Function of Stepwise Solvation

\begin{tabular}{|c|c|c|c|c|c|c|}
\hline $\mathrm{n}$ & \multicolumn{2}{|c|}{$\mathrm{I}_{2}\left(\mathrm{~N}_{2} \mathrm{O}\right)_{\mathrm{n}}$} & \multicolumn{2}{c|}{$\mathrm{I}^{-}\left(\mathrm{CO}_{2}\right)_{\mathrm{n}}$} & \multicolumn{2}{c|}{$\mathrm{Br}^{-}\left(\mathrm{CO}_{2}\right)_{\mathrm{n}}$} \\
\hline & $\mathrm{eKE}(\mathrm{eV})$ & $\Delta \mathrm{E}(\mathrm{meV})$ & $\mathrm{eKE}(\mathrm{eV})$ & $\Delta \mathrm{E}(\mathrm{meV})$ & $\mathrm{eKE}(\mathrm{eV})$ & $\Delta \mathrm{E}(\mathrm{meV})$ \\
\hline \hline 0 & 1.600 & 0 & 2.77 & 0 & 2.460 & 0 \\
\hline 1 & 1.485 & 115 & 2.598 & 172 & 2.230 & 230 \\
\hline 2 & 1.358 & 127 & 2.442 & 156 & 2.011 & 219 \\
\hline 3 & 1.242 & 116 & 2.283 & 159 & 1.792 & 219 \\
\hline 4 & 1.122 & 120 & 2.127 & 156 & 1.568 & 224 \\
\hline 5 & 1.006 & 116 & 1.993 & 134 & 1.387 & 181 \\
\hline 6 & 0.905 & 101 & 1.864 & 129 & 1.214 & 173 \\
\hline 7 & 0.808 & 97 & 1.720 & 144 & 1.056 & 158 \\
\hline 8 & 0.715 & 93 & 1.587 & 133 & 0.897 & 159 \\
\hline 9 & 0.612 & 103 & 1.467 & 120 & 0.820 & 77 \\
\hline 10 & 0.535 & 77 & 1.430 & 37 & 0.741 & 79 \\
\hline 11 & 0.492 & 43 & 1.385 & 45 & 0.720 & 21 \\
\hline 12 & 0.434 & 58 & 1.316 & 70 & - & - \\
\hline 13 & - & - & 1.270 & 46 & - & - \\
\hline
\end{tabular}

Figure 6.7 contains the plot of SSE vs. cluster size. The data show that the first several $\mathrm{CO}_{2}$ and $\mathrm{N}_{2} \mathrm{O}$ molecules added to the cluster provide approximately equal stabilization of the halide charge. This behavior is in agreement with a previous study of $I^{-}\left(\mathrm{CO}_{2}\right)_{n=1-7}$, where 
Markovich et al. also observe approximately equal SSEs for these clusters. ${ }^{10}$ Thus, the first several solvent molecules appear to occupy equivalent sites in the cluster around the halide anion.

However, after a gradual decrease in the stabilization energy, there occurs a sudden drop-off for both the $\mathrm{CO}_{2}$ and $\mathrm{N}_{2} \mathrm{O}$ clusters when the cluster contains from 8-10 solvent molecules. The drop-offs occur after $\mathrm{Br}^{-}\left(\mathrm{CO}_{2}\right)_{n=8}, \Gamma\left(\mathrm{CO}_{2}\right)_{\mathrm{n}=9}$, and $\mathrm{I}^{-}\left(\mathrm{N}_{2} \mathrm{O}\right)_{\mathrm{n}=11}$. For the $I\left(\mathrm{~N}_{2} \mathrm{O}\right)_{n}$ clusters, a small dip occurs in the plot at $\mathrm{n}=9$. It is apparent the the additional solvent molecules bind to the clusters through different, weaker interactions. Most likely, this behavior is an indication that the solvent is forming a shell around the halide anion and effectively shielding the charge from any additional solvent molecules.

As mentioned above Markovich et al. have studied the $\mathrm{I}^{-}\left(\mathrm{H}_{2} \mathrm{O}\right)_{\mathrm{n}=1-60}$ clusters. ${ }^{10}$ In their studies, they observe a relatively consistent SSE for the first six $\mathrm{H}_{2} \mathrm{O}$ molecules added to the $\mathrm{I}^{-}$ anion. The lower SSE values measured for $n \geq 7$ are interpreted as the closing of the first solvation shell around the $\mathrm{I}^{-}$anion by the first six $\mathrm{H}_{2} \mathrm{O}$ molecules. While some calculations have suggested that halide anions reside on the surface of the water clusters, ${ }^{18}$ recent calculations by Combariza et al. ${ }^{19}$ support the interpretation of the PES data in terms of a caged iodide anion. The smaller $\mathrm{H}_{2} \mathrm{O}$ solvation shell, as compared to $\mathrm{CO}_{2}$, is consistent with the relative average binding energies for the first shell $(0.35 \mathrm{eV}$ and $0.15 \mathrm{eV}$, respectively). The stronger interactions will results in shorter halide-solvent separations which, in turn, will result in a smaller solvation shell due to steric effects.

The results presented in Figure 6.7 show that the SSEs for the $\mathrm{I}^{-}\left(\mathrm{N}_{2} \mathrm{O}\right)_{n}$ clusters are consistently lower than those for $\mathrm{I}^{-}\left(\mathrm{CO}_{2}\right)_{\mathrm{n}}$ and $\mathrm{Br}^{-}\left(\mathrm{CO}_{2}\right)_{\mathrm{n}}$ clusters. This is rather surprising in

Figure 6.7. Plot of the stepwise solvation energy for the $\mathrm{I}^{-}\left(\mathrm{N}_{2} \mathrm{O}\right)_{\mathrm{n}}, \mathrm{I}^{-}\left(\mathrm{CO}_{2}\right)_{\mathrm{n}}$, and $\mathrm{Br}^{-}\left(\mathrm{CO}_{2}\right)_{\mathrm{n}}$ clusters as a function of the number of solvent molecules. Energies are determined at $25 \%$ of the full peak height for the highest eKE feature in each spectrum. 


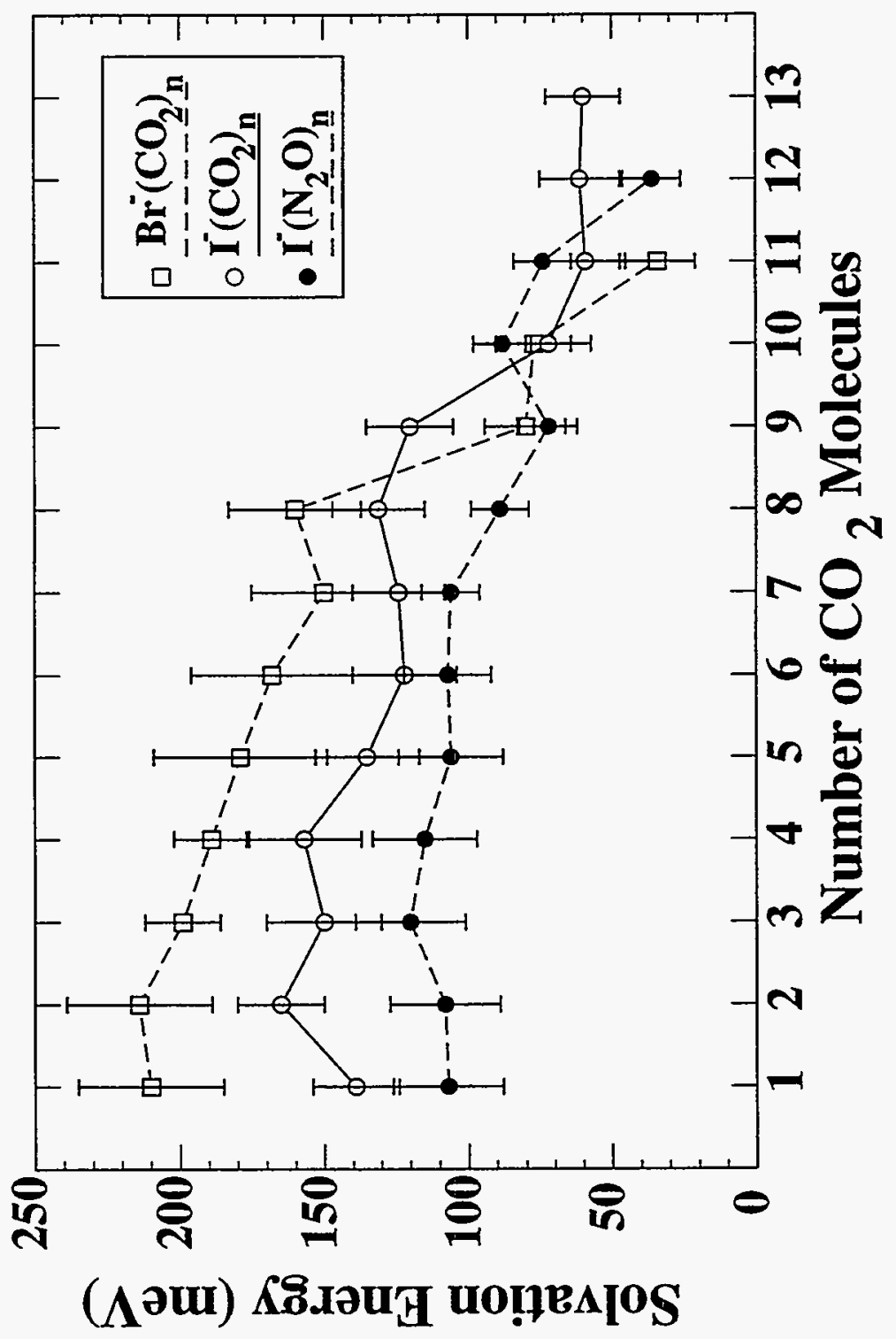

Figure 6.7 
light of the fact that the charge-dipole is a stronger interaction than the charge-quadrupole interaction which is expected to be the dominant term in the $\mathrm{X}^{-}\left(\mathrm{CO}_{2}\right)_{\mathrm{n}}$ clusters. There are several effects which may contribute to this result. Since these clusters are weakly bound, the thermodynamics and geometries are determined primarily by the dominant electrostatic interactions involved. By considering the $\mathrm{X}^{-}\left(\mathrm{CO}_{2}\right)$ and $\mathrm{X}^{-}\left(\mathrm{N}_{2} \mathrm{O}\right)$ long-range electrostatic interactions using the known properties of the $\mathrm{CO}_{2}$ and $\mathrm{N}_{2} \mathrm{O}$ molecules, a qualitative understanding of the observed results is obtained.

While $\mathrm{CO}_{2}$ and $\mathrm{N}_{2} \mathrm{O}$ are isoelectronic molecules, they possess slightly different electrostatic properties which leads to a significantly different solute-solvent interaction. As discussed in detail previously, ${ }^{12}$ the leading term in the $\mathrm{X}^{-}-\mathrm{CO}_{2}$ interaction is the chargequadrupole interaction, $q-\Theta$,

$$
E=\frac{q \cdot \Theta}{2 R^{3}}\left(3 \cdot \cos ^{2}(\xi)-1\right)
$$

where $q=-1$ is the halide charge and $\Theta$ is the quadrupole of the $\mathrm{CO}_{2}$ molecule $\left(\Theta_{\mathrm{CO}_{2}}=-4.3 \mathrm{x}\right.$ $\left.10^{-26} \mathrm{esu} \cdot \mathrm{cm}^{2}\right) .{ }^{20}$ The geometrical variables, $\mathrm{R}$ and $\xi$, are illustrated in Fig. 6.8. The negative sign of $\Theta$ indicates that the carbon atom is positively charged relative to the oxygen atoms. According to Eq. (3), the $\mathrm{X}^{-}\left(\mathrm{CO}_{2}\right)$ cluster will be T-shaped. However, as shown previously, ${ }^{12}$ the charge-quadrupole interaction actually distorts the $\mathrm{CO}_{2}$ molecule from linearity. This distortion results in the observed vibrational structure observed in the $\mathrm{X}^{-}\left(\mathrm{CO}_{2}\right)_{\mathrm{n}}$ spectra. The

Figure 6.8. Schematic diagram of $\mathrm{X}^{-}(\mathrm{M})_{\mathrm{n}}$ complex with coordinates used in the electrostatic calculations. In the figure, $\mathrm{X}=\mathrm{I}$ or $\mathrm{Br}$ and $\mathrm{A}-\mathrm{B}-\mathrm{C}=\mathrm{O}-\mathrm{C}-\mathrm{O}$ or $\mathrm{N}-\mathrm{N}-$ $\mathrm{O}$, in order. Note that for $\mathrm{N}_{2} \mathrm{O}$ the variable, $\mathrm{R}$, refers to the distance from the halide to the center of charge of the molecule, which is not the same as the center of mass. 


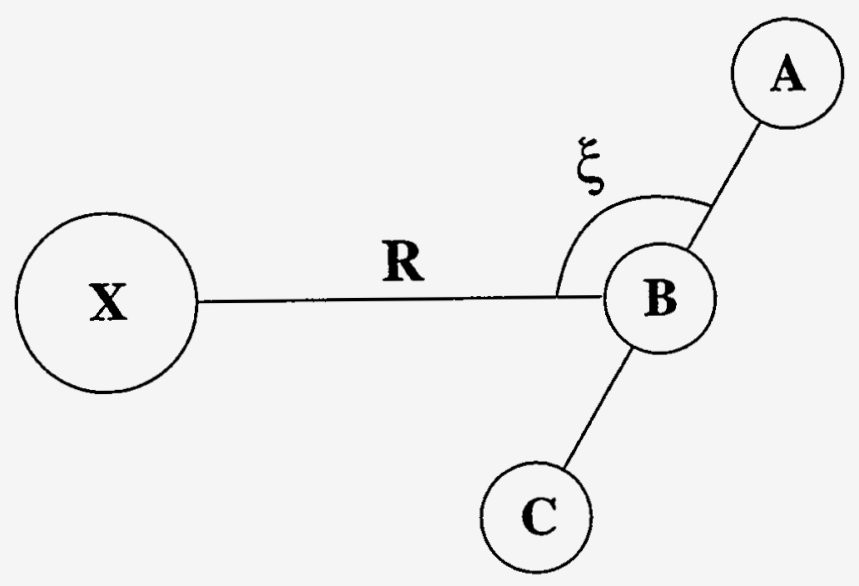

Figure 6.8 
$\mathrm{CO}_{2}$ distortions were determined by a Franck-Condon analysis of the photoelectron spectra $\left(\theta_{\mathrm{OCO}}=174.5 \pm 1.5^{\circ}\right.$ and $172.2 \pm 1.5^{\circ}$ in the $\mathrm{I}^{-}\left(\mathrm{CO}_{2}\right)$ and $\mathrm{Br}^{-}\left(\mathrm{CO}_{2}\right)$ clusters, respectively $)$.

The $\mathrm{I}^{-}\left(\mathrm{N}_{2} \mathrm{O}\right)_{\mathfrak{n}}$ spectra do not contain any features which may be assigned to vibrational motions of the solvent molecules like those observed for $\mathrm{X}^{-}\left(\mathrm{CO}_{2}\right)_{\mathrm{n}}$. This implies that the $\mathrm{N}_{2} \mathrm{O}$ molecules are not significantly distorted by the core ion. To understand the different binding for the $\Gamma\left(\mathrm{N}_{2} \mathrm{O}\right)$ clusters, one must consider the both charge-dipole and charge-quadrupole interactions because $\mathrm{N}_{2} \mathrm{O}$ has a weak dipole moment $\left(\mu_{\mathrm{N}_{2} \mathrm{O}}=0.1608 \mathrm{D} ; \stackrel{+}{\mathrm{N}}-\mathrm{N}-\overline{\mathrm{O}}\right)^{21}$ and a significant quadrupole moment $\left(\Theta_{\mathrm{N}_{2} \mathrm{O}}=-3.36 \pm 0.18 \times 10^{-26} \mathrm{esu} \cdot \mathrm{cm}^{2}\right)^{22}$ which is slightly less than that of the $\mathrm{CO}_{2}$ molecule. The charge-dipole ( $\mathrm{q}-\mu$ ) expression is given in Eq. (4),

$$
E=\frac{q \cdot \mu \cdot \cos (\xi)}{R^{2}}
$$

where $\mu$ is the $\mathrm{N}_{2} \mathrm{O}$ dipole.

Shown in Fig. 6.9(a) and 6.9(b) are surface plots and contour plots for Eq. (3) and (4) as a function of $\xi$ and $\mathrm{R}$ as shown in Fig. 6.8. The values of the $\mathrm{N}_{2} \mathrm{O}$ multipole moments are used to calculate these surfaces. As expected, the q- $\mu$ interaction energetically favors a linear $\mathrm{X}^{-}-\mathrm{N}-\mathrm{N}-\mathrm{O}$ orientation whereas the $\mathrm{q}-\Theta$ interaction, as in the case of $\mathrm{X}^{-}\left(\mathrm{CO}_{2}\right)$, favors a $\mathrm{T}-$ shaped cluster and is repulsive in the linear configurations. By summing Eq. (3) and Eq. (4), we obtain the dominant long range attractive forces between the $\mathrm{I}^{-}$anion and the $\mathrm{N}_{2} \mathrm{O}$ molecule. The result of this summation is shown in Fig. 6.9(c). The figure shows that q- $\Theta$ interaction is the dominant term of the $\mathrm{I}-\mathrm{N}_{2} \mathrm{O}$ electrostatic interaction. The $\mathrm{q}-\mu$ term appears to act only as a slight perturbation. This result suggests that the $\mathrm{I}^{-}\left(\mathrm{N}_{2} \mathrm{O}\right)$ cluster is nearly T-shaped. While

Figure 6.9. Surface plots and contour plots of (a) Eq. (3) and (b) Eq. (4) and (c) the sum of both terms, using the dipole and quadrupole moments of the $\mathrm{N}_{2} \mathrm{O}$ molecule and a charge of $q=-1$ for the halide. Note that the energy axes are different in the three plots. Variables are shown in Fig. 6.8. 

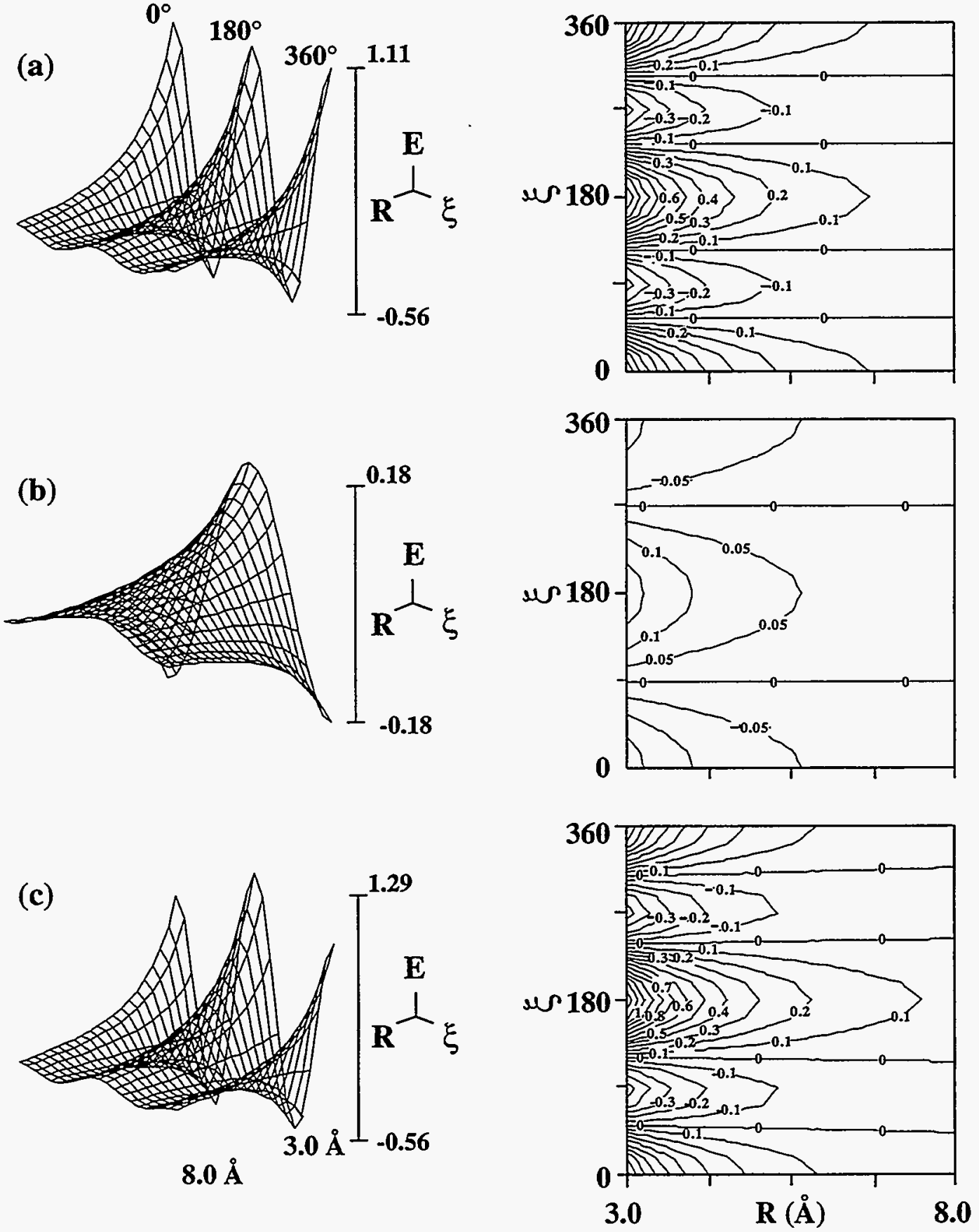

Figure 6.9 
comparison with the $\mathrm{I}^{-}\left(\mathrm{CO}_{2}\right)$ results indicates that the $\mathrm{N}_{2} \mathrm{O}$ molecule could be distorted ${ }^{23}$ by the $\mathrm{q}-\Theta$ electrostatic interaction no evidence exists in the photoelectron spectrum for such an effect. The difference is most likely attributed to the smaller quadrupole of the $\mathrm{N}_{2} \mathrm{O}$ molecule. The small $\mathrm{q}-\mu$ interaction may also hinder solvent distortion.

As the cluster size increases beyond two or more component atoms or molecules, the interactions which determine the geometries obviously become more complex. However, for all of the spectra presented, the thermodynamic data indicate that the solvent molecules add to the halide in nearly equivalent sites. An even distribution of the solvent molecules around the halide minimizes solvent-solvent interactions which become more important as the cluster grows. The relative flatness of the $I\left(\mathrm{~N}_{2} \mathrm{O}\right)_{n}$ curve in Fig. 6.7, as compared to the $\Gamma^{-}\left(\mathrm{CO}_{2}\right)_{n}$ and the $\mathrm{Br}^{-}\left(\mathrm{CO}_{2}\right)_{\mathrm{n}}$ curves, suggests that the $\mathrm{N}_{2} \mathrm{O}$ molecules are able to efficiently locate sites which are nearly equivalent up through nine solvent molecules. The interactions in the $\mathrm{CO}_{2}$ clusters, particularly in the $\mathrm{Br}^{-}\left(\mathrm{CO}_{2}\right)_{\mathrm{n}}$ clusters, appear to gradually decrease with increasing size. Further information about the differences between and similarities of the $I^{-}\left(\mathrm{CO}_{2}\right)_{\mathrm{n}}$ and the $\mathrm{Br}^{-}\left(\mathrm{CO}_{2}\right)_{\mathrm{n}}$ data can be obtained by an analysis of the vibrational structure present in the data which is to be discussed in the following section.

\subsubsection{Vibrational Structure}

As we have shown previousiy, ${ }^{12}$ the relaxation of the $\mathrm{CO}_{2}$ molecule from a bent to linear geometry upon photodetachment produces the vibrational structure observed in the $\mathrm{X}^{-}\left(\mathrm{CO}_{2}\right)_{\mathrm{n}}$ data. The lengthening of this vibrational progression as the cluster increases in size is quite interesting and provides information about the stepwise solvation of the halide anion. In general, the longer progression suggests a larger displacement along the relevant normal coordinate of vibration in the cluster. While a zero-order interpretation of this result suggests that the $\mathrm{CO}_{2}$ subunit is more distorted as the cluster size increases, a more detailed analysis of 
the vibrations reveals that, in fact, the individual $\mathrm{CO}_{2}$ molecules are distorted by an approximately equal or lesser amount as the cluster grows.

We consider the photodetachment of an $\mathrm{X}^{-}\left(\mathrm{CO}_{2}\right)_{\mathrm{n}}$ cluster in which all OCO angles and bond strengths (i.e., force constants) are the same as that of the binary complex, $\mathrm{X}^{-}\left(\mathrm{CO}_{2}\right)$. Photodetachment should primarily excite the collective $\mathrm{CO}_{2}$ bending vibration in which all of the $\mathrm{CO}_{2}$ molecules vibrate in-phase with each other. The extent of the observed vibrational progression in the photoelectron spectrum is determined by $\Delta \mathrm{Q}_{\mathrm{n}}^{\mathrm{CO}_{2}}$, the displacement along the normal coordinate for this in-phase bend between the anion and the neutral clusters. If we consider the $n \mathrm{CO}_{2}$ molecules as uncoupled harmonic oscillators distributed symmetrically around the $I^{-} / I$ atom, the in-phase bend normal coordinate for each cluster can be related to that of the single cluster by an appropriate normalization. ${ }^{24}$

For two clusters, $I^{-}\left(\mathrm{CO}_{2}\right)$ and $\mathrm{I}^{-}\left(\mathrm{CO}_{2}\right)_{n}$, in which identical changes occur in all of the $\angle$ OCO angles, conversion of the Cartesian displacements to normal coordinate displacements ${ }^{25}$ (i.e., $\angle \mathrm{OCO}$ to $\Delta \mathrm{Q}_{\mathrm{n}}^{\mathrm{CO}_{2}}$ ) shows that $\Delta \mathrm{Q}_{\mathrm{n}}^{\mathrm{CO}_{2}}$ is enhanced relative to $\Delta \mathrm{Q}_{1}^{\mathrm{CO}_{2}}$ by

$$
\Delta \mathrm{Q}_{\mathrm{n}}^{\mathrm{CO}_{2}} \approx \sqrt{\mathrm{n}} \cdot \Delta \mathrm{Q}_{1}^{\mathrm{CO}_{2}}
$$

Therefore, if the OCO angle is independent of $\mathrm{n}$, Eq. (5) predicts longer vibrational progressions as $\mathrm{n}$ increases.

Shown in Fig. 6.10 are comparisons of Franck-Condon simulations performed under the constraint of Eq. (5) with the $\mathrm{I}^{-}\left(\mathrm{CO}_{2}\right)_{\mathrm{n}=1-4}$ and $\mathrm{Br}^{-}\left(\mathrm{CO}_{2}\right)_{\mathrm{n}=1-4}$ experimental data. The simulations are compared to the ${ }^{2} \mathrm{P}_{1 / 2}$ region of the spectrum; the ${ }^{2} \mathrm{P}_{3 / 2}$ data is complicated by

Figure 6.10. Comparison of Franck-Condon simulations performed under the constraints of Eq. (5) with the photoelectron spectra of $\mathrm{I}^{-}\left(\mathrm{CO}_{2}\right)_{n}$ and $\mathrm{Br}^{-}\left(\mathrm{CO}_{2}\right)_{n}, \mathrm{n}=1-4$. See text for details. 


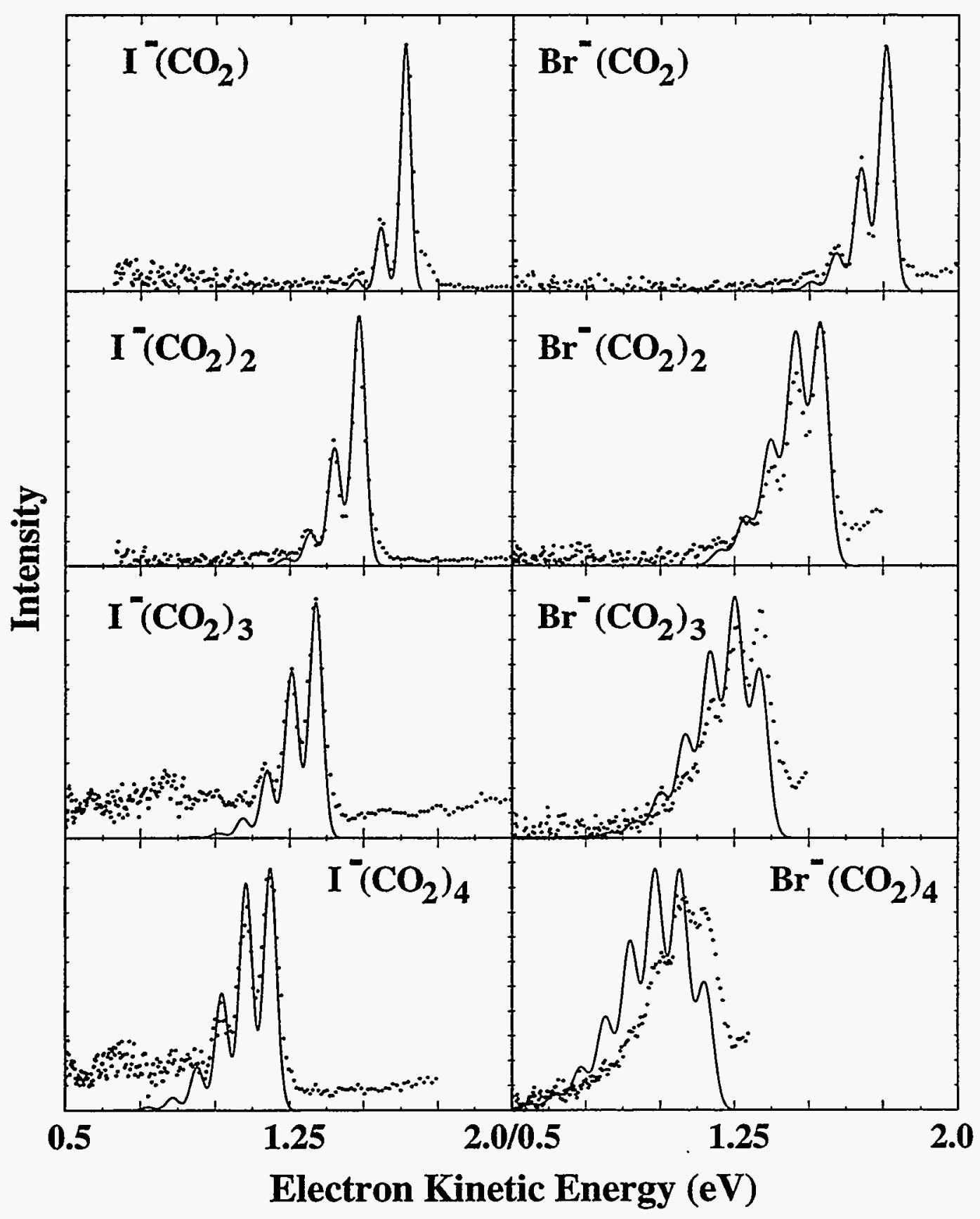

Figure 6.10 
transitions to the two subcomponents of the ${ }^{2} \mathrm{P}_{3 / 2}$ state which are split by the solvent molecule. ${ }^{14}$ The lengths of the $\mathrm{CO}_{2}$ bending progressions are the same for both the ground and excited states since they are primarily determined by the $\mathrm{CO}_{2}$ distortion in the anion cluster. In the photoelectron spectra, the intensities of the vibrational peaks, I, are determined by the Franck-Condon factors (FCFs) as in Eq. (6)

$$
I=v_{e} \cdot\left|\tau_{e}\right|^{2} \cdot\left|\left\langle\psi_{v^{*}}\left(Q_{\mathrm{CO}_{2}}\right) \mid \psi_{v^{*}}\left(Q_{\mathrm{CO}_{2}}\right)\right\rangle\right|^{2}
$$

In Eq. (6) $\tau_{\mathrm{e}}$ is the electronic transition dipole, which is assumed to be constant over the energy range of the vibrational progression and $v_{e}$ is the asymptotic velocity of the photoelectron. ${ }^{26}$ The FCFs are determined by calculation of the spatial overlaps of the anion and neutral vibrational wave functions of harmonic oscillators used to model the bend mode potential surface. The FCFs are convoluted with the experimental resolution function plus an additional Gaussian with a full width at half maximum (FWHM) of $\sim 15 \mathrm{meV}$ (to account for unresolved van der Waals progressions) for comparison to the experimental data. The $\Delta Q_{1}^{\mathrm{CO}_{2}}$ values are determined from fits of the $\mathrm{I}^{-}\left(\mathrm{CO}_{2}\right)$ and $\mathrm{Br}^{-}\left(\mathrm{CO}_{2}\right)$ data. The $\Delta \mathrm{Q}_{\mathrm{n}}^{\mathrm{CO}_{2}}$ values, calculated using Eq. (5), are used to generate the simulations for the $\mathrm{I}^{-}\left(\mathrm{CO}_{2}\right)_{\mathrm{n}=2-4}$ and $\mathrm{Br}^{-}\left(\mathrm{CO}_{2}\right)_{\mathrm{n}=2-4}$ data.

The agreement between data and simulations for $\mathrm{I}^{-}\left(\mathrm{CO}_{2}\right)_{\mathrm{n}}$ is excellent for the four spectra shown suggesting that the above structural considerations are indeed plausible. The agreement of the $\mathrm{I}^{-}\left(\mathrm{CO}_{2}\right)_{\mathrm{n}}$ simulations with the data strongly suggest that the $\mathrm{CO}_{2}$ molecules surround the iodide anion in equivalent positions (at least through $n=4$ ). This is consistent with the results of a Distributed Multipole Analysis presented in the previous paper, illustrating that the solvent $\mathrm{CO}_{2}$ distortion is well modeled by a model which considers only electrostatic interactions. Thus, if there is negligible interaction among the $\mathrm{CO}_{2}$ solvent molecules, each will have an identical interaction with the halide. As a result, all of the $\mathrm{CO}_{2}$ molecules are equally distorted. 
However, the agreement for the $\mathrm{Br}^{-}\left(\mathrm{CO}_{2}\right)_{\mathrm{n}}$ data is not as satisfying. The simulations overestimate the vibrational excitation for the larger clusters. This suggests that different clustering dynamics and interactions are involved for $\mathrm{I}^{-}\left(\mathrm{CO}_{2}\right)_{\mathrm{n}}$ vs. $\mathrm{Br}^{-}\left(\mathrm{CO}_{2}\right)_{\mathrm{n}}$. The most obvious conclusion to be drawn from this result is that the $\mathrm{OCO}$ angles in $\mathrm{Br}^{-}\left(\mathrm{CO}_{2}\right)_{\mathrm{n}}$ become more linear, on average, as a function of cluster size.

By what mechanism does the greater $\mathrm{CO}_{2}$ distortion in the smaller $\mathrm{Br}^{-}\left(\mathrm{CO}_{2}\right)_{\mathrm{n}}$ clusters occur, as compared to the larger clusters? As discussed in the previous paper, if the $\operatorname{Br}^{-}\left(\mathrm{CO}_{2}\right)$ distortion is assisted by a charge-transfer mechanism (CT), then the addition of another solvent molecule will result in a more linear average $\mathrm{CO}_{2}$ molecule because the $\mathrm{CT}$ efficiency decreases. Another possibility is that solvent-solvent interactions favoring more linear $\mathrm{CO}_{2}$ molecules become non-negligible for the multiple clusters, beginning with $\mathrm{Br}^{-}\left(\mathrm{CO}_{2}\right)_{2}$. Both of these possibilities are consistent with the observations. In either case, the data show that the $\mathrm{CO}_{2}$ molecules of the larger clusters are not identical to the $\mathrm{CO}_{2}$ in the $\mathrm{Br}^{-}\left(\mathrm{CO}_{2}\right)$ complex, as in the case of the $\mathrm{I}^{-}\left(\mathrm{CO}_{2}\right)_{\mathrm{n}}$ clusters. This effect is not nearly as clear from the SSE's (Fig. 6.6), illustrating the importance of making spectroscopic as well as energetic measurements.

One of the most intriguing aspects of the $I^{-}\left(\mathrm{CO}_{2}\right)_{n}$ data is the evolution of the vibrational structure in the $\mathrm{I}\left({ }^{2} \mathrm{P}_{3 / 2}\right) \cdot \mathrm{CO}_{2}$ band as the cluster size grows past $\mathrm{n}=9$, as compared to that of the $\mathrm{I}\left({ }^{2} \mathrm{P}_{1 / 2}\right) \cdot \mathrm{CO}_{2}$ band. The ${ }^{2} \mathrm{P}_{3 / 2}$ features are only partially resolved at $\mathrm{n}=2$ in the $4.657 \mathrm{eV}$ data (Fig. 6.3). No resolved features are observed for the ${ }^{2} \mathrm{P}_{3 / 2}$ band in the $5.822 \mathrm{eV}$ data until the cluster size reaches $\mathrm{I}^{-}\left(\mathrm{CO}_{2}\right)_{\mathrm{n}=10}$. At this point, a vibrational progression not only reappears but also becomes better resolved as additional $\mathrm{CO}_{2}$ molecules are added. While the resolution of the apparatus does improve at lower eKE, this is not sufficient to explain the absence of resolved peaks in the $n=9$ spectrum and their re-emergence in the $n \geq 10$ spectra. Of these larger clusters, the most resolved progression is observed in the $\mathrm{I}^{-}\left(\mathrm{CO}_{2}\right)_{13}$ spectrum.

As pointed out previously, ${ }^{12}$ the disappearance and reappearance of the vibrational peaks most likely results from the effects of stepwise solvation on the electronic structure of the 
neutral halogen atom. The approach of the $\mathrm{CO}_{2}$ molecule to the halogen splits the degeneracy of the halogen ${ }^{2} \mathrm{P}_{3 / 2}$ ground state, an effect which accounts for the extra width of the ground state peaks with respect to those of the excited state. However, as the cluster grows large enough for the halogen to be surrounded by solvent molecules, the isotropic spatial symmetry of the isolated halogen atom nearly returns. Thus, the subcomponents of the ${ }^{2} \mathrm{P}_{3 / 2}$ state become nearly degenerate and the peaks narrow allowing resolution of the vibrational progression. It is interesting to note that the size at which the vibrational structure becomes most prominent $(\mathrm{n}=$ 13) is well beyond the solvent shell size predicted by the SSE data $(n=9)$. Further studies on larger clusters may resolve this discrepancy.

Since the bromide anion has a smaller spatial extent than iodide, and thus a higher charge density, a smaller solvation shell is observed, for $\mathrm{Br}^{-}$than for $\mathrm{I}^{-}$. For the same reason, the vibrational structure which appears in the $\mathrm{I}^{-}\left(\mathrm{CO}_{2}\right)_{\mathrm{n}}$ data night be expected to appear at a smaller cluster size than is observed for $\mathrm{I}^{-}\left(\mathrm{CO}_{2}\right)_{\mathrm{n}}$. The drop-off in the stepwise solvation energy occurs at about the same cluster size but no vibrational structure reappears as in the case of $\mathrm{I}^{-}\left(\mathrm{CO}_{2}\right)_{\mathrm{n}}$. Provided the reappearance of vibrational features in the $\mathrm{I}^{-}\left(\mathrm{CO}_{2}\right)_{\mathrm{n}}$ data does, in fact, result from the interaction of the solvent molecules with the iodine ground electronic state, the $\mathrm{Br}^{-}\left(\mathrm{CO}_{2}\right)_{\mathrm{n}}$ data suggest that the $\mathrm{CO}_{2}$ molecules do not form a symmetric structure around the $\mathrm{Br}^{-}$atom, perhaps as a result of solvent-solvent steric effects.

\subsection{Conclusions}

Negative ion photoelectron spectroscopy of the $I\left(\mathrm{CO}_{2}\right)_{n=1-13}, I\left(\mathrm{~N}_{2} \mathrm{O}\right)_{n=1-12}$ and $\mathrm{Br}^{-}\left(\mathrm{CO}_{2}\right)_{\mathrm{n}=1-12}$ clusters has been used to investigate and compare the solvation of $\mathrm{I}^{-}$by $\mathrm{CO}_{2}$ and $\mathrm{N}_{2} \mathrm{O}$ and $\mathrm{Br}^{-}$by $\mathrm{CO}_{2}$. The measured SSEs provide information about the size of the first solvation shell which forms around the halide in the $\mathrm{X}^{-}\left(\mathrm{CO}_{2}\right)_{\mathrm{n}}$ and $\mathrm{I}^{-}\left(\mathrm{N}_{2} \mathrm{O}\right)_{\mathrm{n}}$ clusters. The sudden decrease in the SSE after $\Gamma\left(\mathrm{N}_{2} \mathrm{O}\right)_{\mathrm{n}=11}, \mathrm{I}^{-}\left(\mathrm{CO}_{2}\right)_{\mathrm{n}=9}$ and $\mathrm{Br}^{-}\left(\mathrm{CO}_{2}\right)_{\mathrm{n}=8}$ suggests that the first solvation shells for the $\mathrm{I}^{-}\left(\mathrm{CO}_{2}\right)_{\mathrm{n}}$ and $\mathrm{I}^{-}\left(\mathrm{N}_{2} \mathrm{O}\right)_{\mathrm{n}}$ cluster contain nine and eleven molecules while 
eight $\mathrm{CO}_{2}$ molecules make up the $\mathrm{Br}^{-}\left(\mathrm{CO}_{2}\right)_{\mathrm{n}}$ solvation shell. The size of the $\mathrm{CO}_{2}$ shell determined here is larger than that determined for the $\mathrm{I}^{-}\left(\mathrm{H}_{2} \mathrm{O}\right)_{n}$ cluster by Markovich et al. ${ }^{9}$ as a result of the relatively smaller halide-solvent interaction energies. As a further comparison, Lineberger and co-workers ${ }^{27}$ find solvent shell sizes of $n=14$ and $n=16$ for the $\operatorname{Br}_{2}^{-}\left(\mathrm{CO}_{2}\right)_{\mathfrak{n}}$ and $\mathrm{I}_{2}\left(\mathrm{CO}_{2}\right)_{\mathrm{n}}$ clusters, respectively. All of these results are consistent with the assertion that the solvent shell size correlates with the binding energy of the solute to an individual solvent molecules.

The spectra suggest that different primary structures form when $\mathrm{N}_{2} \mathrm{O}$ and $\mathrm{CO}_{2}$ molecules cluster to a negatively charged ion. This is supported by the appearance of a vibrational progression in the $\mathrm{X}^{-}\left(\mathrm{CO}_{2}\right)_{\mathrm{n}}$ data which are absent in the $I\left(\mathrm{~N}_{2} \mathrm{O}\right)_{\mathrm{n}}$ spectra. The measured SSEs show that the distortion of the $\mathrm{CO}_{2}$ molecules in the $\mathrm{X}^{-}\left(\mathrm{CO}_{2}\right)_{\mathrm{n}}$ clusters provide an additional stabilization of the cluster. Comparison of the $\mathrm{I}$ and $\mathrm{Br}$ data shows a correlation between SSE and the degree of $\mathrm{CO}_{2}$ distortion. The data show that the ion core does not distort the $\mathrm{N}_{2} \mathrm{O}$ molecules, even though the $\mathrm{N}_{2} \mathrm{O}$ quadrupole is comparable to that of $\mathrm{CO}_{2}$ and the $\mathrm{q}-\Theta$ term dominates the electrostatic attractive potential. Thus, the lower SSEs observed from $\Gamma\left(\mathrm{N}_{2} \mathrm{O}\right)_{n}$ (as compared to $\Gamma\left(\mathrm{CO}_{2}\right)_{n}$, result from two effects: 1 ) the slightly smaller quadrupole of $\mathrm{N}_{2} \mathrm{O}$ leads to a weaker $\mathrm{q}-\Theta$ interaction and 2) the $\mathrm{N}_{2} \mathrm{O}$ molecule does not receive additional stabilization through distortion.

The vibrational features in the $\mathrm{X}^{-}\left(\mathrm{CO}_{2}\right)_{\mathrm{n}}$ data, which are assigned to excitation of an inphase $\mathrm{CO}_{2}$ bending vibration upon photodetachment of the anion cluster, are interpreted in terms of a $\mathrm{CO}_{2}$ distortion in the anion clusters. For $\mathrm{I}^{-}\left(\mathrm{CO}_{2}\right)_{\mathrm{n}}$, the vibrational features are observed in the spectra of all the clusters through $\mathrm{I}^{-}\left(\mathrm{CO}_{2}\right)_{13}$. However, for the $\mathrm{Br}^{-}\left(\mathrm{CO}_{2}\right)_{\mathrm{n}}$ spectra, the vibrational features becomes congested and unresolved for the large clusters indicating a difference in the solvation dynamics between $\mathrm{I}^{-}\left(\mathrm{CO}_{2}\right)_{\mathbf{n}}$ and $\mathrm{Br}^{-}\left(\mathrm{CO}_{2}\right)_{n}$. This change in the structure of the solvent shell species upon photodetachment is particularly relevant as a connection between these gas-phase studies and bulk phenomena. Ultrafast 
studies have shown that electronic excitation of solvated molecules can induce a very rapid solvent response which basically involves a reorganization of the first solvent shell. ${ }^{28,29}$

The data show that the halide electronic structure is perturbed by the solvent molecules.

In particular, the data indicate that the halogen ${ }^{2} \mathrm{P}_{3 / 2}$ degeneracy is lifted by interactions with the solvent molecules. In the case of the $\mathrm{I}^{-}\left(\mathrm{CO}_{2}\right)_{\mathrm{n}}$ clusters, the ${ }^{2} \mathrm{P}_{3 / 2}$ degeneracy is nearly regained as the solvent molecules surround the halide. In contrast, the different behavior of the larger $\mathrm{Br}^{-}\left(\mathrm{CO}_{2}\right)_{\mathrm{n}}$ spectra implies a reasonable difference in the solvation of $\mathrm{I}^{-}$and $\mathrm{Br}^{-}$.

Overall, the results suggest that the small differences in the composition of the clusters can results on significantly different clustering geometries and dynamics which will conceivably be carried over into the bulk solvation properties. By studying the evolution of the clustering properties as a function of size, it is possible to investigate the interactions which dominate the solvation of negative ions.

\subsection{Acknowledgments}

This work has been supported by the United States Air Force Office of Scientific Research under Contract No. F49620-94-1-0115. 


\section{References}

${ }^{1}$ Several reviews of ion cluster studies are available: E. J. Bieske and J. P. Maier, Chem. Rev. 93, 2603 (1993); A. W. Castleman, Jr. and R. G. Keesee, Chem. Rev. 86. 589 (1986); P. Kebarle, Ann. Rev. Phys. Chem. 28, 445 (1977).

2O. Cheshnovsky, S. H. Yang, C. L. Pettiette, M. J. Craycraft, Y. Liu, and R. E. Smalley, Chem. Phys. Lett. 138, 119 (1987); S. H. Yang, K. J. Taylor, M. J. Craycraft, J. Conceicao, C. L. Pettiette, O. Cheshnovsky, and R. E. Smalley, Chem. Phys. Lett. 144, 431 (1988); K. J. Taylor, C. L. Pettiette-Hall, O. Cheshnovsky and R. E. Smalley, J. Chem. Phys. 96, 3319 (1992).

${ }^{3}$ T. N. Kitsopoulos, C. J. Chick, A. Weaver, and D. M. Neumark, J. Chem. Phys. 93, 6108 (1990); D. W. Arnold, S. E. Bradforth, T. N. Kitsolopoulos, and D. M. Neumark, J. Chem. Phys. 95, 8753 (1991); C. C. Arnold, Y.Zhao, T. N. Kitsopoulos, and D. M. Neumark, J. Chem. Phys. 97, 6121 (1992); C. C. Arnold, T. N. Kitsopoulos, and D. M. Neumark, J. Chem. Phys. 99, 766 (1993); C. C. Arnold and D. M. Neumark, J. Chem. Phys. 99, 3353 (1993); C. C. Amold and D. M. Neumark, J. Chem. Phys. 100, 1797 (1994).

${ }^{4}$ G. Ganteför. M. Gausa, K. H. Meiwes-Broer, and H. O. Lutz, Faraday Discuss. Chem. Soc. 86, 197 (1988); ibid., J. Chem. Soc. Faraday Trans. 2 6, 2483 (1990).

${ }^{5}$ D. G. Leopold, J. Ho, and W. C. Lineberger, J. Chem. Phys. 86, 1715 (1987); J. Ho, K. M. Ervin, and W. C. Lineberger, J. Chem. Phys. 93, 6987 (1990).

${ }^{6}$ M. J. deLuca, B. Niu, and M. A. Johnson, J. Chem. Phys. 88, 5857 (1988); M. J. deLuca, C. -C. Han, and M. A. Johnson, J. Chem. Phys. 93, 268 (1990).

${ }^{7}$ K. M. McHugh, J. G. Eaton, G. H. Lee, H. W. Sarkas, L. H. Kidder, J. T. Snodgrass, M. R. Manaa, and K. H. Bowen, J. Chem. Phys. 91, 3792 (1989); J. V. Coe, G. H. Lee, J. G. Eaton, S. T. Arnold, H. W. Sarkas, K. H. Bowen, C. Ludewigt, H. Haberland, and D. R. Worsnop, J. Chem. Phys. 92, 3980 (1990).

8J. V. Coe, J. T. Snodgrass, C. B. Friedhoff, M. K. McHugh, and K. H. Bowen, J. Chem. Phys. 83, 3169 (1985); ibid., J. Chem. Phys. 87, 4302 (1987); J. G. Eaton, S. T. Arnold, and K. H. Bowen, Int. J. Mass Spectrom. and Ion Proc. 102, 303 (1990); G. H. Lee, S. T. Arnold, J. G. Eaton, H. W. Sarkas, K. H. Bowen, C. Ludewigt, and H. Haberland, Z. Phys. D 20, 9 (1991).

${ }^{9}$ G. Markovich, R. Giniger, M. Levin, and O. Cheshnovsky, J. Chem. Phys. 95, 9416 (1991); G.

Markovich, S. Pollack, R. Giniger, and O. Cheshnovsky, Z. Phys. D. 26, 98 (1993).

${ }^{10}$ G. Markovich, R. Giniger, M. Levin and O. Cheshnovsky, Z. Phys. D 20, 69 (1991).

${ }^{11}$ D. M. Cyr, G. A. Bishea, M. G. Scarton, and M. A. Johnson, J. Chem. Phys. 97, 5911 (1992); D. M. Cyr, M. G. Scarton, and M. A. Johnson, J. Chem. Phys. 99, 4869 (1993).

${ }^{12}$ D. W. Arnold, S. E. Bradforth, E. H. Kim, and D. M. Neumark, J. Chem. Phys. (to be submitted).

${ }^{13}$ D. W. Arnold, S. E. Bradforth, E. H. Kim, and D. M. Neumark, J. Chem. Phys. 97, 9468 (1992).

14Y. Zhao, C. C. Arnold, and D. M. Neumark, J. Chem. Soc. Faraday Trans. 2 89, 1449 (1993). 
${ }^{15}$ R. B. Metz, A. Weaver, S. E. Bradforth, T. N. Kitsopoulos, and D. M. Neumark, J. Phys. Chem. 94, 1377 (1990).

${ }^{16}$ M. A. Johnson, M. L Alexander, and W. C. Lineberger, Chem. Phys. Lett. 112, 285 (1984).

${ }^{17}$ W. C. Wiley and I. H. McLaren, Rev. Sci. Instrum. 26, 1150 (1955).

${ }^{18}$ L. Perera, and M. L. Berkowitz, J. Chem. Phys. 99, 4222 (1993); L. S. Sremaniak, L. Perera, amd M. L. Berkowitz, Chem. Phys. Lett. 218, 377 (1994); L. Perera and M. L. Berkowitz, J. Chem. Phys. 100, 3085 (1994).

${ }^{19}$ J. E. Combariza, N. R. Kestner, and J. Jortner, J. Chem. Phys. 100, 2851 (1994).

${ }^{20}$ M. R. Battaglia, A. D. Buckingham, D. Neumark, R. K. Pierens, and J. H. Williams, Mol. Phys. 43, 1015 (1981).

${ }^{21}$ L. H. Sharpen, J. S. Muenter, and V. W. Laurie, J. Chem. Phys. 53, 2513 (1970).

22A. D. Buckingham, C. Graham, and J. H. Williams, Mol. Phys. 49, 703 (1983).

${ }^{23} \mathrm{~N}_{2} \mathrm{O}^{-}$has a calculated geometry of $\mathrm{r}_{\mathrm{e}, \mathrm{NN}}=1.22 \AA, \mathrm{r}_{\mathrm{e}, \mathrm{NO}}=1.38 \AA, \theta_{\mathrm{e}}=133^{\circ}$; the measured $\mathrm{EA}\left(\mathrm{N}_{2} \mathrm{O}\right)=$ $0.22 \pm 0.11 \mathrm{eV}$. D. G. Hopper, A. C. Wahl, R. L. C. Wu, and T. O. Tieman, J. Chem. Phys. 65, 5474 (1976).

${ }^{24}$ E. B. Wilson, Jr., J. C. Decius, and P. C. Cross, Molecular Vibrations (Dover, New York, 1980), p.27. The case for two harmonic oscillators is illustrated. We assume this describes the $\mathrm{CO}_{2}$ bending motion for the $n=2$ cluster. The $2^{1 / 2}$ term (and, more generally, the $n^{1 / 2}$ term) results from normal coordinate normalization.

${ }^{25}$ This assumes that the I atom motion is negligible in the single cluster, $\mathrm{I}^{-}\left(\mathrm{CO}_{2}\right)$.

${ }^{26}$ H. S. W. Massey, Negative Ions (Cambridge University Press, Cambridge, 1976); K. Ervin, J. Ho, and W. C. Lineberger, J. Chem. Phys. 91,5974 (1991).

${ }^{27}$ M. L. Alexander, N. E. Levinger, M.A. Johnson, D. Ray, and W. C. Lineberger, J. Chem. Phys. 88, 6200 (1988); J. M. Papanikolas, J. R. Gord, N. E. Levinger, D. Ray, V. Vorsa, and W. C. Lineberger, J. Phys. Chem. 95, 8028 (1991).

${ }^{28}$ M. Maroncelli, J. MacInnis, and G. R. Fleming, Science 243, 1674 (1989); S. Rosenthal, X. Xie, M. Du, and G. R. Fleming, J. Chem. Phys. 95, 4715 (1991).

${ }^{29}$ M. Maroncelli, J. Chem. Phys. 94, 2085 (1991). 


\section{Chapter 7: The Transition State of the $\mathbf{F}+\mathrm{H}_{2}$ Reaction: Agreement Between Theory and Experiment}

\subsection{Introduction}

In the remaining chapters, an application of anion photoelectron spectroscopy is described which is very different from that of the previous chapters. These experiments are aimed at studying chemical reaction dynamics rather than spectroscopy, particularly those which occur in the transition state region of a bimolecular chemical reaction. This application of anion PES has been one of the most exciting recent developments in the study of reaction dynamics.

The characteristics of the potential energy surface for a chemical reaction, particularly in the region of the saddle point, determine the dynamics of chemical reactions and all of the experimental observables, such as reaction rates and the product state distributions. While the asymptotic regions of a reactive potential energy surface (i.e., reactants and products) are usually quite easily studied by spectroscopic means, the characterization of the surface in the transition state region has proven extremely difficult due to the short-lived nature of the "transition state" species.

In the past decade, several studies have been made, in both the time domain and in the frequency domain, in hopes of directly studying the how reactants evolve into products at the transition region. Rather than attempting the "catch" the transition state species during a chemical reaction, these experiments have primarily taken the approach of studying the transitions state regions by means of observing the half-collision, or in other words, by preparing the transition state species from a well-defined precursor molecule which can be produced in reasonably high yield. With the significant advances in the development of femtosecond lasers, experiments have been performed which are able to study the real time evolution of these species.1,2 Typically, the ultrafast experiments are of the 'pump-probe' 
variety in which a reaction is initiated by the first pulse (pump) and monitored by a second time-delayed (probe) pulse. The initialization of the reaction with a first laser pulse is followed by a timed delayed monitoring of the reaction by a second laser pulse. Transition state experiments in the frequency domain also typically begin with a precursor molecule in which a reaction is monitored by fluorescence of the transition state species. $3.4,5$

The experiments to be described below also begin with a precursor molecule. In this case, one collects the photoelectron spectrum of a negative ion with a geometry which approximates that of a "transition state species'" for a bimolecular reaction. This is illustrated schematically in Fig. 7.1. Since the photoelectron spectrum is determined by the FranckCondon overlap between the anion and the neutral species, the photoelectron spectrum, in this case, will serve as a very sensitive probe of the shape of the potential energy surface in the region where the actual transformation from reactants to products occurs in a chemical reaction.

Several bimolecular reactions have been studied using this technique, including heavylight-heavy hydrogen transfer reactions $(\mathrm{X}+\mathrm{HY} \rightarrow \mathrm{XH}+\mathrm{Y} ; \mathrm{X}, \mathrm{Y}=\mathrm{I}, \mathrm{Br}, \mathrm{Cl}, \mathrm{F})^{6}$ and hydrogen abstraction reactions by fluorine $(\mathrm{OH}+\mathrm{F} \rightarrow \mathrm{O}+\mathrm{HF}$; and $\mathrm{RH}+\mathrm{F} \rightarrow \mathrm{R}+\mathrm{HF} ; \mathrm{R}=$ $\left.\mathrm{CH}_{3} \mathrm{O}, \mathrm{C}_{2} \mathrm{H}_{5} \mathrm{O}\right)^{7}$ illustrating that this technique is a very powerful method for obtaining information about reactive potential energy surfaces in the region of the transition state. In addition to the results presented in this chapter, two other applications of anion photoelectron spectroscopy to the study of transition state species will be discussed in the following chapters. Recent transition state studies of fundamental hydroxyl radical reactions will be described in

Figure 7.1: Schematic diagram of the study of the $\mathrm{F}+\mathrm{H}_{2}$ transition state region by photodetachment of the stable $\mathrm{FH}_{2}^{-}$anion. The lower surface is the anion potential energy surface calculated at the MP2 $/ 6-31+\mathrm{G}^{*}$ level of theory and the upper surface is the new $a b$ initio potential energy surface for the $\mathrm{F}+\mathrm{H}_{2}$ reaction calculated by Stark and Werner (see Sec. 7.4.1 for details). The plots represent energy as a function of the $\angle \mathrm{FHH}$ angle and the $\mathrm{F} \cdots \mathrm{H}_{2}$ distance. 


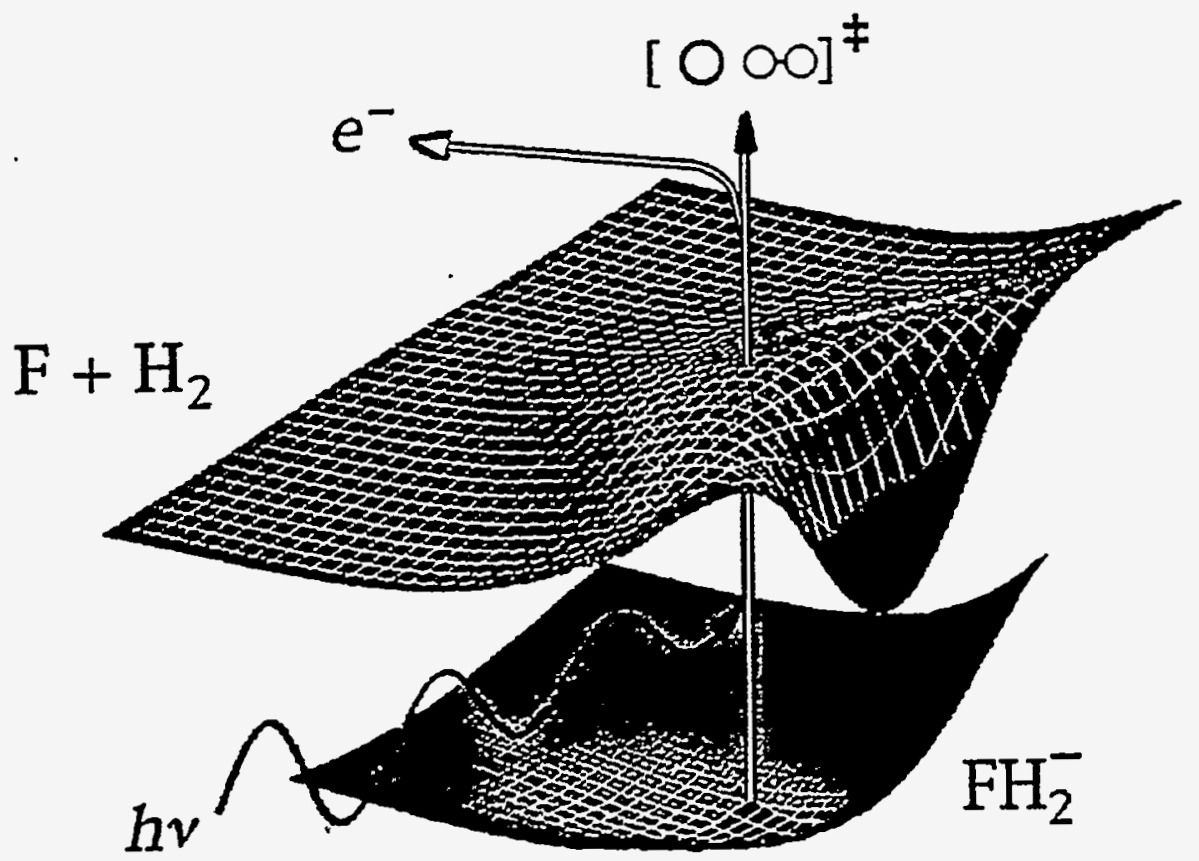

Figure 7.1 
the following chapter and an extension of the method to study reactions in clusters will be described in Chapter 9.

In this study, we address the prototypical reaction

$$
F+H_{2} \rightarrow H+H F
$$

which has been one of the most thoroughly studied reactions in chemical dynamics. This chapter describes the most recent anion photoelectron spectroscopy study of the transition state region of the $\mathrm{F}+\mathrm{H}_{2}$ chemical reaction and makes comparison to the theoretical results of $\mathrm{H}$. $-\mathrm{J}$. Werner, K. Stark and D. M. Manolopoulos. The results represent the refinement of previous studies of this reaction, both experimental and theoretical, leading to a remarkable agreement between experiment and theory for this reaction.

To familiarize the reader with the history of the $\mathrm{F}+\mathrm{H}_{2}$ reaction, a brief outline of the previous work on this reaction will be given in the following section. Included will be a summary of previous anion PES investigations of the $\mathrm{F}+\mathrm{H}_{2}$ reaction carried out in this laboratory. $8,9,10,11$ Full descriptions of these previous studies are included in the dissertations of Alex Weaver ${ }^{12}$ and Steve Bradforth. ${ }^{13}$ Following this, an experimental description will be given in Section 7.3, including a description of the modifications which were made to obtain the improved data set which is presented in Section 7.4. An analysis of the data (Section 7.5) will be followed by a brief summary of the theoretical methods employed by Stark and Werner ${ }^{14}$ to determine an new $a b$ initio potential energy surface (SW) will be given along with an outline of the methods used in the simulation of the PES data by Manolopoulos ${ }^{11}$ in Section 7.6. This will be followed (Section 7.7) by the comparison of the $\mathrm{FH}_{2}^{-}$photoelectron spectrum to simulations generated using several potential energy surfaces including a new fully $a b$ initio potential energy surface for the $\mathrm{F}+\mathrm{H}_{2}$ reaction. 


\subsection{Background}

The appeal of the $\mathrm{F}+\mathrm{H}_{2}$ reaction as a chemical dynamics prototype lies in its accessibility to both experimental and theoretical techniques. While both theory and experiment have progressed together since the early investigation some 35 years ago, it is useful to begin with a summary of the previous experimental results since they serve as the test of theoretical potential energy surface accuracy.

The $\mathrm{F}+\mathrm{H}_{2}$ reaction has several useful properties which include a large exothermicity $(\Delta \mathrm{H}=31.6 \mathrm{kcal} / \mathrm{mole})^{15}$ and a low energy of activation $(\sim 1 \mathrm{kcal} / \mathrm{mole}) .{ }^{16}$ As a result of the early barrier to reaction, a significant portion of the reaction exothermicity is efficiently funneled into vibrational excitation of the HF products. ${ }^{17,18}$ The HF vibrational population inversion which results from the $\mathrm{F}+\mathrm{H}_{2}$ allows a careful study of the vibrational distributions by the chemical laser ${ }^{15,19}$ and infrared chemiluminescence experiments ${ }^{20,21}$ whereby the HF vibrational product distribution was measured to be $[(v=1)=0.31 ;(v=2) 1.00 ;(v=3)=$ $0.47] .{ }^{20}$ The $\mathrm{F}+\mathrm{D}_{2}$ product state distribution peaks at $\mathrm{v}=3$. The rate of reaction has been accurately determined over a large temperature range. ${ }^{16}$ However, the most detailed experimental information obtained to date resulted from the crossed-molecular-beam studies of Lee and co-workers, ${ }^{22,23}$ most notably the results of Neumark et al. ${ }^{23}$ These experiments determined vibrationally state-resolved differential cross-sections for the $F+\mathrm{H}_{2}, \mathrm{~F}+\mathrm{D}_{2}$ and $\mathrm{F}$

$+\mathrm{HD}$ reactions. The $\mathrm{F}+\mathrm{D}_{2}$ results have recently been reinvestigated at higher resolution by Faubel et al. ${ }^{24}$ confirming the previous findings. Along with the angular distributions for the reactions, an interesting observation of forward scattering for $\mathrm{HF}(\mathrm{v}=3)$ and $\mathrm{DF}(\mathrm{v}=4)$ was interpreted in terms of a quantum mechanical dynamical resonance of the transition state complex. ${ }^{23}$

From a theoretical viewpoint, the $\mathrm{F}+\mathrm{H}_{2}$ reaction is very attractive for several reasons. The calculation of electronic structure is simplified by the small number of electrons. The three atom system provides the simplest reactive set of coordinates available for scattering 
calculations. Advancing computational capabilities made it possible to calculate properties of the surface by $a b$ initio methods. The early methods used to test the accuracy of potential energy surfaces, classical trajectory and transition state theory (TST), were complemented by the development of quasiclassical trajectory $(\mathrm{QCT})^{25}$ and full quantum mechanical scattering methods $(\mathrm{QM})^{26,27,28,29,30,31,32}$ which are also used now. These tools enabled the researchers to locate flaws in proposed surfaces more quickly and with greater precision. Nonetheless, the most appealing aspect of the $\mathrm{F}+\mathrm{H}_{2}$ reaction from a theoretical viewpoint, is the amount of experimental information which is available to test newly developed potential energy surfaces.

Table I: Characteristics of Selected $\mathrm{F}+\mathrm{H}_{2}$ Potential Energy Surfaces ${ }^{\mathrm{a}}$

\begin{tabular}{|ccccccc|}
\hline Surface & $\mathrm{V}^{\ddagger}$ & $\mathrm{R}_{\mathrm{F}-\mathrm{H}}$ & $\mathrm{R}_{\mathrm{H}}$ & ZPE & $\mathrm{V}_{2}$ & $\gamma$ \\
M5 & 1.06 & 2.91 & 1.44 & 1.39 & - & 180 \\
T5 & 0.74 & 3.01 & 1.43 & 0.81 & - & 180 \\
T5a & 0.94 & 2.97 & 1.44 & 0.88 & - & 180 \\
5SEC a & 1.57 & 3.17 & 1.44 & - & 317 & 180 \\
b & 0.97 & 3.10 & 1.43 & - & 428 & 104 \\
TS $^{b}$ & 0.78 & 3.13 & 1.42 & - & - & - \\
SW & 1.53 & 2.91 & 1.46 & - & - & 119 \\
\hline
\end{tabular}

a $V$ and $Z P E$ in $\mathrm{kcal} / \mathrm{mol}, \mathrm{R}$ and $\mathrm{R}$ in $\AA$, and $\mathrm{v}_{2}$ in $\mathrm{cm}^{-1}$; b) Collinear saddle point parameters.

While a comprehensive listing of the potential energy surfaces and their unique characteristics will not be given here, several will be discussed in the data analysis section. The most important features of the surfaces are considered to be the location of the saddle point, its energy relative to that of the separated reactants (i.e., the barrier height) and products, and the shape of the bending potential in the transition state region. Table I contains some of the characteristics of the potential energy surfaces to be discussed. Of the several surfaces that have previously been generated, each has been able to reproduce some aspects of the available data but none has found full agreement with the experimental observations. A description of 
four surfaces and their successes will be discussed below beginning with the Muckerman 5 surface $^{33}$ (M5) which was the most widely used surface prior to 1985 . Then we will consider the role which $a b$ initio calculations ${ }^{34}$ played in the development of the series of surfaces produces by Truhlar and co-workers beginning with the T5 surface and continuing to the $\mathrm{T} 5 \mathrm{a}^{35}$ and 5 SEC ${ }^{36}$ surfaces. A brief discussion of a recent modified LEPS (London-Eyring-PolanyiSato $)^{18}$ surface - designated as TS $^{37}$ - will be presented followed by introductory comments on the newly developed $a b$ initio surface of Stark and Werner. ${ }^{38}$

Partly as a result of computer limitations, early surfaces were semi-empirical, mainly based upon the LEPS formalism. $39,40,41,42,43$ The most widely used of these surfaces was known as the 'Muckerman V' or 'M5' surface. ${ }^{33}$ This potential energy surface was designed to match three experimentally observed characteristics of the reaction: 1) the exothermicity of the reaction, 2) the $1.71 \mathrm{kcal} /$ mole activation energy determined by Mercer and Pritchard ${ }^{44}$ (when calculated using classical trajectory calculations), and 3) the observed average vibrational energy of the product HF molecule $(29.5 \mathrm{kcal} / \mathrm{mol}) .{ }^{20}$ While the M5 surface was widely used in QCT and TST calculations, several flaws were soon pointed out by new experimental and theoretical results. More accurate measurements of the activation energy ${ }^{16}$ showed that the surface had been adjusted to match inaccurate experimental results. Additionally, the surface has a fairly strong bending potential at the saddle point (i.e., forcing the reaction through a collinear reaction path). This was inconsistent with the experimental results of Neumark et $a l .{ }^{23}$ who observed significant side scattering of the products in their crossed beam experiments. Trajectory calculations on the M5 surface, when compared to the experimental results, also indicated that the entrance barrier and the vibrationally adiabatic $\mathrm{HF}(\mathrm{v}=3)$ exit barrier were too large on the M5 surface.

$A b$ initio methods were beginning to be applied to the $\mathrm{F}+\mathrm{H}_{2}$ system in the early 1970 's. Schaefer and co-workers ${ }^{34}$ pioneered the investigation of the $\mathrm{F}+\mathrm{H}_{2}$ surface using these methods. The early calculations were incapable of producing a surface of sufficient 
accuracy to use in QCT, TST or QM calculations. However, they did point out early on $(1972)^{45}$ that electron correlation and large basis sets are required to achieve reasonable results. These results, while not used extensively to model experimental data, did point out some deficiencies in the semi-empirical surfaces that were being used. Most importantly, the bending potential of the $\left[\mathrm{FH}_{2}\right]^{\ddagger}$ complex at the saddle point was calculated to be much flatter than that of the M5 surface. Even so, the minimum was still at the linear configuration, encouraging the calculation of reaction surfaces which were constrained to a collinear reaction path for some time. $46,47,48$ As time progressed $a b$ initio calculations played a more important role in determining the $\mathrm{F}+\mathrm{H}_{2}$ reaction surface.

Beginning in 1984, Truhlar and co-workers began an effort to fit a potential energy surface to the available experimental and theoretical results. In general, their procedure consisted of 1) calculating observables on a given analytic reaction surface using QCT and TST methods, 2) identifying the region of the surface responsible for discrepancies, 3) modifying the surface accordingly and 4) repeating the calculations. Their initial efforts focused on modifications of the M5 surface parameters. ${ }^{49}$ After limited success, they introduced additional parameters for flexibility and incorporated the Schaefer's $a b$ initio calculations 50 more quantitatively into the surface fitting procedure. ${ }^{51}$ With their procedure, they produced a surface which matched the activation energy $\left(E_{a}\right)$, the molecular beam threshold energy for reaction $\left(\mathrm{E}_{\mathrm{thr}}\right)$, the exothermicity for the reaction $\left(\Delta \mathrm{H}_{\mathrm{r}}\right)$ and it had a rather flat bending potential in the region of the saddle point that matched Schaefer's calculated bending potential. This potential surface, known as T5, served as the forerunner for the more popular T5a and 5SEC surfaces.

In fact, the only significant difference between the T5 and T5a surfaces is the barrier height which was increased by $0.2 \mathrm{kcal} / \mathrm{mole}$ (to $0.94 \mathrm{kcal} / \mathrm{mol}$ ) to generate the latter surface. This improved the agreement with the reaction rate as calculated by variational TST methods. Thus, the T5a surface also finds agreement with $\mathrm{E}_{\mathrm{a}}, \mathrm{E}_{\mathrm{thr}}$, and $\Delta \mathrm{H}_{\mathrm{r}}$ values. The surface also 
reproduces the HF products angular distributions ${ }^{30,25}$ observed by Neumark et al. ${ }^{23}$ reasonably well. The major flaw in the surface is that it overestimates the product vibrational distributions, where it predicts the major channel to be $\operatorname{HF}(v=3)$ rather than $\operatorname{HF}(v=2)$.

In 1986, Schwenke et al.52 presented the results of $a b$ initio calculations which extrapolated (to the $\mathrm{CI}$ limit) the effects of electron correlation or configuration interaction (CI) on the shape of the $\mathrm{F}+\mathrm{H}_{2}$ potential energy surface. The inclusion of correlation effects through the 'scaled external correlation' (SEC) method had a significant effect on the bending potential which was calculated. Unlike the previous calculations, ${ }^{50}$ Schwenke et al. found that the bending potential at the collinear saddle point had a minimum away from the linear configuration. Thus, the calculations indicated that the reaction surface actually had a nonlinear transition state. These results were supported by even more complete calculations by Bauschlicher et al.53 Their study of electron correlation and CI also resulted in a very flat bending potential and also suggested that the surface had a nonlinear saddle point.

These findings were incorporated into the next modification of the T5/T5a surface, leading to the 5SEC surface in 1991.36 They adjusted the surface to allow for the flat bending potential and the nonlinear saddle point. Additionally, they included long-range dispersion forces in their fit which result in a $[\mathrm{F} \cdots \mathrm{H}-\mathrm{H}]$ van der Waals well in the reactant valley of the surface. This surface was also able to reproduce many observations. However, while an improvement was made in the product vibrational distributions, the 5SEC surface still overestimated the excitation with approximately equal amounts of $\mathrm{HF}(\mathrm{v}=3)$ and $\mathrm{HF}(\mathrm{v}=2)$.

Another reasonably successful reaction surface (TS) was developed by Takayanagi and Sato $^{37}$ in 1988 . The surface is a modified LEPS surface in which the Sato parameter has a functional form which is dependent upon the bond angle of the $\left[\mathrm{FH}_{2}\right]^{\ddagger}$ complex. The surface was generated by fitting the parameters to the observed product state distributions and the angular distributions using quasiclassical trajectory calculations. Their optimized surface has a bent transition state and flat bending potential in accord with the $a b$ initio results. ${ }^{52}$ While the 
product state and angular distributions are well modeled by this surface, the transition state theory rate constant for this surface is twice as large as the experimental rate constant.

Thus, through 1993, none of the proposed reaction surfaces were able to reproduce all of the available experimental data. The general features of the surface were becoming well understood but adjustments of the surface had not yet resulted in quantitative agreement with experiment. At this point a comparison of the bending potentials for the $\left[\mathrm{FH}_{2}\right]^{\frac{\hbar}{}}$ species at the saddle point of each surface serves as an instructional exercise. Figure 7.2 contains calculated the bending potential curves for four of the potential curves considered. Energy is plotted as a function of the $\angle \mathrm{FHH}$ angle, as shown. The curves show illustrate the significant difference between the T5a surface and the 5SEC, TS and SW (to be described below) surfaces. While the latter three surfaces all have minima at non-linear F-H-H angles, the flatness of the three surfaces vary slightly. These small differences along the bending coordinate can have a rather notable effect on the reaction dynamics (i.e., the HF vibrational distributions). As we shall show, they also significantly influence the simulations of the $\mathrm{FH}_{2}^{-}$photoelectron spectrum.

While modifications to the T5 surface continued in the Truhlar group, ${ }^{54}$ another approach was undertaken by Stark and Werner. Using the results of Bauschlicher et al. ${ }^{53}$ as a guide, further $a b$ initio calculations were carried out to study the effects of electron correlation and configuration interaction on the $\mathrm{F}+\mathrm{H}_{2}$ potential energy surface.55 In 1993, a global $a b$ initio potential energy surface was calculated for the $\mathrm{F}+\mathrm{H}_{2}$ reaction and fitted to an analytic function. This surface has turned out to be the most accurate $\mathrm{F}+\mathrm{H}_{2}$ surface to date. Details about the surface construction and simulations calculated on the surface will be presented in a later section when compared to the present results.

While theory advanced significantly since the results of Neumark et al. in 1985, no

Figure 7.2: Plot of the potential energy of the $\left[\mathrm{FH}_{2}\right]^{\frac{\hbar}{5}}$ complex at the transition state as a function of the $\angle F H H$ angle (illustrated at bottom) for the T5a, 5SEC, TS and SW potential energy surfaces. 

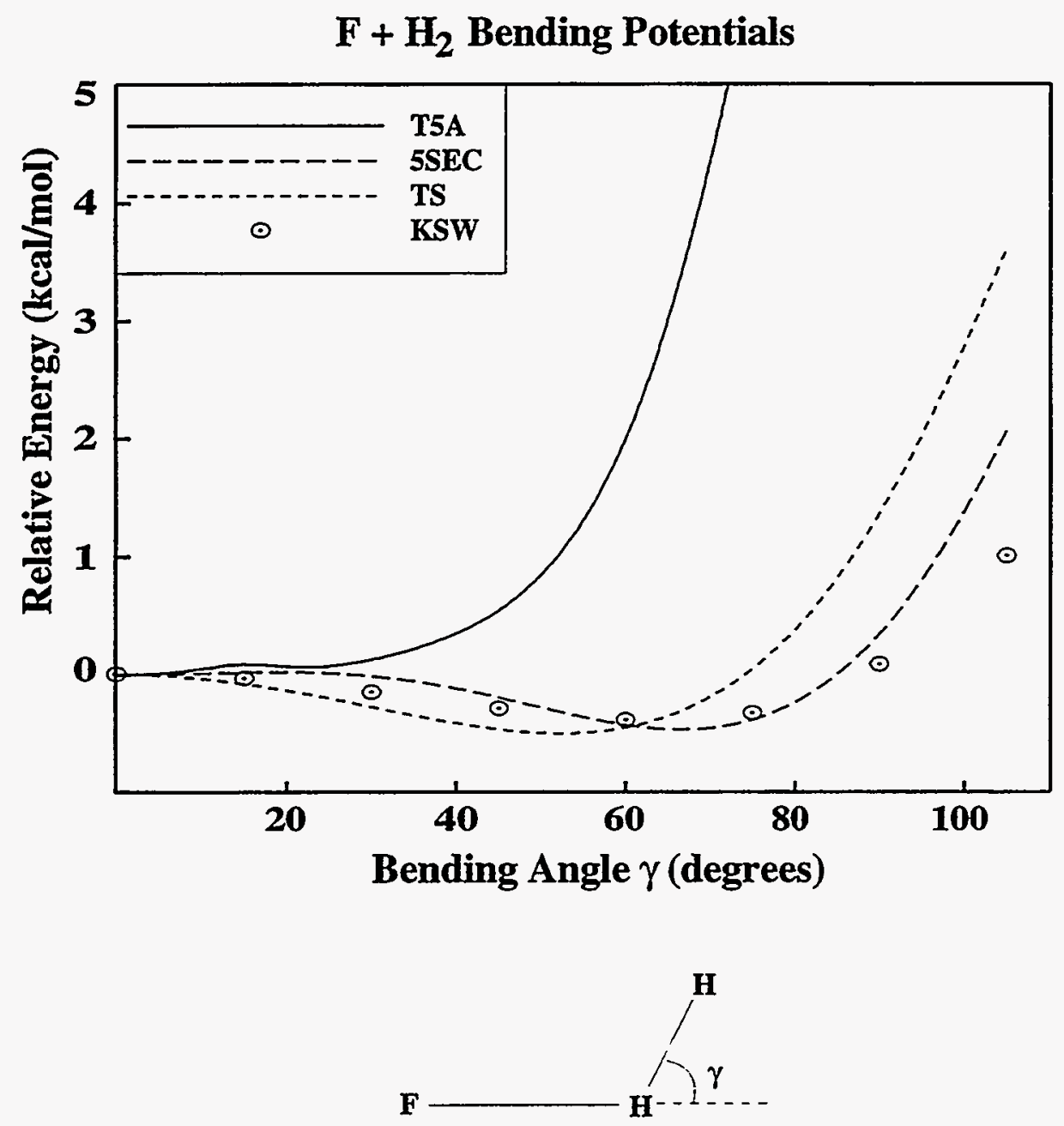

Figure 7.2 
further significant experimental results were obtained until 1990. Additionally, all of the previously available data were obtained by asymptotic methods (i.e., by detection of reactant and product properties). Thus, all of the details of the transition state region had to be extrapolated from the observed data. Since the dynamics of a chemical reaction are greatly determined by properties of the potential energy surface in the transition state region, it is desirable to use a technique which gives detailed information about this very important sector of the surface.

Neumark and co-workers had been developing a new transition state spectroscopy technique which is capable of directly studying the transition state region of bimolecular reactions. ${ }^{6-8,56}$ Several investigations of the $\mathrm{FH}_{2}^{-} \mathrm{PES}$ have been made in this laboratory using this technique. The first two studies made by Weaver et al., began with the collection of the $\mathrm{FH}_{2}^{-}$photoelectron spectrum ${ }^{8}$ in 1990, followed by another study in 1991 in which polarization studies of $\mathrm{FH}_{2}^{-}, \mathrm{FD}_{2}^{-}$, and $\mathrm{FHD}^{-}$were made. ${ }^{9}$ These studies and those which followed provided new information which could be used to test the accuracy of proposed reaction surfaces. In fact, the $\mathrm{FH}_{2}^{-}$PES has turned out to be a very sensitive probe of the $\mathrm{F}+\mathrm{H}_{2}$ transition state region, particularly along the bending coordinate.

Zhang and Miller $^{10}$ calculated the first simulations for the spectra by determining the Franck-Condon overlap of the anion ground state vibrational wave function with the scattering wave functions supported the T5a potential energy surface. The anion parameters were determined from the $a b$ initio calculations of Nichols et al..$^{57}$ While the agreement was initially believed to be reasonable, later experimental and theoretical results showed that, in fact, only a portion of the available photodetachment transitions had been considered in the simulations.

The missing transitions resulted from the incomplete treatment of the $\mathrm{H}_{2}$ nuclear symmetry. Bradforth et al. ${ }^{11}$ collected the photoelectron spectrum of $\mathrm{FH}_{2}^{-}$generated using para $-\mathrm{H}_{2}$ gas as part of the starting material (recall that due to the nuclear symmetry para- $\mathrm{H}_{2}$ only exists in even rotational levels while ortho- $\mathrm{H}_{2}$ only exists in odd rotational levels. 
Normal $-\mathrm{H}_{2}$ is a 3:1 mixture of ortho- $\mathrm{H}_{2}$ to para $-\mathrm{H}_{2}$. Further discussion will be given in Section 7.4). ${ }^{58}$ The appearance of the of the para- $\mathrm{FH}_{2}^{-}$spectrum was significantly different from that of the normal- $\mathrm{FH}_{2}^{-}$spectrum. While most of the observed features were present in both spectra, the intensity profiles were very different. The simulations by Zhang and Miller had actually been for para- $\mathrm{FH}_{2}^{-}$, but compared to the normal- $\mathrm{FH}_{2}^{-}$photoelectron spectrum. When the proper T5a simulation was calculated, the agreement with the experiment was rather poor for both the para- and normal- $\mathrm{FH}_{2}^{-}$spectra.

Better agreement was found between the experimental data and simulations on the TS and 5SEC surfaces, but considerably more resolved structure was predicted by the simulations than was observed in the spectra. ${ }^{11}$ These simulations, which will also be compared to the new data to be shown below, showed that bending motions of the $\left[\mathrm{FH}_{2}\right]^{\ddagger}$ transition state complex are expected to be excited upon photodetachment of the $\mathrm{FH}_{2}^{-}$anion.

Thus, previous investigation of the $\mathrm{FH}_{2}^{-} \mathrm{PES}$ have provided promising results but the agreement with the available theoretical results was questionable. This chapter describes the latest results for the $\mathrm{FH}_{2}^{-} \mathrm{PES}$ and the latest theoretical simulations of the data resulting in a convergence between theory and experiment for the $\mathrm{F}+\mathrm{H}_{2}$ system.

\subsection{Experimental}

The experimental apparatus used to study the $\mathrm{F}+\mathrm{H}_{2}$ transition state region has been described in detail previously. 59 The important features of the apparatus and the details relevant to these experiments will be given here. The source region, in which the $\mathrm{FH}_{2}^{-}$anions are formed, consists of a crossed molecular beam and electron beam. ${ }^{60}$ A mixture of $20 \% \mathrm{NF}_{3}$ / $40 \% \mathrm{H}_{2} / 40 \% \mathrm{~N}_{2}$ is expanded through the 0.020 " orifice of pulsed piezoelectric molecular beam valve operated at $20 \mathrm{~Hz}$ and a stagnation pressure of $\sim 4$ bar. Attachment of low-energy secondary electrons to the $\mathrm{NF}_{3}$ molecules produces $\mathrm{F}^{-}$which subsequently clusters to the $\mathrm{H}_{2}$ carrier gas as in Eq. (2): 


$$
\begin{gathered}
\mathrm{NF}_{3}+\mathrm{e}^{-} \rightarrow \mathrm{F}^{-}+\mathrm{NF}_{2} \\
\mathrm{~F}^{-}+\mathrm{H}_{2} \stackrel{\mathrm{M}}{\longrightarrow} \mathrm{FH}_{2}^{-}
\end{gathered}
$$

In these experiments, both normal- and para- $\mathrm{H}_{2}$ are used to generate normal- and para- $\mathrm{FH}_{2}^{-}$ anions. This portion of the experiment, which has turned out to be the most critical and the most difficult to accomplish successfully, will be discussed in more detail below.

Once the anions are formed, they are extracted into a Wiley-McLaren-type time-offlight mass spectrometer where the $\mathrm{FH}_{2}^{-}$are separated from other anions generated in the source region. The ions are then selectively photodetached by a properly timed 8 ns laser pulse. Photoelectrons which are ejected into the solid angle subtended by the $75 \mathrm{~mm}$ microchannel plate electron detector are energy analyzed by measurement of their time-offlight through the one meter field-free flight tube between the detector and the $-3 \mathrm{~mm}^{3}$ ionlaser interaction region. The spectral resolution of the apparatus is $\sim 10 \mathrm{meV}$ for photoelectrons with an electron kinetic energy (eKE) of $\sim 0.65 \mathrm{eV}$ and degrades as $(\mathrm{eKE})^{3 / 2}$. These experiments were performed using two different photodetachment energies. Most of the results were obtained using the 4 th harmonic $(266 \mathrm{~nm} ; 4.657 \mathrm{eV} ; 15 \mathrm{~mJ} / \mathrm{pulse})$ of a Nd:YAG laser. The other wavelength ( $299 \mathrm{~nm} ; 4.141 \mathrm{eV} ; 6 \mathrm{~mJ} / \mathrm{pulse}$ ) is the first Stokes Raman line generated by focusing the 4 th harmonic ( $50 \mathrm{~mJ} /$ pulse) of the Nd:YAG laser into a high pressure $\left(\sim 20\right.$ bar) $\mathrm{H}_{2}$ cell.

Naturally occurring hydrogen, 'normal- $\mathrm{H}_{2}$ ', consists of a 3:1 ratio of ortho- and para$\mathrm{H}_{2}$. For the production of normal- $\mathrm{FH}_{2}^{-}$, commercial hydrogen (99.99\% purity) was used in the $\mathrm{NF}_{3} / \mathrm{H}_{2} / \mathrm{N}_{2}$ gas mixture. Production of pure (99.7\%) para $-\mathrm{H}_{2}$ is prepared in the U. C. Berkeley Department of Chemistry Low Temperature Laboratory by passivation of normal- $\mathrm{H}_{2}$ on an activated Ni catalyst ("Houdrycat \#197-CP) at $22 \mathrm{~K}$. Under such conditions, a conversion from ortho- $\mathrm{H}_{2}$ to para- $\mathrm{H}_{2}$ occurs. As long as no catalytic surface is provided for the reverse 
conversion from para- to ortho- $\mathrm{H}_{2}$ after preparation, the para- $\mathrm{H}_{2}$ can be used at room temperature for a reasonable period of time. If the para- $\mathrm{H}_{2}$ is stored in aluminum cylinders, the half life for the para $\rightarrow$ ortho conversion is shown to be on the order of a few weeks. ${ }^{61,62}$

While previous data sets have been collected for both normal- and para- $\mathrm{FH}_{2}^{-}$, much valuable information was obscured as a result of the para $\rightarrow$ ortho conversion on the walls of the stainless steel cylinder in which gas mixtures were prepared for the earlier experiments on $\mathrm{FH}_{2}^{-}$. To prevent this conversion, the stainless steel cylinder was replaced by an aluminum cylinder which will not catalyze the conversion as efficiently. As an extra precaution to insure the highest possible purity of the para $-\mathrm{H}_{2}$ in the experiment, Teflon gas lines were used where possible. In the final arrangement the main site available for the conversion was in the stainless steel molecular beam valve housing where it is estimated that the gas has a residence time on the order of ten minutes before escaping into the source region where the ions are formed.

Upon receipt of the para- $\mathrm{H}_{2}$ from the Low Temperature Laboratory, the gas mixture was prepared and allowed to mix for approximately one hour. At this point, data was collected at $20 \mathrm{~Hz}$ in 60,000 laser shot increments for a total of approximately 1.5 million laser shots at $\mathrm{h} v=4.657 \mathrm{eV}$ and 0.9 million laser shots at $\mathrm{hv}=4.141 \mathrm{eV}$. At $\mathrm{hv}=4.657 \mathrm{eV}$, after each 60,000 laser shots, the plane polarized laser was rotated between angles of $0^{\circ}, 54.7^{\circ}$, and $90^{\circ}$. In this way, if any conversion occurred over the 31-hour period during which the data was collected it would be detected in spectra collected at the same polarization. This also prevented any mistaken assignment of peak intensity changes to a polarization dependence when they actually resulted from ortho- $\mathrm{H}_{2}$ contamination.

The results did not show any detectable ortho- $\mathrm{H}_{2}$ contamination under these experimental conditions, wherein all of the $4.657 \mathrm{eV}$ experiments were completed within 36 hours of the preparation of the para- $\mathrm{H}_{2}$. The $4.141 \mathrm{eV}$ data were collected after completion of the $4.657 \mathrm{eV}$ data set collection. Only one polarization $\left(\theta=0^{\circ}\right)$ was used for the $\mathrm{hv}=4.657 \mathrm{eV}$ experiments. Based upon a comparison of the first and last data sets, no significant ortho- $\mathrm{H}_{2}$ 
contamination appears to have occurred over the time of data collection. All of the $4.141 \mathrm{eV}$ para- $\mathrm{FH}_{2}^{-}$were collected within the 36 -hour period following the $4.657 \mathrm{eV}$ data collection.

In addition to the extreme care taken to ensure that the para $\rightarrow$ ortho conversion was minimized during data collection, the data were also improved, with respect to previous data sets, through the use of a more cleanly polarized laser beam. It was previously observed that signal resulting from photodetachment to an excited electronic surface of the $\left[\mathrm{FH}_{2}\right]^{\ddagger}$ complex could effectively removed from the spectrum by collection of the electron signal parallel to the laser polarization. A modification of the optical train decreased the amount of scrambling of the polarization which occurs as the laser beam passes through the optics en route to the photoelectron spectroscopy chamber. This significantly improved the spectrum by more efficiently discriminating against the transitions to the excited state surface. As a result, new features are resolved in the spectrum and the overall signal-to-noise is improved.

\subsection{Results}

Shown in Fig. 7.3 are photoelectron spectra of $\mathrm{FH}_{2}^{-}$collected using a $4.66 \mathrm{eV}$ photodetachment energy. For each spectrum, intensity of electron signal is plotted as a function of electron kinetic energy (eKE). The eKE is related to the internal energy neutral complex, $\mathrm{E}_{\mathrm{int}}^{0}$, by

$$
e K E=h v-D_{o}\left(F^{-} \cdots H_{2}\right)-E A(F)-E_{\mathrm{int}}^{o}+E_{\mathrm{int}}^{-}
$$

Figure 7.3: Photoelectron spectra of $\mathrm{FH}_{2}^{-}$collected at $\mathrm{hv}=4.657 \mathrm{eV}$. (Top) $\mathrm{FH}_{2}^{-}$ions are made using normal $-\mathrm{H}_{2}$ and (bottom) para $-\mathrm{H}_{2}$. Spectra are collected at two different laser polarizations: (left) parallel $\left[\theta=0^{\circ}\right]$ and (right) perpendicular $[\theta=$ $\left.90^{\circ}\right]$ to the direction of electron collection. 


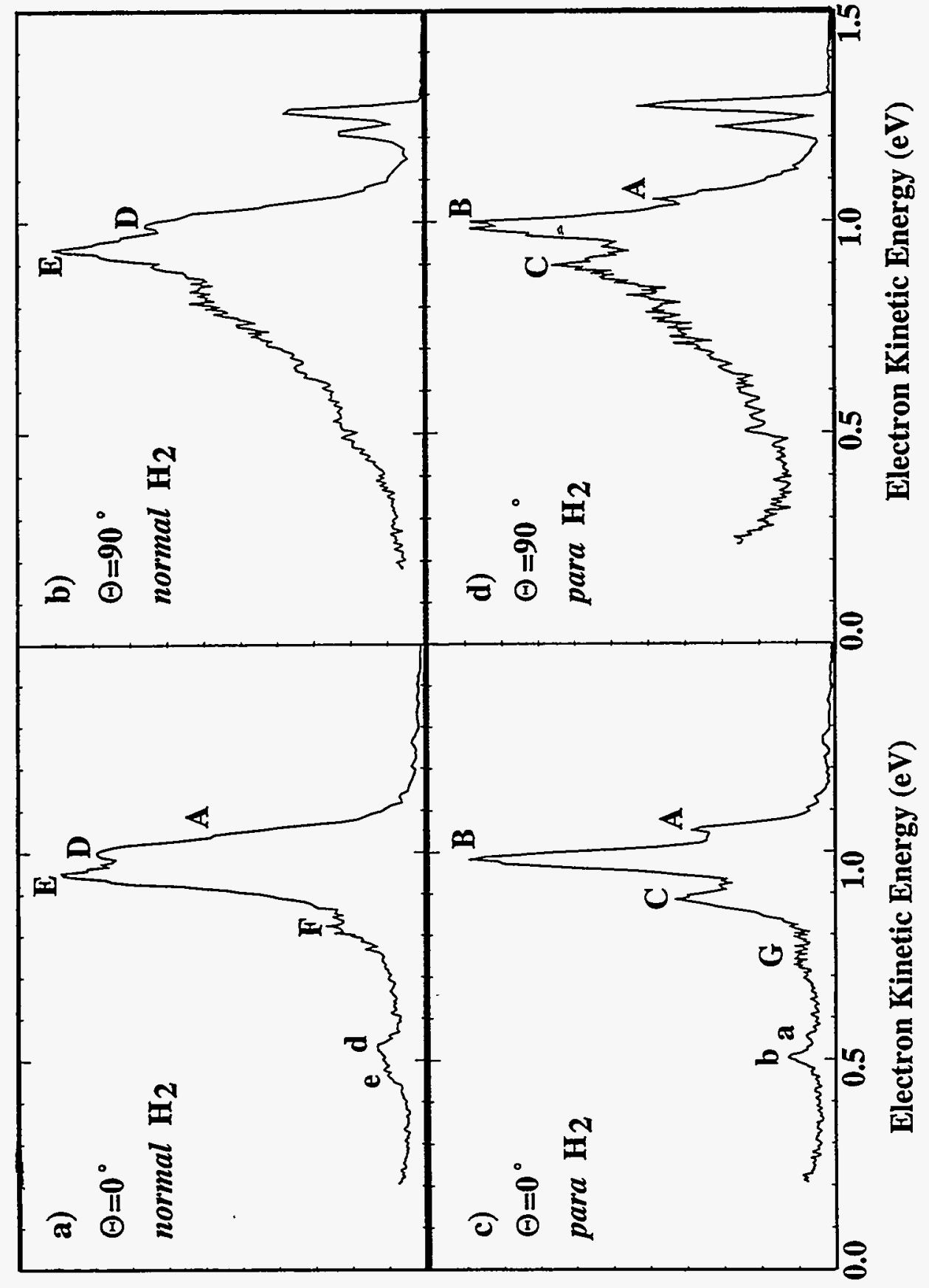

Figure 7.3 
This expression is illustrated in Fig. 7.4, where $h v=4.66 \mathrm{eV}$ is the photon energy, $\mathrm{D}_{\mathrm{o}}\left(\mathrm{FH}_{2}^{-}\right)$is the dissociation energy of the anion and $\mathrm{EA}(\mathrm{F})$ is the fluorine electron affinity. $\mathrm{E}_{\mathrm{int}}^{\circ}$ and $\mathrm{E}_{\mathrm{int}}^{-}$ represent the internal energy of the neutral and anion complexes, respectively. While $E_{\mathrm{int}}^{*}$ is simply the vibrational (and rotational) energy of the anion above the zero-point, $E_{\mathrm{int}}^{o}$ is the energy of the $\left[\mathrm{FH}_{2}\right]^{\ddagger}$ complex above the $\mathrm{F}+\mathrm{H}_{2}$ reactant dissociation asymptote. The four spectra were collected under different experimental conditions. The spectra in Figures 3a and $3 \mathrm{~b}$ were both collected using normal $-\mathrm{H}_{2}$ while the data in Figures $7.3 \mathrm{c}$ and $7.3 \mathrm{~d}$ were collected using para $-\mathrm{H}_{2}$. The direction of laser polarization used for Figures $7.3 \mathrm{a}$ and $7.3 \mathrm{c}$ is changed to a different value for the results shown in Figures $7.3 \mathrm{~b}$ and $7.3 \mathrm{~d}$. Clearly, the spectra are significantly affected by both of these parameters. The positions of the labeled features are summarized in Table II.

Changes which occur with the rotation of the laser polarization results from the anisotropy in the photoelectron angular distribution. The differential cross-section for photodetachment is given by

$$
\frac{d \sigma}{d \Omega}=\frac{\sigma_{t o t}(e K E)}{4 \pi}\left(1+\frac{\beta(e K E)}{2} \cdot\left(3 \cos ^{2} \theta-1\right)\right)
$$

In Eq. (4), $\sigma_{\text {tot }}(\mathrm{eKE})$ is the total cross-section for photodetachment and $\theta$ is the angle between the laser polarization and the direction of electron collection. The asymmetry parameter $(-1 \leq \beta$ $\leq 2$ ), typically has a minor energy dependence over the energy range considered. However, $\beta$ can be significantly different for transitions to two different electronic states, particularly if the

Figure 7.4: Schematic diagram illustrating the energetics involved in $\mathrm{FH}_{2}^{-}$ photodetachment to the $\mathrm{F}+\mathrm{H}_{2}$ transition state region. $\mathrm{D}_{\mathrm{o}}$ is the dissociation energy of the $\mathrm{FH}_{2}^{-}$anion, EA is the electron affinity of either the fluorine or hydrogen atom and $h v$ is the laser photodetachment energy. 


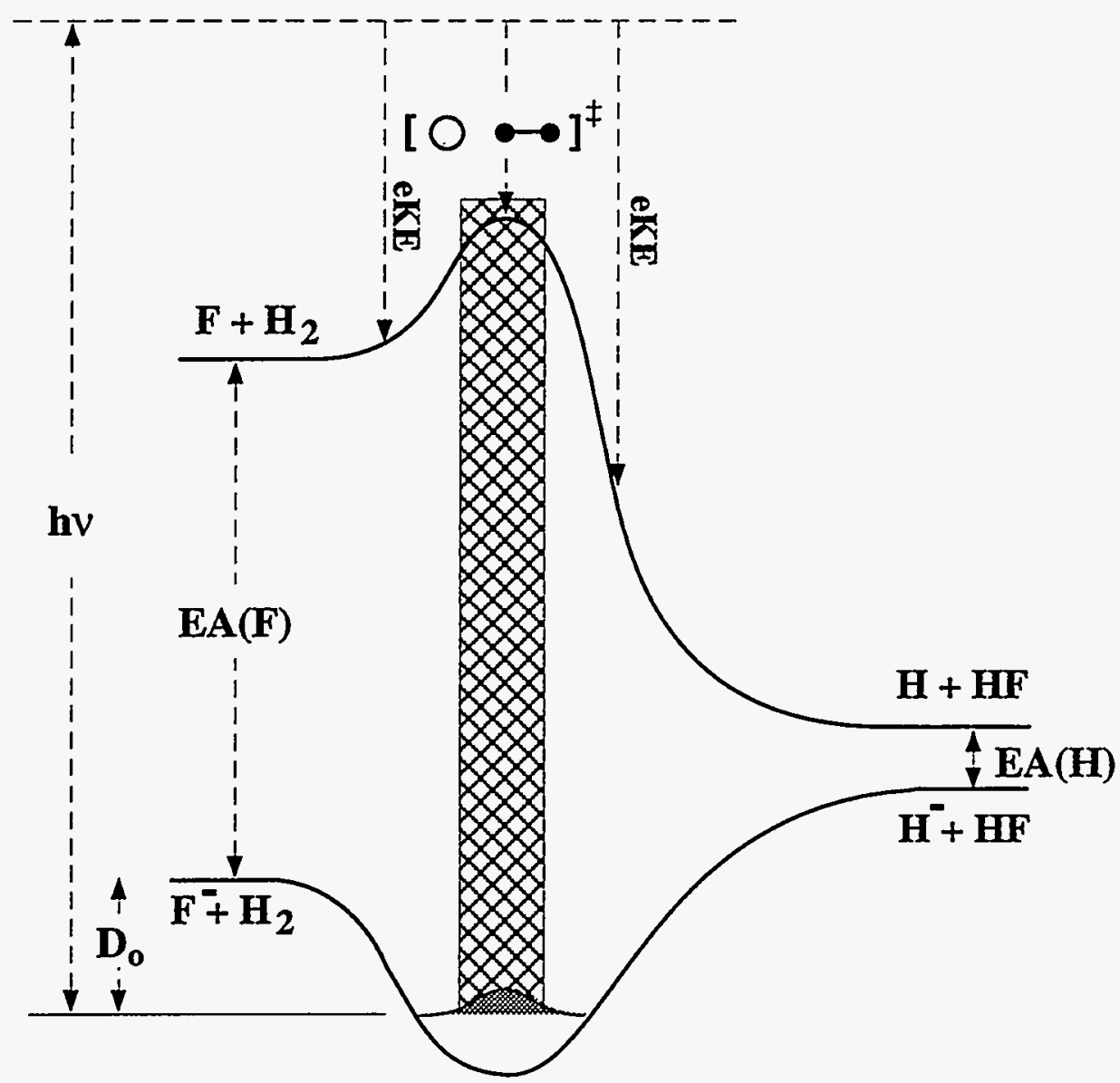

$\mathrm{FH}_{2}^{-}$

Rxn. Coord.

Figure 7.4 
two states result from detachment of electrons from two orbitals of different symmetries. Thus, the study laser polarization dependence allows the detection of transitions to multiple electronic states within a spectrum. This appears to be the case for the $\mathrm{FH}_{2}^{-}$spectra. While the $\theta=0^{\circ}$ spectra contain relatively narrow structured features, rotation of the laser polarization such that $\theta=90^{\circ}$ appears to introduce a broad, primarily unstructured feature into the data due to excited $\mathrm{F}+\mathrm{H}_{2}$ surfaces. The nature of these surfaces have been treated in detail elsewhere. ${ }^{11,13}$

Table II: Observed peak positions in the $\theta=0^{\circ}, 4.657 \mathrm{eV}$ and $4.141 \mathrm{eV}$ $\mathrm{FH}_{2}^{-}$photoelectron spectra.

\begin{tabular}{|c|c|c|}
\hline Peak & eKE (eV) & $\begin{array}{c}\text { Energy (cm } \\
1)\end{array}$ \\
\hline A & 1.044 & 0 \\
B & 0.974 & 565 \\
C & 0.877 & 1347 \\
D & 0.993 & 411 \\
E & 0.943 & 815 \\
F & 0.815 & 1847 \\
G & 0.745 & 2412 \\
a & 0.547 & 4008 \\
b & 0.500 & 4387 \\
d & 0.533 & 4121 \\
e & 0.466 & 4662 \\
\hline
\end{tabular}

Two additional peaks appear at $\sim 1.2-1.3 \mathrm{eV}$ in the $\theta=90^{\circ}$ data. The features result from a two-photon process whereby the $\mathrm{FH}_{2}^{-}$anion is dissociated by the first photon and the second photon detaches the resultant $\mathrm{F}^{-}$anion which is generated. The signal is enhanced in the $\theta=90^{\circ}$ spectra by the asymmetry parameter for the $\mathrm{F}^{-}$photodetachment process.

The difference between the normal- and para- $\mathrm{FH}_{2}^{-}$spectra is remarkable. The features labeled as $B$ and $C$ in the $\theta=90^{\circ}$ spectra lie at energies between the $D$ and $E$ and the $E$ and $F$ 
features, respectively. The features in the para- $\mathrm{FH}_{2}^{-}$spectrum are narrower and more cleanly resolved than those in the normal- $\mathrm{FH}_{2}^{-}$spectrum. Shown in Fig. 7.5 is a comparison of the previously reported para- $\mathrm{FH}_{2}^{-}$data with the present results. As seen, the new data shows clearly resolved features which were not previously observed. This improvement results directly from the care taken to minimize para $\rightarrow$ ortho conversion during the data collection process.

Due to the $\mathrm{eKE}^{3 / 2}$ dependence of the experimental resolution function, it is possible to obtain a spectrum at a higher resolution than that for the $4.657 \mathrm{eV}$ data by decreasing the photon energy used for photodetachment. This is useful to investigate whether there are unresolved features in the spectrum at the higher photon energy. Shown in Fig. 7.6 is the $\mathrm{FH}_{2}^{-}$ photoelectron spectrum collected at $\mathrm{hv}=4.141 \mathrm{eV}$. The peak labeling is consistent with that used in the $4.657 \mathrm{eV}$ data. By decreasing the photon energy from $4.657 \mathrm{eV}$ to $4.141 \mathrm{eV}$ the resolution at the eKE for peak $\mathrm{A}$ in the spectra is improved from $\sim 15 \mathrm{meV}$ to $\sim 10 \mathrm{meV}$. The intensity of peak $\mathbf{C}$ is significantly affected by the cutoff function of the electron detector due to its low eKE. Comparison with the $4.657 \mathrm{eV}$ data does not indicate any significant differences in the spectrum at the higher resolution. Therefore, comparisons to theoretical results will be made with the $4.657 \mathrm{eV}$ data, which are not affected by the electron detector cutoff function.

Figure 7.5: Comparison of new photoelectron spectrum of para- $\mathrm{FH}_{2}^{-}$to the previous data set which suffered from significant ortho- $\mathrm{FH}_{2}^{-}$contamination. The difference results from an improved gas handling procedure to prevent para- to ortho- $\mathrm{H}_{2}$ conversion before data collection. See text for details.

Figure 7.6: Photoelectron spectrum of para- $\mathrm{FH}_{2}^{-}$collected at $\mathrm{hv}=4.141 \mathrm{eV}$ and $\theta=0^{\circ}$. Although the experimental resolution is improved at lower $\mathrm{eKE}$, no further spectral features are resolved in the spectrum as compared to Fig 3. 


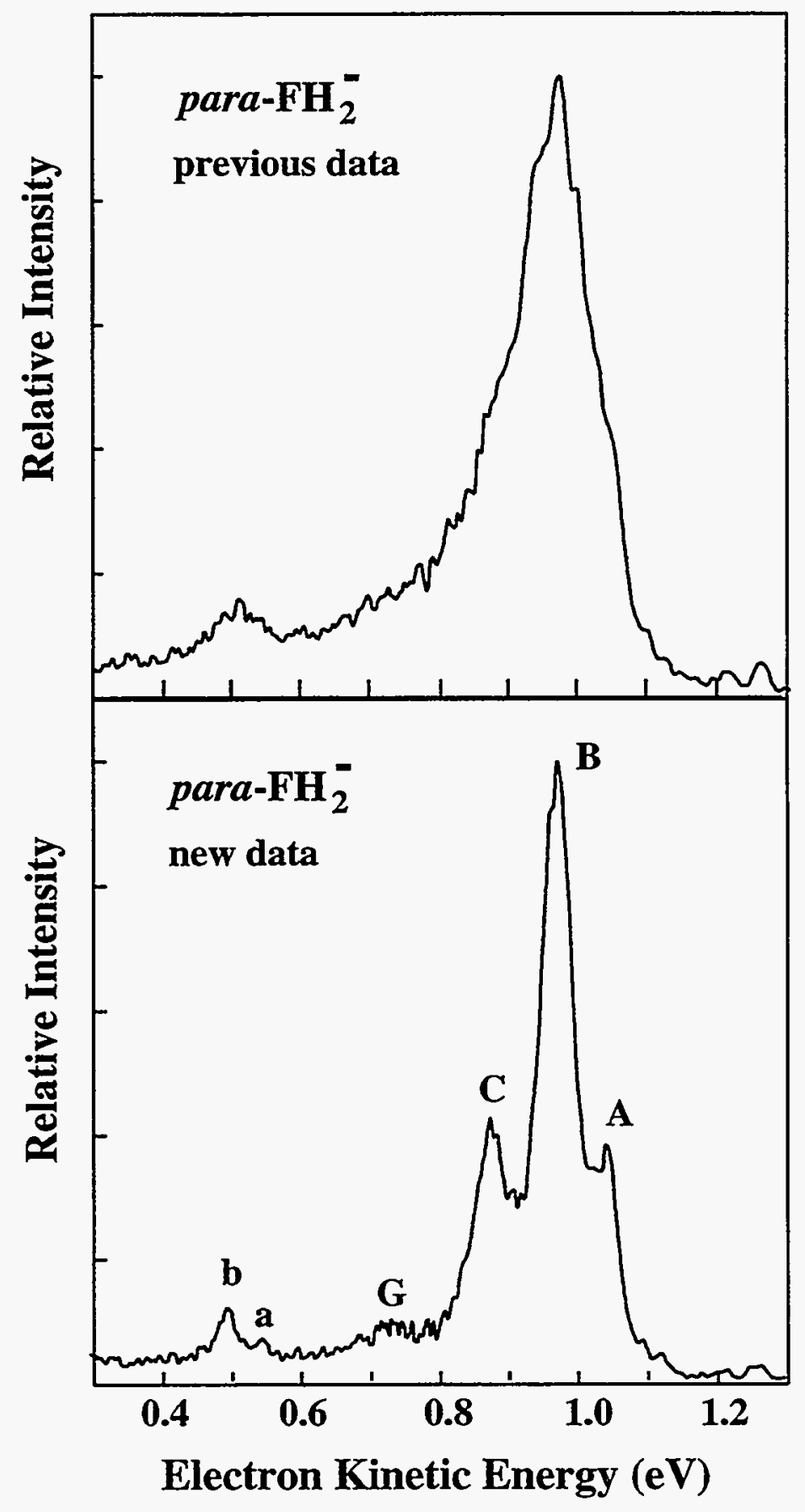

Figure 7.5 


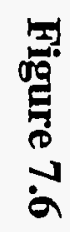

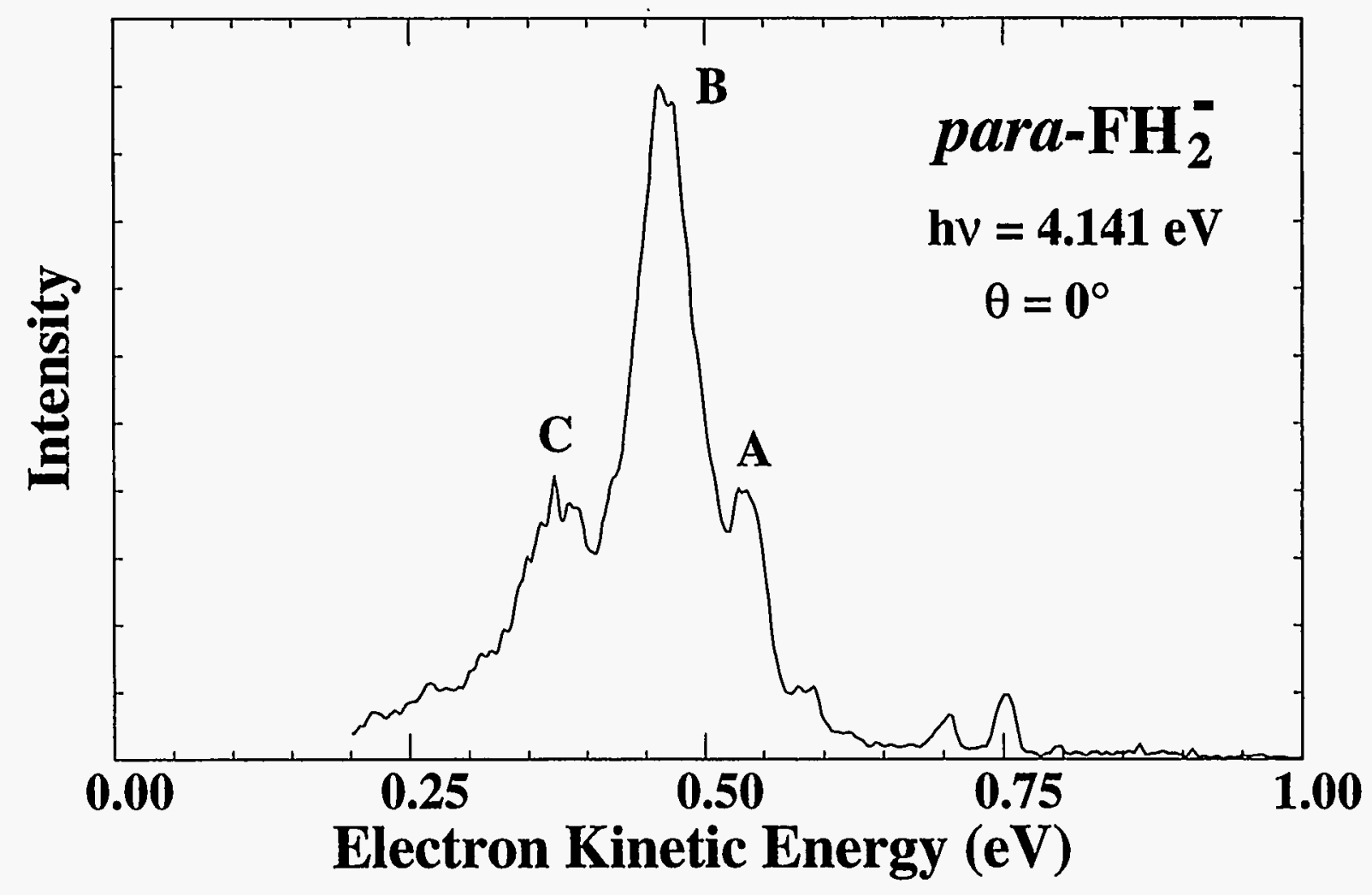




\subsection{Analysis and Discussion}

As a first step in the data analysis, we shall 'decompose' the normal- $\mathrm{FH}_{2}^{-}$spectrum into its ortho- and para- $\mathrm{FH}_{2}^{-}$contributions to assist the assignment of the observed features. Since the photoelectron spectra of para- $\mathrm{FH}_{2}^{-}$only contains transitions between symmetric states and we know the ratio (3:1) of the antisymmetric to symmetric states in the normal- $\mathrm{FH}_{2}^{-}$spectrum, it is a very simple exercise to extract the ortho- $\mathrm{FH}_{2}^{-}$spectrum which can not be directly obtained experimentally. The ortho- $\mathrm{FH}_{2}^{-}$is determined by scaling the para- $\mathrm{FH}_{2}^{-}$spectrum and subtracting it from the normal- $\mathrm{FH}_{2}^{-}$spectrum. The result of this subtraction is shown in Fig. 7.7. The removal of the para- $\mathrm{FH}_{2}^{-}$contribution to the normal- $\mathrm{FH}_{2}^{-}$photoelectron spectrum reveals a much clearer progression which is attributed to the photodetachment of ortho- $\mathrm{FH}_{2}^{-}$. In addition, this calculation shows that two features can be resolved (labeled ' $d$ ' and 'e') near 0.5 eV. These features represent transitions to states which correlate to vibrationally excited $\mathrm{H}_{2}$ ( $\mathrm{v}$ $=1)$.

In both the para- $\mathrm{FH}_{2}^{-}$and 'ortho- $\mathrm{FH}_{2}^{-}$spectra, the peak spacing of the progression increases with the energy of the $\left[\mathrm{FH}_{2}\right]^{\ddagger}$ complex (i.e., as eKE decreases). Additionally, the spacings of the normal- $\mathrm{FH}_{2}^{-}$spectral features are larger than but on the order of the $\mathrm{H}_{2}$ rotational level spacings. ${ }^{63}$ This behavior suggests that the observed progression may be attributed to a bend/hindered rotor progression of the $\left[\mathrm{FH}_{2}\right]^{\ddagger}$ complex formed upon photodetachment.

$A b$ initio calculations by Nichols et al. ${ }^{57}$ show that the $\mathrm{FH}_{2}^{-}$anion is linear. By FranckCondon considerations, the $\left[\mathrm{FH}_{2}\right]^{\ddagger}$ bending excitation upon photodetachment of the linear anion implies that the potential energy surface along the bending coordinate has a minimum at

Figure 7.7: Determination of the ortho- $\mathrm{FH}_{2}^{-}$spectrum by appropriate subtraction of the para- $\mathrm{FH}_{2}^{-}$spectrum from the normal $-\mathrm{FH}_{2}^{-}$spectrum (i.e., $\left[o-\mathrm{FH}_{2}^{-}\right]=\left[n-\mathrm{FH}_{2}^{-}\right]-$ $\left.\left[0.25^{*} p-\mathrm{FH}_{2}^{-}\right]\right)$. 


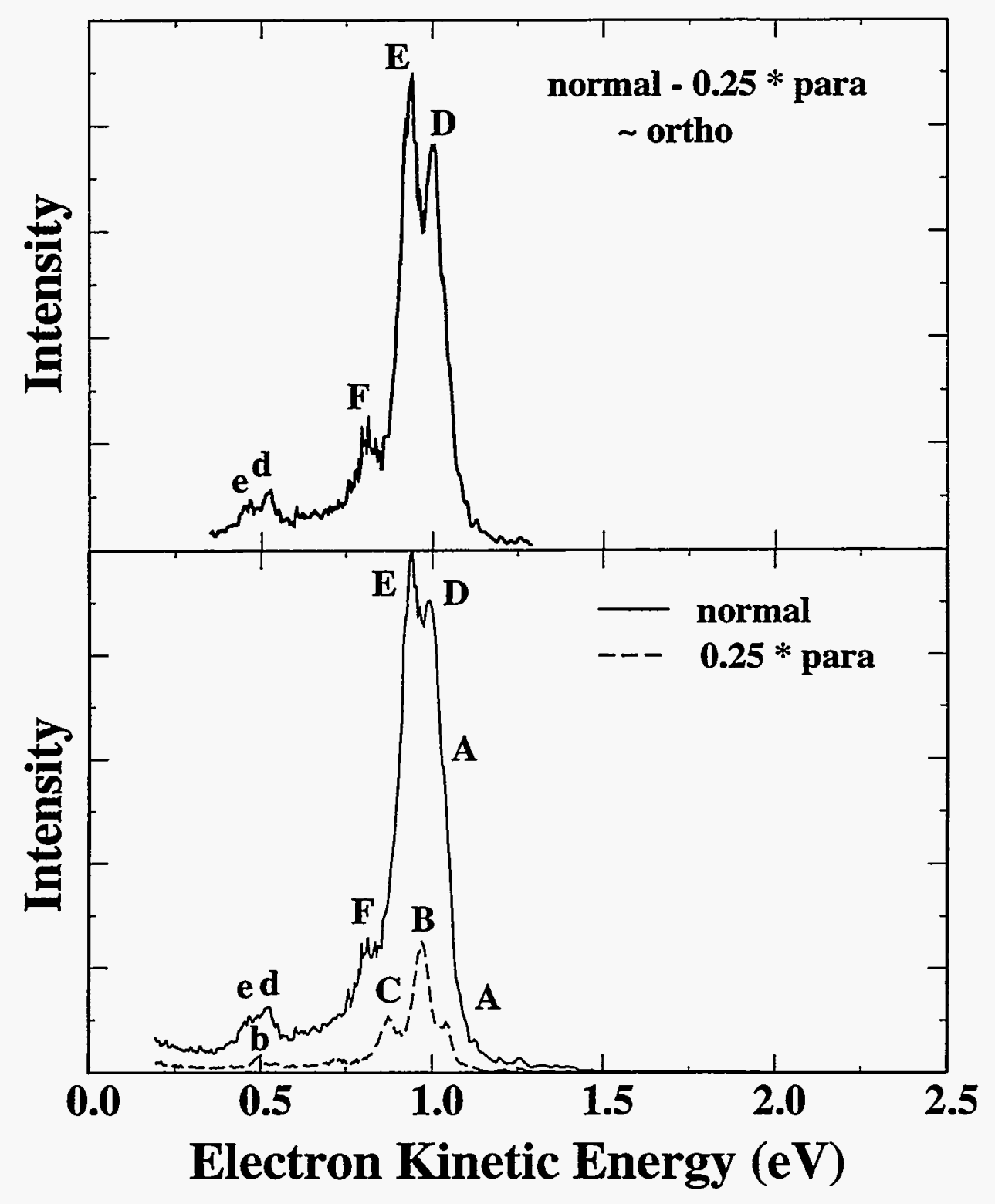

Figure 7.7 
a bent configuration. This is consistent with the observations discussed in Section 7.2.

The assignment to bend/hindered rotor excitation is supported by the dependence of the $\mathrm{FH}_{2}^{-}$photoelectron spectrum on the ortho- vs. para- $\mathrm{H}_{2}$ symmetry. This dependence suggest that the nuclear spin state of the $\mathrm{H}_{2}$ used to form the ions is conserved during the course of the experiment. As a result the $\mathrm{FH}_{2}^{-}$, in its ground vibrational state has either a symmetric or antisymmetric total wave function depending upon whether it was formed from para- or ortho $-\mathrm{H}_{2}$ respectively.

On the neutral reaction surface, the nuclear symmetry of the $\mathrm{H}_{2}$ results two subsets of scattering states. These sets also are distinguished by whether they are symmetric (para) or antisymmetric (ortho) with respect to permutation of the $\mathrm{H}$ nuclei. These symmetry constraints result in the very different appearances of the normal- and para- $\mathrm{FH}_{2}^{-}$photoelectron spectra. Symmetry considerations show that photodetachment of symmetric states of the linear $\mathrm{FH}_{2}^{-}$ anion results exclusively in symmetric states of the neutral $\left[\mathrm{FH}_{2}\right]^{\ddagger}$ complex and likewise for the antisymmetric states. In other words, the photodetachment of the para- $\mathrm{FH}_{2}^{-}$and ortho- $\mathrm{FH}_{2}^{-}$ species will provide transitions to exclusive but fully complementary scattering states of the F+ $\mathrm{H}_{2}$ reaction surface. The different symmetries of the scattering states refer to the asymptotic rotational levels of the $\mathrm{H}_{2}$ reactant. As the $\mathrm{F}$ atom and the $\mathrm{H}_{2}$ molecule approach in the transition state region, these states correlate to hindered rotor or bending vibrational motions of the $\left[\mathrm{FH}_{2}\right]^{\ddagger}$ complex. The simulations, to be shown below, strongly support the assignment of the observed progressions in the $\mathrm{FH}_{2}^{-}$photoelectron spectra to the bend/hindered rotor states of the $\left[\mathrm{FH}_{2}\right]^{\ddagger}$ transition state complex.

The remainder of the discussion is dedicated to comparison of the photoelectron spectra to calculations performed by D. M. Manolopoulos. First comparison will be made to simulations using a few of the potential energy surfaces discussed in the Background section. These will be followed by comparison to simulations on the SW surface. These comparisons not only confirm the assignments but also indicate that the experiment is very sensitive to the 
details of the transition state region of the $\mathrm{F}+\mathrm{H}_{2}$ potential energy surface. Additionally, they indicate that the SW surface is the most accurate surface proposed for the $\mathrm{F}+\mathrm{H}_{2}$ reaction to date.

\subsection{Theoretical Methodology}

For continuity in the comparison between the experiment and theory, a very brief summary of the $a b$ initio potential energy surface calculations performed by $\mathrm{K}$. Stark and H. -J. Werner and the Franck-Condon calculations performed by D. M. Manolopoulos will be given here. For further details, the reader is referred to the references given.

\subsubsection{Calculation of the $A b$ Initio Potential Surface}

In order to calculate an accurate $\mathrm{F}+\mathrm{H}_{2}$ potential energy surface, several important factors must be successfully addressed. Most important among these are the degree of electron correlation, the size of the basis set used and the degree of configuration interaction considered in the calculations. The region of the surface where these effects will be most important is the transition state region. Knowles et al. ${ }^{64}$ studied the degree to which each of these effects contributes to the shape of the $\mathrm{F}+\mathrm{H}_{2}$ potential energy surface. Based upon their findings, Stark and Werner have constructed a global potential energy surface for the reaction. ${ }^{14}$ Beginning with a very large set of complete active space self-consistent field (CASSCF) reference functions, a contracted multireference configuration interaction (MRCI) calculation was used. Effects of higher order excitations were included by use of the Davidson correction (Q). This MRCI $+\mathrm{Q}$ method was used over a large range of $\mathrm{F}-\mathrm{H}_{2}$ geometries $(\sim 600)$ to

determine the globally accurate potential energy surface for the $\mathrm{F}+\mathrm{H}_{2}$ reaction. The surface was then fit parametrically for use in the calculation of various reaction properties, including the Franck-Condon profile for $\mathrm{FH}_{2}^{-}$photodetachment. 


\subsubsection{Calculation of the Franck-Condon Simulations}

The calculation of the Franck-Condon overlap between the $\mathrm{FH}_{2}^{-}$anion and the $\mathrm{F}+\mathrm{H}_{2}$ potential energy surface is similar to, although more complicated than, the calculation of Franck-Condon factors for the bound molecules presented in the previous chapters. Using the methodology developed by Schatz for the analysis of other transition state spectra, ${ }^{64}$ Manolopoulos calculates the theoretical Franck-Condon profile expected upon $\mathrm{FH}_{2}^{-}$ photodetachment according to equation 5:

$$
P(E)=\sum_{n}\left|\left\langle\psi_{n}(E) \mid \Psi_{i}\right\rangle\right|^{2}
$$

The anion vibrational wave function, $\psi_{\mathrm{i}}$, is calculated using the $a b$ initio harmonic frequencies calculated by Nichols et al. ${ }^{57}$ and properly symmetrized to represent either the ortho- or paraform of $\mathrm{FH}_{2}^{-}$. The neutral wave functions, $\Psi_{\mathrm{n}}(\mathrm{E})$, are the energy-normalized scattering wave functions for the $\mathrm{F}+\mathrm{H}_{2}$ potential energy surface. The scattering wave functions which correlate to the $n$ asymptotic quantum numbers are calculated as a function of $E$, using the method developed by Schatz. ${ }^{65}$ They are then substituted into Eq. (5) to simulate the $\mathrm{FH}_{2}^{-}$ photoelectron spectrum.

\subsection{Comparison between Theory and Experiment}

Comparison of the data with simulations performed using several different $\mathrm{F}+\mathrm{H}_{2}$ potential energy surfaces illustrate the sensitivity of the anion photoelectron spectroscopy technique to the shape of the potential energy surface in the region of the transition state. In particular, as we shall show, the experiments are particularly sensitive to the bending coordinate in the transition state region. Of the several potential energy surfaces have been proposed for the $\mathrm{F}+\mathrm{H}_{2}$ reaction, Manolopoulos has performed simulations on the T5a, 5SEC, 
TS surfaces and on the new SW surface. Comparison of these simulations will be made with the data, in this order, below.

We begin the comparison with the T5a potential energy surface, developed by Steckler et al..$^{35}$ as a modification of the earlier T5 surface. The simulation shown in Fig. 7.8a (top), shows the comparison of the $\mathrm{T} 5 \mathrm{a}$ simulation of the $\mathrm{FH}_{2}^{-}$photoelectron spectrum with the para- $\mathrm{FH}_{2}^{-}$results. This simulation is identical to that of Zhang and Miller except for the value assumed for the dissociation energy of $\mathrm{FH}_{2}^{-}$. In this figure, we use $\mathrm{D}_{\mathrm{o}}=0.23 \mathrm{eV}$, since this aligns the most intense peaks in the experimental and simulated spectrum as well as the smaller peaks at $0.5 \mathrm{eV}$. This value is well within the range of $a b$ initio values of Nichols et al. $(0.20 \pm$ $0.10 \mathrm{eV}) .57$

Due to the 3:1 ratio of antisymmetric and symmetric states present in normal $-\mathrm{H}_{2}$, the normal- $\mathrm{FH}_{2}^{-}$photoelectron spectrum contains photodetachment transitions to both the antisymmetric and symmetric scattering states of $\mathrm{F}+\mathrm{H}_{2}$. Thus, the simulation of the spectrum requires that the overlap of the anion ground state wave function with the ortho scattering wave functions and the para scattering wave functions be made and then combined in the appropriate ratio. The result of this calculation is shown in Fig. $7.8 \mathrm{~b}$ (bottom) and compared to the normal- $\mathrm{FH}_{2}^{-}$photoelectron spectrum.

Overall, the simulations on the T5a surface do a poor job of reproducing the

Figure 7.8: (Top) Three-dimensional simulation (dotted) of the $\mathrm{FH}_{2}^{-}$photoelectron spectrum using the T5a surface, considering only symmetric permutation states, of Zhang and Miller (Ref. 10), compared to the $\theta=0^{\circ}$ para- $\mathrm{FH}_{2}^{-}$experimental spectrum (solid). (Bottom) Three-dimensional simulation (dotted) of the $\mathrm{FH}_{2}^{-}$ photoelectron spectrum, considering both symmetric and antisymmetric permutation states compared to the $\theta=0^{\circ}$ normal- $\mathrm{FH}_{2}^{-}$experimental spectrum (solid). The simulation is a weighted sum of the $p-\mathrm{FH}_{2}^{-}$and $o-\mathrm{FH}_{2}^{-}$simulations. Both calculations assume the same anion and neutral parameters and $\mathrm{D}_{0}\left(\mathrm{~F}^{-} \cdots \mathrm{H}_{2}\right)=$ $0.23 \mathrm{eV}$. 


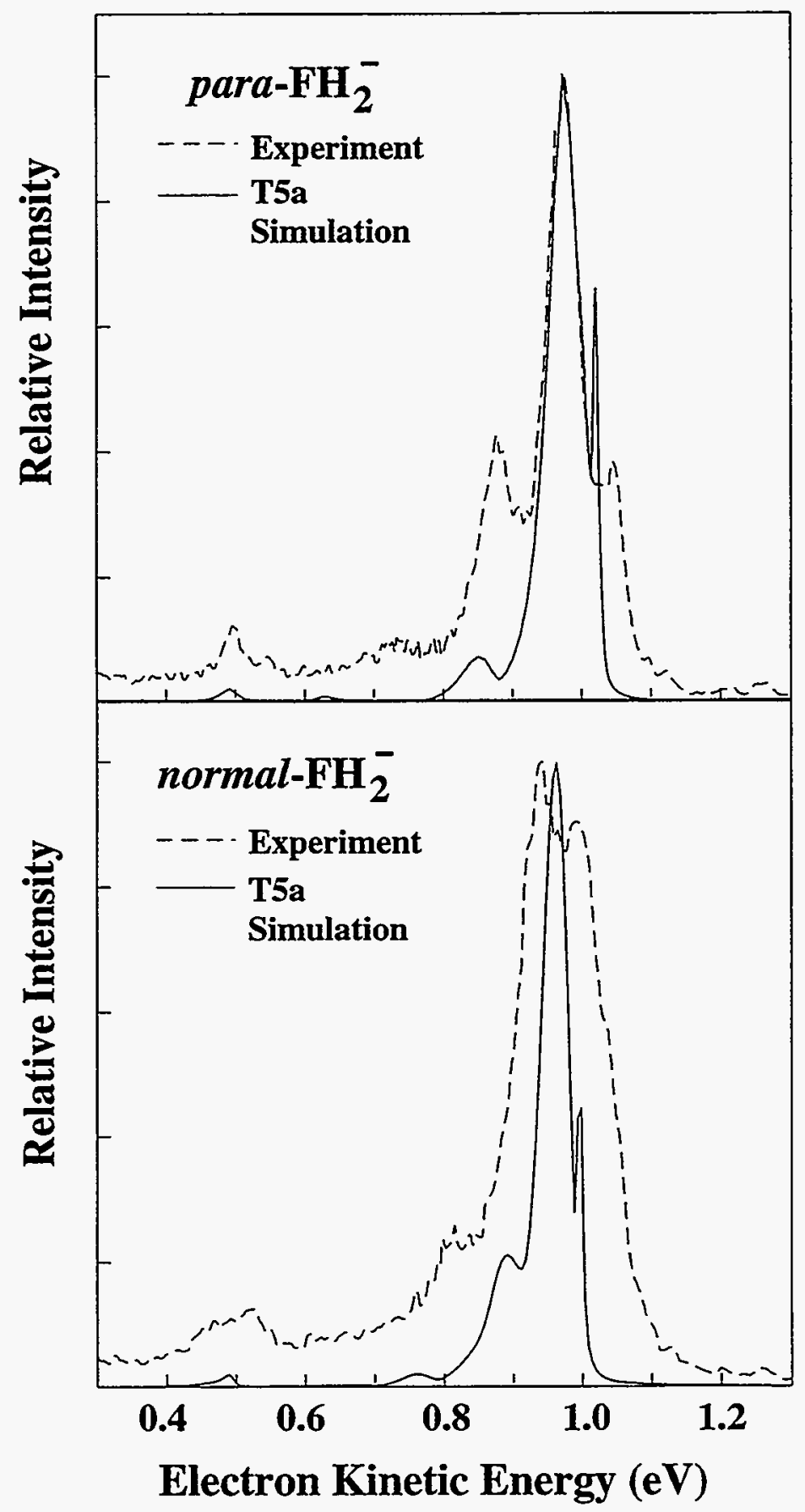

Figure 7.8 
experimentally observed features. However, although the comparison is not exceptionally good, the simulations do show that a significant difference exists between the ortho- $\mathrm{FH}_{2}^{-}$and para- $\mathrm{FH}_{2}^{-}$simulations as a result of the symmetry of the scattering wave functions because all of the other parameters are kept the same in the simulations.

Comparison of the 5SEC simulation to the experimental data is made in Fig. 7.9. The $\mathrm{FH}_{2}^{-}$dissociation energy is assumed to be $\mathrm{D}_{\mathrm{o}}=0.20 \mathrm{eV}$, again for alignment of spectral features. As in the case of the T5a surface, the overall agreement between the experiment and theory is not very good. The simulation predicts feature that do not appear in the experimental data and does not predict features that are observed. There are two separate types of transitions which are observed in the simulations, as summarized in Table III. The two types of transitions are distinguished according to their labels. The lettered peaks result from transitions to bend/hindered rotor states of the $\left[\mathrm{FH}_{2}\right]^{\ddagger}$ complex. The numbered peaks lie below the reactant zero-point energy $(0.269 \mathrm{eV})$. This suggests that these features correspond to scattering states which are localized on the $\mathrm{H}+\mathrm{HF}$ product valley side of the saddle point. Such states are accessible by $\mathrm{FH}_{2}^{-}$photodetachment, provided they have sufficient overlap with the anion.

Somewhat better agreement is found by comparison of the data with simulations performed using the TS potential energy surface. As seen in Fig. 7.10, the peak spacings and profiles agree much better than for the other surfaces. In particular, the agreement for the para- $\mathrm{FH}_{2}^{-}$photoelectron spectrum (top) is quite good. However, the widths of the peaks are underestimated by a significant amount. Note that the transition to the $v=0$ hindered rotor state is located at the nearly same eKE as the $\mathrm{H}+\mathrm{HF}$ scattering state, "1", in the TS simulation.

While the reasonable agreement between theory and experiment suggests that the

Figure 7.9: Simulations of the $p-\mathrm{FH}_{2}^{-}$and $n-\mathrm{FH}_{2}^{-}$photoelectron spectra using the 5SEC surface superimposed on the experimental $\left(\theta=0^{\circ}\right)$ data. $D_{0}=0.24 \mathrm{eV}$ is assumed for $\mathrm{FH}_{2}^{-}$in both simulations. 


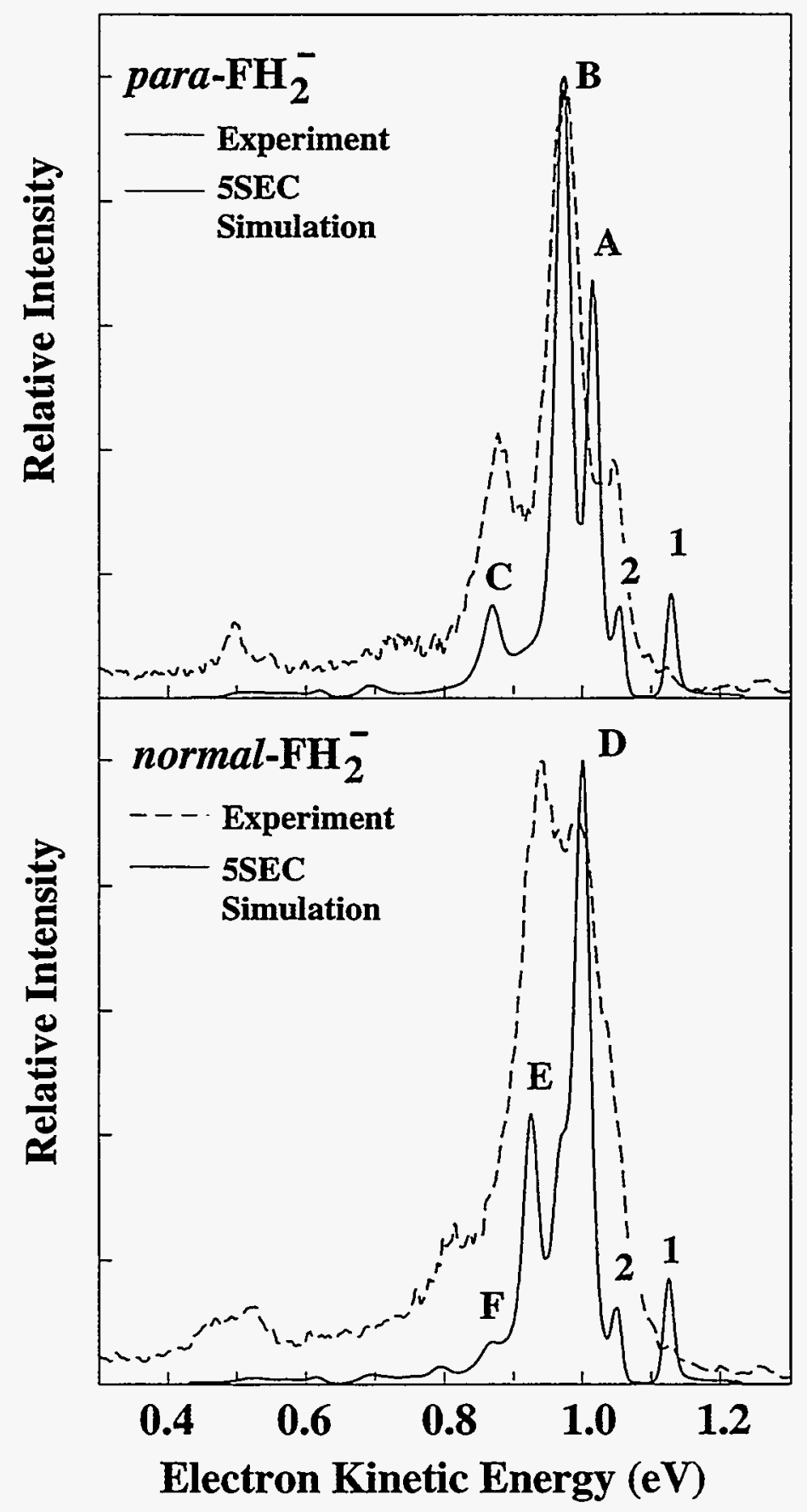

Figure 7.9 
observed spectral features correspond to bend/hindered rotor motion of the $\left[\mathrm{FH}_{2}\right]^{\ddagger}$ complex, its is clear that none of the surfaces discussed above is exactly correct in the transition state region, for which the $\mathrm{FH}_{2}^{-}$anion has excellent Franck-Condon overlap. This is consistent with the disagreement found between previous experimental observations and calculations using these surfaces, as discussed in Section 7.2.

Table III: Positions, widths, and assignments for features in $\mathrm{FH}_{2}^{-}$simulations on the 5SEC and TS potential energy surfaces.

\begin{tabular}{|c|c|c|c|c|}
\hline Simulation & Peak & $\mathrm{eKE}(\mathrm{eV})$ & FWHM (eV) & Assignment \\
\hline \multirow[t]{8}{*}{ 5SEC } & 1 & 1.124 & 0.003 & $\mathrm{H} / \mathrm{HF}$ resonance \\
\hline & 2 & 1.050 & 0.001 & $\mathrm{H} / \mathrm{HF}$ resonance \\
\hline & A & 1.012 & 0.012 & $\mathrm{~F} / \mathrm{H}_{2}(\mathrm{j}=0)$ \\
\hline & $\mathrm{B}$ & 0.968 & 0.019 & $F / H_{2}(j=2)$ \\
\hline & $\mathrm{C}$ & 0.866 & 0.027 & $\mathrm{~F} / \mathrm{H}_{2}(\mathrm{j}=4)$ \\
\hline & $\mathrm{D}$ & 1.002 & 0.016 & $\mathrm{~F} / \mathrm{H}_{2}(\mathrm{j}=1)$ \\
\hline & $\mathrm{E}$ & 0.926 & 0.019 & $\mathrm{~F} / \mathrm{H}_{2}(\mathrm{j}=3)$ \\
\hline & $\mathrm{F}$ & 0.792 & 0.032 & $F / H_{2}(j=5)$ \\
\hline \multirow[t]{7}{*}{ TS } & 1 & 1.030 & 0.008 & $\mathrm{H} / \mathrm{HF}$ resonance \\
\hline & $+\mathrm{A}$ & & & $\mathrm{F} / \mathrm{H}_{2}(\mathrm{j}=0)$ \\
\hline & $\mathrm{B}$ & 0.972 & 0.014 & $F / H_{2}(j=2)$ \\
\hline & $\mathrm{C}$ & 0.874 & 0.010 & $\mathrm{~F} / \mathrm{H}_{2}(\mathrm{j}=4)$ \\
\hline & $\mathrm{D}$ & 1.002 & 0.022 & $\mathrm{~F} / \mathrm{H}_{2}(\mathrm{j}=1)$ \\
\hline & $\mathrm{E}$ & 0.932 & 0.010 & $\mathrm{~F} / \mathrm{H}_{2}(\mathrm{j}=3)$ \\
\hline & $\mathrm{F}$ & 0.800 & 0.014 & $F / H_{2}(j=5)$ \\
\hline
\end{tabular}

The final set of simulations to be compared to the data are performed on the SW

Figure 7.10: Simulations of the $p-\mathrm{FH}_{2}^{-}$and $n-\mathrm{FH}_{2}^{-}$photoelectron spectra using the TS surface superimposed on the experimental $\left(\theta=0^{\circ}\right)$ data. $\mathrm{D}_{0}=0.20 \mathrm{eV}$ is assumed for both of the simulations. 


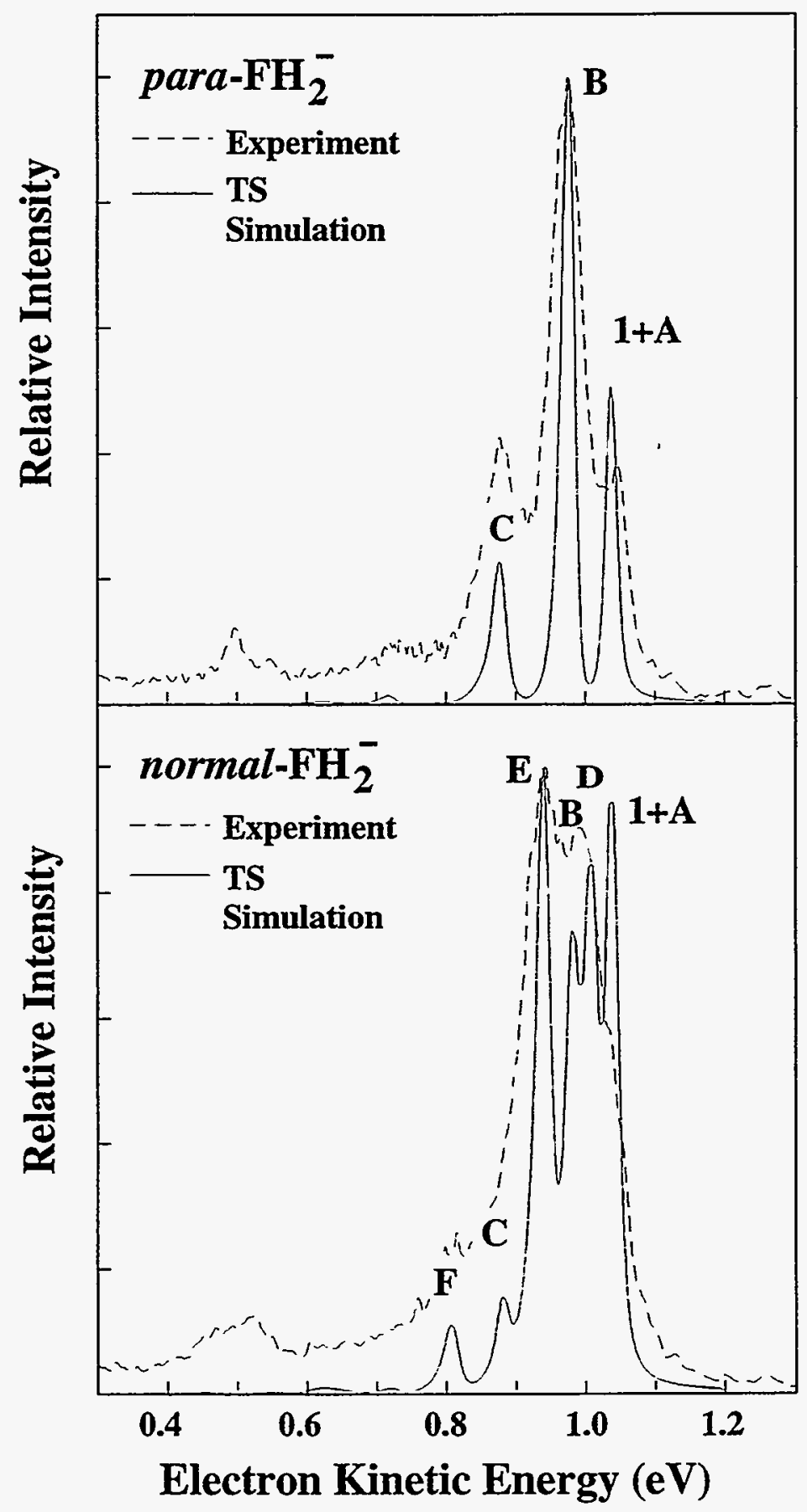

Figure 7.10 
surface. Shown in Fig. 7.11 is a contour plot of the transitions state region of the $a b$ initio calculated potential energy surface. The surface, in bold lines, has dual saddle points (S) on either side of the collinear barrier. The surface agrees very well, energetically, with other accepted values (in parentheses): $D_{e}(H F)=6.188 \mathrm{eV}(6.123 \mathrm{eV}), \mathrm{D}_{\mathrm{e}}\left(\mathrm{H}_{2}\right)=4.740 \mathrm{eV}(4.748$ $\mathrm{eV}), \Delta \mathrm{H}_{\mathrm{r}}=31.77 \mathrm{kcal} / \mathrm{mol}(31.7 \mathrm{kcal} / \mathrm{mol}), \mathrm{EA}(\mathrm{F})=3.393 \mathrm{eV}(3.398 \mathrm{eV}) .66$ The height of the barrier at the bent transition state $\left(\gamma=119^{\circ}\right)$ is $1.53 \mathrm{kcal} / \mathrm{mol}$ with respect to separated reactants. The bond distances at the saddle points are $\mathrm{R}_{\mathrm{F}-\mathrm{H}_{2}}=1.541 \AA$ and $\mathrm{R}_{\mathrm{H}-\mathrm{H}}=0.772 \AA$. Also shown in the figure is the projection of the para- $\mathrm{FH}_{2}^{-}$ground state wave function in thin dotted lines onto the SW surface. This clearly illustrates the excellent Franck-Condon overlap between the anion $\left(\mathrm{R}_{\mathrm{F}-\mathrm{H}_{2}}=2.075 \AA, \mathrm{r}_{\mathrm{H}-\mathrm{H}}=0.770 \AA \text { and } \gamma=0^{\circ}\right)^{57}$ and the transition state region of the $\mathrm{F}+$ $\mathrm{H}_{2}$ surface.

The simulations using the SW surface are shown in Fig. 7.12 superimposed on the

Figure 7.11: The transition state region of the $\mathrm{SW}$ ab initio $\mathrm{F}+\mathrm{H}_{2}$ potential energy surface (bold) and the projection of the $\mathrm{FH}_{2}^{-}$anion ground state wave function onto this surface (thin). The solid lines are contours of the new $\mathrm{F}+\mathrm{H}_{2}$ PES in steps of 0.4 $\mathrm{kcal} / \mathrm{mol}$ from 0.4 to $2.8 \mathrm{kcal} / \mathrm{mol}$, relative to the bottom of the asymptotic $\mathrm{F}+\mathrm{H}_{2}$ valley. The long dashed lines are similar contours from -2.8 to $0.0 \mathrm{kcal} / \mathrm{mol}$. The short dashed lines are contours of the para- $\mathrm{FH}_{2}^{-}$anion wave function, each successive contour representing a decrease in the square modulus of the wave function by a factor of 10 . The coordinates of the plot are the $\mathrm{F}-\mathrm{H}_{2}$ distance and the Jacobi angle, $\gamma$, that are illustrated in the inset; the $\mathrm{H}-\mathrm{H}$ bond length is fixed at 0.771 throughout. The $\mathrm{F}+\mathrm{H}_{2}$ transition state saddle points (S) occur along the minimum energy paths between a $\mathrm{C}_{2 \mathrm{v}}$ symmetry van der Waals well in the $\mathrm{F}+\mathrm{H}_{2}$ reactant valley and the two degenerate $\mathrm{H}+\mathrm{HF}$ product valleys.

Figure 7.12: Simulations of the $p-\mathrm{FH}_{2}^{-}$and $n-\mathrm{FH}_{2}^{-}$photoelectron spectra using the new $a b$ initio surface of Stark and Werner (SW) superimposed on the experimental $(\theta=$ $\left.0^{\circ}\right)$ data. $\mathrm{D}_{\mathrm{o}}=0.205 \mathrm{eV}$ is assumed for both of the simulations. 


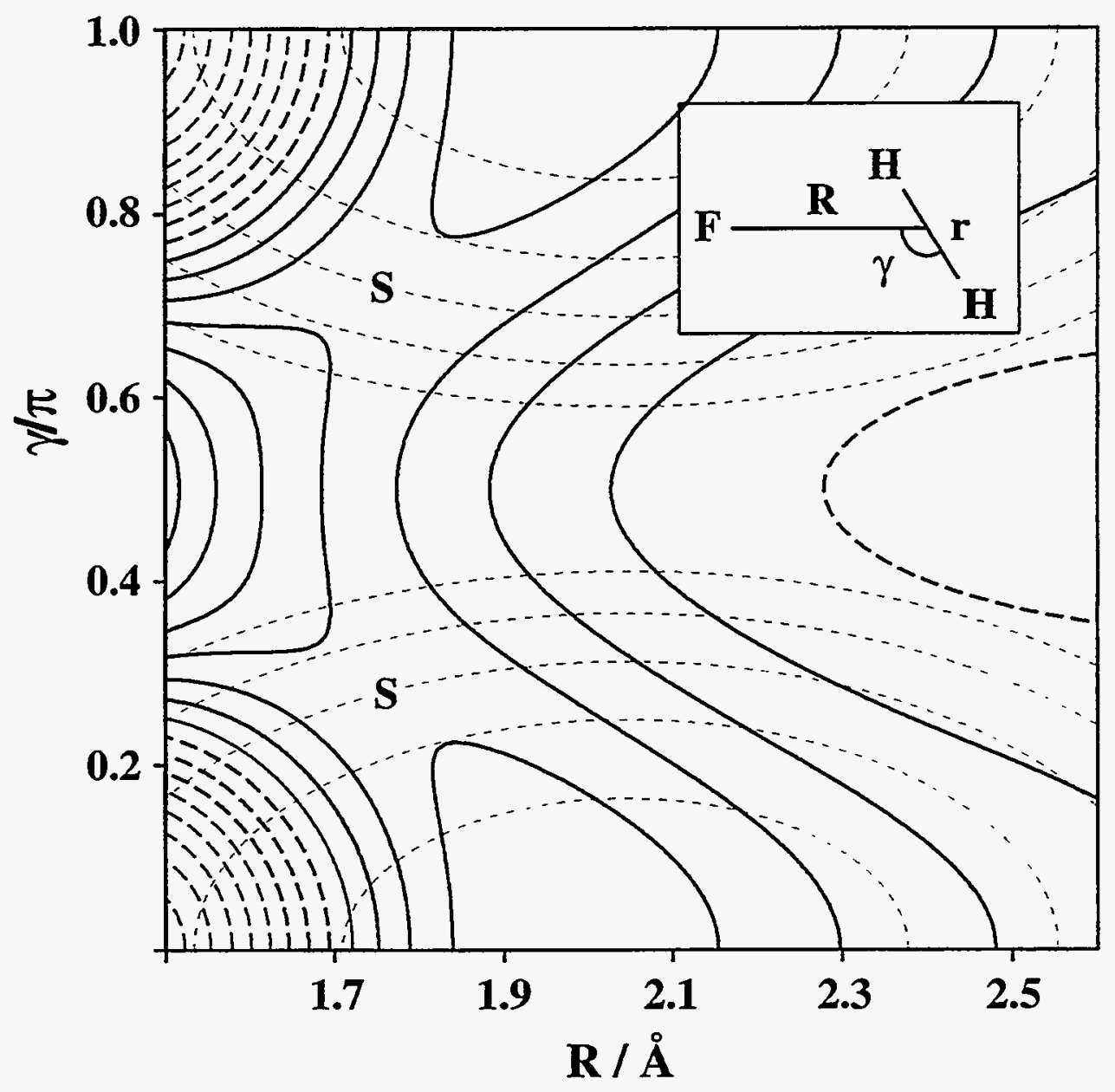

Figure 7.11 


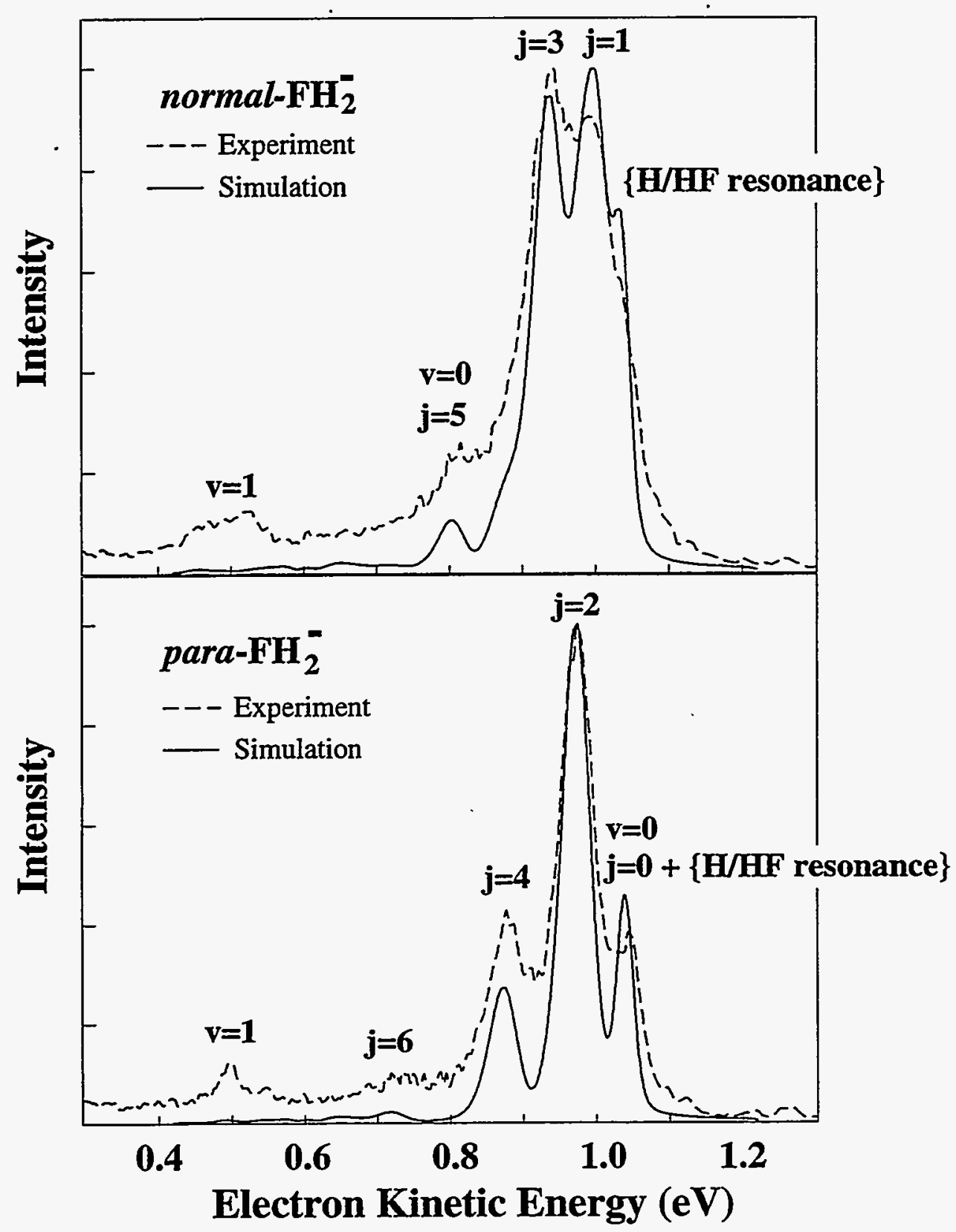

Figure 7.12 
experimental data. The simulations assume that the $\mathrm{FH}_{2}^{-}$dissociation energy is $\mathrm{D}_{\mathrm{o}}=0.205 \mathrm{eV}$. As in the TS simulations there is hindered rotor state which is coincident with the scattering state for the peak labeled ' $v=0$ '. The labels ' $\mathrm{j}$ ' correspond to the asymptotic $\mathrm{H}_{2}$ rotational levels in the reactant valley. The agreement between the simulations and the spectra is excellent. The peak spacings and profiles are very well reproduced. In addition, the simulation, after convolution with the experimental resolution function, also reproduces the observed peak widths. This suggests that the SW potential energy surface is quite accurate in the transition state region, where the Franck-Condon overlap for $\mathrm{FH}_{2}^{-}$photodetachment is also excellent.

The only regions where there is less agreement is in the $v=1$ peak located at $0.5 \mathrm{eV}$. The simulation underestimate the intensity of this peak in both the para and the ortho spectra. The reason for this small discrepancy is believed to result from the harmonic oscillator approximation used for the anion wave function. It is expected that the intensity for that feature will be better reproduced if the anharmonicity of the $\mathrm{FH}_{2}^{-}$vibrations were included in the simulations. Such calculations are planned in which an accurate $a b$ initio $\mathrm{FH}_{2}^{-}$potential surface will be used to determine the anion ground state wave function to be used in the Franck-Condon overlap with the $\mathrm{F}+\mathrm{H}_{2}$ potential energy surface.

\subsection{Conclusions}

In this Chapter, we have presented the results of an experimental reinvestigations of the $\mathrm{F}+\mathrm{H}_{2}$ reaction potential energy surface in the transition state region by the study of the $\mathrm{FH}_{2}^{-}$ photoelectron spectrum. By improved experimental efforts, we have obtained significantly improved $\mathrm{FH}_{2}^{-}$photoelectron spectrum compared to previous results obtained in this laboratory. The improved data show much more clearly how the observed spectral features depend upon the nuclear spin statistics in the $\mathrm{FH}_{2}^{-}$anion. By comparison to beautiful theoretical results of Stark and Werner and Manolopoulos, the observed vibrational progressions have been 
interpreted as photodetachment transition to bend/hindered rotor states of the unstable $\left[\mathrm{FH}_{2}\right]^{\ddagger}$ transition state complex.

The comparison of the experimental data to three potential energy surfaces which have been widely used in the study of the $\mathrm{F}+\mathrm{H}_{2}$ reaction and to the new ab initio potential energy surface has shown that the anion photodetachment technique is a very sensitive method for the study of the transition state species of a bimolecular chemical reaction. While the T5a, 5SEC and TS surfaces were all able to reproduce other experimental results for the $\mathrm{F}+\mathrm{H}_{2}$ reaction, none were able to satisfactorily reproduce the observed $\mathrm{FH}_{2}^{-}$photoelectron spectrum. However, for the first time, quantitative agreement has been obtained between the simulations and data using the new SW surface. The peak spacings, profile and widths are very well reproduced. This agreement is remarkable because the simulation is derived purely from quantum mechanical principles (i.e., an $a b$ initio potential energy surface, exact threedimensional quantum mechanical scattering calculations, and an $a b$ initio calculated geometry and frequencies for the $\mathrm{FH}_{2}^{-}$anion). Thus, this work represents a true convergence of theory and experiment in the description of the $\mathrm{F}+\mathrm{H}_{2}$ transition state region.

Some work still remains to determine whether the SW potential energy surface will possess sufficient accuracy to reproduce the other observed experimental results, such as the product state distributions, product angular distributions and rate constants. Since the publication of this work, some calculations have been performed that indicate that the surface will, indeed, serve as an accurate global potential energy surface for the $\mathrm{F}+\mathrm{H}_{2}$ reaction. Aoiz and co-workers ${ }^{66}$ have recently calculated the product angular distributions using quasiclassical scattering techniques and have determined that the angular distributions are well reproduced, including the forward scattering peaks. More importantly, they show that the surface successfully reproduces the product vibrational distribution, the criterion which other proposed surfaces have failed to meet. Full quantum mechanical calculations of these reactions properties are now underway and preliminary results look promising. ${ }^{67}$ 


\subsection{Acknowledgments}

I greatly thank D. M. Manolopoulos for communication of his simulations and K. Stark and H. -J. Werner for communications of their potential energy surface prior to publication. This work has been sponsored by the United States Air Force Office of Scientific Research under contract No. AFOSR-91-0084 and by the United Kingdom Science and Engineering Research Council. 


\section{References}

${ }^{1}$ C. Jaques, L. Valachovic, S. Ionov, E. Böhmer, Y. Wen, J. Segall, and C. Wittig, J. Chem. Soc. Faraday Trans. 89, 1419 (1993); S. I. Ionov, G. A. Brucker, C. Jaques, Y. Chen., and C. Wittig, J. Chem. Phys. 99, 3420 (1993); S. I. Ionov, G. A. Brucker, C. Jaques, L. Valachovic, and C. Wittig, J. Chem. Phys. 99, 6553 (1993).

2M. Dantus, M. J. Rosker, and A. H. Zewail, J. Chem. Phys.89, 6128 (1988); M. Gruebele and A. H. Zewail, Ber. Bunsen. Ges. - Phys. Chem. 94, 1210 (1990);L. R. Khundkar and A. H. Zewail, Ann. Rev. Phys. Chem. 41, 15 (1990); A. H. Zewail, Faraday Discuss. Chem. Soc. 91, 207 (1991); J. L. Herek, S. Pedersen, T. Baumert, J. L. Herek, and A. H. Zewail, J. Chem. Phys. 99, 4430 (1993);A. H. Zewail, J. Phys. Chem. 97, 12447 (1993).

3P. R. Brooks, Chem. Rev. 88, 407 (1988). This article contains a review of these types of experiments performed through 1987.

${ }^{4}$ C. Jouvet, M. C. Duval, B. Soep, W. H. Breckenridge, C. Whitham, and J. P. Visticot, J. Chem. Soc. Faraday Trans 2 85, 1133 (1989); B. Soep, C. J. Whitham, A. Keller, and J. P. Visticot, Faraday Discuss. Chem. Soc. 91, 191 (1991); B. Soep, S. Abbes, A. Keller and J. P. Visticot, J. Chem. Phys. 96, 440 (1992).

5E. R. Lovejoy, S. K. Kim, and C. B. Moore, Science 256, 1541 (1992); E. R. Lovejoy and C. B. Moore, J. Chem. Phys. 98, 7846 (1993).

${ }^{6}$ S. E. Bradforth, A. Weaver, D. W. Arnold, R. B. Metz, and D. M. Neumark, J. Chem. Phys. 92, 7205 (1990); A. Weaver, R. B. Metz, S. E. Bradforth, and D. M. Neumark, J. Phys. Chem. 92, 5558 (1988); R. B. Metz, A. Weaver, S. E. Bradforth, T. N. Kitsopoulos, and D. M. Neumark, J. Phys. Chem. 94, 1377 (1990).

${ }^{7}$ S. E. Bradforth, D. W. Arnold, R. B. Metz, A. Weaver, and D. M. Neumark, J. Phys. Chem. 95, 8066 (1991).

${ }^{8}$ A. Weaver, R. B. Metz, S.E. Bradforth, and D. M. Neumark, J. Chem. Phys. 93, 5352 (1990).

${ }^{9}$ A. Weaver and D. M. Neumark, Faraday Discuss. Chem. Soc. 91, 5 (1991).

10J. Z. H. Jhang and W. H. Miller, J. Chem. Phys. 92, 1811 (1990); J. Z. H. Jhang, W. H. Miller, A. Weaver, and D. M. Neumark, Chem. Phys. Lett. 182, 283 (1991).

${ }^{11}$ S. E. Bradforth, D. W. Arnold, D. M. Neumark, and D. E. Manolopoulos, J. Chem. Phys. 99, 6345 (1993); D. E. Manolopoulos, K. Stark, H. -J. Werner, D. W. Arnold, S. E. Bradforth, and D. M. Neumark, Science 262, 1852 (1993).

${ }^{12}$ A. Weaver, Ph. D. thesis, University of California, Berkeley (1991).

${ }^{13}$ S. E. Bradforth, Ph. D. thesis, University of California, Berkeley (1991).

${ }^{14} \mathrm{~K}$. Stark and H. -J. Werner, to be published. 
15J. H. Parker and G. C. Pimentel, J. Chem. Phys. 51, (1969); R. D. Coombe and G. C. Pimentel, ibid. 59, 251 (1973).

${ }^{16}$ E. Wurzburg and P. L. Houston, J. Chem. Phys. 72, 4811 (1980); R. F. Heidner, J. F. Bott, C. E. Gardner, and J. E. Melzer, ibid. 72, 4815 (1980).

${ }^{17}$ J. C. Polanyi, Acc. Chem. Res. 5, 161 (1972).

${ }^{18}$ R. D. Levine and R. B. Bernstein, Molecular Reaction Dynamics and Chemical Reactivity, Oxford University Press (New York, 1987).

${ }^{19}$ M. J. Berry, J. Chem. Phys. 59, 6229 (1973).

${ }^{20}$ J. C. Polanyi and D. C. Tardy, J. Chem. Phys. 51, 5717 (1969); J. C. Polanyi and K. B. Woodall, J. Chem. Phys. 57, 1574 (1972).

${ }^{21}$ N. Jonathan, C. M. Melliar-Smith, and D. H. Slater, Mol. Phys. 20, 93 (1971).

${ }^{22}$ T. P. Schaefer, P. E. Siska, J. M. Parson, F. P. Tully, Y. C. Wong, and Y. T. Lee, J. Chem. Phys. 53, 3385 (1970).

${ }^{23}$ D. M. Neumark, A. M. Wodtke, G. N. Robinson, C. C. Hayden, and Y.T. Lee, J. Chem. Phys. 82, 3045 (1985); D. M. Neumark, A. M. Wodtke, G. N. Robinson, C.C. Hayden, and Y.T. Lee, ibid. 82, 3067 (1985).

${ }^{24}$ M. Faubel, S. Schlemmer, F. Sondermann, and J. P. Toennies, J. Chem. Phys. 94, 4676 (1991).

25F. J. Aoiz, V. J. Herrero, M. M. Nogueira, and V. Saez Rabanos, Chem. Phys. 81, 1 (1992).

26J. D. Kress, Z. Bacic, G. A. Parker, and R. T. Pack, Chem. Phys. Lett. 157, 484 (1989); Z. Bacic, J. D.

Kress, G. A. Parker, and R. T. Pack, J. Chem. Phys. 92, 2344 (1990).

${ }^{27}$ C. -H. Yu, D. J. Kouri, M. Zhao, and D. G. Truhlar, Chem. Phys. Lett. 157, 491 (1989); C. -H. Yu, D. J. Kouri, M. Zhao, D. G. Truhlar, and D. W. Schwenke, Int. J. Quantum Chem. 23, 45 (1989).

28J. Z. H. Zhang and W. H. Miller, J. Chem. Phys. 92, 1811 (1990).

${ }^{29}$ D. E. Manolopoulos, M. D'Mello, and R. E. Wyatt, J. Chem. Phys. 93, 403 (1990); M. D'Mello, D. E. Manolopoulos, and R. E. Wyatt, Chem. Phys. Lett. 168, 113 (1990).

30J. M. Launay and M. LeDourneuf, Chem. Phys. Lett. 169, 473 (1990); J. M. Launay, Theor. Chim. Acta 79, $183(1990)$.

${ }^{31}$ G. C. Lynch, P. Halvick, M. Zhao, D. G. Truhlar, C. -H. Yu, D. J. Kouri, and D. W. Schwenke, J. Chem. Phys. 94, 7150 (1991).

32J. D. Kress and E. F. Hayes, J. Chem. Phys. 97, 4881 (1992).

${ }^{33} \mathrm{~J}$. T. Muckerman, Theoretical Chemistry - Advances and Perspectives, Vol.6A, pp. 1 - 77 (Academic Press, New York, 1981). 
${ }^{34} \mathrm{~A}$ nice review of the theoretical efforts through 1985 is given in: H. F. Schaefer, III, J. Phys. Chem. 89, 5336 (1985).

${ }^{35}$ R. Steckler, D. G.Truhlar, and B. C. Garrett, J. Chem. Phys. 82, 5499 (1985).

${ }^{36}$ G. C. Lynch, R. Steckler, D. W. Schwenke, A. J. C. Varandas, and D. G. Truhlar, J. Chem. Phys. 94, 7136 (1991).

${ }^{37}$ T. Takayanagi and S. Sato, Chem. Phys. Lett. 144, 191 (1988); T. Takayanagi, S. Tsunashima and S. Sato, J. Chem. Phys. 93, 2487 (1990).

${ }^{38}$ More details about the $a b$ initio surface will be given in a future publication by: $\mathrm{K}$. Stark and $\mathrm{H}$. -J. Werner, unpublished results.

39 J. T. Muckerman, J. Chem. Phys. 54, 1155 (1971); J. Chem. Phys. 56, 2997 (1972); P. A. Whitlock and J. T. Muckerman, ibid. 61, 4624 (1974).

40R. L. Jaffe and J. B. Anderson, J. Chem. Phys. 54, 2224 (1971).

${ }^{41}$ R. L. Wilkins, J. Chem. Phys. 57, 912 (1972).

${ }^{42}$ D. L. Thompson, J. Chem.Phys. 57, 4170 (1972).

43J. C. Polanyi and J. L. Schreiber, Far. Disc. Chem. Soc. 62, 267 (1977).

${ }^{44}$ P. D. Mercer and H. O. Pritchard, J. Chem. Phys. 63, 1468 (1959).

${ }^{45}$ C. F.Bender, S. V. O'Neill, P. K. Pearson, and H. F. Schaefer, Science 176, 1412 (1972).

${ }^{46}$ S. R. Ungemach, H. F. Schaefer, and B. Liu, Far. Disc. Chem. Soc. 62, 330 (1977).

${ }^{47}$ M. J. Frisch, B. Liu, J. S. Binkley, H. F. Schaefer III, and W. H. Miller, Chem. Phys. Lett. 114, 1 (1985).

48J. S. Wright, R. J. Williams, and R. E. Wyatt, Chem. Phys. Lett. 184, 159 (1991).

${ }^{49}$ D. G. Truhlar, B. C. Garrett, and N. C. Blais, J. Chem. Phys. 80, 232 (1984).

${ }^{50}$ C. F. Bender, P. K. Pearson, S. V. O'Neil, and H. F. Schaefer, J. Chem. Phys. 56, 4626 (1972).

5IF. B. Brown, R. Steckler, D. W Schwenke, D. G Truhlar, and B. C. Garrett, J. Chem. Phys. 82, 188 (1985).

${ }^{52}$ D. W. Schwenke, R. Steckler, F. B. Brown, and D. G. Truhlar, J. Chem. Phys. 84, 5706 (1986); D. W. Schwenke, R. Steckler, F. B. Brown, and D. G. Truhlar, J. Chem. Phys. 86, 2443 (1987).

${ }^{53}$ C. W. Bauschlicher, Jr., S. P. Walch, S. R. Langhoff, P. R. Taylor, and R. L. Jaffe, J. Chem. Phys. 88, 1743 (1988).

${ }^{54}$ Another surface has recently been generated by the Truhlar group known as 6 SEC. 
55P. J. Knowles, K. Stark, and H. -J.Werner, Chem. Phys. Lett. 185, 555 (1991).

${ }^{56}$ D. M. Neumark, Ann. Rev. Phys. Chem. 43, 153 (1992).

57J. A. Nichols, R. A. Kendall, S. J. Cole, and J. Simons, J. Phys. Chem. 95, 1074 (1991).

${ }^{58}$ In that same investigation, Bradforth characterized the purely repulsive excited electronic surfaces of the $\mathrm{F}+\mathrm{H}_{2}$ system via an analysis of the polarization dependence of the spectra.

${ }^{59}$ R. B. Metz, A. Weaver, S. E. Bradforth, T. N. Kitsopoulos, and D. M. Neumark, J. Phys. Chem. 94, 1377 (1990).

60M. A. Johnson, M. L. Alexander, and W. C. Lineberger, Chem. Phys. Lett. 112, 285 (1984).

${ }^{61}$ J. E. Pollard, D. J. Trevor, J. E. Reutt, Y. T. Lee, and D. A. Shirley, J. Chem. Phys. 77, 34 (1982).

${ }^{62}$ R. E. Continetti, Ph. D. Thesis, University of California, Berkeley (1989).

${ }^{63}$ Assuming $\Delta \mathrm{E}_{\mathrm{J}-1, \mathrm{~J}}=2 \mathrm{~B}_{\mathrm{o}} \mathrm{J}, \Delta \mathrm{E}_{0,1}=118 \mathrm{~cm}^{-1}, \Delta \mathrm{E}_{0,2}=355 \mathrm{~cm}^{-1}, \Delta \mathrm{E}_{0,3}=711 \mathrm{~cm}^{-1}, \Delta \mathrm{E}_{0,4}=1186 \mathrm{~cm}^{-1}$, $\Delta \mathrm{E}_{0,5}=1779 \mathrm{~cm}^{-1}, \Delta \mathrm{E}_{0,6}=2491 \mathrm{~cm}^{-1}$. Assumes $\mathrm{B}_{\mathrm{o}}=\mathrm{B}_{\mathrm{e}}-0.5^{*} \alpha_{\mathrm{e}}$ where $\mathrm{B}_{\mathrm{e}}=60.853 \mathrm{~cm}^{-1}$ and $\alpha_{\mathrm{e}}=$ $3.062 \mathrm{~cm}^{-1}$ from K. P. Huber and G. Herzberg, Spectra and Molecular Structure: Constants of Diatomic Molecules, (Van Nostrand, New York 1979).

${ }^{64}$ G. C. Schatz, J. Chem. Phys. 90, 3582 (1990); G. C. Schatz, J. Phys. Chem. 94, 6157 (1990).

${ }^{65}$ G. C. Schatz, Chem. Phys. Lett. 150, 92 (1988).

${ }^{66}$ F. J. Aoiz, L. Bañares, V. J. Herrero, V. Saez Rábanos, K. Stark, and H. -J. Werner. (to be published).

${ }^{67} \mathrm{D}$. M. Manolopoulos, private communication. 


\title{
Chapter 8: Spectroscopy of the Transition State: Elementary \\ Reactions of the Hydroxyl Radical Studied by \\ Photoelectron Spectroscopy of $\mathrm{O}^{-}\left(\mathrm{H}_{2} \mathrm{O}\right)$ and $\mathrm{H}_{3} \mathrm{O}_{2}^{-}$
}

\begin{abstract}
The transition state region of the $\mathrm{OH}+\mathrm{OH} \rightarrow \mathrm{O}\left({ }^{(} \mathrm{P}\right)+\mathrm{H}_{2} \mathrm{O}$ and the $\mathrm{OH}+\mathrm{H}_{2} \mathrm{O} \rightarrow \mathrm{H}_{2} \mathrm{O}+\mathrm{OH}$ reactions are studied by photoelectron spectroscopy of the $\mathrm{O}^{-}\left(\mathrm{H}_{2} \mathrm{O}\right)$ and $\mathrm{H}_{3} \mathrm{O}_{2}^{-}$anions. The observed features are assigned to vibrations of the unstable neutral complex based upon comparison with previous heavy-light-heavy transition state spectra and with the results of $a b$ initio calculations performed here and elsewhere. One dimensional Franck-Condon calculations for $a b$ initio antisymmetric stretch potentials provide an understanding of the change in peak spacings and intensities observed upon isotopic substitution.
\end{abstract}

\section{Introduction}

Negative ion photoelectron spectroscopy is a method which has proven successful in providing direct information about the transition state region of bimolecular chemical reactions. We have studied the transition state species for several bimolecular chemical reactions by photodetachment of a stable negative ion which possesses a geometry approximating that of the neutral transition state species. These reactions include heavy-light-heavy hydrogen transfer reactions $(\mathrm{X}+\mathrm{HY} \rightarrow \mathrm{XH}+\mathrm{Y} ; \mathrm{X}, \mathrm{Y}=\mathrm{I}, \mathrm{Br}, \mathrm{Cl}, \mathrm{F}),{ }^{1,2}$ hydrogen abstraction reactions by fluorine $\left(\mathrm{OH}+\mathrm{F} \rightarrow \mathrm{O}+\mathrm{HF} \text { and } \mathrm{ROH}+\mathrm{F} \rightarrow \mathrm{RO}+\mathrm{HF} ; \mathrm{R}=\mathrm{CH}_{3} \mathrm{O}, \mathrm{C}_{2} \mathrm{H}_{5} \mathrm{O}\right)^{3}$ and the prototypical $\mathrm{F}+\mathrm{H}_{2}$ reaction. ${ }^{4}$ In this paper, we present the results of recent experiments in our laboratory where the photoelectron spectra of the $\mathrm{H}_{3} \mathrm{O}_{2}^{-}$and $\mathrm{O}^{-}\left(\mathrm{H}_{2} \mathrm{O}\right)$ anions are collected to study the unstable neutral complexes which are involved in two fundamental reactions of the hydroxyl radical:

$$
\mathrm{OH}+\mathrm{H}_{2} \mathrm{O} \rightarrow \mathrm{H}_{2} \mathrm{O}+\mathrm{OH} \text { (1). }
$$




$$
\mathrm{OH}+\mathrm{OH} \rightarrow \mathrm{O}\left({ }^{3} \mathrm{P}\right)+\mathrm{H}_{2} \mathrm{O}
$$

The role of the hydroxyl radical as a propagator of chain reactions makes it extremely important in atmospheric chemistry, combustion chemistry, and a wide range of other chemically active environments. The hydroxyl radical is known to play a vital role in the stratospheric ozone chemistry through the $\mathrm{HO}_{\mathrm{x}}$ cycle. ${ }^{5}$ It also acts to remove many chemical species which are important in tropospheric chemistry, including $\mathrm{CO}, \mathrm{H}_{2} \mathrm{~S}, \mathrm{SO}_{2}$, and $\mathrm{CH}_{3} \mathrm{CCl}_{3}{ }^{6}$ The reaction of $\mathrm{O}\left({ }^{1} \mathrm{D}\right)+\mathrm{H}_{2} \mathrm{O}$ serves as a major source of tropospheric $\mathrm{OH}$ radicals. ${ }^{7}$ In combustion, reaction (2) and its reverse reaction serve as termination and propagation steps in the oxidation of hydrogen, respectively. ${ }^{8}$

This set of experiments studying the transition state species of hydroxyl radical reactions not only begins our study of this extremely important class of bimolecular reactions but also continues the extension of our transition state studies to reactions with polyatomic reactant andlor products. Both of the reactions studied here represent quite fundamental chemical reactions that are accessible to accurate study by ab initio methods. To date, however, only a few detailed theoretical studies exist for either reaction (1) or (2).

Figures 1 and 2 show schematic energy diagrams for reactions (1) and (2). Since the anion geometry and energetics play a key role in our experiment, the energetics for the analogous ion-molecule reactions are also shown. The figures represent the energy of the systems as a function of generic reaction coordinates. In Fig. 1, both the anion and neutral reactions are thermoneutral due to symmetry. The $\mathrm{H}_{3} \mathrm{O}_{2}^{-}$anion is calculated to have a single minimum (see Sec III B), ${ }^{9}$ the binding energy of which $\left(\mathrm{D}_{0}\left(\mathrm{OH}^{-} \cdots \mathrm{H}_{2} \mathrm{O}\right)=1.18 \mathrm{eV}\right)^{10}$ has been

Figure 1: Schematic energetics diagram for the $\mathrm{H}_{3} \mathrm{O}_{2}^{-} / \mathrm{OH}+\mathrm{H}_{2} \mathrm{O}$ system. Values in italics are theoretically determined values. References given in text.

Figure 2: Schematic energetics diagram for the $\mathrm{O}^{-}\left(\mathrm{H}_{2} \mathrm{O}\right) / \mathrm{OH}+\mathrm{OH}$ system. Values in italics are theoretically determined values. References given in text. 


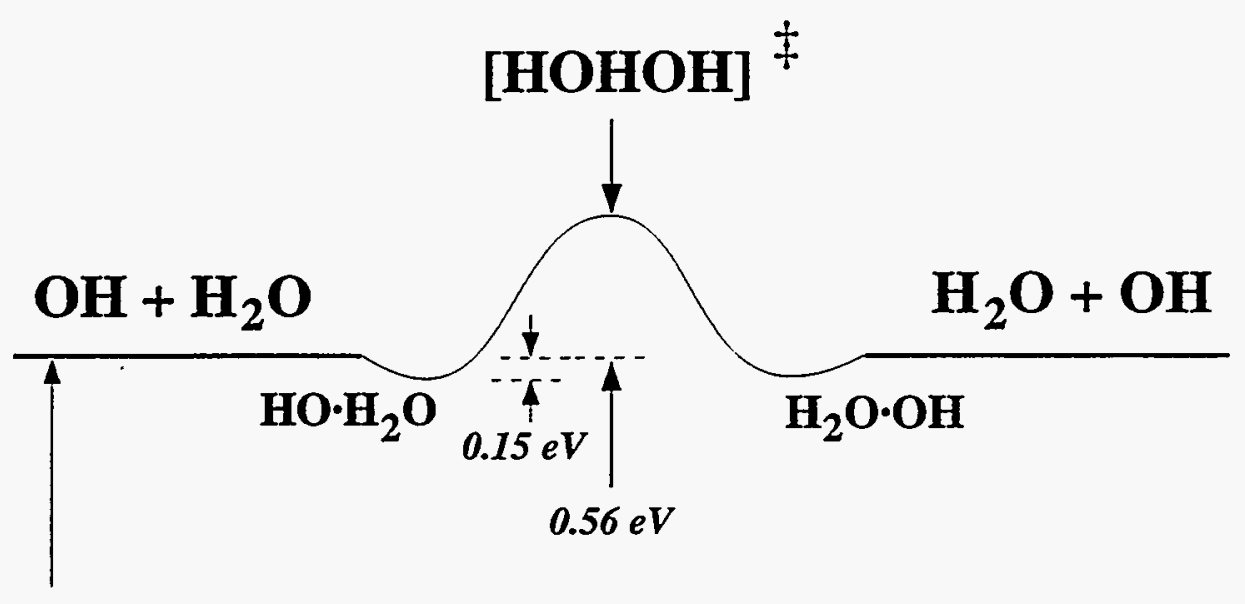

$\mathrm{EA}(\mathrm{OH})=1.825 \mathrm{eV}$

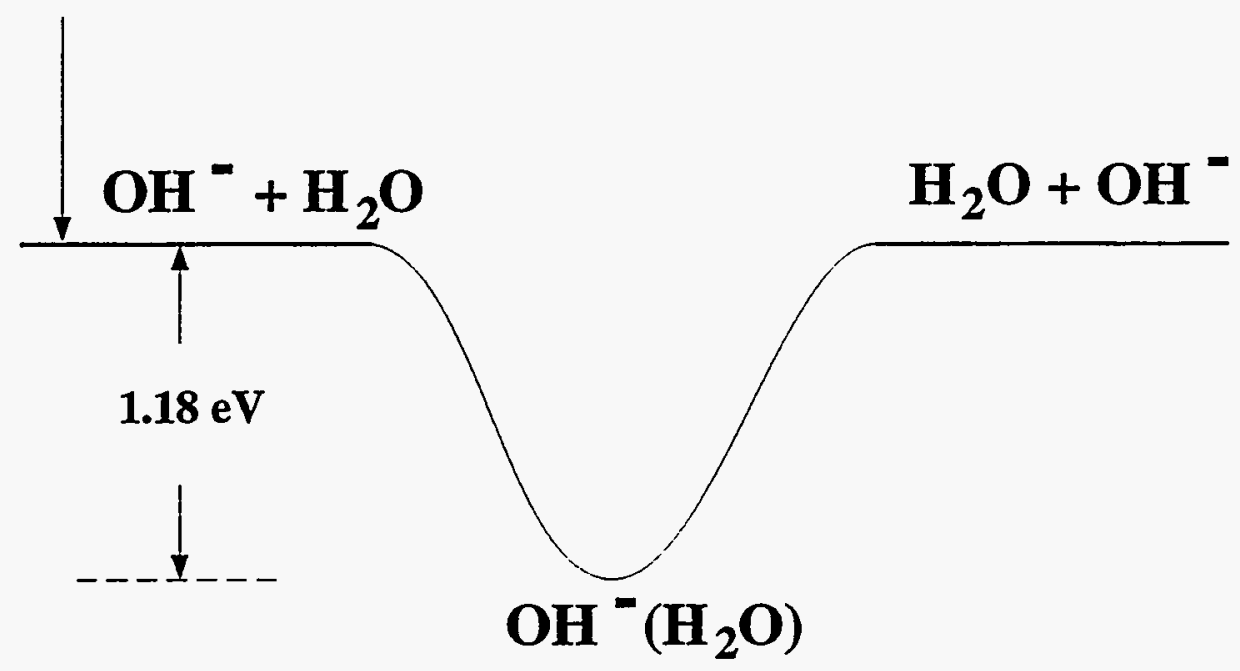

Figure 8.1 

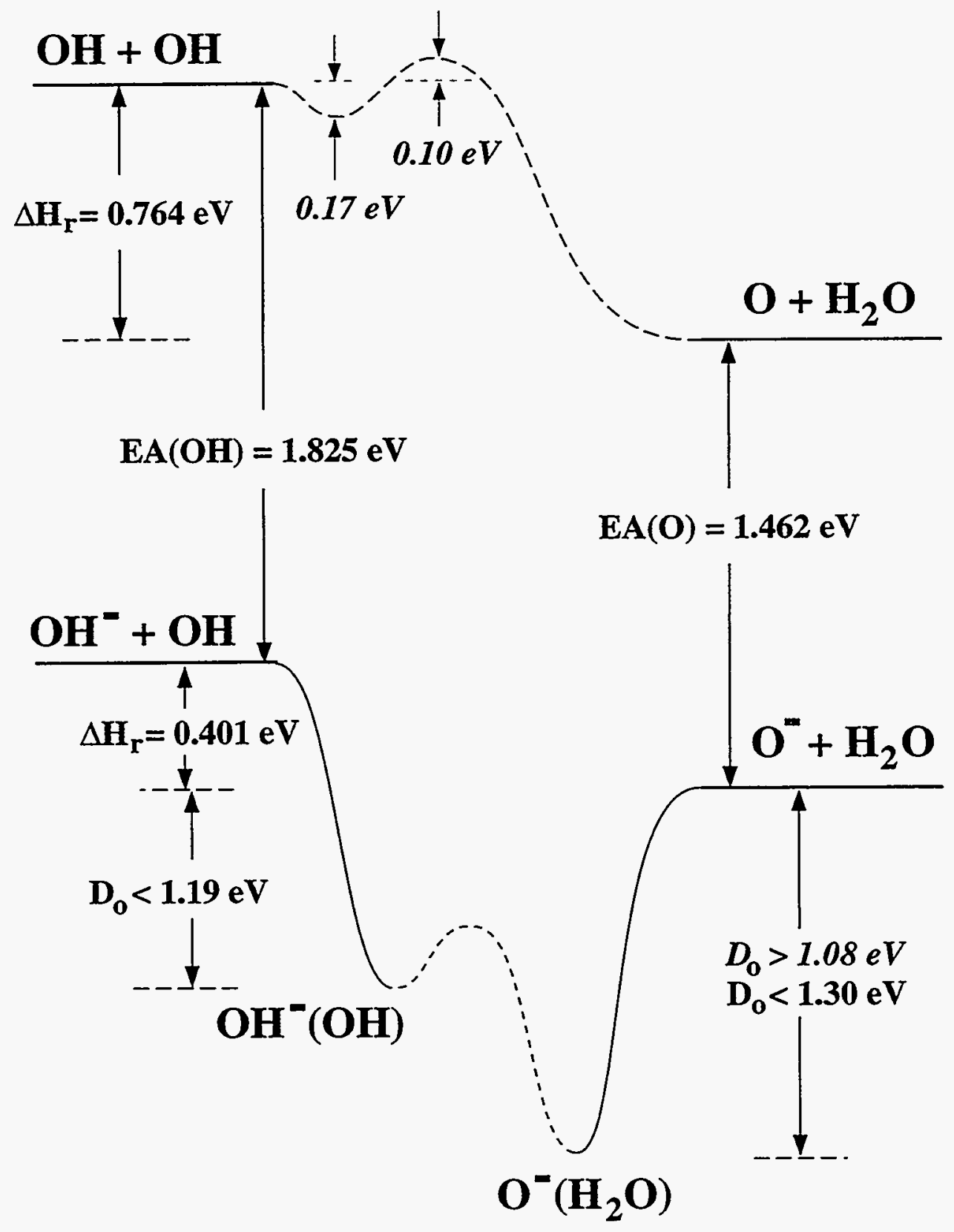

Figure 8.2 
measured by high pressure mass spectrometry. The electron affinity of $\mathrm{OH}(\mathrm{EA}(\mathrm{OH})=1.825$ $\mathrm{eV})^{11}$ has been accurately measured by threshold photodetachment of $\mathrm{OH}^{-}$. The values shown for the neutral potential curve are from $a b$ initio calculations. Schaefer and co-workers ${ }^{12}$ calculated van der Waals minima of $0.15 \mathrm{eV}(3.5 \mathrm{kcal} / \mathrm{mole})$ for the $\mathrm{OH}\left(\mathrm{H}_{2} \mathrm{O}\right)$ complex in the hydrogen-bonded configuration which is applicable to the photodetachment experiments. The global minimum was found to occur in a different intramolecular configuration which is not conducive to hydrogen exchange and lies $0.13 \mathrm{eV}(3.1 \mathrm{kcal} / \mathrm{mole})^{13}$ lower in energy than the local minimum shown in Fig. 1. A barrier height of $0.56 \mathrm{eV}(13 \mathrm{kcal} / \mathrm{mole})$ for the reaction was determined in an ab initio investigation by Nanayakkara et al. ${ }^{14}$

In Fig. 2, the shape of the anion potential is adapted from Lifshift $z^{15}$ and is based upon an anion reaction model developed by Brauman and co-workers. ${ }^{16}$ However, no actual characterization of this anion potential surface has been made (i.e., the height of the barrier between the two geometries, if one exists, has not been determined). Limits for the binding energies of the $\mathrm{O}^{-}\left(\mathrm{H}_{2} \mathrm{O}\right)$ and $\mathrm{OH}^{-}(\mathrm{OH})$ anions have been determined by pulsed electron high pressure mass spectrometry. ${ }^{17,18,19}$ Note that these experimental limits do not indicate whether there are two geometries corresponding to local minima in the potential energy surface separated by a barrier or if the two structures are actually indistinguishable. The electron affinity of oxygen has also been measured by anion threshold photodetachment. ${ }^{20}$ Similarly to $\mathrm{OH}+\mathrm{H}_{2} \mathrm{O}$, a van der Waals minimum is also predicted to exist on the $\mathrm{OH}+\mathrm{OH}$ neutral reaction surface. Fueno ${ }^{21}$ predicts the dipole-dipole complex to be bound by $6.9 \mathrm{kcal} / \mathrm{mole}$. However, Harding suggests that the binding energy is more on the order of $4.2 \mathrm{kcal} / \mathrm{mole} .^{22}$ Harding and Wagner calculate a $2.3 \mathrm{kcal} / \mathrm{mole}$ barrier for the reaction. ${ }^{23}$

In one of the incipient studies of anion photodetachment, $\mathrm{H}_{3} \mathrm{O}_{2}^{-}$was studied by Golub and Steiner over 25 years ago. ${ }^{24}$ The total photodetachment cross-section was measured as a function of photon energy up to $4 \mathrm{eV}$. The monotonic increase observed in their photoelectron signal with increasing photon energy beyond $2.8 \mathrm{eV}$ was interpreted as photodetachment of the 
$\mathrm{OH}^{-}\left(\mathrm{H}_{2} \mathrm{O}\right)$ anion cluster to form a dissociative neutral complex. No underlying structure was resolved in the data obtained from their early study. Since then, however, the $\mathrm{H}_{3} \mathrm{O}_{2}^{-}$anion has been characterized by $\mathrm{x}$-ray structural analysis after its observation in the crystals of transition metal complexes. ${ }^{25}$ This anion has also been observed in the IR spectra of tetraalkylammonium ion hydroxide hydrate complexes. ${ }^{26}$ The photoelectron spectra of $\mathrm{H}_{3} \mathrm{O}_{2}^{-}$presented below show resolved features which contain information about the dynamics near the transition state region of the $\mathrm{OH}+\mathrm{H}_{2} \mathrm{O}$ reaction. No other experimental investigation of this symmetric hydrogen exchange reaction has been found in the literature.

Reaction (2) has been more thoroughly investigated experimentally. Numerous measurements of the rate of reaction (2) over various temperature ranges $27,28,29,30,31,32,33,34,35,36$ show that the hydroxyl radical disproportionation has a non-Arhennius behavior. ${ }^{37}$ Measurements for the rate of the reverse reaction have also been made and found to be consistent with these findings. ${ }^{38}$ The source of this non-Arhennius behavior has been debated with respect to the presence or absence of a potential barrier along the reaction path. Initially, Wagner and Zellner ${ }^{28}$ suggested a barrierless reaction in which the long-range attractive forces affected the temperature dependence of the reaction. Harding and Wagner have since calculated a $2.3 \mathrm{kcal} / \mathrm{mole}$ barrier and conclude that the long range forces do not play a major role in the reaction's temperature dependence. ${ }^{23}$ Recent results by Michael ${ }^{39}$ are consistent with the results of Ref. 23. While the $\mathrm{O}^{-}+\mathrm{H}_{2} \mathrm{O}$ reaction has been studied by several groups ${ }^{40,41,42,43,44,45}$ and the $\mathrm{O}^{-}\left(\mathrm{H}_{2} \mathrm{O}\right)$ has been observed by mass spectrometry in $\mathrm{H}_{2} / \mathrm{O}_{2} / \mathrm{N}_{2}$ flames, ${ }^{46}$ no experimental characterization of the $\mathrm{O}^{-}\left(\mathrm{H}_{2} \mathrm{O}\right)$ anion has been completed which can confirm the results of the $a b$ initio calculations to be presented in Sec III B. A detailed analysis of the $\mathrm{O}^{-}\left(\mathrm{H}_{2} \mathrm{O}\right)$ anion photoelectron spectrum, to be presented below, could provide additional insight into the nature of the barrier for this reaction.

The reverse of reaction (2), may also play a role in experiments studying $\mathrm{O}\left({ }^{1} \mathrm{D}\right)+\mathrm{H}_{2} \mathrm{O}$ $\rightarrow \mathrm{OH}+\mathrm{OH}$ where $\mathrm{O}$ atoms are generated by ozone photolysis. ${ }^{47,48}$ Sauder et al. ${ }^{48}$ estimate 
that as much as $10 \%$ of the observed $\mathrm{OH}$ products may result from reactions of $\mathrm{O}\left({ }^{3} \mathrm{P}\right)$ ground state atoms with the water molecules in these photoinitiated reaction experiments.

In the following sections, we will briefly describe the experiments performed (Sec. II) and present the results obtained (Sec. III A). Ab initio calculations to be used in the analysis of the data are described in Sec. III B. The results of these calculations are used in onedimensional Franck-Condon analyses to qualitatively understand the photoelectron spectra in terms of the dynamics in the transition state region of the reaction. Comparisons will be drawn with our studies of related fluorine atom reactions $\left(\mathrm{OH}+\mathrm{F}\right.$ and $\left.\mathrm{CH}_{3} \mathrm{OH}+\mathrm{F}\right)$ which we have investigated previously. ${ }^{3}$

\section{Experimental}

The apparatus employed in these experiments, described in detail previously, ${ }^{2}$ is a dual time-of-flight anion photoelectron spectrometer. Details relevant to the present results will be summarized here. Anions of interest are generated in the source region at the intersection of a

pulsed molecular beam and a $1 \mathrm{keV}$ electron beam. ${ }^{49}$ A gas mixture $\left(4 \% \mathrm{H}_{2} \mathrm{O}, 96 \% \mathrm{~N}_{2} \mathrm{O}\right)$, at a stagnation pressure of $1 \mathrm{bar}$, is expanded through the molecular beam valve orifice $\left(0.020^{\prime \prime}\right)$ at a repetition rate of $20 \mathrm{~Hz}$. The $1 \mathrm{keV}$ electron beam intersects the molecular beam at the orifice of the molecular beam valve. At this intersection, a variety of chemical processes occur which lead to the formation of $\mathrm{O}^{-}\left(\mathrm{H}_{2} \mathrm{O}\right)$ and $\mathrm{H}_{3} \mathrm{O}_{2}^{-}$anions. $\mathrm{O}^{-}$anions, generated by dissociative attachment of low-energy electrons to $\mathrm{N}_{2} \mathrm{O}, 50,51$ can form $\mathrm{O}^{-}\left(\mathrm{H}_{2} \mathrm{O}\right)$ by termolecular clustering reactions or $\mathrm{OH}^{-}$by $\mathrm{O}^{-}+\mathrm{H}_{2} \mathrm{O} \rightarrow \mathrm{OH}^{-}+\mathrm{OH}$. Hydroxide ions which are generated can also cluster to $\mathrm{H}_{2} \mathrm{O}$ molecules to form the $\mathrm{H}_{3} \mathrm{O}_{2}^{-}$clusters. As shown by Melton ${ }^{52}$ and others, electron bombardment of $\mathrm{H}_{2} \mathrm{O}$ generates $\mathrm{H}^{-}, \mathrm{O}^{-}$, and $\mathrm{OH}^{-}$anions, all of which can contribute to ion formation. As the expansion continues, the molecules relax rotationally and vibrationally by collisions with the carrier gas. 
The cooled ions are extracted into a Wiley-McLaren-type time-of-flight mass spectrometer ${ }^{53}$ where the ions separate, according to mass, from other anions which are formed in the source region. The mass resolution of the apparatus, $M / \Delta M \sim 250$, allows easy separation of the $\mathrm{H}_{3} \mathrm{O}_{2}^{-}$and $\mathrm{O}^{-}\left(\mathrm{H}_{2} \mathrm{O}\right)$ ions. The ion of interest is then selectively photodetached by a properly timed $8 \mathrm{~ns}$ Nd:YAG laser pulse. Photoelectrons, detected with $70 \mathrm{~mm}$ multichannel plates, are energy analyzed after time-of-flight measurements through a 1 meter field-free flight tube oriented perpendicular to the mass spectrometer flight tube. The resolution of the apparatus is $8 \mathrm{meV}$ for electrons with $0.65 \mathrm{eV}$ of electron kinetic energy (eKE) and degrades as (eKE) $)^{3 / 2}$. For these experiments, the fourth harmonic (266 nm; 15 $\mathrm{mJ} /$ pulse) of the Nd:YAG laser was employed for photodetachment. The plane-polarized laser beam can be rotated with a half-wave plate in order to study photoelectron angular distributions.

\section{Results}

\section{A. Experimental Results}

Figure 3 shows the photoelectron spectra collected for $\mathrm{H}_{3} \mathrm{O}_{2}^{-}$and $\mathrm{D}_{3} \mathrm{O}_{2}^{-}$. Figure 4 displays the $\mathrm{O}^{-}\left(\mathrm{H}_{2} \mathrm{O}\right)$ and $\mathrm{O}^{-}\left(\mathrm{D}_{2} \mathrm{O}\right)$ spectra. In these spectra, the relationship between the eKE and the internal energy of the neutral complex is given by

Figure 3: Photoelectron spectra of $\mathrm{H}_{3} \mathrm{O}_{2}^{-}$and $\mathrm{D}_{3} \mathrm{O}_{2}^{-}$collected using a $4.657 \mathrm{eV}$ photodetachment energy. The arrow ' $a$ ' indicates the asymptotic energy for the $\mathrm{OH}$ $+\mathrm{H}_{2} \mathrm{O}$ ground state products.

Figure 4: Photoelectron spectra of $\mathrm{O}^{-}\left(\mathrm{H}_{2} \mathrm{O}\right)$ and $\mathrm{O}^{-}\left(\mathrm{D}_{2} \mathrm{O}\right)$ collected using a $4.657 \mathrm{eV}$ photodetachment energy. The regions $a / a^{\prime}$ and $b / b^{\prime}$ indicate the limits for the asymptotic energies for dissociation of the neutral complex into $\mathrm{O}+\mathrm{H}_{2} \mathrm{O}$ and $\mathrm{OH}$ $+\mathrm{OH}$, respectively. 


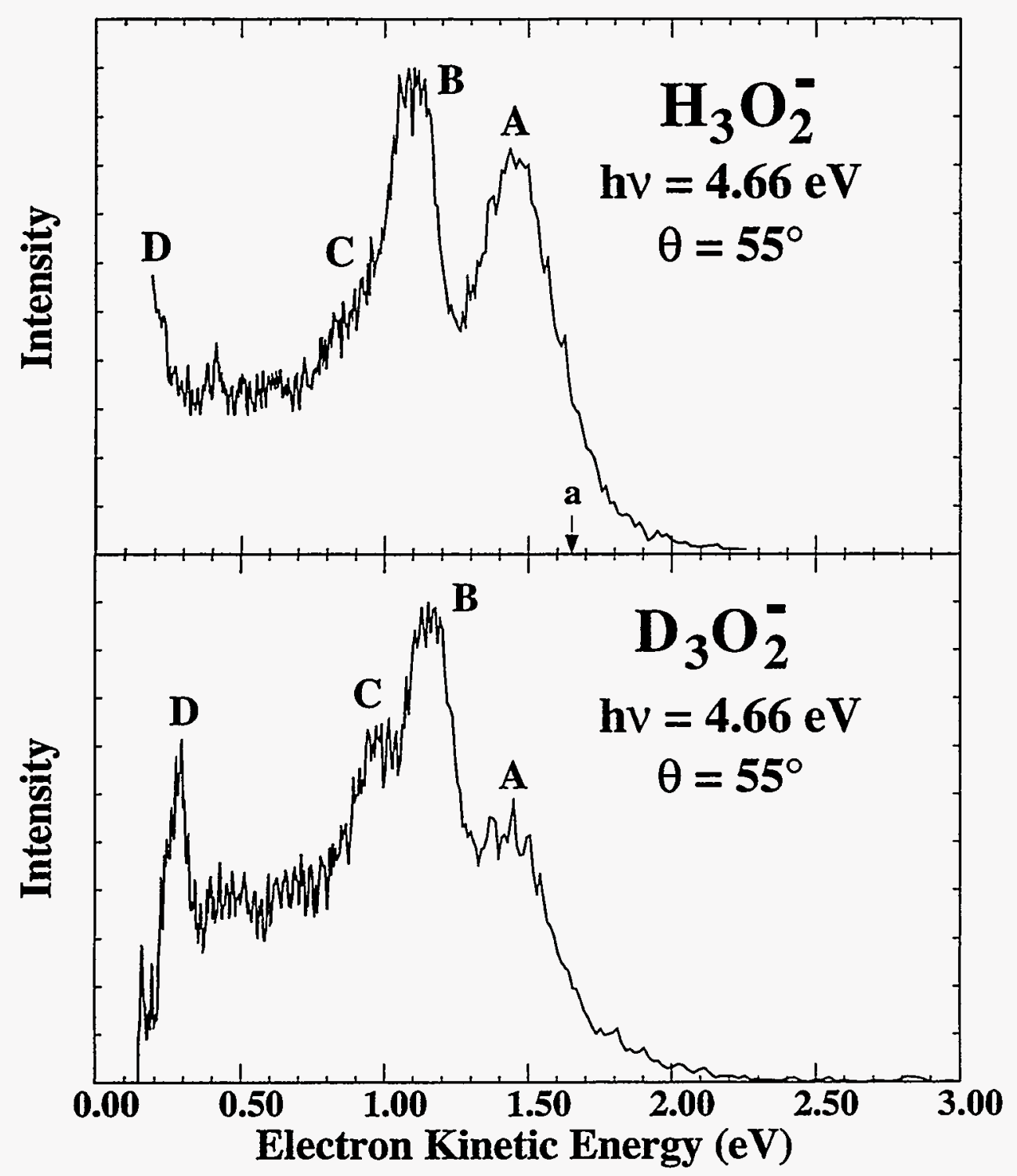

Figure 8.3 


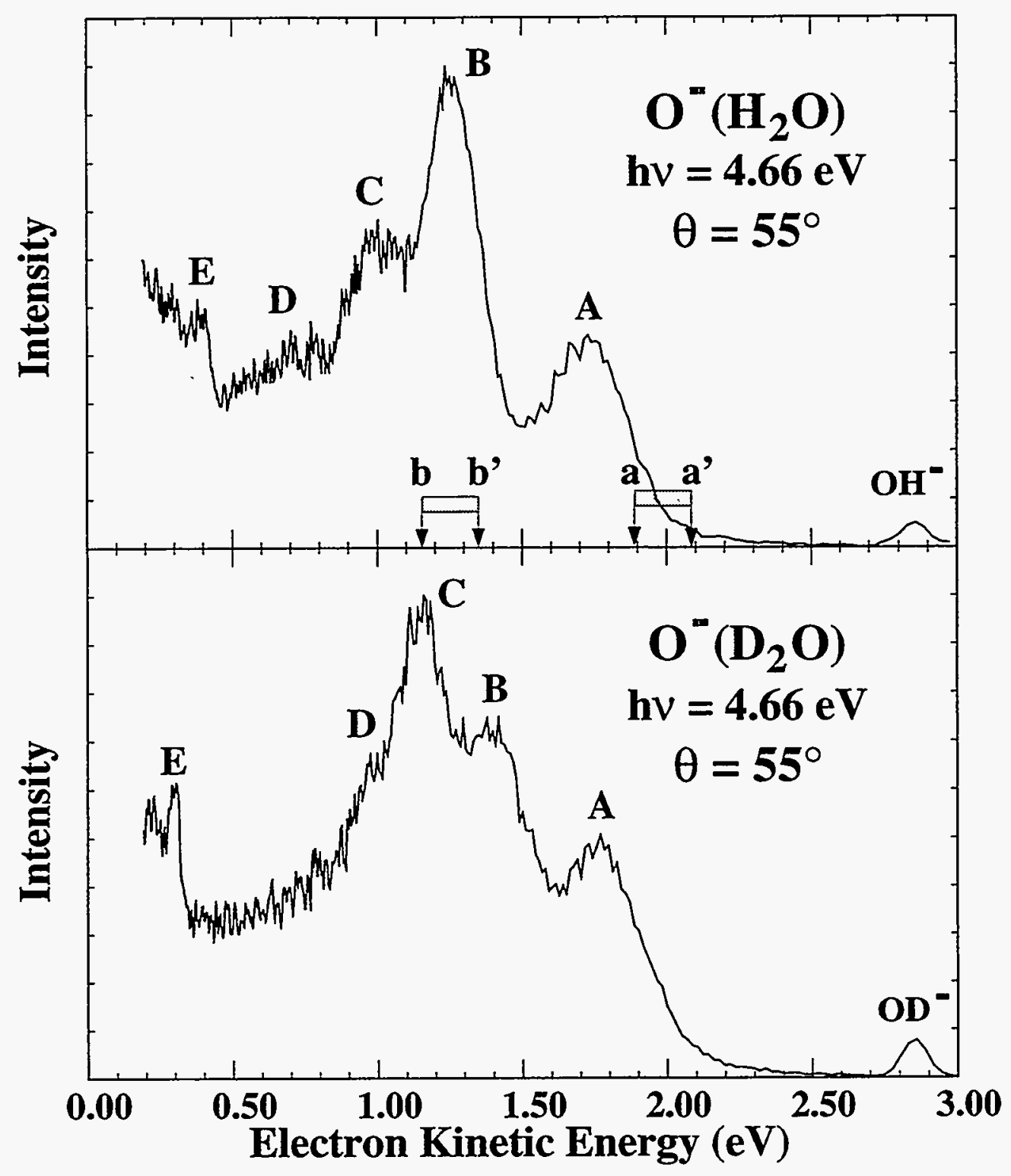

Figure 8.4 


$$
\mathrm{eKE}=\mathrm{hv}-\mathrm{D}_{\mathrm{o}}-\mathrm{EA}-\mathrm{E}_{\mathrm{int}}^{\circ}+\mathrm{E}_{\mathrm{int}}^{-}
$$

In Eq. (3), $\mathrm{D}_{\mathrm{o}}$ is the lowest dissociation energy of the anion complex [i.e., $\mathrm{D}_{\mathrm{o}}\left(\mathrm{OH}^{-} \cdots \mathrm{H}_{2} \mathrm{O}\right)$ or $\mathrm{D}_{0}\left(\mathrm{O}^{-} \cdots \mathrm{H}_{2} \mathrm{O}\right)$ given in Figs. 1 and 2] and EA is the electron affinity of the fragment anion [i.e., $\mathrm{EA}(\mathrm{OH})=1.825 \mathrm{eV}^{11}$ and $\left.\mathrm{EA}(\mathrm{O})=1.462 \mathrm{eV}^{20}\right] . \mathrm{E}_{\mathrm{int}}^{-}$and $\mathrm{E}_{\mathrm{int}}^{\mathrm{o}}$ represent the internal energies if the anion and neutral complexes, respectively. For the anion, $\mathrm{E}_{\mathrm{int}}^{-}$is the internal energy above the zero point. For the neutral $[\mathrm{HOHOH}]$ complex, $\mathrm{E}_{\mathrm{int}}^{\circ}$ is the energy above the separated $\mathrm{OH}+$ $\mathrm{H}_{2} \mathrm{O}$ ground state fragments. For the [HOHO] complex, $\mathrm{E}_{\mathrm{int}}^{\mathrm{o}}$ is the energy above the ground state $\mathrm{O}+\mathrm{H}_{2} \mathrm{O}$ products.

In each of the spectra, the energetic asymptotes for dissociation of the neutral complex are indicated by arrows. In Fig. 3, the arrow marked 'a' indicates the $\mathrm{OH}+\mathrm{H}_{2} \mathrm{O}$ limit while in Fig. 4 the regions bounded by the $a / a^{\prime}$ and $b / b^{\prime}$ arrows indicate the limits for the $\mathrm{O}+\mathrm{H}_{2} \mathrm{O}$ (product) and $\mathrm{OH}+\mathrm{OH}$ (reactant) ground state energetic asymptotes; the large uncertainties reflect the uncertainty in $\mathrm{D}_{0}\left(\mathrm{O}^{-}-\mathrm{H}_{2} \mathrm{O}\right)$. As seen, almost all of the signal in the spectra occurs at energies which correspond to neutral complexes which are energetically unstable with respect to dissociation into either reactants or products.

There are several similarities between the $\mathrm{H}_{3} \mathrm{O}_{2}^{-}$and the $\mathrm{O}^{-}\left(\mathrm{H}_{2} \mathrm{O}\right)$ spectra. The spectra consist primarily of very broad, irregularly spaced features. Isotopic substitution significantly changes not only the positions of the features but also the intensities. The centers, widths and spacings of the broad features in the $\mathrm{H}_{3} \mathrm{O}_{2}^{-}$and $\mathrm{D}_{3} \mathrm{O}_{2}^{-}$spectra are given in Table $\mathrm{I}$. Those for the $\mathrm{O}^{-}\left(\mathrm{H}_{2} \mathrm{O}\right)$ and $\mathrm{O}^{-}\left(\mathrm{D}_{2} \mathrm{O}\right)$ spectra are given in Table II. Based upon previous studies of the heavy-light-heavy hydrogen exchange reactions, ${ }^{1,2}$ we expect these broad features to be related to the antisymmetric stretch motion of the 'transfer' hydrogen atom between the oxygen atoms (i.e., $\mathrm{O} \cdots \stackrel{\leftrightarrow}{\mathrm{H}} \cdots \mathrm{O}$ ). 
Table I: Peak Positions and Widths for the $\mathrm{H}_{3} \mathrm{O}_{2}^{-}$and $\mathrm{D}_{3} \mathrm{O}_{2}^{-}$

4.66 eV Photoelectron Spectra ${ }^{a}$

\begin{tabular}{|c|c|c|c|}
\hline Peak & eKE (eV) & Energy & Width (eV) \\
\hline $\mathrm{H}_{3} \mathrm{O}_{2}^{-}$ & & & \\
\hline $\mathrm{A}$ & 1.45 & 0.0 & 0.33 \\
\hline $\mathrm{B}$ & 1.10 & 2823 & 0.19 \\
\hline $\mathrm{C}$ & 0.91 & 4355 & 0.21 \\
\hline $\mathrm{D}_{3} \mathrm{O}_{2}^{-}$ & & & \\
\hline $\mathrm{A}$ & 1.45 & 0.0 & 0.33 \\
\hline $\mathrm{B}$ & 1.16 & 2339 & 0.20 \\
\hline $\mathrm{C}$ & 0.94 & 4113 & 0.21 \\
\hline $\mathrm{D}$ & $0.293^{\mathrm{b}}$ & --- & -- \\
\hline
\end{tabular}

a) Position of peak center and full width at half-maximum (FWHM) as determined by fit to a Gaussianshaped peak. No consideration is made for superimposed vibrational structure.

b) Intensity severely affected by electron detector cutoff function.

Another common feature to the data sets is the appearance of a feature at very low eKE. The intensity of this feature (labeled as $\mathrm{D}$ in the $\mathrm{H}_{3} \mathrm{O}_{2}^{-}$data and $\mathrm{E}$ in the $\mathrm{O}^{-}\left(\mathrm{H}_{2} \mathrm{O}\right)$ data) is significantly affected by the electron detector cutoff function, thus its absolute intensity is suppressed. The large energy separation from the other features in the spectrum suggests that the low eKE feature represents photodetachment to electronically excited neutral complexes. Another piece of evidence which supports this assignment is the change in position of peak $\mathrm{E}$ upon isotopic substitution. As seen in Fig. 4, peak E moves to lower eKE upon deuteration. This effect is consistent with the assignment of this feature to an excited state which undergoes a smaller zero point energy decrease than the anion upon deuteration.

Also seen in the $\mathrm{O}^{-}\left(\mathrm{H}_{2} \mathrm{O}\right)$ and $\mathrm{O}^{-}\left(\mathrm{D}_{2} \mathrm{O}\right)$ photoelectron spectra is a feature near $2.85 \mathrm{eV}$. This peak occurs at the same energy as signal for $\mathrm{OH}^{-}$photodetachment. We believe that this feature corresponds to a sequential two-photon process. The $\mathrm{O}^{-}\left(\mathrm{H}_{2} \mathrm{O}\right)$ anion is photodissociated into $\mathrm{OH}^{-}+\mathrm{OH}$ followed by photodetachment of the $\mathrm{OH}^{-}$anion during the 
same $8 \mathrm{~ns}$ laser pulse. A less intense signal was also observed at $\sim 3.2 \mathrm{eV}$ which corresponds to $\mathrm{O}^{-}$photodetachment after photodissociation of $\mathrm{O}^{-}\left(\mathrm{H}_{2} \mathrm{O}\right)$ into $\mathrm{O}^{-}+\mathrm{H}_{2} \mathrm{O}$.

Table II: Peak Positions and Widths for the $\mathrm{O}^{-}\left(\mathrm{H}_{2} \mathrm{O}\right)$ and $\mathrm{O}^{-}\left(\mathrm{D}_{2} \mathrm{O}\right) 4.66 \mathrm{eV}$ Photoelectron Spectra ${ }^{\mathrm{a}}$

\begin{tabular}{|c|c|c|c|}
\hline Peak & eKE $(\mathrm{eV})$ & Energy & Width (eV) \\
\hline \hline $\mathrm{O}^{-}\left(\mathrm{H}_{2} \mathrm{O}\right)$ & & & \\
\hline $\mathrm{A}$ & 1.72 & 0.0 & 0.34 \\
\hline $\mathrm{B}$ & 1.27 & 3629 & 0.22 \\
\hline $\mathrm{C}$ & 1.00 & 5807 & 0.27 \\
\hline $\mathrm{D}$ & $\sim 0.7$ & $\sim 8200$ & 0.3 \\
\hline $\mathrm{E}$ & $0.40^{\mathrm{b}}$ & 10646 & -- \\
\hline $\mathrm{O}^{-}\left(\mathrm{D}_{2} \mathrm{O}\right)$ & & & \\
\hline $\mathrm{A}$ & 1.76 & 0.0 & 0.34 \\
\hline $\mathrm{B}$ & 1.41 & 2823 & 0.25 \\
\hline $\mathrm{C}$ & 1.15 & 4920 & 0.22 \\
\hline $\mathrm{D}$ & 0.925 & 6734 & 0.21 \\
\hline $\mathrm{E}$ & $0.297^{\mathrm{b}}$ & 11800 & -- \\
\hline
\end{tabular}

a) Position of peak center and full width at half-maximum (FWHM) as determined by fit to a Gaussianshaped peak. No consideration is made for superimposed vibrational structure.

b) Intensity severely affected by electron detector cutoff function.

\section{B. $A b$ initio Calculations}

For use in the analysis of the data, we have performed $a b$ initio calculations for the anion and neutral complexes involved in the photodetachment processes being studied. The calculations include geometry optimizations and potential energy curve calculations along selected coordinates. While not intended as state-of-the-art calculations for these systems, the results are to be used in simple model calculations to understand, qualitatively, the features observed in the photoelectron spectra. 


\section{Anion Calculations}

Several studies of the hydrogen bonding characteristics of the closed-shell $\mathrm{H}_{3} \mathrm{O}_{2}^{-}$ion have been carried out previously using $a b$ initio methods.9,54,55 However, in most of these studies, only partial geometry optimizations were performed with one or more fixed parameters. The full optimization by Rohlfing et al. ${ }^{9}$ at the MP2/6-31G** level of theory resulted in a complex with a linear, symmetric $\mathrm{O} \cdots \mathrm{H} \cdots \mathrm{O}$ arrangement within a non-planar overall $\mathrm{H}_{3} \mathrm{O}_{2}^{-}$geometry as shown in Fig. 5. From these results, it appears that the strong hydrogen bond which exists in the $\mathrm{H}_{3} \mathrm{O}_{2}^{-}$anion leads to a rather short $\mathrm{O}-\mathrm{O}$ distance $(\sim 2.4 \AA)$ with the hydrogen atom centered between the two oxygen atoms. No further optimizations of the $\mathrm{H}_{3} \mathrm{O}_{2}^{-}$geometry are pursued here.

As seen in Fig. 5, the dihedral angle of $\mathrm{H}_{3} \mathrm{O}_{2}^{-}$is calculated to be $110^{\circ}$. Since the hindered rotation/torsional motion is expected to be somewhat floppy, we have calculated the MP2/6-31++G** energy of the complex as a function of the dihedral angle with all of the other geometrical parameters fixed. The shape of the potential curve and the height of the 'trans' barrier ( $\sim 120 \mathrm{~cm}^{-1}$ at $\pi$ radians), shown in Fig. 6 , agrees with Spirko et al.'s $\mathrm{s}^{55}$ calculations using different fixed parameters, but the 'cis' barrier $\left(\sim 600 \mathrm{~cm}^{-1}\right.$ at 0 radians) is significantly larger than that obtained by Spirko $\left(-300 \mathrm{~cm}^{-1}\right)$. In their analysis, they find a zero point energy of 62 $\mathrm{cm}^{-1}$ for the hindered rotor motion. Thus, while the potential minima occur at dihedral angles

Figure 5: Ab initio calculated geometries for the $\mathrm{H}_{3} \mathrm{O}_{2}^{-}$and $\mathrm{O}^{-}\left(\mathrm{H}_{2} \mathrm{O}\right)$ anions. Values for $\mathrm{H}_{3} \mathrm{O}_{2}^{-}$are from Ref. 9. For $\mathrm{O}^{-}\left(\mathrm{H}_{2} \mathrm{O}\right)$, values 'A' are $M P 2 / 6-31+\mathrm{G}^{*}$ parameters from Ref. 56 and values 'B' are MP2/6-31++G** parameters determined in the present study. See text for details.

Figure 6: $A b$ initio potential energy curve for the $\mathrm{H}_{3} \mathrm{O}_{2}^{-}$hindered rotor motion at the MP2/6-31++G** level of theory. The dihedral angle is varied while the other geometrical parameters are fixed as: $\mathrm{R}(\mathrm{O} 1-\mathrm{O} 2)=2.4 \AA$; $(\mathrm{O} 1-\mathrm{H} 1)=\mathrm{R}(\mathrm{O} 2-\mathrm{H} 3)=$ $0.962 \AA ; \mathrm{R}(\mathrm{O} 1-\mathrm{H} 2)=\mathrm{R}(\mathrm{O} 2-\mathrm{H} 2)=1.2 \AA ; \mathrm{OHO}=180^{\circ} ; \mathrm{HOH}=104.5^{\circ}$. 

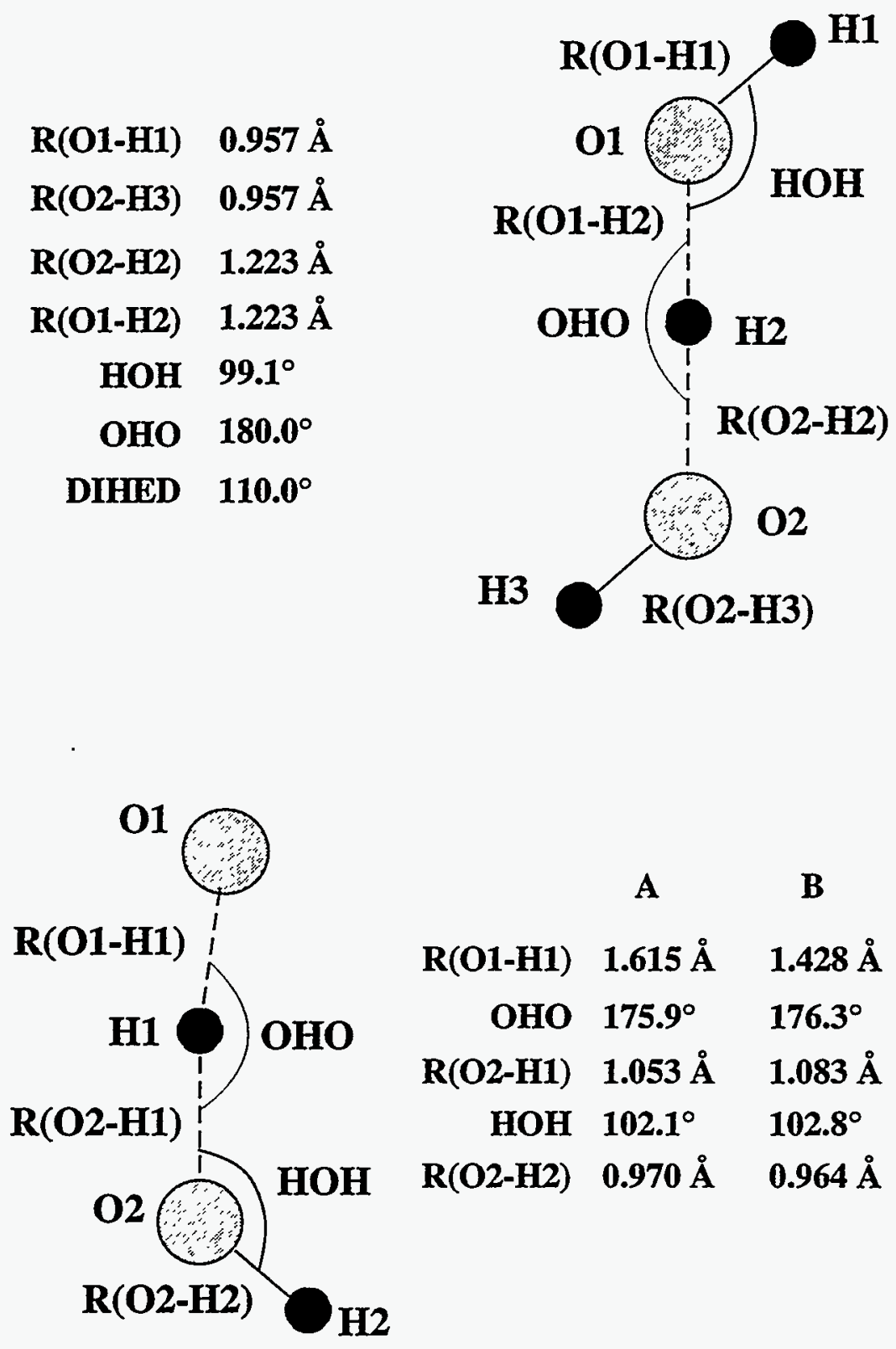

Figure 8.5 


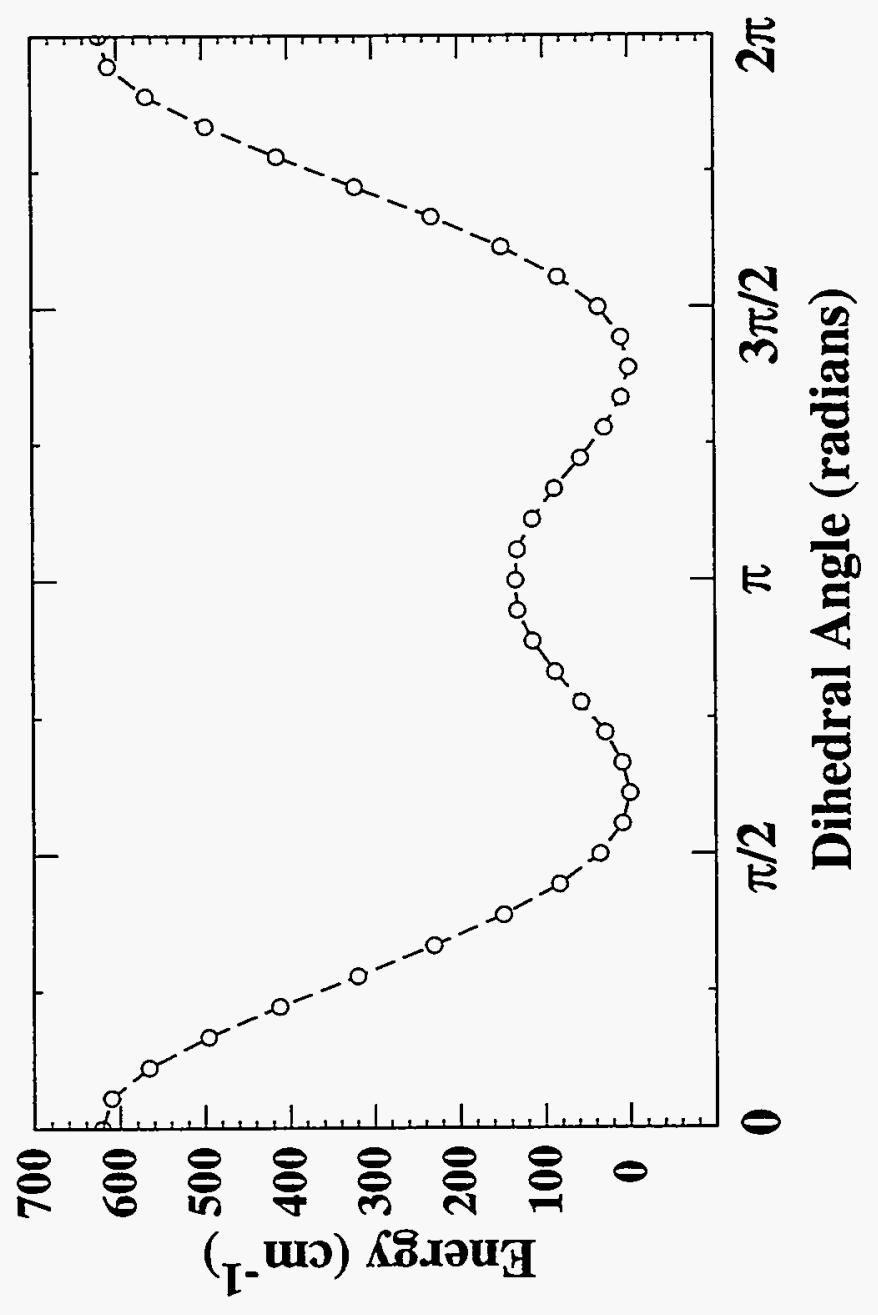

Figure 8.6 
of $110^{\circ}$ and $250^{\circ}$, the anion torsional motion is very floppy with an average dihedral angle of $180^{\circ}$.

With this in mind, potential energy curves for the antisymmetric $\mathrm{O} \cdots \mathrm{H} \cdots \mathrm{O}$ motion of trans $-\mathrm{H}_{3} \mathrm{O}_{2}^{-}$are calculated at the MP2/6-31++G** level of theory using a dihedral angle of $180^{\circ}$ (the 'trans' configuration). In addition to the dihedral angle, several other parameters are frozen to calculate the potential for this coordinate (see caption for Fig. 7). The central hydrogen atom, H2, was allowed to move between two oxygen atoms which were fixed at $\mathrm{R}(\mathrm{O} 1-\mathrm{O} 2)=$ $2.4 \AA$. The resultant potential energy curve (Fig. 7 , bottom) has very flat bottom with a minimum which occurs at the centrosymmetric nuclear configuration.

Fewer theoretical studies of the $\mathrm{O}^{-}\left(\mathrm{H}_{2} \mathrm{O}\right)$ anion have been made as a result of the more complicated open-shell interaction. Roehl et al. ${ }^{56}$ find that the $\mathrm{O}^{-}\left(\mathrm{H}_{2} \mathrm{O}\right)$ anion is most stable in a planar, 'quasilinear' configuration (Fig. 5) at the MP2/6-31+G* level of theory. The geometry of this quasilinear species is reoptimized here at the MP2/6-31++G** level of theory to include additional diffuse functions on the hydrogen atoms. The optimized parameters from both calculations are summarized in Fig. 5. In all of the calculations, the OHO angle is nearly linear while the $\mathrm{HOH}$ angle is slightly more acute than the angle found in $\mathrm{H}_{2} \mathrm{O}$. The extended $\mathrm{R}(\mathrm{O} 2-$ $\mathrm{H} 1$ ) bond length as compared to $\mathrm{R}(\mathrm{O} 2-\mathrm{H} 2)$ indicates that considerable hydrogen bonding occurs between the $\mathrm{O}^{-}$and the $\mathrm{H}_{2} \mathrm{O}$ but not as much as in the $\mathrm{H}_{3} \mathrm{O}_{2}^{-}$anion.

The antisymmetric $\mathrm{O}^{\cdots} \mathrm{H} \cdots \mathrm{O}$ motion of $\mathrm{O}^{-}\left(\mathrm{H}_{2} \mathrm{O}\right)$ is also investigated as in the case of $\mathrm{H}_{3} \mathrm{O}_{2}^{-}$. In this case, the potential energy curves are calculated at the QCISD/6-31++ $\mathrm{G}^{* *}$ level of theory. As for the $\mathrm{H}_{3} \mathrm{O}_{2}^{-}$calculations, several parameters are fixed. While the OHO bond angle is assumed to be linear for simplicity, the remainder of the frozen parameters are based

Figure 7: Ab initio calculated potential energy curves for $\mathrm{H}_{3} \mathrm{O}_{2}^{-}$and $\mathrm{HOHOH}$ along the central hydrogen atom antisymmetric stretch coordinate. The $\mathrm{H}$ atom position is varied while the other parameters are fixed as: $R(\mathrm{O} 1-\mathrm{O} 2)=2.4 \AA ; \mathrm{R}(\mathrm{O} 1-\mathrm{H} 1)=$ $\mathrm{R}(\mathrm{O} 2-\mathrm{H} 3)=0.962 \AA ; \mathrm{OHO}=180^{\circ} ; \mathrm{HOH}=99.1^{\circ} ;$ dihedral $=180^{\circ}$. 


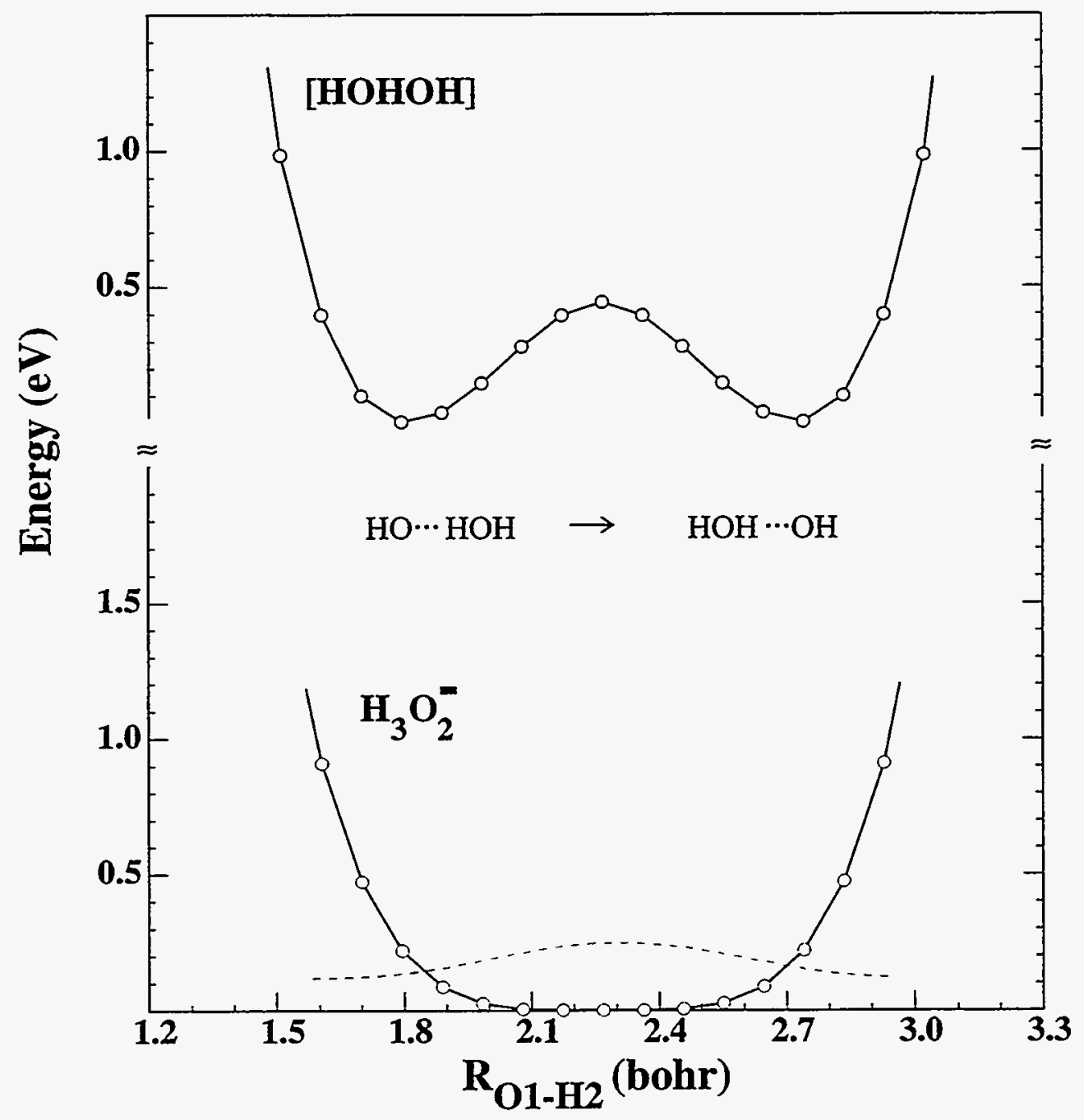

Figure 8.7 
upon the $a b$ initio results and are given in caption for Fig. 8. Along the constrained $\mathrm{O} \cdots \mathrm{H} \cdots \mathrm{O}$ coordinate, the potential energy curve for the ${ }^{2} \mathrm{~A}$ " ground electronic state (Fig. 8, bottom) has a single minimum which occurs at the equilibrium geometry. However, there is a shelf in the potential, corresponding to the $\mathrm{OH}^{-}-\mathrm{OH}$ geometry, which is $<0.1 \mathrm{eV}$ above the minimum in the curve. It is important to note that this is not a calculation of the minimum energy path (MEP) between $\mathrm{OH}^{-}+\mathrm{OH}$ and $\mathrm{O}^{-}+\mathrm{H}_{2} \mathrm{O}$. Along the MEP, changes in the $\mathrm{O}-\mathrm{O}$ distance probably accompany changes in the $\mathrm{H}$-atom position. Therefore, the calculated potential energy curve does not provide any information about whether there is a single minimum or a double minimum along the MEP.

\section{Neutral Calculations}

The potential energy curves calculated in the preceeding section determine the FranckCondon (FC) region for anion photodetachment along the $\mathrm{O} \cdots \mathrm{H} \cdots \mathrm{O}$ coordinate. For use in FC calculations to be presented below, potential energy curves for the antisymmetric motion of the neutral $[\mathrm{HOHOH}]^{\ddagger}$ and $[\mathrm{HOHO}]^{\ddagger}$ complexes are calculated along the same coordinates and using the same fixed parameters which are employed in the anion calculations. The neutral curves are calculated at the same levels of theory as used for the anions. The calculated curves, shown in Figs. 7 and 8 respectively, both contain double minima. Note that these do not correspond to minima on the $\mathrm{O}+\mathrm{H}_{2} \mathrm{O}$ and $\mathrm{OH}+\mathrm{H}_{2} \mathrm{O}$ potential surfaces. Rather, they are one-dimensional (1D) slices through the multidimensional reaction surfaces and the 1D minima correspond to slices through the valleys in the higher dimensional potential surfaces which lead to the reactant and product asymptotes.

Figure 8: Ab initio calculated potential energy curves for $\mathrm{O}^{-}\left(\mathrm{H}_{2} \mathrm{O}\right)$ and $\mathrm{HOHO}$ along the central $\mathrm{H}$ atom antisymmetric stretch vibrational coordinate. The $\mathrm{H}$ atom position is varied while the other geometrical parameters are fixed as: $\mathrm{R}(\mathrm{O} 1-\mathrm{O} 2)=2.5 \AA$; $\mathrm{R}(\mathrm{O} 2-\mathrm{H} 3)=0.962 \AA ; \mathrm{OHO}=180^{\circ} ; \mathrm{HOH}=102.8^{\circ}$. 


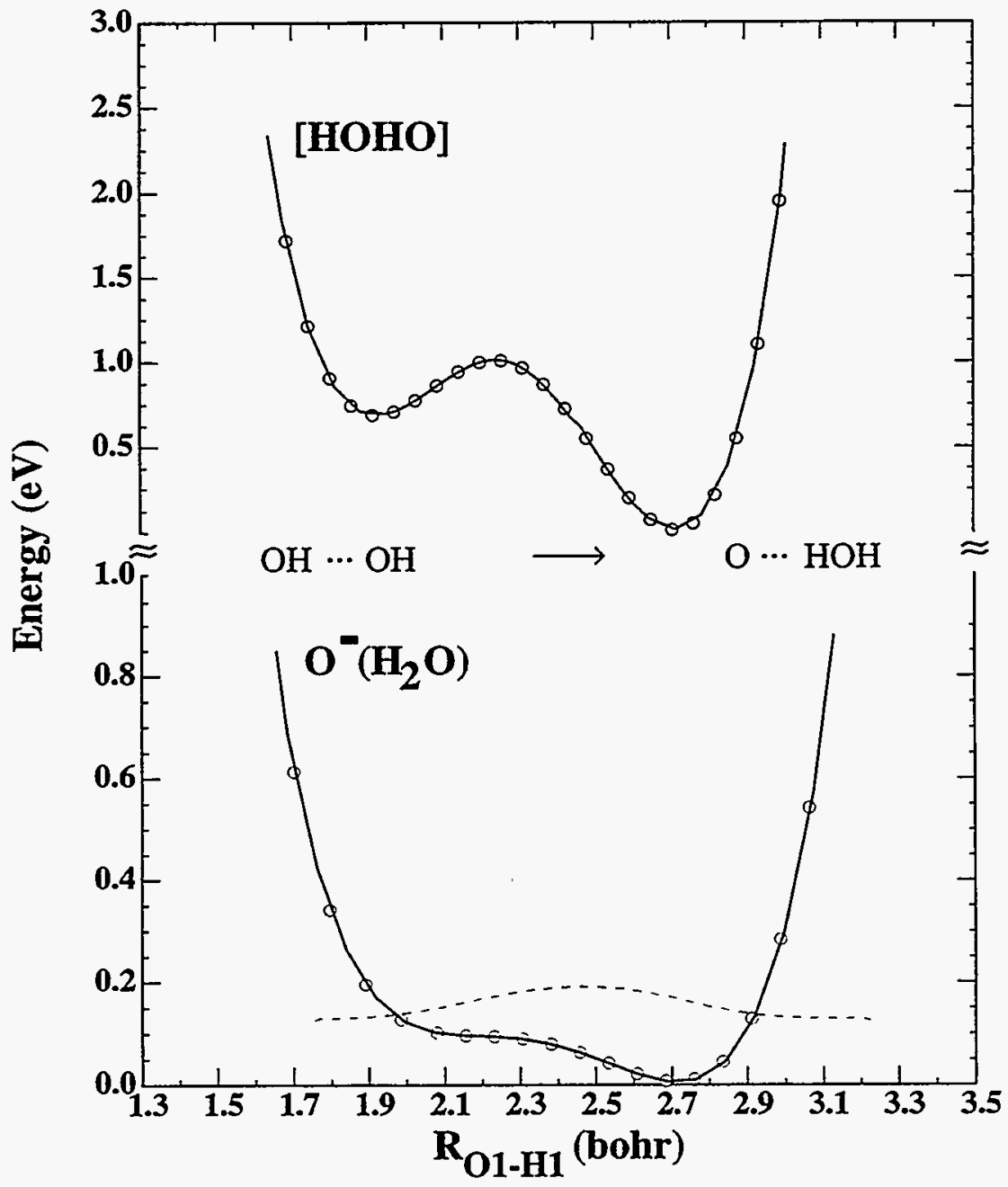

Figure 8.8 
The $\mathrm{H}_{3} \mathrm{O}_{2}^{-}$anion is a closed-shell species with the $\left(. .\left(6 \mathrm{a}^{\prime}\right)^{2}\left(7 \mathrm{a}^{\prime}\right)^{2}\left(1 \mathrm{a}^{\prime \prime}\right)^{2}\left(8 \mathrm{a}^{\prime}\right)^{2}\left(2 \mathrm{a}^{\prime \prime}\right)^{2}\right)$ orbital occupation. Photodetachment of the anion can lead to either a ${ }^{2} \mathrm{~A}$ ' or a ${ }^{2} \mathrm{~A}$ " state, both of which correlate to the ground state $\mathrm{OH}+\mathrm{H}_{2} \mathrm{O}$ dissociation products of the neutral $[\mathrm{HOHOH}]^{\ddagger}$ complex. The surface shown in Figure 7 is the ${ }^{2} \mathrm{~A}^{\prime}$ surface which is calculated to be lowest in energy at the MP2/6-31++G** level of theory in the Franck-Condon region. At the minimum energy anion geometry used in the potential curve calculations, the ${ }^{2} \mathrm{~A}$ ' state is predicted to lie $0.58 \mathrm{eV}$ below the ${ }^{2} \mathrm{~A}^{\prime \prime}$ state at the MP2/6-31++G** level of theory. 57 The barrier to symmetric hydrogen exchange along this restricted $0 \cdots \mathrm{H}^{\cdots} \mathrm{O}$ coordinate is $0.50 \mathrm{eV}$ at the MP2/6$31++G^{* *}$ level of theory.

For $\mathrm{OH}+\mathrm{OH}$, the interaction of two ground state hydroxyl radicals splits the $\mathrm{OH}\left({ }^{2} \Pi\right)$ states into four singlet states and four triplet states. The hydroxyl radical disproportionation, reaction (2), can occur adiabatically on three of the four triplet states along a $C_{s}$ planar reaction path. ${ }^{28}$ Based upon the orbital occupation calculated for the $\mathrm{O}^{-}\left(\mathrm{H}_{2} \mathrm{O}\right)$ anion $\left(. .\left(6 a^{\prime}\right)^{2}\left(1 a^{\prime \prime}\right)^{2}\left(7 a^{\prime}\right)^{2}\left(8 a^{\prime}\right)^{2}\left(2 a^{\prime \prime}\right)^{1}\right)$, one-electron photodetachment can form the ${ }^{1} A^{\prime},{ }^{1} A^{\prime \prime}$ or ${ }^{3} A^{\prime \prime}$ species. Of these possibilities, the lowest energy species at the geometry of the anion is the ${ }^{3} \mathrm{~A} "$ state. It is the ${ }^{3} \mathrm{~A}$ " state on which the $\mathrm{OH}+\mathrm{OH}$ disproportionation is most likely to produce $\mathrm{O}\left({ }^{3} \mathrm{P}\right)+\mathrm{H}_{2} \mathrm{O} .21,22 \mathrm{~A}{ }^{3} \mathrm{~A}^{\prime}$ state is calculated to lie $\sim 2.4 \mathrm{kcal} / \mathrm{mole}^{22}$ above the ${ }^{3} \mathrm{~A}^{\prime \prime}$ state at the saddle point but since this electronic state has unpaired electrons in two different a' orbitals it is not accessible by one-electron photodetachment of the $\mathrm{O}^{-}\left(\mathrm{H}_{2} \mathrm{O}\right)$ anion. Therefore, we have calculated the potential energy curve for the ${ }^{3} \mathrm{~A}$ " state which is shown in Fig. 8. Along the restrained $\mathrm{O} \cdots \mathrm{H} \cdots \mathrm{O}$ coordinate, the $1 \mathrm{D}$ minima are separated by $0.690 \mathrm{eV}$ with a $0.322 \mathrm{eV}$ barrier with respect to the $\mathrm{OH}-\mathrm{OH}$ 'reactant valley well'.

While two hydroxyl radicals can also react to form hydrogen peroxide, no information about this reaction can be obtained from this data due to poor Franck-Condon overlap between the anion and the species involved in the reaction. The peroxide formation occurs on the higher-lying singlet reaction surface, which correlates to $\mathrm{O}\left({ }^{1} \mathrm{D}\right)+\mathrm{H}_{2} \mathrm{O} .58$ It is possible that the 
peak $\mathrm{E}$ in the $\mathrm{O}^{-}\left(\mathrm{H}_{2} \mathrm{O}\right)$ anion photoelectron spectrum marks the onset of transitions to hydrogen bonded region of the singlet surface but further studies at higher photodetachment are necessary to determine the actual identity of this feature.

As a comparison to the anion geometries, the geometry of the neutral complexes at the saddle point of the neutral reaction surface is also of interest. The transition state structure of the $\mathrm{OH}+\mathrm{H}_{2} \mathrm{O}$ reaction has been investigated in detail by Nanayakkara et. al. ${ }^{14}$ Their best calculated transition state geometry (Fig. 9) lies on a barrier estimated to be $0.46 \mathrm{eV}$ above the separated products. No further investigation is made of this species.

For the $\mathrm{OH}+\mathrm{OH}$ reaction, the ${ }^{3} \mathrm{~A}$ " saddle point species has been located previously at the $\mathrm{SCF}^{21}$ and $\mathrm{MCSCF}^{22}$ level of theory. We have located the stationary point at the MP2/6$31++G^{* *}$ and QCISD/6-31++G** levels of theory. The calculated geometry for the $[\mathrm{HOHO}]^{\ddagger}$ structure is also shown in Fig. 9.

\section{Analysis and Discussion}

\section{A. Initial Considerations}

The appearance of the photoelectron spectra are primarily determined by the FranckCondon (FC) overlap between the bound anion ground state wave function and the scattering wave functions on the $\mathrm{OH}+\mathrm{HX} \rightarrow \mathrm{X}+\mathrm{H}_{2} \mathrm{O}(\mathrm{X}=\mathrm{OH}, \mathrm{O})$ reaction surfaces. Thus, the success of negative ion photoelectron spectroscopy as a probe of reaction dynamics in the transition state region is contingent upon having significant Franck-Condon overlap between the anion ground state and the transition state region of the neutral reaction surface. It is therefore useful to compare the calculated geometries for the anions and neutral transition state species. 
Both of the anions used in this study have significant hydrogen bonding character. The calculated equilibrium geometries for these anion species (Fig. 5) indicate that the central hydrogen atom interacts significantly with both oxygen atoms. The very flat antisymmetric stretch potential for both anions (Fig. 7 and 8) results in an extended hydrogen atom motion and a large FC region for the photodetachment process. Based upon the classical turning points for the $v^{\prime}=0$ level of the calculated one-dimensional potential curves, the $\mathrm{R}(\mathrm{O} 1-\mathrm{H} 2)$ distance varies from $0.99 \AA$ to $1.42 \AA$ for $\mathrm{H}_{3} \mathrm{O}_{2}^{-}$and $1.06 \AA$ to $1.56 \AA$ for $\mathrm{O}^{-}\left(\mathrm{H}_{2} \mathrm{O}\right)$.

Comparison of the anion geometries (Fig. 5) to the calculated transition state structures (Fig. 9), finds reasonable agreement of the general anion and neutral structures. The symmetric position of the $\mathrm{H}$ atom in the $[\mathrm{HOHOH}]$ transition state structure is analogous to that of the $\mathrm{H}_{3} \mathrm{O}_{2}^{-}$anion. The $\mathrm{O}-\mathrm{O}$ separation in the transition state species is shorter than that of the ion in both cases. However, as Figs. 7 and 8 show, the flat potential curves for the anions along the antisymmetric $\mathrm{H}$-atom motion still results in significant overlap with both the reactant and product valleys. This means that the FC region will be centered slightly away from the saddle point toward the entrance and exit channels but it is still clearly in the transition state region. This is analogous to several of the $\mathrm{XHX}^{-}$systems studied previously in this laboratory..$^{1,2}$

The major differences between the anion and transition state structures occurs in the OHO bond angles. However, as for the hindered rotor motion described in Sec. III B, the bending motion involving the central $\mathrm{H}$ atom is not expected to be very rigid and will also have a reasonably large zero point motion. In the case of $\mathrm{H}_{3} \mathrm{O}_{2}^{-}$, the dihedral angle is very different between the two species but is not expected to be strongly coupled to the reaction coordinate.

Figure 9: $A b$ initio calculated transition state geometries for the $\mathrm{OH}+\mathrm{H}_{2} \mathrm{O} \rightarrow \mathrm{H}_{2} \mathrm{O}+\mathrm{OH}$ and $\mathrm{OH}+\mathrm{OH} \rightarrow \mathrm{O}+\mathrm{H}_{2} \mathrm{O}$ reactions. Parameters for $[\mathrm{HOHOH}]^{\ddagger}$ are from Ref. 14. For $[\mathrm{HOHO}]^{\ddagger}$, 'A' parameters are UHF/4-31G values from Ref. 21 , 'B' parameters are MCSCF/DZP values from Ref. 23 and 'C' parameters are MP2/6$31++G^{* *}$ values are from the present work. See text for details. 

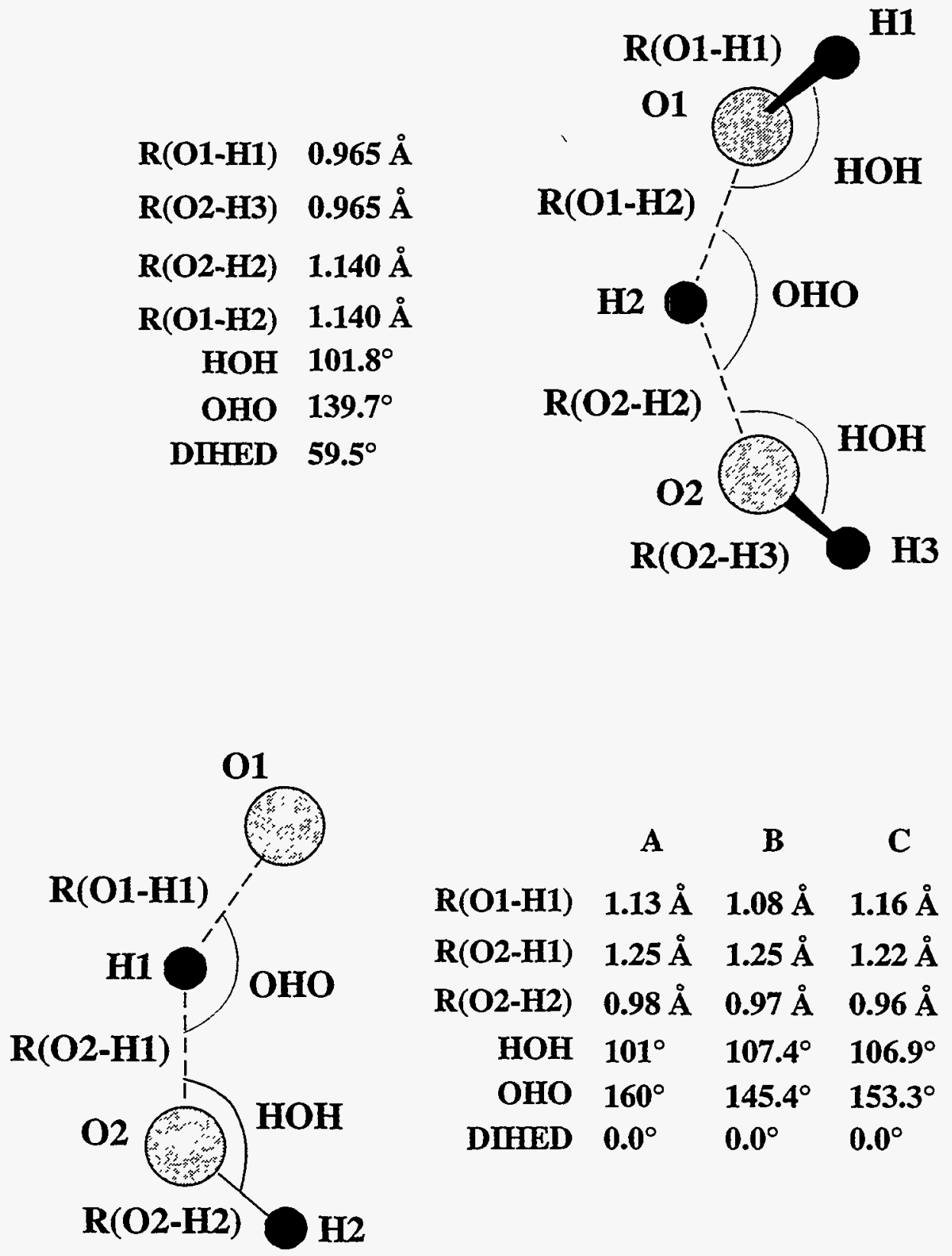

Figure 8.9 
The photodetachment transition from the 'linear' anion to the neutral surface with a bent transition state will most likely produce a 'torqued' neutral complex which will dissociate into rotationally excited dissociation products. The conclusion to be drawn from these rough comparisons is that based upon the ab initio results, photodetachment of the $\mathrm{H}_{3} \mathrm{O}_{2}^{-}$and $\mathrm{O}^{-}\left(\mathrm{H}_{2} \mathrm{O}\right)$ anions will provide information about the transition state regions of reactions (1) and (2).

To assess the relationship of the features observed in the photoelectron spectra to the dynamics which occur at the transition state of reactions (1) and (2), several factors must be considered. A good starting point is the general appearance of the data. All of the data consist of several very broad features (FWHM $\sim 0.2 \mathrm{eV}$ or greater) which are irregularly spaced in energy. The position of the features above the dissociation asymptote suggests that they do not provide information about the portion of the potential energy surface where the van der Waals minima are predicted to exist. ${ }^{12}$ By forming the anions in a molecular beam expansion, we produce anions which are primarily in their ground vibrational state. Thus, the spacings observed between features in the photoelectron spectrum will be representative of the vibrational motions of the unstable neutral complex. These vibrations must correspond to motions which are approximately perpendicular to the reaction coordinate and have vibrational periods which are on a time scale shorter than that of the dissociation process. Otherwise, only a continuum feature would be observed. In the $\mathrm{OH}+\mathrm{H}_{2} \mathrm{O}$ and $\mathrm{OH}+\mathrm{OH}$ systems, the reaction coordinate is described, to a good approximation, by the $\mathrm{O}-\mathrm{O}$ separation.

As mentioned in Sec. II A, the isotopic dependence shows that the vibrations primarily involve hydrogen atom motion. While the observed peak spacings are quite irregular, it is useful to compare them to the observed vibrational frequencies of the 'component' $\mathrm{OH}\left(\omega_{\mathrm{e}}=\right.$ $\left.3735 \mathrm{~cm}^{-1}\right)^{59}$ and $\mathrm{H}_{2} \mathrm{O}\left(\mathrm{v}_{1}=3657 \mathrm{~cm}^{-1} ; \mathrm{v}_{2}=1595 \mathrm{~cm}^{-1} ; \mathrm{v}_{3}=3756 \mathrm{~cm}^{-1}\right)^{60}$ molecules. The observed A-B spacing in the $\mathrm{H}_{3} \mathrm{O}_{2}^{-}$spectrum $\left(\sim 2800 \mathrm{~cm}^{-1}\right)$ does not match well with any of the 'component' frequencies. The B-C spacing is significantly smaller $\left(-1500 \mathrm{~cm}^{-1}\right)$. In the case of the $\mathrm{O}^{-}\left(\mathrm{H}_{2} \mathrm{O}\right)$ data, the A-B spacing $\left(-3600 \mathrm{~cm}^{-1}\right)$ is near the $\mathrm{OH}$ stretching frequencies of the 
$\mathrm{OH}$ radical and the $\mathrm{H}_{2} \mathrm{O}$ molecule. However, as for the $\mathrm{H}_{3} \mathrm{O}_{2}^{-}$spectrum, the $\mathrm{B}-\mathrm{C}$ spacing is significantly smaller $\left(2300 \mathrm{~cm}^{-1}\right)$ and does not match any of the 'component' frequencies. The significantly lower vibrational frequencies observed for the neutral complexes relative to those of the component molecules indicates that the central hydrogen atom interacts significantly with both of the oxygen atoms in the neutral complexes.

This significant perturbation of vibrational frequencies strongly suggests that the experiments are, in fact, probing the transition state region for reactions (1) and (2). Similar effects were observed for several $\mathrm{XHY}^{-}$systems studied previously in this laboratory. ${ }^{1,2}$ This is consistent with the shapes of the calculated potential energy curves for the $[\mathrm{HOHO}]$ and [HOHOH] complexes. The double minima potentials will support irregularly spaced eigenvalues with complex eigenfunctions. The effects on the FC profile will be considered in detail in the following section.

The observed peak widths are at least an order of magnitude greater than the experimental resolution. This observation is similar to that observed in previous transition state spectra of heavy-light-heavy anions. Analysis of the other transition state spectra using accurate multi-dimensional quantum mechanical techniques have shown that observed peak widths in those data result from both homogeneous and inhomogeneous contributions. Additionally, it was shown that in those spectra the widths of adjacent peaks could result from completely different dynamical effects. For the $\mathrm{O}^{-}\left(\mathrm{H}_{2} \mathrm{O}\right)$ and $\mathrm{H}_{3} \mathrm{O}_{2}^{-}$spectra, it is unclear whether lifetime effects or unresolved transitions are primarily responsible for the peak widths. However, based upon the calculated geometries for the anions and the neutral transition state species, we expect a significant amount of bend/hindered rotor excitation of the neutral upon anion photodetachment. These motions, which correlate to product rotational states, are most likely responsible for the fine structure observed on the $v=0$ features in the data. Similar effects have been observed in the analysis of the $\mathrm{FH}_{2}^{-}$and $\mathrm{OHCl}^{-}$photoelectron spectra. ${ }^{4,61}$ The actual contributions to these peak widths must be determined through a combination of efforts 
which include higher resolution experimental measurements using anion zero electron kinetic energy (ZEKE) spectroscopy62 and multi-dimensional calculations which can model neutral bending motions and peak broadening which occurs as a result of short lifetime effects.

As a more quantitative test of these assertions, a Franck-Condon analysis can be compared with the data. A full, accurate calculation of the FC overlap for comparison to the experimental data requires an accurate determination of the anion geometry and an accurate potential energy surface for the neutral reaction. As shown recently for the $\mathrm{F}+\mathrm{H}_{2} \rightarrow \mathrm{H}+\mathrm{HF}$ reaction, such a calculation is a very difficult, but achievable, task for $a b$ initio theory. ${ }^{4}$ However, a full calculation of this sort is beyond the scope of the data analysis to be presented here, and simpler approach will be considered. We have shown previously that analysis within a reduced dimensionality model can provide valuable and insightful information about the photoelectron spectra. ${ }^{1,2,3}$ In heavy-light-heavy triatomic systems studied previously, simple treatments of the data employed one-dimensional (1D) and two-dimensional (2D) slices from semi-empirical potential energy surfaces along coordinates which could possibly be active in the photoelectron spectrum. These simple analyses revealed quite clearly that the major features in the photoelectron spectra of other heavy-light-heavy systems could be assigned to the antisymmetric hydrogen atom motion in the neutral transition state complex. However, such semi-empirical potential energy surfaces have not been constructed for reactions (1) and (2) yet. As an alternative approach, we will use the one-dimensional ab initio potential energy curves along the antisymmetric hydrogen stretch coordinate for the anion and neutral (Sec. III B). These potential energy curves can then be used to calculate one-dimensional stick spectra which may be compared to the data.

\section{B. Franck-Condon Simulations}

The polynomial functions determined from least-squares fit to the $a b$ initio data points for both the anion and the neutral complexes are used to determine 1D Franck-Condon factors 
(FCFs) along the approximate hydrogen atom antisymmetric stretch vibrational coordinate. In this model, both the anion and neutral potential functions support a set of bound quantum states such that, within the FC approximation, the intensity of a photodetachment transition is given by

$$
I \propto v_{e} \cdot\left|\tau_{e}\right|^{2} \cdot\left|\left\langle\psi_{v^{\prime}}\left(Q_{u s}\right) \mid \psi_{v^{m}}\left(Q_{a s}\right)\right\rangle\right|^{2}
$$

In Eq. (4), $\Psi_{v^{\prime \prime}}$ and $\Psi_{v^{\prime}}$ are the vibrational wave functions of the anion and neutral, respectively, along the hydrogen atom antisymmetric stretch vibrational coordinate, $Q_{a s}$, and $\mathrm{v}_{\mathrm{e}}$ is the asymptotic velocity of the photodetached electron. In the simulations, the electronic transition dipole, $\tau_{\mathrm{e}}$, is assumed to be constant as a function of eKE. For each polynomial, eigenvalues and eigenfunctions are determined numerically by standard matrix methods. ${ }^{63}$ The eigenfunctions are used to determine the Franck-Condon factors by numerical integration. An appropriate change in the reduced mass allows calculation of FCF's for the deuterated analogs using the same potential energy curves. In the figures shown below, the stick spectra will be compared directly with the data. In addition, the stick spectra will be convoluted with the experimental resolution function plus an additional Gaussian with FWHM $=200 \mathrm{meV}$ for comparison to the broad features observed in the experimental data.

Shown in Fig. 10 are the results of the $\mathrm{H}_{3} \mathrm{O}_{2}^{-}$and $\mathrm{D}_{3} \mathrm{O}_{2}^{-}$simulations calculated by the above method using the anion and potential energy curves calculated at MP2/6-31++ $\mathrm{G}^{* *}$ level of theory. The simulations are superimposed upon the experimental data for comparison of peak spacings, intensities and isotope dependence. Since the simulations assume that all of the

Figure 10: Experimental data (dotted) and Franck-Condon simulations (solid) for $\mathrm{H}_{3} \mathrm{O}_{2}^{-}$ and $\mathrm{D}_{3} \mathrm{O}_{2}^{-}$. Franck-Condon factors are calculated using the ab initio surfaces shown in Figure 7. 


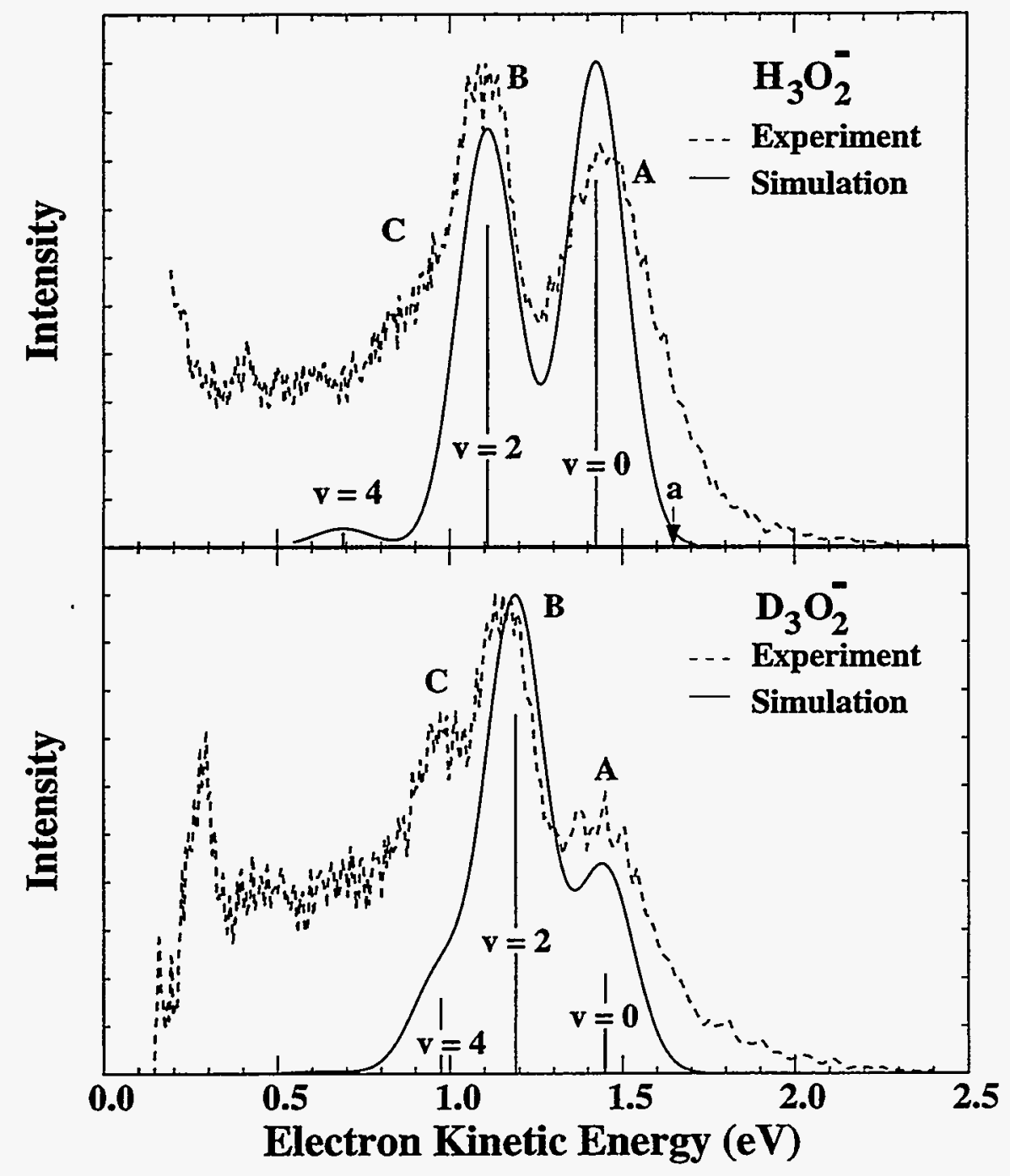

Figure 8.10 
anions are in their ground vibrational state, the peaks spacings in the simulations are indicative of the energy levels which are supported by the neutral potential energy surfaces. Fixing the terminal $\mathrm{OH}$ bond lengths at equal values imposes a symmetry about the center of the potential energy curves. Due to this symmetry, only transitions to even states of the neutral have nonzero intensity. Thus, the features in the simulation represent photodetachment transitions to the $v^{\prime}=0, v^{\prime}=2$ and $v^{\prime}=4$ vibrational states supported by the neutral potential energy curve. In both the $\mathrm{H}_{3} \mathrm{O}_{2}^{-}$and $\mathrm{D}_{3} \mathrm{O}_{2}^{-}$spectra, the observed peak spacings are reasonably well reproduced by the simulations. Although the intensity of peak $\mathrm{C}$ is underestimated in both simulations, the observed change in peak intensities that result from isotopic substitution is very well modeled. The positions of these peaks, their relative integrated intensities and their assignments are given in Table III.

Table III: Simulated Peak positions for the $\mathrm{H}_{3} \mathrm{O}_{2}^{-}$and $\mathrm{D}_{3} \mathrm{O}_{2}^{-}$spectra

\begin{tabular}{|c|c|c|c|c|}
\hline \multirow{2}{*}{$\mathrm{v}^{\prime}$} & \multicolumn{2}{|c|}{$\mathrm{H}$} & \multicolumn{2}{c|}{$\mathrm{D}$} \\
\cline { 2 - 5 } & Energy & Intensity & Energy & Intensity \\
\hline \hline 0 & 0.0 & 1.0 & 0.0 & 0.25 \\
\hline 1 & 524.2 & 0.0 & 125.0 & 0.0 \\
\hline 2 & 3712.5 & 0. & 2547.9 & 1.0 \\
\hline 3 & 6432.2 & 0.0 & 3819.0 & 0.0 \\
\hline 4 & 9954.4 & 0.03 & 5922.5 & 0.23 \\
\hline
\end{tabular}

Shown in Fig. 11 are the results of the $\mathrm{O}^{-}\left(\mathrm{H}_{2} \mathrm{O}\right)$ and $\mathrm{O}^{-}\left(\mathrm{D}_{2} \mathrm{O}\right)$ simulations. In general, there is very good agreement between the convoluted 1D simulations and the photoelectron spectra, particularly for the $\mathrm{O}^{-}\left(\mathrm{H}_{2} \mathrm{O}\right)$ spectrum. Note that since there is no symmetry in these potentials, transitions to all of the neutral vibrational levels are allowed, as indicated. As might

Figure 11: Experimental data (dotted) and Franck-Condon simulations (solid) for $\mathrm{O}^{-}\left(\mathrm{H}_{2} \mathrm{O}\right)$ and $\mathrm{O}^{-}\left(\mathrm{D}_{2} \mathrm{O}\right)$. Franck-Condon factors are calculated using the ab initio surfaces shown in Figure 8. 


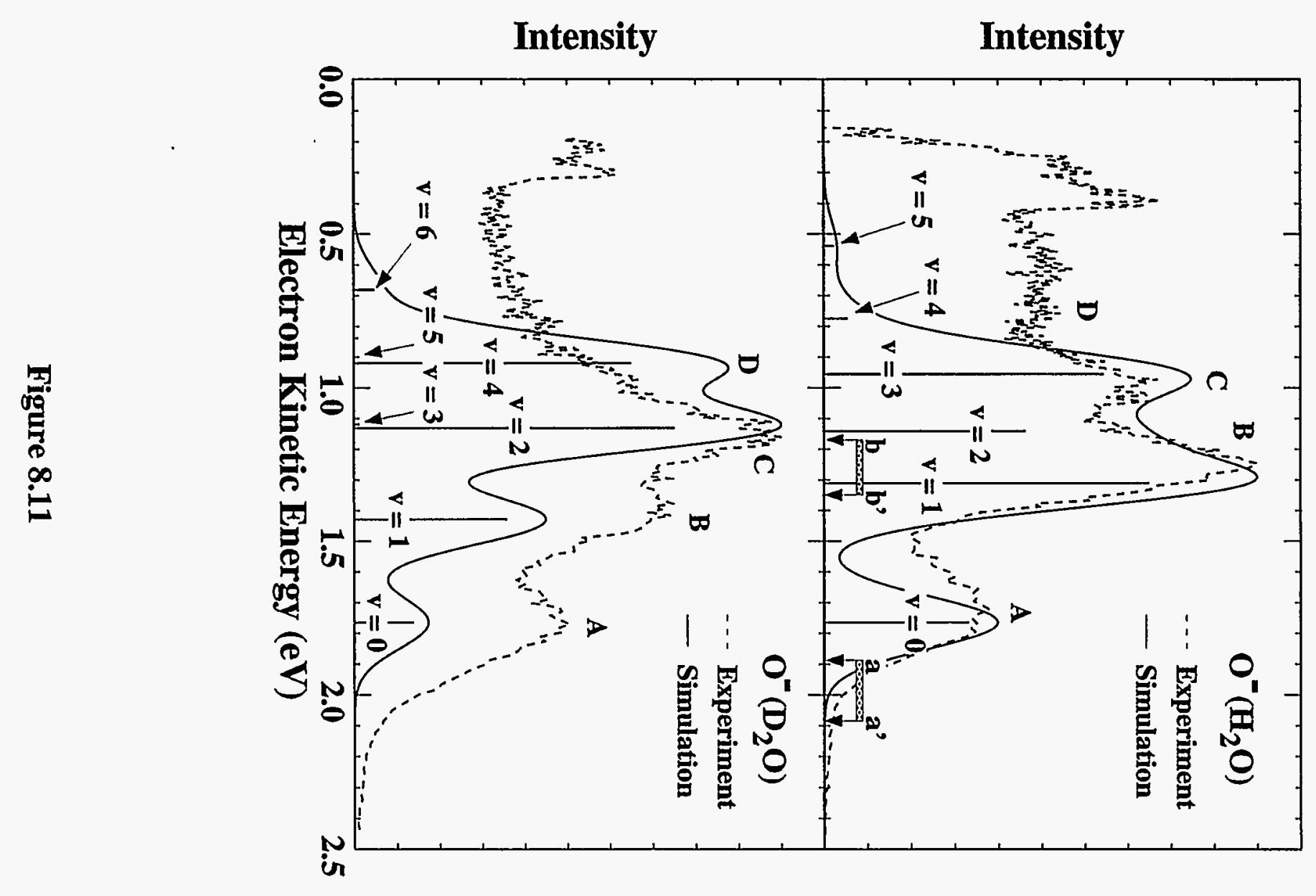


be expected, the peak positions change upon isotopic substitution. The peak shifts are not uniform, however. Inspection of the simulated peak positions given in Table IV, shows that the $v^{\prime}=1$ and the $v^{\prime}=3$ transitions shift by 963 and $1315 \mathrm{~cm}^{-1}$, respectively, to higher eKE while the $v^{\prime}=2$ peak shifts $85 \mathrm{~cm}^{-1}$ to lower eKE.. Although the length of the $\mathrm{O}^{-}\left(\mathrm{D}_{2} \mathrm{O}\right)$ progression is overestimated, the simulations also reproduce the change of relative intensities upon isotopic substitution. However, the intensity pattern is very irregular. This is particularly true for the $\mathrm{O}^{-}\left(\mathrm{D}_{2} \mathrm{O}\right)$ simulation where the $\mathrm{v}^{\prime}=2$ feature is the dominant transitions while $\mathrm{v}^{\prime}=3$ has almost no intensity. A similar situation occurs for the $v^{\prime}=4 / v^{\prime}=5$ pair.

Table IV: Simulated Peak positions for the $\mathrm{O}^{-}\left(\mathrm{H}_{2} \mathrm{O}\right)$ and $\mathrm{O}^{-}\left(\mathrm{D}_{2} \mathrm{O}\right)$ spectra

\begin{tabular}{|c|c|c|c|c|}
\hline \multirow{2}{*}{$\mathbf{v}^{\prime}$} & \multicolumn{2}{|c|}{$\mathrm{c}$} & \multicolumn{2}{c|}{$\mathrm{D}$} \\
\cline { 2 - 5 } & Energy & Intensity & Energy & Intensity \\
\hline \hline 0 & 0.0 & 0.3419 & 0.0 & 0.15 \\
\hline 1 & 3664 & 1.0 & 2701 & 0.42 \\
\hline 2 & 5032 & 0.0042 & 5117 & 1.0 \\
\hline 3 & 6527 & 0.1807 & 5212 & 0.02 \\
\hline 4 & 7989 & 0.0007 & 6814 & 0.96 \\
\hline 5 & - & -- & 7480 & 0.02 \\
\hline 6 & - & - & 8746 & 0.08 \\
\hline
\end{tabular}

In order to understand the significant changes in peak spacings and intensities that occur upon isotopic substitution, it is useful to study the wave functions that are supported by the $1 \mathrm{D}$ potential energy curves calculated for the restricted $\mathrm{O} \cdots \mathrm{H} \cdots \mathrm{O}$ motion of the neutral complexes. As shown in Fig. 7, the $\mathrm{H}_{3} \mathrm{O}_{2}^{-}$anion ground state wave function has the greatest overlap at the barrier of the symmetric neutral potential. In Fig. 12, the calculated neutral potentials for the $[\mathrm{HOHOH}]^{\ddagger}$ and $[\mathrm{DODOD}]^{\frac{1}{*}}$ complexes are shown again with their corresponding eigenvalues and eigenfunctions. The barrier in the neutral potential curve occurs near the first and second vibrational levels supported by the surface. This figure illustrates why

Figure 12: The MP2/6-31++G** neutral potential energy curves for the HOHOH complex (as in Figure 7) with the associated eigenfunctions and eigenvalues. 


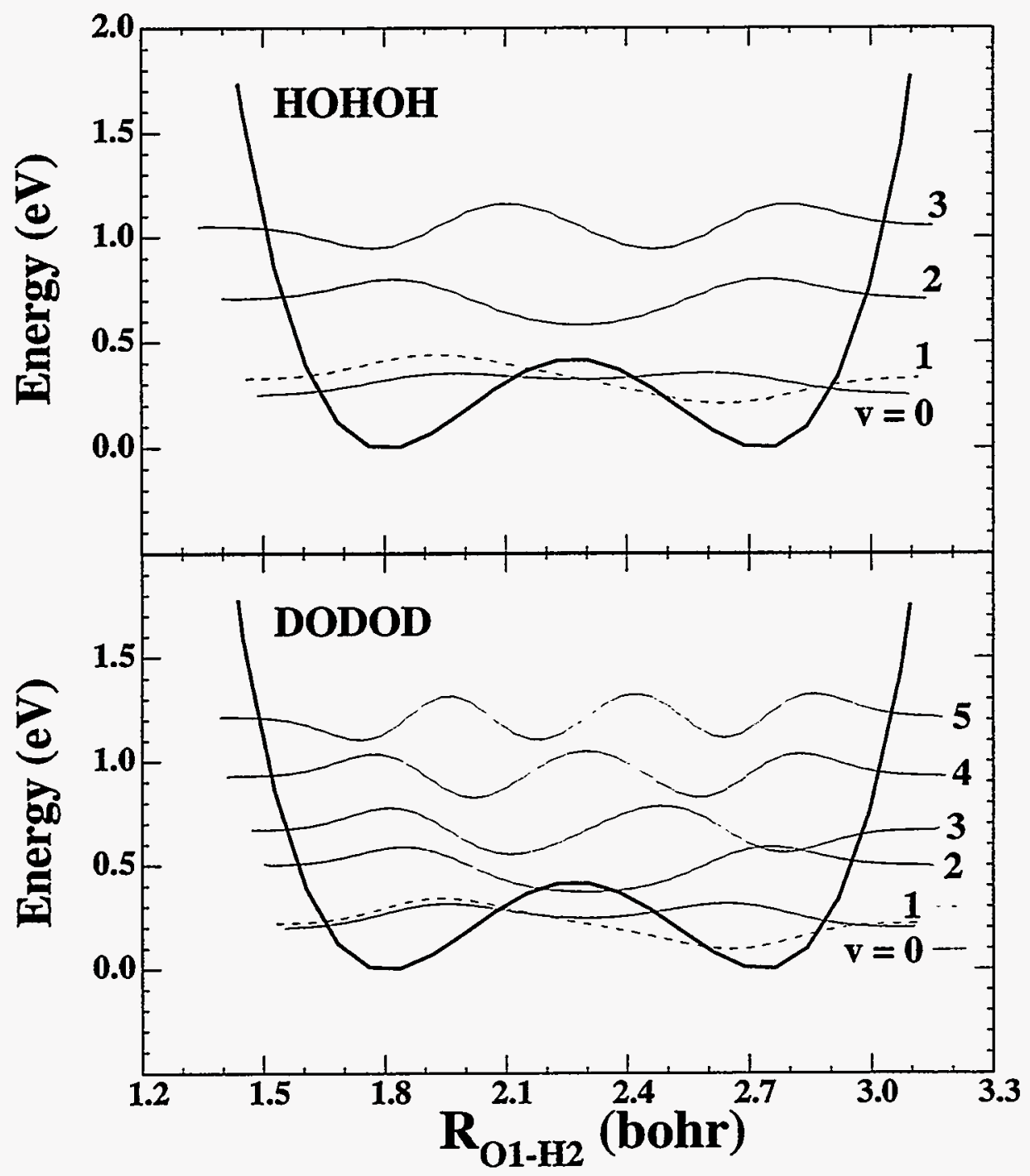

Figure 8.12 
the relative intensity of the $v^{\prime}=0$ level is decreased upon deuteration. The wave function of the deuterated species, which has a lower zero-point energy, is localized away from the center of the potential. Since this is where the anion wave function has its greatest amplitude, the FC overlap for $v^{\prime}=0$ is diminished with respect to the $v^{\prime}=2$ level which is located above the barrier and has considerable amplitude in the center of the potential curve. This effect has also been observed in other $\mathrm{XHX}^{-}$photoelectron spectra.

The $\mathrm{O}^{-}\left(\mathrm{H}_{2} \mathrm{O}\right)$ anion ground state wave function (Fig. 8), while overlapping both the reactant and product wells, has its greatest amplitude at a geometry which corresponds to the product side $\left(\mathrm{O}+\mathrm{H}_{2} \mathrm{O}\right)$ of the barrier. Again, the terms 'reactant' and 'product' relate to the valleys in the multidimensional potential energy surface which correspond to the minima in the 1D potential slices used in this model. The barriers in the $1 \mathrm{D}$ curves do not directly relate to the barrier along the minimum energy reaction path but are instead the barrier separating the reactant and product valleys at a given $\mathrm{O}-\mathrm{O}$ separation.

Shown in Fig. 13 are the neutral curves for HOHO and DODO with the associated eigenvalues and eigenfunctions. The effects of the barrier in this case are more interesting as a result of the asymmetry of the potential functions. The $v^{\prime}=0$ and $v^{\prime}=1$ levels are localized in the product well for both the hydrated and deuterated species. However, the $v^{\prime}=2$ and $v^{\prime}=3$ levels of $\mathrm{HOHO}$ are located at the barrier between the reactant and product wells. Upon isotopic substitution, we see that the $v^{\prime}=2$ and $v^{\prime}=3$ vibrational levels and the $v^{\prime}=4$ and $v^{\prime}=5$ levels of DODO form nearly degenerate pairs. Further inspection of the $v^{\prime}=2 / v^{\prime}=3$ eigenfunction pair for DODO shows that one of the levels $(v=2)$ is primarily localized in the product well while the other $(v=3)$ has most of its intensity in the reactant well. Within each

Figure 13: The MP2/6-31++G** and QCISD/6-31++G** neutral potential energy curves or the HOHO complex (as in Figure 8 ) with the associated eigenfunctions and eigenvalues. 


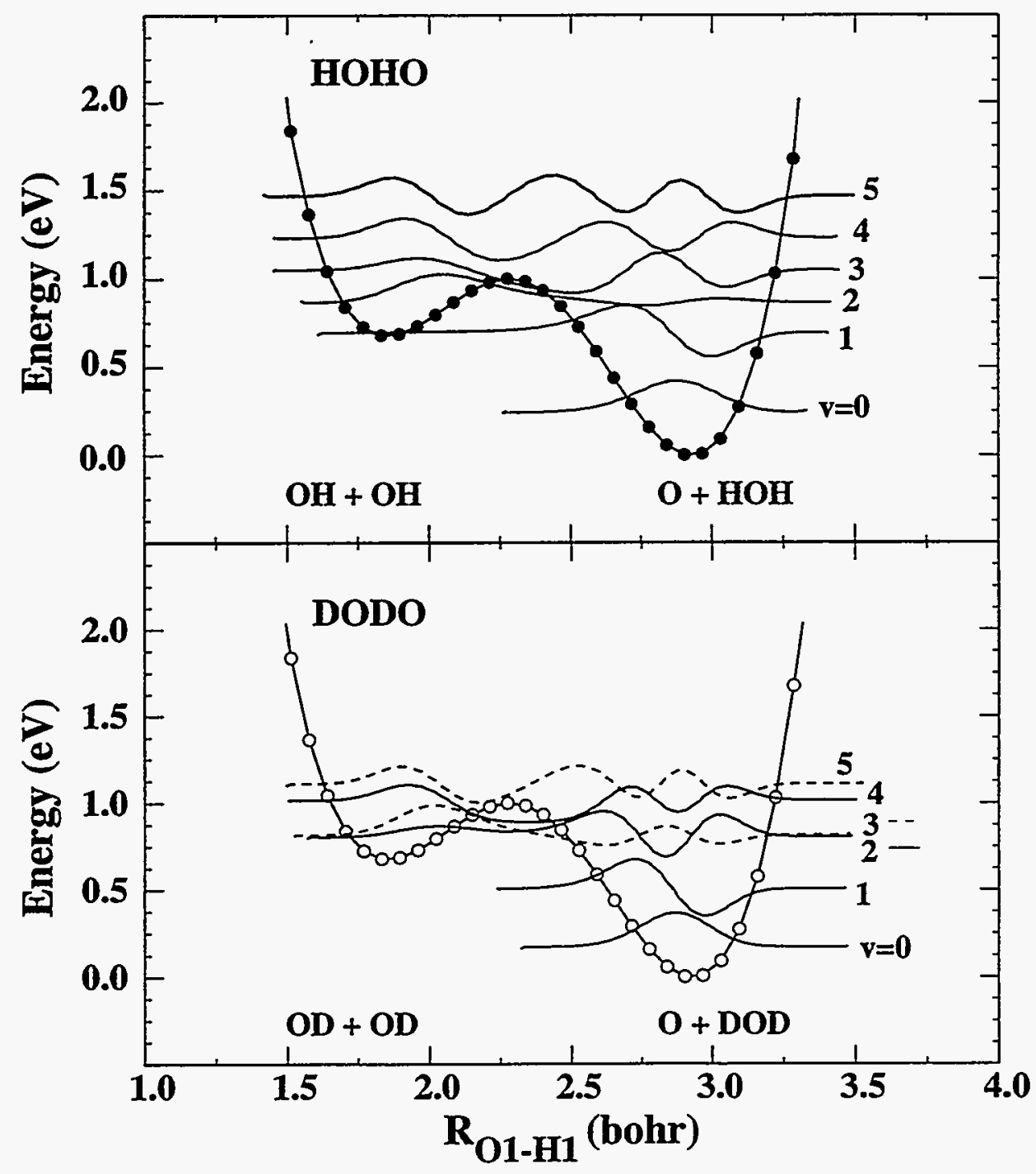

Figure 8.13 
pair of DODO levels, the combination of different nodal structures and localiztion for the wave function results in markedly different FCFs for consecutive states (Table IV and Fig. 11). Thus the $v^{\prime}=2$ state has the greatest FC overlap with the anion function while that of the $v^{\prime}=3$ level is nearly zero. A similar effect occurs for the $v^{\prime}=4$ and $v^{\prime}=5$ levels. For the HOHO complex, the intensity alternation between adjacent levels is less dramatic.

It is interesting to consider how the spectra relate to the asymptotic dynamics as a function of the vibrational 'level' of the unstable neutral complex. The neutral that results from photodetachment of $\mathrm{H}_{3} \mathrm{O}_{2}^{-}$has only one dissociation channel: $\mathrm{OH}+\mathrm{H}_{2} \mathrm{O}$. However, the [HOHO] complex has two accessible dissociation channels at the photodetachment energy used. As indicated by the arrows $a / a^{\prime}$ and $b / b^{\prime}$ in Figs. 4 and 11 the $\mathrm{O}+\mathrm{H}_{2} \mathrm{O}$ product channel is open to all of the observed vibrational levels, but the $\mathrm{OH}+\mathrm{OH}$ reactant channel becomes accessible only at the higher vibrational levels. For example, peak $A$ in both the $\mathrm{O}^{-}\left(\mathrm{H}_{2} \mathrm{O}\right)$ and $\mathrm{O}^{-}\left(\mathrm{D}_{2} \mathrm{O}\right)$ spectra is energetically limited to product dissociation, while peak $\mathrm{C}$ in both spectra can dissociate to both reactant and product. While peak B is limited to product dissociation in $\mathrm{O}^{-}\left(\mathrm{D}_{2} \mathrm{O}\right)$, the uncertainty in the asymptote prevents an assignment for feature $\mathrm{B}$ in the $\mathrm{O}^{-}\left(\mathrm{H}_{2} \mathrm{O}\right)$ spectrum.

The 1D wave functions suggest reactant/product specificity beyond what is energetically allowed. For [DODO], the $v^{\prime}=2$ and $v^{\prime}=4$ states are localized in the product well, while the $v^{\prime}=3$ and $v^{\prime}=5$ states, which have poor FC overlap with the anion, are localized in the reactant well. Thus, within the limits of the wave functions of this $1 D$ model and their FC overlap with the anion, these results suggest that a DODO complex generated from $\mathrm{O}^{-}\left(\mathrm{D}_{2} \mathrm{O}\right)$ photodetachment will preferentially dissociate to products, rather than reactants, even though both are energetically allowed. The vibrational levels of the HOHO complex have a somewhat different reactant/product character. The $\mathrm{HOHO} v^{\prime}=2$ wave function (peak $\mathrm{C}$ ) is primarily a reactant wave function. The figure also shows that even though the uncertainty of the $\mathrm{OH}+\mathrm{OH}$ asymptote may energetically allow the $\mathrm{v}^{\prime}=1$ state of $\mathrm{HOHO}$ (peak $\mathrm{B}$ ) to 
dissociate to reactants, it most likely will be a product state. The character of the $v^{\prime}=1$ wave function for $\mathrm{HOHO}$ (Fig. 13, top) which is located near the minimum of the reactant well suggests that it will remain primarily a product state within the uncertainty of the asymptotic limit.

Similar studies ${ }^{3}$ have been made for the $\mathrm{OH}+\mathrm{F} \rightarrow \mathrm{O}+\mathrm{HF}$ and $\mathrm{CH}_{3} \mathrm{OH}+\mathrm{F} \rightarrow \mathrm{CH}_{3} \mathrm{O}$ $+\mathrm{HF}$ reactions by photodetachment of $\mathrm{OHF}^{-}$and $\mathrm{CH}_{3} \mathrm{OHF}^{-}$. In these photoelectron spectra, there are also two channels for dissociation of the neutral complex which are separated by almost $1.5 \mathrm{eV}$ (c.f. $0.4 \mathrm{eV}$ for $\mathrm{OH}+\mathrm{OH}$ ). Since an $a b$ initio potential energy surface has been calculated for the $\mathrm{OH}+\mathrm{F}$ reaction, ${ }^{64}$ a more detailed analysis was possible. Two-dimensional Franck-Condon analyses ${ }^{65}$ using the available surface, and two slightly modified versions, showed that the simulated photoelectron spectrum was very sensitive to the details of the surface in the transition state region. A one-dimensional analysis coupled with the $2 \mathrm{D}$ analysis showed that features could be assigned to reactant and product states of the neutral [OHF] complex as we have shown for the [HOHO] complex. It was also shown that the different product state features in the spectrum correlated to different HF vibrational levels of the [OHF] dissociation products. A similar correlation is expected in the case of the [DODO] complex where peak $\mathrm{B}$ (and peak $\mathrm{C}$ ) in the $\mathrm{O}^{-}\left(\mathrm{D}_{2} \mathrm{O}\right)$ spectrum will correlate to an oxygen atom and a vibrationally excited $\mathrm{D}_{2} \mathrm{O}$ molecule.

\section{Excited States}

As mentioned above, both the $\mathrm{H}_{3} \mathrm{O}_{2}^{-}$and the $\mathrm{O}^{-}\left(\mathrm{H}_{2} \mathrm{O}\right)$ photoelectron spectra show evidence for photodetachment transitions to excited states of the neutral complex. The features which are assigned as excited states occur at eKE's which are significantly affected by the electron detector cutoff function. In fact, these features most likely represent only thresholds for excited states. Experiments at higher photon energies are necessary to determine the true 
characteristics of these features. As a result, little can be determined from this data about the nature of the excited state surfaces.

As mentioned above several electronic states result from the interaction of two hydroxyl radicals. We have observed the ${ }^{3} \mathrm{~A}$ " state and the ${ }^{3} \mathrm{~A}^{\prime}$ is not accesible by one-electron photodetachment. It is likely that the excited state represent photodetachment to form one of the singlet electronic states that exist. The $26 \mathrm{kcal} / \mathrm{mole}$ endothermicity 22 of the $\mathrm{OH}+\mathrm{OH} \rightarrow$ $\mathrm{H}_{2} \mathrm{O}+\mathrm{O}\left({ }^{1} \mathrm{D}\right)$ places the lower limit for the asymptote at $\mathrm{eKE}=0.225 \mathrm{eV}$. It is quite possible that the feature at eKE $=0.38 \mathrm{eV}$ in the $\mathrm{O}^{-}\left(\mathrm{H}_{2} \mathrm{O}\right)\left(0.30 \mathrm{eV}\right.$ in the $\mathrm{O}^{-}\left(\mathrm{D}_{2} \mathrm{O}\right)$ spectrum) represent the onset of photodetachment to that surface. Fueno calculates the barrier for that reaction to be $35 \mathrm{kcal} / \mathrm{mole}^{21}$

While there are several possible electronic states which arise from the interaction of two $\mathrm{OH}$ radicals, there are a limited number of states available in the $\mathrm{OH}+\mathrm{H}_{2} \mathrm{O}$ interaction. As mentioned above, we find that the ${ }^{2} \mathrm{~A}$ " state of the $\mathrm{HOHOH}$ lies $0.58 \mathrm{eV}$ above the ${ }^{2} \mathrm{~A}$ ' state at the anion gometry at the MP2/6-31++G** level of theory. This compares with the $>1.1 \mathrm{eV}$ spacing between peaks $\mathrm{A}$ and $\mathrm{E}$ in the $\mathrm{H}_{3} \mathrm{O}_{2}^{-}$spectrum. It is unlikely that species correlating to eletronically excited products will be observed because the first excited states of $\mathrm{OH}$ and $\mathrm{H}_{2} \mathrm{O}$ lie are more than $4 \mathrm{eV}$ above their respective ground states. Further experiments at higher photodetachment energies are required to identfy the transitions which are responsible for these features.

\section{Summary}

Negative ion photoelectron spectroscopy of $\mathrm{H}_{3} \mathrm{O}_{2}^{-}$and $\mathrm{O}^{-}\left(\mathrm{H}_{2} \mathrm{O}\right)$ has been used to study the transition state regions of the $\mathrm{OH}+\mathrm{H}_{2} \mathrm{O} \rightarrow \mathrm{H}_{2} \mathrm{O}+\mathrm{OH}$ and $\mathrm{OH}+\mathrm{OH} \rightarrow \mathrm{O}\left({ }^{3} \mathrm{P}\right)+\mathrm{H}_{2} \mathrm{O}$ hydroxyl radical reactions. In both cases, the several broad features, all of which are located above the energetic neutral dissociation asymptote, are assigned to vibrational motions of the unstable neutral complex perpendicular to the reaction coordinate, specifically the H-atom 
antisymmetric stretch. Calculated anion equilibrium geometries and the neutral transition state geometries suggest that the neutral complex studied in each experiments is near the transition state region for its respective reaction.

One dimensional Franck-Condon analyses are performed using $a b$ initio calculated $\mathrm{H}$-atom antisymmetric stretch potential energy curves. Overall, the agreement between the $1 \mathrm{D}$ simulations and the observed photoelectron spectrum supports the assignment of the observed features to $\mathrm{O} \cdots \stackrel{\leftrightarrow}{\mathrm{H}} \cdots \mathrm{O}$ motion of the unstable $[\mathrm{HOHOH}]$ and [HOHO]complexes. The simple 1D model is able to reproduce the rather complicated isotope effects quite well. Slight discrepancies in the spacings and intensities may result from multi-dimensional effects which have not been considered. Better agreement could also conceivably be achieved within this one-dimensional framework by using potential energy curves calculated at different $\mathrm{O}-\mathrm{O}$ separations than those chosen here.

The extension of transition state spectroscopy by anion photodetachment to four and five atom systems offers additional challenges to theoretical methods as a result of the increased degrees of freedom which may play a role in the reaction dynamics. Several recent investigations of the simpler $\mathrm{OH}+\mathrm{H}_{2}$ reaction have been reported.66,67,68,69 In addition to the increased complexity of determining an accurate neutral reaction surface, these systems are also complicated by the nature of the anion potential energy surfaces. The very flat potential energy surface calculated along the central $\mathrm{H}$-atom coordinate for both the $\mathrm{H}_{3} \mathrm{O}_{2}^{-}$and $\mathrm{O}^{-}\left(\mathrm{H}_{2} \mathrm{O}\right)$ anion complexes can have both beneficial and detrimental effects on the analysis of the photoelectron spectra of these species. The flatness of the anion potential complicates the analysis of the spectra because one must determine a reasonably accurate potential surface from which the vibrational wave functions may be calculated. However, the ground state vibrational wave function for this flat potential has very large spatial extents. As such, there is FranckCondon overlap with both of the reactant and product wells in the calculated neutral potential energy surfaces. 
As discussed above, one aspect that cannot be modeled in the $1 \mathrm{D}$ models is the width of the spectral features. Further higher dimensional analyses are required to address this aspect of the data properly. In the $1 \mathrm{D}$ analysis, several parameters were fixed, some at values different from those determined by $a b$ initio geometry optimization methods. In particular, consideration should be given to the role of the O-O separation since it will determine the lifetime of the complex. Additional consideration should also be given to the torsional angle, the $\mathrm{HOH}$ and the $\mathrm{OHO}$ angles which may lead to product rotational excitation upon photodetachment.

\section{Acknowledgments}

This work had been supported by the United States Air Force Office of Scientific Research under contract No. F49620-94-1-0115. 


\section{References}

${ }^{1}$ S. E. Bradforth, A. Weaver, D. W. Arnold, R. B. Metz, and D. M. Neumark, J. Chem. Phys. 92, 7205 (1990); A. Weaver, R. B. Metz, S. E. Bradforth, and D. M. Neumark, J. Phys. Chem. 92, 5558 (1988).

${ }^{2}$ R. B. Metz, A. Weaver, S. E. Bradforth, T. N. Kitsopoulos, and D. M. Neumark, J. Phys. Chem. 94, 1377 (1990).

${ }^{3}$ S. E. Bradforth, D. W. Arnold, R. B. Metz, A. Weaver, and D. M. Neumark, J. Phys. Chem. 95, 8066 (1991).

${ }^{4}$ S. E. Bradforth, D. W. Arnold, D. M. Neumark, and D. E. Manolopoulos, J. Chem. Phys. 99, 6345

(1993); D. E. Manolopoulos, K. Stark, H. -J. Werner, D. W. Arnold, S. E. Bradforth, and D. M. Neumark, Science 262, 1852 (1993).

${ }^{5}$ World Meteorological Organization, Global Ozone Research and Monitoring Project - Report No.16, (1985).

${ }^{6}$ J. A. Logan, M. J. Prather, S. F. Wofsy, and M. B. McElroy, J. Geophys. Res. 86, 7210 (1981); D. Perner, U. Platt, M. Trainer, G. Hübler, J. Drummond, W. Junkermann, J. Rudolph, B. Schubert, A. Volz, D. H. Ehhalt, K. J. Rumpel, and G. Helas, J. Atmos. Chem. 5, 185 (1987).

${ }^{7}$ H. Levy II, Planet. Space. Sci. 20, 919 (1972).

8J. Warnatz, in Combustion Chemistry, ed. by W. C. Gardiner, Jr.,(Springer Verlag, New York, 1984).

${ }^{9}$ C. M. Rohlfing, L. C. Allen, C. M. Cook, and H. B. Schlegel, J. Chem. Phys. 78, 2498 (1983).

${ }^{10}$ Represents the average of the two values $(26.8 \mathrm{kcal} / \mathrm{mol}$ and $27.6 \mathrm{kcal} / \mathrm{mol})$ determined in the following references, respectively: M. Meot-Ner and L. W. Sieck, J. Phys. Chem. 90, 6687 (1986); G. J. C. Paul and P. Kebarle, J. Phys. Chem. 94, 5184 (1990).

11P. A. Schultz. R. D. Mead, P. L. Jones, and W. C. Lineberger, J. Chem. Phys. 77, 1153 (1982).

${ }^{12}$ K. S. Kim, H. S. Kim, J. H. Jang, H. S. Kim, B. -J. Mhin, Y. Xie, and H. F. Schaefer, III, J. Chem. Phys. 94, 2057 (1991).

13Y. Xie and H. F. Schaefer III, J. Chem. Phys. 98, 8829 (1993).

${ }^{14}$ A. A. Nanayakkara, G. G. Balint-Kurti, and I. H. Williams, J. Phys. Chem. 96, 3662 (1992).

${ }^{15}$ C. Lifshitz, J. Phys. Chem. 86, 3634 (1982).

${ }^{16}$ C. R. Moylan, J. A. Dodd, C. -C. Han, and J. I. Brauman, J. Chem. Phys. 86, 5350 (1987).

${ }^{17}$ A. A. Viggiano, R. A. Morris, C. A. Deakyne, F. Dale and J. F. Paulson, J. Phys. Chem. 94, 8193 (1990).

${ }^{18}$ F. C. Fehsenfeld and E. E. Ferguson, J. Chem. Phys. 61, 3181 (1974).

19 J. D. Payzant, R. Yamdgani, and P. Kebarle, Can. J. Chem. 49, 3308 (1971). 
${ }^{20}$ D. M. Neumark, K. R. Lykke, T. Anderson, and W. C. Lineberger, Phys. Rev. A. 32, 1890 (1985).

${ }^{21}$ T. Fueno, in Applied Quantum Chemistry, ed. V. H. Smith, et al., p. 33, (D.Reidel, New York, 1986).

${ }^{22}$ L. B. Harding, J. Phys. Chem. 95, 8653 (1991).

${ }^{23}$ L. B. Harding and A. F. Wagner, Twenty-Second Symposium (International) on Combustion, p. 983, The Combustion Institute, 1988.

${ }^{24}$ S. Golub and B. Steiner, J. Chem. Phys. 49, 5191 (1968).

${ }^{25}$ K. Abu-Dari, K. N. Raymond, and D. P. Freyberg, J. Am. Chem. Soc. 101, 3688 (1979); K. Abu-Dari, D. P. Freyberg, and K. N. Raymond, Inorg. Chem. 18, 2427 (1979).

${ }^{26}$ K. H. Harmon, B. A. Southworth, and P. A. Mounts, J. Mol. Struct. 296, 69 (1993).

${ }^{27}$ F. P. Del Greco and F. Kaufman, Disc. Far. Soc. 33, 128 (1962); F. Kaufman, Ann. Geophys. 20, 106 (1964).

${ }^{28}$ G. Dixon-Lewis, W. E. Wilson and A. A. Westenberg, J. Chem. Phys. 44, 2877 (1966); A. A.

Westenberg, and A. deHaas, J. Chem. Phys. 58, 4066 (1973).

29J. E. Breen and G. P. Glass, J. Chem. Phys. 52, 1082 (1970).

${ }^{30}$ A. McKenzie, M. F. R. Mulcahy, and J. R. Steven, J. Chem. Phys. 59, 3244 (1973).

${ }^{31}$ M. A. A. Clyne and S. Down, J. Chem. Soc. Faraday Trans. 2 70, 253 (1974).

${ }^{32}$ W. T. Rawlins and W. C. Gardiner, Jr., J. Chem. Phys. 60, 4676 (1974).

${ }^{33}$ D. W. Trainor and C. W. von Rosenberg, Jr., J. Chem. Phys. 61, 1010 (1974).

34J. Ernst, H. Gg. Wagner. and R. Zellner, Ber. Bunsenges. Phys. Chem. 81, 1270 (1977).

${ }^{35} \mathrm{G}$. Wagner and R. Zellner, Ber. Bunsenges. Phys. Chem. 85, 1122 (1981); R. Zellner, F. Ewig, R. Paschke, and G. Wagner, J. Phys. Chem. 92, 4184 (1988).

${ }^{36}$ M. T. Woolridge, R. K. Hanson, C. T. Bowman, Int. J. Chem. Kinet. 26, 389 (1994).

${ }^{37}$ R. Zellner, J. Phys. Chem. 83, 18 (1979).

${ }^{38}$ E. A. Albers, K. Hoyermann, H. GG. Wagner, and J. Wolfrum, Thirteenth Symposium (International) on Combustion, p.81, The Combustion Institute, 1971; J. W. Sutherland, P. M. Patterson, and R. B. Klemm, Twenty-Third Symposium (International) on Combustion, p. 51, The Combustion Institute, 1990; A. Lifshitz and J. V. Michael, ibid., p.59.

39J. V. Michael, Prog. Energy Combust. Sci. 18, 327 (1992).

${ }^{40}$ D. Vogt, Adv. Mass Spectrom. 5, 222 (1971).

${ }^{41}$ J. F. Paulson and P. J. Gale, Adv. Mass Spectrom. 7A, 263 (1978). 
${ }^{42}$ M. P. Karnett and R. J. Cross, Chem. Phys. Lett. 82, 277 (1981).

43J. M.Van Doren, S. E. Barlow, C. H. DePuy, and V. M. Bierbaum, Int. J. Mass Spectrom. and Ion Proc. 109, 305 (1991).

${ }^{44}$ D. F. Varley, D. J. Levandier, and J. M. Ferrar, J. Chem. Phys. 96, 8806 (1992).

${ }^{45}$ M. A. Buntine, D. J. Lavrich, C. E. Dessent, M. G. Scaron, and M. A. Johnson, Chem. Phys. Lett. 216, 471 (1993).

46P. F. Knewstubb and T. M. Sugden, Nature 196, 1311 (1962).

${ }^{47}$ C. B. Cleveland and J. R. Wiesenfeld, J. Chem. Phys. 96, 248 (1992); D. S. King, D. G. Sauder, and M. P. Casassa, J. Chem. Phys. 97, 5919 (1992) and references therein.

${ }^{48}$ D. G. Sauder, J. C. Stephenson, D. S. King, and M. P. Casassa, J. Chem. Phys. 97,952 (1992).

${ }^{49}$ M. A. Johnson, M. L. Alexander, and W. C. Lineberger, Chem. Phys. Lett. 112, 285 (1984).

${ }^{50}$ B. E. Knox and B. P. Burtt, J. Chem. Phys. 28, 1256 (1958).

${ }^{51}$ F. C. Fehsenfeld and E. E. Ferguson, J. Chem. Phys. 61, 3181 (1974).

${ }^{52}$ C. E. Melton, J. Phys. Chem. 76, 22 (1972).

${ }^{53}$ W. C. Wiley and I. H. McLaren, Rev. Sci. Instrum. 26, 1150 (1955).

${ }^{54}$ B. O. Roos, W. P. Kraemer, and G. H. F. Diercksen, Theoret. Chim. Acta 42, 77 (1976); S. Ikuta, J. Comput. Chem. 5, 374 (1984); Z. Latajka and S Scheiner, J. Mol. Struct. 234, 373 (1991); G. V.

Yukhnevich, E. G. Kokhanova, A. I. Pavlyuchko, and V. V. Volkov, J. Mol. Struct. 122, I (1985).

55V. Spirko, W. P. Kraemer, and A. Cejchan, J. Mol. Spectrosc. 136, 340 (1989).

${ }^{56}$ C. M. Roehl, J. T. Snodgrass, C. A. Deakyne, and M. T. Bowers, J. Chem. Phys. 94, 6546 (1991).

${ }^{57}$ The energy separation is using the spin projected energies (PMP2). The unprojected values (UMP2) provides a separation of $0.36 \mathrm{eV}$.

${ }^{58}$ The singlet transition state is calculated to lie $32 \mathrm{kcal} / \mathrm{mole}$ higher in energy than the triplet transition state species at the MRDCI/SCF level of theory. See Refs. 21 and 22.

${ }^{59}$ G. Herzberg, Molecular Spectra and Molecular Structure I. Spectra of Diatomic Molecules, Krieger, Malabar (1989).

${ }^{60} \mathrm{G}$. Herzberg, Molecular Spectra and Molecular Structure III. Electronic Spectra and Electronic Structure of Polyatomic Molecules, Krieger, Malabar (1991).

${ }^{61}$ M. J. Davis, H. Koizumi, G. C. Schatz, S. E. Bradforth, and D. M. Neumark, J. Chem. Phys. (submitted for publication).

62T. N. Kitsopoulos, I. M. Waller, J. G. Loeser, and D. M. Neumark, Chem. Phys. Lett. 159, 300 (1989);

Y. Zhao, C. C. Arnold, and D. M. Neumark, J. Chem. Soc. Faraday Trans. 89, 1449 (1993). 
${ }^{63}$ D. O. Harris, G. G. Engerholm, and W. D. Gwinn, J. Chem. Phys. 43, 1515 (1965).

64J. J. Sloan, D. G. Watson, J. M. Williamson, and J. S. Wright, J. Chem. Phys. 75, 1190 (1981).

${ }^{65}$ The simulations assumed the reaction proceeded along a collinear reaction path and the simulations were calculated using a wavepacket propagation method.

${ }^{66}$ D. C. Clary, J. Chem. Phys. 95, 7298 (1991); ibid., J. Chem. Phys. 96, 3656 (1992); G. Nyman and D.

C. Clary, J. Chem. Phys. 99, 7774 (1993).

${ }^{67}$ D. Wang and J. M. Bowman, J. Chem. Phys. 96, 8906 (1992).

${ }^{68}$ U. Manthe, T. Seideman, and W. H. Miller, J. Chem. Phys. 99, 10078 (1993).

${ }^{69}$ D. H. Zhang and J. Z. H. Zhang, J. Chem. Phys. 100, 2697 (1994). 


\title{
Chapter 9: The Study of the I + HI Chemical Reaction Dynamics in Clusters by Photodetachment of $\mathrm{IHI}^{-}(\mathrm{M})_{\mathbf{n}}$
}

\begin{abstract}
Photoelectron spectra have been obtained for several clusters of the type, $\mathrm{IH}^{-}(\mathrm{M})_{\mathrm{n}}$ and IDI $(M)_{n}$, where $M=N_{2} O$ and $\mathrm{CO}_{2}$ and $n \leq 5$. The results of these experiments are interpreted in terms of the solvated anions and the solvated transition state species for the $\mathrm{I}+\mathrm{HI} \rightarrow \mathrm{IH}+\mathrm{I}$ reaction. These experiments, extensions of our previous transition state studies, provide information about the evolution of chemical reaction dynamics in transition state region from the gas phase to the condensed phase. For the single clusters, simulations show that the data can modeled using the bare $\mathrm{I}+\mathrm{HI}$ reaction surface and assuming the anion is slightly distorted by the solvent molecule. The larger clusters are considered more quantitatively in terms of solvent effects on the properties of $\mathrm{IHI}^{-}$and $[\mathrm{IHI}]^{\ddagger}$. In addition to spectral features that strongly resemble those observed in the $\mathrm{IHI}^{-}$spectrum, separate transitions are observed that are assigned to photodetachment of a second isomer of the anion.
\end{abstract}

\subsection{Introduction}

Photoelectron spectroscopy of stable negative ions has been used to study transition state species for gas phase bimolecular chemical reactions previously in this laboratory. The reactions studied include several heavy-light-heavy hydrogen transfer systems, ${ }^{1,2,3}$ hydrogen abstraction reactions by fluorine $e^{4}$ and fundamental hydroxyl radical reactions. ${ }^{5}$ This technique has also been used very successfully to study the transition state region of the prototypical F+ $\mathrm{H}_{2}$ reaction. ${ }^{6}$ In those experiments, the photoelectron spectrum is collected for an anion whose geometry is very similar to that of a transition state species for the chemical reaction of interest. The region of the potential energy surface which is studied by photodetachment of this 
'precursor anion' is determined by the Franck-Condon overlap between the anion and the neutral reaction surface.

However, in addition to gas phase chemical reactions, which play an important role in our environment, solution phase chemistry offers a completely different set of conditions under which chemical reactions can take place. In fact, exploitation of solvent effects on chemical reaction rates is one of the main tools of the synthetic chemist. Therefore, the microscopic effects which solvent molecules have on the dynamics of chemical reactions are of great interest. It has been shown that interactions on the molecular scale, particularly in the first solvent shell, play a vital role in solvation and condensed phase reactions dynamics. ${ }^{7,8,9,10}$

While many studies have used clusters to study the evolution of 'static' chemical properties from the gas phase to the near-bulk phase (see Chapters 4 and 6 for further discussion), less attention has been directed towards the use of cluster experiments to understand how chemical reaction dynamics evolve as solvent interactions become available to act as a catalytic or a quenching agent for a chemical reaction. ${ }^{11,12,13}$ In this paper, we present the results of experiments designed in order to study the reaction dynamics which occur in sizeselected clusters and how they differ from the previous gas-phase dynamical studies. As an extension of our previous transition state studies, we collect the photoelectron spectrum of an anion complex in which the anion is clustered with a controlled number solvent molecules. Photodetachment of the anion initiates a half-reaction in the cluster. By studying the changes which occur in the photoelectron spectrum as a function of cluster size, we are able to study the evolution of the chemical reaction dynamics from the gas phase to the condensed phase.

Another field for which these results are relevant is that of supercritical phenomenon. ${ }^{14}$ Reaction dynamics that occur in supercritical fluids are poorly understood. The high densities attained in the supercritical fluids lead to the solvation of typically insoluble chemicals. Additionally, the different interactions which occur on the microscopic scale can lead to greatly enhanced reaction rates. Models of supercritical reaction phenomena are centered around the 
concept of solute-solvent clustering processes in the supercritical fluid. ${ }^{15}$ However, most of the studies are aimed at determining the static properties of solutes under supercritical conditions. As a result, it is reasonable that the studies of clustered transitions state 'precursor anions' will lend some insight into the reaction dynamics which occur under conditions of high pressure and high temperature where the high densities lead to the formation of clusters throughout the volume of the fluid.

In this initial study of reaction dynamics in clusters, we return to the $\mathrm{IHI}^{-}$precursor anion. It was one of the first transition state species investigated in this laboratory in order to study the transition state region of the prototypical heavy-light-heavy reaction,

$$
\mathrm{I}+\mathrm{HI}^{\prime} \rightarrow \mathrm{IH}+\mathrm{I}^{\prime}
$$

This species has been investigated using anion photoelectron spectroscopy ${ }^{1}$ and threshold photodetachment (ZEKE) spectroscopy. ${ }^{16}$ The spectra show vibrationally resolved features which are assigned to various motions of the unstable $[\mathrm{IHI}]^{\ddagger}$ complex in the transition state region of the $\mathrm{I}+\mathrm{HI}$ reaction. In the experiments presented here, the $\mathrm{IHI}^{-}$anion is clustered with $\mathrm{CO}_{2}$ or $\mathrm{N}_{2} \mathrm{O}$ 'solvent' molecules. By monitoring differences between $\mathrm{IH}^{-}$and the $\mathrm{IHI}^{-}(\mathrm{M})_{\mathrm{n}}$ photoelectron spectra, we expect to learn about the evolution of the $\mathrm{I}+\mathrm{HI}$ reaction dynamics as solvent interactions become increasingly important.

In the following section, the cluster experiments will be described in more detail. In Sec. 9.4.3, the photoelectron spectra of $\mathrm{IHI}^{-}(\mathrm{M})_{\mathrm{n}}$ clusters will be presented, where for $\mathrm{M}=$ $\mathrm{N}_{2} \mathrm{O}, \mathrm{n}=1-5 ; \mathrm{M}=\mathrm{CO}_{2}, \mathrm{n}=1-3$. Spectra are also shown for the isotopic analog, $\mathrm{DI}^{-}(\mathrm{M})_{\mathrm{n}}$, where $\mathrm{M}=\mathrm{N}_{2} \mathrm{O}, \mathrm{n}=1 ; \mathrm{M}=\mathrm{CO}_{2}, \mathrm{n}=1-3$. As in the case of the halide cluster spectra $\left[\mathrm{X}^{-}(\mathrm{M})_{\mathrm{n}}\right.$; $\left.\mathrm{X}=\mathrm{F}, \mathrm{Cl}, \mathrm{Br}, \mathrm{I} ; \mathrm{M}=\mathrm{CO}_{2}, \mathrm{~N}_{2} \mathrm{O}\right]$ reported previously, the spectra retain most of the character of the central chromophore, in this case $\mathrm{IH}^{-}$. This indicates that the precursor anion retains it overall structure. The analysis (Sec. 9.4) is divided into two sections. For the single clusters 
(i.e., $\mathrm{IH}^{-}\left(\mathrm{N}_{2} \mathrm{O}\right)$ and $\mathrm{IHI}^{-}\left(\mathrm{CO}_{2}\right)$ ), differences of the $\mathrm{IHI}^{-}(\mathrm{M})$ spectra from the $\mathrm{IHI}^{-}$spectrum are considered in terms of a perturbed anion and a non-perturbed neutral complex. ${ }^{17}$ Simulations using a semi-empirical potential energy surface for reaction (1) are compared to the data and considered in terms of a change in the Franck-Condon region for the photodetachment of $\mathrm{IHI}^{-}$. For the larger clusters, more qualitative considerations are made concerning the interactions within both the anion and the neutral complexes.

\subsection{Experimental}

The apparatus employed in these experiments, a dual time-of-flight anion photoelectron spectrometer, has been described in detail previously. ${ }^{3}$ Details relevant to these experiments will be summarized here. Anion clusters are generated at the intersection of a pulsed molecular beam and a $1 \mathrm{keV}$ electron beam. ${ }^{18}$ In the ion source, the ions of interest, and several others, are generated by a combination of electron attachment and clustering processes. A mixture of gases $(\sim 1 \% \mathrm{HU} / 49 \% \mathrm{M} / 50 \% \mathrm{He} / \mathrm{Ne} \text {, typically })^{19}$ is expanded through the orifice $\left(0.015^{\prime \prime}\right)$ of a piezoelectric pulsed molecular beam valve run at a stagnation pressure of $\sim 5$ bar and a repetition rate of $20 \mathrm{~Hz}$. Outside the valve orifice, at the intersection with the electron beam, $\mathrm{I}^{-}$ is formed by dissociative attachment of low-energy $(\sim \mathrm{eV})$ secondary electrons ${ }^{20}$ to $\mathrm{HI}$. $\mathrm{IHI}^{-}$ and $\mathrm{IH}^{-}(\mathrm{M})_{\mathrm{n}}$ are formed subsequently by clustering processes as the molecular beam expansion proceeds. The clusters then relax rotationally and vibrationally by further collisions with the carrier gas during the remainder of the supersonic expansion.

The cooled anions are extracted into a Wiley-McLaren-type time-of-flight mass spectrometer ${ }^{21}$ where they separate according to mass. The mass resolution of the spectrometer is $\mathrm{M} / \Delta \mathrm{M} \sim 250$. The ion of interest is selectively photodetached by a properly timed $8 \mathrm{~ns}$ laser pulse. Photoelectron kinetic energies (eKE) are determined by time-of-flight measurements through a 1 meter flight tube. The resolution of the apparatus is $\sim 0.010 \mathrm{eV}$ for electrons with $\mathrm{eKE}=0.65 \mathrm{eV}$ and degrades as $(\mathrm{eKE})^{3 / 2}$. For these experiments, the 4 th $(266 \mathrm{~nm} ; 4.657 \mathrm{eV} ; 15$ 
$\mathrm{mJ} /$ pulse) and 5th (213 nm; $5.822 \mathrm{eV} ; 6 \mathrm{~mJ} /$ pulse) harmonics of a Nd:YAG pulsed laser are employed for photodetachment. The $213 \mathrm{~nm}$ photons generate background signal through interactions with the surfaces of the detector chamber. A background spectrum, collected using the same laser power used during data collection, is fitted to a smooth function which is scaled and subtracted from the data to correct for the moderate level background signal.

\subsection{Results}

Figures 9.1 and 9.2 show the photoelectron spectra of $\mathrm{IHI}^{-}\left(\mathrm{N}_{2} \mathrm{O}\right)_{\mathrm{n}=0-5}$ and $\mathrm{IHI}^{-}\left(\mathrm{CO}_{2}\right)_{\mathrm{n}=0-2}$ collected at $\mathrm{hv}=4.657 \mathrm{eV}$. The $\mathrm{hv}=4.657 \mathrm{eV}$ photoelectron spectra of III, $\mathrm{IDI}^{-}\left(\mathrm{N}_{2} \mathrm{O}\right)$ and $\mathrm{IDI}^{-}\left(\mathrm{CO}_{2}\right)_{n=1,2}$ are shown in Fig. 9.3. Photoelectron spectra of $\operatorname{IHI}^{-}\left(\mathrm{N}_{2} \mathrm{O}\right)_{\mathrm{n}=0-4}$ collected at $h v=5.822 \mathrm{eV}$ are shown in Fig. 9.4. The hv $=5.822 \mathrm{eV}$ photoelectron spectra of $\mathrm{IHI}^{-}\left(\mathrm{CO}_{2}\right)_{\mathrm{n}=0-3}$ and $\mathrm{IDI}^{-}\left(\mathrm{CO}_{2}\right)_{\mathrm{n}=0-3}$ are presented in Fig. 9.5. The $\mathrm{hv}=5.822 \mathrm{eV}$ photoelectron spectra reveal additional structure in the $\mathrm{IH}^{-}(\mathrm{M})_{\mathrm{n}}$ spectra which was not energetically accessible using the $4.657 \mathrm{eV}$ photodetachment energy. However, many of the features are broadened by the lower resolution of the apparatus for higher eKE electrons.

For each spectrum, the intensity of electron signal is plotted as a function of electron kinetic energy. In the photoelectron spectra the eKE is related to the internal energy of the neutral complex by

$$
e K E=h v-D_{o}(\Gamma \cdots H I)-E A(I)-E_{\mathrm{int}}^{o}+E_{\mathrm{int}}^{-}-E_{\mathrm{solv}}^{-}
$$

where $h v$ is the photodetachment energy, $D_{o}\left(I^{-} \cdots H I\right)$ is the dissociation energy of the anion and $E A(I)$ the electron affinity of iodine. $E_{\text {solv }}$ represents the total energy of solvation for the

Figure 9.1: Photoelectron spectra of $\mathrm{IHI}^{-}\left(\mathrm{N}_{2} \mathrm{O}\right)_{\mathrm{n}=0-5}$ collected at $\mathrm{hv}=4.657 \mathrm{eV}$.

Figure 9.2: Photoelectron spectra of $\mathrm{IHI}^{-}\left(\mathrm{CO}_{2}\right)_{\mathrm{n}=0-2}$ collected at $\mathrm{hv}=4.657 \mathrm{eV}$. 


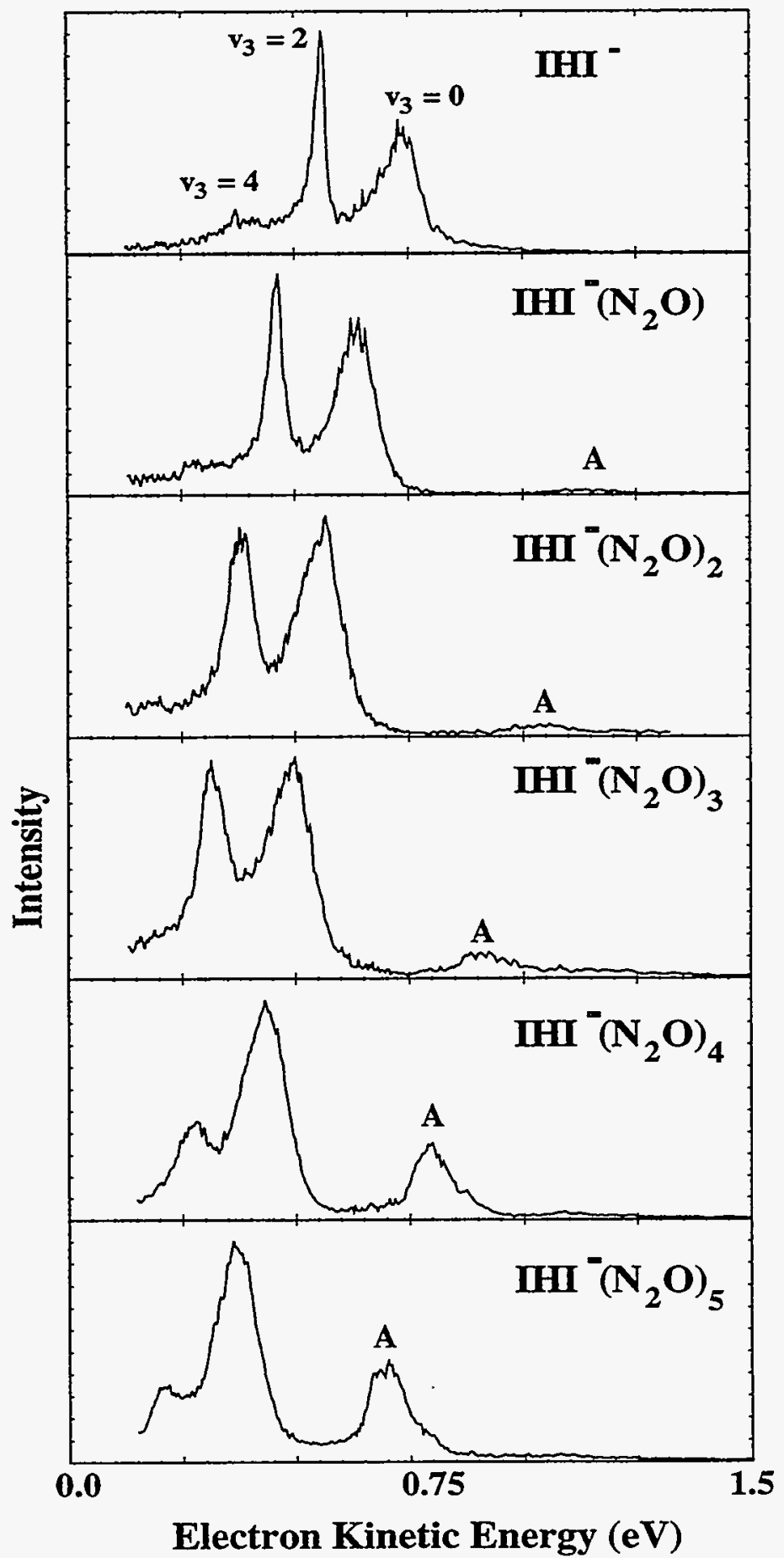

Figure 9.1 


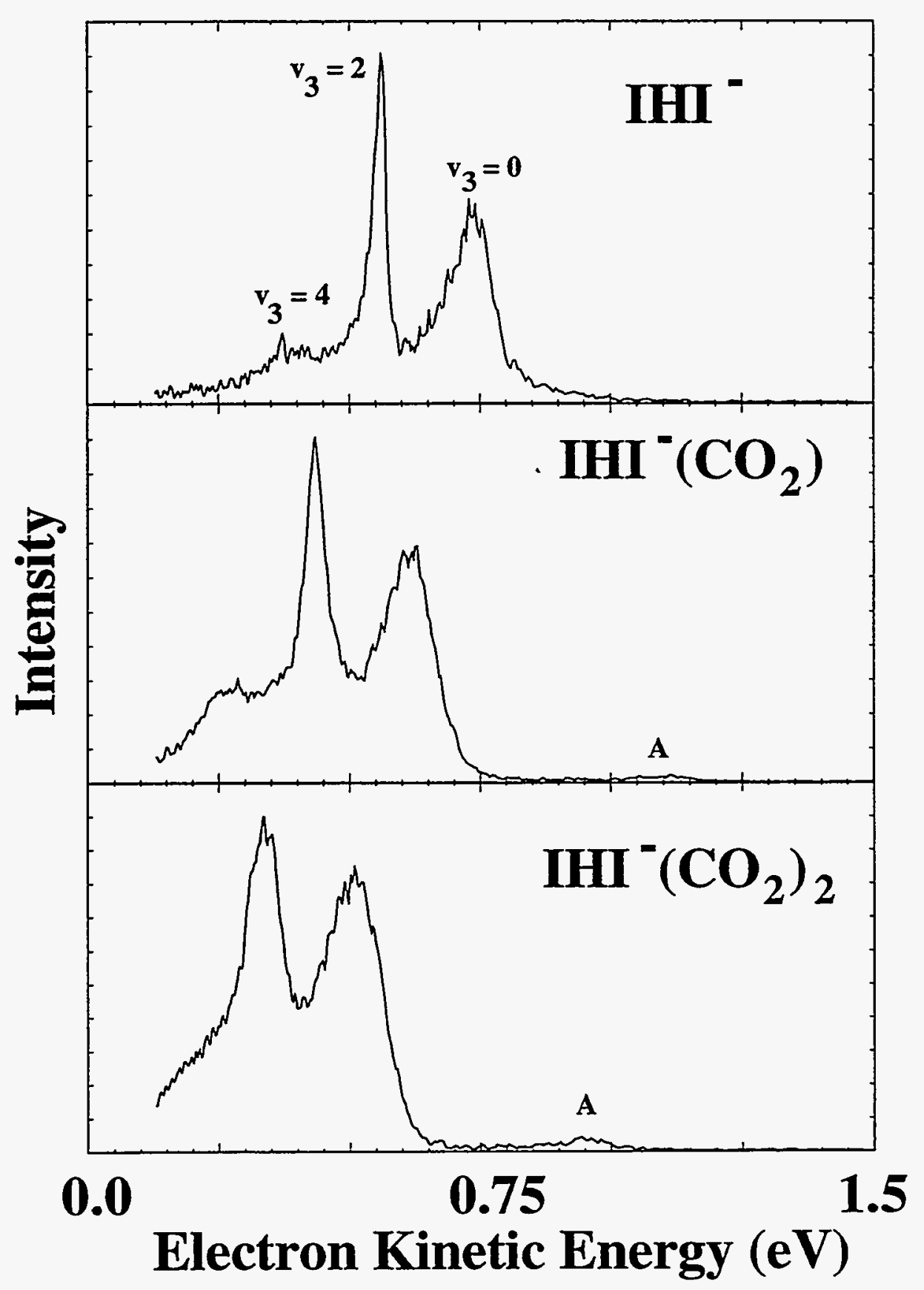

Figure 9.2 
Table I: Peak positions and widths for the $h v=4.657 \mathrm{eV} \mathrm{IHI}^{-}(\mathrm{M})_{\mathrm{n}}$ photoelectron spectra. ${ }^{\mathrm{a}}$

\begin{tabular}{|c|c|c|c|c|c|c|}
\hline $\mathrm{IHI}^{-}(\mathrm{M})_{\mathrm{n}}$ & \multicolumn{3}{|c|}{${\mathrm{M}=\mathrm{N}_{2} \mathrm{O}}^{\mathrm{O}}$} & \multicolumn{3}{c|}{${\mathrm{M}=\mathrm{CO}_{2}}$} \\
\hline & $\mathrm{v}=0$ & $\Delta$ & $\mathrm{v}=2$ & $\mathrm{v}=0$ & $\Delta$ & $\mathrm{v}=2$ \\
\hline $\mathrm{n}=0$ & 0.734 & 171 & 0.563 & 0.734 & 171 & 0.563 \\
& $(74)$ & & $(23)$ & $(74)$ & & $(23)$ \\
$\mathrm{n}=1$ & 0.634 & 174 & 0.460 & 0.613 & 178 & 0.435 \\
& $(120)$ & & $(42)$ & $(110)$ & & $(55)$ \\
$\mathrm{n}=2$ & 0.558 & 181 & 0.377 & 0.508 & 172 & 0.336 \\
& $(210)$ & & $(170)$ & $(240)$ & & $(180)$ \\
$\mathrm{n}=3$ & 0.49 & 170 & 0.32 & $\ldots$ & $\ldots$ & $\ldots$ \\
& $(220)$ & & $(170)$ & & & \\
$\mathrm{n}=4$ & 0.43 & $\ldots$ & $\ldots$ & $\ldots$ & $\ldots$ & $\ldots$ \\
& $(220)$ & & & & & \\
$\mathrm{n}=5$ & 0.367 & $\ldots$ & $\ldots$ & $\ldots$ & $\ldots$ & $\ldots$ \\
& $(220)$ & & & & & \\
\hline
\end{tabular}

a Positions are eKE given in $\mathrm{eV}$. Numbers in parentheses are peak widths in $\mathrm{meV}$. Splittings, $\Delta$, are given in $\mathrm{meV}$.

Figure 9.3: Photoelectron spectra of $\mathrm{DI}^{-}, \mathrm{DI}^{-}\left(\mathrm{N}_{2} \mathrm{O}\right)$, and $\mathrm{IDI}^{-}\left(\mathrm{CO}_{2}\right)_{\mathrm{n}=1,2}$ collected at hv $=$ $4.657 \mathrm{eV}$. 


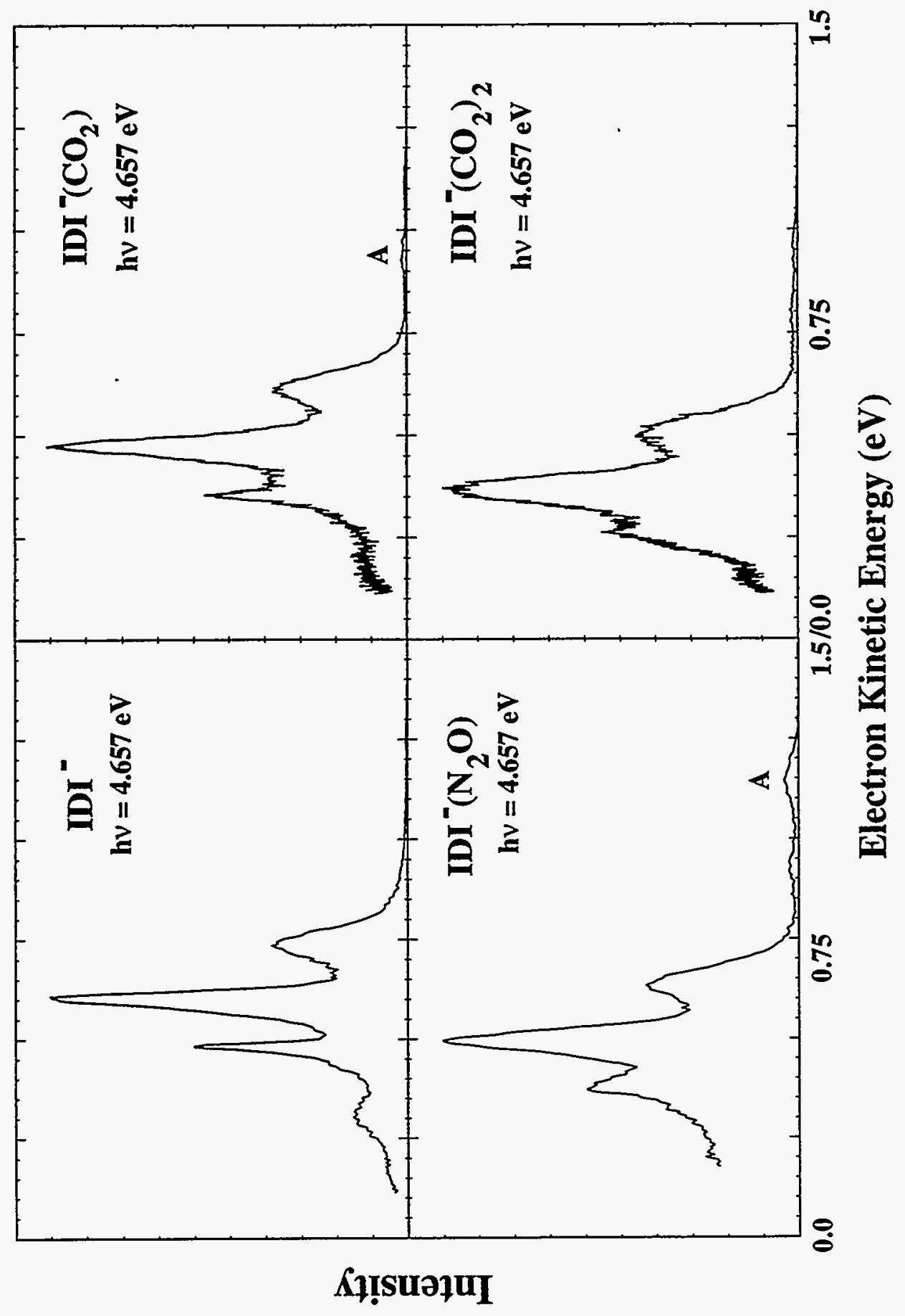

Figure 9.3 
Table II: Peak positions and widths for the $\mathrm{hv}=4.657 \mathrm{eV} \mathrm{IDI}^{-}(\mathrm{M})_{\mathrm{n}}$ photoelectron spectra. $^{\mathrm{a}}$

\begin{tabular}{|c|c|c|c|c|c|c|c|c|c|c|}
\hline $\mathrm{IDI}^{-}(\mathrm{M})_{\mathrm{n}}$ & \multicolumn{7}{|c|}{${\mathrm{M}=\mathrm{N}_{2} \mathrm{O}}^{\mathrm{O}}$} & \multicolumn{5}{c|}{${\mathrm{M}=\mathrm{CO}_{2}}$} \\
\hline & $\mathrm{v}=0$ & $\Delta$ & $\mathrm{v}=2$ & $\Delta$ & $\mathrm{v}=4$ & $\mathrm{v}=0$ & $\Delta$ & $\mathrm{v}=2$ & $\Delta$ & $\mathrm{v}=4$ \\
\hline $\mathrm{n}=0$ & 0.732 & 125 & 0.607 & 121 & 0.486 & 0.732 & 125 & 0.607 & 121 & 0.486 \\
& $(75)$ & & $(45)$ & & $(13)$ & $(75)$ & & $(45)$ & & $(13)$ \\
$\mathrm{n}=1$ & 0.634 & 139 & 0.495 & 115 & 0.380 & 0.612 & 139 & 0.473 & 108 & 0.365 \\
& $(100)$ & & $(80)$ & & $(70)$ & $(100)$ & & $(80)$ & & $(70)$ \\
$\mathrm{n}=2 \mathrm{~b}$ & $\ldots$ & $\ldots$ & $\ldots$ & $\ldots$ & $\ldots$ & 0.503 & 138 & 0.365 & $\ldots$ & $\ldots$ \\
& & & & & & $(240)$ & & $(180)$ & & \\
\hline
\end{tabular}

a Positions are eKE given in $\mathrm{eV}$. Numbers in parentheses are peak widths in $\mathrm{meV}$.

b A third peak was used: $\mathrm{eKE}=0.26 \mathrm{eV} F W H M=85 \mathrm{meV}$; the $\mathrm{v}=4$ peak is significantly affected by electron detector cutoff function.

Figure 9.4: Photoelectron spectra of $\operatorname{IHI}^{-}\left(\mathrm{N}_{2} \mathrm{O}\right)_{\mathrm{n}=0-4}$ at hv $=5.822 \mathrm{eV}$.

Figure 9.5: Photoelectron spectra of $\mathrm{IHI}^{-}\left(\mathrm{CO}_{2}\right)_{n=1-3}$ and $\mathrm{IDI}^{-}\left(\mathrm{CO}_{2}\right)_{\mathrm{n}=1-3}$ at $\mathrm{hv}=5.822 \mathrm{eV}$. 


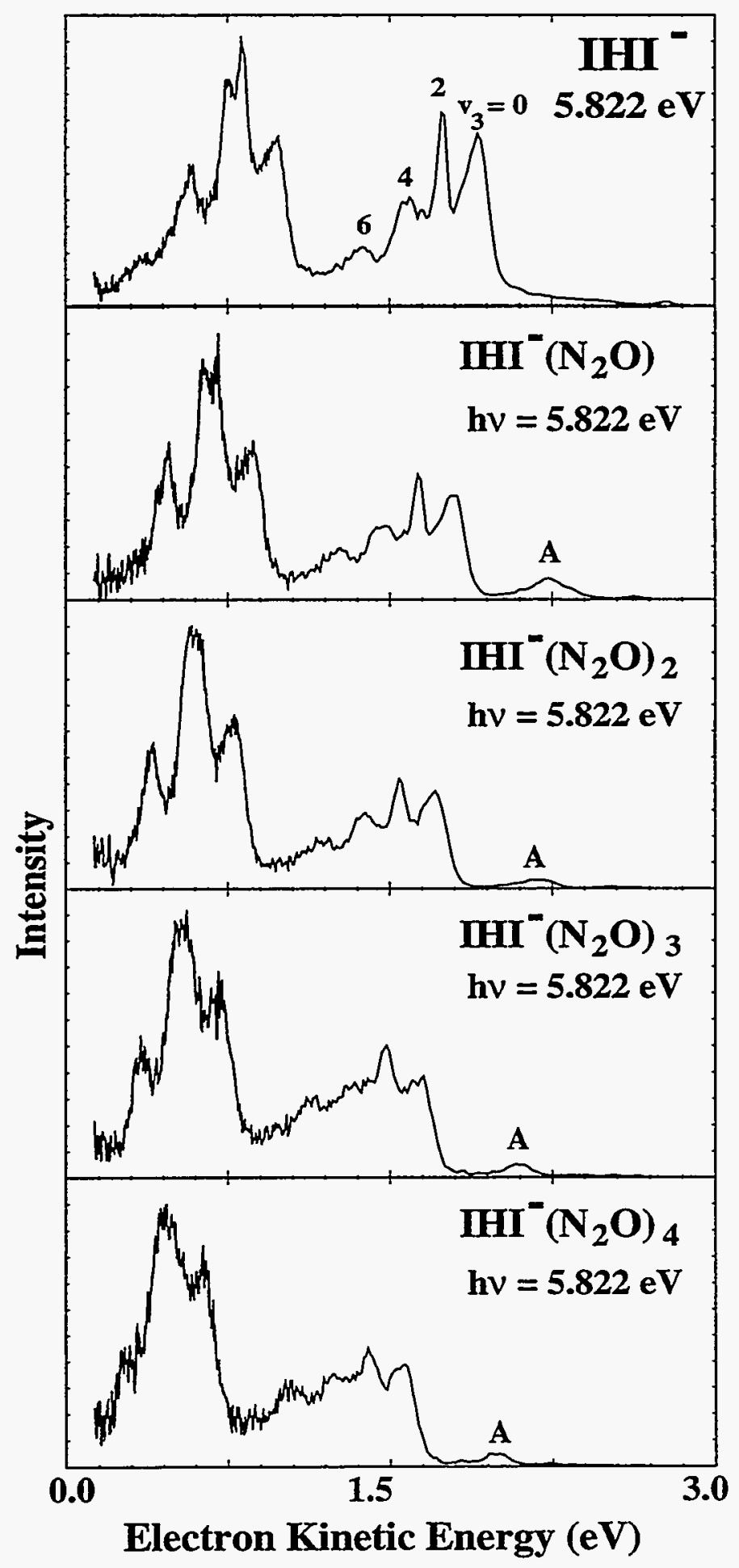

Figure 9.4 


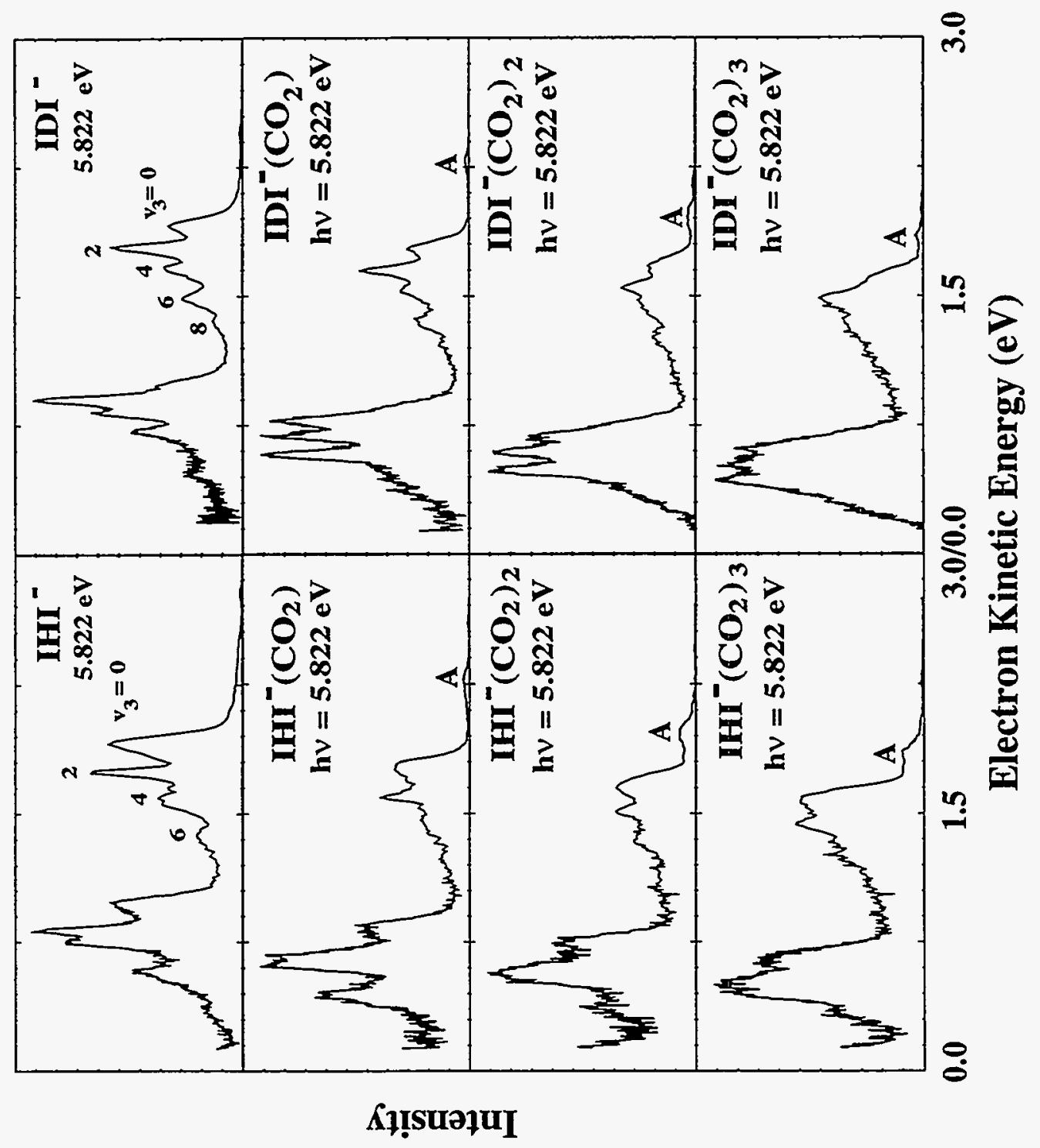

Figure 9.5 
Table III: Peak positions, widths and relative integrated intensities for the $h v=5.822 \mathrm{eV} \operatorname{IHI}^{-}(\mathrm{X})_{\mathrm{n}}$ photoelectron spectra.

\begin{tabular}{|c|c|c|c|c|c|c|c|c|c|c|c|c|c|c|}
\hline $\mathrm{IHI}^{-}(\mathrm{M})_{\mathrm{n}}$ & \multicolumn{10}{|c|}{$\mathrm{M}=\mathrm{N}_{2} \mathrm{O}$} & \multicolumn{1}{c|}{${\mathrm{M}=\mathrm{CO}_{2}}$} \\
\hline & $\mathrm{v}=0$ & $\Delta$ & $\mathrm{v}=2$ & $\Delta$ & $\mathrm{v}=4$ & $\Delta$ & $\mathrm{v}=6$ & $\mathrm{v}=0$ & $\Delta$ & $\mathrm{v}=2$ & $\Delta$ & $\mathrm{v}=4$ & $\Delta$ & $\mathrm{v}=6$ \\
\hline $\mathrm{n}=0$ & 1.91 & 170 & 1.74 & 170 & 1.59 & 220 & 1.37 & 1.91 & 170 & 1.74 & 150 & 1.59 & 220 & 1.37 \\
& $(150)$ & & $(50)$ & & $(200)$ & & $(150)$ & $(150)$ & & $(50)$ & & $(200)$ & & $(150)$ \\
$\mathrm{n}=1$ & 1.79 & 170 & 1.62 & 160 & 1.47 & 210 & 1.26 & 1.77 & 170 & 1.60 & 140 & 1.46 & 230 & 1.23 \\
& $(135)$ & & $(75)$ & & $(180)$ & & $(150)$ & $(140)$ & & $(75)$ & & $(190)$ & & $(180)$ \\
$\mathrm{n}=2$ & 1.7 & 160 & 1.54 & 170 & 1.38 & 200 & 1.18 & 1.66 & 150 & 1.51 & 150 & 1.36 & 230 & 1.13 \\
& $(145)$ & & $(85)$ & & $(170)$ & & $(160)$ & $(165)$ & & $(85)$ & & $(190)$ & & $(190)$ \\
$\mathrm{n}=3$ & 1.63 & 150 & 1.48 & 130 & 1.35 & 210 & 1.14 & 1.57 & 140 & 1.43 & 150 & 1.28 & 230 & 1.05 \\
& $(150)$ & & $(85)$ & & $(190)$ & & $(190)$ & $(125)$ & & $(110)$ & & $(200)$ & & $(200)$ \\
$\mathrm{n}=4$ & 1.55 & 150 & 1.40 & 150 & 1.25 & 220 & 1.03 & $\ldots$ & $\ldots$ & $\ldots$ & $\ldots$ & $\ldots$ & $\ldots$ & $\ldots$ \\
& $(150)$ & & $(95)$ & & $(190)$ & & $(190)$ & & & & & & & \\
\hline
\end{tabular}


Table IV: Peak positions, widths and relative integrated intensities for the hv $=5.822 \mathrm{eV}$ $\operatorname{IDI}(\mathrm{X})_{\mathrm{n}}$ photoelectron spectra.

\begin{tabular}{|c|c|c|c|c|c|c|c|c|c|}
\hline IDI $^{-}(\mathrm{M})_{\mathrm{n}}$ & \multicolumn{10}{c|}{${\mathrm{M}=\mathrm{CO}_{2}}$} \\
\hline & $\mathrm{v}=0$ & $\Delta$ & $\mathrm{v}=2$ & $\Delta$ & $\mathrm{v}=4$ & $\Delta$ & $\mathrm{v}=6$ & $\Delta$ & $\mathrm{v}=8$ \\
\hline $\mathrm{n}=0$ & 1.91 & 130 & 1.78 & 120 & 1.66 & 170 & 1.49 & 150 & 1.34 \\
& $(130)$ & & $(60)$ & & $(110)$ & & $(120)$ & & $(125)$ \\
$\mathrm{n}=1$ & 1.77 & 130 & 1.65 & 120 & 1.53 & 170 & 1.36 & 160 & 1.20 \\
& $(120)$ & & $(75)$ & & $(120)$ & & $(130)$ & & $(130)$ \\
$\mathrm{n}=2$ & 1.66 & 110 & 1.55 & 110 & 1.44 & 180 & 1.26 & & \\
& $(140)$ & & $(80)$ & & $(155)$ & & $(155)$ & & \\
$\mathrm{n}=3$ & 1.59 & 110 & 1.49 & 130 & 1.35 & 180 & 1.18 & & \\
& $(140)$ & & $(95)$ & & $(190)$ & & $(190)$ & & \\
& & & & $75)$ & & & \\
\end{tabular}


$\mathrm{IHI}^{-}$anion by the $n$ solvent molecules. $E_{\text {int }}^{-}$represents the internal energy of the anion complex (i.e., vibrational and rotational energy). $E_{\mathrm{int}}^{o}$ represent the energy of the $[\mathrm{IHI}]^{\ddagger}(\mathrm{M})_{\mathrm{n}}$ species above the energy of the separated $\mathrm{I}+\mathrm{HI}+\mathrm{n}(\mathrm{M})$ products. In the $\mathrm{hv}=5.822 \mathrm{eV}$ data, transitions are also observed to an excited electronic surface corresponding to the $I^{*}+H I$ reaction. Thus, in equation (2) $E_{\mathrm{int}}^{o}$ will also include the I-I* separation for these transitions $(0.943 \mathrm{eV}) .{ }^{22}$

In general, the $\mathrm{IHI}^{-}(\mathrm{M})_{\mathrm{n}}$ spectra appear as broadened, shifted versions of the $\mathrm{IHI}^{-}$ spectrum. The peak positions and widths for the ground state features observed in the hv = 4.657 and $5.822 \mathrm{eV}$ data are provided in Tables I - IV. These are determined by fitting each $\mathrm{IHI}^{-}(\mathrm{M})_{\mathrm{n}}$ spectrum with a set of Gaussian curves, one for each peak in the spectrum. Except for the bare anions, all of the spectra consist of features which are significantly broader than the experimental resolution.

As indicated in the $\mathrm{IHI}^{-}$and $\mathrm{III}^{-}$spectra, the previous analysis of the $\mathrm{IH}^{-}$data assigned the features as photodetachment transitions to the unstable [IHI] ${ }^{\ddagger}$ complex. The progression was assigned to the asymmetric hydrogen vibrational motion $\left(\mathrm{v}_{3}\right)$ of the complex prior to dissociation into $\mathrm{I}+\mathrm{HI}$. The $\mathrm{v}_{3}=4$ feature in the $\mathrm{hv}=4.657 \mathrm{eV} \mathrm{PI}^{-}$spectrum which is slightly broader than the experimental resolution. The fine structure present on top of the $v_{3}$ $=0$ peak in the IHI $^{-}$spectrum is, in fact, reproducible and has been studied in greater detail using threshold photodetachment (ZEKE) spectroscopy and assigned to HI hindered rotor motions of the $[\mathrm{IHI}]^{\ddagger}$ complex. ${ }^{16}$ In the $\mathrm{IHI}^{-}(\mathrm{M})_{\mathrm{n}=1}$ data at $\mathrm{hv}=4.657 \mathrm{eV}$, the fine structure on the $v_{3}=0$ feature is still apparent, although it is not visible in the $\operatorname{IDI}^{-}(M)_{n=1}$ data. The $\mathrm{IHI}^{-}$ peak spacings are: $\Delta(0-2)=1360 \mathrm{~cm}^{-1}$ and $\Delta(2-4)=1540 \mathrm{~cm}^{-1}$. The $\mathrm{DI}^{-}$spacings are: $\Delta(0-2)$ $=1020 \mathrm{~cm}^{-1}$ and $\Delta(2-4)=980 \mathrm{~cm}^{-1}$. The significantly decreased frequency, relative to that of $\mathrm{HI}\left(2309 \mathrm{~cm}^{-1}\right)$ or $\mathrm{DI}\left(1600 \mathrm{~cm}^{-1}\right)^{23}$ indicates that the $\mathrm{H}$-atom interacts strongly with both of the iodine atoms in the neutral complex. 
The spectral features shift to lower eKE as each additional solvent molecule is added. For example, the $v_{3}=0$ feature in the $\mathrm{IHI}^{-}\left(\mathrm{N}_{2} \mathrm{O}\right)$ and $\mathrm{IH}^{-}\left(\mathrm{CO}_{2}\right)$ spectra are shifted by $0.097 \mathrm{eV}$ and $0.111 \mathrm{eV}$, respectively, relative to the $\mathrm{v}_{3}=0$ feature of the $\mathrm{IHI}^{-}$spectrum. The stepwise solvent shift decreases as a function of cluster size. For $n=2$ through $n=5$ in the $\operatorname{IHI}^{-}\left(\mathrm{N}_{2} \mathrm{O}\right)_{n}$ spectra the shifts are $78,68,58$ and $63 \mathrm{meV}$, according to center of the $v_{3}=0$ feature in each of the respective spectra.

The clusters also induce changes in the peak spacings of some of the spectra, again, measured by the peak centers. As seen in Table I, the spacings of the peaks change when the $\mathrm{N}_{2} \mathrm{O}$ molecule is clustered to the $\mathrm{IDI}^{-}$anion. In particular, the $\mathrm{v}_{3}=0-\mathrm{v}_{3}=2$ spacing increases by $14 \mathrm{meV}$ while the $\mathrm{v}_{3}=2-\mathrm{v}_{3}=4$ spacing decreases by $6 \mathrm{meV}$. For the $\mathrm{DI}^{-}\left(\mathrm{CO}_{2}\right)$ spectrum we see that the $v_{3}=0-v_{3}=2$ spacing increases by $14 \mathrm{meV}$ and the $0-4$ spacing decreases by 13 $\mathrm{meV}$. Thus, the position of the $v_{3}=2$ feature in both of these spectra appears to move relative to the other two features in the progression. In the $\mathrm{IHI}^{-}\left(\mathrm{CO}_{2}\right)$ and $\mathrm{IHI}^{-}\left(\mathrm{N}_{2} \mathrm{O}\right)$ spectra at $\mathrm{hv}=$ $4.657 \mathrm{eV}$ only the $v_{3}=0-v_{3}=2$ spacing is well determined. While the $\operatorname{IHI}^{-}\left(\mathrm{N}_{2} \mathrm{O}\right) \mathrm{v}_{3}=0-\mathrm{v}_{3}=$ 2 spacing is the same as that in the $\mathrm{IH}^{-}$spectrum, the spacing between the peaks in the $\mathrm{IHI}^{-}\left(\mathrm{N}_{2} \mathrm{O}\right)_{2}$ spectrum does increase, primarily as a result of the increased $\mathrm{v}_{3}=2$ peak width (i.e., the onset of signal for the $v_{3}=2$ peak is unchanged relative to $\mathrm{IHI}^{-}$and $\mathrm{IHI}^{-}\left(\mathrm{N}_{2} \mathrm{O}\right)$ ). This phenomenon will be treated in more detail below.

As mentioned above, the experiments performed at $\mathrm{hv}=5.822 \mathrm{eV}$ reveal transitions that are not energetically accessible in at $\mathrm{hv}=4.657 \mathrm{eV}$. However, the peak broadening that occurs at higher eKE's results in a significantly greater uncertainty in the determination of the peak spacings. For example, the point spacing at $\mathrm{eKE}=1.5 \mathrm{eV}$, is $12 \mathrm{meV}$, thus the peak centers, and spacings, have an uncertainty of at least this amount. Nonetheless, the addition of clusters to the $\mathrm{IH}^{-}$complex does have noticeable effects on the spectra. The two most noticeable effects are the additional broadening of the peaks, as observed in the $h v=4.657 \mathrm{eV}$ data, and the increase in the integrated intensities of the higher vibrational levels relative to that 
of the lower levels. In the IHI- $\left(\mathrm{N}_{2} \mathrm{O}\right)$ spectrum, the spacing between the centers of the $v_{3}=2$ and $v_{3}=4$ peaks increases slightly, primarily as a result in the additional broadening of the $v_{3}=$ 4 peak over that of the $v_{3}=2$ peak. Unfortunately, due to the peak widths, the uncertainties in the peak centers $(-15-20 \mathrm{meV})$ the changes are not significant. For example, the $\mathrm{IH}^{-}\left(\mathrm{N}_{2} \mathrm{O}\right) \mathrm{v}_{3}$ $=0-v_{3}=2$ spacing decreases in the $5.822 \mathrm{eV}$ according to the peak centers. In that data the uncertaintues are even larger due to the higher eKE's of the photoelectrons. The $v_{3}=2-v_{3}=4$ spacing onset remains constant within the uncertainty. For the larger cluster, the peak spacings are constant within the uncertainty of the peak center determination. Thus, while the observed changes in peak spacings in the $5.822 \mathrm{eV}$ data are consistent with those observed for the 4.657 $\mathrm{eV}$ data, the uncertainties prevent their use as a confirmation of the observed behavior. The spectra do, however, provide information about the change in the intensities of the vibrational features as a function of cluster size.

- In addition to the features which are readily associated with the spectrum of the bare $\mathrm{IHI}^{-}$, another feature, which will generally be referred to as feature A, appears in the $\mathrm{IHI}^{-}(\mathrm{M})_{\mathrm{n}}$ spectra at higher eKE which does not correspond to any feature observed in the $\mathrm{IHI}^{-}$spectrum. The intensity of this feature in the $\mathrm{IH}^{-}\left(\mathrm{N}_{2} \mathrm{O}\right)_{n}$ spectra increases as a function cluster size in the $\mathrm{hv}=4.657 \mathrm{eV}$ data. However, in the $\mathrm{IHI}^{-}\left(\mathrm{N}_{2} \mathrm{O}\right)_{n}$ spectra collected at $\mathrm{hv}=5.822 \mathrm{eV}$ this feature is significantly less intense and does not increase as a function of cluster size. In the $\mathrm{IHI}^{-}\left(\mathrm{CO}_{2}\right)_{\mathrm{n}}$ spectra, the intensity of this feature is independent of the photodetachment energy. The three data sets were collected using slightly different ion source conditions, suggesting that a second isomer of the anion cluster, which is not described appropriately as $\operatorname{IH}^{-}(\mathrm{M})_{\mathrm{n}}$, is contributing to the spectrum. The behavior of this feature as a function of cluster size further supports this assignment. In the $\mathrm{IH}^{-}\left(\mathrm{N}_{2} \mathrm{O}\right)_{\mathrm{n}}$ data, this feature shifts to lower eKE by approximately the same amount as the $\mathrm{IHI}^{-}$features as the cluster size increases. However, in the $\mathrm{IHI}^{-}\left(\mathrm{CO}_{2}\right)_{\mathrm{n}}$ data, the high eKE feature moves to lower eKE more rapidly than the rest of the 
spectrum. Thus, feature A does not appear to be directly associated with species responsible for the peaks at lower $\mathrm{eKE}$.

\subsection{Analysis and Discussion}

\subsubsection{Initial Considerations}

We begin the data analysis with a brief review of previous $\mathrm{IHI}^{-}$results obtained in this laboratory. Here, we will consider only the ground state features which are present in the data. The photoelectron spectrum of $\mathrm{IHI}^{-}$has been studied previously in this laboratory by both photoelectron spectroscopy ${ }^{1}$ and threshold photodetachment (ZEKE) spectroscopy. ${ }^{16}$ Based upon the observed isotope dependence of the spectral features, the progression is assigned to the $v_{3}$ antisymmetric stretch motion of the unstable $[\mathrm{IHI}]^{\ddagger}$ complex. Due to the symmetry, only photodetachment transitions to even $v_{3}^{\prime}$ states of the neutral complex are allowed. Thus, the peaks in the spectrum correspond to the $v_{3}^{\prime}=0, v_{3}^{\prime}=2$, and $v_{3}^{\prime}=4$ states of the complex.

The fine structure on the $v_{3}^{\prime}=0$ feature is assigned to rotational scattering states where the peaks correspond to excited rotational states of the HI product (or reactant) as the complex falls apart. This effect can only be observed in three-dimensional calculations. The shoulders present in under the $\mathrm{v}_{3}^{\prime}=2$ peak are determined to be a progression in the I-I symmetric stretch motion. These features can be observed in two-dimensional calculations as will be shown below. The very sharp feature observed for the $v_{3}^{\prime}=4$ peak results from a transition to a resonance state which is quasi-bound. This upper limit for the width of this feature was determined to be $30 \mathrm{~cm}^{-1}$ by ZEKE spectroscopy. ${ }^{16}$

The analysis of the $\mathrm{IHI}^{-}$photoelectron spectrum revealed that the available reaction surfaces underestimate the gradient along the reaction path from the saddle point to the dissociation asymptotes. The gentle slope of the LEPS (London-Eyring-Polanyi-Sato) reaction path allows the repulsive electronic surface to support quasi-bound vibrational states. These 
states result when the combined zero point energy of the neutral reactants/products changes more rapidly than the electronic potential energy surface in the transition state region.

To analyze the $\mathrm{IHI}^{-}(\mathrm{M})_{\mathrm{n}}$ data presented above, several points need to be considered in more detail. We are interested in understanding the how the solvent molecule interacts with the anion and the neutral complex to produce the observed differences from the $\mathrm{IHI}^{-}$spectrum. We will first consider how the binding within the clusters leads the observed shift of the $\mathbb{H I}^{-}(M)_{n}$ spectra relative to the spectrum of $\mathrm{HH}^{-}$. Then, the change in peak spacings will be addressed in two parts. The spectra of the singly clustered anions will be considered in terms of the photodetachment of an anion cluster containing a geometrically distorted $\mathrm{IHI}^{-}$to a neutral complex in which there is a minimal interaction between the solvent molecule and the $[\mathrm{IHH}]^{\frac{1}{*}}$ species. In this limit the spectra will be interpreted in terms of photodetachment of the $\mathrm{IHI}^{-}(\mathrm{M})$ anion to the unperturbed $\mathrm{I}+\mathrm{HI}$ reaction surface. As a result of the $\mathrm{IHI}^{-}$perturbation the FranckCondon region for $\mathrm{IHI}^{-}(\mathrm{M})$ photodetachment is different from that of $\mathrm{IHI}^{-}$. For the larger clusters, we will consider how the interactions of the solvent molecules with both the anion and the neutral can lead to the observed changes in the $\mathrm{IH}^{-}(\mathrm{M})_{\mathrm{n}}$ photoelectron spectra. Finally, we will consider alternative ion structures that may be responsible for feature $A$ which appears in the cluster spectra.

The basis for the shift of the spectrum to lower $\mathrm{eKE}$ as a function of cluster size is illustrated in Fig. 9.6. Considering the singly clustered anion, the interactions which bind the $\mathrm{N}_{2} \mathrm{O}$ and $\mathrm{CO}_{2}$ solvent molecules to the $\mathrm{IH}^{-}$anion are the charge-dipole and/or chargequadrupole interactions. The solvent molecule serves to stabilize the negative charge. The anion interactions will be significantly stronger than the solvent-neutral interaction. Stabilizing

Figure 9.6: Schematic diagram of the energetic involved in the solvation of $\mathrm{IH}^{-}$by a single solvent molecule. The difference between $E_{\text {solv }}^{-}$and $E_{\text {solv }}^{o}$ leads to the observed solvent shift. 


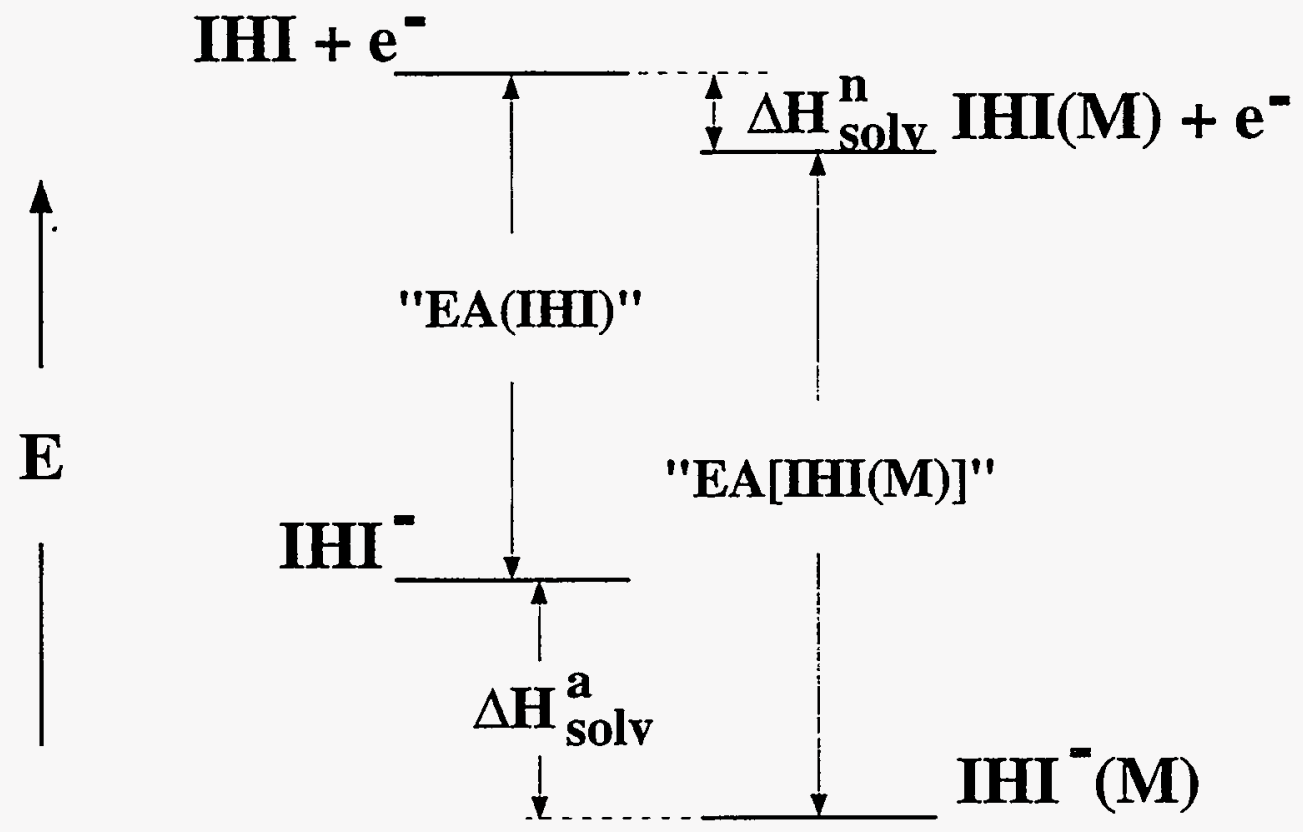

Figure 9.6 
the unstable neutral complex corresponds to altering of the neutral reaction surface in the transition state region (i.e., changing the barrier height). The resultant larger threshold detachment energy for the cluster leads to the observed spectral shift.

Two solvent molecules which interact with the anion identically stabilize the charge by the same amount. If the neutral interactions are also identical the spectra of consecutive clusters will step evenly to lower eKE. In general, however, the spectral shift for the second cluster can be larger or smaller than the first depending upon the relative overall stabilization of the anion and neutral complexes. For example, a decreasing spectral shift as a function of cluster size can result from either weaker anion or stronger neutral interactions with the solvent molecules. These two possibilities cannot be distinguished based upon the observed spectral shift alone.

The $\mathrm{IHI}^{-}(\mathrm{M})_{\mathrm{n}}$ spectra show that a larger spectral shift occurs for the $\mathrm{CO}_{2}$ molecule than for the $\mathrm{N}_{2} \mathrm{O}$ molecule. This is similar to the result obtained for the $\Gamma^{-}\left(\mathrm{CO}_{2}\right)_{n}$ and $\mathrm{I}^{-}\left(\mathrm{N}_{2} \mathrm{O}\right)_{n}$ clusters. As discussed in that study, the similar electrostatic properties of the $\mathrm{CO}_{2}\left(\Theta_{\mathrm{CO}_{2}}=-4.3\right.$ $\mathrm{X} 10^{-26}$ esu $\left.\cdot \mathrm{cm}^{2}\right)^{24}$ and $\mathrm{N}_{2} \mathrm{O}\left(\mu_{\mathrm{N}_{2} \mathrm{O}}=0.1608 ; \Theta_{\mathrm{N}_{2} \mathrm{O}}=-3.6 \times 10^{-26} \text { esu } \cdot \mathrm{cm}^{-2}\right)^{25,26}$ molecules ${ }^{27}$ suggest that the interaction the $\mathrm{N}_{2} \mathrm{O}$ molecule would have a slightly weaker interaction with a charged species than the $\mathrm{CO}_{2}$ molecule. Additionally, in the $\Gamma\left(\mathrm{CO}_{2}\right)_{\mathrm{n}}$ clusters, the $\mathrm{CO}_{2}$ molecule acquires an additional stabilization by distorting from linearity. This distortion was identified by the $\mathrm{CO}_{2}$ bending progression which resulted upon photodetachment of the $I^{-}\left(\mathrm{CO}_{2}\right)_{\mathrm{n}}$ cluster. While no bending progression is observed in the $\mathrm{IH}^{-}\left(\mathrm{CO}_{2}\right)_{\mathrm{n}}$ data, its presence cannot be ruled out because there is considerable congestion in the spectra and the intensity of transitions to excited bending levels of $\mathrm{CO}_{2}$ is expected to be small.

Since both the $\mathrm{IHI}^{-}(\mathrm{M})_{\mathrm{n}}$ and the $\mathrm{I}^{-}(\mathrm{M})_{\mathrm{n}}$ clusters bound primarily by electrostatic interactions, one might expect the size-dependence of the solvation energy to be quite similar for the two data sets. However, a significantly different behavior of the solvation shifts is observed for the data. The SSEs observed for the $\operatorname{IHI}^{-}\left(\mathrm{N}_{2} \mathrm{O}\right)_{n}$ clusters $(97,79,68$, and $60 \mathrm{meV}$ 
for the first four $\mathrm{N}_{2} \mathrm{O}$ molecules) are less than those observed for the $\mathrm{I}^{-}\left(\mathrm{N}_{2} \mathrm{O}\right)_{n}$ clusters $(107$, 108,120 and $115 \mathrm{meV}$ for the first four $\mathrm{N}_{2} \mathrm{O}$ molecules). A similar comparison is made for the $\mathrm{CO}_{2}$ clusters. (i.e., 111 and $95 \mathrm{meV}$ for $\mathrm{IHI}^{-}\left(\mathrm{CO}_{2}\right)_{\mathrm{n}=1-2} ; 139$ and $165 \mathrm{meV}$ for $\left.\mathrm{I}^{-}\left(\mathrm{CO}_{2}\right)_{\mathrm{n}=1-2}\right)$. The difference in the magnitude of the SSEs is not surprising because the larger spatial extent of $\mathrm{IHI}^{-}$relative to $\mathrm{I}^{-}$leads to a lower charge density and a weaker anion interaction.

However, in addition to the overall smaller shifts in the $\mathrm{IHI}^{-}(\mathrm{M})_{\mathrm{n}}$ spectra, there is also a quicker decrease in the SSE as a function of cluster size indicating that consecutive solvent molecules encounter different interactions in the either the anion or the neutral clusters. This effect was not apparent in the $I^{-}(M)_{n}$ clusters until several solvent molecules had been added to the cluster. Whether the changes occur in the anion or the neutral cluster is very important to the analysis of the data but cannot be determined from the energetics alone. Further consideration is given to the possible interactions and geometries in Sections 9.4.3 and 9.4.4.

\subsubsection{Change in Peak Spacings and Franck-Condon Simulations}

Another of the effects observed in the data is the change in peaks spacings and intensities in the $\mathrm{IHI}^{-}(\mathrm{M})_{\mathrm{n}}$ spectra with respect to the $\mathrm{IHI}^{-}$peak spacings. The change in the

vibrational pattern of the $[\mathrm{IHI}]^{\ddagger}$ complex can result from two possible effects. The solvent molecules can induce changes in the spectrum either by distortion of the anion, whereby the Franck-Condon region for photodetachment is changed to a different part of the reaction surface, or by a direct interaction with the neutral complex, changing the potential energy surface on which the $I+H I$ reaction proceeds. Most likely, the changes result from a combination of the two effects, the latter becoming more important as the cluster size increases.

While, in general, a different peak spacings in various clusters is not surprising, the shift of one vibrational peak with respect to the other members of a progression is unusual. This is the observation made for the $\operatorname{IDI}^{-}\left(\mathrm{N}_{2} \mathrm{O}\right)$ and $\mathrm{III}^{-}\left(\mathrm{CO}_{2}\right)$ spectra. For the $\operatorname{DI}^{-}\left(\mathrm{N}_{2} \mathrm{O}\right)$ and $\mathrm{DI}^{-}\left(\mathrm{CO}_{2}\right)$ spectra, the $v_{3}=2$ peak shifts with respect to the $\mathrm{v}_{3}=0$ and $\mathrm{v}_{3}=4$ peaks. In order to 
understand the physical basis for this phenomenon, it is useful to consider the Franck-Condon overlap between the $\mathrm{IHI}^{-}$vibrational wave functions and $\mathrm{I}+\mathrm{HI}$ scattering wave functions. As a first approximation, we will assume that for the $\mathrm{IDI}^{-}(\mathrm{M})$ photodetachment transition, the solvent interaction is strong enough to invoke an anion distortion but the interaction of the $M$ with the $[\mathrm{IDI}]^{\ddagger}$ complex is negligible. Thus, the neutral reaction surface for $\mathrm{I}+\mathrm{HI}$ can be used without including distortion arising from solvent interactions. In this case, Franck-Condon factor calculations will be very much like those for bare $\mathrm{IHI}^{-}$but the region of Franck-Condon overlap has been shifted as a result of the different anion geometry.

As will be seen, we find that the behavior can be reproduced by assuming that the anion distortion occurs along the $\mathrm{R}_{\mathrm{I}-\mathrm{I}}$ coordinate while keeping a central equilibrium position for the $\mathrm{H}$-atom. Within these constraints, one-and two-dimensional Franck-Condon simulations are presented in the following sections. These simulations and considerations of the potential energy surface provide a qualitative understanding of the observed behavior of the $v_{3}=2$ peak in the $\mathrm{IDI}^{-}\left(\mathrm{N}_{2} \mathrm{O}\right)$ and the $\mathrm{IDI}^{-}\left(\mathrm{CO}_{2}\right)$ spectra.

\subsubsection{One-dimensional Simulations}

We have shown previous chapters that the use of one-dimensional models can be very useful in understanding the qualitative nature of the transition state spectra even though the reaction has additional degrees of freedom available. As shown previously, the profile of the $\mathrm{IHI}^{-}$photoelectron spectrum can be modeled by calculating 1D FCFs along the antisymmetric stretch coordinate for that system. This is similar to the analysis used for the $\mathrm{OH}+\mathrm{H}_{2} \mathrm{O}$ reaction in Chapter 8. The Franck-Condon factor is calculated according to Eq. (3),

$$
I \propto v_{e} \cdot\left|\tau_{e}\right|^{2}\left|\left\langle\Psi_{v^{\prime}}\left(Q_{3}\right) \mid \Psi_{v^{\prime \prime}}\left(Q_{3}\right)\right\rangle\right|^{2}
$$


In Eq. (3), $\Psi_{\mathrm{v}^{\prime \prime}}$ and $\Psi_{\mathrm{v}^{\prime}}$ are the vibrational wave functions of the anion and neutral, respectively, along the $v_{3}$ asymmetric stretch coordinate and $v_{e}$ is the asymptotic velocity of the photodetached electron. The electronic transition dipole, $\tau_{e}$, is assumed to be constant as a function of eKE.

For the $\mathrm{IHI}^{-}$photodetachment studies, the 1D potential energy curves were obtained by taking slices of the semi-empirical, two-dimensional, collinear LEPS-A potential energy surface $^{28}$ at various fixed interiodine distances. In that analysis, the best agreement of peak spacings and integrated intensities was found with the data when $R_{\mathrm{I}-\mathrm{I}}$ was assumed to be 3.88 $\AA$. In a similar analysis of the $\mathrm{IH}^{-}\left(\mathrm{N}_{2} \mathrm{O}\right)$ and $\mathrm{IH}^{-}\left(\mathrm{CO}_{2}\right)$ spectra, the same variables were adjusted and the best agreement was found using $\mathrm{R}_{\mathrm{I}-\mathrm{I}^{\prime}}=3.925 \AA$ in both cases. The stick spectra which result from the calculations are convoluted with the experimental resolution and shown in Fig. 9.7 along with the $\mathrm{IDI}^{-}$and $\mathrm{IDI}^{-}\left(\mathrm{N}_{2} \mathrm{O}\right)$ spectra.

An understanding of why the $v_{3}=2$ peak shifts relative to the $v_{3}=0$ and the $v_{3}=4$ peaks is found by studying the one-dimensional potential energy curves at the interiodine distance employed in the $\mathrm{IDI}^{-}$and the $\mathrm{DI}^{-}(\mathrm{M})$ simulations. Shown in Fig. 9.8 are the onedimensional slices from the collinear $\mathrm{I}+\mathrm{HI}$ reaction surface at $\mathrm{R}_{\mathrm{I}-\mathrm{I}^{\prime}}=3.88$ and $3.925 \AA$. As the Figure shows, the separation of the two wells and the height of the barrier between them increases as a function of $\mathrm{R}_{\mathrm{II}}$. The eigenvalues shown are the $\mathrm{v}_{3}=0,2$ and 4 for the [IDI] species at the two different interiodine separations.. The position of the $v_{3}=2$ level near the top of the barrier results in a significant energy change for that level when the barrier height is

Figure 9.7: One-dimensional simulations of the $\operatorname{IDI}^{-}\left(\mathrm{N}_{2} \mathrm{O}\right)$ and the $\operatorname{mI}^{-}\left(\mathrm{CO}_{2}\right)$ photoelectron spectra collected at hv $=4.657 \mathrm{eV}$ using the LEPS-A semi-empirical potential energy surface. The interiodine distance used is $\mathrm{R}_{\mathrm{I}-\mathrm{I}^{\prime}}=3.925 \AA$.

Figure 9.8: One-dimensional potential energy curves from the $I+H I$ LEPS-A reaction surface, shown with the eigenvalues determined for the deuterated analog, at two different interiodine distances $\left(\mathrm{R}_{\mathrm{I}-\mathrm{I}^{\prime}}=3.88\right.$ and $3.925 \AA$ ). 


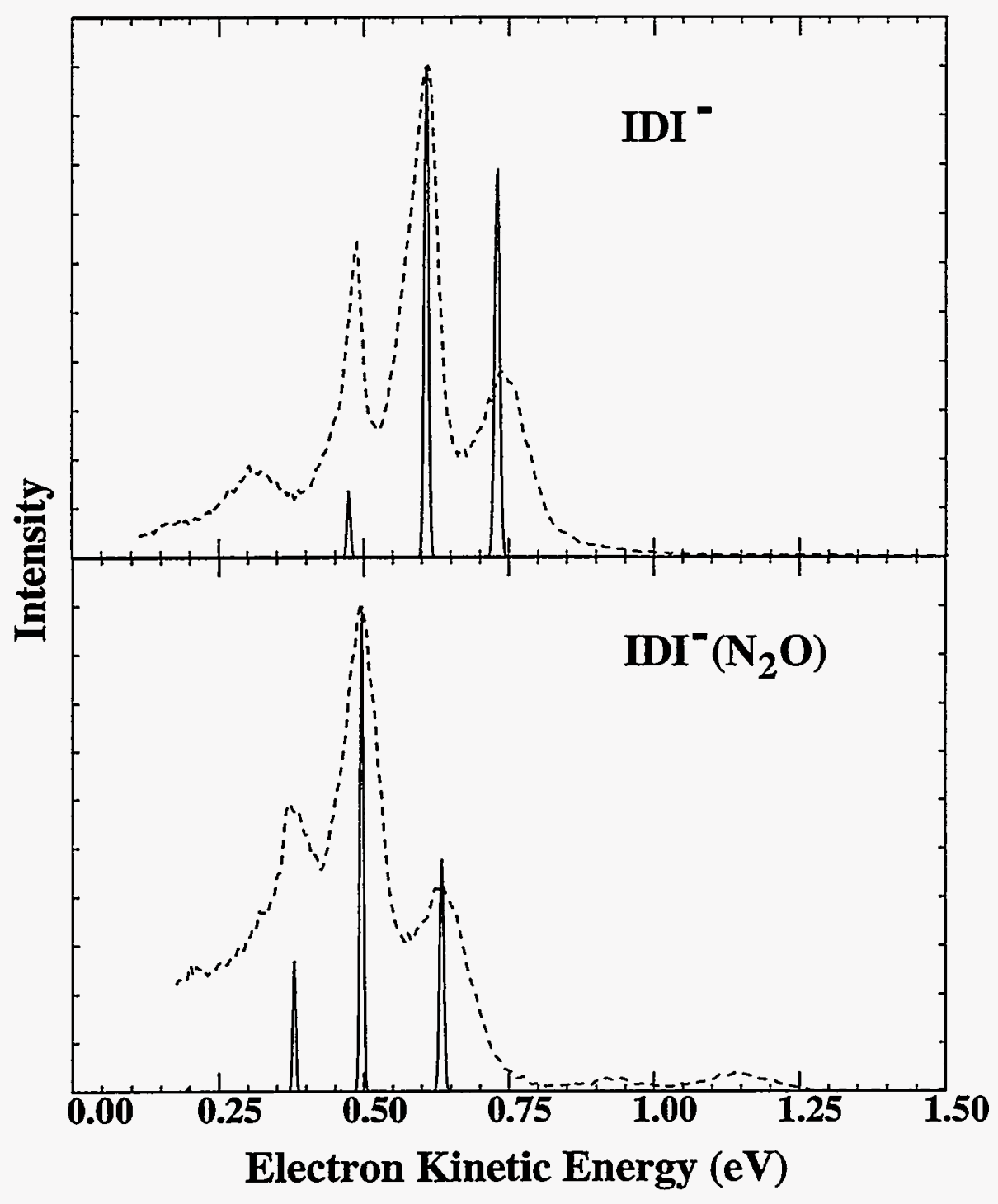

Figure 9.7 


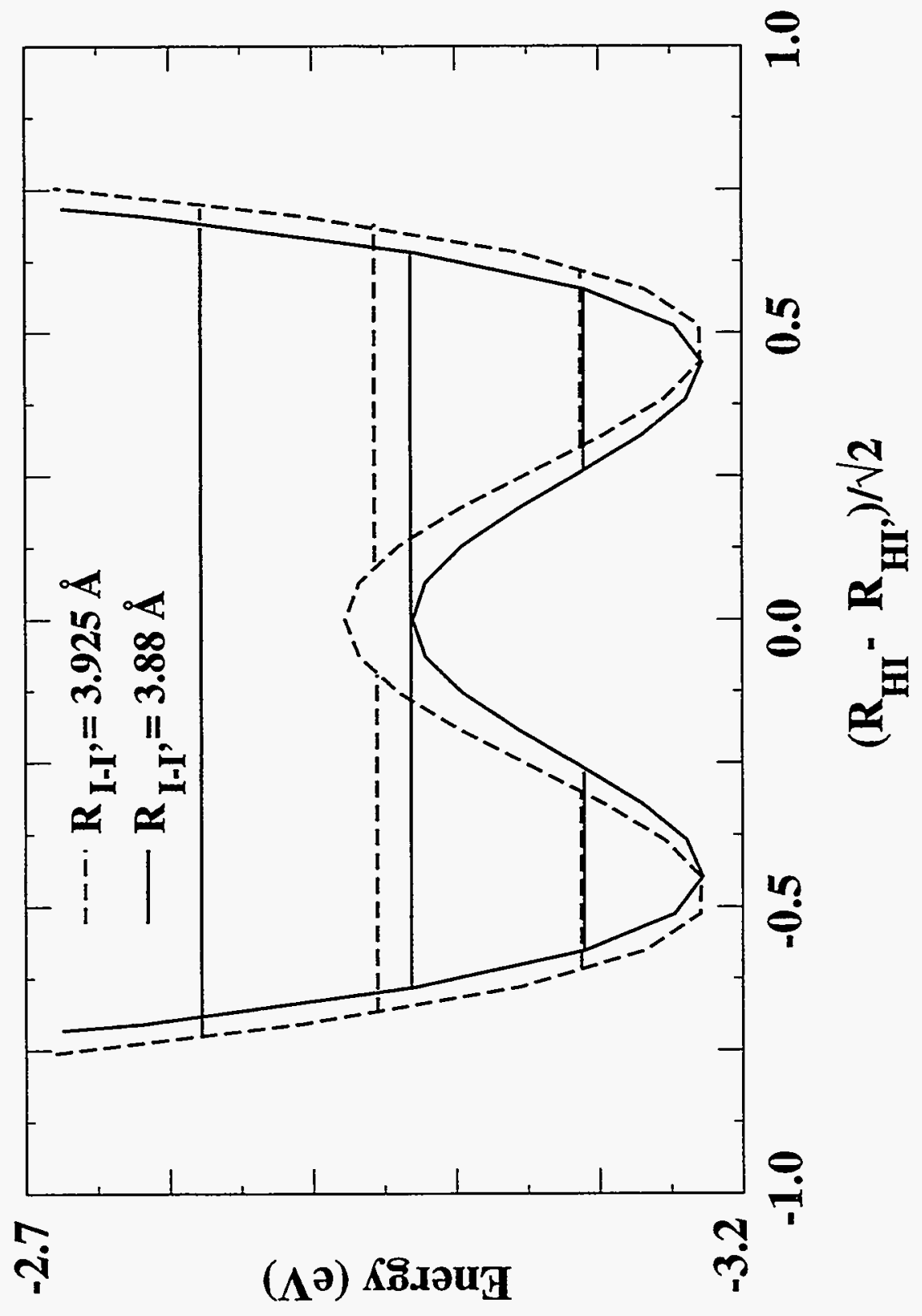

Figure 9.8 
changed. The $v_{3}=0$ and the $v_{3}=4$ levels are far enough below and above the barrier, respectively, that they do not experience significant shifts as a result of the change in barrier height. The result is that the $v_{3}=2$ level shifts with respect to the other two allowed transitions as observed in the photoelectron spectrum.

\subsubsection{Two-dimensional Simulations}

Two-dimensional simulations using the LEPS-A collinear potential energy surface have also been performed to determine what differences might be expected if the symmetric stretch is included in the Franck-Condon calculation. These calculations are determined by a timedependent wave packet propagation method, based upon the methods of Kosloff and Kosloff ${ }^{29}$ that has been adapted by S. E. Bradforth to simulate photoelectron spectra of linear a triatomic, $\mathrm{AHB}^{-}{ }^{-30,31}$ The calculation is quantum mechanically exact for the given two-dimensional collinear potential surface.

In the calculations, the anion ground state wave function is treated as a twodimensional separable harmonic oscillator, where the two frequencies are the symmetric and antisymmetric stretches of the anion $\left(\mathrm{IHI}^{-}-\omega_{1}=121 \mathrm{~cm}^{-1} ; \omega_{3}=682 \mathrm{~cm}^{-1} ; \mathrm{DI}^{-}-\omega_{1}=124\right.$ $\left.\mathrm{cm}^{-1} ; \omega_{3}=470 \mathrm{~cm}^{-1}\right)$. Figure 9.9 shows a projection of the wave functions onto the LEPS-A potential energy surface for the $\mathrm{I}+\mathrm{HI}$ reaction. The only difference between the two wave functions is their assumed interiodine distance, $\mathrm{R}_{\mathrm{I}-\mathrm{I}}=3.88 \AA$ vs. $\mathrm{R}_{\mathrm{I}-\mathrm{I}}=3.925 \AA$. This illustrates how the change in geometry affects the Franck-Condon region for photodetachment.

Figure 9.9: London-Eyring-Polanyi-Sato semi-empirical potential energy surface for the I

$+\mathrm{HI}$ reaction, plotted in mass weighted coordinates. Contours are at $0.05,0.2$, $0.5,0.9,1.4,1.9,2.1,2.5$ and $2.9 \mathrm{eV}$ with respect to the $\mathrm{I}+\mathrm{HI}$ asymptote. The $\mathrm{X}$ indicates the saddle point for the surface and the shaded region indicates the $95 \%$ limits of the Franck-Condon region for photodetachment of the $\mathrm{IHI}^{-}$anion assuming $\mathrm{R}_{\mathrm{I}-\mathrm{I}}=3.88 \AA$ and $\mathrm{RI}-\mathrm{I}=3.925 \AA$. 


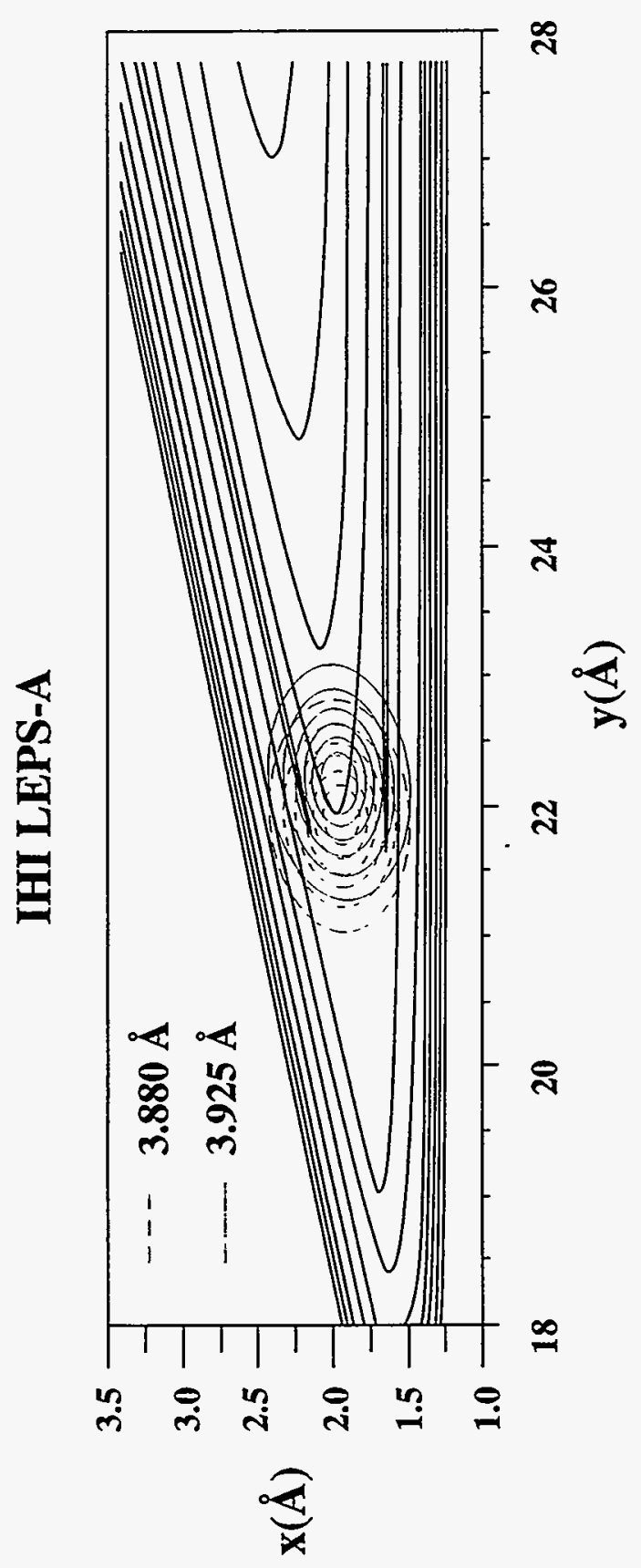

Figure 9.9 
This wave function serves as the initial wave packet which is propagated on the neutral reaction potential energy surface using the time-dependent Schrodinger equation, the solution of which is given in Eq. (4),

$$
|\phi(t)\rangle=e^{-i \hat{H} t / \hbar}|\phi(0)\rangle
$$

In Eq. (4), $e^{-i \hat{H} t / \hbar}$ is the time evolution operator and $\hat{H}$ is the Hamiltonian for the neutral surface. The overlap of $\phi(t)$ with $\phi(0)$ is known as the time autocorrelation function, $C(t)$,

$$
C(t)=\langle\phi(0) \mid \phi(t)\rangle
$$

which yields the photoelectron spectrum through a Fourier transformation, ${ }^{32}$

$$
\sigma(E)=\int_{-\infty}^{\infty} e^{i E t / \hbar} C(t) d t
$$

The $I+H I$ potential energy surface used for these calculations is the LEPS-A surface with a bending vibration zero-point (ZPB) energy correction added at each point on the surface. The wave packet is propagated for a total of 0.75 ps, yielding a best possible resolution (or narrowest possible peak) of $\sim 45 \mathrm{~cm}^{-1}$ for the simulation.

Shown in Fig. 9.10 are the 2D simulations which result from propagation of the wave

Figure 9.10: Franck-Condon calculations for $\mathrm{IHI}^{-}$and $\mathrm{II}^{-}$photodetachment using the time-dependent wave packet propagation technique. The LEPS-A + ZPB surface is used for the $\mathrm{I}+\mathrm{HI}$ reaction surface. The simulations are calculated at two interiodine distances, $3.88 \AA$ and $3.925 \AA$. 


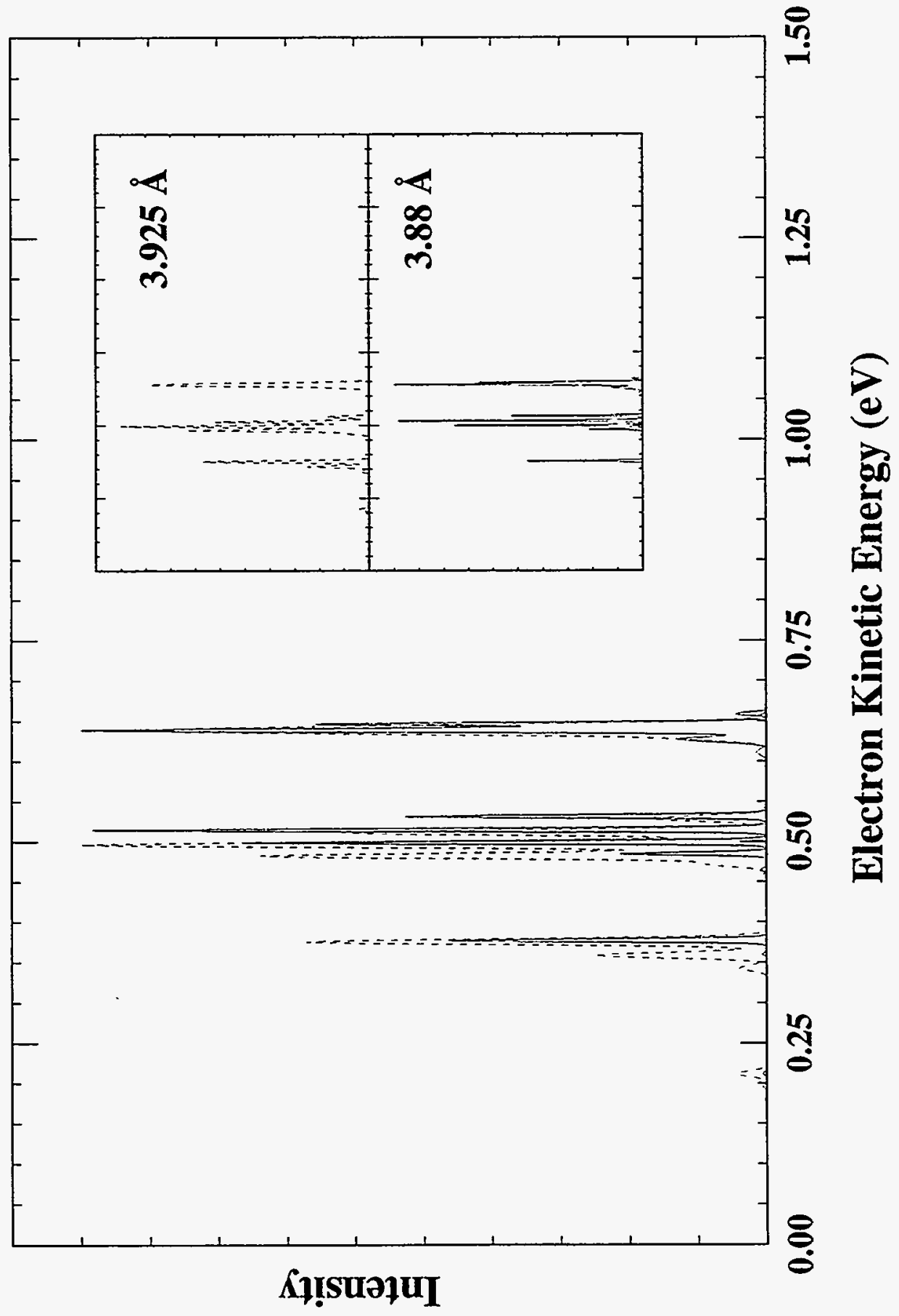

Figure 9.10 
packets shown in Fig. 9.9. Figure 9.11 compares the data to the simulations for the clustered $\mathrm{IHI}^{-}$and $\mathrm{IDI}^{-}$species. The simulations predict excitation of both the symmetric and antisymmetric stretch motions of the $[\mathrm{IDI}]^{\ddagger}$ complex. As observed in the $1 \mathrm{D}$ simulations and in the photoelectron spectra, the $v_{3}$ progression becomes more extended as the interiodine distance is increased. A similar trend is predicted for the I-I symmetric stretch progression.

However, while the effect is very noticeable for the $v_{3}=2$ band, the $v_{3}=0$ and $v_{3}=4$ are less sensitive to the change in $R_{\mathrm{I}-\mathrm{l}}$. Thus, the center of the $v_{3}=2$ band will move with increasing $R_{I-I}$ while the $v_{3}=0$ an the $v_{3}=4$ band centers will remain relatively stationary. The frequency of the symmetric stretch motion increases as a function of the $v_{3}$ level, due to a coupling between the two motions, increasing $v_{3}=0-v_{3}=2$ separation even further. In agreement with the 1D simulations, these results indicate that the uneven changes in peak spacings result from the solvent perturbation of the $\mathrm{IHI}^{-}$anion to an increased $\mathrm{R}_{\mathrm{I}-\mathrm{I}}$ separation relative to the bare $\mathrm{IHI}^{-}$anion.

The agreement between the $1 \mathrm{D}$ and the $2 \mathrm{D}$ simulations strongly supports the interpretation of the data as the photodetachment of a solvent-distorted anion. The changes in the spectrum provide information about the degree to which the solvent and the anion interact. This validation will be important to the qualitative interpretation of the spectra for the larger clusters in the next section.

\subsubsection{Larger Clusters}

As seen in Figs. 1 - 5 the features of the $\mathrm{IH}^{-}(\mathrm{M})_{\mathrm{n}}$ spectra become broaden significantly as the cluster size increases. The increasing peak width (as a function of cluster size) most likely results from unresolved intermolecular vibrational bands. Although the uncertainty in

Figure 9.11: Comparison of the $\mathrm{IHI}^{-}(\mathrm{M})$ and $\mathrm{IDI}^{-}(\mathrm{M})$ experimental data to Franck-Condon simulations using the time-dependent wave packet propagation technique. 


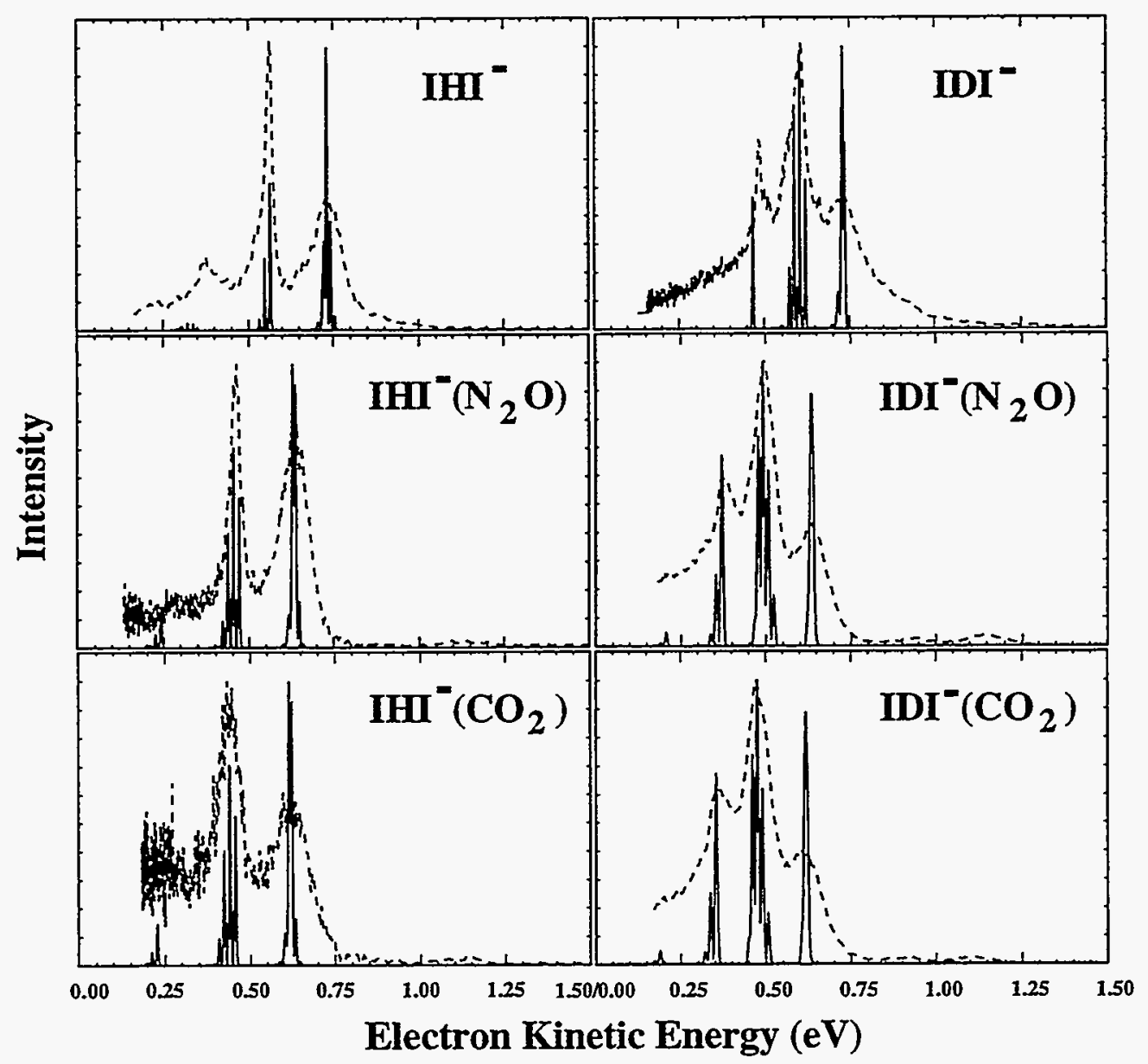

Figure 9.11 
the peak centers and integrated intensities for such broad overlapping peaks limits the quantitative analysis which can be performed, it is clear that the higher $v_{3}$ levels gain intensity, relative to the lower levels, as a function of cluster size. Due to the large uncertainties for the peak centers, changes in peak spacings for the larger clusters are less obvious.

This observation can be considered qualitatively in terms of solvent effects on the I+ HI reaction surface. The most critical property of the surface which can be modified by the solvent is the barrier height. By simple electrostatic arguments, it seems that the interactions of solvent molecules with a polar $H$ molecule (reactant or product) will be stronger than the interactions with the transition state complex. These unequal interactions result in an increase in the barrier height for the reaction as compared to that for the gas-phase reaction.

This interpretation is consistent with the change in peak intensities as a function of cluster size discussed in the previous section. The $\mathrm{DI}^{-}(\mathrm{M})$ 1D simulation showed that as the barrier height (along the $v_{3}$ coordinate) increased, the $v_{3}$ progression becomes more extended. However, it is possible that the changes in the observed spectral profile result from a distortion of the anion potential energy surface which accompanies solvation. Perhaps the shape of the surface changes in addition to the shift in the position of the equilibrium configuration, as we have assumed. It is quite difficult to separate the effects of anion-solvent and neutral-solvent effects at this level of analysis.

A more quantitative treatment is limited by the complexity of the system. As the cluster size increases, the interactions of the solvent molecules with the neutral complex and with each other become more important. As a result, for the larger clusters, the experiments can not simply be approximated as the photodetachment of the anion cluster to a neutral species which moves on the $I+H I$ potential energy surface. Instead, the $(I+H)(M)_{n}$ surface becomes fully established and must be considered in the data analysis. Since the $\mathrm{IHI}^{-}$anion efficiently localizes the extra electron electron when clustered with either $\mathrm{N}_{2} \mathrm{O}$ or $\mathrm{CO}_{2}$ (as evidenced by the general appearance of the data), the photoelectron spectra provide information about this 
solvated reaction surface. As evidenced by the effort required to calculate an accurate $a b$ initio surface for the much simpler $\mathrm{F}+\mathrm{H}_{2}$ reaction (Chapter 7), it does not seem feasible to approach the problem from this brute force direction initially. This is especially true when one considers the increased number of degrees of freedom which must be considered for the solvent molecules. Rather, it is probably more useful to begin with a perturbative approach.

As a first step, perhaps an effective potential energy surface can be constructed by calculating the effect of a static solvent field on the reaction surface. Modifications of the solvent field can be made to model different cluster sizes and geometries. This effective reaction surface can then be used to compare with the data. ${ }^{33}$ At this point, it will be possible to determine 1) what region of the 'solvated potential energy surface' is being probed by photodetachment of the clustered anion and 2) how the reaction surface differs from the gas phase bimolecular reaction surface.

\subsubsection{Feature "A" and Possible Anions Geometries}

As mentioned above, a broad structureless feature appears in the $\operatorname{IH}^{-}(\mathrm{M})_{\mathrm{n}}$ data, designated as "A", which does not have a corresponding feature in the $\mathrm{IHI}^{-}$spectrum. The position of peak "A" is each spectrum is summarized in Table V. Since these experiments include mass-selection prior to photodetachment, it is known that the species responsible for feature ' $A$ ' has the same chemical composition as $\mathrm{IHI}^{-}(\mathrm{M})_{\mathrm{n}}$ as it enters the laser interaction region. The intensity of this peak is dependent upon the ion source conditions, implying that it is best attributed to the photodetachment of a second isomer of the cluster ion. Feature 'A' consistently lies to higher eKE than the those peaks which are readily associated with the $\mathrm{IHI}^{-}$ spectrum. In fact, for $\mathrm{IHI}^{-}(\mathrm{M})_{\mathrm{n}=1}$ and $\mathrm{HH}^{-}(\mathrm{M})_{\mathrm{n}=2}$, feature 'A' lies to higher eKE than the spectrum of the bare $\mathrm{IHI}^{-}$spectrum. Thus, this feature results from the photodetachment to a neutral with an threshold photodetachment energy which is significantly lower than that of the $[\mathrm{IHI}]^{\dot{亠}}$ complex. 
Table V: Position of feature ' $\mathrm{A}^{\prime}$ in the $\mathrm{IHI}^{-}(\mathrm{M})_{\mathrm{n}}$ photoelectron spectra

\begin{tabular}{|c|cc|c|c|}
\hline \multirow{2}{*}{} & \multicolumn{2}{|c|}{$\mathrm{IHI}^{-}\left(\mathrm{N}_{2} \mathrm{O}\right)_{\mathrm{n}}$} & $\mathrm{IHI}^{-}\left(\mathrm{CO}_{2}\right)_{\mathrm{n}}$ & $\mathrm{IDI}^{-}\left(\mathrm{CO}_{2}\right)_{\mathrm{n}}$ \\
\cline { 2 - 5 } & $4.657 \mathrm{eV}$ & $5.822 \mathrm{eV}$ & $5.822 \mathrm{eV}$ & $5.822 \mathrm{eV}$ \\
\hline $\mathrm{n}=1$ & 1.15 & 2.24 & 2.26 & 2.26 \\
$\mathrm{n}=2$ & 1.05 & 2.20 & 1.95 & 1.95 \\
$\mathrm{n}=3$ & 0.90 & 2.10 & 1.80 & 1.82 \\
$\mathrm{n}=4$ & 0.80 & 2.00 & $\ldots$ & $\ldots$ \\
$\mathrm{n}=5$ & 0.70 & $\ldots$ & $\ldots$ & $\ldots$ \\
\hline
\end{tabular}

The behavior of the feature as a function of cluster size provides a clue to its identity. In the $\mathrm{IHI}^{-}\left(\mathrm{N}_{2} \mathrm{O}\right)_{\mathrm{n}}$ data collected at $\mathrm{hv}=4.657 \mathrm{eV}$, feature 'A' shifts an average of $100 \mathrm{meV}$ per solvent molecule without a continuous decrease in the SSE. These values are close to those observed for the solvation of $\mathrm{I}$ by $\mathrm{N}_{2} \mathrm{O}$. For the $\mathrm{IHI}^{-}\left(\mathrm{CO}_{2}\right)_{n}$ data, the two observed solvent shifts are significantly larger than those observed for $\mathrm{IH}^{-}\left(\mathrm{CO}_{2}\right)_{n^{*}}$. Thus, peak "A" in each spectrum represents photodetachment of a cluster in which the charge carrier, or chromophore, is better described as $\mathrm{I}^{-}$rather than $\mathrm{IH}^{-}{ }^{34}$ Beyond this observation, the data do not provide any further geometrical information about species " $\mathrm{A}$ ".

However, it is interesting to consider possible geometries for the $\operatorname{IH}^{-}(\mathrm{M})_{\mathrm{n}}$ species by comparison other results. The IHI- anion is believed to be a centrosymmetric species ${ }^{35,36}$ in which the negative charge is delocalized over both iodine atoms. Therefore, the solvent molecules, bound primarily by electrostatic forces, will encounter a fairly diffuse charge distribution. Some insight into the geometries of these species can be gained by comparison to the results for $\mathrm{CO}_{2}$ and $\mathrm{N}_{2} \mathrm{O}$ cluster to the iodide anion in Chapter 6 and to the studies of Lineberger and co-workers in which time-resolved measurements of $\mathrm{I}_{2}^{-}$photodissociation and recombination was measured in $\mathrm{I}_{2}^{-}\left(\mathrm{CO}_{2}\right)_{n}$ clusters. ${ }^{11}$ Briefly, the $\mathrm{I}^{-}(\mathrm{M})_{\mathrm{n}}$ PES studies showed that the $\mathrm{CO}_{2}$ sub-units within the $\mathrm{I}^{-}\left(\mathrm{CO}_{2}\right)_{\mathrm{n}}$ cluster were distorted from linearity by the chargequadrupole interaction while the $\mathrm{N}_{2} \mathrm{O}$ molecules in the $\mathrm{I}^{-}\left(\mathrm{N}_{2} \mathrm{O}\right)_{\mathrm{n}}$ clusters showed no such 
distortion. This distortion of the $\mathrm{CO}_{2}$ leads to a stronger interaction between the $\mathrm{CO}_{2}$ and the $\mathrm{I}^{-}$ with respect to that of $\mathrm{N}_{2} \mathrm{O}$. The larger $\mathrm{CO}_{2}$ solvation energy observed in the $\mathrm{IH}^{-}(\mathrm{M})$ spectra suggest that a similar distortion may also occur in the $\mathrm{IH}^{-}\left(\mathrm{CO}_{2}\right)$ clusters. While no $\mathrm{CO}_{2}$ progression, like that observed for the $\mathrm{I}^{-}\left(\mathrm{CO}_{2}\right)_{\mathbf{n}}$ data is observed in the $\mathrm{IHI}^{-}\left(\mathrm{CO}_{2}\right)_{\mathbf{n}}$ data, its presence cannot be ruled out because there is considerably more congestion in the $\mathrm{IH}^{-}(\mathrm{M})_{\mathrm{n}}$ spectra.

Lineberger and co-workers, as part of their analysis calculated possible geometries for several of the $\mathrm{I}_{2}^{-}\left(\mathrm{CO}_{2}\right)_{\mathrm{n}}$ clusters which they studied. ${ }^{37}$ Of importance to the present results, they found that the first three or four $\mathrm{CO}_{2}$ molecules formed a 'belt' around the $\mathrm{I}_{2}^{-}$anion which can be imagined as an equator around the $\mathrm{I}_{2}^{-}$bond axis. The similarity of the $\mathrm{I}_{2}^{-}$and $\mathrm{IHI}^{-}$ electrostatic properties would suggest that the same type of structure would result for the $\mathrm{IHI}^{-}\left(\mathrm{CO}_{2}\right)_{\mathbf{n}}$ clusters. In fact, although the calculations have not been performed, it is very likely that the $\mathrm{IHI}^{-}\left(\mathrm{N}_{2} \mathrm{O}\right)$ n clusters would roughly be described in the same manner due to the similar electrostatic properties of the $\mathrm{N}_{2} \mathrm{O}$ and $\mathrm{CO}_{2}$ molecules.

\subsection{Conclusions}

The photoelectron spectra of $\mathrm{IHI}^{-}(\mathrm{M})_{\mathrm{n}}$ have been collected at two photodetachment energies, $h v=4.657$ and $5.822 \mathrm{eV}$, where $\mathrm{M}=\mathrm{Xe}, \mathrm{N}_{2} \mathrm{O}$ and $\mathrm{CO}_{2}$ and $\mathrm{n} \leq 5$. The spectra contain two components. The first set of features, although broadened and shifted in eKE, is easily associated with the photoelectron spectrum of $\mathrm{HI}^{-}$. The second feature, which occurs at much higher eKE, has an ion source condition dependence and behaves differently from the rest of the spectrum as a function of cluster size. This feature is assigned to the photodetachment of another cluster isomer in which the $\mathrm{I}^{-}$anion is the main charge carrier.

For the single clusters $\left(\mathrm{IHT}^{-}(\mathrm{M})_{\mathrm{n}=1}\right.$ and $\left.\mathrm{IDI}^{-}(\mathrm{M})_{\mathrm{n}=1}\right)$ we assume that the anion-solvent interactions is much stronger than the neutral-solvent interaction which is assumed to be negligible. The data are interpreted in terms of photodetachment of a distorted anion cluster to 
the unstable $[\mathrm{IHI}]^{\ddagger}$ complex on an unperturbed $\mathrm{I}+\mathrm{HI}$ potential surface. In the treatment of the data, Franck-Condon simulations using the LEPS-A surface for the reaction show that this assumption is indeed plausible. The differences observed between the $\mathrm{IH}^{-}$spectrum and the $\mathrm{IH}^{-}\left(\mathrm{N}_{2} \mathrm{O}\right)$ and $\mathrm{HH}^{-}\left(\mathrm{CO}_{2}\right)$ spectra are qualitatively reproduced by simulations in which the $\mathrm{IH}^{-}$ interiodine separation is increased relative to the unclustered $\mathrm{IHI}^{-}$anion.

The spectra of the larger clusters become congested by unresolved transitions to closely spaced intermolecular vibrational levels of the neutral complex. The stepwise solvation energies determined from the shift of the spectra to lower eKE as the cluster size increases show that the solvent molecules do not interact equally in a stepwise fashion. For the large clusters, this technique is presented as a means of studying how solvent molecules change the potential energy surface for a chemical reaction, perhaps in the transition state region, in a stepwise fashion. The effects of solvation on the rates of chemical reactions are dependent upon how the solvent molecules influence the potential energy surface of the reaction, particularly in the transition state region. It is hoped that these results on a fairly simple reaction will inspire efforts to determine the effects of clustered solvent molecules on the reaction surface.

\subsection{Acknowledgments}

This work has been supported by the United States Air Force Office of Scientific Research under contract number F49620-94-1-0115. 


\section{References}

${ }^{1}$ A. Weaver, R. B. Metz, S. E. Bradforth, and D. M. Neumark, J. Phys. Chem. 92, 5558 (1988).

${ }^{2}$ S. E. Bradforth, A. Weaver, D. W. Arnold, R. B. Metz, and D. M. Neumark, J. Chem. Phys. 92, 7205 (1990).

${ }^{3}$ R. B. Metz, A. Weaver, S. E. Bradforth, T. N. Kitsopoulos, and D. M. Neumark, J. Phys. Chem. 94, 1377 (1990).

${ }^{4}$ S. E. Bradforth, D. W. Arnold, R. B. Metz, A. Weaver, and D. M. Neumark, J. Phys. Chem. 95,8066 (1991).

5D. W. Arnold, E. H. Kim, C. Xu, and D. M. Neumark, J. Chem. Phys. (to be submitted).

${ }^{6}$ S. E. Bradforth, D. W. Arnold, D. M. Neumark, and D. E. Manolopoulos, J. Chem. Phys. 99, 6345

(1993); D. E. Manolopoulos, K. Stark, H. -J. Werner, D. W. Arnold, S. E. Bradforth, and D. M.

Neumark, Science 262, 1852 (1993).

7J. Troe, J. Phys. Chem. 90, 357 (1986).

${ }^{8}$ M. Maroncelli, J. MacInnis, and G. R. Fleming, Science 243, 1674 (1989); M. Maroncelli, J. Chem. Phys. 94, 2084 (1991).

9J. D. Simon, Pure and App. Chem. 62, 2243 (1990).

${ }^{10}$ P. F. Barbara, G. C. Walker, and T. P. Smith, Science 256, 975 (1992); J. C. Alfano, Y. Kimura, P. K. Walhout, and P. F. Barbara, Chem. Phys. 175, 147 (1993).

${ }^{11}$ D. Ray, N. E. Levinger, J. M. Papanikolas, and W. C. Lineberger, J. Chem. Phys. 91, 6533 (1989); J. M. Papanikolas, J. R. Gord, N. E. Levinger, D. Ray, V. Vorsa, and W. C. Lineberger, J. Phys. Chem. 95, 8028 (1991); J. M. Papanikolas, V. Vorsa, M. E. Nadal, P. J. Campagnola, H. K. Buchenau, and W. C. Lineberger, J. Chem. Phys. 99, 8733 (1993).

12J. Steadman and J. A. Syage, J. Phys. Chem. 95, 10326 (1991); J. A. Syage, J. Phys. Chem. 97, 12523 (1993); ibid., Z. Phys. D 30, 1 (1994).

${ }^{13}$ M. Gutmann, D. M. Willberg, and A. H. Zewail, J. Chem. Phys. 97, 8048 (1992); E. D. Potter, Q. Liu, and A. H. Zewail, Chem. Phys. Lett. 200, 605 (1992); Q. Liu, J. -K. Wang, and A. H. Zewail, Nature 364, 427 (1993).

${ }^{14}$ R. W. Shaw, T. B. Brill, A. A. Clifford, C. A. Eckart, and E. U. Franck, Chem. and Engin. News 69 (No. 15) 26 (1991); H. D. Cochran, P. T. Cummings, and S. Karaborni, Fluid Phase Equilibria 71, 1 (1992).

${ }^{15}$ H. D. Cochran, R. S. Wu, and L. L. Lee, Sep. Sci. and Tech. 25, 2017 (1990); P. T. Cummings, H. D. Cochran, J. M. Simonson, R. E. Mesmer, and S. Karaborni, J. Chem. Phys. 94, 5606 (1991); J. I. Siepman, S. Karaborni, and B. Smit, Nature 365, 330 (1993).

${ }^{16}$ I. M. Waller, T. N. Kitsopoulos, and D. M. Neumark, J. Phys. Chem. 94, 2240 (1990). 
${ }^{17}$ D. M. Neumark, In Advances in Molecular Vibrations and Collisional Dynamics, Vol 1A, pp. 165-185, (JAI Press, New York, 1991).

18M. A. Johnson, M. L. Alexander, and W. C. Lineberger, Chem. Phys. Lett. 112, 285 (1984).

${ }^{19} \mathrm{~A}$ mixture of $80-90 \% \mathrm{Ne}$ and $10-20 \%$ He known as 'first run Ne' available from Airco Products. Other carrier gases included pure $\mathrm{He}, \mathrm{Ar}$, and $\mathrm{N}_{2}$.

${ }^{20}$ The low energy secondary electrons result from ionization of the gases in the expansion by the high energy electrons during which a low energy electron, a high energy electron and a positive ion are generated.

${ }^{21}$ W. C. Wiley and I. H. McLaren, Rev. Sci. Instrum. 26, 1150 (1955).

${ }^{22}$ C. E. Moore, Atomic Energy Levels, Vol. I, NSRDS-NBS 35, (1971).

${ }^{23}$ K. P. Huber and G. Herzberg, Molecular Spectra and Molecular Structure. IV. Constants of Diatomic Molecules. Von Nostrand (New York, 1979).

${ }^{24}$ M. R. Battaglia, A. D. Buckingham, D. Neumark, R. K. Pierens, and J. H. Williams, Mol. Phys. 43, 1015 (1981).

${ }^{25}$ L. H. Sharpen, J. S. Muenter, and V. W. Laurie, J. Chem. Phys. 53, 2513 (1970).

${ }^{26}$ A. D. Buckingham, C. Graham, and J. H. Williams, Mol. Phys. 49, 703 (1983).

${ }^{27}$ The negative sign of $\Theta$ indicates that the center atom is positively charged with respect to the outer atoms.

28J. Manz and J. Römelt, Chem. Phys. Lett. 81, 179 (1981).

${ }^{29}$ D. Kosloff and R. Kosloff, J. Comput. Chem. 52, 35 (1983); R. Kosloff, J. Phys. Chem. 92, 2087 (1988).

${ }^{30}$ S. E. Bradforth, A. Weaver, D. W. Arnold, R. B. Metz, and D. M. Neumark, J. Chem. Phys. 92, 7205 (1990).

${ }^{31}$ S. E. Bradforth, Ph. D. thesis, University of California, Berkeley (1992).

32E. J. Heller, Acc. Chem. Res. 14, 368 (1981).

${ }^{33}$ Such studies have recently begun in the research group of B. Gerber (private communication).

${ }^{34}$ In the $\mathrm{IHI}^{-}\left(\mathrm{N}_{2} \mathrm{O}\right)$ and $\mathrm{IHI}^{-}\left(\mathrm{CO}_{2}\right)$ spectra, peak "A" is separated from the eKE of $\mathrm{I}^{-}$photodetachment transitions $(\mathrm{eKE}=2.76 \mathrm{eV}$ at hv $=5.822 \mathrm{eV})$ by $\sim 0.5 \mathrm{eV}$.

${ }^{35}$ C. M. Ellison and B. S. Ault, J. Phys. Chem. 83, 832 (1979).

${ }^{36}$ K. Kawaguchi and E. Hirota, J. Chem. Phys. 87, 6838 (1987); ibid., 84, 2953 (1986); K. Kawaguchi, ibid., 88, 4186 (1988). 


\section{Appendix A: PES: The Franck-Condon Analysis Program for Anion Photoelectron Spectra}

\section{Introduction}

As demonstrated thoughout this thesis, the analysis of data collected using the anion photoelectron spectroscopy technique requires the ability to calculate the Franck-Condon factors (FCF's) for the photodetachment process being studied. This appendix describes and contains the source code for a computer program, PES, which has been developed to compute the Franck-Condon overlap between the eigenvectors of two general one-dimensional potential energy surfaces (one for the anion and one for the neutral in the case of anion photoelectron spectroscopy). While the core of the code calculates only one-dimensional FCF's, combination band calculations can be made within the separable normal coordinate approximation. In addition, the code is set up to calculate FCF's for photodetachment transitions to multiple electronic states and convolute them all together for direct comparison with the experimental data specified by the user.

The code is currently set up to be used in the analysis of two types of experimental data: 1) that output by the anion photoelectron spectrometer (aka "Stonehenge") and 2) the data output by the threshold photodetachment spectrometer (aka "The ZEKE Machine" or "The Acropolis"). Options are available for easy installation of future data formats as they are created.

With the exception of the $\mathrm{F}+\mathrm{H}_{2}$ data presented in Chapter 7 , all of the data presented in previous Chapters has been analyzed with this code (or previous, more primitive versions).

The PES program is designed to be very user friendly and reasonably flexible. The concept behind the program is that the user will be attempting to fit the data by an iterative 
simulation process. For the first-run data analysis, the program prompts the user for several variables needed to perform the simulation. As part of the input, the user provides a FILENAME. The program saves all of the information provded in the first calculation in the file-'FILENAME.par'. These values can then be adjusted manually with a favorite text editor and then as one of the options at the beginning of the selection menu, these new parameters are read into the program automatically and used for the next simulation. This allows for a reasonably quick turn-around time per iteration. The code also has the capability to do a final numerical fit to the spectrum. HOWEVER, the input parameters must produce a simulation that is very close to the data set before allowing the Chi-Square analysis take over. This part of the routine will NOT fit the data, it will only polish the edges at the end. In fact, this part of the routine has been recently completed (i.e., they may be buggy) and the optimization methods will require improvements.

There are many features of PES which have been developed over the last six years. Initially, they were installed to address specific problems but have eventually become general features. Following is a summary of the capbiliies of the code, PES.

1. Multiple Electronic States - The overall relative intensity of each state can scaled, corresponding to the relative photodetachment cross-sections (or the electronic transition dipoles) for each state.

2. Multiple Vibrational Modes (for each electronic state) - PES treats multiple vibrational modes in the separable normal mode approximation. For multi-mode simulations, combination bands arise. The positions are calculated by summation of terms for individual mode vibrational levels and the intensities are determined by taking the product of individual mode FCFs. 
3. Degenerate Vibrational Modes - The code treats doubly degenerate vibrational modes (for example, a linear triatomic bending mode) if requested in the input. Basically, it calculates the intensity like the calculation of combination bands but is designed to do this upon request without the need for including a second mode of identical frequency.

4. Different Temperatures for Individual Vibrational Modes - Allows you to account for anion vibrational excitation which is not equilibrated between the different normal modes.

5.Harmonic / Morse Oscillator Defaults - PES asks for $\omega_{e}$ and $x_{e} \omega_{e}$ values. If $x_{e} \omega_{e}=$ 0.0 , the program calculates the HO FCFs analytically. If $x_{e} \omega_{e} \neq 0.0$, then a Morse potential, is generated using the given frequency and anharmonicity and the FCFs are determined numerically.

6. General Potential Functions possible - Since the program is now based around a numerical method, general potential functions may be used in the code. For this purpose, the user will need to modify the pot.f and the pescont.f files such that the potentials are used. This will be discussed in more detail below.

\section{Data/Simulation Plotting capability - PES uses NCAR (National Center for} Atmospheric Research) graphics routines to draw the simulations and the data for comparison.

8. Stonehenge and ZEKE format compatible - The program reads two data formats at present (and is easily modified for comparison to other data formats). Both data sets are X-Y format. The routine reads Stonehenge data as (electron counts*55) vs. eKE (eV). and ZEKE data and electron counts vs nanometers. Plots are made accordingly using NCAR routines. 
9. Diagnostic Output Features - The program provides several files which summarize the FCF calculations and serve as diagnostics for assignment of spectral features. A more detailed discussion is given below.

10. Chi-Square Optimization - Although not fully streamlined, PES is able to adjust fitting parameters to obtain better agreement between simulation and data based upon a chisquare minimization.

\section{Usage}

PES is indeed user-friendly. To begin the program, type "pes4". There are a minumum of six questions asked at the beginning of each calculation. The first question, "Are you an Acropolite or a Stonehenger?", is asked in order to determine the experimental data format. The two terms correspond to ZEKE and photoelectron spectrometer data, respectively. ${ }^{1}$ The code then asks about the calculations to be done. Either a manual fit or a chi-square optimization can be chosen. Then you can choose to read the input from a file or from the keyboard. If you choose the manual input the program will create a file with the parameters which you enter to be used later. The file will be called "FILENAME.par". Included in the file is a line-by-line description of the parameters, at the bottom. This file can be modified for future use and will be read in automatically by PES.

The input information you need to give the program is shown in sample input files shown below (Stonehenge and ZEKE) with the line description included:

sample1.par - Stonehenge

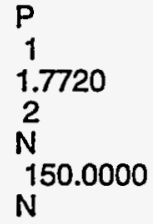




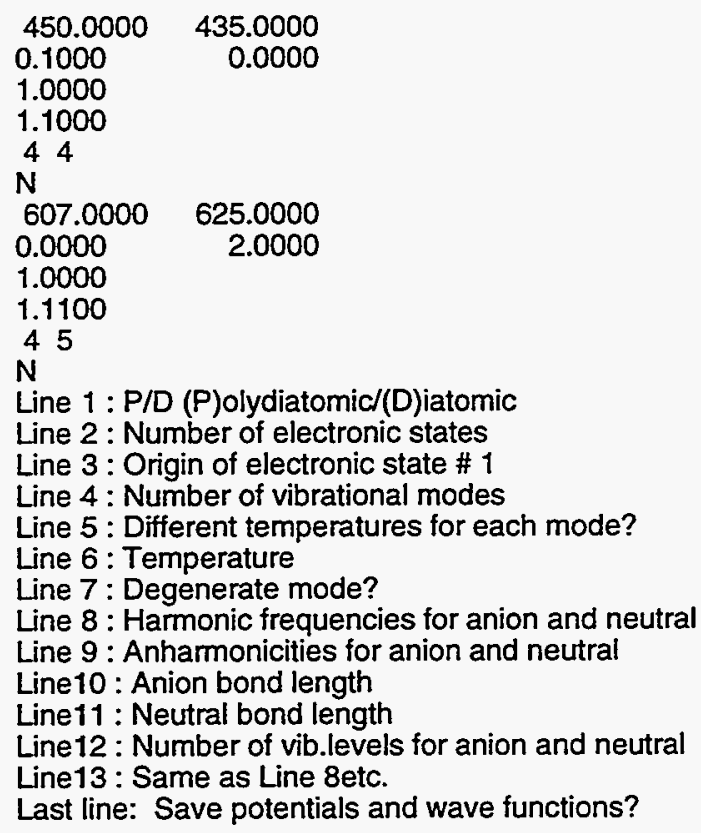

sample2.par - ZEKE

P

1

452.0000

1

50.0000

$\mathrm{N}$

$100.0000 \quad 95.0000$

$1.0000 \quad 0.3000$

1.0000

1.2000

36

N

Line 1 : P/D (P)olydiatomic/(D)iatomic

Line $2:$ Number of electronic states

Line 3: Origin of electronic state \# 1

Line 4 : Number of vibrational modes

Line 5 : Different temperatures for each mode?

Line $6:$ Temperature

Line 7 : Degenerate mode?

Line 8 : Harmonic frequencies for anion and neutral

Line 9: Anharmonicities for anion and neutra!

Line10: Anion bond length

Line11: Neutral bond length

Line12: Number of vib.levels for anion and neutral

Last line: Save potentials and wave functions?

The only difference in the input for the Stonehenge and ZEKE calculations is that energies are in $\mathrm{eV}$ and $\mathrm{nm}$, respectively, for the origin of the spectrum. Additional information is saved in the "plot.par" file if you want to plot the data on the computer screen using the NCAR graphics routines. Samples are shown below: If the chi-square optimization is chosen, similar files - 
"FILENAME.opt"- are generated indicating which variables are to be varied as the optimization

proceeds.

PLOT.PAR - Stonehenge

sample.ene

1000000

8.0000

12.0000

2.000

1.000

$\circ$

Line 1: Experimental file

Line 2: Number of counts in main peak

Line 3: Time Width - ns

Line 4: Energy Width - meV

Line 5: Maximum Energy to display

Line 6: Simulation Scaling Factor

\section{PLOT.PAR - ZEKE}

samzeke.ene

3000.00

5.0000

500.0000

1.000

0

Line 1: Experimental file

Line 2: Number of counts in main peak

Line 3: Resolution - $\mathrm{nm}$

Line 4: Maximum Energy to display

Line 5: Simulation Scaling Factor

Line 6: Terminal type

PES provides output for the analysis of the simulation results in two files: "fort.12" and

"FILENAME.ass". The fort.12 file contains a summary of the input parameters and all of the one-dimensional FCFs that are calculated:

\section{FORT.12}

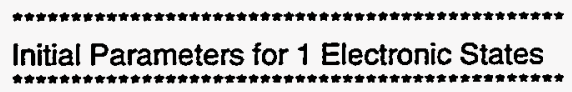

Parameters for Electronic State \# 1

State \# 1 scaled by 1.000

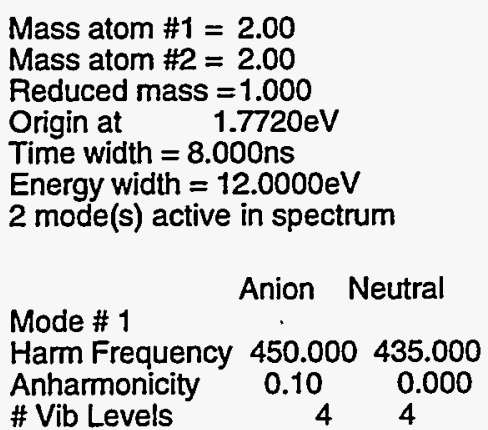




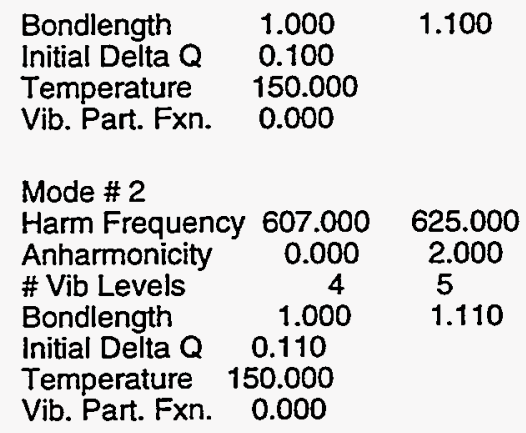

\section{Mode \# 2}

Harm Frequency $607.000 \quad 625.000$

Anharmonicity $\quad 0.000 \quad 2.000$

\# Vib Levels $\quad 4 \quad 5$

Bondlength $\quad 1.000 \quad 1.110$

Initial Delta Q 0.110

Temperature 150.000

Vib. Part. Fxn. 0.000

\section{N A Position}

A Position

$101.7181 \quad 0.0609$

$\begin{array}{lll}2 & 01.6641 & 0.0011\end{array}$

$\begin{array}{lll}3 & 01.6102 \quad 0.0000\end{array}$

$\%$ Population $=98.6626$

$\begin{array}{llll}0 & 11.8278 & 0.0594 & 0.0008 \\ 1 & 11.7738 & 0.8931 & 0.0119 \\ 2 & 11.7199 & 0.1069 & 0.0014\end{array}$

$\begin{array}{llll}3 & 11.6660 & 0.0025 & 0.0000\end{array}$

$\%$ Population $=1.3195$

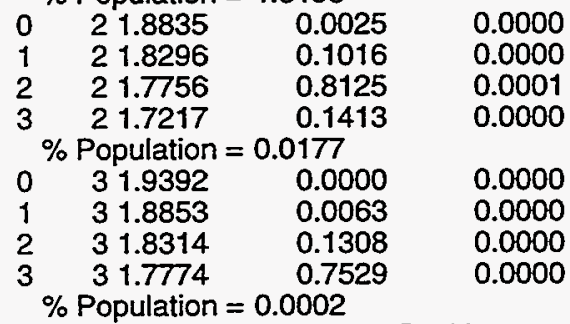

Peak FCF

11.0000000

20.0609089

$3 \quad 0.0010539$

$\begin{array}{ll}4 & 0.0007948\end{array}$

$\begin{array}{ll}5 & 0.0119444\end{array}$

$\begin{array}{ll}6 & 0.0014294\end{array}$

$\begin{array}{lll}N & \text { A } & \text { Position } \\ 0 & 0 & 1.7720\end{array}$

Position

1.7720000

1.7180664

1.6641328

1.8277685

1.7738349

1.7199014

1.6950

1.6185

1.5425

1.4670

$\%$ Population $=99.7039$

$\begin{array}{lll}0 & 1 & 1.8473\end{array}$

$\begin{array}{lll}0 & 1 & 1.8473 \\ 1 & 1 & 1.7703 \\ 2 & 1 & 1.6938\end{array}$

$\begin{array}{lll}2 & 1 & 1.6938\end{array}$

$\begin{array}{lll}3 & 1 & 1.6178\end{array}$

4111.5423

$\%$ Population $=0.2952$

$0 \quad 2 \quad 1.9225$

$\begin{array}{lll}1 & 2 & 1.8455\end{array}$

$2 \quad 2 \quad 1.7690$

321.6930

$\%$ Population $=0.0009$

$\begin{array}{lll}0 & 3 & 1.9978 \\ 1 & 3 & 1.9208 \\ 2 & 3 & 1.8443 \\ 3 & 3 & 1.7683 \\ 4 & 3 & 1.6928\end{array}$

$\%$ Population $=0.0000$

Peak FCF Position

71.00000001 .7720000
FCF Intensity(T)

1.0000

0.0000

0

FCF

1.0000

0.1331

0.0149

0.0026

0.0005

0.1435

0.6867

0.2568

0.0490

0.0113

0.0072

0.2968

0.3729

0.3320

0.0991

Intensity(T)

1.0000

0.1331

0.0149

0.0026

0.0005

0.0004

0.0020

0.0008

0.0001

0.0000

0.0000

0.0000

0.0000

0.0000

0.0000

$0.0005 \quad 0.0000$

$0.0309 \quad 0.0000$

$0.4146 \quad 0.0000$

$0.1290 \quad 0.0000$

$\begin{array}{ll}0.1290 & 0.0000 \\ 0.3312 & 0.0000\end{array}$ 


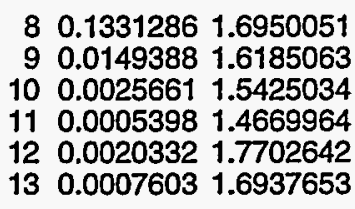

The FILENAME.ass output is very useful since it orders all of the transitions (above a threshold value of $0.02 \%$ intensity) according to energy and writes them out with the assignment according to the anion (A) and neutral (N) vibrational quanta involved in the partivular photodetachment transition:

\section{FILENAME.ass}

Line Position and Intensity Summary for Electronic State \#1

2 Vibrational Modes Used for Calculation

11 Lines Calculated with Intensity Greater than 0.0002

\begin{tabular}{|c|c|c|c|c|c|c|c|}
\hline \multirow[t]{2}{*}{ Mode } & \multicolumn{2}{|c|}{1} & \multicolumn{2}{|c|}{2} & $\begin{array}{l}\text { Position } \\
\text { (eV) }\end{array}$ & FCF & FCFS \\
\hline & $\begin{array}{l}0 \\
0 \\
0 \\
0 \\
0 \\
1 \\
0 \\
1 \\
0 \\
0 \\
1\end{array}$ & $\begin{array}{l}0 \\
0 \\
1 \\
2 \\
0 \\
1 \\
1 \\
2 \\
0 \\
0 \\
1\end{array}$ & $\begin{array}{l}0 \\
0 \\
0 \\
0 \\
0 \\
0 \\
0 \\
0 \\
1 \\
0 \\
0\end{array}$ & $\begin{array}{l}3 \\
2 \\
1 \\
0 \\
1 \\
1 \\
0 \\
0 \\
1 \\
0 \\
0\end{array}$ & $\begin{array}{l}1.5425 \\
1.6185 \\
1.6411 \\
1.6641 \\
1.6950 \\
1.6968 \\
1.7181 \\
1.7199 \\
1.7703 \\
1.7720 \\
1.7738\end{array}$ & $\begin{array}{l}0.0026 \\
0.0149 \\
0.0081 \\
0.0011 \\
0.1331 \\
0.0016 \\
0.0609 \\
0.0014 \\
0.0020 \\
1.0000 \\
0.0119\end{array}$ & $\begin{array}{l}0.0026 \\
0.0149 \\
0.0081 \\
0.0011 \\
0.1331 \\
0.0016 \\
0.0609 \\
0.0014 \\
0.0020 \\
1.0000 \\
0.0119\end{array}$ \\
\hline
\end{tabular}

In this file the FCF values are the intensity for each state relative to each other and FCFS

values are the intensities of the transitions for after they have been scaled according to electronic state by the user.

Other file output by PES are the fort. 7 and fort.11 files. Fort.7 is a listing of the positions and intensities (ordered in increasing energy) in a single column format. This format can be read by older codes (READFCF15.f) that the group used in ancient times. It can also be used to generate a stick spectrum using the separate code "STICK.f" which will be included at the end of the APPENDIX. The fort.11 file contains the simulated spectrum in a two column 
format (eKE vs. intensity or wavelength vs. intensity) which can be read into a favorite program to generate figures for publication.

\section{fort. 7 \\ 1 \\ 11 \\ 0.003187 \\ 1.542503 \\ 0.019005 \\ 1.618506 \\ 0.010388 \\ 1.641072 \\ 0.001360 \\ 1.664133 \\ 0.173323 \\ 1.695005 \\ 0.002071 \\ 1.696840 \\ 0.079836 \\ 1.718066 \\ 0.001875 \\ 1.719901 \\ 0.002705 \\ 1.770264 \\ 1.331165 \\ 1.772000 \\ 0.015908 \\ 1.773835

\author{
IArchaic scheme kept for readfcf15 program \\ Number of transitions \\ IFCF of transition \#1 \\ !Position of transition \#1 \\ IFCF of transition \#2 \\ !Position of transition \#2 \\ -$$
\text { ( }
$$

\section{Advanced usage/Modifications}


MAKEPES4

FFLAGS=-Bstatic - $\mathrm{cg} 89$-O3 -dalign

pes4: pescont4.0 input1.0 params3.o chisqs5.o plot.o calcfef7.o hofff1. o fcfnum1.0 dofcf2.o qfxn5.0 dgnits3.o hunts.o indexx.0 sims4.0 writout.o deriv1.0 makespec2.o tidyup3.o matinv. conv.o rs.o rsb.o matrix.o pot1.o grad1.o banner.o

ncargi77 -Bstatic -cg89-03 -dalign pescont4.0 input1.0 params3.0 chisq55.o plot.o calcfff7.o hofcf1.0 fcfnum1.o doff 2.0 qfxn5.0 dgnits3.o hunts. o indexx.0 sims 4.0 writout.o deriv1.o makespec2.o tidyup3.o matinv.o conv.o rs.o rsb.o matrix.o pot1.o grad1.o banner.o -o pes4

The ncargf77 compiler must be used for use with the NCAR graphics package. If the graphics routines are changed, a different compiler will be needed.

\section{General potentials-}

If you want to use potential energy surface in a general funtional form, this is possible by substituting the function into the file pot1.f, or better yet, creating a new subroutine that will provide an energy at a "bond length" that is sent from the FCFNUM routine. (The simulations in Chapter 8 were made using polynomial fits to $a b$ initio calculated data points along the same anion and neutrla coordinate. This set of values, the basis, is determined from the setdvr subroutine. The energy should be in atomic units (hartrees). If a general function is used, you have to have both an anion and neutral curve in the the same coordinates, with careful consideration given to the reduced mass and the equilibrium positions. One modification must be made to the FCFNUM routine where the POT1.f file is called to make sure the correct subroutine is chosen. When the general potentials are used, you must input the anion and neutral vibrational frequencies carefully because they determine the frequency of the basis functions. You should try to input frequencies that will reasonably cover the spatial extent of the general potential and hopefully have somewhat the same nodal structure as the wavefunctions supported by your potential surface. These basis functions are HO functions are when the Morse potentials are used as a default, the basis is chosen as a fraction ( 0.75 now) of the average of the anion and neutral frequencies.

\section{List of routines used in the PES program (most recent version is indicated):}

pescont4.f: Main controller routine calls other routines for calculations. 
banner.f: Displays program name.

calcfcf7.f: Given the FCFs for individual separable vibrational modes, this routine calculates combination bands which result. This code also sorts all of the lines within an electronic state, indexes them according to the transition responsible for the line and writes them in a file named "FILENAME.ass". Also makes electron velocity correction and outputs positions and FCFs for calculated transitions to file fort. 7 for future use.

chisqs5.f: Calculates a chi-square value for fitting of simulations to data conv.f: Checks for convergence of chi-square fit deriv1.f: Calculate derivative of chi-square value with respect to each variable being adjusted in fit of simulation to data.

dgnrts3.f: Accounts for a doubly degenerate vibrational mode.

dofcf2.f: This routine is called by fcfnum1.f to determine the wavefunctions and the FCF's from the eigenvectors and the $\mathrm{HO}$ basis set. Wavefunctions can be written out to files for viewing or graphical presentation purposes.

fcfnum1.f: This file contains several routines used for the numerical calculation of FCFs using HEG quadrature. Can calculate FCFs for Morse and HOpotentials witout modification using the frequency and anharmonicity input. For more complicated potential functions, modification is required.

grad.f: Calculates gradient for gradient search method of non-linear least squares fitting of the data.

hofcf1.f: This routine calculates analytic FCFs for harmonic oscillators (HOs). hunts.f: Routine from Numerical Recipes used to sort transitions according to energy.

indexx.f: Routine from Numerical Recipes used to assist in the indexxing of 
combination bands according to the transition associated with a line. input1.f: Routine to read input and convert letter case for later usage. makespec2.f: Subroutine does a 'barebones' FCF calculation during NLLSCSC. Makes calls to all necessary subroutines with minimal output. matinv.f: Routine from Bevington inverts a symmetric matrix and calculates the determinant. Used in the Marquardt-Levenberg portion of the NLLSCSC. matrix.f: Various subroutines for matrix multiplication, transposing and display.

params3.f: Prompts user for input to code. Collects parameters for calculations and plotting of data and simulation. Will read input from a formatted input deck files if they exist. If the files are non-existant, this routine will create the files with a user-defined FILENAME.

plot.f: Routines for plotting simulation and data using the NCAR graphics package. Two routines - one for Stonehenge data, one for Acropolis data. pot.f: Contains the routine for the $\mathrm{HO}$ and Morse potentials used in the numerical FCF calculations.

qfxn.f: Calculates partition functions for specified temperature (assumes Boltzman distribution).

rs.f: EISPACK routine for diagonaling a real symmetric matrix in numerical FCF calculations.

rsb.f: EISPACK routine for diagonaling a real symmetric banded matrix in the numerical FCF calculations.

sims4.f: Convolutes the FCF stick spectrum with the user specified resolution function. For Stonehenge data, this includes a time and energy contribution; for Acropolis data, only an energy contribution. Also, determines the energy range over which to calculate the simulation. 
tidyup2.f: Secondary "controller" routine which takes over the program if the user requests a non-linear least squares fit (NLLSF) to the experimental data.

writout.f: Generates output from calculations to terminal and/or files for

future user perusal.

\section{Other files needed - \\ FCFNUM.inc}

C

C

C
c
c
c
c
c
c
C

include file for fcfnum $1 . f$

parameter (MAXQBASIS=100, nad $=12, n X p t s=100, M A X I Q=20$ )

parameter (NFVMAXA=20,NFVMAXN=30)

MAXQBASIS = largest quantum number for basis fcn

nad $=$ max \# ad. curves

MAXQBASIS $=$ max \# pts. in tho

nXpts = same as above

$M A X I Q=\max \#$ of pts between pairs of pts in tho

for tho integral (overlaps)

$\&$

common/units/pi,harev,evwn,hbar,a0,amu,emu,clight,

harwn, amass, harkj

common/params6/HARMA,HARMN,XEWEA,XEWEN,REA,REN,REDMASS

common/params7/NQBASIS,IVIBA,IVIBN

common/params8/E00

common/vpot/vpoten(MAXQBASIS)

common/gauss2/xgs(MAXQBASIS), t(MAXQBASIS,MAXQBASIS)

common/basis/beta, re, eharm, basisk, atomicmu

common/debug/iverbose, ipot

common/arrays/a(MAXQBASIS,MAXQBASIS),s1(MAXQBASIS),s2(MAXQBASIS)

common/savepot/NO(MAXQBASIS),Varray(MAXQBASIS)

common/inits/QMIN,QMAX,DELQ,ORIGIN

common/anionNECA(MAXOBASIS,MAXQBASIS)

common/neutral/VECN(MAXQBASIS,MAXQBASIS)

common/vpsin/VPSIN(NFVMAXN,MAXQBASIS,MAXIQ)

common/vpsiaNPSIA(NFVMAXN,MAXQBASIS,MAXIQ)

common/fcfs/TFCF(NFVMAXN,NFVMAXA), ENE(NFVMAXN,NFVMAXA)

common/fcf2/Q(MAXQBASIS)

common/rint/NIQ

FCFNUM2.inc

parameter(NST2 $=5$, NMDS2 $=15$, NVMXA2 $=100$ )

parameter(NVMXN2 $=100)$

common/eiga/EIGVALA(NST2,NMDS2,NVMXA2)

common/eign/EIGVALN(NST2,NMDS2,NVMXN2)

\section{PARAMS.inc}

parameter(NMDSMX $=15$, NVMAXA $=20, N V M A X N=30, N L I N E S=2500$ )

parameter(NPTSEXP $=1000$, NPTSFIT $=2000, N S T S M X=5$ )

parameter(NPRSMX $=90$, ITRSMX $=30)$

${ }^{1}$ The terms Acropolis and Stonehenge refer to the developmental days of the experiments. Theo Kitsopoulos affectionately named the ZEKE machine after the Acropolis. The PES operators, Steve Bradforth and Alex Weaver, had to find and older historical site for naming the photoelectron spectrometer (since it was built first)...thus, the term Stonehenge. 


\title{
Stick Spectra generation -
}

The fort. 7 file can be used to generate a stick spectrum using the following routine. To compile "f77 -O stick.f -o stick". To use - type "stick". The stick spectrum will be saved in fort.14.

\section{STICK.f}

\author{
Program Stick \\ implicit double precision (a-h,o-z)
}

c

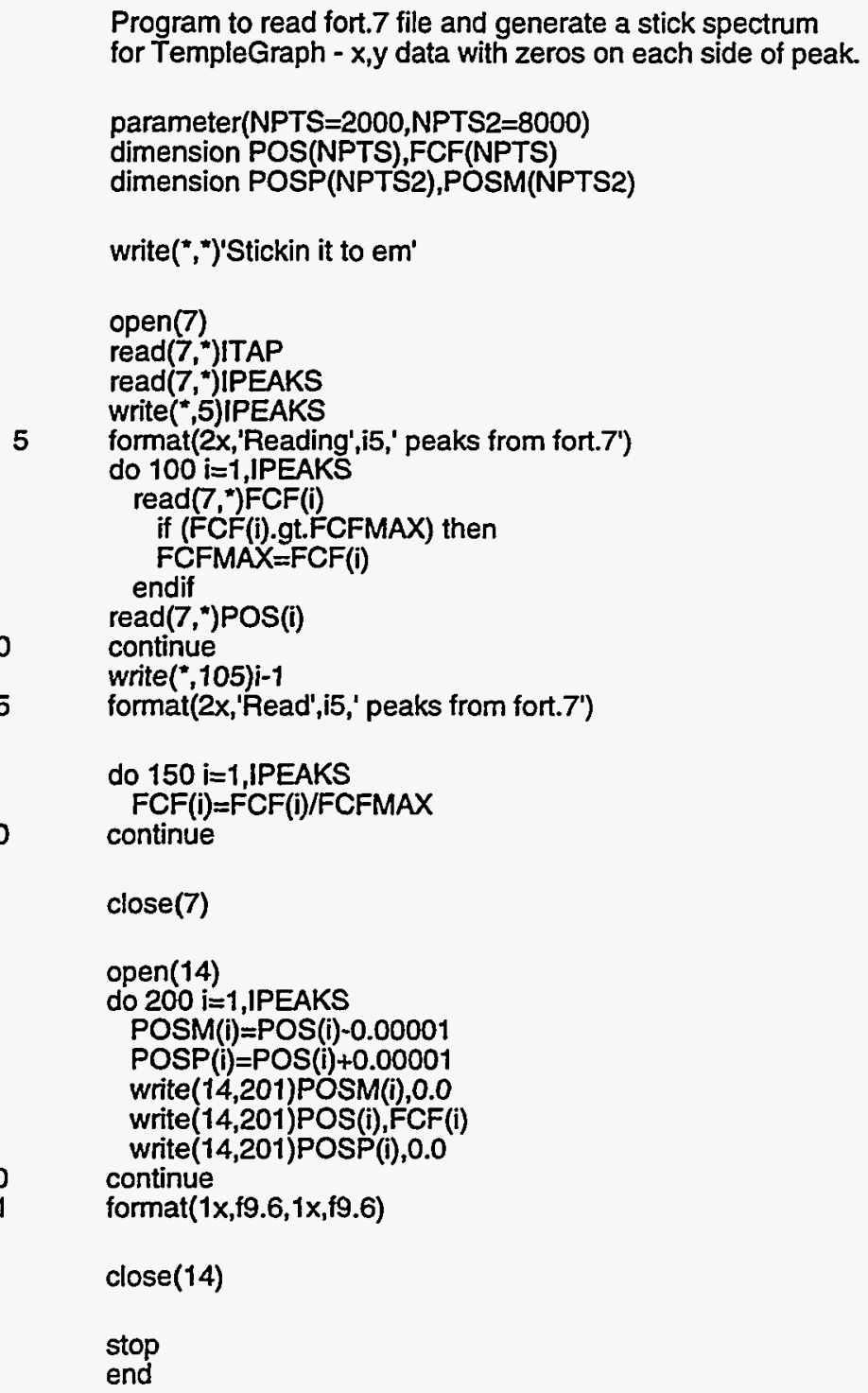


Fortran listing of codes used in PES - in alphabetical order

\section{BANNER.f}

Subroutine Banner0

implicit double precision (a-h,o-z)

c Fun routine

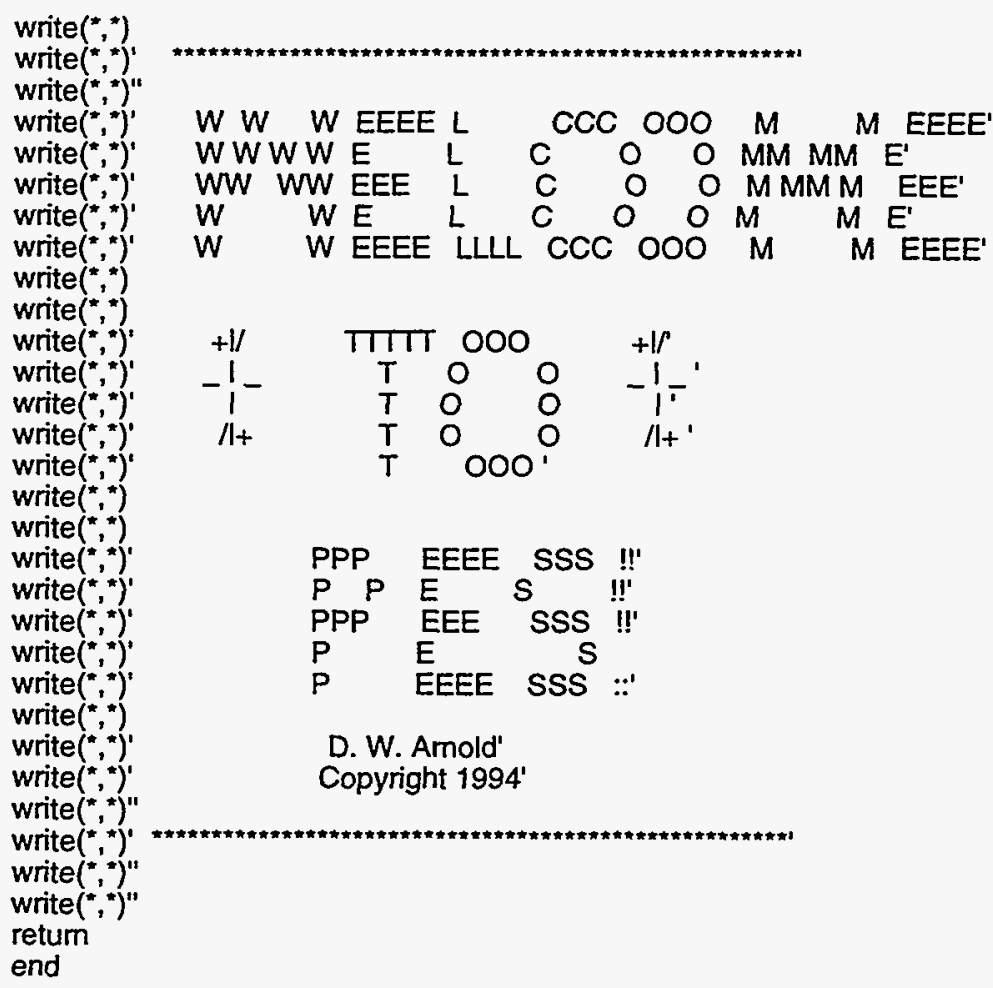

$\begin{array}{llll}P P P & \text { EEEE } & \text { SSS } \\ P & \text { !!' } \\ P & \text { E } & \text { S } & \text { !!' } \\ \text { PPP } & \text { EEE } & \text { SSS } & \text { !!' } \\ P & \text { E } & \text { S } \\ P & \text { EEEE } & \text { SSS } & : !\end{array}$

D. W. Amold'

Copyright 1994'

\section{CALCFCF7.f}

Subroutine CALCFCF(ISTATE,ISTATES,FCF,POS,HFREQA,HFREQN, \&ANHARMA,ANHARMN,IVMAXA,IVMAXN,MODES,SCALE,ORIGIN,ASSFILE, \&EVSTRT,NMSTRT,CMACH)

Implicit Double Precision(a-h,o-z)

include "params.inc"

include "fcfnum2.inc"

C

****LOCAL ARRAYS AND VARIABLES*****

dimension RELFCF(NLINES),POSITION(NLINES)

dimension IC(NMDSMX),NC(NMDSMX)

dimension WKSP(NLINES)

c

*****INDIVIDUAL STATE ARRAYS (TMP)********

dimension POSTMP(NLINES),FCFTMP(NLINES),FCFTMPS(NLINES)

dimension MODECNT(NLINES,NMDSMX)

dimension IVIBCNT(NLINES,NMDSMX),NVIBCNT(NLINES,NMDSMX) dimension POSTMPA(NLINES)

real*8 NMSTRT

character $\mathrm{CMACH}$

character*20 ASSFILE,ASSOUT

integer IWKSP(NLINES),NWKSP(NLINES)

integer CBCNT 


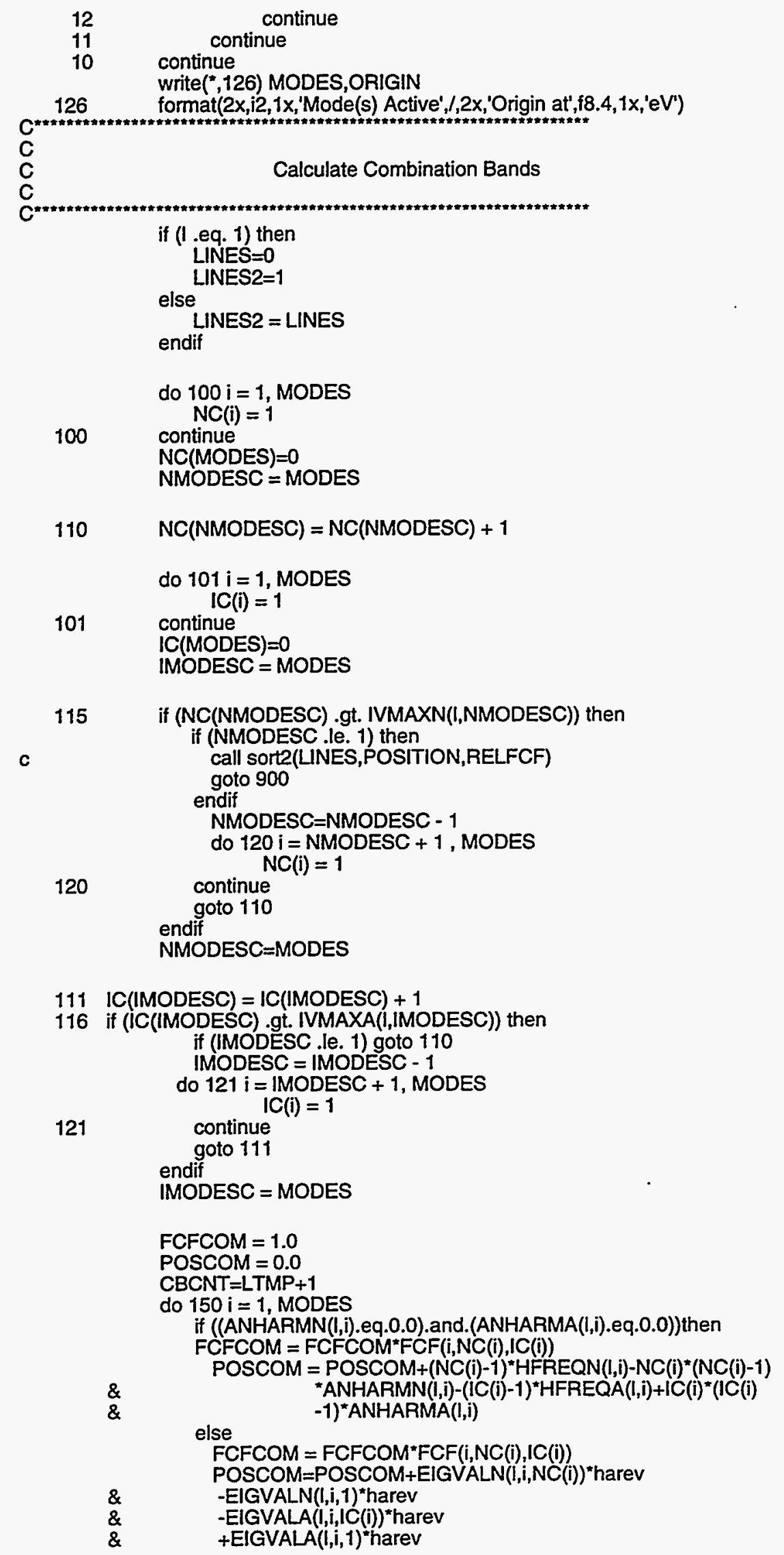


dimension FCF(NMDSMX,NVMAXN,NVMAXA),POS(NMDSMX,NVMAXN, \&NVMAXA)

dimension ANHARMN(NSTSMX,NMDSMX),ANHARMA(NSTSMX,NMDSMX)

dimension HFREQN(NSTSMX,NMDSMX),HFREQA(NSTSMX,NMDSMX) dimension IVMAXA(NSTSMX,NMDSMX), IVMAXN(NSTSMX,NMDSMX)

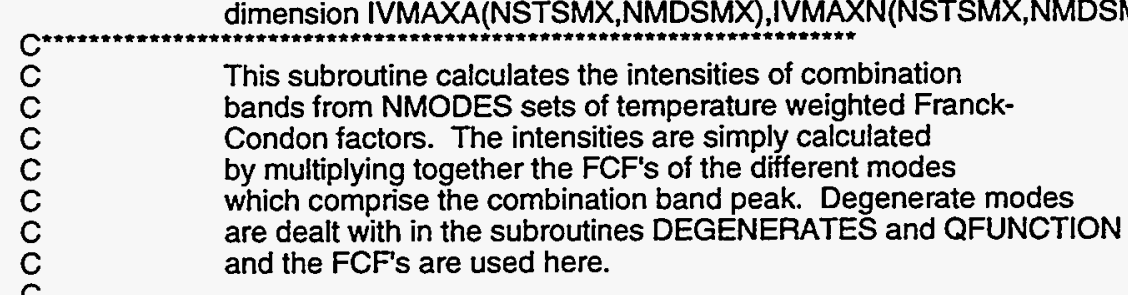

Definition of Variables

MODES-NMODES from main routine; number of modes active ORIGIN-EVSTRT" " " ; energy of spectral origin (eV) HFREQN,HFREQA-harmonic frequencies of anion and neutral ANHARMN,ANHARMA-anharmoniticites of anion and neutral

FCF(i,j,k)-Franck-Condon factor;ith mode, jth anion level, kth neutral level

LINES-total number of combination bands with FCF $>0.005$ and position $>0.15 \mathrm{eV}$

RELFCF-FCF of calculated combination band

POSITION-position of calculated combination bands

NMODESC,IMODESC-counter for modes of neutral and anion

NC,IC-counter for vib. levels in a mode of neut. and anion

CORR-correction to relative FCF with elec. vel. term.(more below)

4/92-The CALCFCF code has been modified so that the subroutine now keeps track of the modes which contribute to each combination band. The arrays

\section{MODECNT,IVIBCNT,NVIBCNT,CBCNT}

are used to keep track of these. The INDEXX subroutine is called to order these by energy and contributing modes, FCF, and position are outputted to a file with the name [FILENAME.ass] The WKSP,IWKSP,NWKSP arrays are needed for the INDEXX routine and the following lines to index the comb. bands.-DWA

5/23/92-The CALCFCF subroutine has been modified [now calcfcf7.f] so that the large set of common blocks present the previous version are no longer present. This was necessary to move on to non-linear least squares fitting because the code needs to be more independent in this application. Also to make it easier to change the default array sizes with the parameter statements, the parameter values are now being sent rather than hard-wired in each subroutine.-DWA

C

write $\left({ }^{*},\right)^{\prime}+$ CALCulating Combination Band FCFs'

harev $=27.211608$

I=ISTATE

LTMP $=0$

do $125 \mathrm{i}=1$, MODES

HFREQN $(1, \mathrm{i})=$ HFREON $(1, \mathrm{i}) / 8065.479$

HFREQA $(1, \mathrm{i})=$ HFREQA $(1, \mathrm{i}) / 8065.479$

ANHARMN $(1, i)=$ ANHARMN $(1, i) / 8065.479$

125 continue

ANHARMA $(1, i)=A N H A R M A(1, i) / 8065.479$

c Normalize all modes to the $(0,0)$ band so that all modes have the $F C F(0,0)$

c equal to 1(one)

FCFNORM $=F C F(1,1,1)$

do $10 i=1$, MODES

do $11 \mathrm{j}=1$, IVMAXA(1,i)

do $12 \mathrm{k}=1$, IVMAXN $(1, \mathrm{i})$

$F C F(i, k, j)=F C F(i, k, j) / F C F N O R M$ 
150 continue

$$
\begin{aligned}
& \text { endif } \\
& \text { if (FCFCOM .It. 0.001) then } \\
& \text { endif } \\
& \text { goto } 111 \\
& \text { if (POSCOM .gt. ORIGIN) then } \\
& \text { endif } \\
& \text { goto } 111 \\
& \text { MODECNT(CBCNT, } i)=i \\
& \text { NVIBCNT(CBCNT, i) }=\text { NC }(i) \\
& \text { IVIBCNT(CBCNT,i)=IC(i) }
\end{aligned}
$$

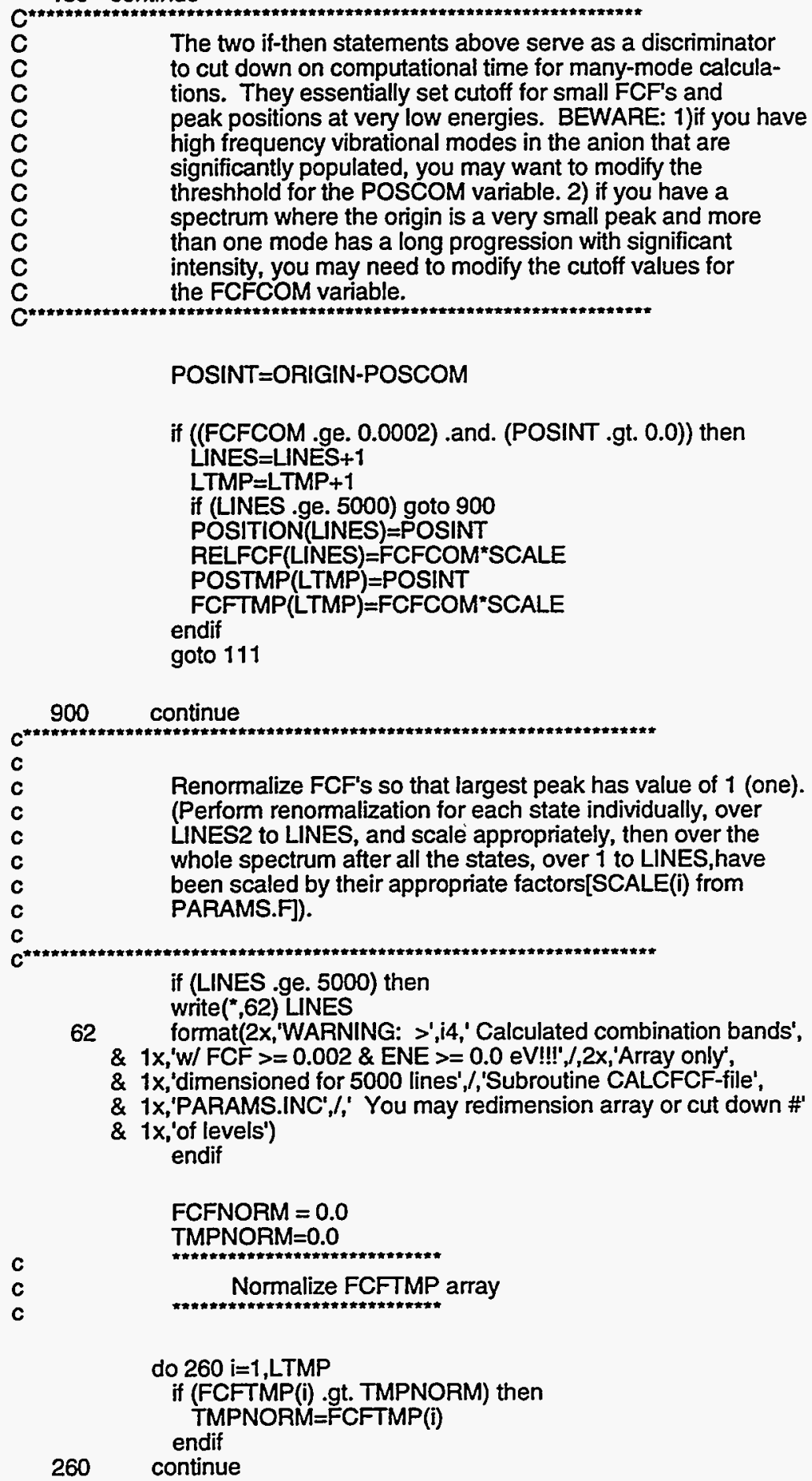




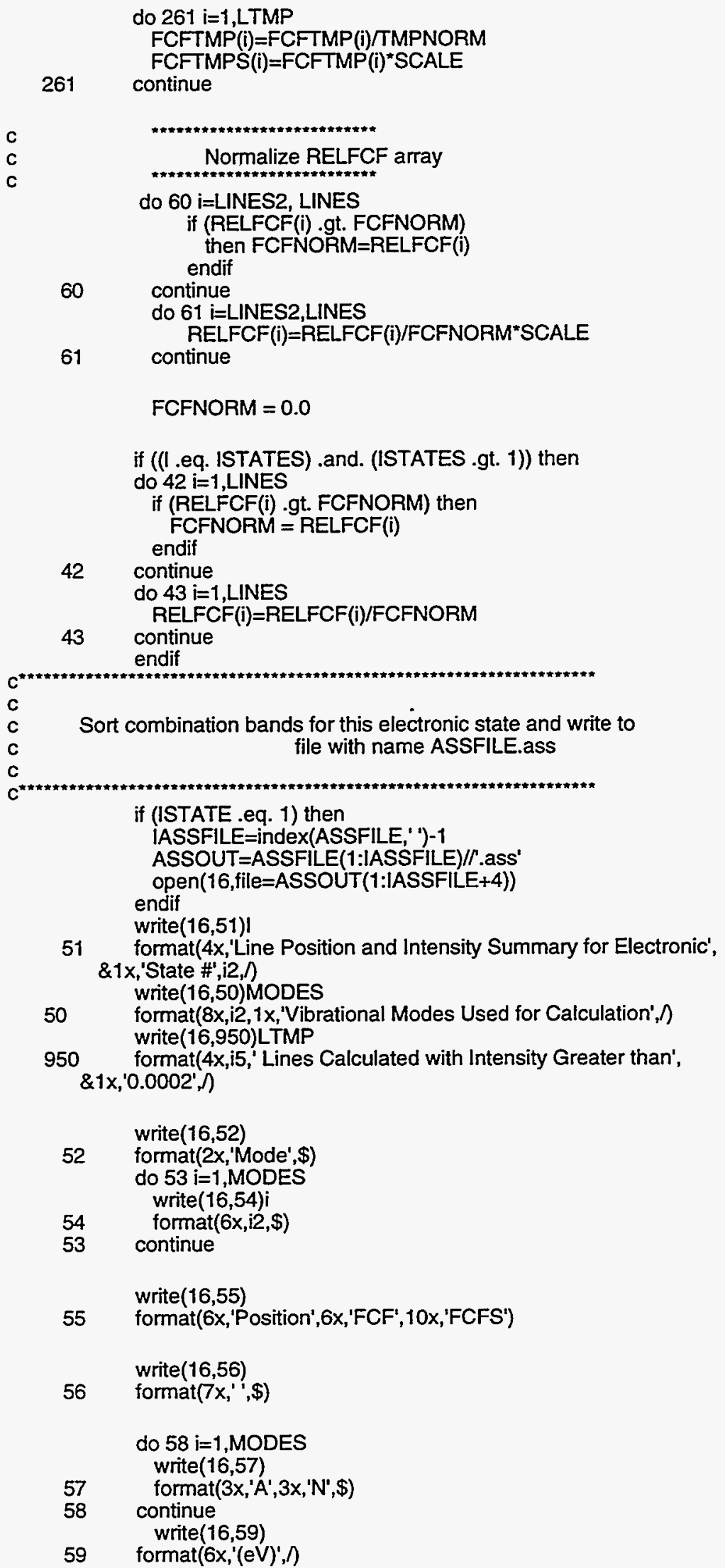


20

21

24

3

27

28

26

30

31

29

32

33

1232

1233

c

c call INDEXX(LTMP,POSTMP,IWKSP)

do $20 \mathrm{i}=1$, LTMP

WKSP $(i)=$ POSTMP(i)

continue

do $21 \mathrm{i}=1, \mathrm{LTMP}$

POSTMP $(i)=W K S P(I W K S P(i))$

continue

do $23 \mathrm{j}=1$, MODES

do $24 \mathrm{i}=1$, LTMP

NWKSP $(\mathrm{i})=$ MODECNT $(\mathrm{i}, \mathrm{j})$

continue

do $25 \mathrm{i}=1, \mathrm{LTMP}$

、 MODECNT $(i, j)=N W K S P(I W K S P(i))$

continue

continue

do $26 \mathrm{j}=1$, MODES

do $27 i=1$, LTMP

NWKSP(i)=IVIBCNT(i,j)

continue

do $28 \mathrm{i}=1, \mathrm{LTMP}$

IVIBCNT (i,j) $=$ NWKSP $($ IWKSP(i))

continue

continue

do $29 \mathrm{j}=1$, MODES

do $30 \mathrm{i}=1$, LTMP NWKSP $(i)=N V I B C N T(i, j)$

continue

do $31 \mathrm{i}=1, \mathrm{LTMP}$

NVIBCNT $(i, j)=N W K S P(I W K S P(i))$

continue

continue

do $32 \mathrm{i}=1$, LTMP

WKSP $(i)=F C F T M P(i)$

continue

do $33 i=1$, LTMP

FCFTMP(i) $=$ WKSP $($ IWKSP(i))

continue

do $1232 \mathrm{i}=1$, LTMP

WKSP $(i)=$ FCFTMPS(i)

continue

do $1233 i=1$, LTMP

FCFTMPS $(i)=$ WKSP $($ IWKSP $(i))$

continue

write $(*$, , $)$ CMACH,EVSTRT,NMSTRT

if (CMACH .eq. 'A') then

write $\left(*^{*},\right)^{\prime}$ 'Converting $\mathrm{eV}$ to $\mathrm{nm}$ for .ass file'

endif

do $34 i=1, L T M P$

if ((FCFTMP(i) .gt. 0.0002) .and. (POSTMP(i) .gt. 0.0)) then write $(16,37)$

37 format $(7 x, ", \$)$

do $35 j=1$, MODES

write $(16,36) I V I B C N T(i, j)-1, N V I B C N T(i, j)-1$

format $(2 x, 12,2 x, i 2, \$)$

continue

if (CMACH .eq. ' $A$ ') then

POSTMPA $(i)=\left(1 /\left(\left(\right.\right.\right.$ EVSTRT-POSTMP $\left.(i)+\left(1 /\left(N M S T R T^{*} 1.0 e-07 * 8065.479\right)\right)\right)$

$\& * 8065.479))^{*} 1 e 07$

else

write(16,38)POSTMPA(i),FCFTMP(i),FCFTMPS(i)

write(16,38)POSTMP(i),FCFTMP(i),FCFTMPS(i) 


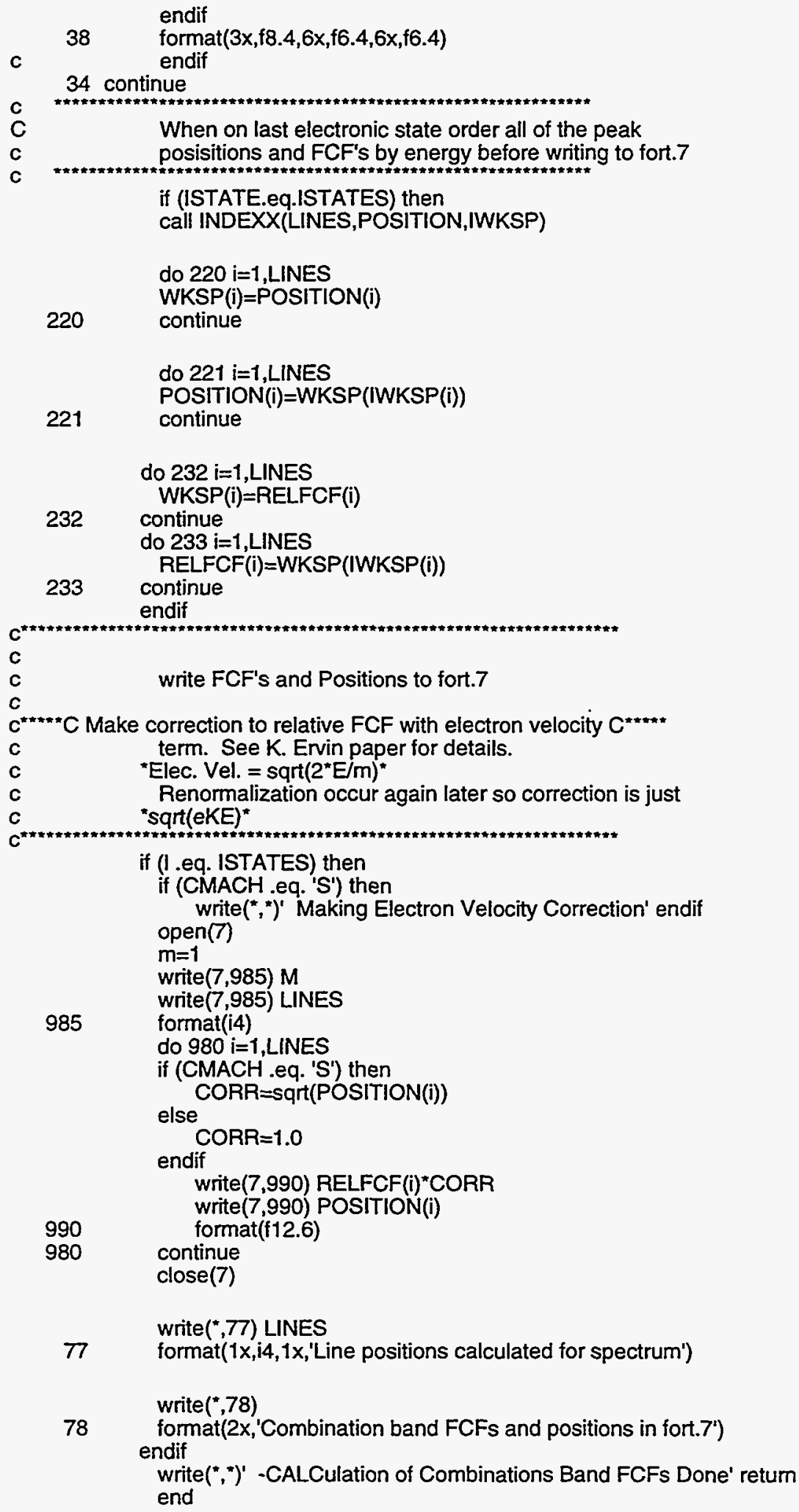

\section{CHISQS5.f}

Subroutine CHISQ(EXPCNTS,CHIOPT,ISTATES,NMODES,CHICALC) implicit double precision $(a-h, 0-z)$

This subroutine calculates the reduced chi square fit to 
data in the EXPCHI() array vs. the simulation in the

SIMCHI() array. The algorithm is based upon Bevington's.

To give a more meaningful value for the chi-square value

the experimental file intensities are converted to "true" electron counts and the simulation is scaled according to the intensity of the largest peak. The number of degrees of freedom is automatcally determined by reading (Function CNTVAR) the number of inputs in the OPTFILE (FILENAME.opt) and then subtracting this value from the number of experimental data points.

Program variables are:

$\operatorname{EXPCHI}(800,2)=$ experimental data

SIMCHI $(800,2)=$ simulated spectrum that has been 'sorted by the 'sorts.f' routine to match energy pts. SIGEXP $(800,2)=a r r a y$ of uncertainties of peak intensities (i.e., the sqit of the intensities)

IPTSEXP=number of points in EXPT and SIMCHI arrays

NVAR=number of parameters being adjusted to get fit to spectrum

WEIGHT $(800)=$ weighting function array

RESID=residual values

OPTFILE=FILENAME.opt which contains information about which variables are to be optimized in fit

CNTVAR=total number of variables (from OPTFILE)

EXPCNTS=number of counts in main channel of exp. data NFREE=number of degrees of freedom

CHIMIN,CHIMAX=range in energy $(\mathrm{nm})$ over which the chi-square calculation is to be performed.

include "params.inc"

character*20 OPTFILE

character ${ }^{*} 1$ CHIOPT

integer CNTVAR

common/chi/EXPCHI(NPTSEXP,2),SIGEXP(NPTSEXP)

common/chi2/IPTSEXP,NVAR

common/chi3/FCHISQ,SIMCHI(NPTSEXP,2)

common/chi4/OPTFILE

common/wgtWEIGHT(NPTSEXP)

dimension NMODES(NSTSMX)

dimension SIMCHI2(NPTSEXP, 2)

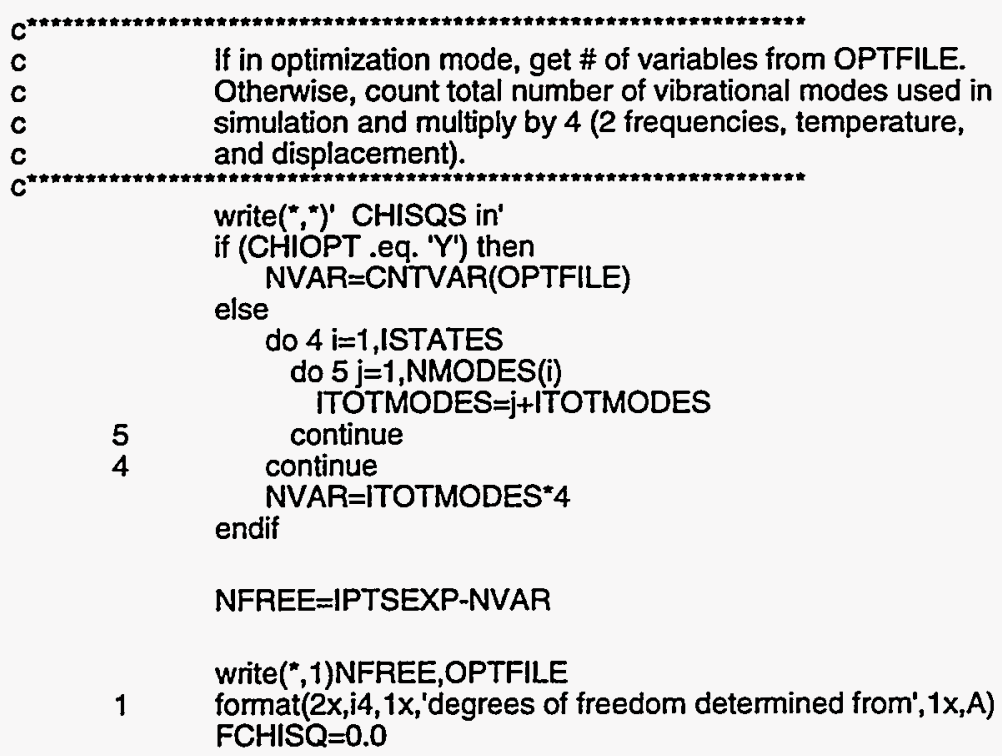




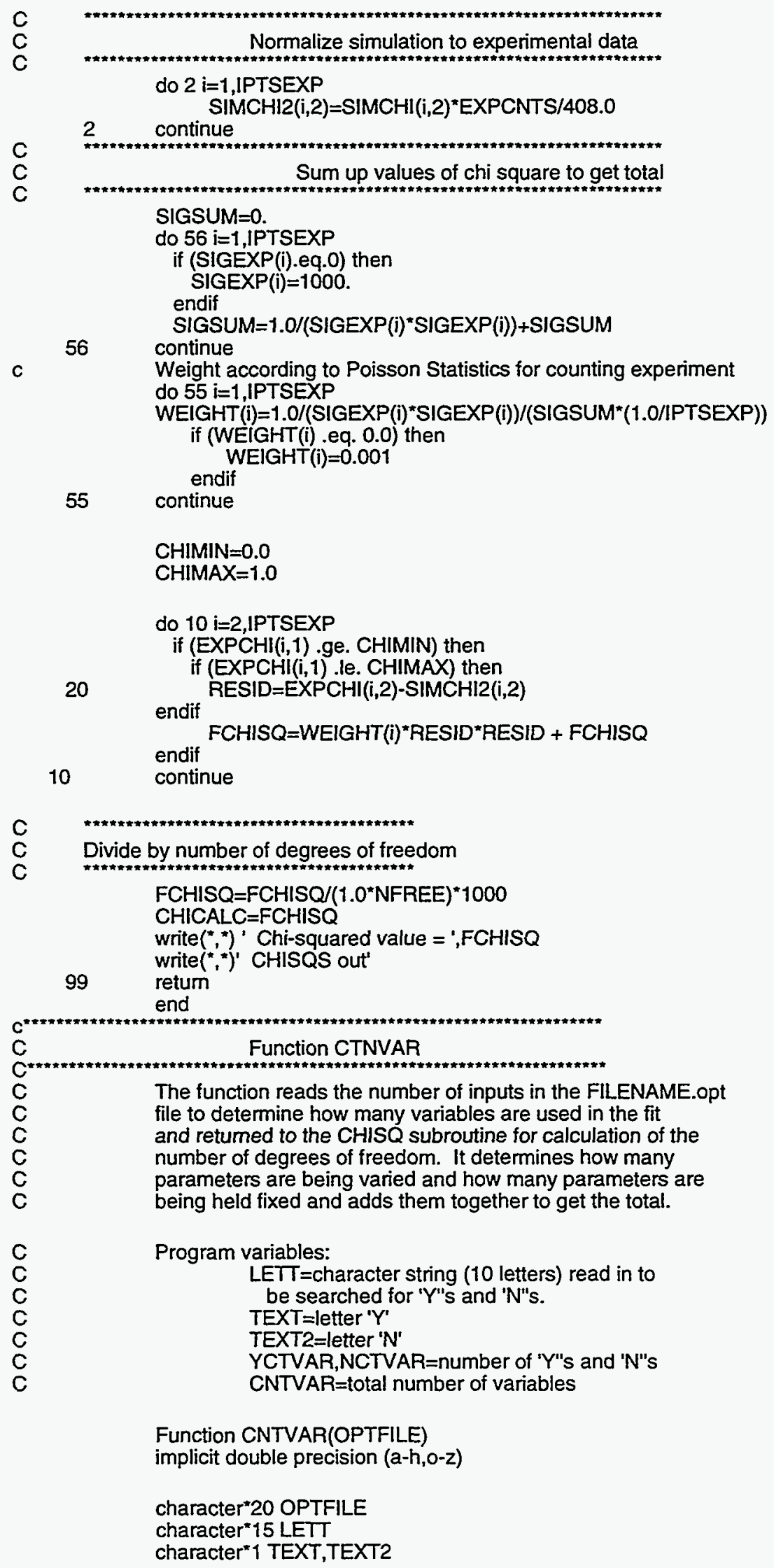


integer YCTVAR,NCTVAR,CNTVAR

YCTVAR $=0$

NCTVAR $=0$

CNTVAR $=0$

$m=0$

$k=0$

TEXT $=' Y{ }^{\prime}$

TEXT2 $=$ ' $N$ '

open(28, file=OPTFILE)

do $10 \mathrm{i}=1,100$

$\operatorname{read}(28,2$, end $=19)$ LETT

LORIG $=-1$

LSUB $=0$

do $11 \mid=1,50$

LSUB=index (LETT (LORIG+1:),TEXT)

if (LSUB .eq. 0) goto 17

$k=k+1$

11

LORIG $=$ LORIG+LSUB

continue

17 YCTVAR $=k$

LORIG $=-1$

LSUB $=0$

do $13 \mid=1,50$

LSUB $=$ index(LETT(LORIG+1:), TEXT2)

if (LSUB .eq. 0 ) goto 14

$m=m+1$

13

LORIG=LORIG+LSUB

continue

14 NCTVAR $=m$

10 CNTVAR=NCTVAR+YCTVAR

2 format(A15)

19 close(28)

retum

end

\section{CONV.f}

Subroutine CONCHECK(CHI1,CHI2,CONV,IT,ISTAT, ITMAX)

Implicit Double Precision(a-h,o-z)

C This routine takes the chi-square values for the last two iterations and checks to see if the convergence criterion has been met for

the optimization being performed. It also checks to see if the

maximum number of iterations has been performed.

write $(",)^{\prime}$ 'CONCHECK in'

if $(($ abs $((\mathrm{CHI} 2-\mathrm{CHI}) /((\mathrm{CHI}+\mathrm{CHI}) / 2))$.$) .le. CONV then$

ISTAT $=2$

else

ISTAT $=1$

endif

if (IT .gt. ITMAX) then

ISTAT $=0$

endif

if ((CHI1-CHI2) .eq. 0 ) then

ISTAT $=3$

endif

write $\left({ }^{*},{ }^{*}\right)$ 'CONCHECK out'

retum

end 


\section{DERIV1.f}

Subroutine DERIVATIVE(QPARAMS,NPAR,NPARV,CMACH,DERIV) Implicit Double Precision(a-h,o-z)

This subroutine calculates the ist derivatives of the simulation, point by point, with respect to each parameter. These derivatives are used to fill the alpha and beta matrices which are used to determine the new parameters used in the optimization via chisquares. This is based upon the Marquardt-Levinberg method, described in both Bevington and Numerical Recipes.

Actually, the code does not use the ML method now...only the steepest descent...needs a bit of work to get to use both optimally.6/25/94 DWA

include "params.inc"

character*1 QPARAMS(NPRSMX)

dimension PLUS(NPTSEXP)

real MINUS(NPTSEXP)

dimension DERIV(NPRSMX,NPTSEXP)

dimension BETA(NPRSMX)

dimension ALPHA(NPRSMX,NPRSMX)

common/chi/EXPCHI(NPTSEXP,2),SIGEXP(NPTSEXP)

common/chi2/IPTSEXP,NVAR

common/chi3/FCHISQ, SIMCHI(NPTSEXP,2)

common/chi4/OPTFILE

common/opt1/PARAMS(NPRSMX,ITRSMX),DELTA(NPRSMX,ITRSMX)

common/opt2/IT

$\mathrm{C}$
$\mathrm{C}$
$\mathrm{C}$
$\mathrm{C}$

Zero the alpha and beta matrices

write $\left({ }^{*},{ }^{*}\right)$ 'DERIV in'

do $100 \mathrm{i}=1$, NPARV

$B E T A(i)=0.0$

do $110 j=1, i$

110 continue

$\operatorname{ALPHA}(i, j)=0.0$

c

continue

C
C
C
C
C

Add delta and calculate simulation, then subtract delta and repeat, then calculate slope $=$ derivative .

NPARVCHK $=0$

do $120 i=1$,NPAR

write $\left.\left({ }^{*},\right)^{\prime}\right)$ nparv $=$ ',i

if (QPARAMS $(i)$.eq. ' $Y$ ') then

PARAMS $(i, I T)=$ PARAMS $(i, I T)+D E L T A(i, I T)$

call MAKESPEC(NPAR,CMACH,PLUS)

C

write $\left({ }^{*},{ }^{*}\right)$ 'iptsexp $=$ ',IPTSEXP

do $6 \mathrm{k}=1,1000,100$

write $(*, ")$ ' plus = ',PLUS(k)

continue

write $(*, *)$ 'expchi i2 = ',EXPCHI $(k, 2)$

PARAMS $(i, I T)=$ PARAMS $(i, I T)-$

2*DELTA $(i, I T)$ call

MAKESPEC(NPAR,CMACH,MINUS)

write $\left({ }^{*},{ }^{*}\right)$ 'iptsexp = ',IPTSEXP

do $7 \mathrm{k}=1,1000,100$

write $\left(",{ }^{*}\right) k$,' minus= ',MINUS(k) 
c

c

130 continue continue
write $\left(^{*}, *\right)$
$i$,' values in DERIV NPARVCHK $=$ NPARVCHK +1 endif

120 continue

if (NPARVCHK .ne. NPARV) then write(",121)NPARVCHK,NPARV

121 format(2X,'ERROR: \# Varied parameters differs between', $1 x$, \&'DERIVATIVE (',i2,') and TIDYUP (',i2,')')

endif

write $\left.\left({ }^{*},\right)^{\prime}\right)$ DERIV out'

retum

end

\section{DGNRTS3.f}

Subroutine Degenerates(ISTATE,FCF,POS,HFREQA,ANHARMA,

\&ANHARMN,MTMP, IVMAXA,IVMAXN,NMODES,DEGMODE)

implicit double precision(a-h,o-z)

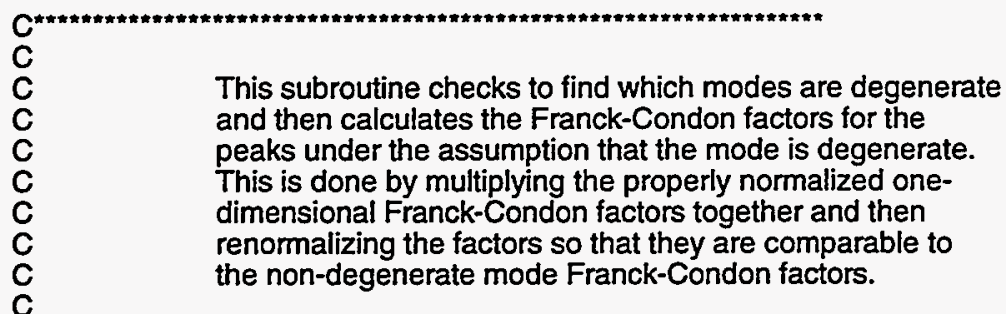

5/23/92-The DEGENERATES subroutine has been modified [now dgnits3.f] so that the large set of common blocks present the previous version are no longer present. This was necessary to move on to non-linear least squares fitting because the code needs to be more independent in this application. Also to make it easier to change the default array sizes with the parameter statements, the parameter values are now being sent rather than hard-wired in each subroutine.-DWA

include "params.inc" include "fcfnum.inc" include "fcinum2.inc" character VERBOSE

real"8 MTMP(NSTSMX,NMDSMX)

dimension SUMDEG(50),DFCF(NMDSMX,NVMAXN,NVMAXA)

dimension FCF(NMDSMX,NVMAXN,NVMAXA),POS(NMDSMX,NVMAXN, \&NVMAXA)

dimension HFREQA(NSTSMX,NMDSMX),ANHARMA(NSTSMX,NMDSMX)

dimension ANHARMN(NSTSMX,NMDSMX)

dimension IVMAXA (NSTSMX,NMDSMX), IVMAXN(NSTSMX,NMDSMX)

dimension NMODES(NSTSMX),DEGMODE(NSTSMX,NMDSMX)

common/verb/VERBOSE

do $114 i=1$,NMODES(ISTATE)

if (DEGMODE(ISTATE,i) .eq. 1.0) then

if (VERBOSE .eq. ' $y$ ' .or. VERBOSE .eq. ' $Y$ ') then write $(", 107) i$ 
endif

write $(12,107) i$

107 format(2x,'FCFs for Degenerate Mode',1x,i1)

c Renormalize FCF's so they sum to $\mathbf{1 . 0}$

DSUM $=0.0$

do $34 k=1$, IVMAXA(ISTATE, $i)$

$k k=k-1$

do 36 l=1, IVMAXN(ISTATE,i)

36

$D S U M=D S U M+F C F(i, l, k)$

continue

do $35 \mid=1$,IVMAXN(ISTATE, $i)$

II $=\mid-1$

$\operatorname{DFCF}(\mathrm{i}, \mathrm{l}, \mathrm{k})=\mathrm{FCF}(\mathrm{i}, \mathrm{l}, \mathrm{k}) / \mathrm{DSUM}$

C Need to reweight for temperature since values were renormalized $c^{* * *}$

$c$

if (II .eq. O) then

if ((ANHARMN(I,i).eq.0.0). and.(ANHARMA(l,i).eq.0.0)) then

EVA=HFREQA(ISTATE, $\mathrm{i}) / 8065.479^{*} \mathrm{kk}$

else

EVA $=(E I G V A L A(I S T A T E, i, k)-E I G V A L A(I S T A T E, i, 1))^{*}$ harev

endif

endif

Z0=EVA $($ MTMP(ISTATE,i)*8.61735E-05)

if $(Z 0 . g e .50 .0)$ then

$\mathrm{ZO}=50.0$

endif

$\operatorname{DFCF}(i, I, k)=\operatorname{DFCF}(i, l, k)^{*} \exp (-Z 0)^{*} k$

35 continue

34 continue

c Multiply FCFs together to get the right degenerate intensities

do $30 \mathrm{k}=1, \mathrm{IVMAXA}($ ISTATE, $\mathrm{i})$
do $39 \mathrm{l}=1,20$

SUMDEG $(l)=0.0$

continue

do 31 I=1, IVMAXN(ISTATE,

do $32 \mathrm{~m}=1$,IVMAXN(ISTATE,i) $n=m+l-1$

SUMDEG(n)=SUMDEG(n)+DFCF $(i, l, k) * D F C F(i, m, k)$

32 continue

31 continue

do 33 I=1, IVMAXN(ISTATE,i)

FCF $(i, l, k)=S U M D E G(l)$

33 continue

30 continue

$C^{* * * *}$ Renormalize such that largest peak has intensity equal to $1.0^{* * * *}$

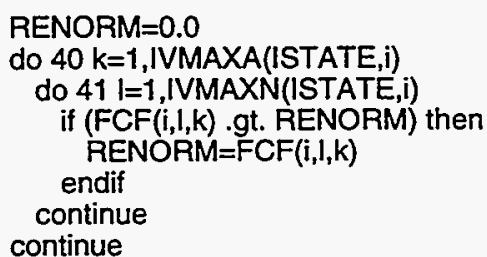

41
40

continue

if (VERBOSE .eq. ' $y$ ' .or. VERBOSE .eq. ' $Y$ ') then write $(*, 108)$

write $\left({ }^{\star}, 117\right) \mathrm{i}$

endif

write $(12,108)$

write $(12,117) \mathrm{i}$

117

format(2x,'Mode',1x,i1)

108

format $\left(1 x,{ }^{\prime} N^{\prime}, 3 x,{ }^{\prime} A ', 4 x\right.$, 'DEGFCF',4x, 'POSITION') 


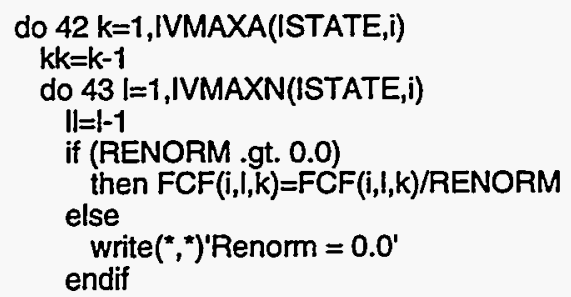

c Need to reweight for temperature since values were renormalized $c^{\star * *}$

$c^{* * *}$

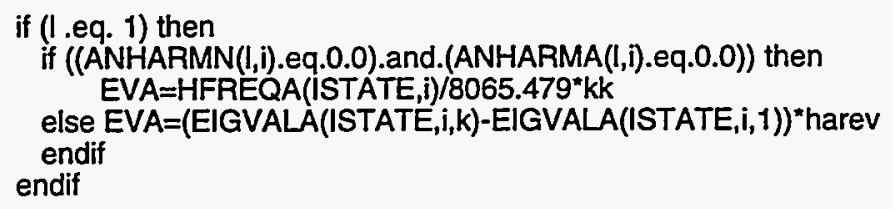

c

$n=k-1$

$m=l-1$

if (FCF $(\mathrm{i}, l, k) \cdot g e \cdot 0.005)$ then

if (VERBOSE .eq. ' $y$ ' .or. VERBOSE .eq. ' $Y$ ') then write $(*, 109) m, n, F C F(i, l, k), \operatorname{POS}(i, l, k)$

$$
\begin{aligned}
& \text { endif } \\
& \text { write }(12,109) m, n, F C F(i, l, k), P O S(i, l, k)
\end{aligned}
$$

109 format $(i 2,2 x, i 2,2 x, f 8.4,2 x, f 8.4)$

c

$$
\begin{aligned}
& 43 \\
& 42
\end{aligned}
$$
endif

42 continue

114 endif

write(",")'Done"

retum

\begin{tabular}{|c|c|}
\hline$c$ & File : dofcf.f \\
\hline C & Find FCFs of transitions in photoelectron spectrum. \\
\hline $\begin{array}{l}\mathbf{C} \\
\mathbf{C}\end{array}$ & 9/12/93 - Completely hacked apart from code originally \\
\hline c & developed by R. B. Metz and rebuilt to \\
\hline c & use in PES fitting code as the numerical routine \\
\hline c & for anharmonic frequencies-DWA \\
\hline c & includes: \\
\hline c & dofcf \\
\hline c & doweight \\
\hline c & qoverlap \\
\hline c & findwf \\
\hline c & donw \\
\hline c & testover \\
\hline & subroutine dofcf(NVA,NVN,WFX) \\
\hline & implicit double precision $(a-h, o-z)$ \\
\hline & Finds FCFs from all 1...NVA levels of anion to all \\
\hline
\end{tabular}

end

\section{DOFCF2.f}


1..NVN levels of neutral.

include "fcfnum.inc"

common/integ/WEIGHT(MAXQBASIS)

common/rinteg/QWEIGHT(MAXIQ)

common/save/SAVEFUNC

dimension WFX(MAXQBASIS,MAXQBASIS,MAXIQ)

dimension WEIGHT(MAXQBASIS), QWEIGHT(MAXIQ)

dimension VPROD(NFVMAXN,NFVMAXA,MAXQBASIS*MAXIQ)

dimension VTEST(MAXQBASIS,MAXQBASIS,MAXIQ)

dimension WFINT(NFVMAXN)

character SAVEFUNC

if (VERBOSE .eq. ' $Y$ ') then

write $\left.(",)^{\prime}\right)$ +dofcf'

endif

C Calculate positions of the lines(for harmonic and morse

oscillators only)

c

do $150 \mathrm{ii}=1$,NVA

$\mathrm{i}=\mathrm{ji}-1$

do $160 \mathrm{jj}=1$, NVN

$\mathrm{j}=\mathrm{jj}-1$

ENE(ij,ii) $=$ EOO- $($ HARMN*i)/ewwn+(HARMA*i $) / e v w n+$

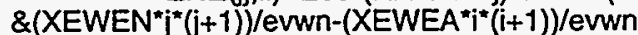

160 continue

150 continue

C

C

c

10

Calculate wavefunctions for anion and neutral vibrational levels

do $10 i=1$, NVA

call VPSIACALC $(i, W F X)$

continue

if (SAVEFUNC .eq.' $Y$ ') then

write $(*, 170)$ NVA,char(NVA+63)

170 format(2x,i2, Anion wave functions written to afx.A - afx.',A) endif

if (iverbose .eq. 1) then do $32 i=1$, NVA

do $33 \mathrm{j}=1$, NIQ

do $34 k=1$,NQBASIS

$\operatorname{VTEST}(i, k, j)=\operatorname{VPSIA}(i, k, j$

)

continue

continue

continue

call

testover(wfint, VTEST,NVA)

write $\left({ }^{*},{ }^{\prime}\right.$ ' Wavefunction overlaps : should all $=1$ ' do $31 \mathrm{j}=1$,NVA

31 write $(*, 1090) \mathrm{j}$, wint $(\mathrm{j})$

1090

format $(i 3, \cdots, 19.6)$

endif

do $20 \mathrm{i}=1, \mathrm{NVN}$

call VPSINCALC $(i, W F X)$

20 continue

if (SAVEFUNC.eq.' $Y$ ') then write $\left({ }^{*}, 180\right)$ NVN, char(NVN+63)

180

format(2x,i2,' Neutral wave functions written to afx.A - afx.',A) endif

if (iverbose .eq. 1) then

do $35 i=1$, NVN

do $36 \mathrm{j}=1, \mathrm{NIQ}$ 


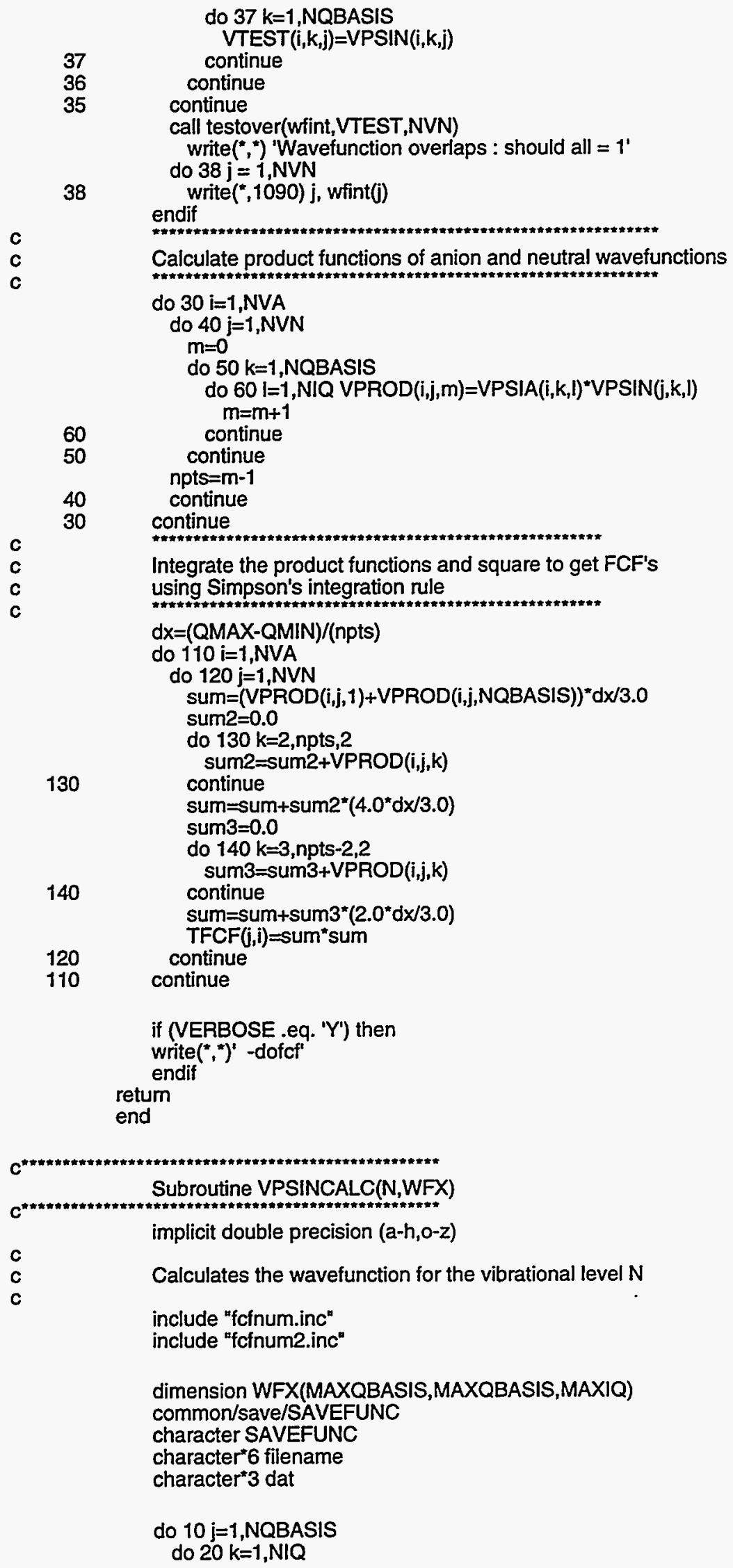

Calculate product functions of anion and neutral wavefunctions $\operatorname{VTEST}(i, k, j)=\operatorname{VPSIN}(i, k, j)$ continue continue continue

call testover(wint,VTEST,NVN)

write $(*, ")$ 'Wavefunction overlaps : should all $=1$ ' 


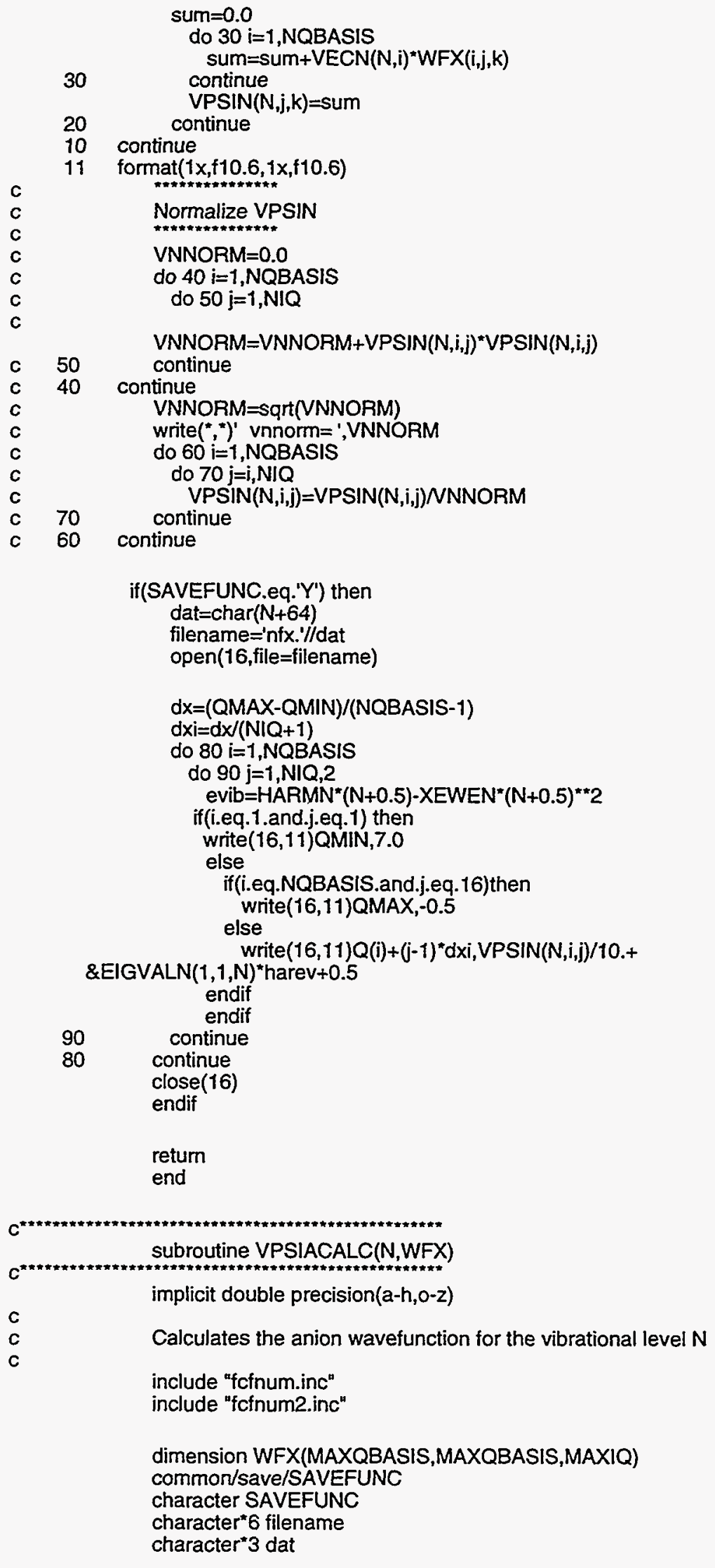


30

20

10

11

do $10 \mathrm{j}=1$, NQBASIS

do $20 \mathrm{k}=1$, NIQ

sum $=0.0$

do $30 \mathrm{i}=1$, NQBASIS

sum $=$ sum+VECA $(N, i)^{*} W F X(i, j, k)$

continue

VPSIA $(N, j, k)=s u m$

continue

continue

format( $1 x, f 10.6,1 x, f 10.6)$

*****************

Normalize VPSIA

VANORM $=0.0$

do $40 \mathrm{i}=1$,NQBASIS

do $50 \mathrm{j}=1, \mathrm{NIQ}$

VANORM $=$ VANORM+VPSIA $(N, i, j){ }^{*} \operatorname{VPSIA}(N, i, j)$

continue

continue

VANORM $=$ sqit(VANORM)

write (", ")' vanorm = ',VANORM

do $60 \mathrm{i}=1$,NQBASIS

do $70 \mathrm{j}=\mathrm{i}, \mathrm{NIQ}$

$\operatorname{VPSIA}(N, \mathrm{i}, \mathrm{j})=\operatorname{VPSIA}(\mathrm{N}, \mathrm{i}, \mathrm{j})$ )NANORM

continue

continue

if (SAVEFUNC.eq. 'Y') then

dat $=\operatorname{char}(\mathrm{N}+64)$

filename='afx.'///dat

open(16,file=filename)

$d x=(Q M A X-Q M I N) /(N Q B A S I S-1)$

$d x i=d x /(N I Q+1)$

do $80 \mathrm{i}=1$,NQBASIS

do $90 j=1, N I Q, 2$

evib=HARMA"(N+0.5)-XEWEA"$(\mathrm{N}+0.5)^{* * 2}$

if((i.eq.1.and.j.eq.1)) then

write $(16,11) \mathrm{QMIN}, 7.0$

else

if(i.eq.NQBASIS.and.j.eq.16)then write $(16,11) \mathrm{QMAX},-0.5$

else

write $(16,11) Q(i)+(j-1){ }^{\star} d x i, V P S I A(N, i, j) / 10 .+$

\&EIGVALA(1,1,N)*hareV

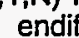

90

endif

continue

close(16)

endif

return

end

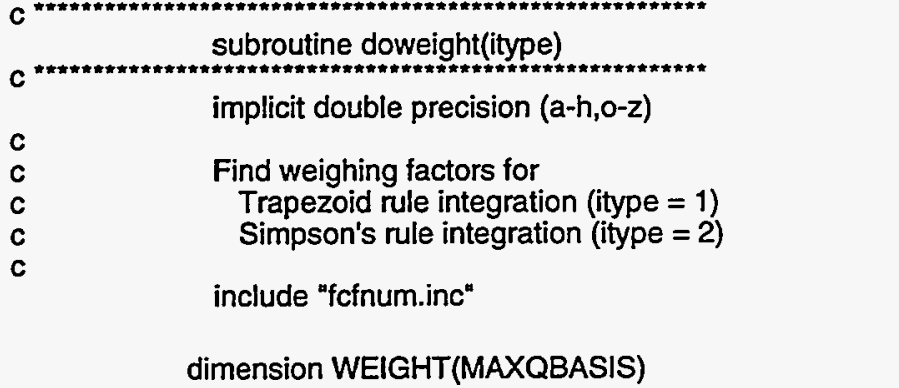


10

common/integ/WEIGHT(MAXQBASIS)

if (iverbose.eq.1) then

write $\left.\left({ }^{*},\right)^{\prime}\right)^{\prime}+$ doweight'

endif

QSTEP $=($ QMAX-QMIN)/(NQBASIS-1)

if (itype .eq. 1) then

WEIGHT $(1)=$ QSTEP $/ 2.0$

WEIGHT(NQBASIS) $=$ QSTEP $/ 2.0$

do $10 \mathrm{i}=2$, NQBASIS-1 endif

WEIGHT(i) = QSTEP

if (itype .eq. 2) then

$N Q=$ NQBASIS

WEIGHT(1) $=$ QSTEP*17.0/48.0

WEIGHT(2) $=$ QSTEP $* 59.0 / 48.0$

WEIGHT(3) $=$ QSTEP $* 43.0 / 48.0$

WEIGHT(4) = QSTEP ${ }^{*} 49.0 / 48.0$

WEIGHT(NQ) $=$ WEIGHT(1)

WEIGHT(NQ-1) = WEIGHT(2)

WEIGHT(NQ-2) $=$ WEIGHT(3)

WEIGHT(NQ-3) $=$ WEIGHT(4)

do $20 i=5, N Q-4$

20 endif

$$
\text { WEIGHT }(i)=\text { QSTEP }
$$

if (iverbose.eq.1) then endif

write $(*, *)^{\prime}$-doweight'

retum

end

c *******************************************************

subroutine findwf(NV,WFX)

c ***************************t

implicit double precision (a-h,o-z)

C

Find value of wavefunctions.

ie, calculate Hermite polynomials explicitly -

do this using recursion relation in

Handbook of Mathematical Functions

Abramowitz + Stegun Sec. 22.7

include "fcfnum.inc"

dimension WFX(MAXQBASIS,MAXQBASIS,MAXIQ),XNORM(MAXQBASIS)

dimension XVAL(MAXQBASIS,MAXIQ)

common/save/SAVEFUNC

character SAVEFUNC

character ${ }^{*} 15$ filename

character ${ }^{*} 7$ dat

if (iverbose.eq.1) then

write $\left({ }^{*},\right)^{\prime}+$ find w'

endif

$R E B=(R E A+R E N) / 2.0$

$\mathrm{dx}=(\mathrm{QMAX}-\mathrm{QMIN}) /(\mathrm{NQBASIS}-1) \mathrm{dxi}=\mathrm{dx} /(\mathrm{NIQ}+1)$

do $7 \mathrm{j}=1, \mathrm{NIQ}$

do $5 \mathrm{i}=1$, NQBASIS

$X V A L(i, j)=Q M I N+\left((i-1)^{\star} d x\right)+\left((j-1)^{\star} d x i\right)-(d x / 2.0)-R E B$

5 continue

7 continue

c

c

first set ho and hi

do $15 \mathrm{j}=1, \mathrm{NIQ}$

do $10 i=1$,NQBASIS

WFX $(1, i, j)=1.0$

WFX $(2, i, j)=2.0^{*} B E T A^{*} X V A L(i, j)$

10

continue

continue 
Now get the rest of the hermite polynomials recursively, using $h[n+1]=2^{*} y^{*} h[n]-2^{*} n^{*} h[n-1]$ where $y=B E T A^{*}\left(x \min +i^{*} x h-r e\right)$

do $30 \mathrm{k}=3$,NQBASIS do $25 j=1, N I Q$

do $20 \mathrm{i}=1$,NQBASIS

WFX $(k, i, j)=2.0^{*} B E T A^{*} X V A L(i, j) *$

\& WFX(k-1,i,j)-2.0* $(k-2) * W F X(k-2, i, j)$

20 continue

25 continue

30 continue

c

Include normalization and $\exp \left(-\mathrm{y}^{*} \mathrm{y} / 2\right)$

$\operatorname{XNORM}(1)=$ sqrt $(B E T A / s q r t(p i))$

do $40 \mathrm{k}=2$, NQBASIS

XNORM $(k)=X N O R M(k-1) /$ sqrt $\left(2.0^{*}(k-1)\right)$

40 continue

do $65 \mathrm{j}=1, \mathrm{NIQ}$

do $60 i=1$,NQBASIS

$y=B E T A^{*} X V A L(i, j)$

yexp=exp $\left(-y^{*} y / 2.0\right)$

do $50 \mathrm{k}=1$, NQBASIS

WFX(k,i,j)=WFX(k,i,j)"XNORM $(k)^{*}$ yexp

continue

continue

continue

if (SAVEFUNC.eq.' $Y$ ') then

do $97 \mathrm{k}=1$, NV

dat=char $(k+64)$ filename='basis. $/ / / d a t$

open (16,file=filename)

do $99 \mathrm{i}=1$, NQBASIS

do $250 \mid=1, \mathrm{NIO}$

write $\left(16,{ }^{*}\right) Q(i)+(l-1)^{\star} d x i, W F X(k, i, l)$ continue

99 continue

continue
close $(16)$

97 continue

write( $\left.{ }^{*}, 100\right) \mathrm{NV}-1$, char $(\mathrm{NV}+63)$

100 format(2x,i2,' Basis functions written to basis. $A$ - basis. ',A)

endif

if (iverbose.eq.1) then write $\left({ }^{*},\right)^{\prime}$-findwf' endif

retum

end

c

subroutine dorw

c

implicit double precision (a-h,o-z)

include "fcfnum.inc"

dimension QWEIGHT(MAXIQ

common/rinteg/QWEIGHT(MAXIQ)

if (iverbose.eq.1) then

endif write $\left(",{ }^{*}\right)^{\prime}+$ don $w^{\prime}$

QWEIGHT(1) $=0.5 /(\mathrm{NIQ}-1)$

QWEIGHT(NIQ) = QWEIGHT(1)

do $10 \mathrm{i}=2$, NIQ-1

QWEIGHT(i) $=1.0 /(\mathrm{N} \mid \mathrm{Q}-1)$

c write $\left({ }^{*},{ }^{,}\right)$QWEIGHT(i)

10 continue 


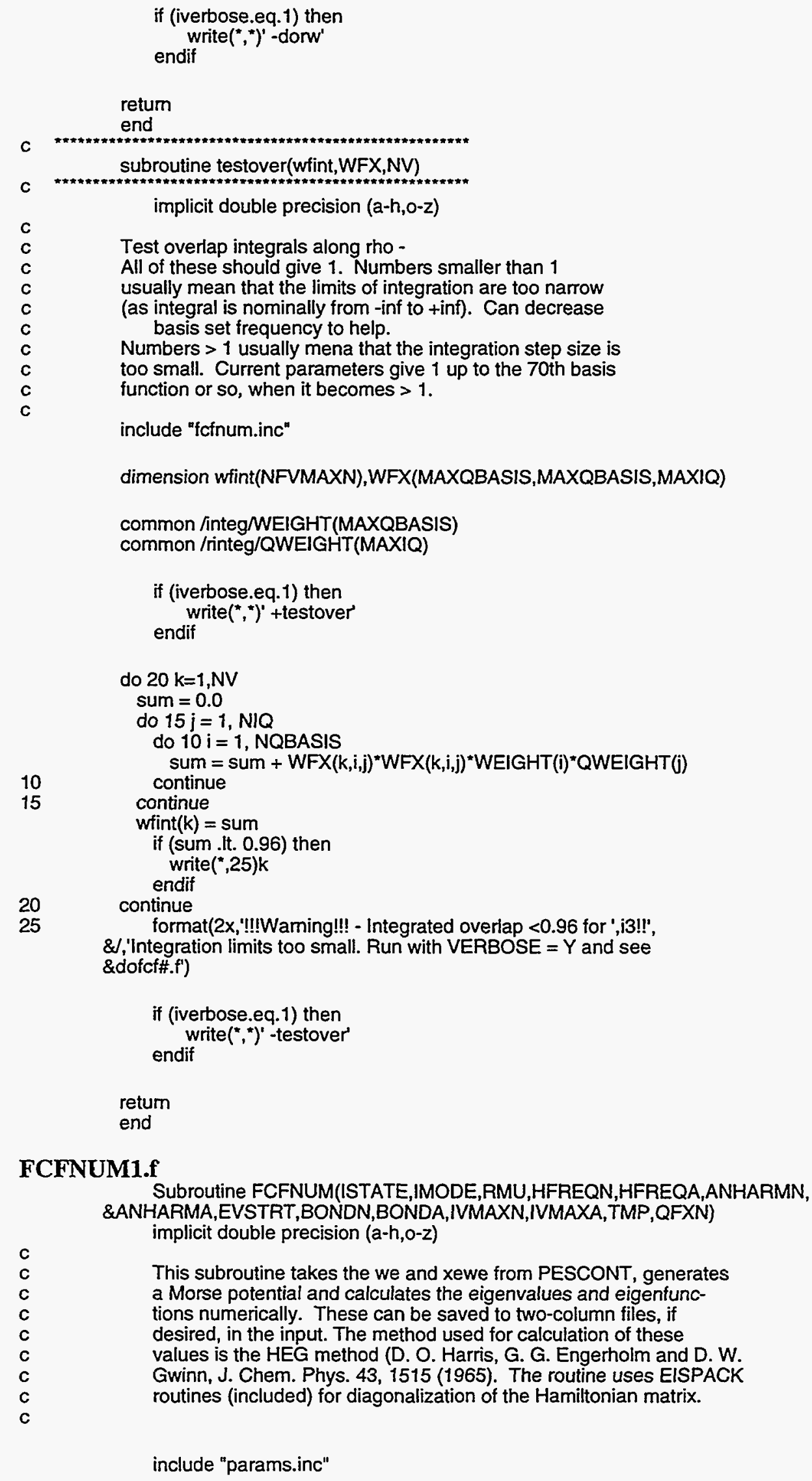


include "fcfnum.inc"

include "fcfnum2.inc"

character*1 VERBOSE,CHIOPT,TERMINAL

character*20 EXPFILE

common/comb3/EVQQ(NVMAXN,NVMAXA),QQ(NVMAXN,NVMAXA),

\&QQB(NVMAXN,NVMAXA),XM1,XM2

common/verbNERBOSE

common/deg/DEGMODE(NSTSMX,NMDSMX),MTMP(NSTSMX,NMDSMX)

common/params/CHIOPT,TERMINAL,EXPFILE

common/integ/weight(MAXQBASIS)

common/saverho/tsave(30,MAXQBASIS,MAXQBASIS),

\&xgssave(30,MAXQBASIS)

dimension TEMPVAL(MAXQBASIS),TEMPVEC(MAXQBASIS,MAXQBASIS)

dimension WFX(MAXQBASIS,MAXQBASIS,MAXIQ), wiint(NFVMAXN)

dimension TOTFCF(NVMAXA)

**"Local ARRAYS*****

dimension NQUAD(NMDSMX)

definitions of variables

XM1,XM2-mass of atoms in amu

REDMASS-reduced mass of diatomic with $X M 1$ and $X M 2$, in grams

HARMN,HARMA-harmonic frequencies neutral and anion, respectively, in wavenumbers

HARMN,HARMA-harm freq of neutral and anion in eV

XEWEN,XWEWCC-anharmonicities of neutral and anion in wavenumbers

XEWEP,XEWEPP-anharmonicities of neutral and anion in $\mathrm{eV}$

ORIGIN-origin of photoelectron spectrum

REN,REA-equilibrium separation of neutral and anion, in Angstroms

ROP,ROPP-equil. sep. of neutral and anion in centimeters

NPMAX,NPPAMX-number of vibrational levels in neutral and anion

TMP-temperature, in Kelvins

TEV-temperature, in $\mathrm{eV}$

MM-neutral

NN-anion

NQBASIS - number of basis functions to be used.

nshow - number of wavefunctions to be saved.

QMIN - Smallest value of $Q$.

QMAX - Largest value of $Q$.

DELQ - Spacing between Q points.

NQBASIS - Number of points in Q.

NIQ - For overlaps in $Q$ the number of points BETWEEN

each pair of points in $Q$. Use an even number.

Contains :

main program

setconst

setdvr

setupA

Finda

findeig

setvo

setv

optimize

findnup

setbeta

findtrac

saveall

readall

ShowArr

Also uses: 
$\begin{array}{ll}\text { c } & \text { dofcf.f } \\ \text { c } & \text { fcfnum.inc (include file with global common blocks) }\end{array}$

- write $\left({ }^{*}, 2\right)$ IMODE,ISTATE

2 format(2x,'FCFNUM used for anharmonic mode calculation of mode',

C \&i2,' in state',i2)

C

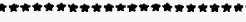

Do a few conversions

call setconst

REDMASS=RMU/1.6605655e-24

HARMN=HFREQN

HARMA $=$ HFREQA

XEWEN=ANHARMN

XEWEA=ANHARMA

$E O O=E V S T R T$

ORIGIN=EVSTRT

REN=BONDN/aO

REA $=$ BONDA $/ \mathrm{OO}$

IVIBN=IVMAXN

IVIBA $=$ IVMAXA

$T E V=T M P * 8.61735 E-05$

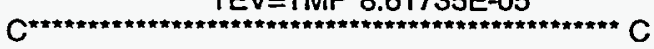

C 1)Determine \# pts and spacing along NC

2) Set up grid, basis functions and

C reference potential

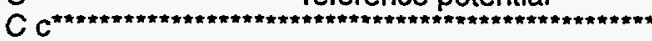

C

C Calculate Morse parameters

C

C RMU $=1.0$

if (XEWEN .gt. 0.0) then

DEN=(HARMN**2/XEWEN**4)/8065.479

$B N=H A R M N N^{*} 1.355977 e$

endif 3*sqrt(REDMASS/DEN)

if (XEWEA g. . 0.0) then

DEA $=\left(\right.$ HARMA ${ }^{\star *} 2 /$ EWEA $\left.^{\star \star} 4\right) / 8065.479$

$B A=H A R M A * 1.355977 e-$ endif

3*sqrt(REDMASS/DEA)

C

if (VERBOSE .eq. ' $Y$ ') then

c write $(*, 42)$ ISTATE,IMODE,DEA,BA,DEN,BN

42 format(2x,'Morse Parameters for State \#',i2,', Mode \#', \&i2, ':',l,4x,'Anion: De = ',f8.4,l,12x,'Beta = ',f8.4, I,4x,

\&'Neutral: De = ',f8.4,/,12x,'Beta = ',f8.4) endif

C $\mathrm{C}$ Initialize intermediate constants

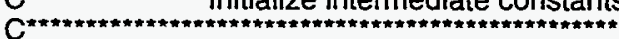

VBETAN $=0.121777 D 0^{\star 2} 2.00^{\star}$ sqrt(REDMASS ${ }^{*}$ XEWEN)

VBETAA $=0.12177700^{* 2}$.0D0*sqrt(REDMASS ${ }^{*}$ XEWEA)

if (VERBOSE .eq. ' $Y$ ') THEN

write $\left({ }^{*}, 93\right)$ ISTATE, IMODE

write $(*, 94)$ VBETAA, VBETAN

endif

93 format(2x,'State \# ',i2,' Mode \# ',i2)

94 format(2x,'Anion Beta: ',f8.4,/,2x,'Neutral Beta: ',f8.4)

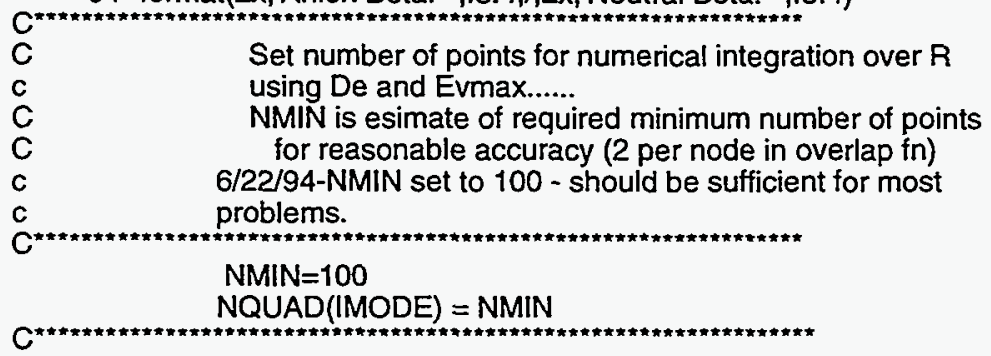




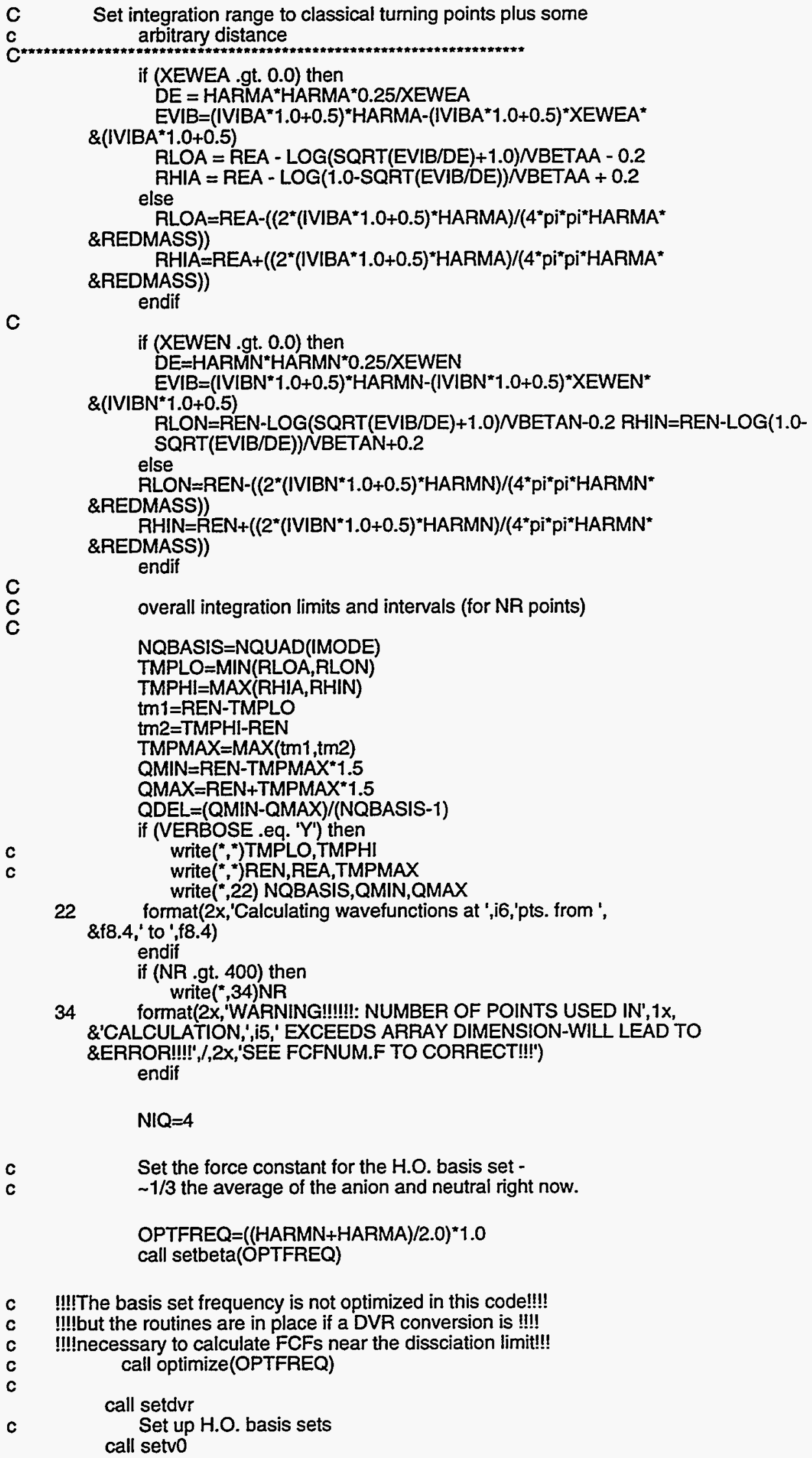

Set the force constant for the H.O. basis set $\sim 1 / 3$ the average of the anion and neutral right now. 
$\mathrm{QMIN}=\mathrm{Q}(1)$

QMAX $=\mathrm{Q}(\mathrm{NQBASIS})$

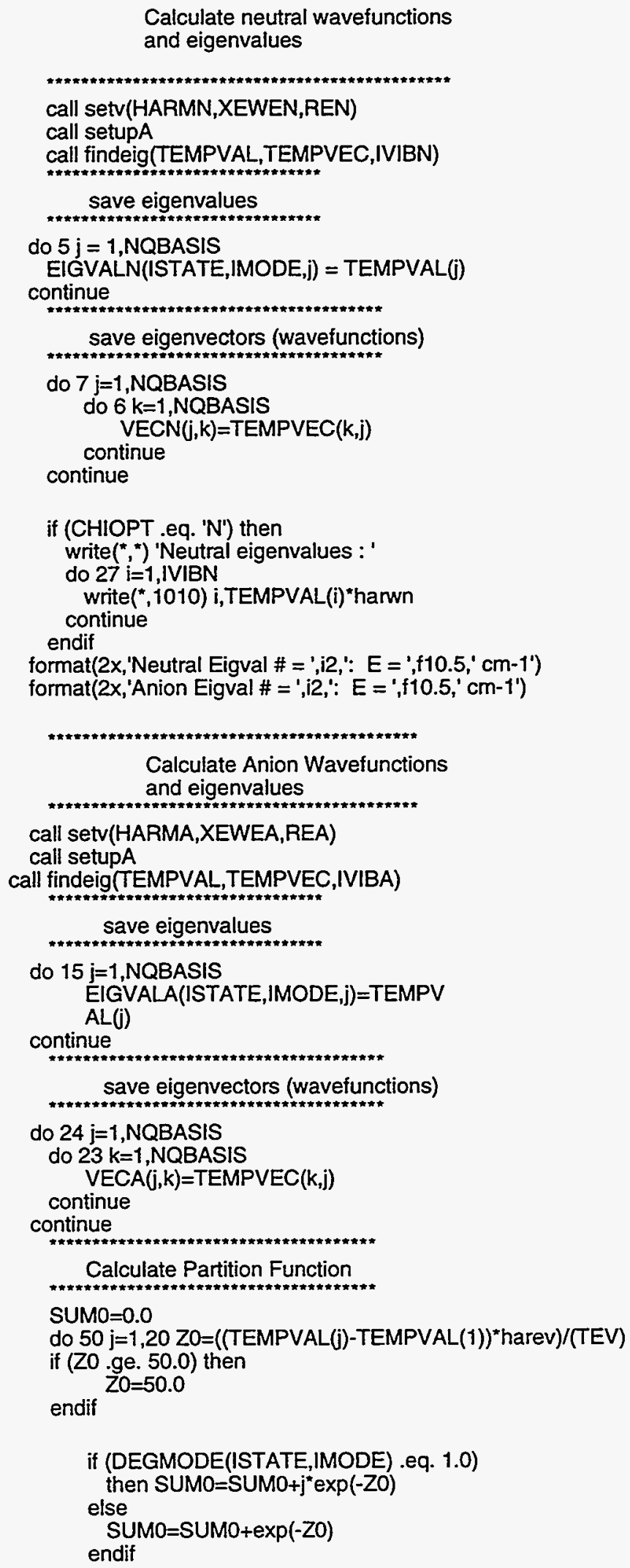

Calculate neutral wavefunctions and eigenvalues 


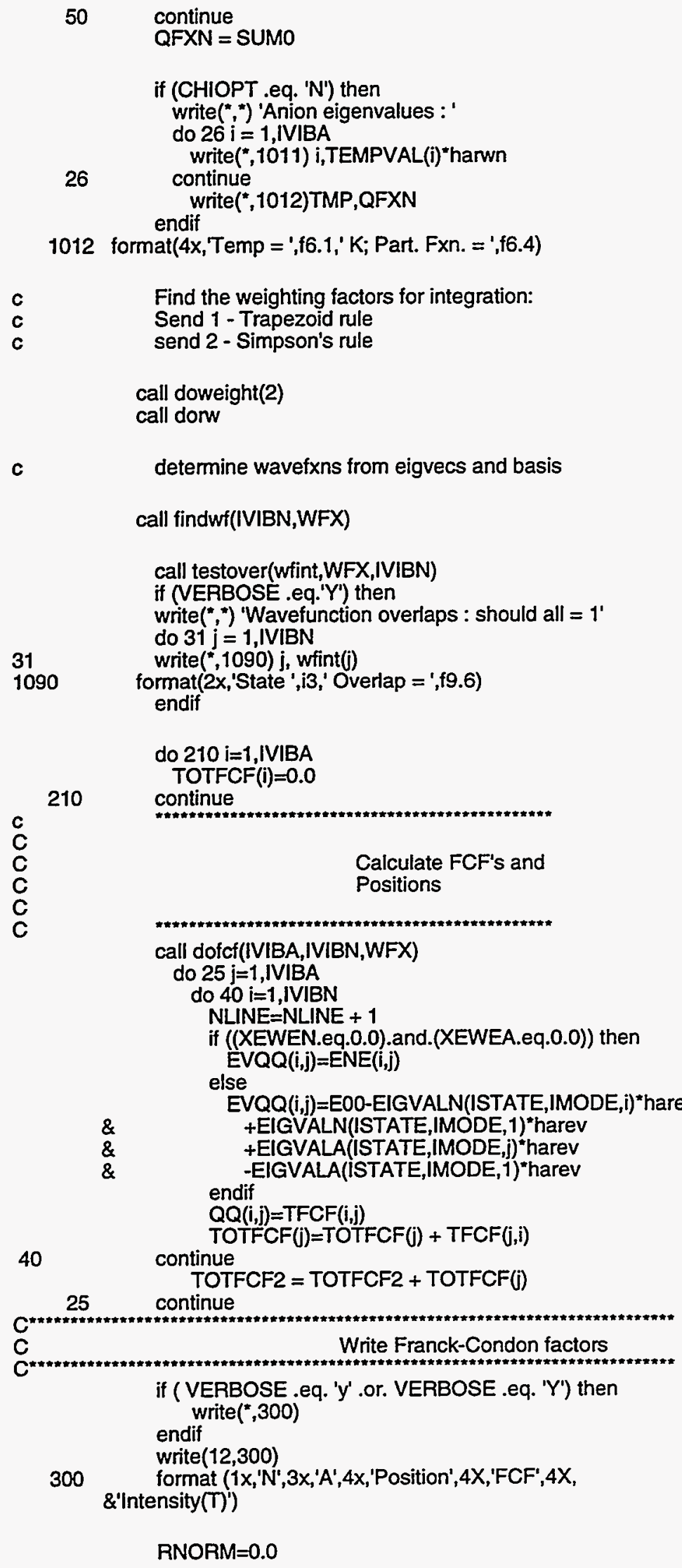

RNORM $=0.0$ 


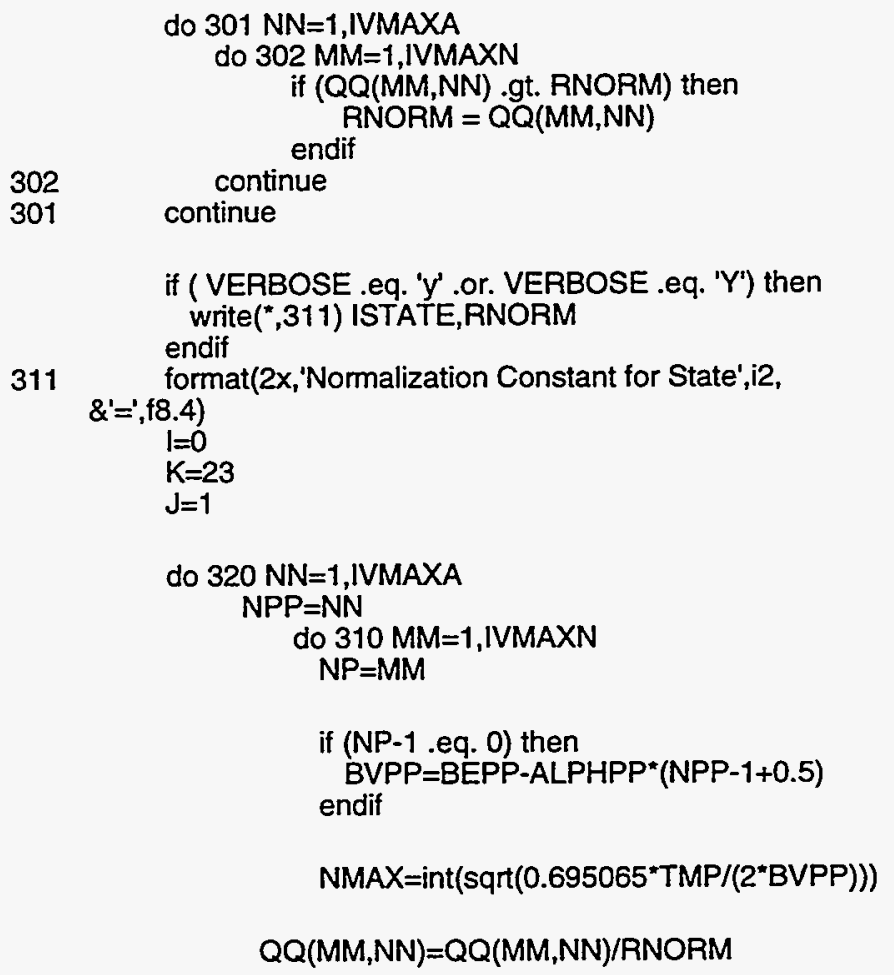


320

104
$P C T=(\exp (-Z 1) / Q F X N) * 100$

endif

if (VERBOSE .eq. ' $y$ ' .or. VERBOSE .eq. ' $Y$ ') then

endif write $(*, 312)$ PCT

write(12,312) PCT

format $(2 x, \%$ Population $=', 1 x, f 8.4)$

continue

write(12,104)

format(2x,'Peak',6x,'FCF',6x,'Position')

retum

end

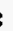

subroutine setconst

define fundamental constants

implicit double precision (a-h,o-z)

include "fcfnum.inc"

if (VERBOSE.eq.' $Y$ ') then

write $\left({ }^{*},{ }^{*}\right)$ ' setconst'

endif

iverbose $=0$

pi $=3.141592654$

hbar $=1.0545887 \mathrm{e}-34$

harev $=27.211608$

evwn $=8065.479$

$\mathrm{aO}=0.52917706$

$\mathrm{amu}=1822.882$

$\mathrm{emu}=9.109534 \mathrm{e}-31$

clight $=2.997925 \mathrm{e} 10$

harwn $=$ harev"ewn

amass $=1.66056 \mathrm{e}-27$

harkj $=2.625504 \mathrm{e} 3$

retum

end

subroutine setdvr

Set up for dvr-type quadrature.

Use either HEG quadrature or DVR method

procedure is as follows :

1) Find $<Q n|x| Q m>=$ MAXQBASIS and form matrix $R$.

2) Find eigenvalues ri and eigenvectors.

Use (orthonormalized) eigenvectors to

construct $T$, where

$T R T(-1)=\operatorname{diag}(\mathrm{ti})$, and

$T(-1)=T$ (transpose)

In HEG quadrature,

3) Find $<$ QnIVIQm> = MAXQBASIS by sum $(i=1, n)$ of Tni V(ri) Tmi. This is equivalent to a Gaussian Quadrature.

Notes: For harmonic oscillators $<Q n|x| Q m>$ can be found analytically, so the Hermite polynomials need never be evaluated !

implicit double precision (a-h,o-z)

include "fcfnum.inc"

dimension wgs(MAXQBASIS,MAXQBASIS),r(MAXQBASIS,MAXQBASIS)

dimension $t$ (MAXQBASIS,MAXQBASIS)

$<Q n|x| Q n+1>=\operatorname{sqr}(n+1) /($ beta*sqrt(2))

all other matrix elements are 0

( $x$ can be written in terms of raising/lowering operators..)

see Atkins, Molecular QM p. 102

if ((CHIOPT.eq.' $\left.N^{\prime}\right)$.and.(NERBOSE.eq.' $\left.Y^{\prime}\right)$ )then

write $\left({ }^{*},\right)^{\prime}+$ setdvr'

endif

do $47 i=1$,NQBASIS 
47

c

c

c

c

50

60

70

80

100

$r(i, 2)=0.0$

$r(i, 1)=\operatorname{sqrt}\left(1.0^{*}(i-1)\right)$

continue

Diagonalize $R$. $R$ is real, tridiagonal, so

use eispack routine for banded matrices

call rsb(MAXQBASIS,NQBASIS, 2,r,xgs, 1, wgs,s1,s2,ierr)

if ((CHIOPT.eq.' 'N').and.(NERBOSE.eq.' $Y$ ' $)$ )then endif

write $\left.(",)^{\prime}\right)$ ' First diagonalization done

eigenvectors are orthogonal, now normalize

do $70 i=1$, NQBASIS

xnorm $=0.0$

do $50 \mathrm{j}=1$,NQBASIS

xnorm $=x$ norm $+w g s(j, i)^{*} w g s(j, i)$

continue

xnorm $=$ sqrt $($ xnorm $)$

do $60 j=1$, NQBASIS

$t(j, i)=w g s(j, i) / x n o r m$

continue

continue

sqrt2 $=$ sqrt(2.0)

do $80 i=1$,NQBASIS

$\mathrm{xgs}(\mathrm{i})=\mathrm{xgs}(\mathrm{i}) / \mathrm{sqr} 2$

continue

format(f12.6)

if ((CHIOPT.eq.' $N$ ').and.(VERBOSE.eq.' $Y$ ')) then

write $\left(^{*},{ }^{*}\right)^{\prime}$-setdur'

endif

return

end

c *********************************************************

subroutine setupA

c Construct the A (Hamiltonian) matrix in HEG quadrature.

The elements of A (ie, Aij) are

Aij $=\langle$ QildeltaVIQj $\rangle+$ ei delta(i,j), where

Qi and Qj are basis functions (harmonic oscillator,

in this case), and deltaV $=V$ - Vref, where

$V$ is the potential which we desire to find

eigenvalues to, and Vref is the (h.o.) potential

that $\{$ Qi\} are exact solutions to. ei is the

ith eigenvalue for the reference problem, and

delta $(i, j)$ is 1 if $i=j$ and 0 otherwise.

The integral is done using DVR methods - this is

equivalent to using an NQBASIS point gauss-hermite

quadrature, where NQBASIS is the number of basis functions

(ie, $A$ is an NQBASIS by NQBASIS matrix).

Note that $A$ is symmetric, as deltaV contains no operators.

implicit double precision (a-h,o-z)

include "fcinum.inc"

if (iverbose.eq. 1) then

write $\left({ }^{*},{ }^{*}\right)$ 'setupa'

endif

call

zero(MAXQBASIS,A,MAXQBASIS,MAXQBASIS)

do $20 \mathrm{i}=1$, NQBASIS

do $10 \mathrm{j}=1$,

$$
A(i, j)=F i n d a(i, j)
$$

$A(j, i)=A(i, j)$

10

20

continue

continue

format("trace $=$ ',, 111.5 )

return 
end

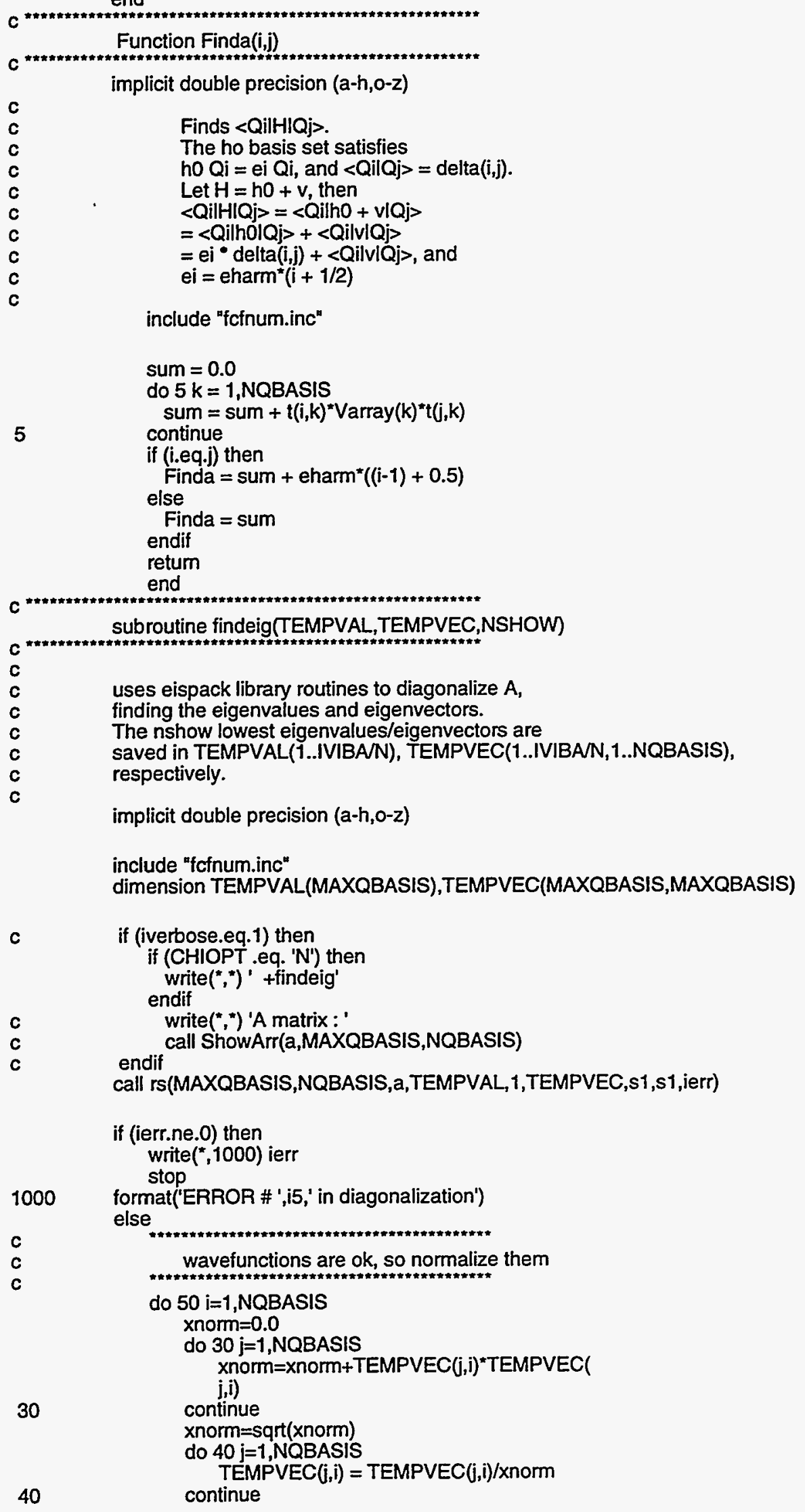




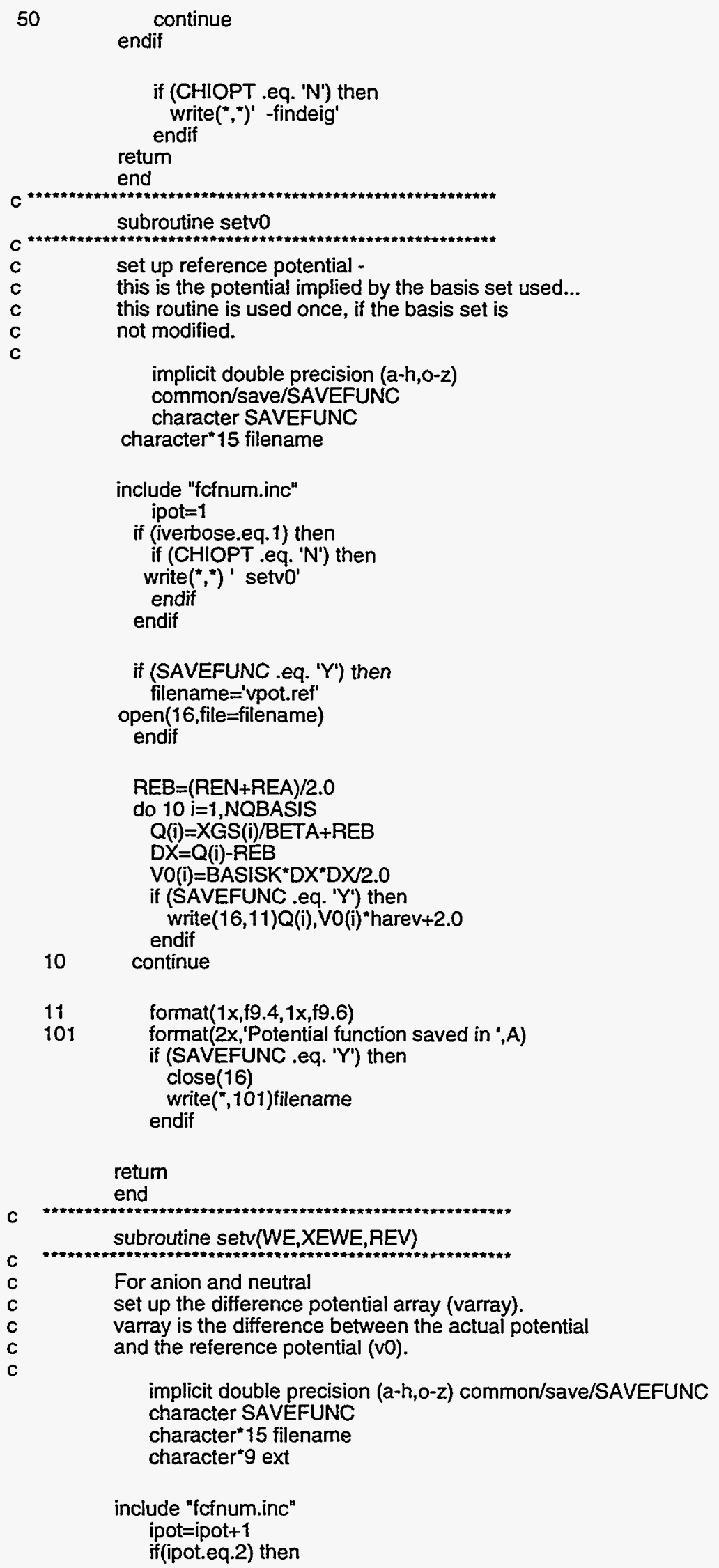




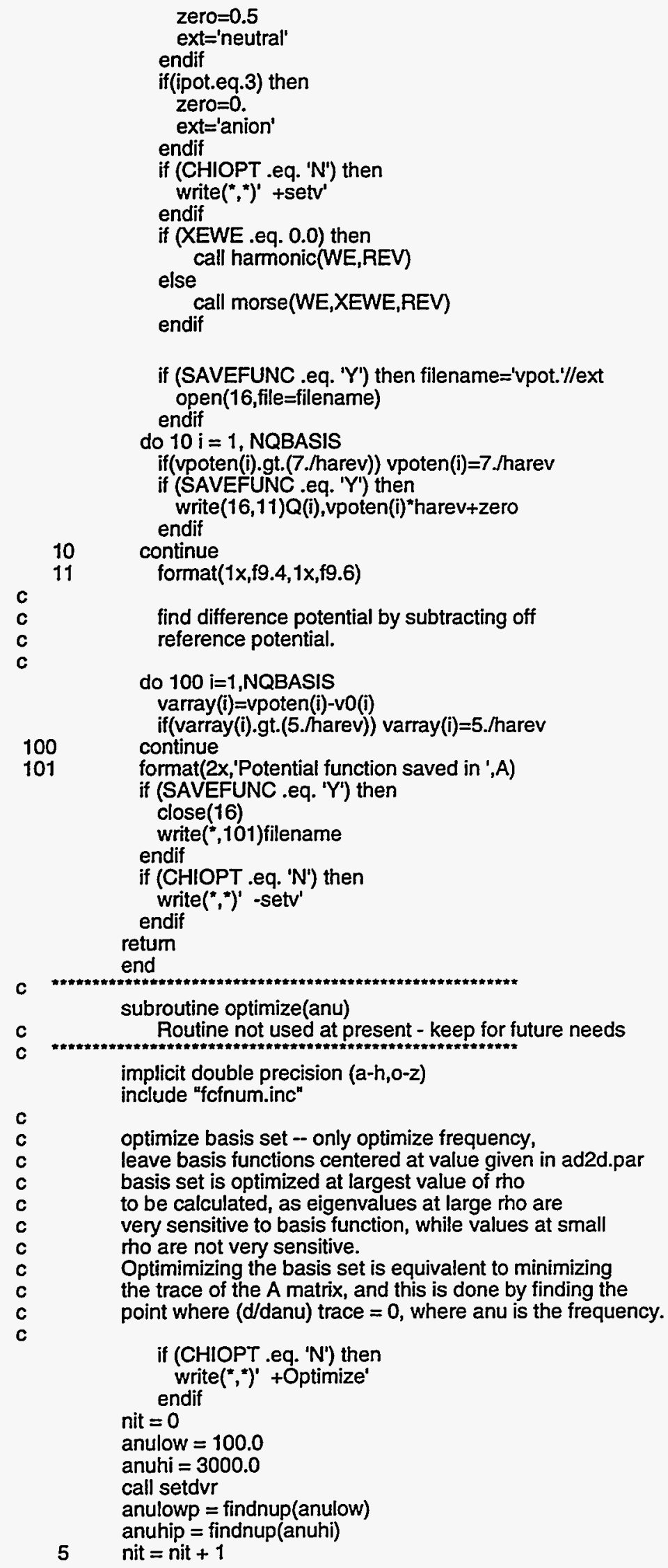


anunew $=$ anulow + anulowp $*$ (anulow - anuhi)/(anuhip - anulowp)

anunew $=($ anuhi + anulow $) / 2.0$

anunewp $=$ findnup(anunew)

if (anunewp.It.0.0) then

anulow $=$ anunew

else

anulowp $=$ anunewp

anuhi $=$ anunew

endif

anuhip $=$ anunewp

if (iverbose.eq.1) then

write $(*, 100)$ nit, anulow,

endif anuhi

100 format('it : ',i3,' $\min :$ ',f8.3,' $\max :$ ',f8.3)

if ((nit.lt.20) .and. ((anuhi-anulow) .gt. 5.0)) goto 5

anu $=($ anuhi + anulow $) / 2.0$

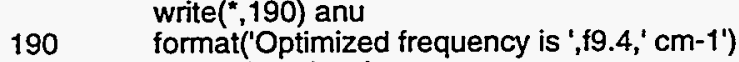

call setbeta(anu)

retum

end

c ***********************************************************

function findnup(anu)

c Not presently used - keep for future needs

c *********************************************************

c find derivative of trace w.r.t. the frequency anu.

c

implicit double precision (a-h,o-z)

include "fcfnum.inc"

write $\left({ }^{*},{ }^{*}\right)^{\prime}+$ +findnup'

hanu $=0.001$

call setbeta(anu-hanu)

write $\left({ }^{*}, 160\right)$ beta,anu,eharm, eharm*harev*evwn

160 format('beta : ',f9.6,' nu : ',f9.3,' e : ',f9.7

$\$,{ }^{\prime}$ or ', f13.4' $\left.\mathrm{cm}-1^{\prime}\right)$

call setvo

call setv(anu-hanu,Zero,REN)

call findtrac(trace1)

write $\left({ }^{*},{ }^{*}\right)$ trace 1

call setbeta(anu+hanu)

call setvo

call setv(anu+hanu,Zero,REN)

call findtrac(trace2)

write $\left(^{*}, *\right)$ trace2

findnup $=($ trace 2 -trace 1$) /$ hanu/2.0

write $(*, 180)$ anu,findnup

180 format(' nu: ',f13.7,' deriv : ',f16.7)

write ("; )' -findnup'

retum

cend

subroutine setbeta(anu)

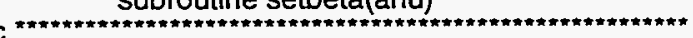

C Input basis function frequency and calculate beta in atomic units

beta $=\left\{\left(k^{*} m u\right) / h b a r^{\wedge} 2\right\}^{\wedge}(1 / 4)$

where $k$ is the force constant for a harmonic oscillator

of this frequency, and

$k=\left[\left(2^{*} \text { pi }^{*} c^{*} \text { freq }\right)^{\wedge} 2\right]^{*} m u$

implicit double precision $(a-h, 0-z)$

include "fcinum.inc"

if (VERBOSE .eq. ' $Y$ ') then

write $\left({ }^{*},\right)^{\prime}+$ setbeta' 


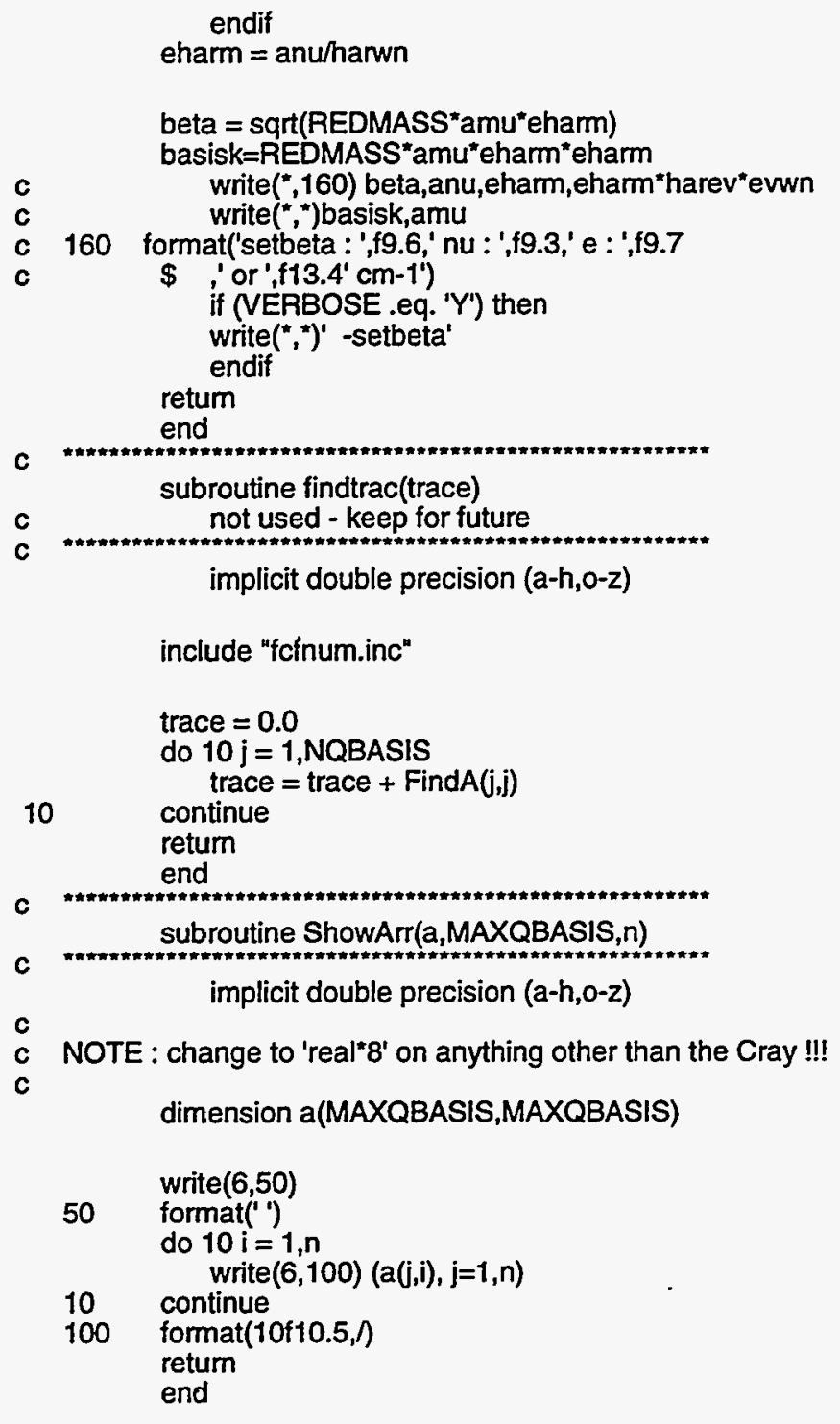

\section{GRAD1.f}

Subroutine GRADIENT(QPARAMS,NPAR,NPARV,CMACH,GRAD,CHIST,EXPCNTS, \&ISTATES,NMODES)

Implicit Double Precision(a-h,o-z)

C
C
C
C
C
C
C
C

This subroutine calculates the 1st derivatives of the simulation, point by point, with respect to each parameter. These derivatives are used to fill the alpha and beta matrices which are used to determine the new parameters used in the optimization via chisquares. This is based upon the Marquardt-Levinberg method, described in both Bevington and Numerical Recipes.

NOT USED FOR ML METHOD AT PRESENT-ONLY STEEPEST DESCENT METHOD... STILL USE THIS ROUTINE TO CALCULATE GRADIENT - 6/26/94 -DWA

include "params.inc"

character*1 QPARAMS(NPRSMX)

character*1 CHIOPT

c

character*20 PARNAMES(NPRSMX)

dimension GRADCHI(NPTSEXP) 
dimension DERIV(NPRSMX,NPTSEXP)

dimension BETA(NPRSMX),GRAD(NPRSMX)

dimension ALPHA(NPRSMX,NPRSMX)

dimension NMODES(NSTSMX)

common/chi/EXPCHI(NPTSEXP,2),SIGEXP(NPTSEXP)

common/chi2/IPTSEXP,NVAR

common/chi3/FCHISQ,SIMCHI(NPTSEXP,2)

common/chi4/OPTFILE

common/opt1/PARAMS(NPRSMX,ITRSMX),DELTA(NPRSMX,ITRSMX)

common/opt2/IT

common/write/PARNAMES(NPRSMX),QPARAMS(NPRSMX)

$\mathrm{C}$
$\mathrm{C}$
$\mathrm{C}$
$\mathrm{C}$
$\mathrm{C}$
$\mathrm{C}$
$\mathrm{C}$

Add delta and calculate simulation, then subtract delta and repeat, then calculate slope $=$ derivative.

120

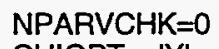

write $\left({ }^{*},\right)^{\prime}$ Calculating GRADient of Chi-Square'

call OPTWRITE()

do $120 \mathrm{i}=1$,NPAR

if (QPARAMS(i) .eq. ' $Y$ ') then

PARAMS $(i, I T)=$ PARAMS $(i, I T)+D E L T A(i, I T) * 0.1$

call MAKESPEC(NPAR,CMACH,GRADCHI)

PARAMS $(i, I T)=$ PARAMS $(i, I T)$-DELTA $(i, I T)^{*} 0.1$

call CHISQ(EXPCNTS,CHIOPT,ISTATES,NMODES,CHIGRAD)

GRAD $(i)=$ CHIST - CHIGRAD

SUM = SUM + GRAD (i)**2

endif

NPARVCHK=NPARVCHK +1

continue

do $130 \mathrm{i}=1$,NPAR

if (QPARAMS(i) .eq. ' $Y$ ') then

GRAD(i) = DELTA $(i, I T)^{*}$ GRAD(i)/sqrt(SUM)

write $(*, 131)$ i,GRAD(i)

131 format(2x,'Parameter ',i2,' has gradient of ',f9.6)

endif

130 continue

if (NPARVCHK .ne. NPARV) then write $(*, 121)$ NPARVCHK,NPARV

121 format(2x,'ERROR: \# Varied parameters differs between',1x,

\&'DERIVATIVE $\left(\left(, \mathrm{i} 2,{ }^{\prime}\right)\right.$ and TIDYUP $\left.\left({ }^{\prime}, \mathrm{i} 2,{ }^{\prime}\right) '\right)$

endif

write(",")' GRADient of Chi-Square calculated'

retum

end

\section{HOFCF1.f}

Subroutine hofcf(ISTATE,MODE,RMU,VOP,VOPP,XEWEC,XEWECC,EVSTRT, \& REOP,REOPP,NPMAX,NPPMAX,TMP,QFXN)

implicit double precision (A-H,O-Z)

C $6 / 90$ - This program will calculate the Franck-Condon factors for a diatomic

C molecules using a harmonic oscillator potential and corrections to

C harmonic FCF's for anharmonicities. This program routine has been

C adapted from pascal to FORTRAN in $6 / 90$ by Don $W$. Arnold. The

C original program was based upon Hutchisson, Phys. Rev., 36, 410, (1930). 
Note:'float' command is to convert integer to real...sometimes just multiplied the integer by 1.0 in the program.

Code has been modified to deal with only harmonic oscillators on $11 / 13 / 92$ by DWA. The first version to do this is ANHFCF5.F while all previous versions made a correction to the harmonic wave function to account for anharmonicity. At this stage, I am implementing the LAG.F code to calculate the FCF's for systems which are anharmonic. It uses Laguerre polynomial wavefunctions which are appropriate for Morse oscillators and a numerical integration of those functions. All variables are essentially unchanged but the QQANH has been dropped and the LAG.F now uses a consistent set of arrays.-11/13/92 DWA

9/11/93-Now this code is called HOFCF1.F. The LAG.F code has problems with small anharmonicities...it blows up at the gamma function calculation. Instead, a numerical method is employed to calculate FCF's for general potentials fcinum1.f-DWA

definitions of variables

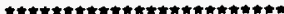

XM1,XM2-mass of atoms in amu

RMU-reduced mass of diatomic with $X M 1$ and $X M 2$, in grams

VOP, VOPP-harmonic frequencies neutral and anion, respectively, in wavenumbers

EVVOP,EVVOPP-harm freq of neutral and anion in $\mathrm{eV}$

EVSTRT-origin of photoelectron spectrum

REOP,REOPP-equilibrium separation of neutral and anion, in Angstroms

ROP,ROPP-equil. sep. of neutral and anion in centimeters NPMAX,NPPAMX-number of vibrational levels in neutral and anion

TMP-temperature, in Kelvins

TEV-temperature, in eV

MM-neutral

NN-anion

parameter(NMDSMX =15,NVMAXA =20,NVMAXN=30,NLINES=2500) parameter(NPTSEXP $=1000, N P T S F I T=1000, N S T S M X=5$ ) dimension $Q Q(30,30), Q Q I N T(30,30)$

character VERBOSE

real*8 MTMP(NSTSMX,NMDSMX)

common/comb3/EVQQ(NVMAXN,NVMAXA),QQ(NVMAXN,NVMAXA), \&QQB(NVMAXN,NVMAXA), XM1,XM2

common/verb/NERBOSE

C common/deg/DEGMODE(NSTSMX,NMDSMX),MTMP(NSTSMX,NMDSMX)

$\mathrm{pi}=3.141592654$

$c=2.99792458 E+10$

$h=6.626176 E-27$

C

${ }_{* * * * * *}$ Convert from wavenumbers to $\mathrm{eV}^{* * * * *}$

EVVOP=VOP/8065.479

EVVOPP $=$ VOPP $/ 8065.479$

$X E W E P=X E W E C / 8065.479$

C

$X E W E P P=X E W E C C / 8065.479$

ROP $=$ REOP*1.0E-8

ROPP $=$ REOPP $1.0 \mathrm{E}-8$

******"Convert temperature from kelvins to $\mathrm{eV}^{* * * * *}$

$T E V=T M P * 8.61735 E-05$

C

$A L F=s q r($ (VOPP NOP)

$D E N=1.0+A L F^{*} A L F$

XNUM=1.0-ALF*ALF

NPMAX $=N P M A X+1$

NPPMAX $=$ NPPMAX +1

$D E L=(R O P-R O P P)^{\star} 2.0^{*} \mathrm{pi}^{*} \mathrm{sqr}\left(\mathrm{c}^{*} \mathrm{VOPP}{ }^{*} \mathrm{RMU} / \mathrm{h}\right)$ 


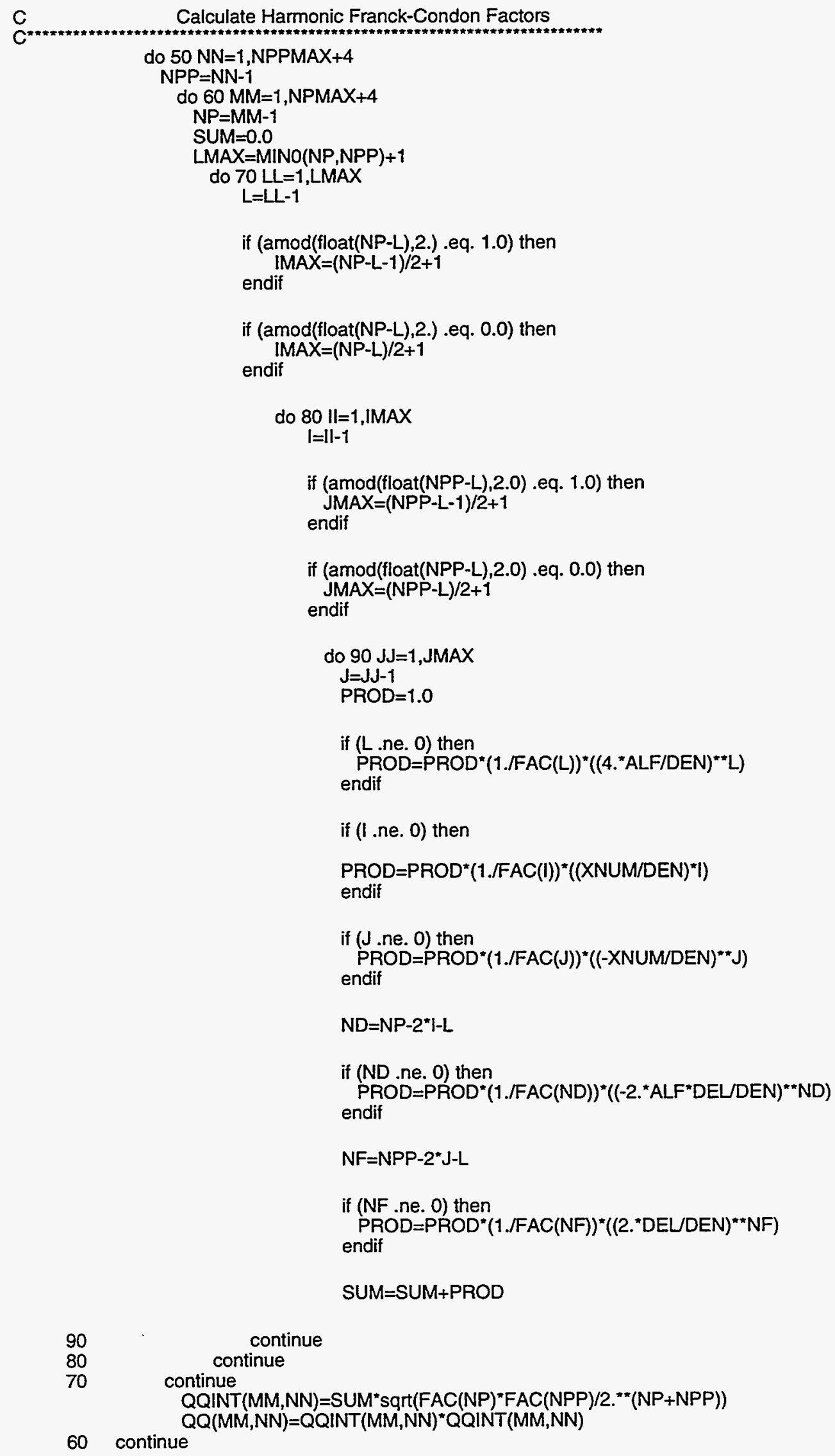


50 continue

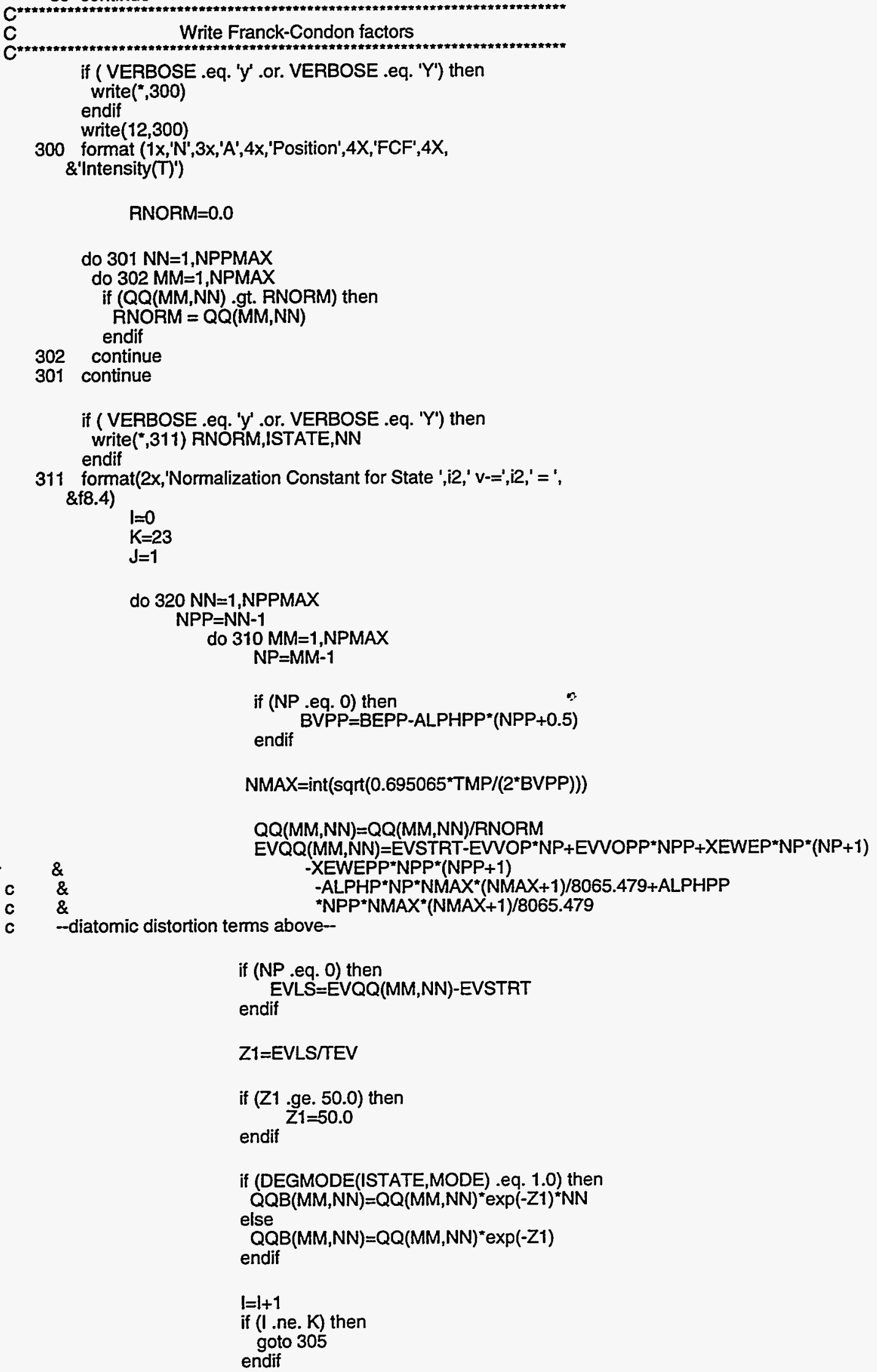

if (NP .eq. 0) then endif 
$J=J+1$

$K=K^{*} J$

305

if (VERBOSE .eq. ' $y$ ' .or. VERBOSE .eq. ' $Y$ ') then

write $\left.{ }^{*}, 303\right) N P, N P P, Q Q(M M, N N), E V Q Q(M M, N N), Q Q B(M M, N N)$ endif

write(12,303)NP,NPP,QQ(MM,NN),EVQQ(MM,NN),QQB(MM,NN)

310

continue

if (DEGMODE(ISTATE,MODE) .eq. 1.0) then

$P C T=\left(\exp (-Z 1)^{*} N N / Q F X N\right)^{*} 100$

else

$P C T=(\exp (-Z 1) / Q F X N) * 100$

endif

if (VERBOSE .eq. ' $y$ ' .or. VERBOSE .eq. 'Y') then

write $(*, 312)$ PCT

endif

320 continue

write $(12,104)$

NPMAX $=$ NPMAX-1

NPPMAX=NPPMAX-1

104

303

format(2x,'Peak',6x,'FCF',6x,'Position')

format $(12,2 x, 12,2 x, f 8.4,2 x, f 8.4,2 x, f 8.4)$

format $(f 12.6, f 12.6)$

format $(2 x, \%$ Population $=', 1 x, f 8.4)$

return
end

C
C

end

\section{Subroutine for calculating factorials}

FUNCTION FAC(N)

implicit double precision $(A-H, O-Z)$

if ( $N$.eq. 0$)$ then

go to 400

endif

if $(N$.eq. 1$)$ then

go to 400

endif

result $=1.0$

if ( $N$.It. 0$)$ then

$N=-1 * N$

endif

do $410 i=2, N$

result=result ${ }^{*} i$

410

continue

$\mathrm{FAC}=$ result

return

$400 \quad F A C=1.0$

retum

end

\section{HUNTS.f}

Subroutine HUNT(N,X,JLO)

implicit double precision(a-h,o-z)

C

From Numerical Recipes -Press, Teukolsky, Flannery, and Vetterling 
c

c

1

2

3

ENDIF

$$
\text { ELSE }
$$$$
\text { ENDIF }
$$

Cambridge University Press, 1989.

include "params.inc"

common/hunt $1 / X X(N P T S F I T)$

DIMENSION XX(N)

ASCND $=X X(N) \cdot G T \cdot X X(1)$

IF(JLO.LE.0.OR.JLO.GT.N)THEN

$\mathrm{JLO}=0$

$\mathrm{JHI}=\mathrm{N}+1$

ENDIF

INC=1

IF(X.GE.XX(JLO).EQV.ASCND)THEN

$J H I=J L O+I N C$

IF(JHI.GT.N)THEN $\mathrm{JH} I=\mathrm{N}+1$

ELSE IF(X.GE.XX(JHI).EQV.ASCND)THEN

$$
\text { JLO=JHI }
$$

INC=INC+INC

GO TO 1

$J H I=J L O$

$J L O=J H I-I N C$

IF(JLO.LT.1)THEN JLO $=0$

ELSE IF(X.LT.XX(JLO).EQV.ASCND)THEN

JHIILJO

INC=INC+INC

$$
\text { GO TO } 2
$$

ENDIF

$$
\text { IF(JHI-JLO.EQ.1)RETURN }
$$

$\mathrm{JM}=(\mathrm{JHI}+\mathrm{JLO}) / 2$

IF(X.GT.XX(JM).EQV.ASCND)THEN

$$
J L O=J M
$$

ELSE

$J H I=J M$

ENDIF

GO TO 3

END

\section{INDEXX.f}

SUBROUTINE INDEXX(N,ARRIN,INDX)

implicit double precision $(a-h, o-z)$

From Numerical Recipes - Press, Flannery, Teukolsky and Vetterling

c $\quad$ Cambridge University Press, 1989.

DIMENSION ARRIN(N),INDX(N)

write $(*, 5) \mathrm{N}$

5 format(2x,'Indexing', 1x,i6,' Combination Bands')

DO $11 \mathrm{~J}=1, \mathrm{~N}$

INDX $(J)=J$

11 CONTINUE

$L=N / 2+1$

IR=N

10 CONTINUE

IF(L.GT.1)THEN

$L=L-1$

INDXT $=$ INDX(L)

$Q=A R R I N(I N D X T)$

ELSE

INDXT $=$ INDX(IR)

$Q=A R R I N(I N D X T)$

INDX(IR)=INDX(1)

IR $=$ IR -1

IF(IR.EQ.1)THEN

INDX(1)=INDXT

ENDIF

RETURN

ENDIF

$\mathrm{I}=\mathrm{L}$ 
$J=L+L$

IF(J.LE.IR)THEN

IF(J.LT.IR)THEN

IF(ARRIN(INDX(J)).LT .ARRIN(INDX(J+1))) $J=J+1$

ENDIF

IF(Q.LT.ARRIN(INDX(J)))THEN

INDX $(\mathrm{I})=\mathrm{INDX}(\mathrm{J})$

$I=J$

$\mathrm{J}=\mathrm{J}+\mathrm{J}$

ELSE

$J=i R+1$

ENDIF

GO TO 20

ENDIF

INDX(I)=INDXT

GO TO 10

END

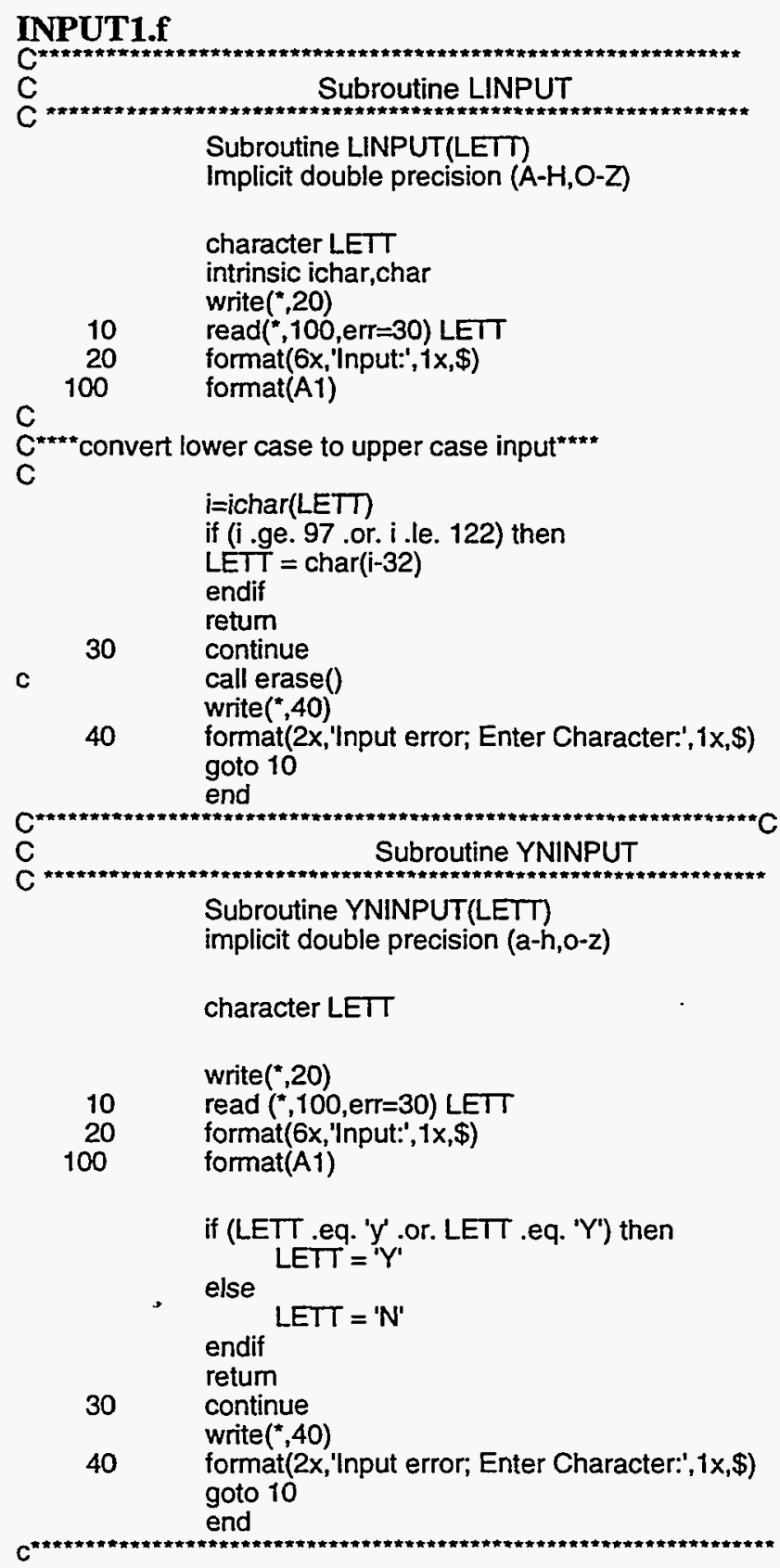


c

Subroutine REALINPUT

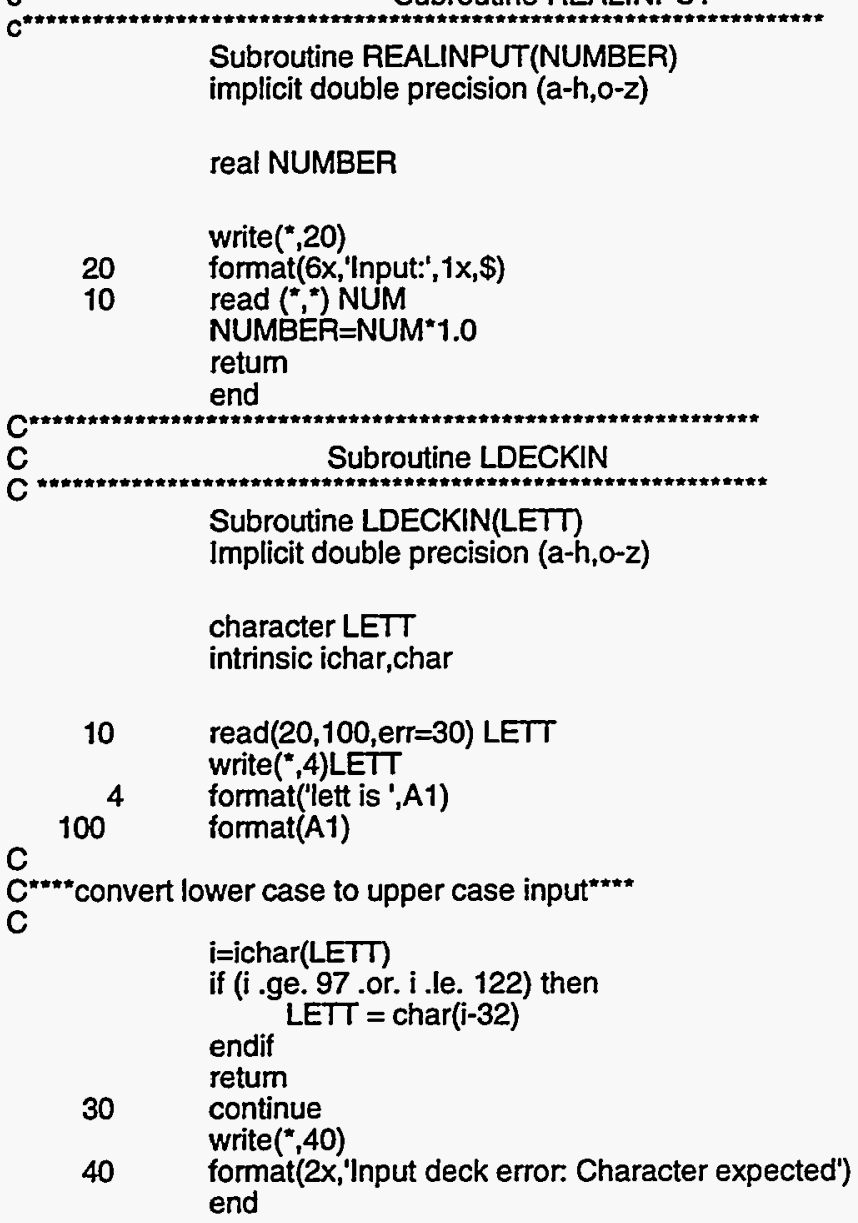

MAKESPEC2.f

Subroutine MAKESPEC(NPAR,CMACH,BDERIV)

Implicit Double Precision(a-h,o-z)

C This is a subroutine to do a bare bones simulation of data during

C optimization routine TIDYUP. It basically makes the same calls as

C during a manual fit but without the output being generated until

C the last step. Parameters for each step are kept so that manual

C checks of the output can be made if so desired. The spectra will

C be generated so that periodic plotting can be done, during the

C fitting procedure, if so desired (and If we can find a suitable

C plotting routine).

C include "params.inc"

character LDEGMODE(NSTSMX,NMDSMX), QTMP(NSTSMX,NMDSMX)

character ${ }^{*} 1$ VERBOSE, TERMINAL

character"1 CHIOPT,CMACH

character*20 DECKTITLE,OPTFILE,EXPFILE

character*20 PARNAMES(NPRSMX)

character*1 QPARAMS(NPRSMX)

real*8 NMSTRT(NSTSMX),EVSTRT(NSTSMX)

real"8 NMSTRT1

common/comb1/FCF(NMDSMX,NVMAXN,NVMAXA),POS(NMDSMX, NVMAXN,NVMAXA),DEGFLAG

common/comb3/EVQQ(NVMAXN,NVMAXA),QQ(NVMAXN,NVMAXA), 
common/comb5/IVMAXA(NSTSMX,NMDSMX),IVMAXN(NSTSMX,NMDSMX) common/sim/TW,EW,ORIGIN,A(NPTSFIT),SIM(NPTSFIT,2), EMAX,

\&EXPCNTS

common/hunt1/XX(NPTSFIT) common/chi/EXPCHI(NPTSEXP,2),SIGEXP(NPTSEXP)

common/chi2/PTSEXP,NVAR

common/chi3/FCHISQ,SIMCHI(NPTSEXP,2)

common/chi4/OPTFILE

common/verb/NERBOSE

common/deg/DEGMODE(NSTSMX,NMDSMX),MTMP(NSTSMX,NMDSMX)

common/params/CHIOPT,TERMINAL, EXPFILE

common/params2/RESOLUTION,ITERATION,ISTATES

common/params $3 /$ EVSTRT(NSTSMX),SCALE(NSTSMX),NMODES(NSTSMX) common/params4/BONDN(NSTSMX,NMDSMX),DELQ1(NSTSMX,NMDSMX),

\&LDEGMODE(NSTSMX,NMDSMX),QTMP(NSTSMX,NMDSMX)

common/params5/NMSTRT(NSTSMX)

common/plot/EXPT(NPTSEXP,2)

common/out/RMU2,QFXN(NSTSMX,NMDSMX)

common/opt1/PARAMS(NPRSMX,ITRSMX),DELTA(NPRSMX,ITRSMX)

common/opt2/IT

common/write/PARNAMES(NPRSMX), QPARAMS(NPRSMX)

common/flag/ITFLAG

real"8 MTMP(NSTSMX,NMDSMX)

dimension PARAMS(NPRSMX,ITRSMX)

dimension HFREQA(NSTSMX,NMDSMX),HFREQN(NSTSMX,NMDSMX)

dimension ANHARMA(NSTSMX,NMDSMX),ANHARMN(NSTSMX,NMDSMX)

dimension BONDA(NSTSMX,NMDSMX)

dimension BDERIV(NPTSEXP)

NPTS $=2000$

$\stackrel{\mathrm{C}}{\mathrm{C}}$

array

C

write $\left({ }^{*},\right)^{\prime}+$ MAKESPEC'

Initialize SIMCHI

do $29 \mathrm{i}=1$,IPTSEXP

BDERIV $(i)=0.0$

C
C
C
C

continue

Set up variable arrays for calculation

NPARCHK $=0$

do $100 \mathrm{i}=1$,ISTATES

do $110 j=1, N M O D E S(i)$

HFREQA $(i, j)=$ PARAMS (NPARCHK +1, IT)

HFREQN $(i, j)=$ PARAMS(NPARCHK $+2,1 T)$

ANHARMA $(\mathrm{i}, \mathrm{j})=$ PARAMS (NPARCHK $+3, \mathrm{IT})$

ANHARMN $(i, j)=$ PARAMS(NPARCHK +4, IT)

BONDA $(\mathrm{i}, \mathrm{j})=$ PARAMS $(\mathrm{NPARCHK}+5, \mathrm{IT})$

BONDN $(i, j)=$ PARAMS(NPARCHK $+6, I T)$

MTMP $(i, j)=$ PARAMS(NPARCHK $+7, I T)$

110 continue NPARCHK $=$ NPARCHK +7

EVSTRT $(i)=$ PARAMS(NPARCHK $+1, I T)$

NPARCHK=NPARCHK +1

100

continue

if (ITFLAG .eq. 1) then

call OPTWRITE() 


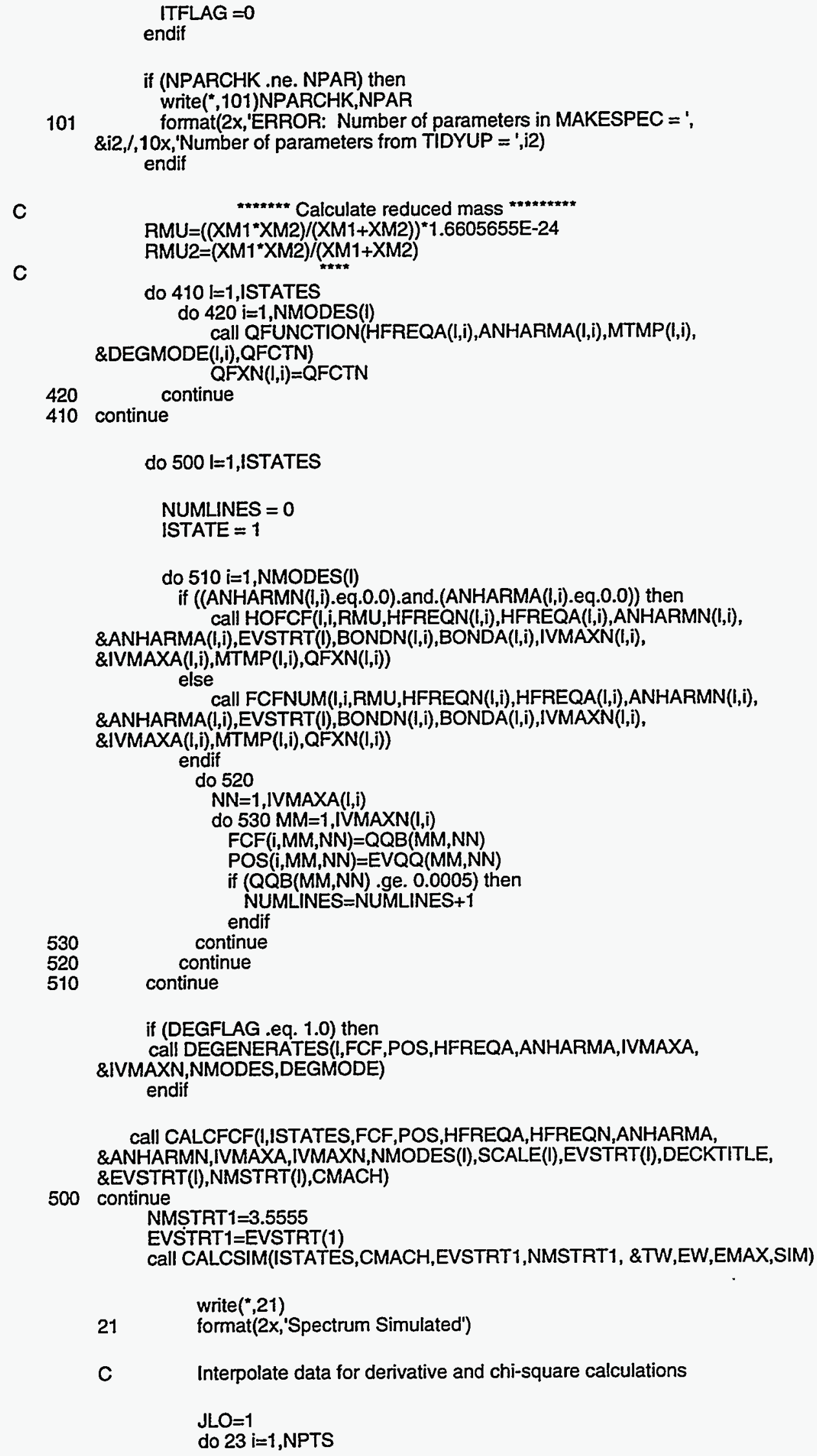

call CALCFCF(I,ISTATES,FCF,POS,HFREQA,HFREQN,ANHARMA, \&ANHARMN,IVMAXA,IVMAXN,NMODES(l),SCALE(l),EVSTRT(l),DECKTITLE, \&EVSTRT(I),NMSTRT(I),CMACH)

500 continue

NMSTRT $1=3.5555$

EVSTRT1=EVSTRT(1)

call CALCSIM(ISTATES,CMACH,EVSTRT1,NMSTRT1, \&TW,EW,EMAX,SIM)

21 write(",21) format(2x,'Spectrum Simulated')

C Interpolate data for derivative and chi-square calculations

$\mathrm{JLO}=1$

do $23 i=1$,NPTS 
$X X(i)=\operatorname{SiM}(i, 1)$

continue

do $22 \mathrm{i}=1$,IPTSEXP

FIND=EXPT(i,1)

call hunt(NPTS,FIND,JLO)

SIMCHI $(i, 1)=\operatorname{SIM}(J L O, 1)$

SIMCHI(i,2)=SIM(JLO,2)

BDERIV $(i)=\operatorname{SIM}(J L O, 2)$

22

$\mathrm{JLO}=\mathrm{JLO}+1$

continue

write (",")' MAKESPEC out"

return

end

\section{MATINV.f \\ Subroutine MATINV(ARRAY,NORDER,DET) \\ Implicit Double Precision(a-h,o-z)}

This routine, taken from Bevington, inverts
C calculates its determinant.-DWA-5/27/92.

This routine, taken from Bevington, inverts a symmetric matrix and

$\mathrm{C}$
$\mathrm{C}$
$\mathrm{C}$
$\mathrm{C}$
$\mathrm{C}$

dimension ARRAY (15,15), IK(15), JK(15)

Find largest element ARRAY $(i, j)$ in rest of matrix

write $(", *)$ 'MATINV in

$\mathrm{DET}=1$.

do $100 \mathrm{k}=1$, NORDER

AMAX $=0$.

do $30 \mathrm{i}=\mathrm{k}$,NORDER

do $30 j=k$,NORDER

if (abs(AMAX)-abs(ARRAY(i,j))) $24,24,30$

24

$A M A X=A R R A Y(i, j)$

$\mathrm{IK}(\mathrm{K})=\mathrm{i}$

$J K(k)=$

30

continue

$\stackrel{\mathrm{C}}{\mathrm{C}}$ Interchange rows and columns to put in matrix $\operatorname{ARRAY}(k, k)$

if (AMAX) 41,32,41

$\mathrm{DET}=0$.

goto 140

$\mathrm{i}=\mathrm{IK}(\mathrm{k})$

if (i-k) $21,51,43$

do $50 \mathrm{j}=1$, NORDER

SAVE=ARRAY $(\mathrm{i}, \mathrm{j})$

ARRAY $(k, j)=A R R A Y(i, j)$

50

ARRAY $(i, j)=-S A V E$

$j=J K(k)$

if $(j-k) 21,61,53$

do $60 i=1$,NORDER

SAVE=ARRAY $(i, k)$

ARRAY $(i, k)=A R R A Y(i, j)$

$\mathrm{C}$
$\mathrm{C}$
$\mathrm{C}$

$\operatorname{ARRAY}(\mathrm{i}, \mathrm{j})=-\mathrm{SAVE}$

Accumulate Elements of Inverse Matrix

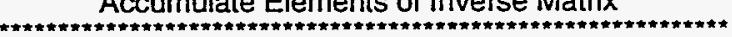

do $70 \mathrm{i}=1$,NORDER

if (i-k) $63,70,63$

ARRAY $(i, k)=-A R R A Y(i, k) / A M A X$

continue

do $80 \mathrm{i}=1$, NORDER

do $80 \mathrm{j}=1$,NORDER 


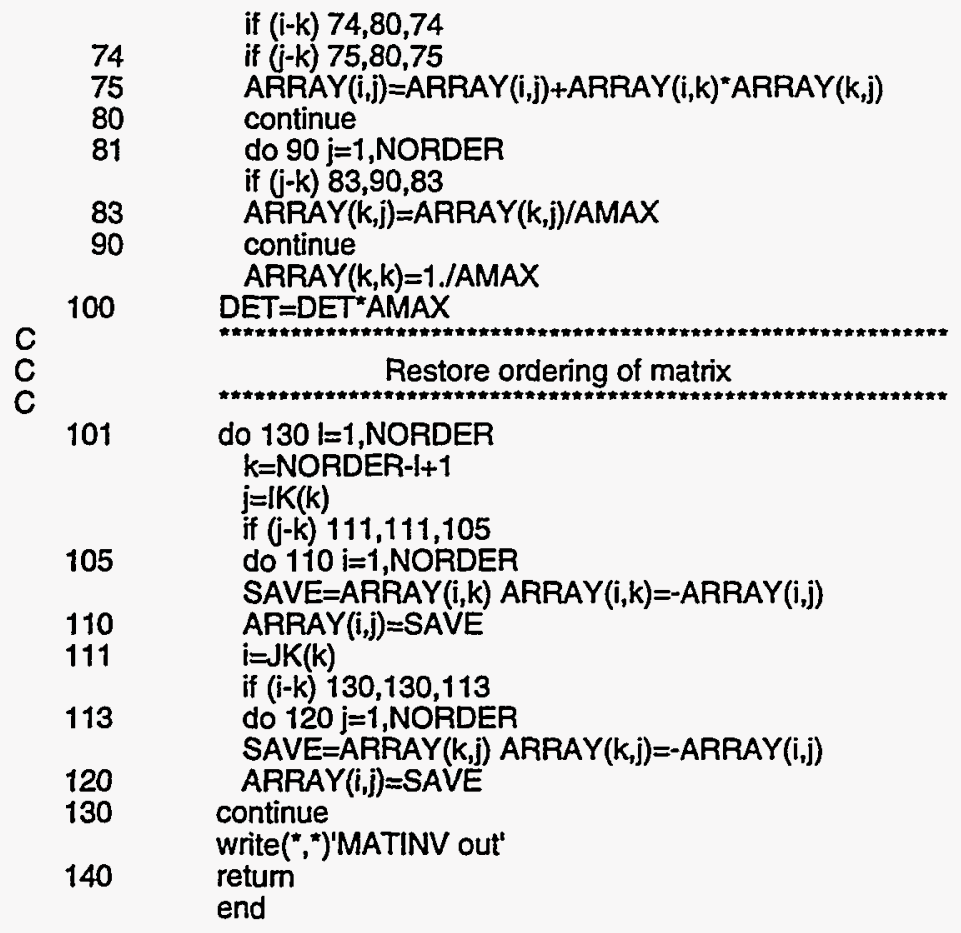

\section{MATRIX.f}

Matrix.f : matrix subroutines:

Written by RBM 1989

Includes:

matmult : multiply matrices

transpose : find the transpose of a matrix

showarr1, showarr2: print out a matrix

showarrs : print out a symmetric matrix

zero: set all elements of a matrix to be 0

Note: The Cray has more efficient subroutines for

transpose, matmult, and zero, but these are used anyhow.

Showarrs has a nice way of printing out a symmetric matrix.

subroutine matmult(ndim,a,nra,nca,b,nrb,ncb,c,nrc,ncc,ierr)

Finds $C=A B$, where $A$ and $B$ are matrices.

all matrices are dimensioned to ndim $x$ ndim

The actual size of $A$ is nra $\times$ nca, $B$ is

nib $\times$ ncb, etc.

Note that nca must be equal to nrb, or the matrices

cannot be multiplied. If they are not equal, the

routine will exit with ierr $=1$. If they are

equal, the routine will exit with ierr $=0$.

implicit double precision (a-h,o-z)

dimension a(ndim,ndim), b(ndim,ndim), c(ndim,ndim)

ierr $=0$

if (nca.ne.nrb) then

ierr $=1$

endif

goto 999
gotr 1

do 30 ir $=1$, nra

do $20 \mathrm{icb}=1, \mathrm{ncb}$

sum $=0.0$ 


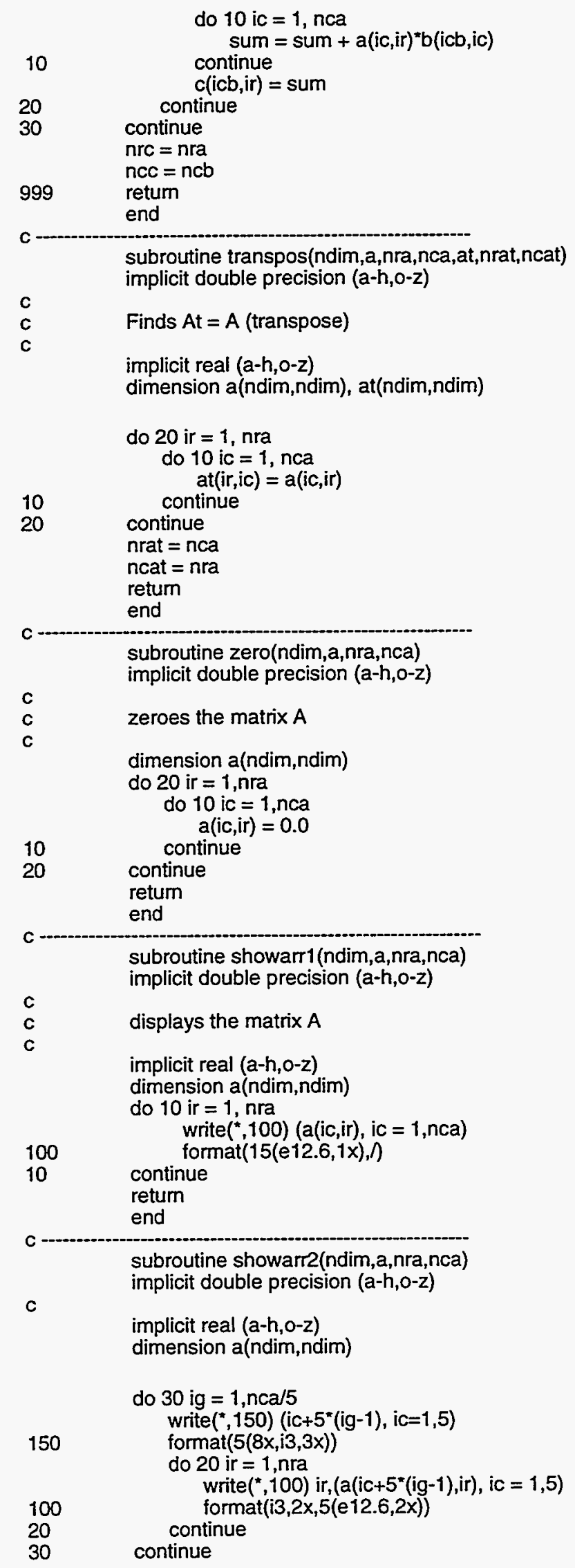



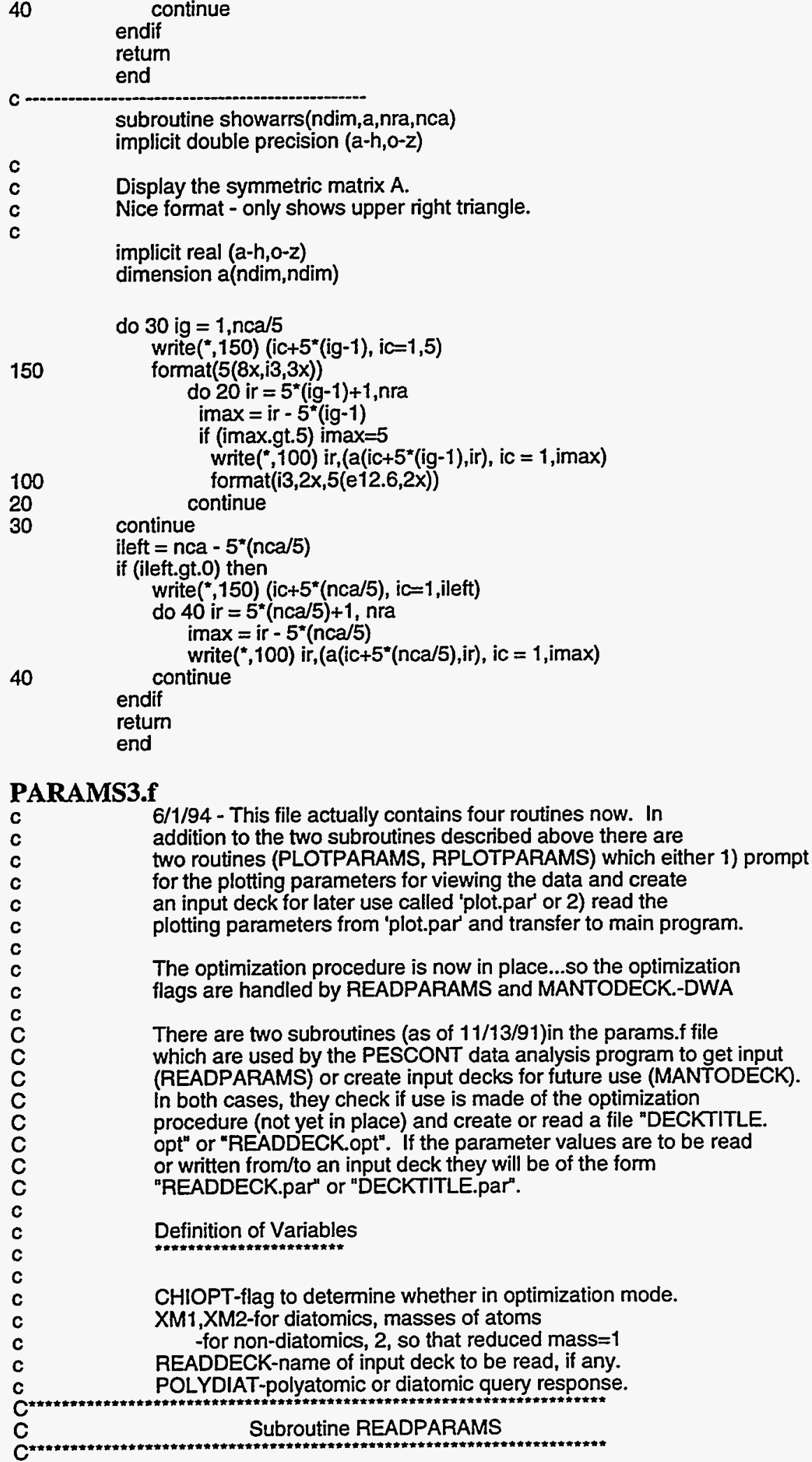
Subroutine READPARAMS(READDECK,CMACH)

implicit double precision $(a-h, o-z)$

This subroutine is to read in the parameters for the PESCONT data analysis program (under construction 11/13/91) from an input deck. The MANTODECK subroutine will write the input deck in the appropriate form, thus if modifications are made to this subroutine, the appropriate changes should be made to the MANTODECK routine.

include "params.inc"

dimension DEV(NSTSMX)

character LDEGMODE(NSTSMX,NMDSMX),QTMP(NSTSMX,NMDSMX) character QFREQA(NSTSMX,NMDSMX), QFREQN(NSTSMX,NMDSMX)

character QANHARMA(NSTSMX,NMDSMX), QANHARMN(NSTSMX,NMDSMX) character QBONDA(NSTSMX,NMDSMX), QBONDN(NSTSMX,NMDSMX) character QEVSTRT (NSTSMX)

character QMTMP(NSTSMX)

character SAVEFUNC

character"20 READDECK,PARFILE,OPTFILE,EXPFILE character*1 TERMINAL,CHIOPT,CMACH,POLYDIAT,LETT

real $^{*} 8$ MTMP(NSTSMX,NMDSMX),TMP

real*8 NMSTRT(NSTSMX)

common/comb1/FCF(NMDSMX,NVMAXN,NVMAXA),POS(NMDSMX, NVMAXN,NVMAXA),DEGFLAG

common/comb2/ANHARMN(NSTSMX,NMDSMX),ANHARMA(NSTSMX,NMDSM $\mathrm{X})$ common/comb3/EVQQ(NVMAXN,NVMAXA),QQANH(NVMAXN,NVMAXA),

\& QQANHB(NVMAXN,NVMAXA),XM1,XM2

common/comb4/HFREQN(NSTSMX,NMDSMX),HFREQA(NSTSMX,NMDSMX) common/comb5/IVMAXA(NSTSMX,NMDSMX),IVMAXN(NSTSMX,NMDSMX) \&EXPCNTS common/sim/TW,EW,ORIGIN,A(NPTSFIT),SIM(NPTSFIT,2), EMAX,

common/qfet/BONDA(NSTSMX,NMDSMX),QFCTN common/deg/DEGMODE(NSTSMX,NMDSMX),MTMP(NSTSMX,NMDSMX) common/params/CHIOPT,TERMINAL,EXPFILE common/params2/RESOLUTION,ITERATION,ISTATES common/params3/EVSTRT(NSTSMX),SCALE(NSTSMX),NMODES(NSTSMX) common/params4/BONDN(NSTSMX,NMDSMX),DELQ1(NSTSMX,NMDSMX),

\&LDEGMODE(NSTSMX,NMDSMX),QTMP(NSTSMX,NMDSMX)

common/params5/NMSTRT(NSTSMX)

common/chi2/IPTSEXP,NVAR

common/chi4/OPTFILE

common/save/SAVEFUNC

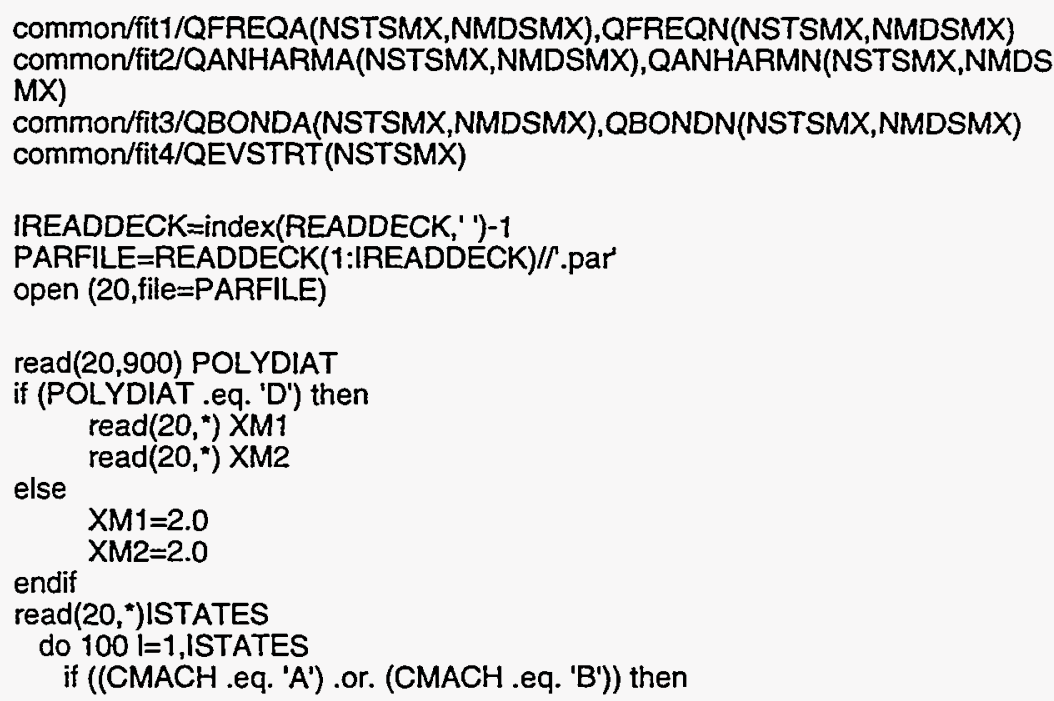


110

77 format(2x,'Sorry : $\wedge$ - Can only save functions when calculating ',

write(*,111)PARFILE

111 format(2x,'Molecular parameters read from', $1 \times, A$ )

$\stackrel{\mathrm{C}}{\mathrm{C}}$

mon to see if in optimization

if (CHIOPT .eq. ' $\left.Y^{\prime}\right)$ then

OPTFILE=READDECK(1:IREADDECK $) / /^{\prime} . \mathrm{opt}^{\prime}$

open(21, file=OPTFILE)

do $200 \mathrm{l}=1$,ISTATES

if (QMTMP(I) .eq. ' $N$ ') then

read(21,") LEIT

do $130 i=1$,NMODES(l)

130

continue

$\operatorname{QTMP}(1, \mathrm{i})=\mathrm{LETT}$

endif

if (QMTMP(I) .eq. ' $Y$ ') then

do $140 \mathrm{i}=1$, NMODES(I)

140

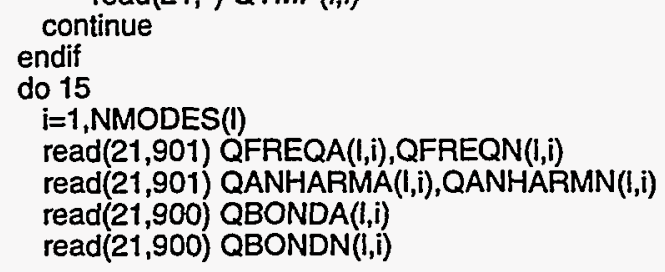


15

200

20

901 format(A1,1x,A1)

902 format $(f 9.4,+9.4)$

return

end
read(21,900)QEVSTRT(I)

continue

$\operatorname{read}(21,902)$ CHIMIN,CHIMAX

close(21)

write $(*, 20)$ OPTFILE

format(2x,'Optimization parameters read from',1x,A) endif

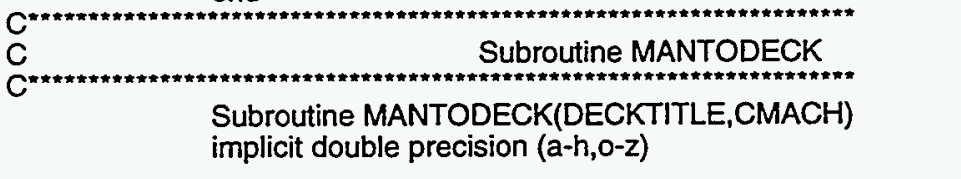

C This subroutine is designed to read in a manual input and

C write an input that is of the proper form to be read by

C READPARAMS (above). Thus, changes to this routine should

C be followed by according changes to the READPARAMS routine.

include "params.inc"

real NUMBER

dimension DEV(NSTSMX)

character LDEGMODE(NSTSMX,NMDSMX)

character QFREQA(NSTSMX,NMDSMX), QFREQN(NSTSMX,NMDSMX)

character QANHARMA(NSTSMX,NMDSMX),QANHARMN(NSTSMX,NMDSMX)

character QBONDA(NSTSMX,NMDSMX), QBONDN(NSTSMX,NMDSMX)

character QEVSTRT(NSTSMX)

character QTMP(NSTSMX,NMDSMX)

character QMTMP(NSTSMX)

character QTMP,LETT,POLYDIAT

character SAVEFUNC

character*20 DECKTITLE,PARFILE,OPTFILE,EXPFILE

character*1 TERMINAL,CHIOPT,CMACH

real*8 MTMP(NSTSMX,NMDSMX),TMP

real*8 NMSTRT(NSTSMX)

\&

common/comb1/FCF(NMDSMX,NVMAXN,NVMAXA),POS(NMDSMX, NVMAXN,NVMAXA),DEGFLAG

common/comb2/ANHARMN(NSTSMX,NMDSMX),ANHARMA(NSTSMX,NMDSMX) common/comb3/EVQQ(NVMAXN,NVMAXA),QQANH(NVMAXN,NVMAXA), QQANHB(NVMAXN,NVMAXA), XM1,XM2

\& common/comb4/HFREQN(NSTSMX,NMDSMX),HFREQA(NSTSMX,NMDSMX) common/comb5/IVMAXA(NSTSMX,NMDSMX), IVMAXN(NSTSMX,NMDSMX) common/sim/TW, EW, ORIGIN,A(NPTSFIT),SIM(NPTSFIT,2), EMAX,

\&EXPCNTS

common/gfct/BONDA(NSTSMX,NMDSMX),QFCTN

common/deg/DEGMODE(NSTSMX,NMDSMX),MTMP(NSTSMX,NMDSMX)

common/params/CHIOPT,TERMINAL,EXPFILE

common/params2/RESOLUTION,ITERATION,ISTATES

common/params3/EVSTRT(NSTSMX),SCALE(NSTSMX),NMODES(NSTSMX)

common/params4/BONDN(NSTSMX,NMDSMX),DELQ1(NSTSMX,NMDSMX),

\&LDEGMODE(NSTSMX,NMDSMX),QTMP(NSTSMX,NMDSMX)

common/params5/NMSTRT(NSTSMX)

common/chi2/IPTSEXP,NVAR

common/chi4/OPTFILE

common/save/SAVEFUNC 
common/fit1/QFREQA(NSTSMX,NMDSMX), QFREQN(NSTSMX,NMDSMX)

common/fit2/QANHARMA(NSTSMX,NMDSMX), QANHARMN(NSTSMX,NMDSMX)

common/fit3/QBONDA(NSTSMX,NMDSMX),QBONDN(NSTSMX,NMDSMX)

common/fit4/QEVSTRT (NSTSMX)

write $(*, 10)$

10 format(2x,'(D/d)iatomic or (P/p)olyatomic?',2x)

call LINPUT(LETT)

POLYDIAT $=$ LETT

if (POLYDIAT .eq. 'D') then

write $\left.{ }^{*}, 15\right) 1$

call REALINPUT(NUMBER)

$X M 1=$ NUMBER

write $\left.{ }^{*}, 15\right) 2$

call REALINPUT(NUMBER)

$X M 2=N U M B E R$

15 else

format(2x,'What is the mass of atom \#',i1,'(amu)',2x)

17

$X M 1=2.0$

$X M 2=2.0$

write $\left.{ }^{*}, 17\right)$

endif

format(2x,'Assuming reduced mass of 1.0 for norm. coord.')

format(2x,'How many electronic states? ',\$)

read $\left({ }^{*},{ }^{*}\right)$ ISTATES

do $4 l=1$, ISTATES

if (ISTATES .gt. 1) then

write $\left({ }^{*}, 28\right) !$

28

format(2x,'Enter parameters for electronic state \#',i2,/)

endif

30

if ( $\mathrm{CMACH}$.eq. ' $A$ ') then

write $(*, 30)$

format(2x,'What is origin of spectrum?(nm)')

call REALINPUT(NUMBER)

NMSTRT $(I)=$ NUMBER

if (I .eq. 1) then

else

EVSTRT $(i)=0.50$

$\operatorname{DEV}(I)=\left(1 /\left(\text { NMSTRT }(I)^{\star} 1 \mathrm{e}-7^{\star} 8065.479\right)^{*} 1 \mathrm{e}-7^{\star} 8065.479\right)$

endif

EVSTRT $(I)=E V S T R T(1)-D E V(I)$

endif

if (CMACH .eq. ' $S$ ') then

write $(*, 31)$

31

format( $2 x$, 'What is origin of spectrum?(eV)')

read(",")NUMBER

EVSTRT $(1)=$ NUMBER

NMSTRT (1) $=0.0$

endif

40

write $(*, 40)$

format(2x,'How many vibrational modes active in spectrum?',l,6x, \&'Input:',\$)

read $(*, 41)$ NMODES $(l)$

41 format(i2)

write $(*, 42)$

42

format(2x,'Different temperatures for each vibrational mode? ', $1 x$ )

call YNINPUT(LETT)

QMTMP $(l)=L E T T$

if (QMTMP(I) .eq. ' $N$ ') then

write $(*, 43)$ 
call REALINPUT(NUMBER)

TMP=NUMBER

do $44 i=1$, NMODES(I)

$\operatorname{MTMP}(1, \mathrm{i})=\mathrm{TMP}$

endif

do $75 \mathrm{i}=1$, NMODES(l)

write $(*, 45) i$

45

tormat(2x,'Is mode',i2,' a degenerate mode?', $1 x$ )

call YNINPUT(LETT)

LDEGMODE $(\mathrm{l}, \mathrm{i})=$ LET

if (LETT .eq. ' $Y$ ') then

DEGMODE $(1, i)=1.0$

DEGFLAG $=1.0$

else

DEGMODE $(l, i)=0.0$

endif

if (QMTMP(I) .eq. ' $Y$ ') then write $(", 47) i$

format(2x,'What is temperature for mode \#',i2,'?(K) ')

call REALINPUT(NUMBER)

$\operatorname{MTMP}(1, \mathrm{i})=$ NUMBER

endif

write $(*, 50) i$

format(2x, 'What are freq. of mode',i2,'?(anion, neutral,cm-1)',1x) read(**)NUMBER

HFREQA $(I, i)=$ NUMBER

read $(*, *) N U M B E R$

HFREQN $(l, i)=N U M B E R$

write $(*, 55) \mathrm{i}$

format(2x,'What are anharm. of mode',i2,'?(anion, neutral,cm-1)',1x)

read(",")NUMBER

ANHARMA $(1, i)=$ NUMBER

$\operatorname{read}\left(*,{ }^{*}\right)$ NUMBER

ANHARMN $(I, \mathrm{i})=$ NUMBER

write $(*, 60)$

format(2x,' What is anion bond length?(Ang)',1x)

$\operatorname{read}(*, *)$ NUMBER

$\operatorname{BONDA}(1, \mathrm{i})=$ NUMBER

write $\left.{ }^{\star}, 65\right)$

format(2x,'What is neutral bond length?(Ang)',1x)

read(",") NUMBER

BONDN $(1, i)=N U M B E R$

$\operatorname{DELQ1}(1, i)=\operatorname{abs}(B O N D A(l, i)-B O N D N(1, i))$

write $\left({ }^{*}, 70\right) i$

format(2x,'How many vib. levels for mode',i2,'?(anion, neutral)', 1x)

read $(*, 41)$ IVMAXA $(J, j)$

$\operatorname{read}(*, 41) I V M A X N(l, i)$

75

continue

write $(*, 76)$

76 format(2x,'Save potentials and wavefunctions for plotting?',1x)

call YNINPUT(LETT)

SAVEFUNC=LETT

if ((SAVEFUNC .eq.'Y'). and.((ISTATES.gt.1).or.(NMODES(1).gt.1))) then write $\left.{ }^{*}, 77\right)$

SAVEFUNC $=' N '$

endif

77 format(2x,'Sorry :^( - Can only save functions when calculating ', 


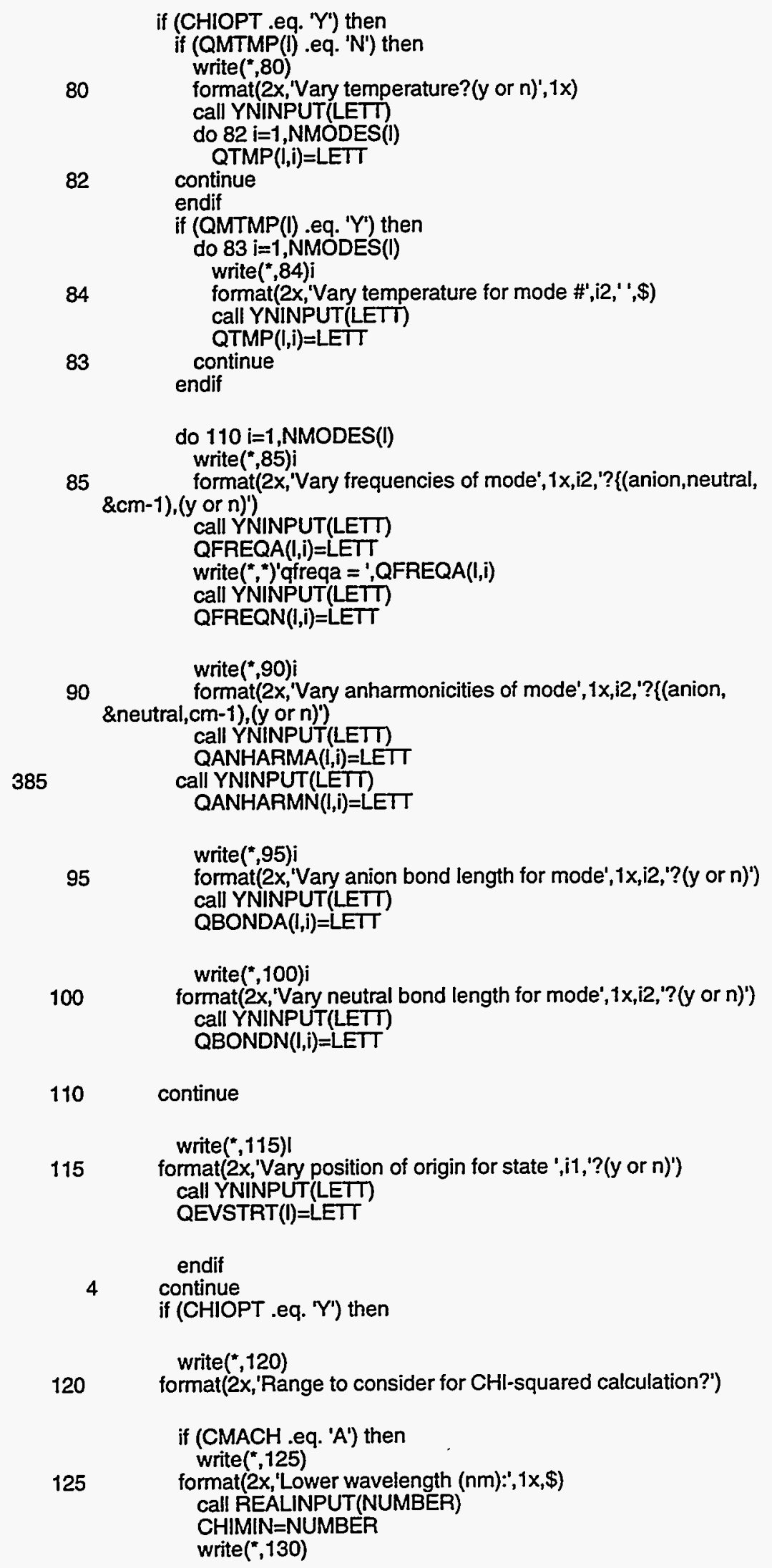

83 
format(2x,'Upper wavelength $\left.(\mathrm{nm}):^{\prime}, 1 \mathrm{x}, \$\right)$

read $(*, ")$ CHIMAX

endif

if (CMACH .eq. ' $S$ ') then

write $(*, 135)$

135

format(2x,'Lower electron kinetic energy $(e V): ', 1 \times, \$)$

call REALINPUT(NUMBER)

CHIMIN=NUMBER

write $(*, 140)$

140

format(2x,'Upper electron kinetic energy $(e V): ', 1 x, \$)$

$\operatorname{read}\left({ }^{*}, *\right)$ CHIMAX

endif

endif

IDECKTITLE=index(DECKTITLE,' ')-1

PARFILE=DECKTITLE(1:IDECKTITLE)/r.par

open(23,file=PARFILE(1:IDECKTITLE+4))

write $(23,900)$ POLYDIAT

if (POLYDIAT .eq. 'D') then

write $(23,910)$ XM1

endif write $(23,910)$ XM2

write $(23,920)$ ISTATES

do $150 \mid=1,1$ TSATES

if ((CMACH .eq. 'A') .or. (CMACH .eq. ' $B$ ')) then write $(23,910)$ NMSTRT(I)

else

write $(23,910)$ EVSTRT(I)

endif

write $(23,920)$ NMODES(l)

write $(23,900)$ QMTMP(l)

if (QMTMP(l) .eq. ' $N$ ')

then write

endif

$(23,910)$ TMP

do $160 i=1$,NMODES(I)

write $(23,900)$ LDEGMODE $(1, i)$

if (QMTMP(I) .eq. ' $Y$ ') then

write $(23,910)$

endif

write $(23,930)$ HFREQA $(l, j)$, HFREQN $(I, j)$

write $(23,930)$

ANHARMA(l,i),ANHARMN $(1, i)$ write

$(23,910)$ BONDA(l,i)

write $(23,910)$ BONDN $(I, i)$

160

150 continue

write $(23,940)$ IVMAXA $(1, i), I V M A X N(1, i)$

continue

write $(23,900)$ SAVEFUNC

$i=1$

do $1001:=1$,ISTATES

write $(23,500) \mathrm{i}$

500

format(2x, 'Line',i2,' : P/D (P)olydiatomic/(D)iatomic')

$i=i+1$

if (POLYDIAT .eq. 'D') then write $(23,505)$

505

format(2x,'Line',i2,' : Mass atom \#1')

$$
\mathrm{i}=\mathrm{i}+1
$$

write $(23,510) i$

$510 \quad$ format(2x,'Line',i2,' : Mass atom \#2')

endif $i=i+1$

write $(23,512) i$

format(2x,'Line',i2,' : Number of electronic states') 
$i=i+1$

write $(23,515) \mathrm{i}, \mathrm{l}$

515 format(2x,'Line',i2,' : Origin of electronic state \#',i2)

$\mathrm{i}=\mathrm{i}+1$

write $(23,535) \mathrm{i}$

535 format(2x,'Line',i2,' : Number of vibrational modes')

$i=i+1$

write $(23,520) i$

520

format(2x,'Line',i2,' : Different temperatures for each mode?')

$\mathrm{i}=\mathrm{i}+1$

write $(23,525) i$

525

530

(2x, 'Line',i2, : Temperature'

$i=i+1$

write $(23,530) \mathrm{i}$

format(2x, 'Line',i2,' : Degenerate mode?')

$i=i+1$

write $(23,540) \mathrm{i}$

540 format(2x,'Line',i2,' : Harmonic frequencies for anion and neutral')

$i=i+1$

write $(23,545) \mathrm{i}$

545 format(2x,'Line',i2, : Anharmonicities for anion and neutral')

$i=i+1$

write $(23,550) \mathrm{i}$

550 format(2x,'Line',i2,' : Anion bond length')

$i=i+1$

write $(23,555)$

555

560

565

format(2x,'Line',i2,' : Neutral bond length')

$i=i+1$

write $(23,560) i$

format(2x,'Line',i2,' : Number of vib.levels for anion and neutral')

if (NMODES $(l)$.gt. 1) then

write $(23,565) i+1, i-4$

endif

format(2x,'Line',i2,' : Same as Line',i2,'etc.')

1001

continue

write $\left.(23,)^{\prime}\right)$ Last line: Save potentials and wave functions?' close(23)

c

c

240

230

231

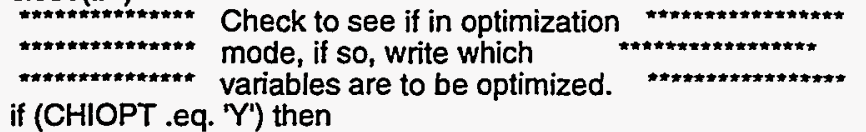

OPTFILE=DECKTITLE(1:IDECKTITLE)/r.opt

open (27, file=OPTFILE)

do $231 \mathrm{l}=1$, ISTATES

if (QMTMP(I) .eq. ' $N$ ') then

write $(27,900)$ QTMP $(1,1)$

endif

if (QMTMP(I) .eq. ' $Y$ ') then

do $240 \mathrm{i}=1$, NMODES(I)

write $(27,900)$ QTMP(I,i)

continue

endif

do $230 i=1$,NMODES $(I)$

write $(27,950)$ QFREQA(l,i), QFREQN $(1, \mathrm{i})$

write $(27,950)$ QANHARMÁ(l,i), QANHARMN $(1, i$

) write $(27,900)$ QBONDA $(1, i)$

write $(27,900)$ QBONDN $(1, i)$

continue

write(27,900)QEVSTRT(I)

continue

write $(27,930)$ CHIMIN,CHIMAX

$i=1$

write $(27,600) \mathbf{i}$

600

format(2x,'Line',i2,' : Temperature')

$i=i+1$

write $(27,605) i$

605 format(2x,'Line',i2,' : Harmonic frequencies of anion and neutral')

$\mathrm{i}=\mathrm{i}+1$

write $(27,610) \mathrm{i}$ 
610 format(2x,'Line',i2,' : Anharmonicities of anion and neutral') $i=i+1$ write $(27,615) i$

615 format(2x,'Line',i2,' : Bond length of anion') $i=i+1$ write $(27,620) i$

620 format(2x,'Line',i2,' : Bond length of neutral') $i=i+1$ write $(27,630)$

630 format(2x,'Line',i2,' : CHI-squared calculation range')

625

if (NMODES(1) .gt. 1) then write(27,625)i+1,i-4
format(2x,'Line',i2,' : Same as Line ',i2,' etc.') close(27)

endif

else

endif goto 999

900format(A1)

910format ( $\$ 9.4)$

920format(i2)

930 format $(f 9.4,1 \times, f 9.4)$

940 format(i2, 1x,i2)

950format $(A 1,1 \times, A 1)$

999retum

end

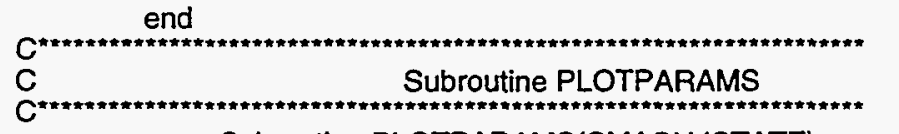

Subroutine PLOTPARAMS(CMACH,ISTATE) implicit double precision $(\mathrm{a}-\mathrm{h}, \mathrm{O}-\mathrm{z})$

$\mathrm{C}$
$\mathrm{C}$
$\mathrm{C}$
$\mathrm{C}$
$\mathrm{C}$
$\mathrm{C}$
$\mathrm{C}$
$\mathrm{C}$

This subroutine is to read in the parameters to be used in the plotting of the simulations and the experimental file. It also will write these parameters into a file titled 'plot.par'. This file will be read in during the future runs as part of the input deck and modifications to these plotting parameters can be made by simple editing. If the option is selected read the parameters from an input deck another subroutine, CPLOTPARAMS, will read in the plotting parameters.

real $^{*} 8$ NUMBER real*8 NMSTRT(NSTSMX)

include "params.inc"

character*20 EXPFILE,OPTFILE character ${ }^{*} 1 \mathrm{CMACH}, \mathrm{CHIOPT}, \mathrm{TERMINAL}$

dimension ACRORAW(1000,2)

common/params/CHIOPT,TERMINAL,EXPFILE

common/params2/RESOLUTION,ITERATION,ISTATES common/params3/EVSTRT(NSTSMX),SCALE(NSTSMX),NMODES(NSTSMX) common/params5/NMSTRT(NSTSMX) common/chi/EXPCHI(NPTSEXP,2),SIGEXP(NPTSEXP) common/chi2/IPTSEXP,NVAR common/chi3/FCHISQ,SIMCHI(NPTSEXP,2) common/chi4/OPTFILE common/plot/EXPT(NPTSEXP,2) common/sim/TW,EW, ORIGIN,A(NPTSFIT),SIM(NPTSFIT,2), EMAX, \&EXPCNTS

if (CHIOPT .eq. ' $Y$ ') then write $(*, 5)$ 
5

6

else

format(2x,'Plot after each nth iteration?(n=integer)')

read $(*, 6)$ ITERATION

ormat(i2)

endif

\section{write $(*, 10)$}

10 format(2x,'What experimental file shall I plot?')

$\operatorname{read}\left({ }^{*},{ }^{*}\right)$ EXPFILE

write $(*, 20)$
$20 \quad$ format( $2 x, 1$

$\operatorname{read}(", ")$ EXPCNTS

if (CMACH .eq. ' $A$ ') then

open $(1$, file=EXPFILE,err $=97)$

do $12 i=1,1000$

read $(1$, , end $=13$, err $=99$ ) (ACRORAW $(i, j), j=1,2)$

12

write $(10,18)$ ACRORAW(i,1),ACRORAW(i,2)

13

19

c

18

else

IPTSEXP $=\mathrm{i}-1$

write $(*, 19)$ IPTSEXP,EXPFILE

format $(2 x, 14$, points read from ',A)

close (1)

close $(10)$

C

c

3

7

14

15

else

format $(\$ 9.5,1 \times, f 9.5)$

if (CMACH .eq. ' $S$ ') then

open $(1$,file=EXPFILE, err=95)

do $3 i=1,1000$

read $\left(1,{ }^{*}\right.$, end $=14$, err $\left.=93\right)(\operatorname{EXPT}(i, j), j=1,2)$

write $(10,7)$ (EXPT(i,j),j=1,2)

if (CHIOPT .eq. ' $Y$ ') then

$\operatorname{EXPCHI}(i, 1)=\operatorname{EXPT}(i, 1)$

Divide by $55^{* *}(3 / 2)=408$ to get the actual number of counts in the main channel of the spectrum.

EXPCHI(i,2)=EXPT(i,2)*EXPCNTS $/ 408.0$

write $(22,8)(\operatorname{EXPCH}(i, j), j=1,2)$

endif $\operatorname{SIGEXP}(i)=\operatorname{sqrt}(\operatorname{EXPCHI}(\mathrm{i}, 2) * 1.0)$

continue

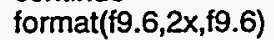

format $(f 12.6,2 x, f 12.6)$

IPTSEXP=i-1

close (1)

close (10)

write $\left({ }^{*}, 15\right)$

format(2x,'No experimental data will be plotted')

endif endif

if (CMACH .eq. 'S') then

write $(*, 21)$

21 format( $2 x$, 'What is time width for spectrum?(nsec)')

read $(*, ") T W$

write $(", 25)$

25

else format $(2 x$, 'What is energy width for spectrum?(meV)') $\operatorname{read}\left({ }^{*},{ }^{*}\right) \mathrm{EW}$

$\mathrm{TW}=0.0$

write (",27)

27

format $\left(2 x\right.$, 'What resolution for simulation? $\left.(\mathrm{cm}-1)^{\prime}\right)$ read $\left({ }^{*},{ }^{*}\right)$ NUMBER 
if (CMACH .eq. ' $A$ ') then

write $(*, 40)$

RESOLUTION=NUMBER

$E W=N U M B E R / 8065.479$

endif

read $\left({ }^{*}, *\right)$ NUMBER

WAVEMAX $=$ NUMBER

EMAX $=1.0 /\left(\right.$ WAVEMAX $\left.{ }^{*} 1 e-7 * 8065.479\right)$

else

if (CMACH .eq. 'S') then

50 write $\left(^{*}, 50\right)$ format $(2 x$, 'What is the maximum energy to display? $(\mathrm{eV})$ ') read $(*, *)$ NUMBER

$$
\text { endif }
$$

do $59 i=1$,ISTATES

write $(*, 60) i$

60 format(2x, 'Scale peak intensities for state',i2,1x,'

c

\&by how much?(0.0...1.0)')

call REALINPUT(NUMBER)

read( $\left(^{*}\right.$, ")NUMBER

59

SCALE $(i)=N U M B E R$

format(2x,'Teminal type:',/,'(x)term,(o)penwindows,

\&(s)unview, or (t)ektronix?',\$)

$\operatorname{read}\left(*,{ }^{*}\right)$ TERMINAL

open(18,file='plot.par')

if (CHIOPT .eq. ' $Y$ ') then

write $\left(18,{ }^{*}\right)$ ITERATION

endif

write $\left(18,{ }^{*}\right)$ EXPFILE

write $(18,67)$ EXPCNTS

if (CMACH .eq. 'S') then

write $(18,62)$ TW

write $(18,62) \mathrm{EW}$

endif

if (CMACH eq. ' $A$ ') then

write $(18,62)$ RESOLUTION

endif

if (CMACH .eq. 'A') then write $(18,62)$ WAVEMAX

else

if (CMACH .eq. 'S') then write $(18,63)$ EMAX

endif

do $81 \mathrm{i}=1$, ISTATES

81

write $(18,63)$ SCALE(i)

continue

write $(18,68)$ TERMINAL

62 format( $(\mathbf{8 . 4})$

67 format( $\$ 9.2)$

68 format(A2)

63 format(\$5.3)

$i=1$

if (CHIOPT.eq. 'Y') then write $(18,990) i$

990 format(2x,'Line ',i1,': No. of iterations between plots') 
$i=i+1$

endif

write $(18,991) i$

991

format(2x,'Line ',i1,': Experimental file')

$\mathrm{i}=\mathrm{i}+1$

992

write $(18,992) i$

format( $2 x$, 'Line ',i1,': Number of counts in main peak')

$\mathrm{i}=\mathrm{i}+1$

if (CMACH.eq.'S')then

write $(18,993) i$

993 format(2x,'Line ',i1,': Time Width - ns')

$\mathrm{i}=\mathrm{i}+1$

format(2x,'Line ',i1,:: Energy Width - meV')

$i=i+1$

endif

if (CMACH.eq.' $A$ ') then

write $(18,995)$

995 format(2x,'Line ',i1,:: Resolution - cm-1')

$j=i+1$

endif

format(2x,'Line ',i1,:: Maximum Energy to display')

$i=i+1$

format(2x,'Line ',i1,': Simulation Scaling Factor')

retum

93 write $(*, 92)$

92 format(2x,'ERROR: Data read error - PLOTPARAMS - Stonehenge')

$95 \quad$ write $(*, 94)$

94 format(2x,'ERROR: File read error - PLOTPARAMS - Stonehenge')

97 write $(*, 96)$

96 format(2x,'ERROR: File read error - PLOTPARAMS - Acropolis')

99 write $(*, 98)$

98 format(2x,'ERROR: Data read error - PLOTPARAMS - Acropolis')

stop

end

$\begin{array}{ll}C^{\prime} & \text { Subroutine RPLOTPARAMS } \\ C^{n} & \text { Subroutine RPLOTPARAMS(CMACH, ISTATE) } \\ & \text { implicit double precision }(a-h, 0-2)\end{array}$

$C$
$C$
$C$
$C$
$C$
$C$
$C$

This subroutine is to read in the parameters to be used in the plotting of the simulations and the experimental file. It also will write these parameters nto a file title 'plot.par'. This routine is called if the RDECK flag is ' $Y$ '(i.e., the parameters are being read from an input deck rather than being input manually. If the parameters are entered manually, the subroutine PLOTPARAMS is called instead.

include "params.inc"

character*20 EXPFILE,OPTFILE 
character*1 CMACH,CHIOPT,TERMINAL

dimension ACRORAW(NPTSEXP,2),EXPT(NPTSEXP,2)

real"8 NMSTRT(NSTSMX)

common/params/CHIOPT,TERMINAL,EXPFILE

common/params2/RESOLUTION,ITERATION,ISTATES

common/params3/EVSTRT(NSTSMX), SCALE(NSTSMX),NMODES(NSTSMX)

common/params5/NMSTRT (NSTSMX)

common/chi/EXPCHI(NPTSEXP,2),SIGEXP(NPTSEXP)

common/chi2//PTSEXP,NVAR

common/chi3/FCHISQ,SIMCHI(NPTSEXP,2)

common/chi4/OPTFILE

common/plot/EXPT(NPTSEXP,2)

common/sim/TW,EW,ORIGIN,A(NPTSFIT),SIM(NPTSFIT,2), EMAX, \&EXPCNTS

open(19,file='plot.par')

if (CHIOPT .eq. 'Y') then

read $(19,10)$ ITERATION

10

endif

format(i2)

$\operatorname{read}\left(19,{ }^{*}\right)$ EXPFILE

$\operatorname{read}(19, *)$ EXPCNTS

if (CMACH .eq. ' $S$ ') then

read(19,")TW

$\operatorname{read}\left(19,{ }^{*}\right)$ EW

endif

if (CMACH .eq. ' $A$ ') then

read(19,")RESOLUTION

$E W=R E S O L U T I O N / 8065.479$

endif

if (CMACH .eq. ' $A$ ') then

read $(19,15$,err $=99)$ WAVEMAX

EMAX $=1.0 /($ WAVEMAX*1e-7*8065.479)

endif

if (CMACH .eq. 'S') then

$\operatorname{read}(19,16, e \pi=99)$ EMAX

endif

do $40 \mathrm{i}=1$,ISTATES

read(19,")SCALE(i)

40 continue

read(19,")TERMINAL

format (f8.4)

format( $\{5.3)$

format $(\$ 5.3)$

close (19)

if (CMACH .eq. 'A') then

open $(1$, file=EXPFILE, err $=97)$

do $12 \mathrm{i}=1,1000$

read $\left(1,{ }^{*}\right.$,end $=13$, err $=91$ ) (ACRORAW $\left.(i, j), j=1,2\right)$

write $(10,19)$ ACRORAW $(i, 1), A C R O R A W(i, 2)$

12

continue

13

IPTSEXP $=\mathrm{i}-1$

write $\left({ }^{*}, 17\right)$ IPTSEXP,EXPFILE

17 format $(2 x, i 4$, points read from ',A)

close(1)

close (10)

19

forme

if (CMACH .eq. 'S') then

open $(1$, file $=$ EXPFILE,err $=95)$

do $3 i=1,1000$

read $\left(1,{ }^{*}\right.$,end $=14$, err $\left.=93\right)($ EXPT $(i, j), j=1,2)$

write $(10,7)(\operatorname{EXPT}(i, j), j=1,2)$ 
C

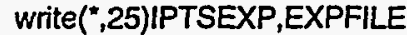
endif endif

format $(99.6,2 x, f 9.6)$

format $(f 12.6,2 x,+12.6)$

IPTSEXP $=\mathrm{i}-1$

close (1)

close (10)

write $(", 11)$

format(2x,'No experimental data will be plotted')

write $\left({ }^{*}, 20\right)$

format(2x,i4,1x,'Data points read from',1x,A)

return

99 write $(*, 98)$

format(2x,'ERROR: Format error in plot.par deck')

97 write(",96)

96 format(2x,'ERROR: File read error - RPLOTPARAMS - Acropolis')

$95 \quad$ write $(*, 94)$

94 format(2x,'ERROR: File read error - RPLOTPARAMS - Stonehenge')

93 write $\left({ }^{*}, 92\right)$

92 format(2x,'ERROR: Data read error - RPLOTPARAMS - Stonehenge')

91 write $(*, 90)$

90 format(2x,'ERROR: Data read error - RPLOTPARAMS - Acropolis')

stop

end

\section{PESCONT4.f}

Program PESFIT

Implicit double precision $(A-H, O-Z)$

PESFIT is a main controller program which oversees the FCF calculations and calls the appropriate subroutines according to the requests and input parameters of the user.

The code is designed to calculate Franck-Condon factors within the separable normal mode approximation for as many uncoupled modes as necessary, then convolute the resultant stick spectrum with the experimental resolution function for comparison to anion photoelectron spectra or threshold photodetachment (ZEKE) spectra).

For harmonic vibrational modes, the FCFs are calculated analytically and for anharmonic vibrations the program, by default generates a Morse potential curve using the input harmonic frequency and anharmonicity adn solves the Schrodinger eqn by numerical grid based methods. Since a numerical method is used, the code is fairly easily adapted for usage with a general potential energy function. Accordingly, the partition functions are calculated 
numerically in both cases. For harmonic systems, QFXN is called before FCF calculations; for anharmonic systems, the part. fxns. are calculated 'on-the-fly' in FCFNUM.

For a more thorough description of this routine,the subroutines it calls and the capabilities of the package, see the Ph.D thesis of Don W. Arnold - 1994.

Address:

\author{
Neumark Group \\ Department of Chemistry \\ University of Califomia \\ Berkeley, CA 94720 \\ email: neumark@violet.berkeley.edu
}

This program is also meant to calculate a non-linear least squares best fit for a spectrum starting $w /$ the frequencies that are found to give the best fit to the positions by hand. You can have anharmonic frequencies for any of the modes (as much as that means for polyatomic molecules).

OLD COMMENTS BELOW - 6/1/94 - DWA

11/17/91-The input deck format has been changed slightly from the format used in the PESFIT or MSTFIT programs. Now a few questions are asked by the main controller program and the rest of the queries are made by the subroutines READPARAMS or MANTODECK depending on whether an input deck is to be read or created for future runs.

9/11/93-PESCONT3.F is modified to PESCONT4.F in order to allow anharmonicities to be handled more accurately. If an anharmonicity is entered during input, the normal analytic HO FCF calculation bypassed and a numerical routine (HEG) is used to calculate the FCF's which then are handled in the same fashion as before to calculate the combination bad intensities.-DWA

include "params.inc"

character LDEGMODE(NSTSMX,NMDSMX), QTMP(NSTSMX,NMDSMX)

character*1 VERBOSE,TERMINAL

character*1 LETT,QPLOT,CMACH,CHIOPT,RDECK

character*20 DECKTITLE,READDECK,OPTFILE,EXPFILE

character LDEGMODE(NSTSMX,NMDSMX),QTMP(NSTSMX,NMDSMX) character QFREQA(NSTSMX,NMDSMX), QFREQN(NSTSMX,NMDSMX)

character QANHARMA(NSTSMX,NMDSMX), QANHARMN(NSTSMX,NMDSMX) character QBONDA(NSTSMX,NMDSMX), QBONDN(NSTSMX,NMDSMX) character QEVSTRT (NSTSMX)

real*8 MTMP(NSTSMX,NMDSMX)

real"8 NMSTRT(NSTSMX)

real*4 REMAX

common/comb1/FCF(NMDSMX,NVMAXN,NVMAXA),POS(NMDSMX, NVMAXN,NVMAXA),DEGFLAG

common/comb2/ANHARMN(NSTSMX,NMDSMX),ANHARMA(NSTSMX,NMDSMX) common/comb3/EVQQ(NVMAXN,NVMAXA), QQANH(NVMAXN,NVMAXA),

QQANHB(NVMAXN,NVMAXA),XM1,XM2 common/comb4/HFREQN(NSTSMX,NMDSMX),HFREQA(NSTSMX,NMDSMX) common/comb5/IVMAXA(NSTSMX,NMDSMX),IVMAXN(NSTSMX,NMDSMX) common/sim/TW, EW, ORIGIN,A(NPTSFIT), SIM(NPTSFIT,2), EMAX,

\&EXPCNTS

common/hunt1/XX(NPTSFIT) common/chi/EXPCHI(NPTSEXP,2),SIGEXP(NPTSEXP) common/chi2/IPTSEXP,NVAR common/chi3/FCHISQ,SIMCHI(NPTSEXP,2) common/verb/NERBOSE common/deg/DEGMODE(NSTSMX,NMDSMX),MTMP(NSTSMX,NMDSMX) common/params/CHIOPT,TERMINAL,EXPFILE 
common/params2/RESOLUTION,ITERATION,ISTATES common/params3/EVSTRT(NSTSMX), SCALE(NSTSMX),NMODES(NSTSMX) common/params4/BONDN(NSTSMX,NMDSMX),DELQ1(NSTSMX,NMDSMX), \&LDEGMODE(NSTSMX,NMDSMX),QTMP(NSTSMX,NMDSMX) common/params5/NMSTRT(NSTSMX) common/plot/EXPT(NPTSEXP,2)

common/out/RMU2,QFXN(NSTSMX,NMDSMX) common/qfet/BONDA(NSTSMX,NMDSMX), QFCTN

common/fit1/QFREQA(NSTSMX,NMDSMX),QFREQN(NSTSMX,NMDSMX) common/fit2/QANHARMA(NSTSMX,NMDSMX),QANHARMN(NSTSMX,NMDSMX) common/fit3/QBONDA(NSTSMX,NMDSMX), QBONDN(NSTSMX,NMDSMX) common/fit4/QEVSTRT(NSTSMX)

common/title/OPTFILE

NPTS $=1000$

C

C

Check experimental file format

call banner0

1 write $(*, *)$ 'Are you an (A/a)cropolite or a (S/s)tonehenger?' call LINPUT(LETT) CMACH $=$ LETT

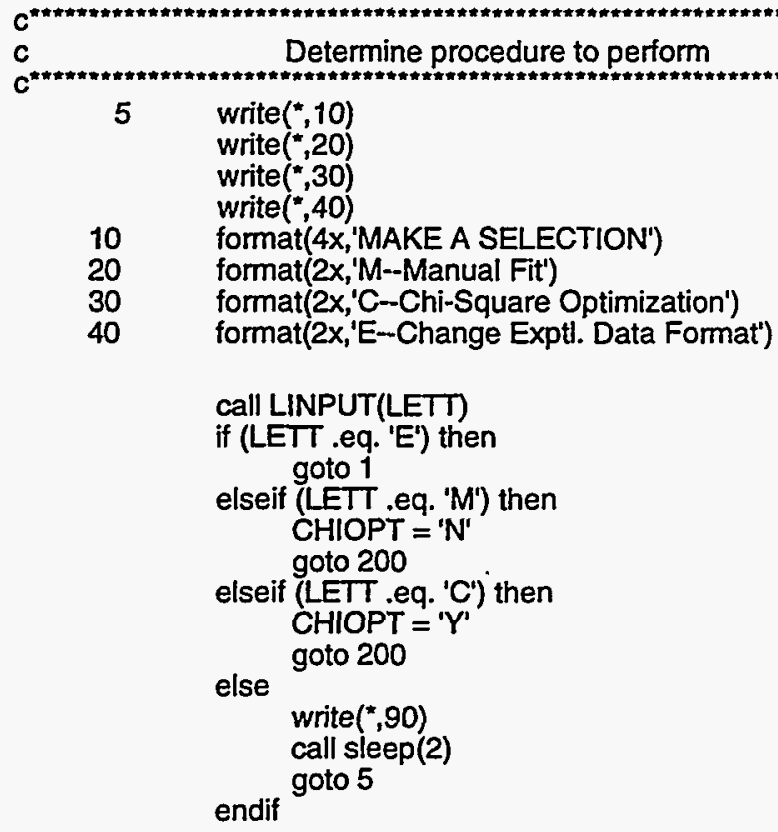

90 format('Input error')

200 write $(*, 210)$

write $(", 220)$

write $(*, 230)$

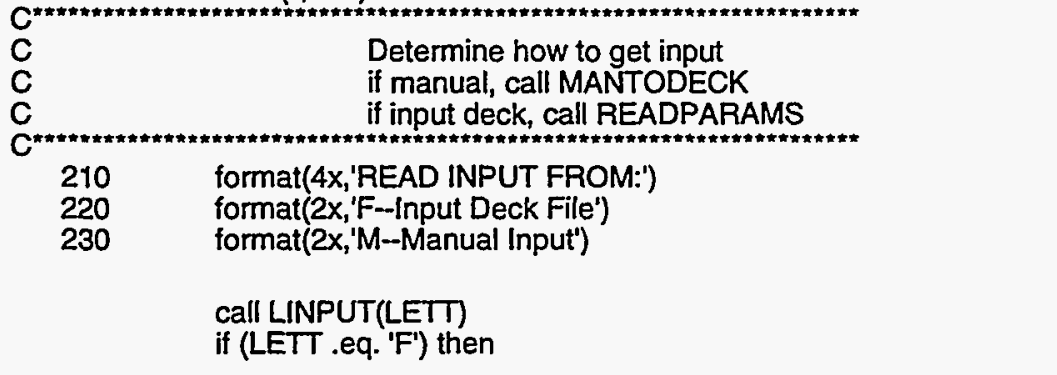




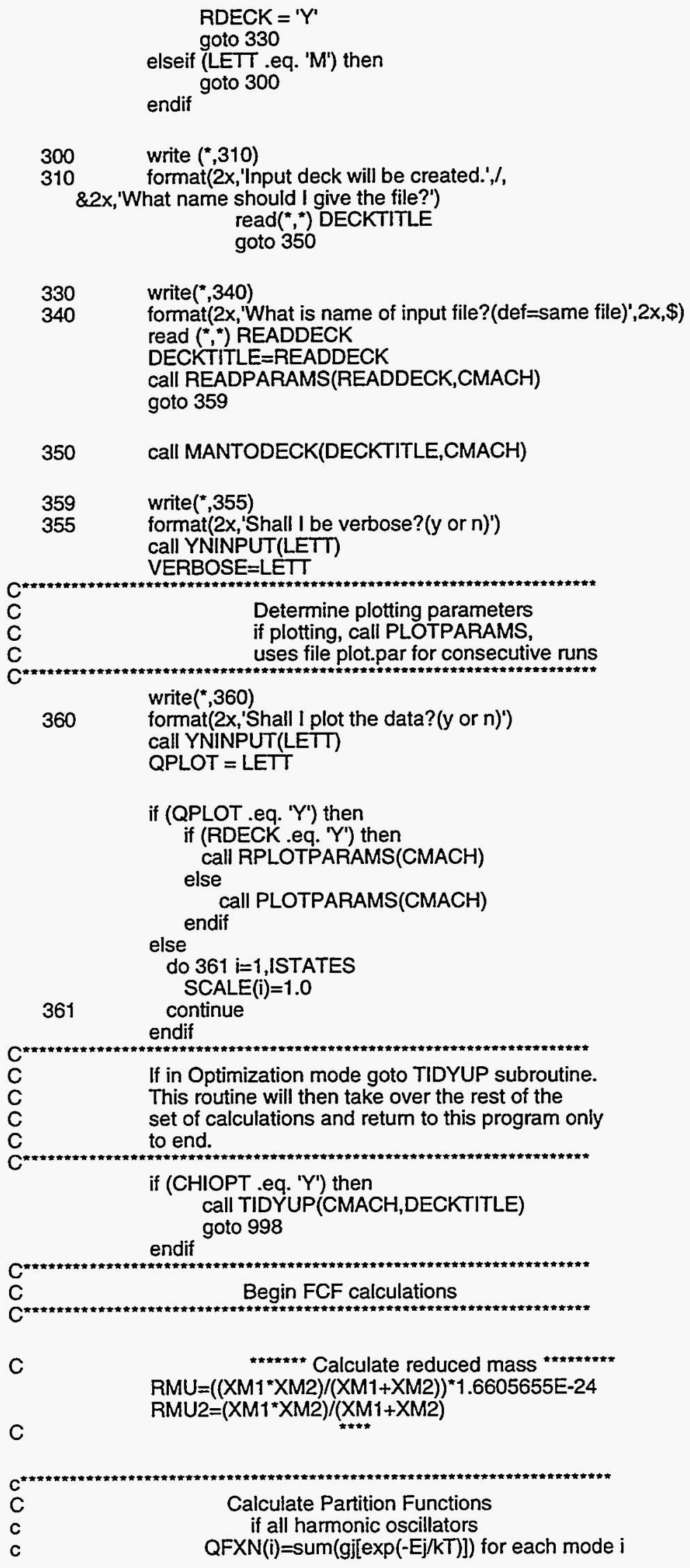




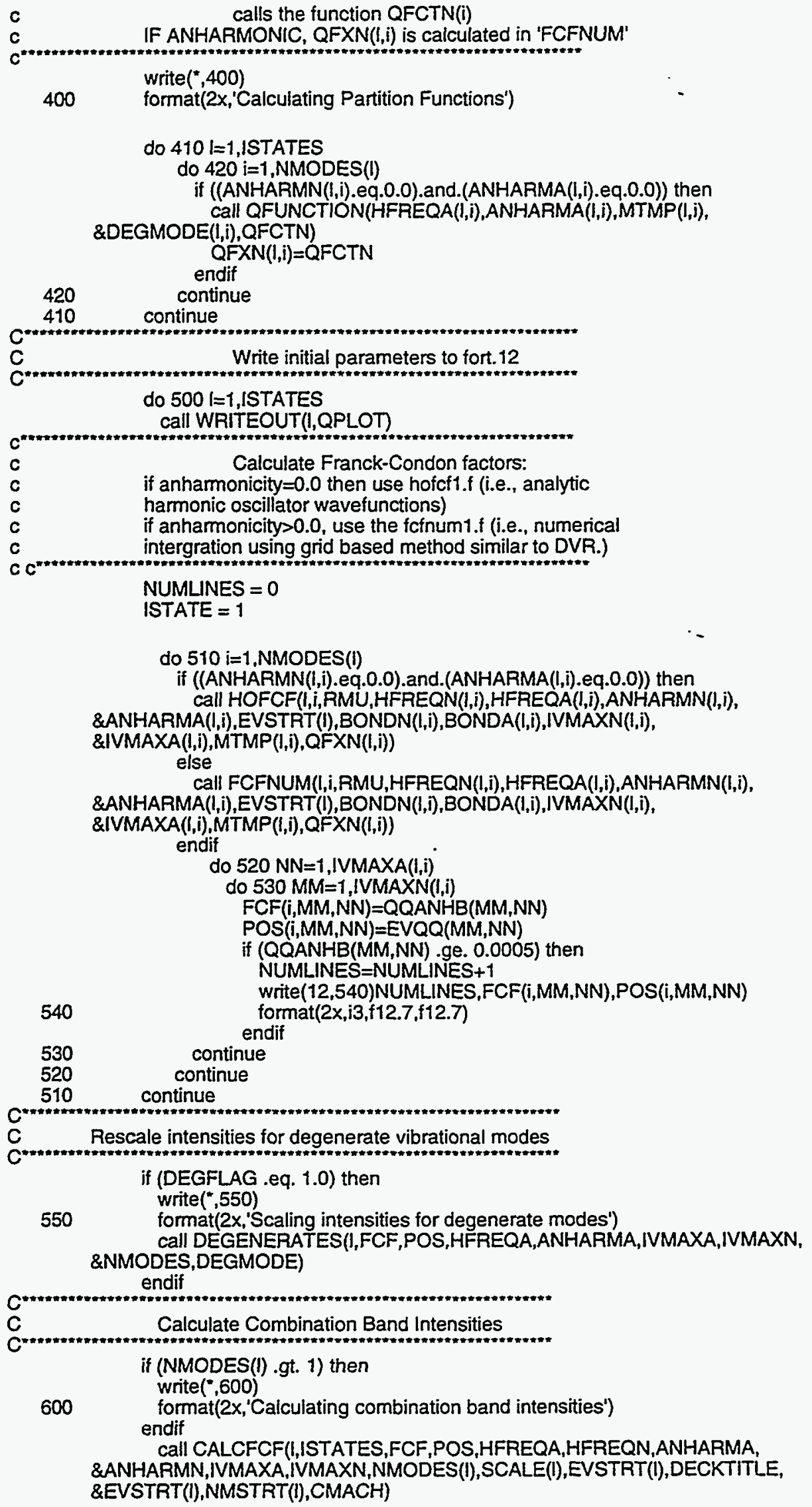




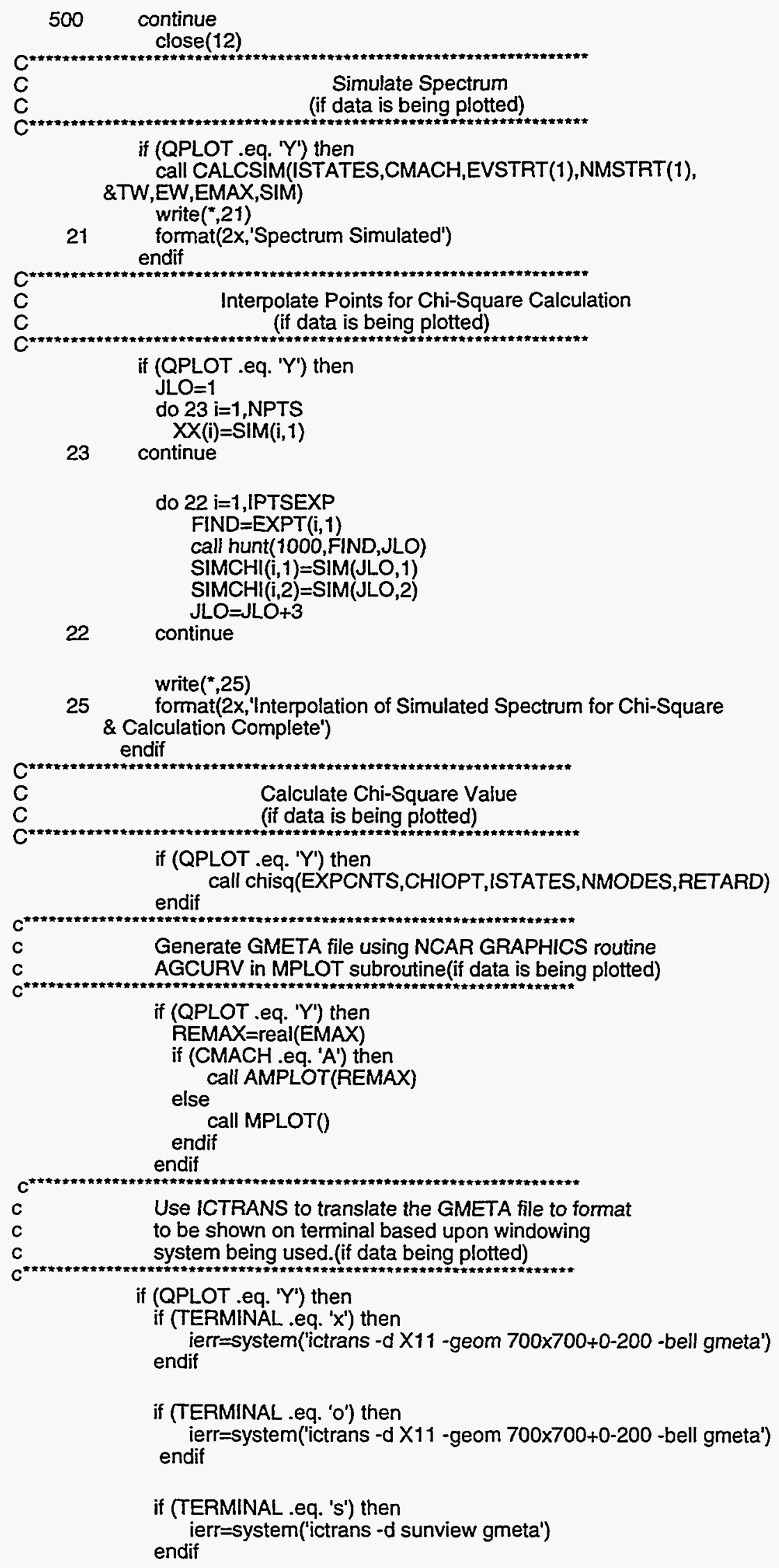



endif

if (TERMINAL .eq. ' $t$ ') then

ierr=system('ictrans - d tek gmeta')

998

write $\left({ }^{*}, 997\right)$

format(2x,'Done')

stop

end

\section{PLOT.f}

6/1/92-The plot.f file contains two programs which plot the data which is output from the PESFIT code. There are two codes:

MPLOT which plots Stonehenge data and AMPLOT which plots Acroppolis

data. Now the programs just use the basic AGCURV command

and its corresponding setup commands since the EZMXY does not work for two curves with differing numbers of data points.-DWA

These programs are designed to take the simulations from the PESFIT program and plot them using the NCAR (National Center for Atmospheric Research) graphics routines available on many machines. The first attempt at this is using the EZMXY routine which is available so that the both the simulation and the experimental data may be plotted. A separate routine, EZXY will be used for just plotting the simulation without the experimental data.-March/92-DWA

Subroutine MPLOTO

Implicit real*4 (A-H,O-Z)

C

For drawing Stonehenge data and simulations

C

parameter(NPTSEXP=1000,NPTSFIT $=2000$, NPLOTS $=10$ )

Define the data arrays

dimension XDRA(NPTSEXP,NPLOTS),YDRA(NPTSEXP,NPLOTS)

dimension XDRASIM(NPTSFIT),YDRASIM(NPTSFIT)

dimension XDRAEXP(NPTSEXP), YDRAEXP(NPTSEXP)

real*4 XMAXD,XMIND,YMAXD,YMIND,XMAX,XMIN,YMAX,YMIN

\&EXPCNTS

common/sim/TW,EW,ORIGIN,A(NPTSFIT),SIM(NPTSFIT,2),EMAX,

write( $\left({ }^{*},\right)^{\prime}$ MPLOTting data'

c

Initialize GKS

call OPNGKS

call SETUSV('PB',2)

c

c

Initialize parameters for plotting max and min

$X M A X=0$.

$X M I N=10000$

YMAX $=0$.

$Y M I N=0$.

c

Fill data arrays

do $12 i=1,2000$

read $\left(11,{ }^{*}\right.$, end $\left.=14\right)$ XDRASIM(i), YDRASIM(i)

12

continue

IPTSSIM=i-1

do $10 i=1,1000$

$\operatorname{read}\left(10,{ }^{*}\right.$, end $\left.=15\right)$ XDRAEXP(i),YDRAEXP(i)

10 continue

15 IPTSEXP=i-1 
do $13 i=1$, IPTSSIM

XDRASIM(i)=real(XDRASIM(i))

YDRASIM(i)=real(YDRASIM(i))

Find minima and maxima in data

(Leave YMIN equal to zero in all cases)

do $20 \mathrm{i}=1$,IPTSSIM

if (XDRASIM(i) .gt. XMAX) then

$X M A X=X D R A S I M(i)$

endif

if (XDRASIM(i) .It. XMIN) then

XMIN = XDRASIM(i)

endif

if (YDRASIM(i) .gt. YMAX) then

YMAX = YDRASIM(i)

20

$$
\text { endif }
$$

continue

21

do $21 \mathrm{i}=1$,IPTSEXP

if (XDRAEXP(i) .gt. XMAX) then

$X M A X=X D R A E X P(i)$

endif

if (XDRAEXP(i) .It. XMIN) then

$X M I N=$ XDRAEXP(i)

endif

if (YDRAEXP(i) .gt. YMAX) then

YMAX = YDRAEXP(i)

$$
\text { endif }
$$

Set min and max for drawing routine

XMAXD $=X M A X+0.05^{*}(X M A X-X M I N)$

$X M I N D=X M I N-0.05^{*}(X M A X-X M I N)$

$Y M A X D=Y M A X+0.01 *(Y M A X-Y M I N)$

YMIND $=$ YMIN

draw a boundary

call BNDARY

define maximum and minimum in the plot

call AGSETF('X/MINIMUM.',XMIND)

call AGSETF('XMAXIMUM:', XMAXD)

call AGSETF('Y/MINIMUM:',YMIND)

call AGSETF('Y/MAXIMUM.', YMAXD)

adjust autograph parameters to read two dimensional arrays for both XDRA and YDRA so that the energy points do not have to be the same for both arrays.

call DISPLA $(0,2,0)$

call AGSTUP(XDRASIM(1), 1,1,IPTSSIM,1,YDRASIM(1),1,1,IPTSSIM,1) c Label $x$ and $y$ axis labels

call ANOTAT('Electron Kinetic Energy (eV)','Intensity',0,0,0,' ')

call AGSETC('LABEL/NAME.','B'

call AGSETF('LINE/NUMBER:'-100.)

call AGSETF('LINE/DEFINITION/CHARACTER.',0.30) 
call AGSETC('LINE/TEXT.','Electron Kinetic Energy(eV)\$')

call AGSETC('LABELNNAME.','L')

call AGSETF('LINENUMBER.',100.)

call AGSETF('LINE/DEFINITION/CHARACTER.',0.30)

C call AGSETC('LINE/TEXT.','Intensity\$')

Give the graph a title

call AGSETC('LABELNAME.','T')

call AGSETF('LINENUMBER:',100.)

call AGSETF('LINE/DEFINITION/CHARACTER:',0.30)

call AGSETC('LINE/TEXT.','Photoelectron Spectrum\$')

Set definitions for line properties

call AGSETI('DASH/SELECTOR.',5)

call AGSETI('DASH/PATTERNS/2:'52428)

52428 in binary is 1100110011001100

call AGSETI('DASH/PATTERNS/3.',43690)

43690 in binary is 1010101010101010

call AGSETI('DASH/PATTERNS/4.',61166)

61166 in binary is 1110111011101110

call AGSETI('DASH/PATTERNS/5.',61680)

61680 in binary is 1111000011110000

Draw the background

call AGBACK

draw graph, using AGCURV

44

write $(*, 44)$ IPTSEXP,IPTSSIM \&points')

format(2x,'Plotting', 1x,i4,1x,'exptl and', 1x, i4, 1x'simulated

call AGCURV(XDRASIM(1),1,YDRASIM(1),1,IPTSSIM,1) call AGCURV(XDRAEXP(1), 1,YDRAEXP(1),1,IPTSEXP,2)

close GKS

call CLSGKS

write $\left({ }^{*},\right)^{\prime}$ MPLOTted'

retum

end

Subroutine AMPLOT

For drawing Acropolis data and simulations Implicit real* 4 (A-H,O-Z)

parameter(NPTSEXP=1000,NPTSFIT=2000,NPLOTS=10)

Define the data arrays

dimension XDRA(NPTSEXP,NPLOTS),YDRA(NPTSEXP,NPLOTS)

dimension XDRASIM(NPTSFIT), YDRASIM(NPTSFIT)

dimension XDRAEXP(NPTSEXP), YDRAEXP(NPTSEXP)

real*4 XMAXD,XMIND, YMAXD, YMIND,XMAX,XMIN, YMAX, YMIN

real*4 ANMMIN,ANMMAX

common/plot2/ANMMIN,ANMMAX

common/sim/TW,EW,ORIGIN,A(NPTSFIT),SIM(NPTSFIT,2),EMAX, \&EXPCNTS

Initialize GKS

call OPNGKS

call SETUSV('PB',2) 
Initialize parameters for plotting max and min

$X M A X=0$.

$X M I N=10000$.

YMAX $=0$.

$\mathrm{YMIN}=0$.

Fill data arrays

do $12 \mathrm{i}=1,2000$ $\operatorname{read}(11, *$,end $=14)$ XDRASIM(i),YDRASIM(i)

continue

IPTSSIM=i-1

do $10 i=1,1000$

read $\left(10,{ }^{*}\right.$,end $\left.=15\right)$ XDRAEXP(i),YDRAEXP(i)

continue

IPTSEXP $=\mathrm{i}-1$

Convert all numbers to single precision for the ncar code

do $11 i=1$,IPTSEXP

XDRAEXP $(i)=$ real $(X D R A E X P(i))$

YDRAEXP(i)=real(YDRAEXP(i))

continue

do $13 \mathrm{i}=1$,IPTSSIM

XDRASIM(i)=real(XDRASIM(i))

YDRASIM $(i)=$ real(YDRASIM(i)) continue

Find minima and maxima in data (Leave YMIN equal to zero in all cases)

do $20 i=1$,IPTSSIM

if (YDRASIM(i) .gt. YMAX) then YMAX $=$ YDRASIM(i)

endif

if (XDRASIM(i) .gt. XMAX) then $X M A X=X D R A S I M(i)$

endif

if (XDRASIM(i) .It. XMIN) then endif

continue

do $21 i=1$,IPTSEXP

if (YDRAEXP(i) .gt. YMAX) then

endif YMAX $=$ YDRAEXP(i)

if (XDRAEXP(i) .gt. XMAX) then $X M A X=X D R A E X P(i)$ endif

if (XDRAEXP(i) .It. XMIN) then $X M I N=X D R A E X P(i)$

continue endif

write $(*, 400) \times M I N, X M A X$

Set min and max for drawing routine

$X M A X D=X M A X+0.05^{*}(X M A X-X M I N)$

$X M I N D=X M I N-0.05^{*}(X M A X-X M I N)$

$Y M A X D=Y M A X+0.01^{*}(Y M A X-Y M I N)$

$Y M I N D=Y M I N$

XMIND=ANMMIN

XMAXD=ANMMAX 
c

44 \&points'

adjust autograph parameters to read two dimensional arrays for both XDRA and YDRA so that the energy points do not have to be the same for both arrays.

call DISPLA $(0,2,0)$

call AGSTUP(XDRASIM(1),1,1,IPTSSIM,1,YDRASIM(1),1,1,IPTSSIM,1)

Label $x$ and $y$ axis labels

call ANOTAT('Electron Kinetic Energy (eV)','Intensity', 0,0,0,' ')

call AGSETC('LABELNAME.','B')

call AGSETF('LINE/NUMBER:',-100.)

call AGSETF('LINE/DEFINITION/CHARACTER.',0.30)

call AGSETC('LINE/TEXT.','Wavelength $(\mathrm{nm}) \$$ ')

call AGSETC('LABELNAME:','L')

call AGSETF('LINE/NUMBER.',100.)

call AGSETF('LINE/DEFINITION/CHARACTER.',0.30)

call AGSETC('LINETTEXT.','Intensity\$')

Give the graph a title

call AGSETC('LABEL/NAME.' 'T')

call AGSETF('LINE/NUMBER:';100.)

call AGSETF('LINE/DEFINITION/CHARACTER:',0.30)

call AGSETC('LINETTEXT.','ZEKE Spectrum\$')

Set definitions for line properties

call AGSETI('DASH/SELECTOR:',5)

call AGSETI('DASH/PATTERNS/2.',52428)

52428 in binary is 1100110011001100

call AGSETI('DASH/PATTERNS/3.',43690)

43690 in binary is 1010101010101010

call AGSETI('DASH/PATTERNS/4.',61166)

61166 in binary is 1110111011101110

call AGSETI('DASH/PATTERNS/5.',61680)

61680 in binary is 1111000011110000

Draw the background

call AGBACK

draw graph, using AGCURV

write $\left({ }^{*}, 44\right)$ IPTSEXP,IPTSSIM

format(2x, 'Plotting', 1x, i4, $1 x$, 'expt) and', $1 x, 14,1 \times$ 'simulated call AGCURV(XDRASIM(1), 1,YDRASIM(1),1,IPTSSIM,1) call AGCURV(XDRAEXP(1), 1,YDRAEXP(1),1,IPTSEXP,2)

close GKS

call CLSGKS

return

end 
c

Subroutine BNDARY
C Routine to draw the plotter-frame edge.

C

CALL PLOTIT $(0,0,0)$

CALL PLOTIT $(32767,0,1)$

CALL PLOTIT $(32767,32767,1)$

CALL PLOTIT $(0,32767,1)$

CALL PLOTIT $(0,0,1)$

RETURN

END

\section{POT1.f}

c

subroutine harmonic(HWE,HREV)

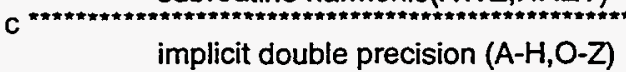

include "fcfnum.inc"

if (VERBOSE .eq. ' $Y$ ') then

write $(", ")^{\prime}$ ' + Harmonic'

endif

POTK = REDMASS"amu*(HWE/harwn)**2

do $20 \mathrm{i}=1$, NQBASIS

$R T=Q(i)-H R E V$

20

Vpoten(i)=POTK*RT"RT/2.0

continue

if (VERBOSE .eq. ' $Y$ ') then

write $(*,)^{*}$ '-Harmonic'

endif

retum

end

c ************************************************** Subroutine morse(WE, XEWE, REV),

implicit double precision $(\mathrm{A}-\mathrm{H}, \mathrm{O}-\mathrm{Z})$

include "fcfnum.inc"

dmorse $(x x, x 0$, dee,$x a l p h a)=\operatorname{dee}^{*}\left(1.0-\exp \left(-x a \mid p h a^{*}(x x-x 0)\right)\right)^{* * 2}$

XALPHAV $=$ sqrt(2.0*REDMASS*amu*XEWE/harwn)

$\mathrm{XDEV}=(\mathrm{WE} / \mathrm{harwn})^{* *} 2 /\left(4.0^{*} \mathrm{XEWE} / \mathrm{harwn}\right)$

do $30 i=1$, NQBASIS

Vpoten $(i)=$ dmorse (Q(i),REV,XDEV,XALPHAV)

30 continue

retum

end

C

$\mathrm{C}$

(a)

Subroutine Lennard-Jones

implicit double precision (A-H,O-Z)

if (ipottypB .eq. 5) then

$\operatorname{read}(1, *) \operatorname{lc}(2,1), \operatorname{Ic}(2,3), \mathrm{VOB}$

$\operatorname{lc}(2,1)=\operatorname{lc}(2,1)$

$\operatorname{lc}(2,3)=\operatorname{lc}(2,3)$

$\operatorname{lc}(2,2)=0$.

$\operatorname{lc}(2,4)=0$.

vOB $=$ VOB $/$ harev

shelf $=0.5$ harev

write $(6,)^{*}$ 'Shelf at ', shelf*harev,' eV'

do 866 ix=1,nXpts

$x i=x \min +(i x-1)^{\star} d x$

$z$ pot $(i x, 2)=$ potenli $(2, x i)+v O B$

if $($ real $(z$ pot $(i x, 2))$-v0B.gt.shelf $) z$ pot $(i x, 2)=$ shelf $+v O B$ continue

endif 


\section{Subroutine Exponential}

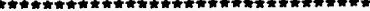

implicit double precision $(A-H, O-Z)$

read $\left(1,{ }^{\prime}\right)$ beta, A, vOB

beta=beta*a0

$A=A$ harev

$\mathrm{VOB}=\mathrm{vOB} / \mathrm{harev}$

shelf $=3.0 /$ harev

write $\left.(6,)^{\prime}\right)^{\prime}$ Establishing shelf in Repulsive potential',ipot

write $\left(6,{ }^{\prime}\right)$ 'so that full range of potential energy '

write $(6,99)$ 'is no greater than ', shelf*harev,' eV' $^{\prime}$

format $(a, 15.1, a)$

write $\left(6,{ }^{,}\right)$

do 844 ix $=1, n \times p t s$

$x i=x \min +(i x-1)^{\star} d x$

$z p o t(i x, 2)=A^{*} \operatorname{dexp}\left(-b e t a^{*} x i\right)+v 0 B$

if (real(zpot(ix,2))-vOB.gt.shelf) zpot(ix,2)=shelf $+v 0 B$

continue

endif

\section{QFXN5.f}

Subroutine QFUNCTION(HFREQA,ANHARMA,TMP,DEGMODE,QFCTN)

implicit double precision(a-h,o-z)

NOTE (5/23/92): This version of QFUNCTION [qfxn5.f] has had

the centrifugal distortion parameters removed for tidiness.

The qfxn 4.f still has the parameters which have just been

commented out in case a diatomic molecule should need to

be simulated will all factors considered. The new form of

usage makes this a more independent subroutine.

parameter(NMDSMX =15,NVMAXA $=20, N V M A X N=30, N L I N E S=2500$ )

parameter(NPTSEXP $=1000$, NPTSFIT $=2000, N S T S M X=5$ )

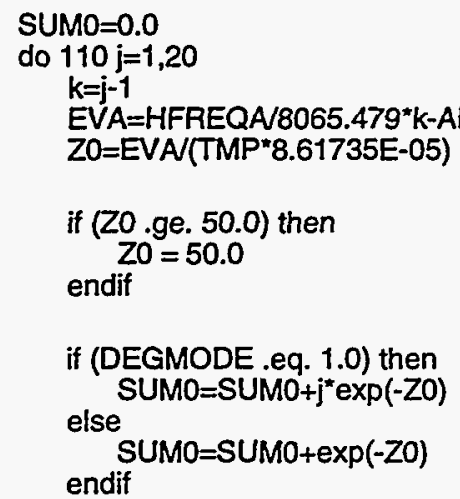

110 continue

QFCTN=SUMO

return

end

RS.f

c

c

c

subroutine rs(nm,n,a,w, matz,z,fv1,fv2,ierr)

integer $n, n m$,ierr, matz

double precision $a(n m, n), w(n), z(n m, n), f v 1(n), f v 2(n)$

this subroutine calls the recommended sequence of

subroutines from the eigensystem subroutine package (eispack)

to find the eigenvalues and eigenvectors (if desired)

of a real symmetric matrix.

on input 
$\mathrm{nm}$ must be set to the row dimension of the two-dimensional array parameters as declared in the calling program dimension statement.

$n$ is the order of the matrix a.

a contains the real symmetric matrix.

matz is an integer variable set equal to zero if only eigenvalues are desired. otherwise it is set to any non-zero integer for both eigenvalues and eigenvectors.

on output

$w$ contains the eigenvalues in ascending order.

$z$ contains the eigenvectors if matz is not zero.

ierr is an integer output variable set equal to an error completion code described in the documentation for tqliat and tql2. the normal completion code is zero.

fv1 and fv2 are temporary storage arrays.

questions and comments should be directed to burton s. garbow, mathematics and computer science div, argonne national laboratory

this version dated august 1983.

if $(\mathrm{n}$.le. $\mathrm{nm})$ go to 10

ierr $=10^{*} n$

go to 50

10 if (matz .ne. 0) go to 20

......... find eigenvalues only

call tred1(nm,n,a, w, fv1,fv2)

tqlat encounters catastrophic underflow on the Vax

call tqlrat(n,w,fv2,ierr)

call tql1 $(n, w, f v 1, i e r r)$

go to 50

C

20 call tred2( $\mathrm{nm}, \mathrm{n}, \mathrm{a}, \mathrm{w}, \mathrm{fv} 1, \mathrm{z})$

50 return call tql2(nm,n,w,fv1,z,ierr) end subroutine tql2(nm,n,d,e,z,ierr)

integer i,j,k,l,m,n,ii,l1,l2,nm,mml,ierr double precision $d(n), e(n), z(n m, n)$

\& double precision $c, c 2, c 3$,dli,el1,f,g,h,p,

this subroutine is a translation of the algol procedure tq 12 , num. math. 11, 293-306(1968) by bowdler, martin, reinsch, and wilkinson.

handbook for auto. comp., vol.ii-linear algebra, 227-240(1971).

this subroutine finds the eigenvalues and eigenvectors

of a symmetric tridiagonal matrix by the ql method. the eigenvectors of a full symmetric matrix can also be found if tred2 has been used to reduce this full matrix to tridiagonal form.

on input

$\mathrm{nm}$ must be set to the row dimension of two-dimensional array parameters as declared in the calling program dimension statement. 
$\mathrm{n}$ is the order of the matrix.

$d$ contains the diagonal elements of the input matrix.

e contains the subdiagonal elements of the input matrix in its last $n-1$ positions. $e(1)$ is arbitrary.

$z$ contains the transformation matrix produced in the reduction by tred2, if performed. if the eigenvectors of the tridiagonal matrix are desired, $z$ must contain the identity matrix.

on output

d contains the eigenvalues in ascending order. if an error exit is made, the eigenvalues are correct but unordered for indices $1,2, \ldots$, ierr-1.

e has been destroyed.

$z$ contains orthonormal eigenvectors of the symmetric tridiagonal (or full) matrix. if an error exit is made, $z$ contains the eigenvectors associated with the stored eigenvalues.

ierr is set to

zero for normal return,

$j$ if the j-th eigenvalue has not been determined after 30 iterations.

calls pythag for dsqri $\left(a^{*} a+b^{*} b\right)$.

questions and comments should be directed to burton s. garbow, mathematics and computer science div, argonne national laboratory

this version dated august 1983.

ierr $=0$

if (n .eq. 1) go to 1001

c

do $100 i=2, n$

$100 \mathrm{e}(\mathrm{i}-1)=e(i)$

$f=0.0 \mathrm{~d} 0$

tst $1=0.0 \mathrm{~d} 0$

$e(n)=0.0 d 0$

c

do $240 I=1, n$

$\mathbf{j}=0$

$h=\operatorname{dabs}(d(l))+\operatorname{dabs}(e(l))$

if (tst1 .It. $h$ ) tst $1=h$

c look for small sub-diagonal element

do $110 \mathrm{~m}=1, \mathrm{n}$

tst2 $=$ tst $1+\operatorname{dabs}(\mathrm{e}(\mathrm{m}))$

if (tst2 .eq. tst1) go to 120

c

$e(n)$ is always zero, so there is no exit

110

continue

c

120

if $(m$.eq. $l)$ go to 220

if $(j$.eq. 30) go to 1000

$\mathbf{j}=\mathbf{j}+1$

c

form shitt

$11=1+1$

$12=11+1$

$\mathrm{g}=\mathrm{d}(\mathbf{l})$

$\mathrm{p}=(\mathrm{d}(11)-\mathrm{g}) /\left(2.0 \mathrm{~d} 00^{*} \mathrm{e}(\mathrm{l})\right)$

$r=\operatorname{pythag}(p, 1.0 \mathrm{~d} 0)$

$d(l)=e(l) /(p+d \operatorname{sign}(r, p))$ 


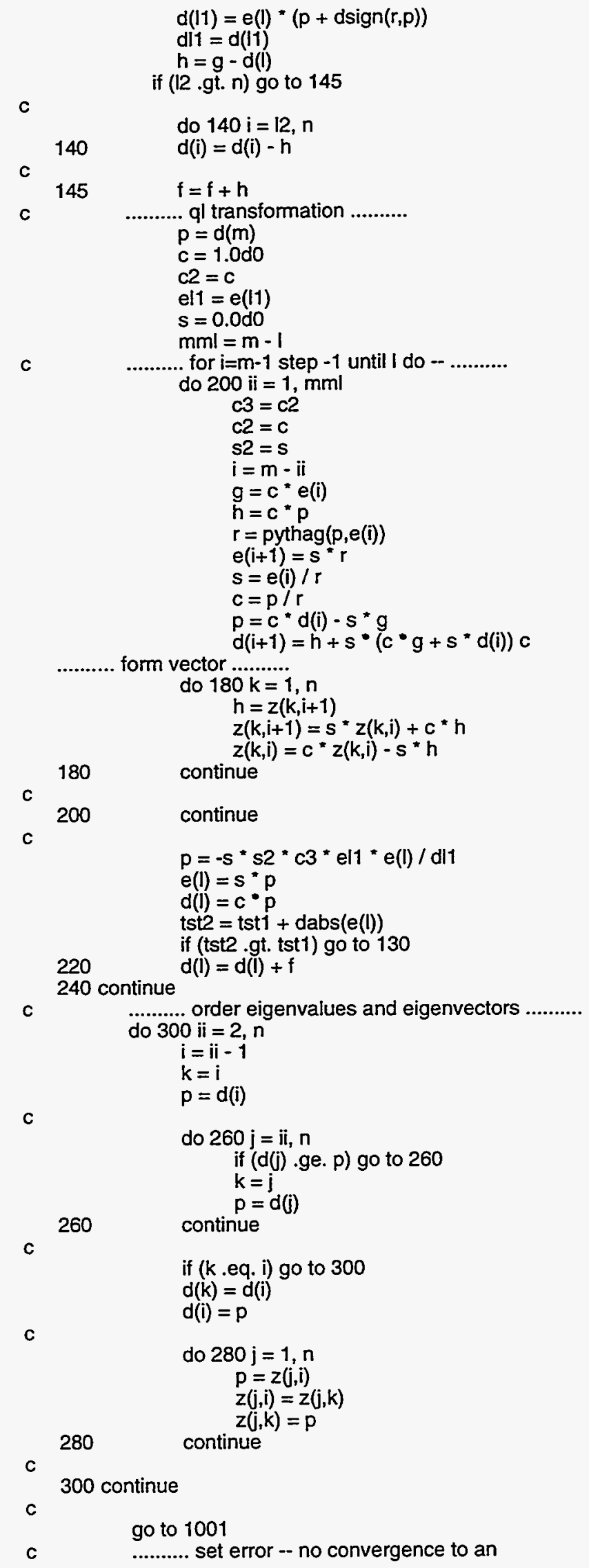




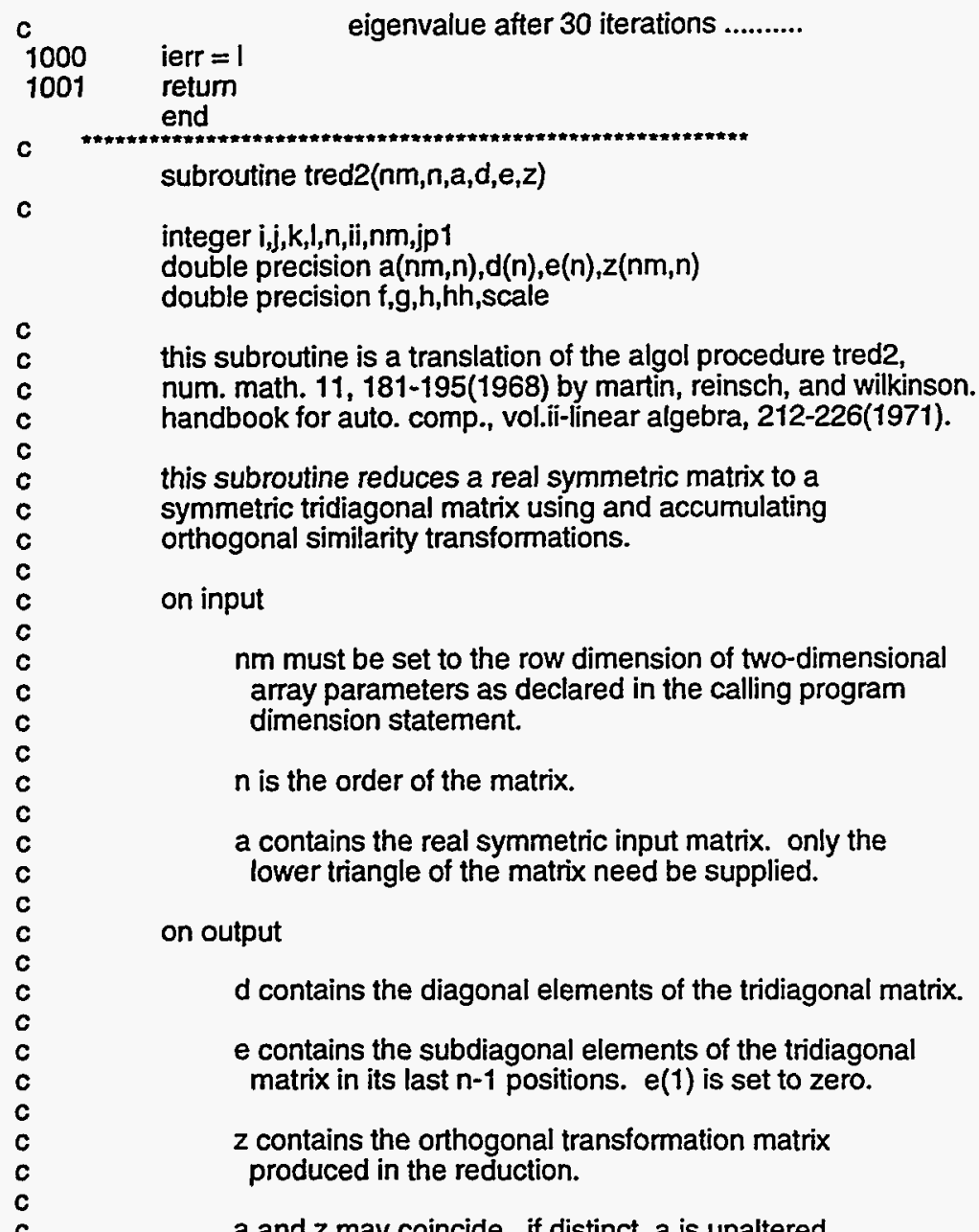

$a$ and $z$ may coincide. if distinct, $a$ is unaltered.

questions and comments should be directed to burton s. garbow, mathematics and computer science div, argonne national laboratory

this version dated august 1983.

do $100 \mathrm{i}=1, \mathrm{n}$

c

c

80

do $80 j=i, n$

$d 00$ continue $d(i)=a(n, i)$

c

c

if ( $n$.eq. 1) go to 510

......... for $i=n$ step -1 until 2 do -

do $300 \mathrm{ii}=2, n$

$\mathrm{i}=\mathrm{n}+\mathbf{2}-\mathrm{ii}$

$l=i-1$

$h=0.0 \mathrm{do}$

scale $=0.0 \mathrm{do}$

if (l. It. 2) go to 130

c scale row (algol tol then not needed)

$120 \quad \begin{aligned} & \text { do } 120 k=1,1 \\ & \text { scale }=\text { scale }+ \text { dabs }(d(k))\end{aligned}$

c

if (scale ne. $0.0 \mathrm{d0}$ ) go to 140

c

$$
e(i)=d(l)
$$


c

C

.

135

140 do $150 k=1,1$

150

200

220

240

c

C

245

C

c

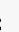

c

continue

$f=d(l)$

$e(i)=$ scale $* g$

$h=h-f^{*} g$

$d(l)=f-g$

.......... form $a^{*} u$..........

do $170 \mathrm{j}=1$,

$170 \quad e(j)=0.0 \mathrm{~d} 0$

do $240 \mathrm{j}=1, \mathrm{I}$

$f=d(j)$

$z(\mathrm{j}, \mathrm{i})=\mathrm{f}$

jp1 $=j+1$

continue

$h h=f /(h+h)$

do...... form $q$.........

$250 \quad e(j)=e(j)-h h^{*} d(j)$

........... form reduced a

do $280 \mathrm{j}=1$,

$f=d(j)$

c$$
\mathrm{g}=\mathrm{e}(\mathrm{j})
$$

$\mathrm{g}=-\mathrm{dsign}(\operatorname{dsqrt}(\mathrm{h}), \mathrm{f})$

$g=e(j)+z(j, j) * f$

if (I.It. jp1) go to 220

do $200 \mathrm{k}=\mathrm{jp} 1$,

$g=g+z(k, j) \cdot d(k)$

$e(k)=e(k)+z(k, j) * f$

$$
\begin{aligned}
& \text { do } 260 k=j, 1 \\
& z(k, j)=z(k, j)-f^{*} e(k)-g * d(k)
\end{aligned}
$$

$d(j)=z(1, j)$

$c$

$290 \quad d(i)=h$

$c$

(a)

$$
\text { do } 500 i=2, n
$$$$
I=i-1
$$$$
z(n, l)=z(1, l)
$$$$
z(l, I)=1.0 \mathrm{do}
$$$$
h=d(i)
$$

c

$$
\begin{aligned}
& \text { if (h eq. } 0.0 d 0) \text { go to } 380 \\
& \text { do } 330 k=1,1
\end{aligned}
$$


c

C 340

c

360

c

380

400

500 continue

520

510 do $520 i=1, n$

$$
d(i)=z(n, i)
$$$$
z(n, i)=0.0 \mathrm{do}
$$

$$
z(n, n)=1.00
$$

$\mathrm{e}(1)=0.0 \mathrm{do}$

return

end

c

c

$$
\text { subroutine tql1(n,d,e,ierr) }
$$

integer $\mathrm{i}, \mathrm{j}, l, \mathrm{~m}, \mathrm{n}, \mathrm{ii}, 11,12, \mathrm{mml}$, ,ierr

double precision $d(n), e(n)$

double precision $\mathrm{c}, \mathrm{c2}, \mathrm{c3}, \mathrm{dl} 1, \mathrm{el1}, \mathrm{f}, \mathrm{g}, \mathrm{h}, \mathrm{p}, \mathrm{r}, \mathrm{s}, \mathrm{s} 2, \mathrm{tst1}$,tst2,pythag $\mathrm{c}$

this subroutine is a translation of the algol procedure tql1,

num. math. 11, 293-306(1968) by bowdler, martin, reinsch, and wilkinson.

handbook for auto. comp., vol.ii-linear algebra, 227-240(1971).

this subroutine finds the eigenvalues of a symmetric

tridiagonal matrix by the ql method.

on input

$\mathrm{n}$ is the order of the matrix.

$d$ contains the diagonal elements of the input matrix.

e contains the subdiagonal elements of the input matrix in its last $n-1$ positions. $e(1)$ is arbitrary.

on output

$d$ contains the eigenvalues in ascending order. if an error exit is made, the eigenvalues are correct and ordered for indices $1,2, \ldots$ ierr-1, but may not be the smallest eigenvalues.

e has been destroyed.

ierr is set to zero for normal retum,

$j$ if the j-th eigenvalue has not been determined after 30 iterations.

calls pythag for $\operatorname{dsqrt}\left(a^{*} a+b^{*} b\right)$.

questions and comments should be directed to burton s. garbow, mathematics and computer science div, argonne national laboratory

this version dated august 1983 . 
c ierr $=0$

if ( $n$.eq. 1) go to 1001

c

$100 \quad(i-1)=2, n$

c $e(i-1)=e(i)$

$f=0.0 \mathrm{do}$

tst1 $=0.0 \mathrm{~d} 0$

C

$e(n)=0.0 \mathrm{~d} 0$

do $290 I=1, n$

$\mathrm{j}=0$

$h=\operatorname{dabs}(d(I))+\operatorname{dabs}(e(l))$

if (tst1. It. $h$ ) tst $1=h$

c

do $110 \mathrm{~m}=1, \mathrm{n}$

tst2 $=$ tst $1+\operatorname{dabs}(e(m))$

if (tst2 .eq. tst1) go to 120

$c$

$e(n)$ is always zero, so there is no exit

c

110 continue

c

120 if (m.eq. I) go to 210

130 if $(j . e q .30)$ go to 1000

$j=j+1$

c

form shift .

$11=1+1$

$12=11+1$

$\mathrm{g}=\mathrm{d}(\mathrm{I})$

$\mathrm{p}=(\mathrm{d}(11)-\mathrm{g}) /\left(2.0 \mathrm{~d} 0^{\circ} \mathrm{e}(\mathrm{l})\right)$

$r=$ pythag $(\mathrm{p}, 1.0 \mathrm{~d} 0)$

$\mathrm{d}(l)=\mathrm{e}(\mathrm{l}) /(\mathrm{p}+\mathrm{dsign}(\mathrm{r}, \mathrm{p}))$

$\mathrm{d}(11)=\mathrm{e}(\mathrm{l}) *(\mathrm{p}+\mathrm{dsign}(\mathrm{r}, \mathrm{p}))$

$\mathrm{dl} 1=\mathrm{d}(11)$

$h=g-d(i)$

c

if (12.gt. $n$ ) go to 145

do $140 i=12, n$

$140 \quad d(i)=d(i)-h$

$145 \quad f=f+h$

c

ql transformation ...........

$$
\begin{aligned}
& p=d(m) \\
& c=1.0 \mathrm{~d} 0 \\
& c 2=c \\
& \text { el } 1=e(11) \\
& s=0.0 \mathrm{do} \\
& \mathrm{mml}=\mathrm{m}-1
\end{aligned}
$$

c

for $i=m-1$ step -1 until I do do $200 \mathrm{ii}=1, \mathrm{mml}$

$$
\begin{aligned}
& c 3=c 2 \\
& c 2=c \\
& s 2=s \\
& i=m-i \\
& g=c^{*} e(i) \\
& h=c^{*} p \\
& r=p y t h a g(p, e(i)) \\
& e(i+1)=s^{*} r \\
& s=e(i) / r \\
& c=p / r \\
& p=c * d(i)-s^{*} g \\
& d(i+1)=h+s^{*}\left(c^{*} g+s^{*} d(i)\right)
\end{aligned}
$$
continue

c

$p=-s^{*} s 2^{*} c 3^{*}$ el1 * $e(l) /$ dl1 e $(l)=s^{*} p$

$d(I)=c^{*} p$

tst2 $=$ tst $1+\operatorname{dabs}(e(I))$

if (tst2 .gt. tst1) go to 130

$210 \quad p=d(l)+f$

c order eigenvalues

if (1 .eq. 1) go to 250 
c

230

$250 \quad i=1$

$270 \quad d(i)=p$

290 continue

C

go to 1001

c

1000 ierr $=1$

1001 retum

end

c

double precision function pythag $(a, b)$

double precision $a, b$

c

c

c$$
10 \text { continue }
$$

finds dsqrt $\left(a^{* *} 2+b^{* *} 2\right)$ without overflow or destructive underllow

double precision $p, r, s, t, u$

$\mathrm{p}=\operatorname{dmax} 1(\operatorname{dabs}(\mathrm{a}), \operatorname{dabs}(\mathrm{b}))$

if (p .eq. 0.0do) go to 20

$r=(\operatorname{dmin} 1(\operatorname{dabs}(a), \operatorname{dabs}(b)) / p) * * 2$

$t=4.0 d 0+r$

if (t .eq. 4.0d0) go to 20

$S=r / t$

$u=1.0 \mathrm{~d} 0+2.0 \mathrm{~d}^{*} \mathrm{~s}$

$\mathrm{p}=\mathrm{u}^{*} \mathrm{p}$

go to 10

$$
r=(s / u) * * 2 * r
$$

20 pythag $=p$

retum

end

c

subroutine tred1 (nm,n,a,d,e,e2)

c

integer $i, j, k, l, n, i, n m, j p 1$

double precision $a(n m, n), d(n), e(n), e 2(n)$

double precision $\mathrm{f}, \mathrm{g}, \mathrm{h}, \mathrm{scale}$

this subroutine is a translation of the algol procedure tred1,

num. math. 11, 181-195(1968) by martin, reinsch, and wilkinson.

handbook for auto. comp., vol.ii-linear algebra, 212-226(1971).

this subroutine reduces a real symmetric matrix

to a symmetric tridiagonal matrix using

orthogonal similarity transformations.

on input

$\mathrm{nm}$ must be set to the row dimension of two-dimensional array parameters as declared in the calling program dimension statement.

$\mathrm{n}$ is the order of the matrix.

a contains the real symmetric input matrix. only the lower triangle of the matrix need be supplied.

on output

a contains information about the orthogonal transformations used in the reduction in its strict lower triangle. the full upper triangle of $a$ is unaltered. 
$d$ contains the diagonal elements of the tridiagonal matrix.

e contains the subdiagonal elements of the tridiagonal matrix in its last $n-1$ positions. $e(1)$ is set to zero.

$e 2$ contains the squares of the corresponding elements of $e$. $\mathrm{e} 2$ may coincide with $\mathrm{e}$ if the squares are not needed.

questions and comments should be directed to burton s. garbow, mathematics and computer science div, argonne national laboratory

this version dated august 1983.

100 continue

do $100 i=1, n$

$d(i)=a(n, i)$

$a(n, i)=a(i, i)$

c

......... for $i=n$ step -1 until 1 do --

do 300 ii $=1, n$

$i=n+1-i i$

$\mathrm{i}=\mathrm{i}-1$

$\mathrm{h}=0.0 \mathrm{do}$

scale $=0.0 \mathrm{do}$

if (I .It. 1) go to 130

c scale row (algol tol then not needed) ........... do $120 \mathrm{k}=1$, !

c scale $=$ scale $+\operatorname{dabs}(d(k))$

c

if (scale .ne. 0.0d0) go to 140

130

$$
\text { do } \begin{aligned}
125 j & =1,1 \\
d(j) & =a(l, j)
\end{aligned}
$$$$
a(l, j)=a(i, j)
$$

continue

$$
a(i, j)=0.0 d 0
$$

$e(i)=0.0 \mathrm{do}$

$\mathrm{e} 2(i)=0.0 \mathrm{d0}$

go to 300

140

do $150 k=1,1$

$d(k)=d(k) /$ scale

continue

$h=h+d(k)$ * d(k)

c

$e 2(i)=$ scale * scale ${ }^{*} h$

$f=d(I)$

$g=-d \operatorname{sign}(d s q r t(h), f)$

$\mathrm{e}(i)=$ scale * $\mathrm{g}$

$h=h-f * g$

$d(l)=f-g$

if (1) eq. 1) go to 285

c

form $a^{*}$

170

do $170 j=1, j$

c $e(j)=0.0 \mathrm{~d} 0$

$$
\begin{gathered}
\text { do } 240 j=1, l \\
f=d(j) \\
g=e(j)+a(j, j) * f \\
\text { jp } 1=j+1 \\
\text { if }(1 . \text { It. jp } 1) \text { go to } 220 \\
\text { do } 200 k=j p 1,1 \\
g=g+a(k, j) * d(k) \\
e(k)=e(k)+a(k, j) * f \\
\text { continue }
\end{gathered}
$$

c

$$
e(j)=g
$$

continue 


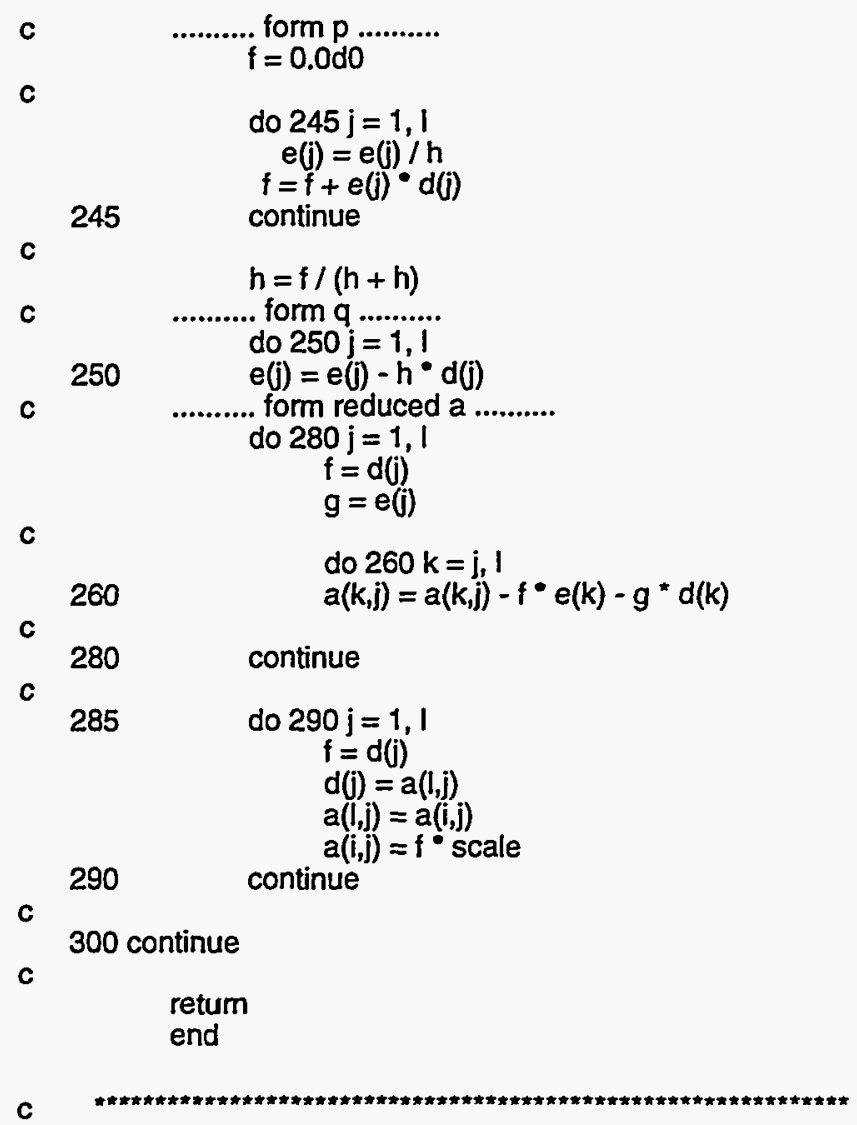

\section{RSB.f}

C

c

subroutine rsb(nm,n,mb,a,w, matz,z,fv1,fv2,ierr)

integer $n, m b, n m$,ierr,matz double precision $a(n m, m b), w(n), z(n m, n), f v 1(n), f v 2(n)$ logical tf

this subroutine calls the recommended sequence of subroutines from the eigensystem subroutine package (eispack) to find the eigenvalues and eigenvectors (if desired) of a real symmetric band matrix.

on input

$\mathrm{nm}$ must be set to the row dimension of the two-dimensional array parameters as declared in the calling program dimension statement.

$\mathrm{n}$ is the order of the matrix $\mathrm{a}$.

$\mathrm{mb}$ is the half band width of the matrix, defined as the number of adjacent diagonals, including the principal diagonal, required to specify the non-zero portion of the lower triangle of the matrix.

a contains the lower triangle of the real symmetric band matrix. its lowest subdiagonal is stored in the last $n+1-m b$ positions of the first column, its next subdiagonal in the last $n+2-m b$ positions of the second column, further subdiagonals similarly, and finally its principal diagonal in the $n$ positions of the last column. contents of storages not part 
of the matrix are arbitrary.

matz is an integer variable set equal to zero if

only eigenvalues are desired. otherwise it is set to

any non-zero integer for both eigenvalues and eigenvectors.

on output

$w$ contains the eigenvalues in ascending order.

$z$ contains the eigenvectors if matz is not zero.

ierr is an integer output variable set equal to an error completion code described in the documentation for tqliat and tql2. the normal completion code is zero.

fv1 and fv2 are temporary storage arrays.

questions and comments should be directed to burton s. garbow, mathematics and computer science div, argonne national laboratory

this version dated august 1983.

if ( $\mathrm{n}$.le. $\mathrm{nm}$ ) go to 5

ierr $=10^{*} n$

go to 50

5 if (mb .gt. 0) go to 10

ierr $=12^{\circ} \mathrm{n}$

go to 50

10 if (mb .le. n) go to 15

ierr $=12 * n$

go to 50

15 if (matz .ne. 0) go to 20

$\mathrm{t} f=$. false.

call bandr(nm,n,mb,a,w,fv1,fv2,tf,z)

call tqlrat( $n, w, f v 2$,ierr)

go to 50

$20 \mathrm{tf}=$.true

call bandr(nm,n,mb,a,w,fv1,fv1,tf,z)

50 return call tql2(nm,n,w,fv1,z,ierr)

C

end

SUBROUTINE TQLRAT(N,D,E2,IERR)

subroutine tqlrat(n,d,e2,ierr)

C

INTEGER I,J,L,M,N,II,L1,MML,IERR

DOUBLE PRECISION D(N), E2(N)

DOUBLE PRECISION B,C,F,G,H,P,R,S,T,EPSLON,PYTHAG

$C$
$C$
$C$
$C$
$C$
$C$
$C$
$C$
$C$
$C$
$C$
$C$
$C$
$C$
$C$
$C$
$C$

This subroutine is a translation of the Algol procedure tqlrat,

Algorithm 464, Comm. ACM 16, 689(1973) by Reinsch.

This subroutine finds the eigenvalues of a symmetric tridiagonal matrix by the rational QL method.

On input

$N$ is the order of the matrix.

D contains the diagonal elements of the input matrix.

E2 contains the squares of the subdiagonal elements of the input matrix in its last $\mathrm{N}-1$ positions. E2(1) is arbitrary. 


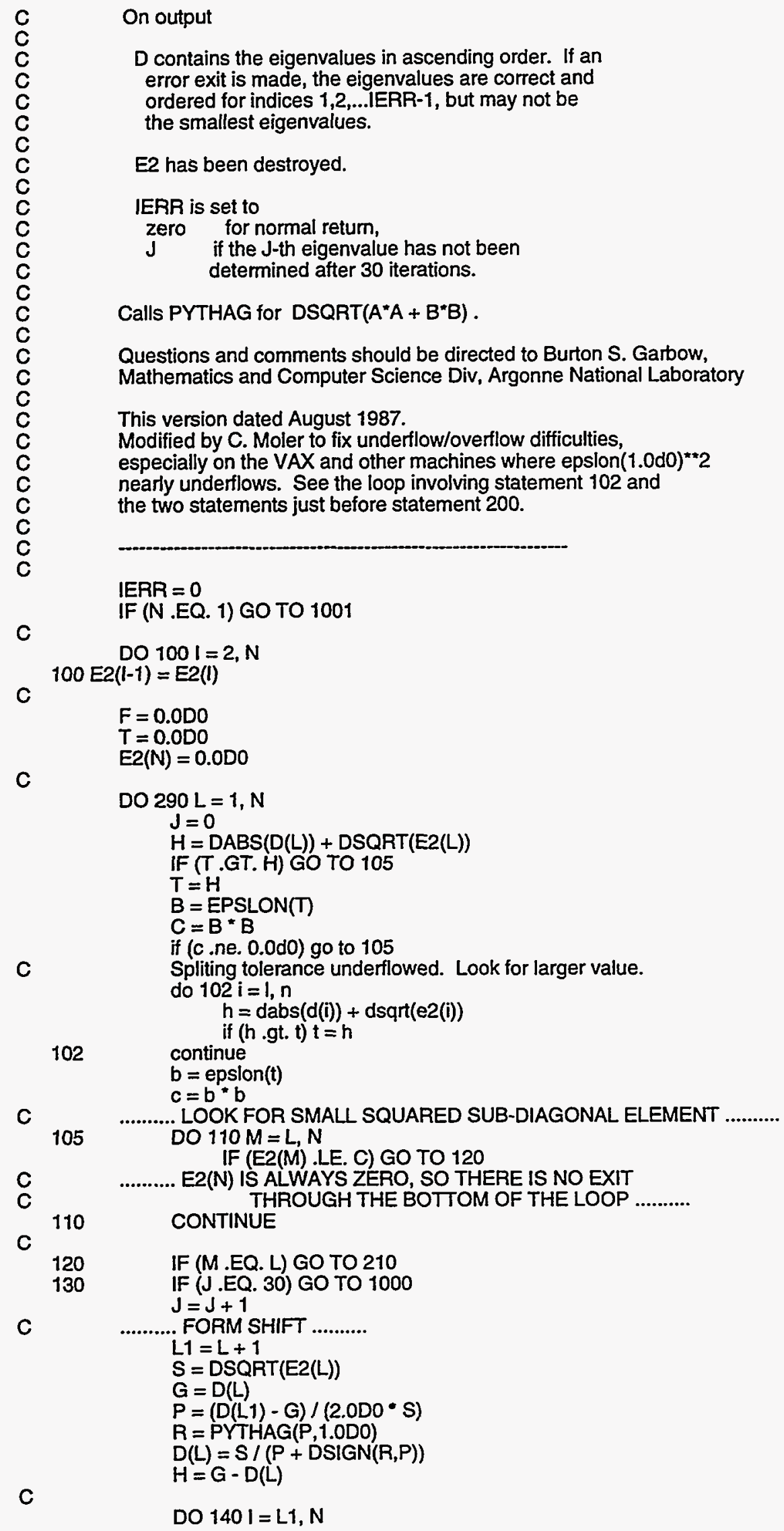


C

140

$D(I)=D(l)-H$

c

$F=F+H$

$G=D(M)$

IF (G.EQ. 0.0D0) $G=B$

$H=G$

$S=0.0 D 0$

$M M L=M-L$

C

FOR $I=M-1$ STEP -1 UNTIL L DO --

DO $200 \|=1, M M L$

$I=M-\|$

$P=G * H$

$R=P+E 2(l)$

$E 2(1+1)=S * R$

$S=E 2(I) / R$

$D(I+1)=H+S^{*}(H+D(I))$

$G=D(I)-E 2(l) / G$

C Avoid division by zero on next pass

if $(g$.eq. $0.0 d 0) \mathrm{g}=$ epsion(d(i)) $\mathrm{h}=\mathrm{g}$

200 CONTINUE

C

$E 2(L)=S^{*} G$

$D(L)=H$

C

GUARD AGAINST UNDERFLOW IN CONVERGENCE TEST

IF (H.EQ. 0.0DO) GO TO 210

IF (DABS(E2(L)).LE. DABS(C/H)) GO TO 210

$E 2(L)=H^{\star} E 2(L)$

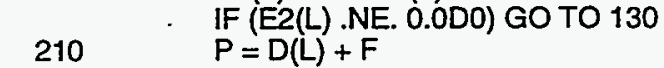

C

IF (L.EQ. 1) GO TO 250

C FOR I=L STEP -1 UNTIL 2 DO -DO $230 \|=2, L$ $I=L+2-I I$

IF (P.GE. D(I-1)) GO

TO $270 \mathrm{D}(\mathrm{I})=\mathrm{D}(\mathrm{l}-1)$

C

230 CONTINUE

$250 \quad l=1$

$270 \quad \mathrm{D}(\mathrm{l})=\mathrm{P}$

C

290 CONTINUE

C $\quad$.......... SET ERROR - NO CONVERGENCE TO AN

1000 IERR $=L$

1001 RETURN

C

END

c double precision function pythag $(a, b)$

c double precision $a, b$

CC

CC

C

C

C

10 continue

finds dsqrt(a**2+b**2) without overflow or destructive underflow

double precision $p, r, s, t, u$

$\mathrm{p}=\operatorname{dmax1}(\operatorname{dabs}(\mathrm{a}), \operatorname{dabs}(\mathrm{b}))$

if (p .eq. 0.0d0) go to 20

$r=(\operatorname{dmin} 1(\operatorname{dabs}(a), \operatorname{dabs}(b)) / p)^{* * 2}$

c $t=4.0 \mathrm{~d} 0+r$

c if (t .eq. 4.0d0) go to 20

c $s=r / t$

$u=1.0 \mathrm{~d} 0+2.0 \mathrm{~d} 0 * \mathrm{~s}$

$p=u^{*} p$

c go to 10

$\mathrm{p}=\mathrm{u}^{*} \mathrm{p}$
$\mathrm{r}=(\mathrm{s} / \mathrm{u})^{* *} \mathrm{2}^{*} \mathrm{r}$

20 pythag $=p$

retum 
c

c

$a=$

$=a-1.0 d 0$

$c=b+b+b$

eps $=\operatorname{dabs}(c-1.0 \mathrm{~d} 0)$

if (eps .eq. 0.0d0) go to 10

epslon $=$ eps*dabs $(x)$

retum

end

ssumption 2.

assumptions, it should be true that,

$a$ is not exactly equal to four-thirds,

b has a zero for its last bit or digit,

$c$ is not exactly equal to one,

eps measures the separation of 1.0 from

the next larger floating point number.

the developers of eispack would appreciate being informed

about any systems where these assumptions do not hold.

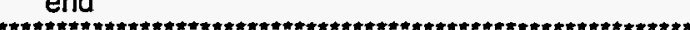

subroutine bandr(nm,n,mb,a,d,e,e2,matz,z)

C

c

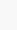

REFORMULATED S2 IN LOOP 500 TO AVOID OVERFLOW. (9/29/89 BSG)

integer $\mathrm{j}, \mathrm{k}, \mathrm{l}, \mathrm{n}, \mathrm{r}, \mathrm{i} 1, \mathrm{i} 2, \mathrm{j} 1, \mathrm{j} 2, \mathrm{kr}, \mathrm{mb}, \mathrm{mr}, \mathrm{m1}, \mathrm{nm}, \mathrm{n2}, \mathrm{r1}$,ugl,maxl,maxr double precision $a(n m, m b), d(n), e(n), e 2(n), z(n m, n)$ double precision $\mathrm{g}, \mathrm{u}, \mathrm{b} 1, \mathrm{~b} 2, \mathrm{c} 2, \mathrm{f1}, \mathrm{f2}, \mathrm{s} 2, \mathrm{dmin}, \mathrm{dmin} \mathrm{t}$ logical matz

this subroutine is a translation of the algol procedure bandrd, num. math. 12, 231-241(1968) by schwarz.

handbook for auto. comp., vol.ii-linear algebra, 273-283(1971).

this subroutine reduces a real symmetric band matrix to a symmetric tridiagonal matrix using and optionally accumulating orthogonal similarity transformations.

on input

$\mathrm{nm}$ must be set to the row dimension of two-dimensional array parameters as declared in the calling program dimension statement.

$\mathrm{n}$ is the order of the matrix.

$\mathrm{mb}$ is the (half) band width of the matrix, defined as the number of adjacent diagonals, including the principal diagonal, required to specify the non-zero portion of the lower triangle of the matrix. 
a contains the lower triangle of the symmetric band input matrix stored as an $n$ by $m b$ array. its lowest subdiagonal is stored in the last $n+1-m b$ positions of the first column, its next subdiagonal in the last $n+2-m b$ positions of the second column, further subdiagonals similarly, and finally its principal diagonal in the n positions of the last column. contents of storages not part of the matrix are arbitrary.

matz should be set to .true. if the transformation matrix is to be accumulated, and to false. otherwise.

on output

a has been destroyed, except for its last two columns which contain a copy of the tridiagonal matrix.

d contains the diagonal elements of the tridiagonal matrix.

e contains the subdiagonal elements of the tridiagonal matrix in its last $n-1$ positions. $e(1)$ is set to zero.

e2 contains the squares of the corresponding elements of e. e2 may coincide with e if the squares are not needed.

$z$ contains the orthogonal transformation matrix produced in the reduction if matz has been set to .true. otherwise, $z$ is not referenced.

questions and comments should be directed to burton s. garbow, mathematics and computer science div, argonne national laboratory

this version dated september 1989.

$\mathrm{dmin}=2.0 \mathrm{~d} 0 * *(-64)$

dmint $=2.0 \mathrm{dO}^{* *}(-32)$

c

do $30 \mathrm{j}=1, \mathrm{n}$

$30 \mathrm{~d}(\mathrm{j})=1.0 \mathrm{~d} 0$

if (.not. matz) go to 60

40

do $50 \mathrm{j}=1, n$

do $40 \mathrm{k}=1, \mathrm{n}$

50 continue $z(j, j)=1.0 \mathrm{~d} 0$

$$
z(j, j)=1.0 \mathrm{do}
$$

$60 m 1=m b-1$

if $(m 1-1) 900,800,70$

$70 n 2=n-2$

do $700 k=1, n 2$

$\max r=\min 0(m 1, n-k)$

c

for $r=\operatorname{maxr}$ step -1 until 2 do --

do $600 r 1=2$, $\max r$

$r=\max r+2-r 1$

$k r=k+r$

$m r=m b-r$

$\mathrm{g}=\mathrm{a}(\mathrm{kr}, \mathrm{mr})$

$a(k r-1,1)=a(k r-1, m r+1)$

$\mathrm{ugl}=\mathrm{k}$

$$
\begin{gathered}
\text { do } 500 \mathrm{j}=k r, n, m 1 \\
\mathrm{j} 1=\mathrm{j}-1 \\
\mathrm{j} 2=\mathrm{j} 1-1 \\
\text { if }(\mathrm{g} . \mathrm{eq} \cdot 0.0 \mathrm{~d} 0) \text { go to } 600 \\
\mathrm{~b} 1=\mathrm{a}(\mathrm{j} 1,1) / g \\
\mathrm{~b} 2=\mathrm{b} 1{ }^{*} \mathrm{~d}(\mathrm{j} 1) / \mathrm{d}(\mathrm{j})
\end{gathered}
$$


c

200

c

300

c

350

c

400

c

c

450

c

c

j1)
IF (ABS(B1) .GT. 1.0DO) THEN

$$
U=1.0 \mathrm{DO} / \mathrm{B1}
$$

ELSE

$$
S 2=U /(U+B 2)
$$

B2) ENDIF

$$
\mathrm{S} 2=1.0 \mathrm{DO} /(1.0 \mathrm{DO}+\mathrm{B1} \text {. }
$$

if (s2 .ge. 0.5do) go to

$450 \mathrm{~b} 1=\mathrm{g} / \mathrm{a}(\mathrm{j} 1,1)$

$b 2=b 1 \cdot d(j) / d(j 1)$

$\mathrm{c} 2=1.0 \mathrm{~d} 0-\mathrm{s} 2$

$d(i 1)=c 2^{\circ} d(j 1)$

$d(j)=c 2^{*} d(j)$

$\mathrm{f} 1=2.0 \mathrm{~d} 0$ * $\mathrm{a}(\mathrm{j}, \mathrm{m} 1)$

$\mathrm{t} 2=\mathrm{b} 1 * a(j 1, \mathrm{mb})$

$a(j, m 1)=-b 2 *(b 1 * a(j, m 1)-a(j, m b))-f 2+a(j, m 1) a(j 1, m b)=b 2 *(b 2 *$

$a(j, m b)+f 1)+a(j 1, m b) a(j, m b)=b 1 *(f 2-f 1)+a(j, m b)$

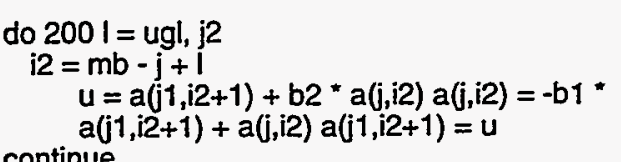

$$
\begin{aligned}
u g l= & j \\
& a(j 1,1)=a(i 1,1)+b 2 \\
& * g \text { if }(j . e q . n) \text { go to } \\
& 350 \operatorname{maxl}= \\
& \min 0(m 1, n-j 1)
\end{aligned}
$$

do $300 \mathrm{l}=2$, $\max l$

$i 1=j 1+1$

$\mathrm{i} 2=\mathrm{mb}-1$

$u=a(i 1, i 2)+b 2 * a(i 1, i 2+1) a(i 1, i 2+1)=-b 1 *$

continue $a(i 1, i 2)+a(i 1, i 2+1) a(i 1, i 2)=u$

$$
i 1=j+m 1
$$

if (i1 .gt. $n)$ go to 350

$g=b 2 * a(i 1,1)$

if (.not. matz) go to 500

$$
\begin{aligned}
\text { do } 400 \mathrm{I} & =1, n \\
u & =z(l, j 1)+b 2 * z(1, j) z(1, j)=- \\
b 1 \cdot z(1, j 1)+z(1, j) z(l, j 1)=u &
\end{aligned}
$$

continue

go to 500

$u=d(j 1)$

$d(j 1)=s 2 * d(i)$

$\mathrm{d}(\mathrm{i})=\mathrm{s} 2 \mathrm{x}^{*} \mathrm{u}$

$\mathrm{f} 1=2.0 \mathrm{do} * \mathrm{a}(\mathrm{j}, \mathrm{m} 1)$

$\mathrm{t} 2=\mathrm{b} 1 * \mathrm{a}(\mathrm{j}, \mathrm{mb})$

$u=b 1 *(f 2-f 1)+a(11, m b)$

$a(j, m 1)=b 2 *(b 1 * a(j, m 1)-a(j 1, m b))+f 2-a(j, m 1) a(j 1, m b)=b 2 *(b 2 *$

$a(j 1, m b)+f 1)+a(j, m b) a(j, m b)=u$

do $460 \mathrm{l}=\mathrm{ugl}$, j2

$\mathrm{i} 2=\mathrm{mb}-\mathrm{j}+\mathrm{i}$

$\mathrm{u}=\mathrm{b} 2 * a(\mathrm{j} 1, \mathrm{i} 2+1)+\mathrm{a}(\mathrm{j}, \mathrm{i} 2) \mathrm{a}(\mathrm{j}, \mathrm{i} 2)=-$

continue $a(j 1, i 2+1)+b 1-a(j, i 2) a(j 1, i 2+1)=u$

$u g l=j$

$a(j 1,1)=b 2 * a(j 1,1)$

$+g$ if $(j$.eq. $n)$ go to

480

$\max 1=\min 0(m 1, n-$

do $470 \mathrm{I}=2$, $\max I$ 
c

470 continue

$i 1=j 1+1$

$\mathrm{i} 2=\mathrm{mb}-1$

$u=b 2 * a(i 1, i 2)+a(i 1, i 2+1) a(i 1, i 2+1)=-a(i 1, i 2)$

$+b 1^{*} a(i 1, i 2+1) a(i 1, i 2)=u$

$$
i 1=j+m 1 \text { if (i1 .gt. n) go to }
$$

$480 \mathrm{~g}=\mathrm{a}(\mathrm{i1}, 1)$

$a(i 1,1) 480$

$a(i 1,1)=b 1$ *

if (.not. matz)

go to 500

do $490 \mathrm{I}=1, \mathrm{n}$

$u=b 2 \cdot z(l, j 1)+z(1, j) z(1, j)=-$

490

continue $z(l, j 1)+b 1^{*} z(l, j) z(l, j 1)=u$

500

continue

c

600

continue

c

c

if $(\bmod (k, 64)$.ne. 0$)$ go to 700 do $650 \mathrm{j}=\mathrm{k}$, n

if (d(j).ge. dmin) go to 650

C

610

$\operatorname{maxl}=\max O(1, m b+1-j)$

c

do $610 \mathrm{I}=\operatorname{maxl}, \mathrm{m} 1$

$a(j, l)=$ dmint ${ }^{*} a(j, l)$

c

if $(j$ eq. $n)$ go to 630

$\max 1=\operatorname{mino}(m 1, n-j)$

do $6201=1, \operatorname{maxl}$

$i 1=\mathbf{j}+1$

$\mathrm{i} 2=\mathrm{mb}-1$

620 continue

$a(i 1, i 2)=d \min r t * a(i 1, i 2)$

c

630

if (.not. matz) go to 645

c

do $6401=1, n$

645

$z(l, j)=d$ minit $* z(l, j)$

650

$a(j, m b)=d m i n * a(j, m b)$

continue $d(j)=d(j) / d m i n$

c

700 continue

C

800 do $810 j=2, n$

$810 e(j)=\operatorname{dsqrt}(d(j))$

c

if (.not. matz) go to 840

do $830 \mathrm{j}=1, \mathrm{n}$

do $820 \mathrm{k}=2, \mathrm{n}$

$820 \quad z(j, k)=e(k) \cdot z(j, k)$

830 continue

c

$840 \mathrm{u}=1.0 \mathrm{~d} 0$

do $850 j=2, n$

$a(j, m 1)=u^{*} e(j) * a(j, m 1) u=$

e(j)

$e 2(j)=a(j, m 1) * 2$

$a(j, m b)=d(j) * a(j, m b)$

$\mathrm{d}(\mathrm{j})=\mathrm{a}(\mathrm{j}, \mathrm{mb})$

$e(j)=a(j, m 1)$ 
850 continue

c

$$
\begin{aligned}
& d(1)=a(1, \mathrm{mb}) \\
& e(1)=0.0 \mathrm{dD} \\
& e 2(1)=0.0 \mathrm{do} \\
& \text { go to } 1001
\end{aligned}
$$

c

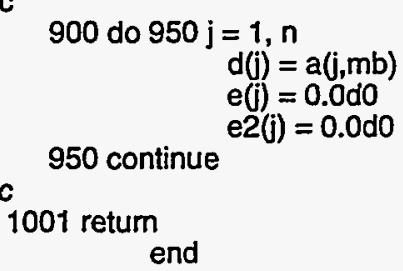

c

\section{SIMS4.f}

Subroutine CALCSIM(ISTATES,CMACH,EVSTRT1,NMSTRT, \&TW,EW,EMAX,SIM)

c
c
C
c
C

This subroutine reads the positions and FCFs from the fort.7

file and convolutes with the resolution function specified

in the input.

implicit double precision (a-h,o-z)

include "params.inc"

common/gauss1/A(NPTSFIT) common/write/PARNAMES(NPRSMX),QPARAMS(NPRSMX)

common/opt1/PARAMS(NPRSMX,ITRSMX),DELTA(NPRSMX,ITRSMX)

common/params3/EVSTRT(NSTSMX),SCALE(NSTSMX),NMODES(NSTSMX)

common/opt2/IT

dimension SIM(NPTSFIT,2)

character $\mathrm{CMACH}$

real*8 NMSTRT,EVSTRT1

if (CMACH .eq. 'S') then

$E W 2=E W / 1000$

else

$E W 2=E W$

endif

NPTS $=2000$

PSLOPE $=2840000.0$

write $(", ")^{\prime}+$ CALCSIM"

do $11 i=1$,NPTS

$11 \quad \operatorname{SIM}(1,2)=0$.

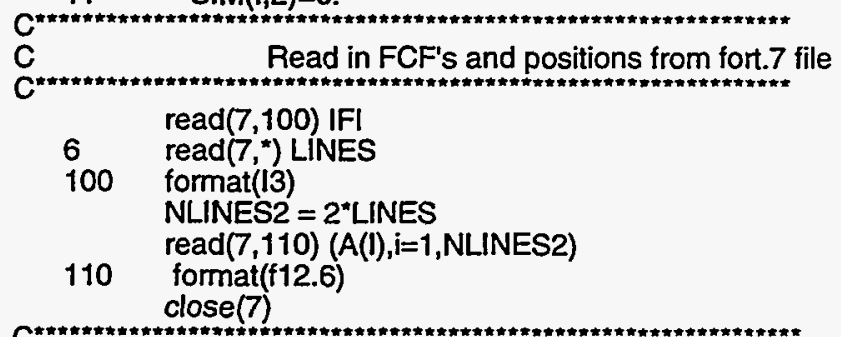

C Determine Range of Energy over which to Simulate

C data based upon fort.7 file since it is ordered

C by energy of peak position.-6/1/92-DWA

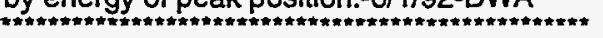

$E M I N=A(2)$

$E M A X=A(N L I N E S 2)$

$E M I N=E M I N-E M I N{ }^{*} 0.20$ 
$E M A X=E M A X+E M A X * 0.20$

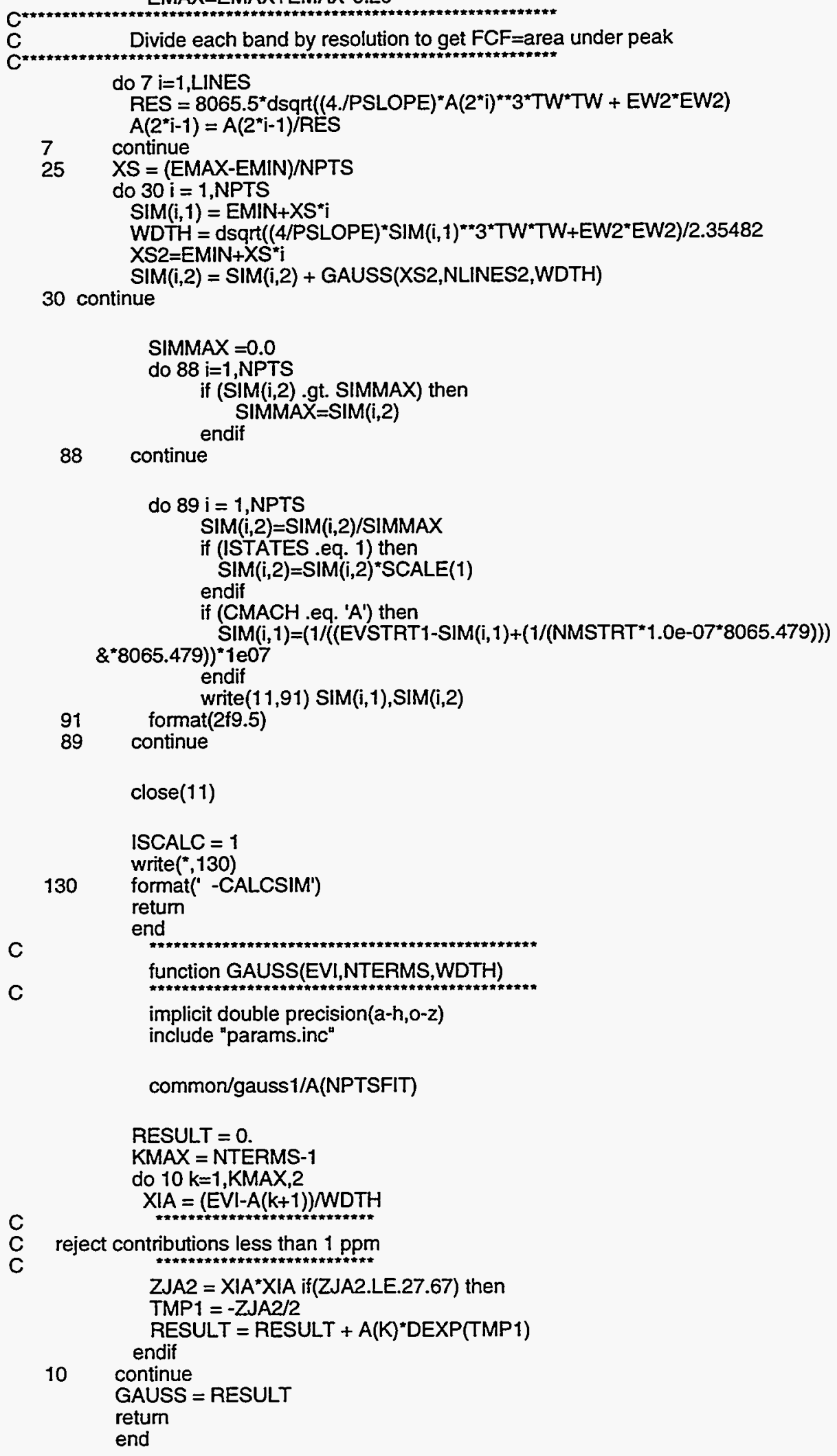

88 continue

SIMMAX $=0.0$

do $88 \mathrm{i}=1$,NPTS

if (SIM(i,2) .gt. SIMMAX) then

$\operatorname{SIMMAX}=\operatorname{SIM}(i, 2)$ 
TWYUP3.f

Subroutine TIDYUP(CMACH)

implicit double precision (a-h,o-z)

C This subroutine is called if the CHIOPT flag is set to ' $Y$ '. From

C this point the code is run in the chi-squares optimization mode using

C the same set of subroutines which the PES code calls during a single simulation.

include "params.inc"

$C^{* * * * * * * * * * * * * * * *}$ From pescont3.4******************

character QFREQA(NSTSMX,NMDSMX),QFREQN(NSTSMX,NMDSMX)

character QANHARMA(NSTSMX,NMDSMX), QANHARMN(NSTSMX,NMDSMX)

character QBONDA(NSTSMX,NMDSMX), QBONDN(NSTSMX,NMDSMX)

character QEVSTRT(NSTSMX)

character LDEGMODE(NSTSMX,NMDSMX), QTMP(NSTSMX,NMDSMX)

character" 1 VERBOSE, TERMINAL

character*1 CMACH,CHIOPT

character"20 OPTFILE,EXPFILE

real"8 MTMP(NSTSMX,NMDSMX)

real"8 NMSTRT(NSTSMX)

common/comb1/FCF(NMDSMX,NVMAXN,NVMAXA),POS(NMDSMX,

\& NVMAXN,NVMAXA),DEGFLAG

common/comb2/ANHARMN(NSTSMX,NMDSMX),ANHARMA(NSTSMX,NMDSMX)

common/comb3/EVQQ(NVMAXN,NVMAXA),QQ(NVMAXN,NVMAXA),

\& QQB(NVMAXN,NVMAXA),XM1,XM2

common/comb4/HFREQN(NSTSMX,NMDSMX),HFREQA(NSTSMX,NMDSMX)

common/comb5/IVMAXA(NSTSMX,NMDSMX), IVMAXN(NSTSMX,NMDSMX)

C Keep sim

common/sim/TW,EW,ORIGIN,A(NPTSFIT), SIM(NPTSFIT,2), EMAX,

\&EXPCNTS

common/hunt1 $1 X X$ (NPTSFIT)

C Keep (chi,chi2)? common/chi/EXPCHI(NPTSEXP,2),SIGEXP(NPTSEXP)

common/chi2/IPTSEXP,NVAR

common/chi3/FCHISQ,SIMCHI(NPTSEXP,2)

common/chi4/OPTFILE

common/verbNERBOSE

common/deg/DEGMODE(NSTSMX,NMDSMX),MTMP(NSTSMX,NMDSMX)

common/params/CHIOPT, TERMINAL,EXPFILE

common/params2/RESOLUTION,ITERATION,ISTATES

common/params3/EVSTRT(NSTSMX),SCALE(NSTSMX),NMODES(NSTSMX)

common/params4/BONDN(NSTSMX,NMDSMX),DELQ1(NSTSMX,NMDSMX),

\&LDEGMODE(NSTSMX,NMDSMX), QTMP(NSTSMX,NMDSMX)

common/params5/NMSTRT(NSTSMX)

common/plot/EXPT(NPTSEXP,2)

common/out/RMU2,QFXN(NSTSMX,NMDSMX)

common/qfct/BONDA(NSTSMX,NMDSMX), QFCTN

common/fit1/QFREQA(NSTSMX,NMDSMX),QFREQN(NSTSMX,NMDSMX)

common/fit2/QANHARMA(NSTSMX,NMDSMX), QANHARMN(NSTSMX,NMDSMX) common/fit3/QBONDA(NSTSMX,NMDSMX), QBONDN(NSTSMX,NMDSMX) common/fit4/QEVSTRT(NSTSMX)

common/write/PARNAMES(NPRSMX),QPARAMS(NPRSMX)

common/flag/ITFLAG

character"20 PARNAMES(NPRSMX)

character"1 QPARAMS(NPRSMX)

dimension PARAMS(NPRSMX,ITRSMX)

dimension DERIV(NPRSMX,NPTSEXP)

dimension CHI(ITRSMX)

dimension BETA(NPRSMX), SIGMAA(NPRSMX)

dimension ALPHA(NPRSMX,NPRSMX),ARRAY(NPRSMX,NPRSMX)

dimension PARNEW(NPRSMX), PARLAST(NPRSMX), GRAD(NPRSMX) 
common/opt1/PARAMS(NPRSMX,ITRSMX),DELTA(NPRSMX,ITRSMX)

common/opt2/IT

common/wgt/WEIGHT(NPTSEXP)

write $(", "))^{\prime T I D Y U P '}$

IT $=1$

RMU2 $=\left(X M 11^{*} X M 2\right) /(X M 1+X M 2)$

Set up array of parameter names

do $10 i=1$,ISTATES

do $20 \mathrm{j}=1$,NMODES(i)

PARNAMES(NPAR+1)='Anion Vib. Frequency'

PARNAMES(NPAR+2)='Neut. Vib. Frequency'

PARNAMES(NPAR+3)='Anion Anharmonicity

PARNAMES(NPAR+4)='Neut. Anharmonicity '

PARNAMES(NPAR+5)='Anion Bondlength

PARNAMES(NPAR+6)='Neutral Bondlength '

PARNAMES $(N P A R+7)=$ Temperature

20 $\mathrm{NPAR}=\mathrm{NPAR}+7$

continue

PARNAMES(NPAR+1)='Origin

10

$N P A R=N P A R+1$

$\mathrm{C}$
$\mathrm{C}$
$\mathrm{C}$
$\mathrm{C}$
$\mathrm{C}$

continue

Set up an array of variable parameters

NPAR $=0$

do $40 \mathrm{i}=1$, ISTATES

do $50 j=1$,NMODES(i)

PARAMS(NPAR $+1, I T)=H F R E Q A(i, j)$

PARAMS(NPAR $+2, I T)=$ HFREQN $(i, j)$

PARAMS(NPAR+3,IT)=ANHARMA $(i, j)$

PARAMS $(N P A R+4, I T)=$ ANHARMN $(i, j)$

PARAMS $(N P A R+5, I T)=B O N D A(i, j)$

PARAMS(NPAR+6,IT)=BONDN $(i, j)$

PARAMS(NPAR $+7, I T)=M T M P(i, j)$

NPAR $=N P A R+7$

50

continue

PARAMS(NPAR $+1, \mathrm{IT}$ ) $=$ EVSTRT $(\mathrm{i})$

$N P A R=N P A R+1$

40

continue

$\mathrm{C}$
$\mathrm{C}$
$\mathrm{C}$
$\mathrm{C}$

Set up an array of variable flags

NPAR $=0$

do $70 i=1$,ISTATES

do $80 j=1$,NMODES(i)

QPARAMS(NPAR+1)=QFREQA $(i, j)$

QPARAMS(NPAR+2)=QFREQN(i,j)

QPARAMS $(N P A R+3)=$ QANHARMA $(i, j)$

QPARAMS(NPAR+4)=QANHARMN $(i, j)$

QPARAMS $(N P A R+5)=$ QBONDA $(i, j)$

QPARAMS (NPAR+6) $=$ QBONDN $(i, j)$

QPARAMS(NPAR +7$)=$ QTMP $(i, j)$

NPAR $=N P A R+7$

80

continue

QPARAMS(NPAR +1 ) $=$ QEVSTRT(i)

$N P A R=N P A R+1$

70 continue

$\mathrm{C}$
$\mathrm{C}$
$\mathrm{C}$
$\mathrm{C}$

Set up array of delta values

NPAR $=0$

do $83 \mathrm{i}=1$, ISTATES 


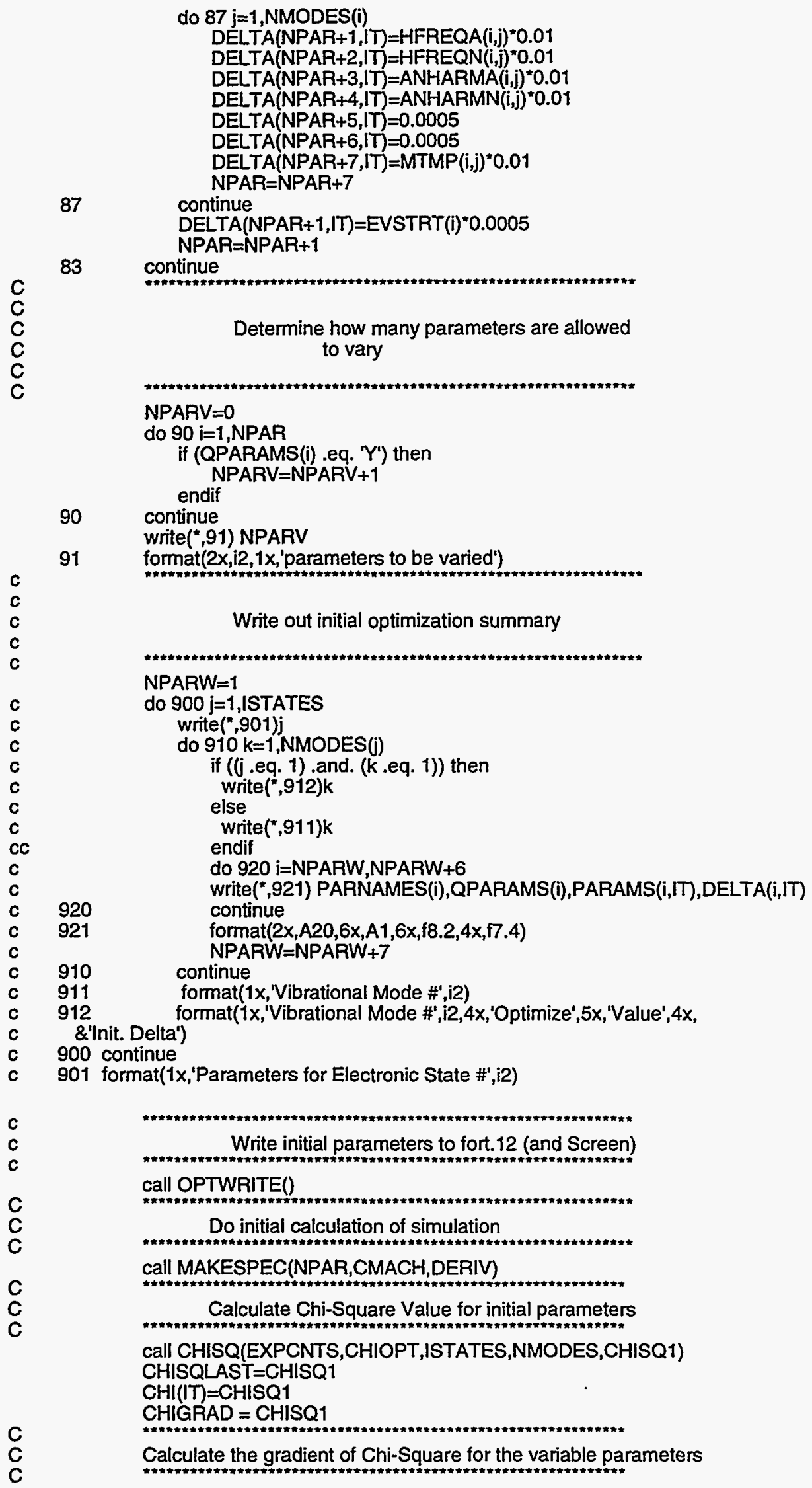

Determine how many parameters are allowed to vary

Write out initial optimization summary

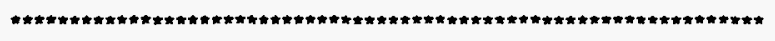

NPARW $=1$

do $900 \mathrm{j}=1$, ISTATES

write (",901)j

do $910 k=1$,NMODES(j)

if $((j$. eq. 1$)$. and. $(k$. eq. 1$))$ then

write $(", 912) \mathrm{k}$

else

write $(", 911) \mathrm{k}$

endif

do $920 \mathrm{i}=N$ PARW,NPARW +6

Write initial parameters to fort.12 (and Screen)

call OPTWRITE()

Do initial calculation of Simulation call MAKESPEC(NPAR,CMACH,DERIV)

$\mathrm{C}$
$\mathrm{C}$

Calculate Chi-Square Value for initial parameters call CHISQ(EXPCNTS,CHIOPT,ISTATES,NMODES,CHISQ1) CHISQLAST $=$ CHISQ1

$\mathrm{CHI}(I T)=\mathrm{CHISQ} 1$

CHIGRAD $=$ CHISQ1 
401

402

C
C
C

340

C

C

C

call GRADIENT(QPARAMS,NPAR,NPARV,CMACH,GRAD,CHIGRAD,EXPCNTS,

\&ISTATES,NMODES)

do $401 \mathrm{i}=1$,NPAR

if (QPARAMS(i) .eq. 'Y') then

write $(*, 402) i, G R A D(i)$

endif

continue

format('Gradient for parameter \#',i2,': ',f7.4)

IGRAD $=0$

ISLOPECHK $=1$

ICHKFLAG $=0$

ICNT $1=0$

ICNT2 $=0$

Use gradient to calculate new parameters

write $\left({ }^{*},\right)^{\prime \prime}$ 'new PARAMETERS'

do $300 i=1$,NPAR

PARLAST $(i)=$ PARAMS $(i, I T)$

PARAMS $(i, I T+1)=$ PARAMS $(i, I T)$

DELTA $(i, I T+1)=D E L T A(i, I T)$

if (QPARAMS(i) .eq. ' $Y$ ') then

PARAMS $(i, I T+1)=$ PARAMS $(i, I T)+$ GRAD $(i)$

write $(*, 302)$ PARAMS $(i, I T+1)$

endif

format $(2 x, f 11.6)$

continue

Simulate w/new parameters; check for reduction of Chi_Square

$I T=I T+1$

call MAKESPEC(NPAR,CMACH,DERIV)

IT $=$ IT -1

call CHISQ(EXPCNTS,CHIOPT,ISTATES,NMODES,CHISQ1)

c

if past parabola step, check for convergence

if(ICHKFLAG eq. 1) then

ITMAX $=25$

CONV $=0.0001$

write( $\left.{ }^{*}, 322\right)$ CHISQ1,CHISQLAST

call CONCHECK(CHISQ2,CHISQ1,CONV,IT,ISTAT,ITMAX)

if (ISTAT .eq. 0) goto 1000 !Too many iterations, kick out

c

if (ISTAT .eq. 1) goto 399 !Recalculate gradient ...

and start again from here

if (ISTAT .eq. 2) goto 1002 !Converged

if (ISTAT eq. 3 ) goto 9999 iff $=0$, problem, kick out endif

c

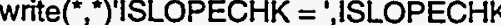

if (ISLOPECHK .eq. 3) then

write $(", *)^{\prime}$ ISLOPECHK $=3$

if ((CHISQ1-CHISQLAST).It.0) then

goto 310

else

if ((CHISQ1-CHISQLAST).eq.0) then

goto 320

else

if ((CHISQ1-CHISQLAST).gt.0) then goto 360

$$
\text { endif }
$$

endif

endif

else

if (ISLOPECHK .eq. 2) then

write(", ")'ISLOPECHK=2'

if ((CHISQ1-CHISQLAST).it.0) then

ISLOPECHK = 3 goto 310

else

if ((CHISQ1-CHISQLAST).eq.0) then goto 320 
358

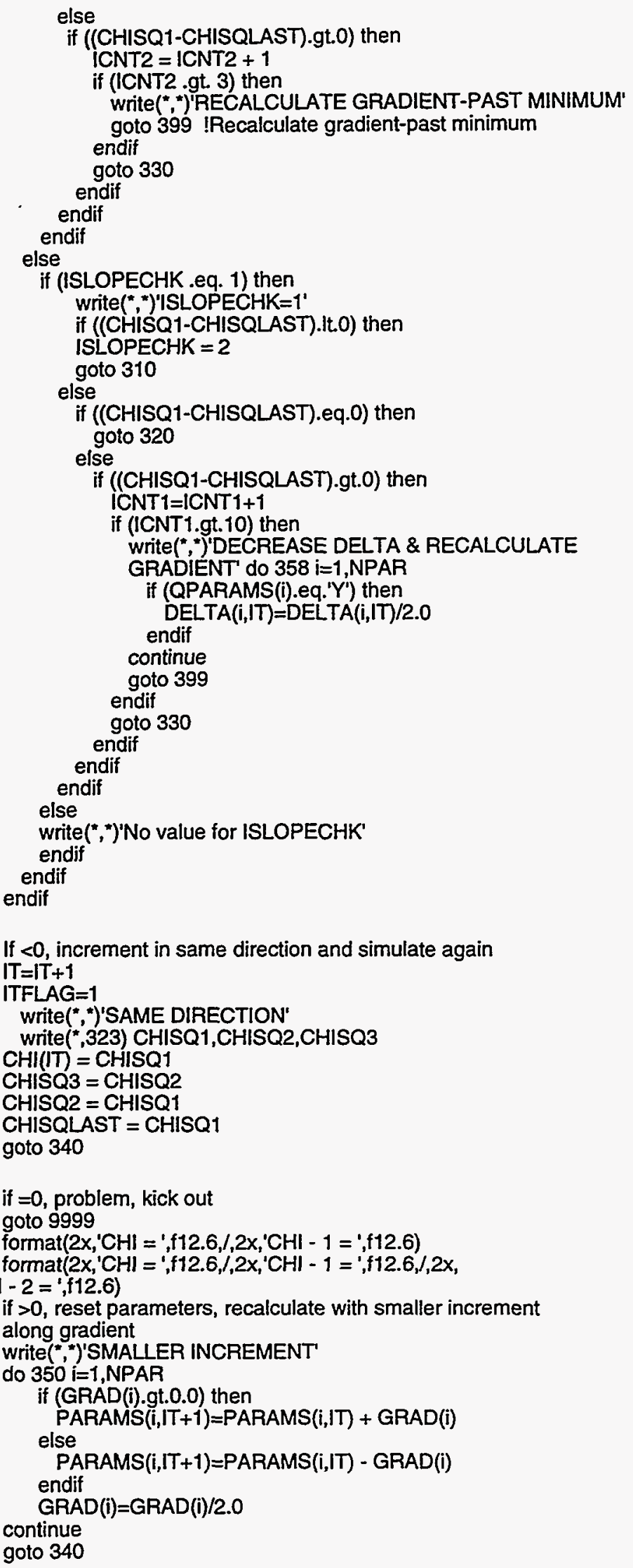


c

370

c

99

97

98

96

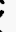

c

c.

c 190

c 180

C

C

C

c

C

c if $>0$, find minimum of parabola formed by last three points

$\mathrm{IT}=\mathrm{IT}+1$

write $\left.{ }^{*},{ }^{*}\right) '$ ' PARABOLA'

DPARAB $=1 . /(1 .+($ CHISQ1-CHISQ2 $) /($ CHISQ3-CHISQ2 $))+0.5$

write $\left({ }^{*}, 323\right) \mathrm{CHISQ} 1, \mathrm{CHISQ} 2, \mathrm{CHISQ3}$

write $(*, ")$ 'dparab=',DPARAB

do $370 i=1$,NPAR

PARAMS $(i, I T+1)=$ PARAMS $(i, I T+1)$-DPARAB*GRAD(i)

\section{continue}

ICHKFLAG $=1$

goto 343

Comment out matrix method temporarily

Set initial value of FLAMBDA

FLAMBDA $=0.001$

Calculate 1st derivative for each parameter at each simulated point

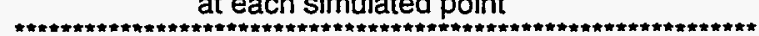

call DERIVATIVE(QPARAMS,NPAR,NPARV,CMACH,DERIV)

do $98 \mathrm{i}=1$, NPARV

do $97 j=1$, NPARV

write $(*, 96) i, j$, DERIV(i,j)

continue

continue

format(2x,'deriv(',i1, ',',i1,') = ',,f12.6)

Fill the alpha and beta matrices

do $150 \mathrm{i}=1$,IPTSEXP

do $160 j=1$,NPARV

BETA $(j)=B E T A(i)+(E X P C H I(i, 2)-S I M C H I(i, 2))^{*} W E I G H T(i){ }^{*}$ DERIV(i,j) do $170 \mathrm{k}=1$,j

ALPHA $(j, k)=A L P H A(i, k)+W E I G H T(i){ }^{*}$ DERIV $(i, j){ }^{*}$ DERIV $(i, k)$

continue

continue

continue

do $180 \mathrm{i}=\mathrm{i}$,NPARV

do $190 j=1, i$

$\operatorname{ALPHA}(k, j)=A L P H A(j, k)$

continue

continue

Invert modified curvature matrix to find new parameters

do $210 j=1$,NPARV

do $220 \mathrm{k}=1$, NPARV

ARRAY $(j, k)=A L P H A(j, k) / s q r\left(A L P H A(j, j)^{*} A L P H A(k, k)\right)$

ARRAY $(j, j)=1.0+F L A M B D A$

write( $\left({ }^{*}, 201\right)$ ARRAY $(j, j)$, ARRAY $(j, k)$

format(2x,'arraysetjj = ',f12.6,' arraysetjk = ',f12.6) continue

continue

call MATINV(ARRAY,NPARV,DET)

do $240 i=1$,NPAR

PARNEW(i)=PARAMS $(\mathrm{i}, \mathrm{IT})$

write $(*, 248)$ PARNEW(i)

format $(2 x$, 'parnew1 $=$ ',f12.6)

if (QPARAMS(i) .eq. ' $Y$ ') then do $250 \mathrm{j}=1, \mathrm{NPARV}$

\&*ALPHA(j,j))

PARNEW(i)=PARNEW(i)+BETA(i)*ARRAY $(i, j) /$ sqrt(ALPHA(i,i)

write $(*, 249)$ PARNEW(i),BETA(i),ARRAY(i,j),ALPHA(i,i),ALPHA(j,j)

format(2x,'parnew = ',f12.6,' beta = ',f12.6,' array = ',f12.6,

$2 x_{1}^{\prime}$ alpha1 $=$ ',f12.6,' alpha2 $=$ ',f12.6)

continue

endif

continue

Do simulation with new parameters

call MAKESPEC(NPAR,CMACH,DERIV) 


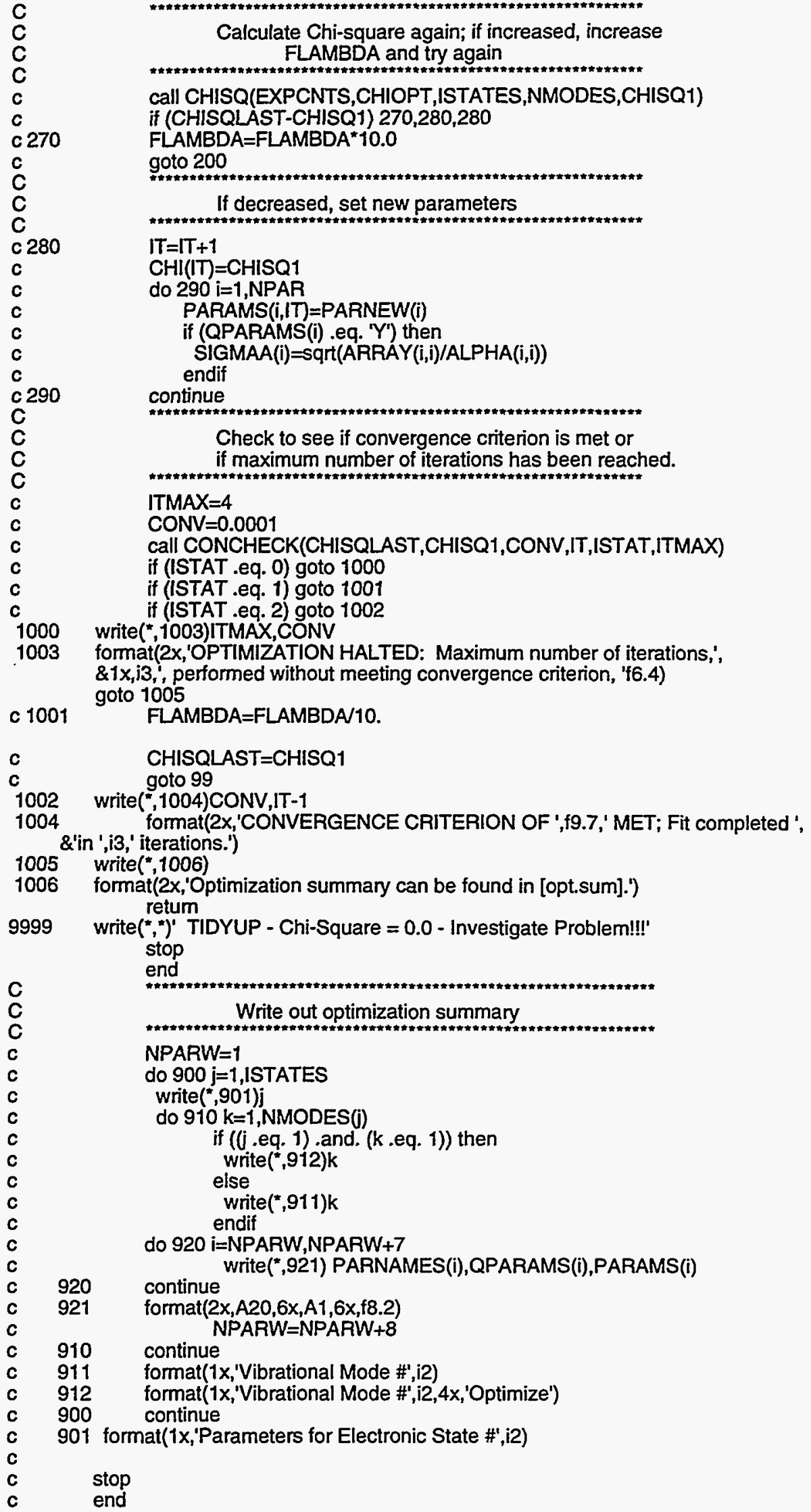




\section{WRITOUT.f}

Subroutine WRITEOUT(ISTATE,QPLOT)

Implicit Double Precision(a-h,o-z)

include "params.inc"

character* 1 CHIOPT,QPLOT,TERMINAL

character"20 EXPFILE

real*8 MTMP(NSTSMX,NMDSMX)

real ${ }^{*} 8$ NMSTRT (NSTSMX)

character LDEGMODE(NSTSMX,NMDSMX),QTMP(NSTSMX,NMDSMX)

common/params/CHIOPT,TERMINAL,EXPFILE

common/params2/RESOLUTION,ITERATION,ISTATES

common/params3/EVSTRT(NSTSMX),SCALE(NSTSMX),NMODES(NSTSMX)

common/params4/BONDN(NSTSMX,NMDSMX),DELQ1(NSTSMX,NMDSMX),

\&LDEGMODE(NSTSMX,NMDSMX),QTMP(NSTSMX,NMDSMX)

common/params5/NMSTRT(NSTSMX)

common/comb2/ANHARMN(NSTSMX,NMDSMX), ANHARMA(NSTSMX,NMDSMX)

common/comb4/HFREQN(NSTSMX,NMDSMX), HFREQA(NSTSMX,NMDSMX)

$\&$ common/comb3/EVQQ(NVMAXN,NVMAXA),QQ(NVMAXN,NVMAXA), QQB(NVMAXN,NVMAXA), XM1, XM2 common/comb5/VMAXA(NSTSMX,NMDSMX),IVMAXN(NSTSMX,NMDSMX) common/sim/TW, EW, ORIGIN,A(NPTSFIT),SIM(NPTSFIT,2), EMAX,

\section{\&EXPCNTS}

common/deg/DEGMODE(NSTSMX,NMDSMX),MTMP(NSTSMX,NMDSMX)

common/afct/BONDA(NSTSMX,NMDSMX), QFCTN

common/out/RMU2,QFXN(NSTSMX,NMDSMX)

if (ISTATE .eq. 1) then

write $(12,90)$

write $(12,91)$ ISTATES

write $(12,90)$

90

format $(12 x$,

format(12x,'Initial Parameters for',i2,1x,'Electronic States',

endif

write $(12,10)$ ISTATE

format(10x,l,15x,'Parameters for Electronic State \#',i2)

11

if (QPLOT .eq. ' $Y$ ') then write(12,11)ISTATE,SCALE(ISTATE)

11 form

format(20x,'State \#',i2,1x,'scaled by ', $15.3,1)$

write $\left(12,{ }^{*}\right) 35$

endif

write(12,92)XM1,XM2,RMU2

92 format (3x,'Mass atom \#1 =', $f 5.2, /, 3 x$,'Mass atom \#2

$\&=', f 5.2, /, 3 x,{ }^{\prime}$ Reduced mass $=', f 6.3$ )

write(12,93)EVSTRT(ISTATE)

93 format $\left(3 x,{ }^{\prime}\right.$ Origin at', $\left.f 8.4,{ }^{\prime} \mathrm{eV}^{\prime}\right)$

write $(12,95)$ TW, EW

95 format(3x,'Time width $=', f 6.3$, 'ns', $/, 3 x$, 'Energy width =',

\&f8.4,'eV')

write $(12,96)$ NMODES(ISTATE)

96 format(2x,i2,1x,'mode(s) active in spectrum', )

write $(12,97)$

97 format(19x,'Anion', $4 x$, 'Neutral')

do $1000 i=1$,NMODES(ISTATE) write $(12,99) \mathrm{i}$

write $(12,98)$ HFREQA(ISTATE,i),HFREQN(ISTATE,i)

write $(12,100)$ ANHARMA(ISTATE,i),ANHARMN(ISTATE,i)

write $(12,101)$ IVMAXA(ISTATE,i), IVMAXN(ISTATE,i) 


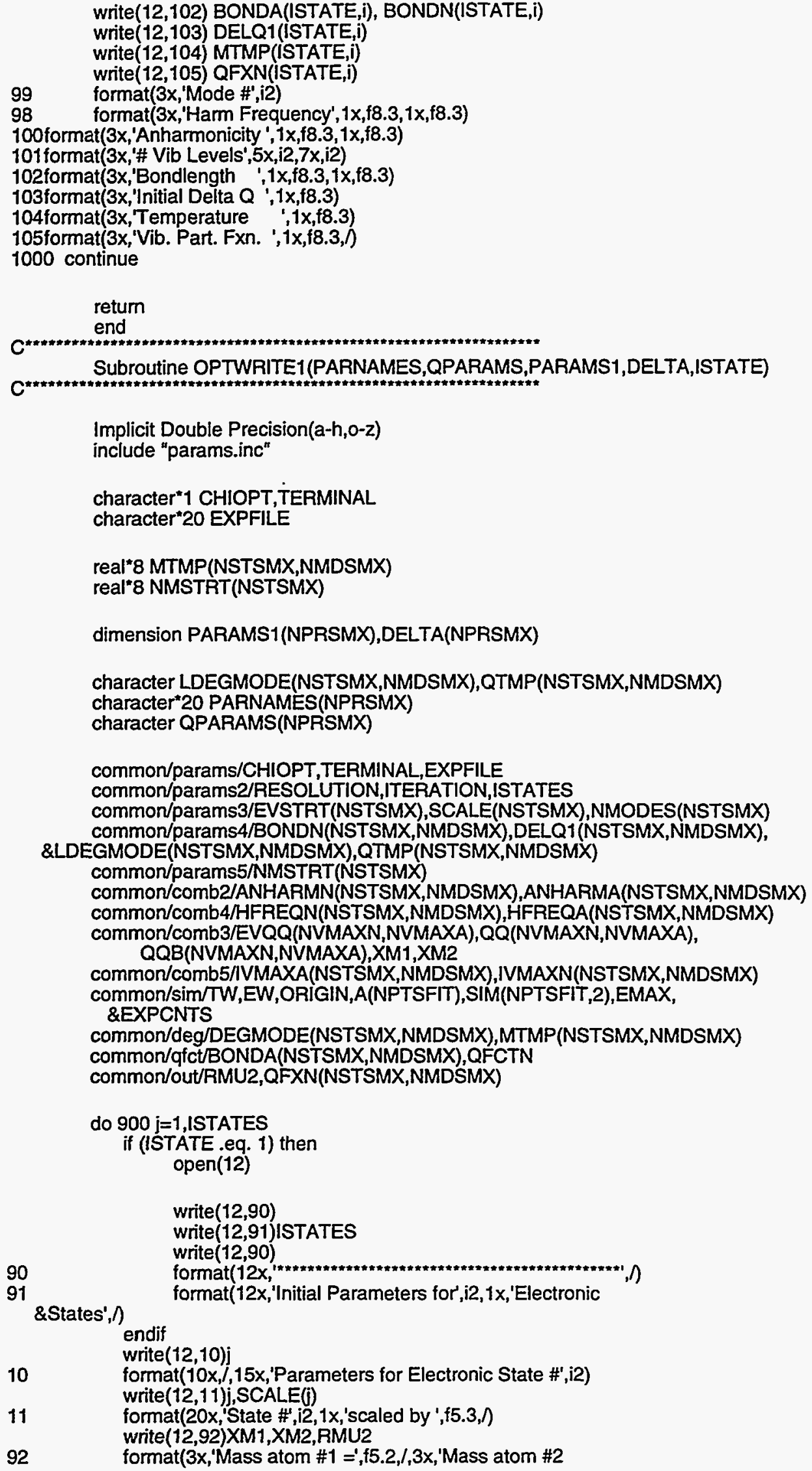


$\&=',\left\{5.2, /, 3 x,{ }^{\prime}\right.$ Reduced mass $='$, t6.3) write (12,93)EVSTRT(j)

format (3x,'Origin at', f8.4,'eV')

write $(12,95) T W, E W$

95 format(3x, 'Time width =',f6.3,'ns', /,3x, 'Energy width =',

$\&\left\{8.4,{ }^{\prime} e V '\right)$

write (12,96)NMODES(j)

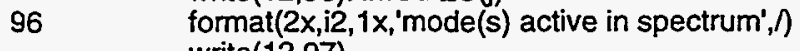

write $(12,97)$

97 format(19x,'Anion',4x,' 'Neutral')

NPARW $=1$

do $910 \mathrm{k}=1$,NMODES $(\mathrm{i})$

if $(j$.eq. 1$)$.and. $(k$.eq. 1$))$ then

else write $(*, 912) \mathrm{k}$

endif

write $\left({ }^{*}, 911\right) \mathrm{k}$

do $920 \mathrm{i}=N P A R W, N P A R W+7$

write(",921) PARNAMES(i),QPARAMS(i),PARAMS1(i),DELTA(i) 920

921 continue

format $(2 x, A 20,6 x, A 1,6 x, f 8.2,4 x, f 6.4)$

NPARW $=$ NPARW +8

910 continue

911 format( $1 x$, 'Vibrational Mode \#', i2)

912 format(1x,'Vibrational Mode \#',i2,4x,'Optimize',5x,'Value',4x, 900 continue \&'Init. Delta')

retum

end

Subroutine OPTWRITE()

Implicit Double Precision (a-h,o-z)

include "params.inc"

This subroutine is called if for writing out parameters

in optimization after the optimization process has begun.

Written initially as a debugging tool

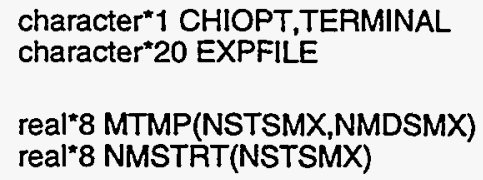

common/params/CHIOPT,TERMINAL,EXPFILE

common/params2/RESOLUTION,ITERATION,ISTATES

common/params3/EVSTRT(NSTSMX),SCALE(NSTSMX),NMODES(NSTSMX)

common/params4/BONDN(NSTSMX,NMDSMX),DELQ1(NSTSMX,NMDSMX),

\&LDEGMODE(NSTSMX,NMDSMX),QTMP(NSTSMX,NMDSMX)

common/params5/NMSTRT(NSTSMX)

common/comb2/ANHARMN(NSTSMX,NMDSMX),ANHARMA(NSTSMX,NMDSMX)

common/comb4/HFREQN(NSTSMX,NMDSMX), HFREQA(NSTSMX,NMDSMX)

\& common/comb3/EVQQ(NVMAXN,NVMAXA),QQ(NVMAXN,NVMAXA), QQB(NVMAXN,NVMAXA),XM1,XM2 common/comb5/VMAXA(NSTSMX,NMDSMX),IVMAXN(NSTSMX,NMDSMX)

\section{\&EXPCNTS} common/sim/TW, EW, ORIGIN,A(NPTSFIT), SIM(NPTSFIT,2), EMAX,

common/deg/DEGMODE(NSTSMX,NMDSMX),MTMP(NSTSMX,NMDSMX)

common/qfet/BONDA(NSTSMX,NMDSMX), QFCTN

common/out/RMU2,QFXN(NSTSMX,NMDSMX)

character*20 PARNAMES(NPRSMX)

character QPARAMS(NPRSMX) 
common/write/PARNAMES(NPRSMX),QPARAMS(NPRSMX)

common/opt1/PARAMS(NPRSMX,ITRSMX),DELTA(NPRSMX,ITRSMX)

common/opt2/TT

write $\left(*^{*},\right)^{\prime}$ OPTWRITE in'

write $(12,5)$ IT

write $(*, 5) I T$

5 format(4X,'PARAMETERS FOR ITERATION \# ',i2)

NPARW $=1$

do $900 \mathrm{j}=1$, ISTATES

write(12,10)j

10 format (10x,l,15x,'Parameters for Electronic State \#',i2)

write(12,11), SCALE(j)

write $(*, 11) j$, SCALE(j)

11 format(20x,'State \#', $12,1 \mathrm{x}$, 'scaled by ', $, 5.3,1)$

write(12,92)XM1,XM2,RMU2

92 format(3x,'Mass atom \#1 =',f5.2,/,3x,' Mass atom \#2

$\&=', f 5.2,(, 3 x, '$ 'Reduced mass $=', 66.3$ )

write $(12,93)$ EVSTRT $(j)$

write $(*, 93)$ EVSTRT(i)

93 format(3x,'Origin at', $\left.18.4,{ }^{\prime}, \mathrm{eV}\right)$

write $(12,95) \mathrm{TW}, \mathrm{EW}$

write $\left.{ }^{*}, 95\right) T W, E W$

95 format(3x,'Time width =',f6.3,'ns',/,3x,'Energy width =',

\&f8.4,'eV')

write $(12,96)$ NMODES $(j)$

96 format(2x,i2,1x,'mode(s) active in spectrum', ()

do $910 \mathrm{k}=1$,NMODES(i)

if ((i.gt. 1$)$.and.(k.eq.1)) then

INCREMENT $=7$

else

INCREMENT $=6$

enơlif

if (k .eq. 1) then

write $(12,912) \mathrm{k}$

write $(*, 912) \mathrm{k}$

else

write $(12,911) \mathrm{k}$

write $\left(^{*}, 911\right) \mathrm{k}$

endif

do 920 i=NPARW,NPARW+INCREMENT

write(12,921)PARNAMES(i),QPARAMS(i),PARAMS $(i, I T), D E L T A(i, I T)$

920 write(",921) PARNAMES(i),QPARAMS(i),PARAMS(i,IT),DELTA(i,IT)

921 continue

format $(2 x, A 20,6 x, A 1,6 x, 18.2,4 x, 77.4)$

910 continue

911 format(1x,'Vibrational Mode \#', i2)

\&'Init. Delta')

900 continue

$$
\begin{aligned}
& \text { write(",") 'OPTWRITE out' } \\
& \text { return } \\
& \text { end }
\end{aligned}
$$




\section{Appendix B. Publications from Graduate Work}

1. D. W. Arnold, C. Xu, E. H. Kim, and D. M. Neumark. "Spectroscopy of the Transition State: Elementary Reactions of the Hydroxyl Radical Studied by Photoelectron Spectroscopy of $\mathrm{O}^{-}\left(\mathrm{H}_{2} \mathrm{O}\right)$ and $\mathrm{H}_{3} \mathrm{O}_{2}^{-}$", Journal of Chemical Physics, (to be submitted).

2. D. W. Amold and D. M. Neumark. "Study of $\mathrm{N}_{2} \mathrm{O}_{2}$ by Anion Photoelectron Spectroscopy of $\mathrm{N}_{2} \mathrm{O}_{2}^{-}$", Journal of Chemical Physics, (to be submitted).

3. D. W. Amold, S. E. Bradforth, E. H. Kim, and D. M. Neumark. "Study of $\mathrm{FCO}_{2}{ }^{-}$and $\mathrm{X}^{-}$ $\left(\mathrm{CO}_{2}\right)[\mathrm{X}=\mathrm{Cl}, \mathrm{Br}$, and I] Using Negative Ion Photoelectron Spectroscopy", Journal of Chemical Physics (to be submitted).

4. D. W. Arnold, S. E. Bradforth, E. H. Kim, and D. M. Neumark. "Study of I" $\left(\mathrm{CO}_{2}\right)_{\mathrm{n}}$, $\mathrm{Br}^{-}\left(\mathrm{CO}_{2}\right)_{n}$, and $\mathrm{I}^{-}\left(\mathrm{N}_{2} \mathrm{O}\right)_{n}$ Clusters by Anion Photoelectron Spectroscopy", Journal of Chemical Physics (to be submitted).

5. E. H. Kim, S. E. Bradforth, D. W. Arnold, and D. M. Neumark. "Study of HCOO and DCOO by Negative Ion Photoelectron Spectroscopy", Journal of Chemical Physics (in preparation).

6. C. Xu, E. de Beer, D. W. Arnold, C. C. Amold, and D. M. Neumark, "Study of $\operatorname{In}_{x} P_{y}$ Clusters by Anion Photoelectron Spectroscopy", Journal of Chemical Physics (accepted for publication).

7. D. W. Arnold, C. Xu, E. H. Kim, and D. M. Neumark. "Study of Low-Lying Electronic States of Ozone by Photoelectron Spectroscopy of $\mathrm{O}_{3}{ }^{-"}$, Journal of Chemical Physics 100, July 15 issue, (1994). 
8. D. E. Manolopoulos, K. Stark, H. -J. Werner, D. W. Amold, S. E. Bradforth, and D. M. Neumark. "The Transition State of the $\mathrm{F}+\mathrm{H}_{2}$ Reaction", Science 262, 1852 (1993).

9. S. E. Bradforth, D. W. Arnold, D. M. Neumark, and D. E. Manolopoulos. "Experimental and Theoretical Studies of the $\mathrm{F}+\mathrm{H}_{2}$ Transition State via Photoelectron Spectroscopy of $\mathrm{FH}_{2}{ }^{-"}$, Journal of Chemical Physics 99, 6345 (1993).

10. S. E. Bradforth, E. H. Kim, D. W. Amold, and D. M. Neumark. "Photoelectron Spectroscopy of $\mathrm{CN}^{-}, \mathrm{NCO}^{-}$, and $\mathrm{NCS}^{-}$", Journal of Chemical Physics 98, 800 (1993).

11. D. W. Arnold, S. E. Bradforth, E. H. Kim, and D. M. Neumark. "Anion Photoelectron Spectroscopy of Iodine-Carbon Dioxide Clusters", Journal of Chemical Physics 97, 9468 (1992).

12. D. W. Amold, S. E. Bradforth, T. N. Kitsopoulos, and D. M. Neumark. "Vibrationally Resolved Spectra of $\mathrm{C}_{2}-\mathrm{C}_{11}$ by Anion Photoelectron Spectroscopy", Journal of Chemical Physics 95, 8753 (1991).

13. S. E. Bradforth, D. W. Arnold, R. B. Metz, A. Weaver, and D. M. Neumark. "Spectroscopy of the Transition State - Hydrogen Abstraction Reactions of Fluorine". Journal of Physical Chemistry 95, 8066 (1991).

14. A. Weaver, D. W. Arnold, S. E. Bradforth, and D. M. Neumark. "Examination of the ${ }^{2} \mathrm{~A}_{2}{ }^{\prime}$ and ${ }^{2} \mathrm{E}$ " States of $\mathrm{NO}_{3}$ by Ultraviolet Photoelectron Spectroscopy of $\mathrm{NO}_{3}{ }^{-"}$. Journal of Chemical Physics 94, 1740 (1991). 
15. S. E. Bradforth, A. Weaver, D. W. Arnold, R. B. Metz, and D. M. Neumark. "Examination of the $\mathrm{Br}+\mathrm{HI}, \mathrm{Cl}+\mathrm{HI}$, and $\mathrm{F}+\mathrm{HI}$ Hydrogen Abstraction Reactions by Photoelectron Spectroscopy of $\mathrm{BrHI}^{-}, \mathrm{ClHI}^{-}$, and $\mathrm{FHI}^{-2}$, Journal of Chemical Physics 92, 7205 (1990). 